\title{
Laboratory Directed Research and Development LDRD-FY-2011
}

March 2012

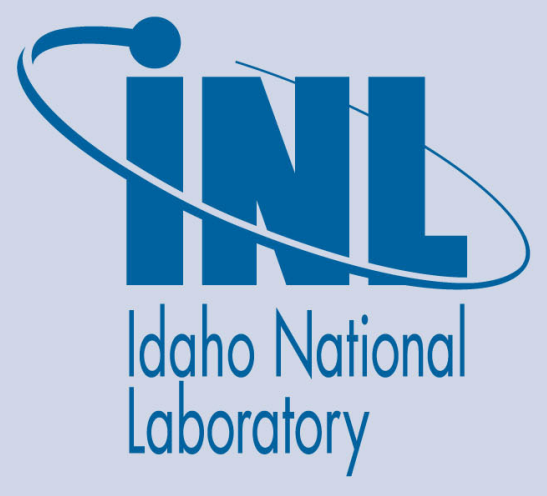

The INL is a U.S. Department of Energy National Laboratory operated by Battelle Energy Alliance 
INL/EXT-11-23890

\section{Laboratory Directed Research and Development LDRD-FY-2011}

March 2012

Idaho National Laboratory

Idaho Falls, Idaho 83415

http://www.inl.gov

Prepared for the

U.S. Department of Energy

Office of Nuclear Energy

Under DOE Idaho Operations Office

Contract DE-AC07-05ID14517 



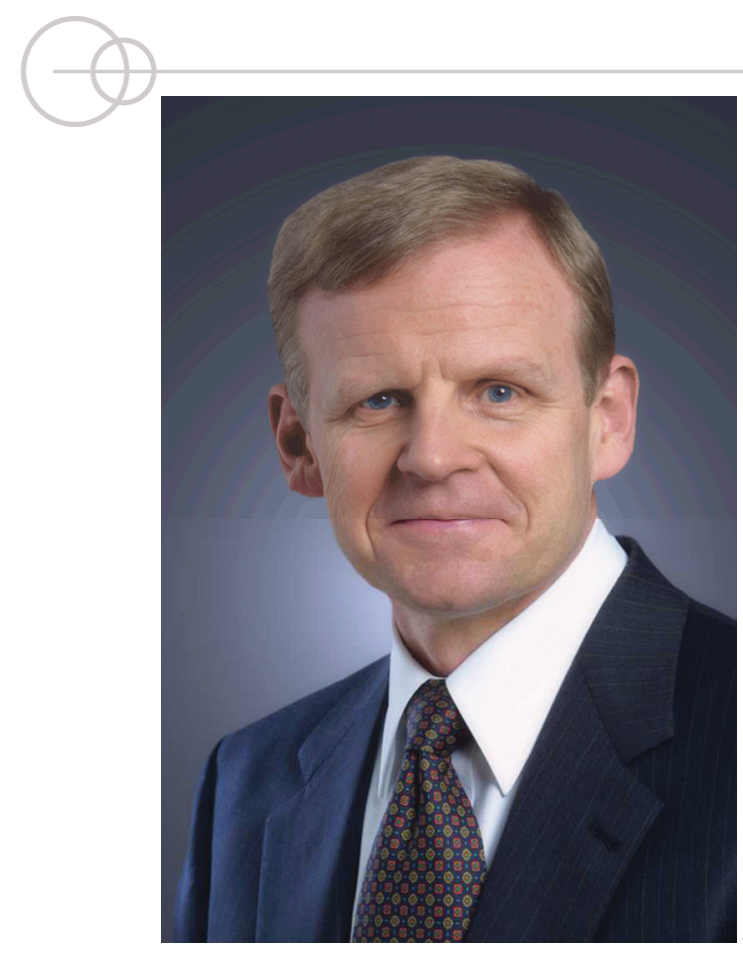

It is my pleasure to present the Idaho National Laboratory (INL) FY 2011 LaboratoryDirected Research and Development (LDRD) Annual Report. This report demonstrates the types of cutting-edge research INL is undertaking to mature our technical capabilities and help ensure the nation's energy security. INL's LDRD Program is operated in compliance with Department of Energy (DOE) Order 413.2B. This work is aligned with INL's strategic plan and benefits current and future missions of the DOE. This report summarizes how the INL LDRD Program is being used to maintain INL's technical vitality while advancing and maturing the DOE Office of Nuclear Energy (DOE-NE) mission.

This report provides a glimpse into our diverse research and development portfolio, which encompasses both advanced nuclear science and technology and underlying technologies. In keeping with the mission, INL's LDRD Program fosters technical capabilities necessary to support current and future DOE-Office of Nuclear Energy research and development needs. In 2011, LDRD projects have benefitted nuclear energy research in fuel cycle separations, characterization of sustainable materials, and improving reliability and validation of nuclear systems.
As a DOE-NE multi-program laboratory, INL research also serves the nation through research and development that helps support national homeland security and critical infrastructure protection. These research areas include nuclear non-proliferation, explosives detection, electric grid reliability, and wireless technologies. INL's isolated site and test-bed infrastructure are ideal for experimentation and demonstrations that help protect the nation's resources and advance energy security.

INL's science base is further augmented by research to advance alternative fuels and energy systems that reduce greenhouse-gas emissions and lessen the nation's dependence on petroleum-based fuels. INL's applied science and real-world assessments of improved energy systems and natural resource development help secure the country's future and protect the environment.

This year's LDRD projects offer a cross section of the distinct creativity and expertise residing at INL. The portfolio offers a robust state-of-the-art contribution in meeting the nation's needs for energy supply, energy integration, energy security, and environmental integrity while addressing the unique challenges encountered in these core strategic areas. Several projects open the door of innovation to new experimental approaches. In short, these projects help INL maintain scientific and technical vitality while fostering creativity and stimulating exploration at the forefront of science and technology.

I am proud of the accomplishments, advancements, and opportunities that INL's LDRD projects provide to the nation. I encourage you to take the time to review these project descriptions and reflect on their contributions.

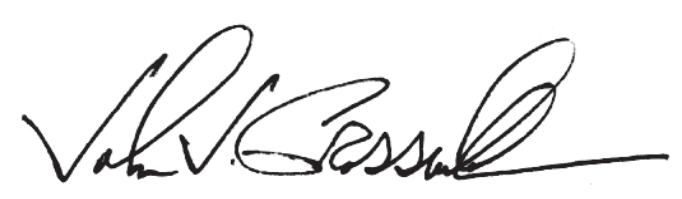

John J. Grossenbacher Laboratory Director 
INL/EXT-11-23890

\section{Acknowledgments}

This annual report provides an overview of the Laboratory Directed Research and Development (LDRD) Program at Idaho National Laboratory (INL). Summarized in this report are 115 technical research projects conducted in FY 2011. The LDRD Program at INL is managed for the Laboratory by John J. Grossenbacher, Laboratory Director; David Hill, Deputy Laboratory Director for Science and Technology; and Anita Gianotto, LDRD Program Manager.

Many people contributed to this report; however, particular thanks go to the principal investigators who conduct the cutting-edge research so important to this laboratory.

Managing Editor: Dena Tomchak

Science Writers: Gordon Holt Caitlin Lanier

Lisa Plaster

Text Processing: $\quad$ Mary Bates

Cover Design: Christine White

Prepared for the U.S. Department of Energy

Director, Nuclear Energy, Science \& Technology

Under DOE Idaho Operations Office

Contract Number: DE-AC07-05ID14517

\section{Disclaimer}

This report was prepared as an account of work sponsored by an agency of the United States Government. Neither the United States Government nor any agency thereof, makes any warranty, express or implied, or assumes any legal liability or responsibility for the accuracy, completeness, or usefulness of any information, apparatus, product, or process disclosed, or represents that its use would not infringe privately owned rights. Reference herein to any specific commercial product, process, or service by trade name, trademark, manufacturer, or otherwise does not necessarily constitute or imply its endorsement, recommendation or favoring by the United States Government or any agency thereof. The views and opinions of authors expressed herein do not necessarily state or reflect those of the United States Government or any agency thereof. 


\section{Table of Contents}

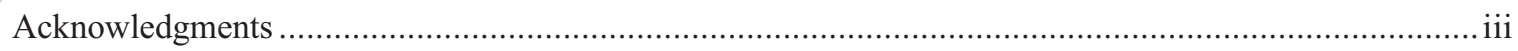

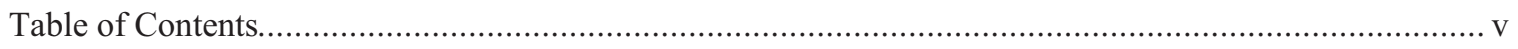

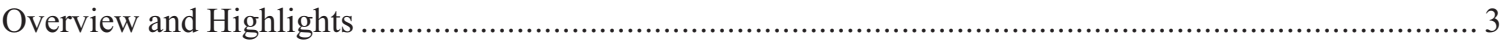

\section{Highlights}

New Facility Makes it Easier for Collaborators to Test Irradiated Materials ......................................... 7

INL Researchers Develop Tool to Better Understand Network Communication Paths ............................ 9

INL Advances Laser-Induced Breakdown Spectroscopy System................................................ 11

Student Research Project Takes First Step to Fabricate Dual Hardness Titanium for Armor

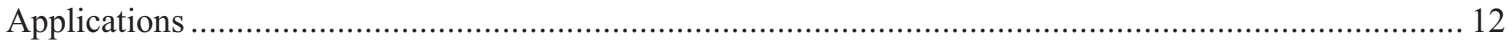

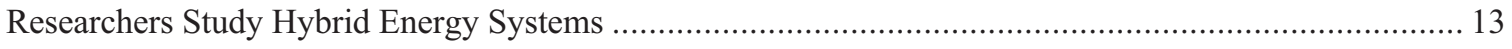

Creating Hydrogeophysics Models for Subsurface Understanding ........................................... 15

Collaborative Project Aims to Model the Recovery of Valuable Materials Locked in Used Nuclear Fuel.

\section{Nuclear Science and Technology}

Development of Reactor Physics Sensitivity Analysis, Uncertainty Quantification, and Data Assimilation Capability at INL for Validation Applications

Development of a Next-Generation Production Code for Nuclear Reactor System Analysis and

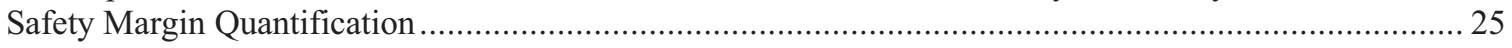

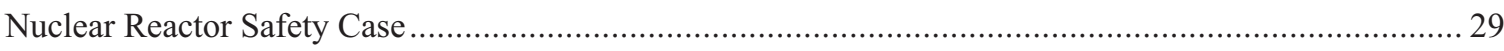

Spatially Resolved Positron Analysis of Hydride Formation in LWR Fuels with GE-Global

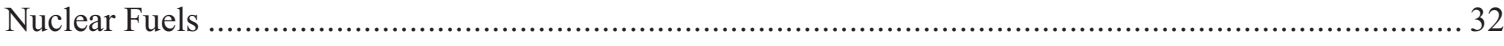

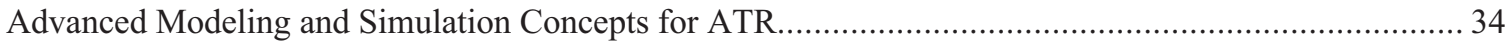

Characterization of a Consolidated Electrochemical Technique for Separation and Recovery of Actinides from Fission Products in Oxide Fuels ................................................................... 36

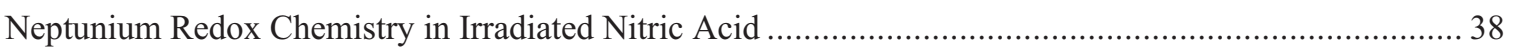

Reversible Gas Phase Reactions for Recovery of Graphite from Recycled HTGR TRISO Fuel............... 39

Comprehensive Thermodynamic Models for the Aqueous Partitioning of Actinides from Used

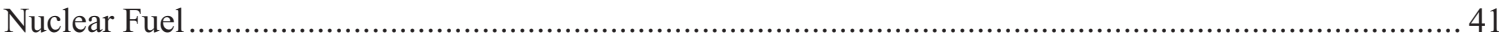

Speciation Behavior of Americium Oxidation States for the Separation of Americium from Curium in Nuclear Processing ...................................................................................... 43

Development of a Simplified Soft Donor Technique for Trivalent Lanthanide Actinide Separations......... 45

Development of a Small Sample Volume Mechanical Properties Testing Technique for Irradiated

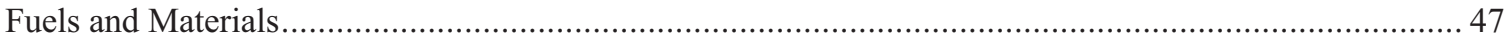

Multiscale Modeling and Simulation of Nuclear Fuel Performance .............................................. 49

Irradiation Testing and Molecular Modeling of Irradiation-Assisted Diffusion and Microstructural

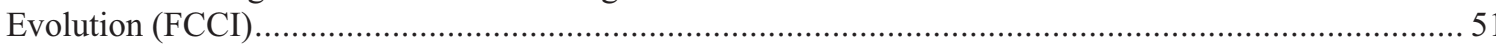


Spatially Correlated Microstructural Characterization: From Centimeters to Nanometers

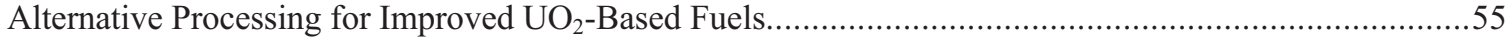

Instrumentation for In-pile Detection of Thermal Conductivity ............................................5

Ultrasonic Transducer Sensors for In-pile Detection of Dimensional Changes .................................61

Haptic Tele-Operated End Effector for Hazardous Environments ...............................................65

Development of In-Reactor Experiment Configuration for Laser-Based Material Properties

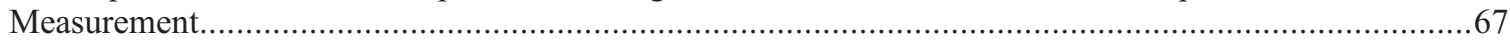

Human Simulation Laboratory for Nuclear Power Plant Human Performance Studies..........................69

Smart Grid Impact on Commercial Nuclear Plants ............................................................. 71

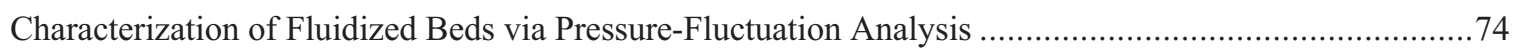

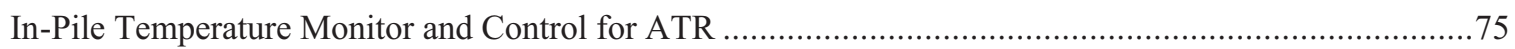

Dissolution and Extraction Studies of Fission Products in Room Temperature Ionic Liquids

(RTILs) and in Supercritical Fluid $\mathrm{CO}_{2}\left(\mathrm{ScCO}_{2}\right)$ and Determination of Radiolytic Stability ....................76

\section{National \& Homeland Security}

Methodologies for the Design, Analysis, and Validation for Operation of Complex Resilient

Networks.

Next Generation Control System “Smart Grid” Simulation Environment ...........................................85

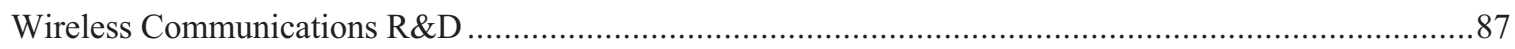

Electronic Warfare Digital Communications and Network Traffic Modeling ...................................89

Experimental Cyber Security: Vulnerability Prediction, Discovery, and Mitigation..............................91

Industrial Control Systems Threat Axis Analysis to Characterize and Quantify Malicious Intent

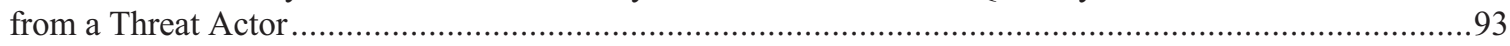

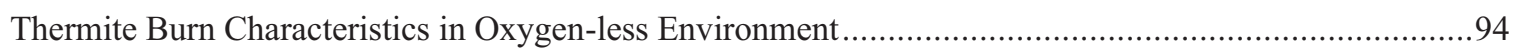

Prototype INL Wildland Fire Early Warning and Situational Awareness System ...............................96

SMC Advanced Armor Materials and Systems R\&D......................................................... 97

Cognitive Network Engine and Simulation Framework .................................................... 99

Enabling Dynamic Spectrum Access in Long-Term Evolution Networks.........................................100

Multi-Rate Shock Physics Simulation of Blast and Penetration Events in Concrete.............................. 101

\section{Energy \& Environmental Science \& Technology}

Advanced Remote Sensing for Energy and Environmental Applications using Unmanned Aerial Vehicles 105

Addressing the Spectrum of Nuclear-Related Non-Destructive Evaluation Needs ............................. 108

Water and Energy System Interdependency Modeling for Multi-criteria Decision Analysis ..................110

Degradation and Conversion of Lignin using Extremophilic Systems .......................................... 112 
Innovative Mathematical Morphology Approach to Optical Color Image Analysis and Pattern Recognition .....

Network Interaction in the Thermoacidophile Alicyclobacillus Acidocaldarius in Response to

Nano Metal Oxides.

Specific Biological Responses to Nano Metal Oxides.

Development of Non-Lethal Methods for Enhanced Lipid Recovery from Microalgae

Development of Thermally Generated In Situ Precipitation Barriers due to Subsurface Heat Injection

Hybrid Osmosis Water Purification Systems Research

Biological Fixation of $\mathrm{CO}_{2}$ for Fuel and Chemical Production using Acetogen Intermediary Metabolism

Electrochemical Production of Synthesis Gas from $\mathrm{CO}_{2}$

Multiscale Coupled Hydrogeophysics Modeling: Advancing Understanding of Reactive Transport and Effective Subsurface Environmental Monitoring

Mechanisms of Self Inhibition of Catalytic Conversion of $\mathrm{CO}_{2}$ to Methanol ......................................... 132

Dynamic Thermo-Mechanical Energy Conversion Simulation ................................................................. 134

Reduction and Retention of Technetium Using Fe(II) Hydroxides ...................................................... 137

Ammonia-based Extraction Methods for the Separation and Purification of Metals ............................... 138

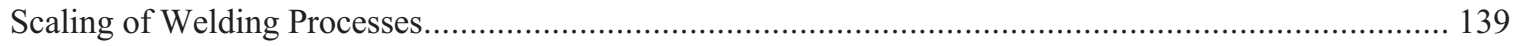

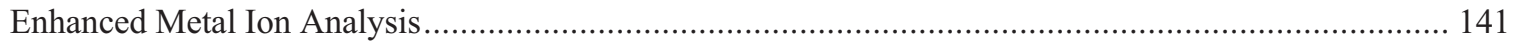

Effect of Glycosylation on the Activity and Stability of Bacterial Enzymes.......................................... 143

Kolbe Electrolysis of Bio-Oils for the Production of Diesel and Aviation Fuels ..................................... 144

Dissolution, Extraction, and Separation of Rare Earth Elements using Soft Donor Ligands in

Conjunction with Ionic Liquids and Supercritical Carbon Dioxide .................................................... 146

Aleatory vs. Epistemic Uncertainty in Seismic Hazard Analyses........................................................... 148

Particle-Discrete Element Model Simulation of the Coupling between Material

Failure/Deformation and Fluid Generation/Flow.....

\section{Energy Security Initiative}

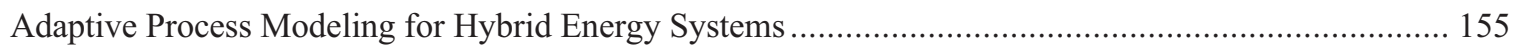

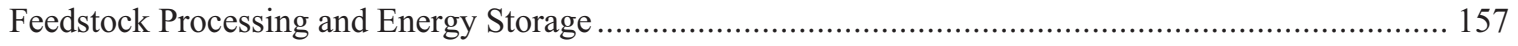

Mesosilicate Supported $\mathrm{CO}_{2}$ Capture Material............................................................................ 159

Membrane Separation System Research for HYTEST …............................................................... 161

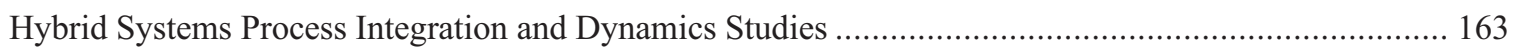

Conversion of Light Hydrocarbons to Fuels and Chemicals .................................................................. 166

Integration of Renewable Energy Resources through Energy Storage and SuperGrid ........................... 167 


\section{Nuclear Nonproliferation Initiative}

Developing a Next-Generation, Risk-Informed Approach for Robust and Resilient Design Development.

Radionuclide Collection-Detection Device for the In Situ Remote Monitoring of ${ }^{99} \mathrm{Tc}$ as a

Proliferation Indicator..... 172

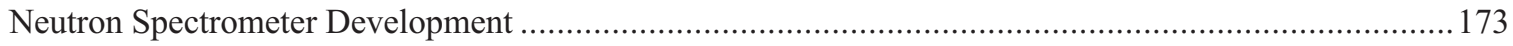

On-line Monitoring of Actinide Concentrations for Advanced Aqueous Separations................................176

Develop a Safeguards Approach for INL Pyroprocessing Lines

Laser-Induced Breakdown Spectroscopy: Development and Application of In Situ Elemental Analysis for Process Streams in Spent-Fuel Reprocessing Facilities

Object Reconstruction Technique for Use in Radiography....

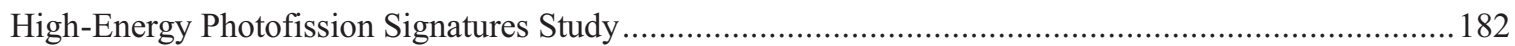

Treaty Verification with Photo-Induced Prompt Signatures

Unconventional Radiation Detection Methods for Nuclear Nonproliferation, Safeguards, and Treaty Verification

Pyro Processing Facility Nuclear Material \& Environmental Radiological Signature

Characterization in the Hot Cell

\section{Institute for Nuclear Energy Science \& Technology}

Evaluation of Covalent Interactions in Actinide-Coordination Compounds

Measurement of Degradation Products of Irradiated Ligands Used in Separations Measuring Actinide Speciation in High-pH Solutions. 195

Isotope Ratio Measurements Methods for Direct Analysis of Samples 198

Irradiation-Induced Evolution of Defect and Microstructure in Nanocrystalline Body-Centered-

Cubic Mo

Microstructural Evolution of $\mathrm{UO}_{2}$ and $\mathrm{U}$ under Irradiation

In Situ Characterization of an Oxide Film for Prediction of Stress Corrosion Cracking Susceptibility

Investigation of Molten Bromide Salt Systems for Separation and Recovery of Actinides from Fission Products

In Situ Micro-Raman Spectroscopy and Modeling of Breakaway Oxidation of Zircaloy Cladding...........206

Characterization of Nanomechanical Response of Material to Determine Key Variables in Stress Corrosion Cracking. 208

Supercritical Fluid Extraction of Actinides from TRISO Reactor Fuels.................................................209

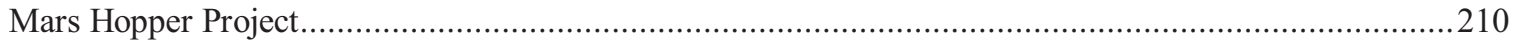

Science-Based Simulation Model of Human Performance for Human Reliability Analysis 


\section{Center for Advanced Energy Studies}

Development of Lignocellulosic Ethanol Production Potential in Idaho

Optimization of Ceramic Waste Forms Used for Electrochemical Processing of Spent Nuclear Fuel

Prediction and Monitoring of $\mathrm{CO}_{2}$ Behavior in Deep Reactive Geologic Formations

Fabrication of Advanced ODS Alloys using Field-Assisted Sintering.

Small Specimen Test Techniques for Evaluating Radiation-Induced Changes in Mechanical Properties

Design and Operational Improvements and LCA in Anaerobic Digestion of Fermented Dairy

Manure using a Two-Stage Process

Transmission Site Suitability Decision Support Technology

CAES Vertical Axis Wind Turbine Project.

Development and Demonstration for National Fuel Efficiency. 238

Targeted Energy Management Toolset Support 240

\section{Center for Advanced Modeling \& Simulation}

Preconditioning Methods for Hybrid Neutronics Calculations......

Fickian and Thermal Diffusion in Nuclear Materials from Linear Response Theory and Multiscale Simulations.

Error Estimation for Stochastic Uncertainty Quantification.

Advanced Visualization using Immersive Environments......

Uncertainty Analysis for Multiscale Models of Nuclear Fuel Performance.

Uncertainty Analysis of Mesoscale Models of Radiation Effects....

Exploratory Nuclear Reactor Safety Analysis and Visualization via Integrated Topological and Geometric Techniques

\section{Instrumentation, Control, and Intelligent Systems}

Integrated Control Systems Data Fusion 259

Wireless Sensor Testing. 261

Anomaly Detection, Diagnosis, and Resilient Control in Complex Engineered Systems 263

3D Spatial Representation in Support of Design Inspection \& Verification 267

Resilient Condition Assessment Monitoring (ReCAM) System.................................................. 268

Automated Differential-Equation-Based Identification ..................................................... 271

Known Secure Sensor Measurements for Detecting Unauthorized Process Manipulation and Falsification of State. 


\section{Appendices}

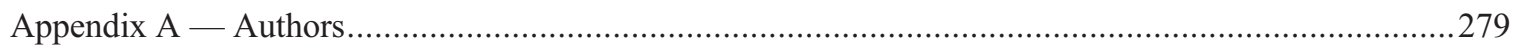

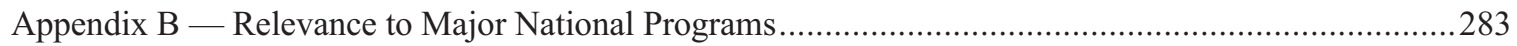

\section{COMMON INL ACRONYMS}

$\begin{array}{ll}\text { ATR } & \text { Advanced Test Reactor } \\ \text { BIGHORN } & \text { Neutronic Solver for Prismatic Geometry Computer Code } \\ \text { BISON } & \text { Implicit Simulation of Nuclear Fuel Computer Code } \\ \text { CAES } & \text { Center for Advanced Energy Studies } \\ \text { CAMS } & \text { Center for Advanced Modeling and Simulation } \\ \text { DOE } & \text { U.S. Department of Energy } \\ \text { DOE-NE } & \text { U.S. Department of Energy Office of Nuclear Energy, Science, and Technology } \\ \text { FCCI } & \text { Fuel Cladding Chemical Interactions } \\ \text { HES } & \text { Hybrid Energy Systems } \\ \text { HTGR } & \text { High Temperature Gas Reactor } \\ \text { HY-TEST } & \text { Hybrid Energy Systems Testing } \\ \text { INL } & \text { Idaho National Laboratory } \\ \text { IRC } & \text { Idaho National Laboratory Research Center } \\ \text { JFNK } & \text { Jacobian-Free Newton-Krylov } \\ \text { LCA } & \text { Life Cycle Analysis } \\ \text { LWR } & \text { Light Water Reactor } \\ \text { MARMOT } & \text { Mesoscale Model Application Computer Code } \\ \text { MFC } & \text { Materials and Fuels Complex } \\ \text { MOOSE } & \text { Multiphysics Object Oriented Simulation Environment Computer Code } \\ \text { ODS } & \text { Oxide Dispersion-Strengthened Steel } \\ \text { PUREX } & \text { Plutonium-Uranium Extraction } \\ \text { R\&D } & \text { Research and Development } \\ \text { SMC } & \text { Specific Manufacturing Capability } \\ \text { TRISO } & \text { Tristructural-Isotropic } \\ \text { VHTR } & \text { Very High Temperature Reactor } \\ & \end{array}$




\section{$\Rightarrow$ Overview and Highlights}

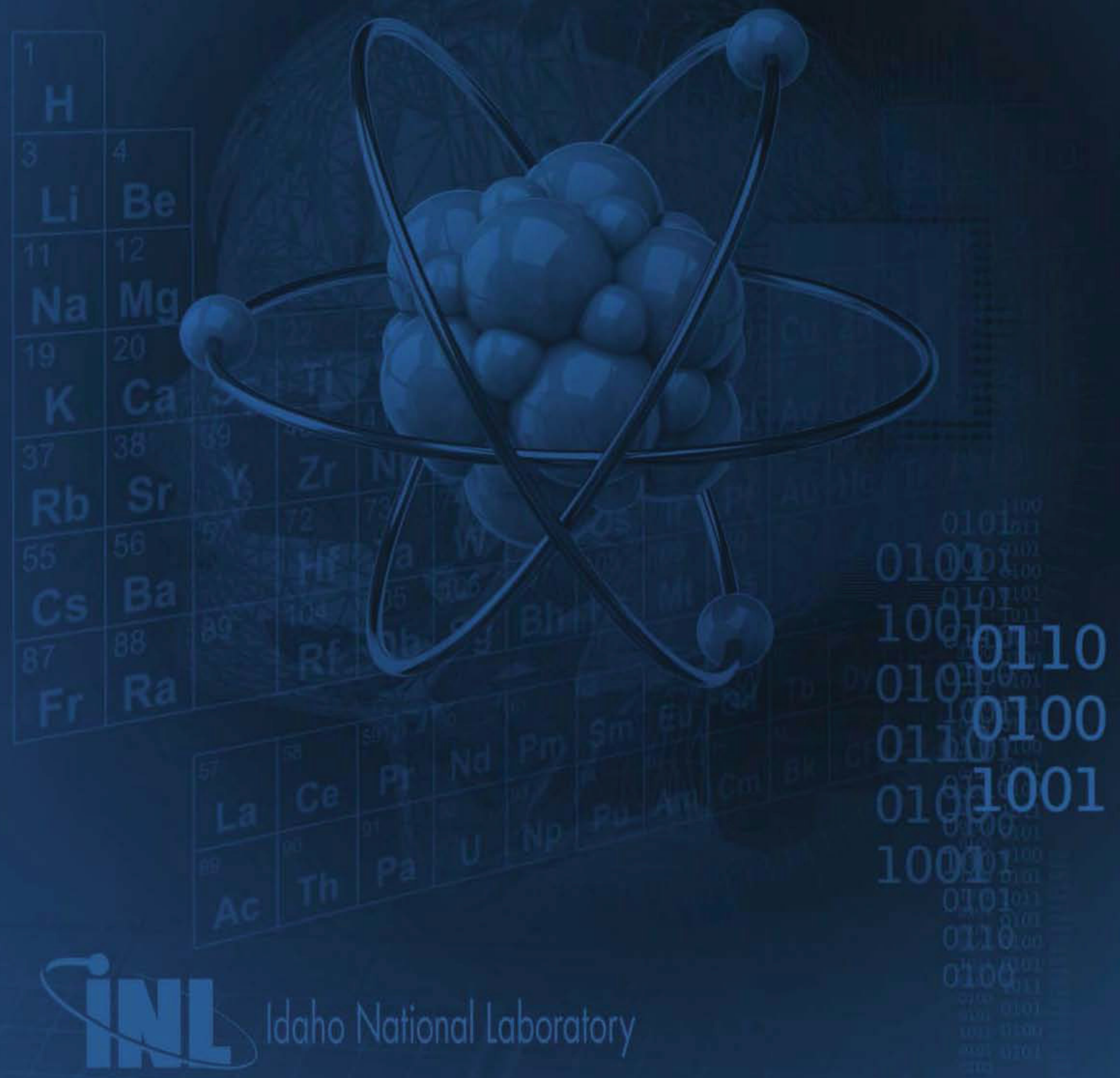




\section{Overview and Highlights}

The FY 2011 LDRD Annual Report is a compendium of the diverse research performed to develop and ensure the INL's technical capabilities support the current and future DOE missions and national research priorities. LDRD is essential to INL-it provides a means for the Laboratory to maintain scientific and technical vitality while funding highly innovative, highrisk science and technology research and development $(\mathrm{R} \& \mathrm{D})$ projects. The program enhances technical capabilities at the Laboratory, providing scientific and engineering staff with opportunities to explore proof-of-principle ideas, advanced studies of innovative concepts, and preliminary technical analyses.

Established by Congress in 1991, the LDRD Program proves its benefit each year through new programs, intellectual property, patents, copyrights, national and international awards, and publications.

\section{Benefits of LDRD to INL}

INL consistently sees significant benefits of the LDRD Program to the Laboratory. One of three FY 2010 INL R\&D 100

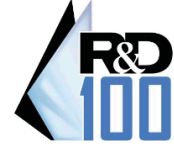
awards, the "Supercritical/Solid Catalyst (SSC) Biodiesel Production Process," was an innovative LDRD initially funded in FY 1995. The research provided a means to use discarded and environmentally unfriendly wastes and convert them into biodiesel fuels using a chemistry breakthrough called Supercritical Solid Catalyst, or SCC. INL scientists Daniel Ginosar, Robert Fox, Lucia Petkovic, and Dan Wendt worked to find ways to create liquid fuels from a variety of waste streams, including municipal wastewater and food processing waste. SSC mixes fat or oil feedstock with supercritical fluid solvents and alcohols at specific temperatures and pressures to completely dissolve the materials during a single supercritical phase. This approach overcomes a key barrier - the polar liquid phase in conventional biodiesel production, which previously required multiple steps to achieve.
Also in FY 2010, this initially LDRDfunded research project received the Federal Laboratory Consortium Far West Region Outstanding Technology Development Award, the Battelle Innovation Award, the Platts Global Energy Award, and was honored as a Wall Street Journal Technology Innovation Award runnerup. In addition, this technology was demonstrated on a pilot scale and manufactured 300,000 gallons of biodiesel fuel in FY 2010.

Twelve patents issued in FY 2011 were attributable to LDRD funding and 18 of the total invention disclosures submitted in FY 2011 were initiated with LDRD research. Sixty-two journal articles and conference proceedings were published during the fiscal year as a result of LDRD research. The FY 2011 metrics are shown in the tables below.

\begin{tabular}{|l|c|}
\hline \multicolumn{2}{|c|}{ FY 2011 LDRD Metrics } \\
\hline National/International Awards & 5 \\
\hline Invention Disclosures & 18 \\
\hline Patents & 12 \\
\hline Copyrights & 0 \\
\hline Refereed Publications & 62 \\
\hline INL Post Docs & 13 \\
\hline INL Students & 21 \\
\hline New Scientific \& Engineering Hires & 10 \\
\hline
\end{tabular}

\begin{tabular}{|l|c|}
\hline \multicolumn{2}{|c|}{ FY 2011 LDRD Program Statistics } \\
\hline Total LDRD Program Cost & $\$ 31,346.8 \mathrm{~K}$ \\
\hline Number of Projects & 115 \\
\hline Largest Project Allocation & $\$ 1,236 \mathrm{~K}$ \\
\hline Smallest Project Allocation & $\$ 40 \mathrm{~K}$ \\
\hline
\end{tabular}




\section{The LDRD Process at INL}

The LDRD Program is administered in accordance with requirements set in DOE Order 413.2B, accompanying contractor requirements, and other DOE and federal requirements invoked through the INL contract. The LDRD Program is implemented in accordance with the annual INL LDRD Program Plan, which is approved by the DOE, Nuclear Energy Program Secretarial Office. This plan outlines the method the Laboratory uses to develop its research portfolio, including peer and management reviews, and the use of other INL management systems to ensure that quality, financial, safety, security, and environmental requirements and risks are appropriately handled. The LDRD Program is assessed annually for both output and process efficiency to ensure the investment is providing expected returns on technical capability enhancement.

The call for proposals and project selection process for the INL LDRD FY 2011 program began in early April, with preliminary budget allocations, and a call for pre-proposals. Preparation of full proposals followed in June and closed in July. The technical and management reviews followed, and the portfolio was submitted for the DOE Idaho Operations Office concurrence in late August/early September. Project initiation was in early October.

The technical review process is independent of and in addition to the management review. These review processes are very stringent and comprehensive, ensuring technical viability and suitable technical risk are encompassed within each project that is selected for funding. Each proposal is reviewed by two or three anonymous technical peers, and the reviews are consolidated into a cohesive commentary of the overall research based on criteria published in the call for proposals. A grade is assigned to the technical review and the review comments and grade are released back to the principal investigators and the managers interested in funding the proposals. Management criteria are published in the call for proposals, and management comments and selection results are available for principal investigator and other interested management as appropriate. The DOE Idaho Operations Office performs a final review and concurs on each project prior to project authorization and on major scope/budget changes should they occur during the project's implementation.

\section{Report Organization}

This report begins with several research highlights that exemplify the diversity of scientific and engineering research performed at INL in FY 2011. Progress summaries for all projects are organized into sections reflecting the major areas of research focus at INL. These sections begin with the DOE-NE Nuclear Science and Technology mission support area, followed by the National and Homeland Security and the Energy and Environmental Science and Technology areas. Major INL initiatives and the distinctive signature area complete the project summaries. The appendices provide an author index and information on project relevance to major national programs. 


\section{Highlights}




\section{New Facility Makes it Easier for Collaborators to Test Irradiated Materials}

Bulent Sencer

10-024

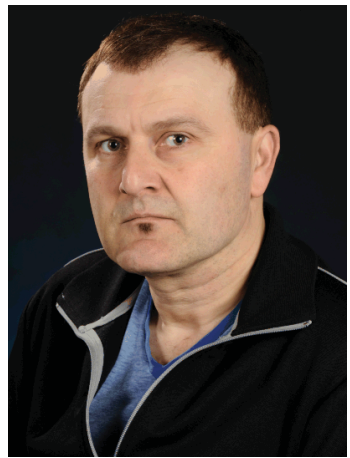

Modern insights about material properties and behavior are enabling the design of new reactor materials that may be stronger under prolonged irradiation. Before such materials can be used to construct new reactor components, they must be rigorously tested and qualified by the Nuclear Regulatory Commission.

To evaluate the robustness of new materials, the Nuclear Regulatory Commission uses standards defined by the American Society for Testing and Materials, which defines a number of characteristics that must be tested and quantified for new materials. For nuclear reactor materials, such tests require expensive and timeconsuming measurements of irradiated materials, which often must be handled inside a hot cell.

This LDRD project created a new capability that makes this sort of testing far more accessible to INL researchers and university collaborators. Specifically, the project created a flexible station for testing small specimens in the Center for Advanced Energy Studies at INL. Collaborators on the project include Boise State University's Dr. Frank Garner, who helped advise the work, and INL's Mitchell Meyer, who helped identify radioactive samples for testing. Developing nuclear materials with significantly improved performance is a key objective of INL's nuclear programs, and this project enhances nuclear materials testing capabilities that support such work. It also makes these capabilities accessible to university collaborators and students affiliated with INL's Advanced Test Reactor National Scientific User Facility and the Center for Advanced Energy Studies (CAES) - a partnership between INL and Idaho's three public research universities.
The new testing station includes equipment for tensile and shear punch tests - two mechanical testing techniques that are well suited for small specimens behind light shielding. There are also provisions for additional nondestructive test equipment to study properties such as void swelling, transmutation, and hardness. The facility can test very small samples (for example, a 3-mm-diameter and 0.25 -mm-thick shear punch sample), which makes testing cheaper, faster, and easier. Since these small samples take up less space inside test reactors, more samples can be irradiated for the same cost. Finally, because the samples are so small, they emit less radiation and can be tested with less shielding than larger samples require. This may eliminate the need for a hot cell and potentially allow such testing to be accomplished in a university setting.

Following the equipment selection process, an Instron tester, which is enclosed to reduce potential furnace and or radioactivity hazards, was purchased and installed in the CAES Materials Lab. The apparatus was also connected to the existing ventilation system at CAES in order to create negative pressure. Initial shearpunch and tensile testing proved successful. A micro-density measurement cell was manufactured, assembled, and added to the existing set-up so that small radioactive samples could be handled.

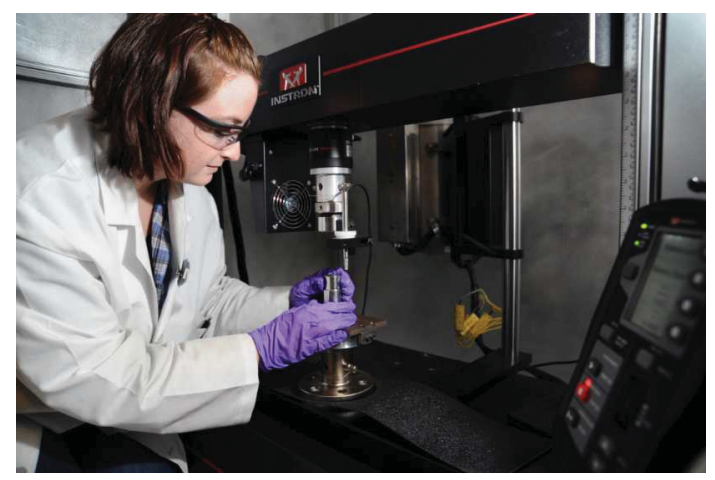

Graduate student Ellen Rabenberg working at the project's shear-punch apparatus.

The final step involved obtaining permits for shipping small samples of radioactive materials from ATR and MFC to CAES. Five shear-punch discs of ATR-irradiated oxide dispersionstrengthened 304 stainless steel and a number of 
shear-punch discs from Westinghouse were procured. Under guidance from Boise State University professor Darryl Butt, graduate student Ellen Rabenberg has been conducting tests on these discs to study deformation and strain effects on the samples. She is preparing her Master's thesis based on this data and is scheduled to present her findings at the Minerals, Metals, \& Materials Society annual meeting in Orlando, Florida, in March 2012. 


\section{INL Researchers Develop Tool to Better Understand Network Communication Paths}

\section{Kurt Welker}

11-022

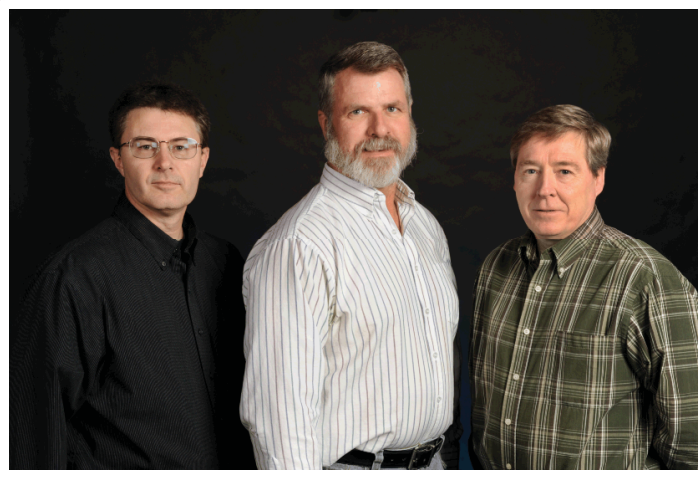

Information networks surround us. Some are simple; others are extremely complex. Whether part of a command and control system, telecommunications network, information system computer network, mobile ad hoc communications network, social network or military supply network, understanding information operations is critical to mission success. In addition, a technology solution gap exists in map-centric, first-order, effects-based mission planning analysis tools that provide a multi-network, cross-domain, traffic-flow modeling capability.

In October 2010, INL initiated an LDRD project to create a software prototype that demonstrates proof-of-concept capabilities that can help bridge the gap between missionplanning analysis tools and multi-network, crossdomain, traffic-flow modeling.

Principal Investigator Kurt Welker and his colleagues Lance Murri and Tim Thompson successfully demonstrated a new software technology prototype, called NetMuster, which will provide the ability to assemble network data from various static and dynamic sources, graphic data sources, and real-time feeds to assist the network analyst in determining probable communication paths from one network component to another. The software also considers existing and potential vulnerabilities of the communication network.

This LDRD project leveraged more than 20 years of INL-developed software to create the NetMuster prototype. NetMuster is a simple, user-friendly software tool that analyzes network traffic flow by combining logical and physical network representations with domain-specific business rules using physics-based models and mathematical graph theory. The NetMuster algorithms could accommodate cell phone, first responder communication, industrial control system, and military communication networks, among many others.

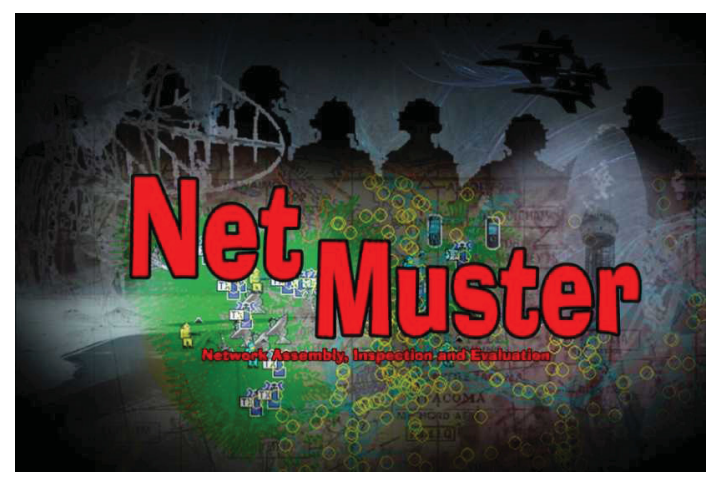

The methodology and algorithms have been developed and implemented as a software proofof-concept prototype for lashing together logical and physical network data with domain-specific, mission-centric business rules to construct a mathematical graph that can be used to determine potential traffic/communications paths through the network. This LDRD provides a new radiofrequency network modeling and simulation capability at INL that supports Wireless Test Bed activities and customers.

"NetMuster includes a capability for ingesting communications network data from sources such as government and public databases, ESRI'TM Shapefiles, and others," said Welker. "A network analyst is then able to aggregate the network data and perform network traffic-flow analyses that can be used to characterize, refine, optimize, and protect network infrastructure."

The LDRD project also established a proofof-concept working software prototype and technical collaboration between INL's National $\&$ Homeland Security Electronic Warfare and 
Wireless Test Bed personnel. For technology demonstration purposes, the researchers selected a simple cell phone network for future applicability to a national wireless test bed. The project provides a new radiofrequency network modeling and simulation capability that can support INL's Wireless Test Bed.
Welker concludes, "In 2012, the team plans on extending NetMuster to include modeling capabilities for other network configurations, including radiofrequency/cyber hybrid networks, and networks with acoustic components."

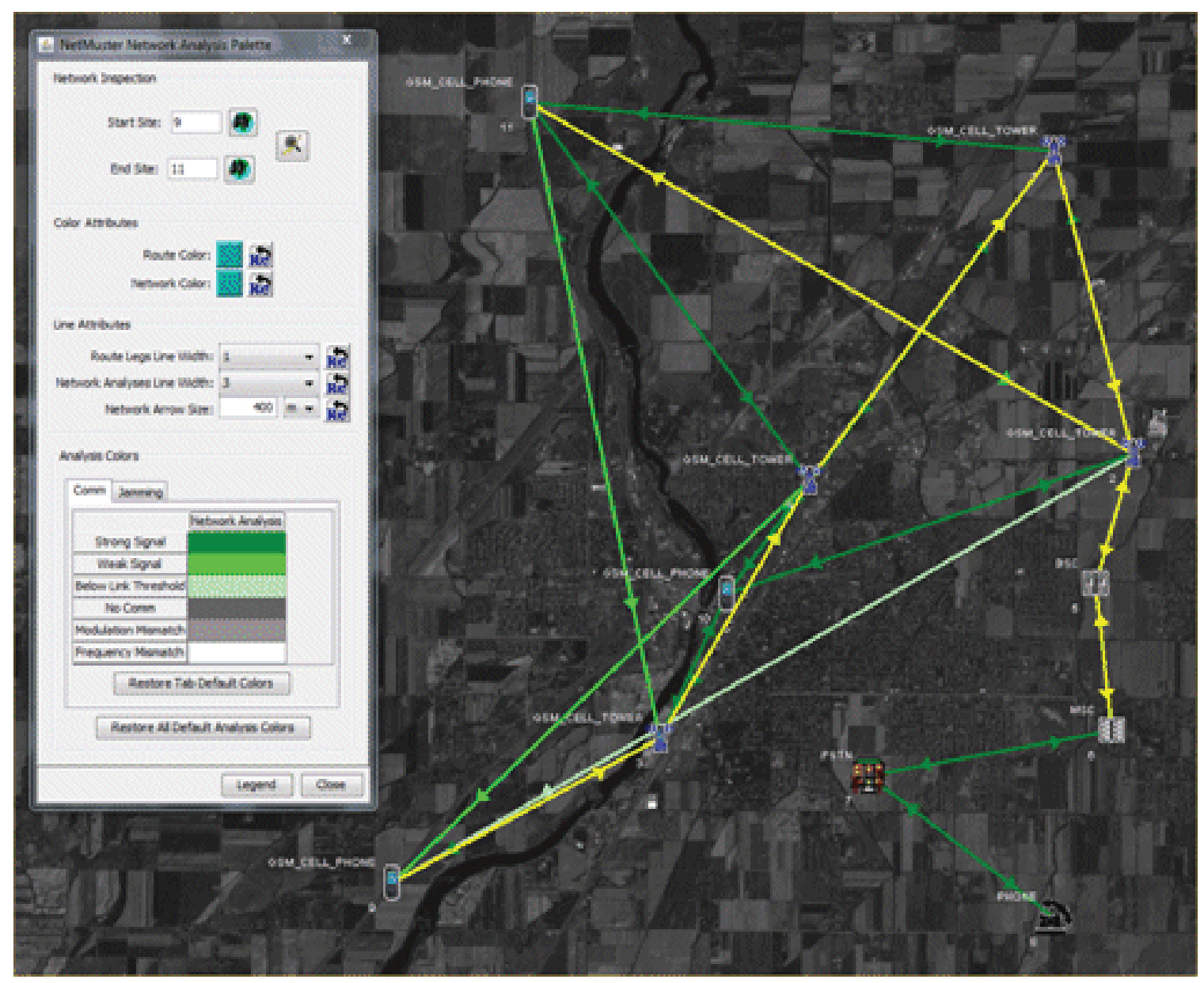

NetMuster analyzes the path information travels as it goes from Point A to Point B. 
INL Advances Laser-Induced Breakdown Spectroscopy System

Jill Scott

07-052

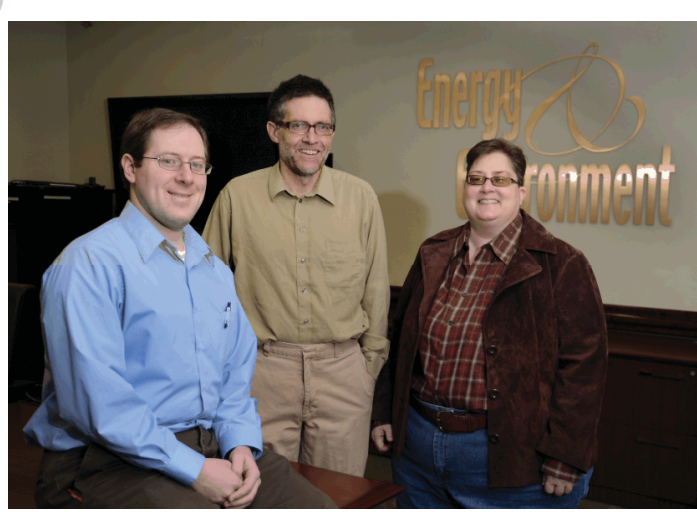

The International Atomic Energy Agency (IAEA) has been tasked with monitoring nuclear fuel processing activities worldwide. However, the current state-of-the-art for monitoring such materials requires time-consuming sample preparation techniques and procedures. DOE is greatly concerned with reducing the risk of nuclear material proliferation and providing IAEA with the technology to improve nuclear safeguards both in the U.S. and in the world. In fact, DOE has gone on record that an improved method for monitoring nuclear fuel processing activities is greatly needed to ensure not just U.S. security, but world security as well.

INL principal investigator Jill Scott and her team have developed a technology to remotely measure and analyze the real-time concentrations of special nuclear materials in process streams in spent fuel reprocessing facilities. This unique analytical technique, the "Laser-Induced Breakdown Spectroscopy System," or LIBS, is an elemental analysis method based on the emission from plasma generated by focusing a laser beam at the interface of the medium to be sampled. This technology would theoretically be applicable to solids, liquids (including molten metals), and gases, and would greatly enhance the transparency of reprocessing facility operations, improve material control and accountability, and provide indications of any illicit reprocessing activities.

In FY 2010, the experimental apparatus for conducting LIBS on molten salts was developed using equipment and facilities at the University of San Diego. Then, during FY 2011, necessary components were acquired to produce benchscale mockups of a LIBS system at INL with the goal of taking into consideration the radiation facility constraints at INL facilities. A portable LIBS system with fiber-optic laser and light collection was designed for conducting LIBS analyses on radioactive uranium and plutonium samples at MFC and CFA. The performance of INL's LIBS system was also evaluated and optimized during the mockup stages.

Scott states, "We've made some key advances during this LDRD project. We have developed a system that has high-resolution LIBS spectra, it's small and compact, low cost, and outputs algorithm integration."

This research has provided INL with a plausible methodology for rapid, real-time elemental analysis of metals in molten salts for monitoring processes related to spent nuclear fuels. Successful completion of this project will help DOE reduce the risk of nuclear material proliferation and provide technology to improve nuclear safeguards. Improved monitoring of nuclear fuel processing will enable DOE, IAEA, and other interested parties to control nuclear materials worldwide and ensure U.S. security in the process.

In addition, these activities may also prove useful to INL and DOE for developing more efficient actinide recycling techniques under the Global Nuclear Energy Partnership or to support Next Generation Nuclear Plant project activities. The LIBS system, especially if coupled with other INL LIBS technology, could open the door for smaller, portable analysis instruments that could be used to monitor levels of uranium and/or plutonium, including isotopic measurements, to help ensure that these elements are accounted for and not diverted.

There is great market potential for the LIBS system INL is developing. It will enable isotope measurements to be done in the field or in situ. And since it already works, the LIBS technology is a small risk especially because the LIBS market is expanding. One patent on this technology is currently pending and it is anticipated that two invention disclosure records will be filed in FY 2012. 


\section{Student Research Project Takes First Step to Fabricate Dual Hardness Titanium for Armor Applications}

\section{Henry Chu, Michael Bakas}

11-060

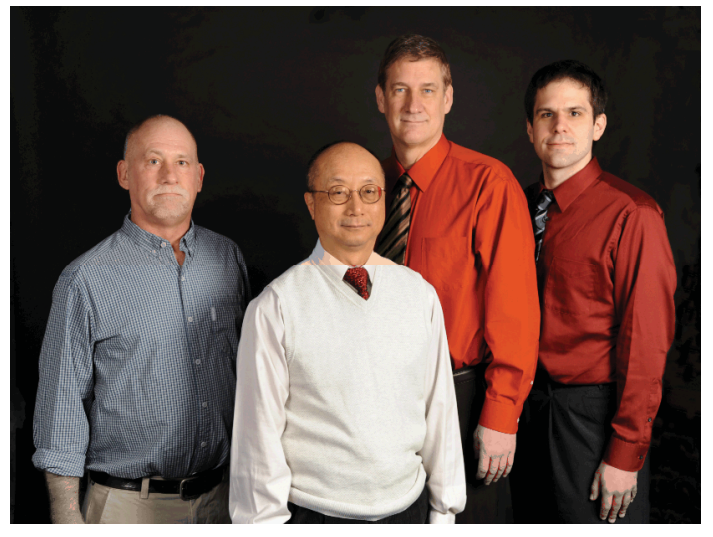

Advanced armor materials must be hard, lightweight, and robust. Although advanced ceramics offer sufficient hardness at a fraction of the weight of steels, most are brittle and cannot withstand multiple impacts. A dual-hardness titanium (Ti) laminate composite could provide a weight-efficient ballistic solution, yet creating a high-hardness Ti alloy has proven difficult using traditional alloying methods.

Student researchers at INL are investigating methods for overcoming this challenge. This LDRD project entailed an initial experiment to determine if a self-propagating high-temperature synthesis (SHS) process could create a highhardness Ti composite that performs with both ceramic and metallic properties.

INL has long supported national security missions through its National \& Homeland Security Directorate and Specific Manufacturing Capability. This LDRD project, led by Drs. Henry Chu and Michael Bakas, reinforces INL's reputation in material science and engineering, and process technology development. Other significant research contributions were made by David Anderson and Bryan Ashton of the U.S. Air Force Academy during their internship tenures at INL.

"For an armor material to be effective against a lethal armor-piercing projectile, it must shatter and/or blunt its hard core, prevent the passage of any fragments, and absorb a sufficient amount of the projectile's energy," said the investigators in a research paper drafted for publication. "In order to meet the first requirement, the armor material must have hardness comparable to that of the hardened core of the projectile."

Ceramic-based armor systems often use a layered laminate configuration in which the ceramic front (impact) face is adhesively bonded to a ductile (metal or organic fiber) backing layer to mitigate the inherent brittleness. Along similar lines, a dual-hardness Ti laminate composite system would behave as if it consists of a highhardness front layer and a ductile, tough back layer. Previous research has demonstrated that materials' behavior problems arise when creating Ti alloys with sufficient hardness to meet the requirement of an effective front impact face.

This LDRD project aimed to create a highhardness Ti composite using an innovative SHS process. The SHS process exploits the induced heat from a reaction between $\mathrm{Ti}$ and other chemical components to form in situ the requisite hard phases.

The team's SHS process successfully produced a composite, as confirmed by x-ray diffraction and scanning electron microscope analysis. The method will need to be refined to obtain sufficient quantity and quality of material for armor applications and ballistic testing. This work is part of a multi-phase experimental study in the attempt to form multiple-layer, variablehardness titanium.

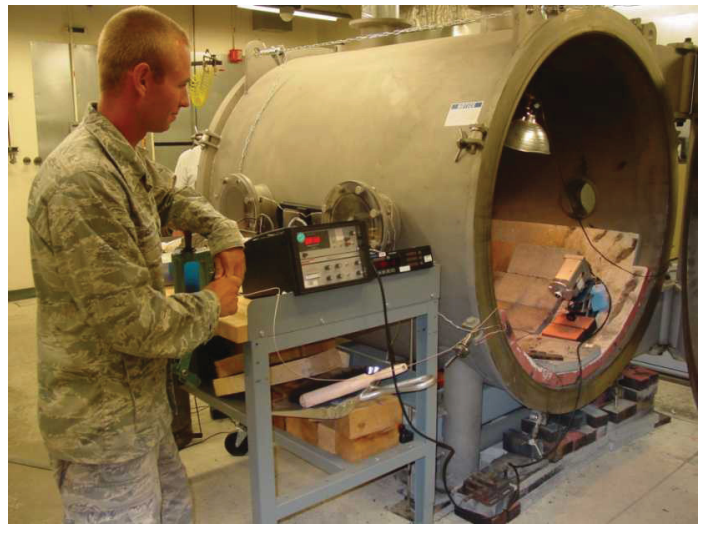

U.S. Air Force Academy cadet Bryan Ashton. 


\section{Researchers Study Hybrid Energy Systems}

\section{Robert Cherry}

10-067

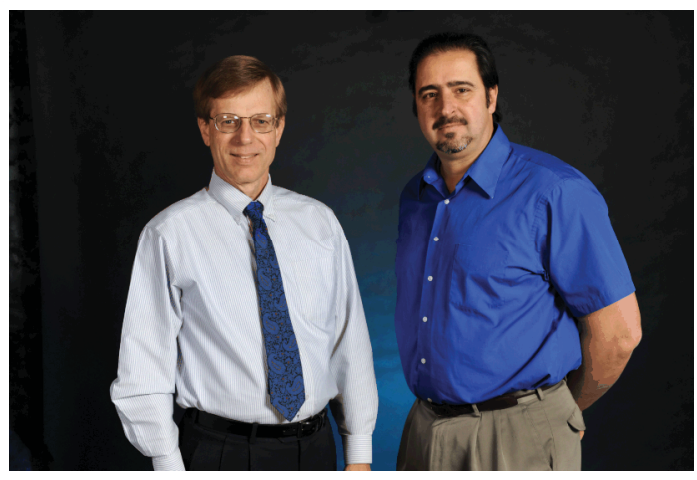

Two concerns about America's energy security are having a secure supply of transportation fuels and reducing greenhouse gases. INL researchers have been studying how to address both of these concerns.

Entering the third and final year of a LDRDfunded project, INL's Robert S. Cherry and Humberto Garcia, along with the Massachusetts Institute of Technology's Charles Forsberg, have studied hybrid energy systems (HESs) and the challenges of integrating dynamic renewable energy sources.

Cherry explained that, "Hybrid energy systems take advantage of the complementary characteristics of different energy conversions to improve a combined system's environmental and energy security performance. For INL, the concept includes making more than just electricity as the product, because we want to make domestic synthetic vehicle fuels too. We also have a special, but not exclusive, interest in using nuclear reactors as an energy source."

Noting that many combinations are possible, Cherry elaborated, "For instance, a nuclear power plant can supply low- $\mathrm{CO}_{2}$ electricity during the day when demand is high and then shift its excess output at night to the production of stored hydrogen for use in a synfuels plant running around the clock."

Co-generation systems have been built in the past and are a form of HES, but they have been limited to fossil fuel combustion for the production of power and steam heat only. The INL hybrid energy concept is more broadly useful because it includes synthetic fuel as a product during off-peak demands.

One specific goal of this research is to understand how choosing the components in a large hybrid energy system can increase the amount of renewable or nuclear energy that can be used in place of fossil energy. Successful development of such hybrid systems would help ensure America's energy supply while reducing its environmental impacts.

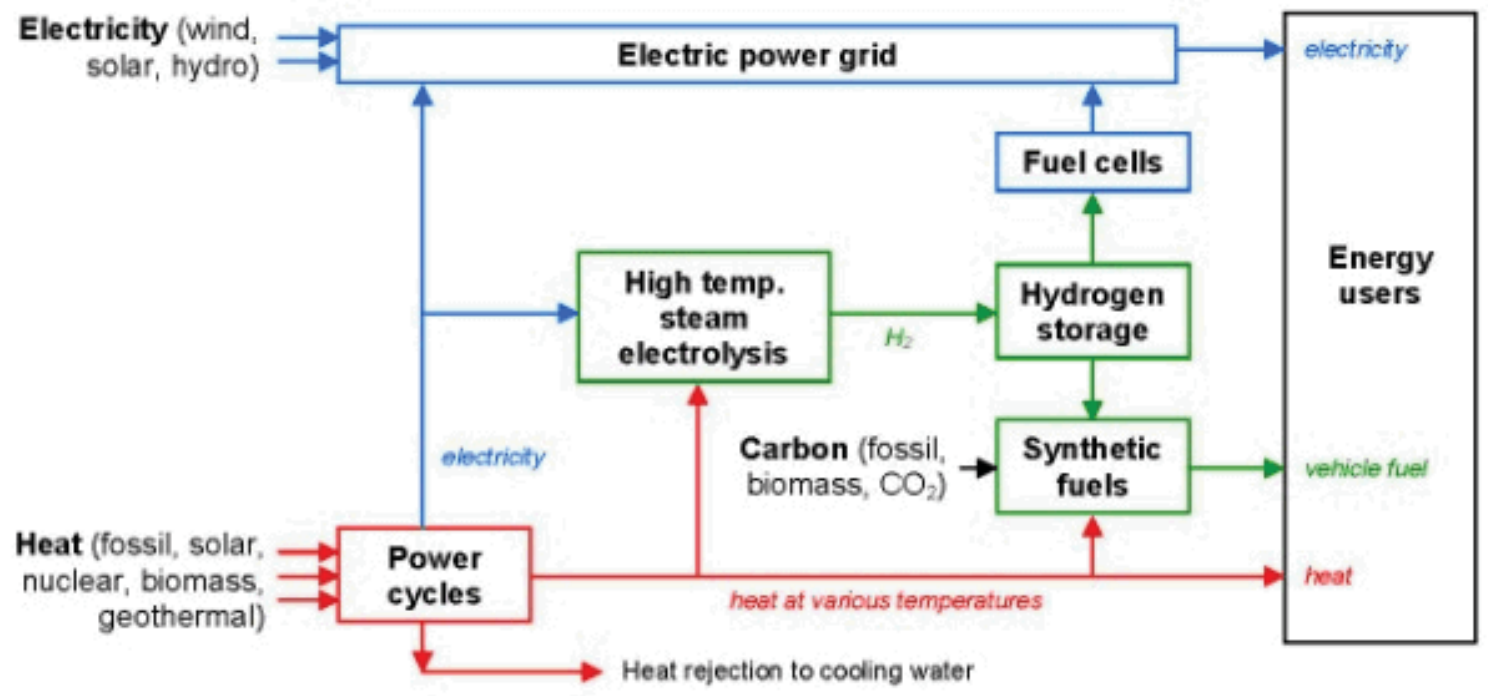

Energy conversions in an example hybrid energy system. 
The key challenge is matching these energy supplies to energy demand, since wind, solar, and, on a seasonal scale, hydropower, are available in quantities and on schedules set by the weather.

Nuclear energy has the opposite problem of needing to run at constant high rates for technical and economic reasons, limiting its use to base load supply rather than economically responding to time-varying demands. Making synthetic fuels for transportation use as a second product can be a tool for balancing electricity supply and demand. It may also aid in reducing carbon dioxide emissions by reusing captured carbon dioxide.

"Effectively combining energy production from various sources into hybrid energy systems and having it available when needed are major challenges," said Cherry. "Our recent work has focused on dynamic analysis of the integration of variable renewable energy into systems making both power and synthetic fuels."

One line of work demonstrated that hybrid systems can provide, from intermittently available renewable power generation capacity, enough energy to make methanol equivalent to five million barrels per day of gasoline.
Methanol can be easily converted into gasoline. This amount is about equal to the quantity of petroleum imported from OPEC. If renewable biomass rather than natural gas is the carbon feed, the energy equivalent of about 4.3 million barrels of oil per day can be made.

Dynamic models of various unit operations were developed and integrated to evaluate the performance of two different HES options. One HES option, termed "traditional," produces electricity only and consists of a primary heat generator, a steam turbine generator, a wind farm, and a battery storage. The other HES option, termed "advanced," includes not only the components present in the traditional solution but also a chemical plant complex to use excess energy for non-electricity services, such as the production of synthetic fuel.

Dynamic performance analyses of these highly coupled energy configurations were completed, as well as comparative dynamic cost analyses. Preliminary results identified that the advanced HES option becomes increasingly more economical than a traditional electricitygeneration-only solution when teamed with more than 20 percent of renewable power generation. This is about the upper limit of current power grids. 


\section{Creating Hydrogeophysics Models for Subsurface Understanding \\ Hai Huang \\ 11-045}

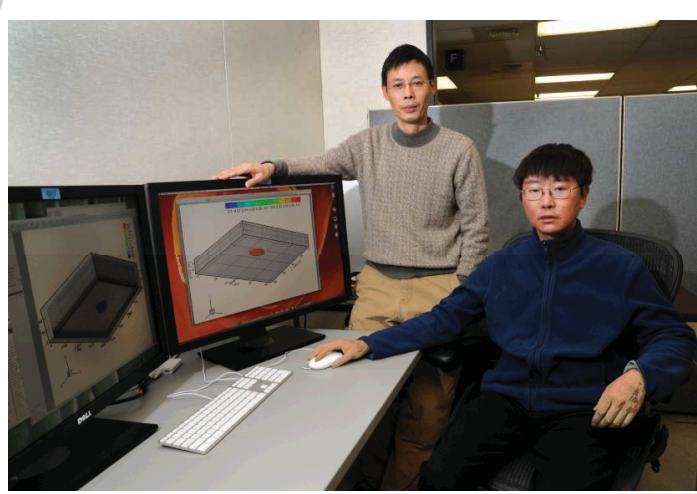

Predicting the future is attempted with great uncertainty. Ask any Wall Street stock analyst.

Multiply the stock market several fold and you get an idea of what scientists are dealing with when they want to predict the behavior of fluids moving in the subsurface. Understanding where and how fast oil, gas, water, and contaminants move through the geologic media are important for many energy and environmental purposes.

At INL, scientists have found that reactive transport models, when calibrated by real-time, high-resolution geophysical measurements, can significantly reduce the uncertainty of predictions, more accurately represent long-term behavior, and aid in the design of energy developments.

Regulators want the ability to more reliably predict and more effectively monitor remediation processes at contaminated DOE sites to reduce risk to communities, protect natural resources, and enhance treatment success. Industry wants the ability to better design well drilling and hydraulic fracturing programs to be more effective at extracting resources from the earth. Coupled hydrogeophysics models may offer some solutions to predicting conditions. By measuring electrical, hydraulic, and transport properties of fluids simultaneously, the feedback can be used to better refine numerical simulations of long-term system behavior.

INL's Hai Huang and Chuan Lu, joined by Tim Johnson of Pacific Northwest National Laboratory, are working to create predictive hydrogeophysics models during a two-year LDRD project titled "Multiscale Coupled Hydrogeophysics Modeling-Advancing Understanding of Reactive Transport and Effective Subsurface Environmental Monitoring."

The main objective of the project is ambitious. The team is seeking "to develop a parallel, fully coupled, multiscale, reactive transport and complex resistivity model that works at both pore and continuum scales." This is being accomplished by unifying three software components: information from a reactive transport simulator, a pore-scale complex conductivity predictor, and a 3D complex resistivity inversion code.

"A validated software capability like this will enable us to better understand complex conductivity signals in terms of the fundamental properties of geochemical and geophysical processes," Principal Investigator Huang said.

One year into the research, Huang touted some success: "Within FY 2011, we developed a methodology for coupling a reactive transport model with time-elapse electric resistivity tomography (ERT) data inversion that provides real-time effective monitoring of the migration of contaminant plume." During FY 2011, the team also conducted numerical assessments for use as the approach for monitoring - for instance, tracking the injection of $\mathrm{CO}_{2}$ into the subsurface for sequestration or optimizing the extraction of oil and gas from a subsurface reservoir.

As a demonstration of the approach, the team investigated the possibility of using ERT coupled with the hydrogeophysics model for monitoring $\mathrm{CO}_{2}$ geological sequestration. Figures 1-3 show results from the simulation. Figure 1 shows the growth of a pocket of $\mathrm{CO}_{2}$ in the subsurface over a 10-year period. Figure 2 shows the locations of electrodes that could hypothetically be employed to monitor the growth of the $\mathrm{CO}_{2}$ over time. Figure 3 shows what the hypothetical ERT network would have measured; there is excellent quantitative agreement. 
Huang observed that "this novel introduction of ERT inversion to [the] CCS community could guide INL's subsurface modeling group into this frontline research field."

During FY 2012, work will be focused on pore-scale reactive transport processes and volume-averaged complex resistivity. Activities include performing coupled continuum-scale reactive transport and complex resistivity simulations under conditions representing fieldand laboratory-scale experiments. This will allow the model to be validated and calibrated to "real" data. Success will be the development of a finite-element-based 3D pore-scale complex resistivity model and implementation of the model within INL's MOOSE framework. The goal is to create better understanding of the relationships between volume-averaged complex resistivity signals and pore-scale reactions.

This research will:

1. Lead to a better understanding of the fundamental physics of pore-scale processes, improving conceptual models for more accurate field-scale contaminant fate and transport predictions

2. Place INL as one of the leaders in quantitative linkage between the pore-scale biogeochemical reaction processes and the complex electrical properties of the porous media

3. Aid INL in developing critical coupled hydrogeophysical modeling capabilities

4. Develop a physics-based process for realtime monitoring of evolution of fossil energy reservoirs during resource extraction and development.
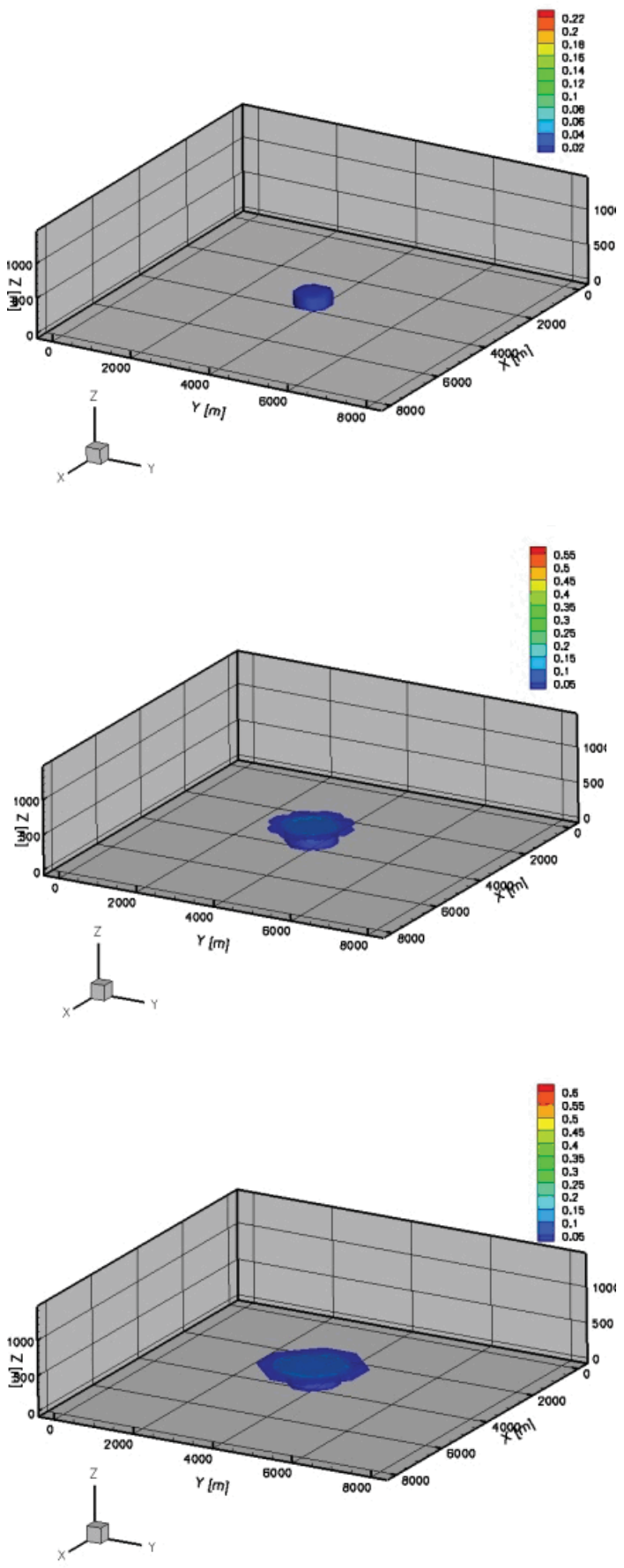

FIGURE 1. Simulated $\mathrm{CO}_{2}$ plume migration in deep saline reservoir at 1, 3, and 10 years. 


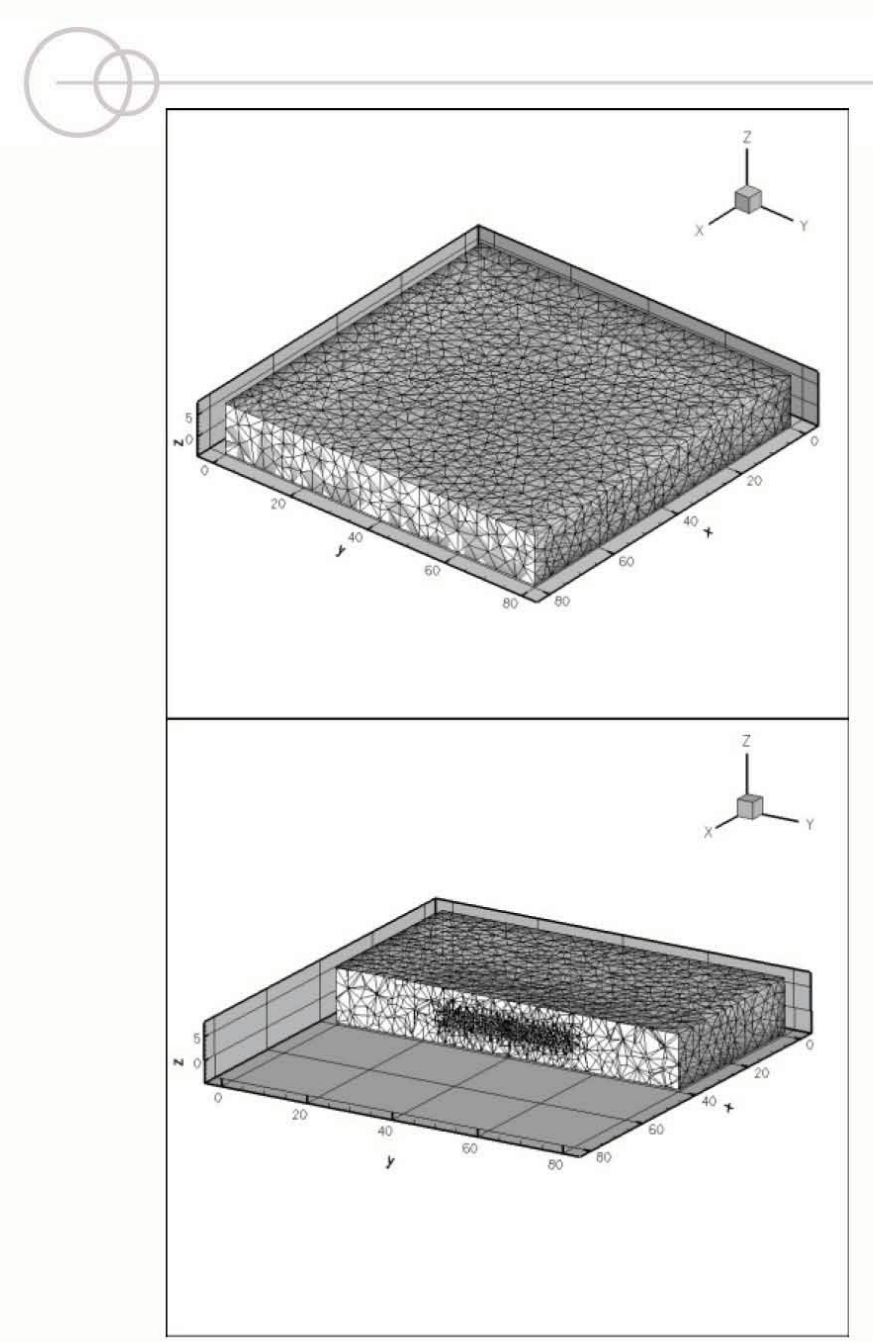

FIGURE 2. Finite element mesh used in ERT forward and inversion calculations.

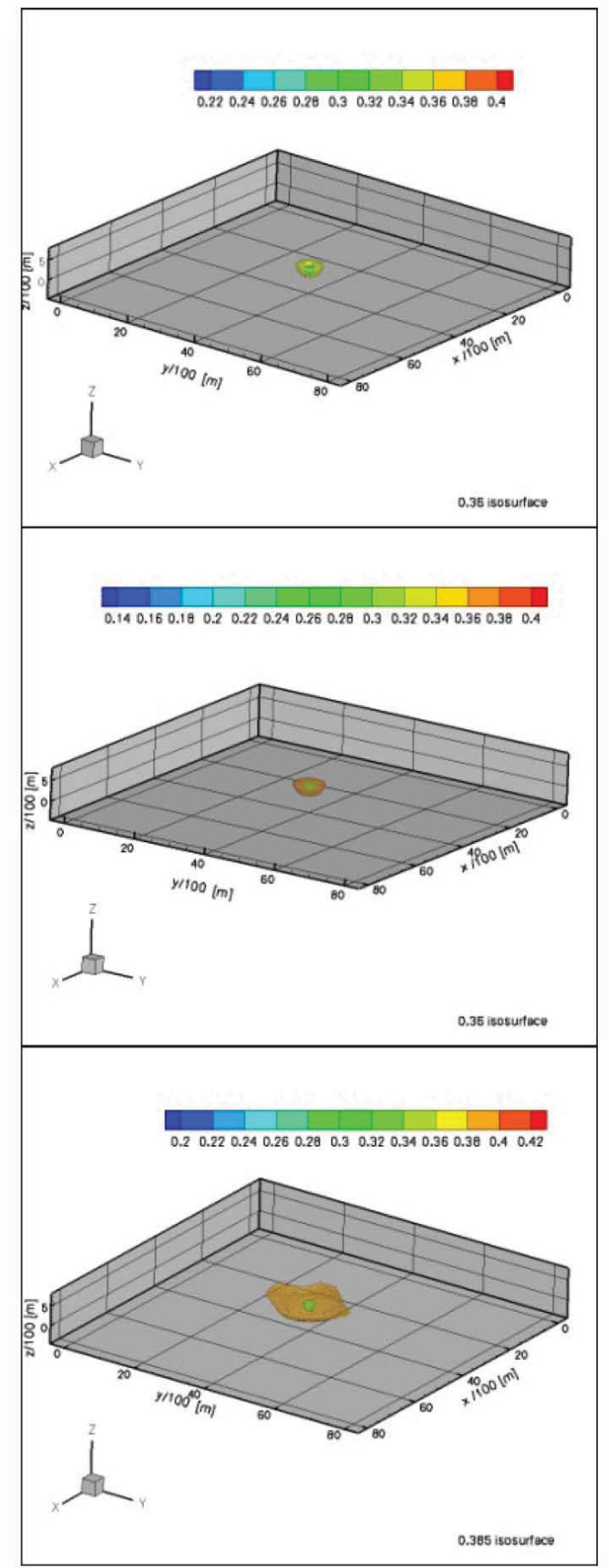

FIGURE 3. Inverted electric conductivity fields from ERT inversion show the growth and migration of the $\mathrm{CO}_{2}$ plume. 


\section{Collaborative Project Aims to Model the Recovery of Valuable Materials Locked in Used Nuclear Fuel}

\section{Peter Zalupski}

$10-027$

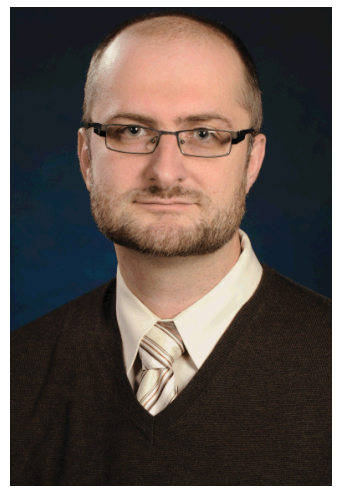

Nuclear fuel recycling technologies could help make use of the valuable energy resource residing inside used fuel rods. To retrieve that resource from used fuel, materials that jump-start the chain reaction must be separated from the components that interfere with fission. Although existing techniques to separate uranium and plutonium are well understood, new approaches will be needed to recover other materials that can fuel advanced fast reactors. However, new aqueous separations methods can be challenging to develop and test.

Recovering valuable nuclear material from used fuel requires complex chemistry involving highly radioactive materials. Design and development of new approaches involves experimentation and testing that is exceptionally challenging because of the materials involved. INL - the nation's nuclear energy research labis uniquely qualified in advanced separations research and development, and this project exemplifies a more fundamental approach to advancing the nuclear fuel cycle.

The aim of this LDRD project is to streamline development of new separations techniques by devising a computational model that accurately predicts how molecules behave in the complex chemical separations environment. Such a model would allow researchers to finetune new extraction processes before heading into the lab to test them. This project adds to INL's existing separations capabilities and supports DOE's mission to develop, test, and ensure the reliability of advanced nuclear fuel cycles.
"Twenty-first century computational science is becoming a powerful tool when predicting outcomes of aqueous separations," said INL research scientist Peter Zalupski, the project's principal investigator. "Spent fuel is a very complex mixture; roughly one third of the periodic table is in there. What we're looking to do is to come up with a way to computationally mimic that type of environment to be able to predict final destinations of uranium, plutonium, and other actinides when we perform separations."

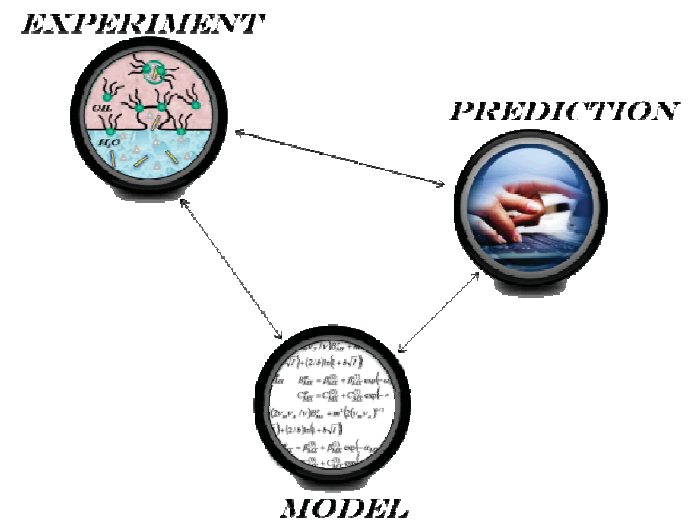

Experimental methods and theoretical modeling can be combined to computationally predict separation of valuable resources from used nuclear fuel.

Separations scientists aim to recover the actinide elements (such as uranium and plutonium) and leave behind the members of the lanthanide family, which capture neutrons and poison the fission process. However, actinides and lanthanides are chemically similar to each other and require complex chemistry to separate. The complicated, concentrated mixtures force molecules to behave in "non-ideal" ways that differ from behavior in more simple solutions.

"In order to predict where actinides go in a typical two-phase separation - whether they stay in the aqueous mixture or travel to the organic solution-you need to have a very, very good grasp on how to theoretically handle non-ideal solution behavior," Zalupski said. 
Two collaborators have partnered with Zalupski to develop a model that accurately describes such behavior. Simon Clegg, at England's University of East Anglia, develops mathematical models. In the first stage of the project, Zalupski and Clegg constructed an initial model to describe molecular interactions in aqueous mixtures that resemble dissolved used nuclear fuel solution. They have also identified the types of interactions that are most important to understand.

Zalupski is collecting experimental data, which Clegg feeds into the model and uses to refine and revise it. Laetitia Delmau at Oak Ridge National Laboratory is using a computational package called Solvent Extraction Fit (SXFIT) to test the accuracy of the model and its predictions.

"That's sort of the end point that demonstrates we can then use that methodology as we progress to design new separation processes," Zalupski said. "If we are able to prove that we can computationally predict with good accuracy, then we have this capability that we can use whenever a new separations scheme is proposed."

Zalupski presented these initial results at the June 2011 American Nuclear Society meeting in Hollywood, Florida. Similar approaches are used to analyze atmospheric chemistry and make climate predictions, but the effort to devise a nuclear fuel separations model is unique to INL. A computational capability such as this could provide a powerful tool to accelerate and simplify development of advanced approaches for recycling used nuclear fuel.

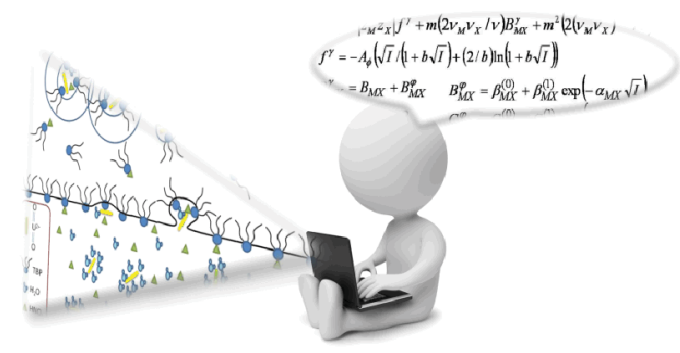

The project builds numerical representation of solutions of dissolved nuclear fuel to project complex chemical behavior on a computer screen. 
$\Rightarrow$ Nuclear Science and Technology

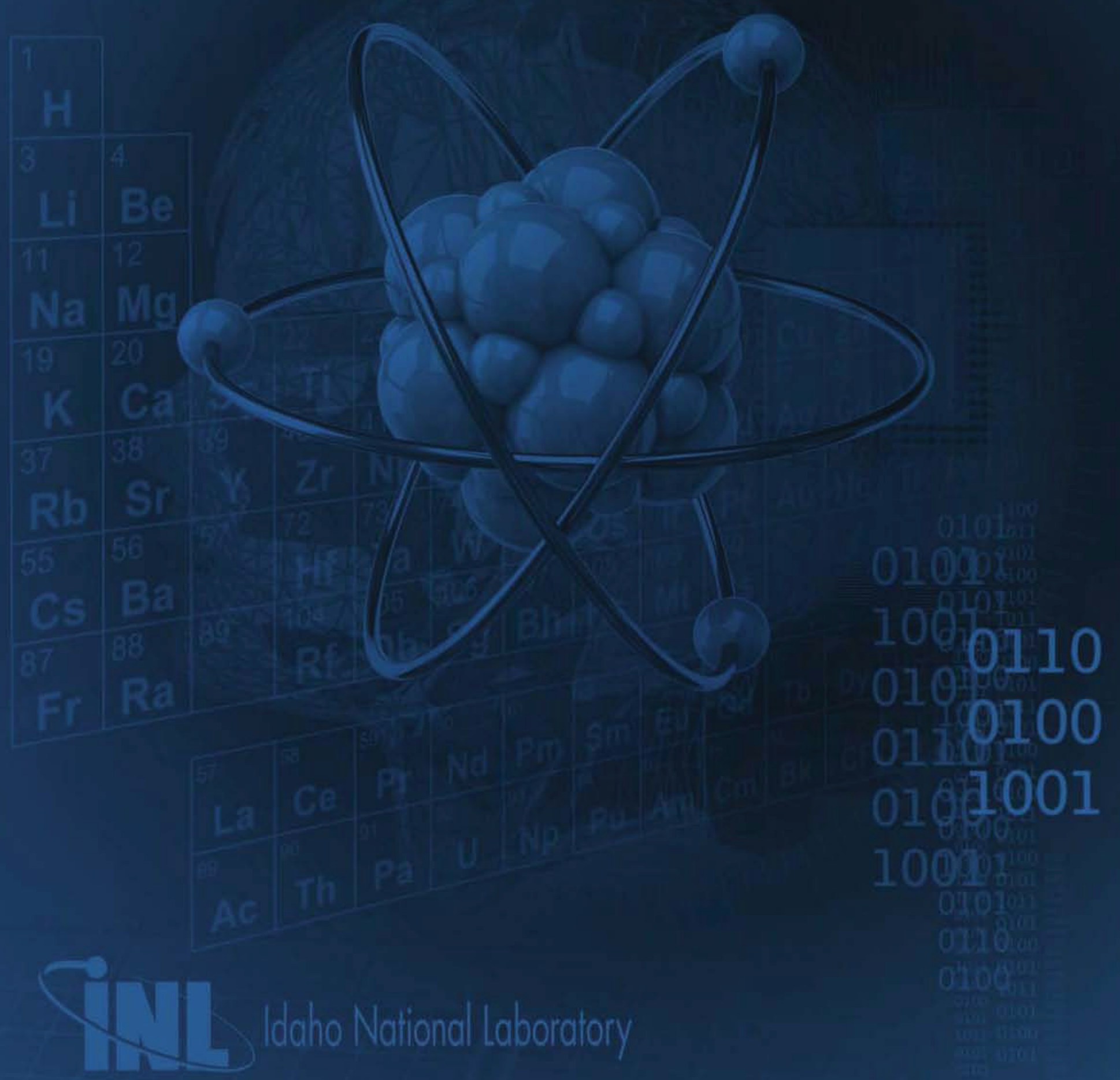




\section{Development of Reactor Physics Sensitivity Analysis, Uncertainty Quantification, and Data Assimilation Capability at INL for Validation Applications Cristian Rabiti, Giuseppe Palmiotti 09-013}

This project's ultimate goal is to provide a reactor physics design environment in which the tools and methodologies to perform uncertainty quantifications are embedded in the environment itself.

The requirements to achieve such a goal are twofold: (1) the acquisition of and/or development of tools to perform reactor physics simulations, and (2) the development of new algorithms and theory to be able to extend the capability to perform uncertainty quantifications in non-linear phenomena.

\section{Project Summary}

The major outcome of this project has been the creation of a computational framework to perform reactor physics simulations in which the basic capability to perform sensitivity analyses based on the adjoint methodology is embedded. This software has been named PHISICS (Parallel Highly Innovative Simulation INL Code System) and was recently submitted for copyright application. PHISICS is a modular environment to perform reactor physics simulations. The environment is built by data-type containers, kernels, and drivers.

Data-type containers are used to define a common standard among the different computational units of the environment (kernels) to exchange data. Data-type containers are usually contained within modules that also provide the capability to handle such data like input/output functions and data mining. Currently, we have such structures to contain mesh, geometry, cross sections, and dependent variables defined by the mesh.

Kernels are instead solver dedicated to the solution of a specific physical aspect of the simulation. The calculations performed by the kernels are driven by the drivers that essentially coordinate the kernels to perform the overall calculation. Presently, we have a kernel performing the solution of the transport equation based on hybrid finite element in space and spherical harmonics in angle, called the Intelligent Nodal Semi-structured Transport Nodal, or INSTANT. The hybrid elements supported are cartesian 2/3D, hexagonal 2D and extruded, unstructured triangular 2D and extruded. During FY 2011, we have added the capability to perform adjoint calculations for all of the supported geometries discussed previously. The adjoint capability is one of the enabling features needed for the embedding of uncertainty quantification capability in the framework.

A second kernel added in FY 2011, called the multi-reactor transmutation analysis utility, or MRTAU, is the one solving the Bateman equation, which is accomplished by using an arbitrary order Taylor or rational Chebyshev approximation of the exponential matrix.

The above kernels are driven by several drivers (two funded under this project and an additional one established previously). The drivers supported under this project realize source or $\mathrm{k}_{\text {eff }}$ core calculation (using the INSTANT kernel), multi-fuel recycle (zero dimensional) using MRTAU stand alone, and whole core depletion calculation (using INSTANT and MRTAU).

\section{Benefits to DOE}

This project focuses on reactor physics simulation enhancement. After three years, we have created a tool that increases the simulation capability available at DOE in terms of both flexibility and accuracy. The environment developed here has already been used by the Next-Generation Nuclear Power campaign to perform analysis for very high temperature gas reactors. The flexibility of the framework and the general approach will easily allow extension of its capabilities and, consequently, meeting future requirements.

\section{Relevant Publications and Presentations}

Kennedy, C., C. Rabiti, and H. Abdel-Khalik, 2011,"GPT-Free Sensitivity Analysis for Eigenvalue Problems," Proceedings of the American Nuclear Society 2011 Annual Winter Meeting, Washington, DC, USA, October 30-November 3, 2011.

Kennedy, C., C. Rabiti, and H. Abdel-Khalik, 2011, "On Rank Determination for Subspace Methods," Proceedings of the American Nuclear Society 2011 Annual Winter Meeting, Washington, DC, USA, October 30-November 3, 2011. 
Rabiti, C., Y. Wang, G. Palmiotti, H. Hiruta, J. Cogliati, A. Alfonsi, A. Epiney, and T. Grimmet, 2011, "PHISICS: New Features and Advancements," Proceedings of the American Nuclear Society 2011 Annual Winter Meeting, Washington, DC, USA, October 30-November 3, 2011.

Rabiti, C., Y. Wang, G. Palmiotti, H. Hiruta, J. Cogliati, and A. Alfonsi, 2011, "PHISICS: A New Reactor Physics Analysis Toolkit," Proceedings of the American Nuclear Society 2011 Annual Meeting, Hollywood, Florida, USA, June 27-30, 2011.
Wang, Y., C. Rabiti, and G. Palmiotti, 2011, "Parallelization of the Red-Black Algorithm on Solving the Second-Order PN Transport Equation with the Hybrid Finite Element Method," Proceedings of the American Nuclear Society 2011 Annual Meeting, Hollywood, Florida, USA, June 27-30, 2011.

Kennedy, C., C. Rabiti, and H. Abdel-Khalik, 2011, "GPT-Free Sensitivity Analysis for Eigenvalue Problems," Nuclear Science and Engineering (submitted). 


\section{Development of a Next-Generation Production Code for Nuclear Reactor System Analysis and Safety Margin Quantification}

T. N. Dinh; R. Nourgaliev, B. Smith (Utah State University), S. Shkoller (University of California-Davis), V. Mousseau, H. Zhao, K. Mousseau, R. Barry, B. Briggs, A. Bui, S. Kadioglu, A. Ougouag 09-033

During the first year of this project (FY 2009), our goal was to investigate the requirements for the next generation of reactor system safety analysis tools and evaluate advanced solution algorithms for thermal hydraulics that can meet those requirements. During FY 2010 and FY 2011, the project was directed toward basic research on models and methods needed to enable development, demonstration, and validation of next-generation system simulation and safety analysis codes. The research focus is placed on fluid flow homogenization and high-order-accurate solution schemes, two-phase flow hyperbolicity, validation data, and uncertainty quantification.

\section{Project Summary}

Research conducted on the next-generation safety analysis code in FY 2009 significantly improved a basic understanding of objectives and functionality of a future "reactor system safety analysis" tool. The improved understanding paves the way for formulation of $\mathrm{R} 7$ as a computational engine for a risk-informed safety margin characterization process and greatly influences the R7 software's requirements.

A novel single-phase flow modeling and homogenization method, called the Lagrangian averaging model, was also developed, which successfully removes small-scale spurious oscillations from smooth flows and offers an order-of-magnitude scale-up in the computational mesh. This includes an extension, the alpha-beta model, which uses the proposed homogenization approach in the smooth-flow regime, and a new shock-capturing technique in the discontinuous flow regime. A new nonlinear method was developed and demonstrated for the theoretical maximum accuracy and stability; this method significantly outperformed classical eddy viscosity methods.

Different approaches to two-phase flow modeling hyperbolization were studied as well, with a focus on testing the potential of a sevenequation model and discrete element method. Applicability of the models to describe steamwater flows in a reactor safety code was also analyzed. The study suggests that a modified sixequation model with adjustment for consistent closure relations is a preferred option. As such, a novel approach for the hyperbolization of the two-phase, six-equation flow model was introduced and analyzed.

Advanced numerical solution algorithms research focuses on high-order-accurate numerical methods in all-speed (all-Mach) compressible flow. The results confirmed the hypothesis about the potential for significant improvement of computational efficiency while maintaining solution accuracy by means of a fully implicit Jacobian Free Newton-Krylov algorithm and an rDG scheme.

Experimental uncertainty quantification and validation support activity focused on the development of automatic uncertainty quantification for particle image velocimetry and the design and construction of a facility for computational fluid dynamics validation. The work demonstrated convincingly that particle image velocimetry uncertainty varies in space and can be computed with minimal expense to the user.

Computational fluid dynamics verification and validation data management was initiated at INL as part of FY 2010 activity in this project. This has led to the development of a proof-ofconcept configuration, acquiring and loading the first set of high-quality experimental data (supplied by Bettis and Utah State University) to the system. The activity has attracted a broad base of attention from industry, academia, and regulators.

\section{Benefits to DOE}

This research provided the technical capabilities and expertise needed to develop analytical tools that transform the current practice in system simulations and safety analysis, as critically needed to support safety and economic improvements and life extension of the aging fleet of light water reactor nuclear power plants. The insights generated are instrumental to the formulation of research and development scope and planning for DOE's Light Water Reactor Sustainability Program's risk-informed safety margin characterization pathway. The project also supported training of graduate students and postdoctoral researchers at Idaho State University, University of California- 
Davis, and Utah State University. The outcome of this research was used to train nearly 100 young researchers and engineers in the field of reactor thermal hydraulics and reactor safety at the 2009 and 2010 Nuclear Engineering Summer School, the "Modeling, Experimentation and Validation in Nuclear Thermal Hydraulics and Safety Analysis (MeV)."

\section{Relevant Publications and Presentations}

Luo, H., L. Luo, A. Ali, R. Nourgaliev, and C. Cai, 2011, “A Parallel, Reconstructed Discontinuous Galerkin Method for the Compressible Flows on Arbitrary Grids," Communication in Computational Physics, Vol. 9, No. 2, pp. 363-389.

Harris, J., D. Nani, K. Jones, M. Khodier, and B. L. Smith, 2011, "Investigation of the Uncertainty of a Validation Experiment due to Uncertainty in its Boundary Conditions," NURETH 14-546, The 14th International Topical Meeting on Nuclear Reactor Thermalhydraulics, NURETH-14, Toronto, Ontario, Canada, September 25-30, 2011.

Wilson, B., and B. L. Smith, 2011, "Errors in Mean and Fluctuating Velocity Due to PIV Bias and Precision Uncertainties," NURETH 14-616, The 14th International Topical Meeting on Nuclear Reactor Thermalhydraulics, NURETH-14, Toronto, Ontario, Canada, September 25-30, 2011.

Dinh, T.-N., R. Nourgaliev, and R. W. Youngblood, 2011, "A System Simulation Code to Support the Safety Case in the LWR Life Extension: Selection, Development, and Testing of Code Architecture and Solution Algorithms," Invited panel speaker, ANS Annual Meeting, Hollywood, Florida, USA, June 26-30, 2011.

Bui, A., R. Nourgaliev, and T.-N. Dinh, 2011, "On Physics Modeling and Components Development in R7," ANS Annual Meeting, Hollywood, Florida, USA, June 26-30, 2011.

Nourgaliev, R., T.-N. Dinh, and R. Nelson, 2011, "Lessons Learned from an Initial VUAssessed Code Development Effort," ANS Annual Meeting, Hollywood, Florida, USA, June 26-30, 2011.
Luo, H., Y. Xia, R. Nourgaliev, and C. Cai, 2011, "A Reconstructed Discontinuous Galerkin Methods for Compressible Flows on Tetrahedral Grids," AIAA-2011-3410, The 20th AIAA Computational Fluid Dynamics Conference, Honolulu, Hawaii, USA, June 27-30, 2011.

Luo, H., H. Xiao, R. Nourgaliev, and C. Cai, 2011, "A Comparative Study of Different Reconstruction Schemes for Reconstructured Discontinuous Galerkin Methods for Compressible Flows on Arbitrary Grids," AIAA-2011-3839, The 20th AIAA Computational Fluid Dynamics Conference, Honolulu, Hawaii, USA, June 27-30, 2011.

Nourgaliev, R., H. Luo, and Y. Xia, 2011, “A Class of Reconstructed Discontinuous Galerkin Methods in Computational Fluid Dynamics," The International Conference on Mathematics Numerical Methods Applied to Nuclear Science and Engineering, Rio de Janeiro, Brazil, May 8-12, 2011.

Smith, B. L., B. H. Timmins, and P. Vlachos, 2011, "A Method for Automatic Estimation of Instantaneous Local Uncertainty in Particle Image Velocimetry Measurements," The PIV Uncertainty Workshop, Las Vegas, Nevada, USA, May 11, 2011.

Luo, H., Y. Xia, R. Nourgaliev, and C. Cai, 2011, "A Class of Reconstructed Discontinuous Galerkin Methods for the Compressible Flows on Arbitrary Grids," AIAA-2011-0199, The 49th AIAA Aerospace Sciences Meeting including the New Horizons Forum and Aerospace Exposition, Orlando, Florida, USA, January 4-7, 2011.

Luo, H., L. Luo, Segawa, H., H. Luo, and R. Nourgaliev, 2011, “A Diagonally Implicit Runge-Kutta Method for the Discontinuous Galerkin Solutions of the Navier-Stokes Equations," AIAA-2011-0685, The 49th AIAA Aerospace Sciences Meeting including the New Horizons Forum and Aerospace Exposition, Orlando, Florida, USA, January 4-7, 2011. 
Wilson, B. M., B. L. Smith, and R. E. Spall, 2011, "Examples of Unsteady CFD Validation Quantities in a Cylinder Array," Nuclear Technology, submitted.

Smith, B. L., B. H. Timmins, and P. Vlachos, 2011, "A Method for Automatic Estimation of Instantaneous Local Uncertainty in Particle Image Velocimetry Measurements," Experiments in Fluids, submitted.

Serencsa, J., and S. Shkoller, 2011, “A Nonlinear Artificial Diffusion Method for Hyperbolic Systems," submitted.

Coutand, D., and S. Shkoller, 2011, "WellPosedness in Smooth Function Spaces for Moving-Boundary 3D Compressible Euler Equations in Physical Vacuum," submitted.

Coutand, D., J. Hole, and S. Shkoller, 2011, "Well-Posedness of the Free-Boundary Compressible 3D Euler Equations with Surface Tension," submitted.

Berry, R. A., R. Saurel, and O. LeMetayer, 2010, "The Discrete Equation Method (DEM) for Fully Compressible, Two-phase Flows in Ducts of Spatially Varying Cross-sections," Nuclear Engineering and Design, Vol. 240, pp. 3797-3818.

Nourgaliev, R., V. A. Mousseau, and N. Dinh, 2010, "A Reconstructed Discontinuous Galerkin Method for the Compressible Navier-Stokes Equations on Arbitrary Grids," Journal of Computational Physics, Vol. 229, pp. 6961-6978, doi: 10.1016/j.jcp 2010.05.033.

Zhao, H., and P. F. Peterson, 2010, “An Overview of Modeling Methods for Thermal Mixing and Stratification in Large Enclosures for Reactor Safety Analysis," The 8th International Topic Meeting on Nuclear Thermal-Hydraulics, Operation and Safety (NUTHOS-8), Shanghai, China, October 10-14, 2010.

Mousseau, K., 2010, "Development of Database to Support V\&V of CFD Codes," Keynote Address, The 3rd Workshop on Computational Fluid Dynamics for Nuclear Reactor Safety (CFD4NRS), Washington, DC, USA, September 14-16, 2010.
Wilson, B., J. Harris, B. L. Smith, and R. E. Spall, 2010, "Unsteady Validation Metrics for CFD in a Cylinder Array," The $3^{\text {rd }}$ Workshop on Computational Fluid Dynamics for Nuclear Reactor Safety (CFD4NRS), Washington, DC, USA, September 14-16, 2010.

Timmins, B. H., B. L. Smith, and P. P. Vlachos, 2010, "Automatic Particle Image Velocimetry Uncertainty Quantification," Paper 2010-30724, ASME Fluids Engineering Conference, Montreal, Quebec, Canada, August 1-4, 2010. (Winner, American Society of Mechanical Engineers Lewis F. Moody Award.)

Wilson, B., J. Harris, B. L. Smith, and R. E. Spall, 2010, "Validation of Unsteady CFD in a Confined Row of Cylinders," Paper 2010-30720, ASME Fluids Engineering Conference, Montreal, Quebec, Canada, August 1-4, 2010.

Nourgaliev, R., H. Luo, and L. Luo, 2010, "A Reconstructed Discontinuous Galerkin Method for the Compressible Flows on Arbitrary Grids," The Sixth International Conference on Computational Fluid Dynamics, St. Petersburg, Russia, July 12-16, 2010

Luo, H., L. Luo, and R. Nourgaliev, 2010, "A Reconstructed Discontinuous Galerkin Method for the Compressible Flows on Arbitrary Grids," The Fifth European Conference on Computational Fluid Dynamics, Lisbon, Portugal, June 14-17, 2010.

Dinh, T.-N., 2010, “Modeling, Simulation, and Validation to Support LWR Plant Life Extension," The 2nd Workshop on V\&V of Nuclear System Analysis, Myrtle Beach, South Carolina, USA, May 24-28, 2010.

Nourgaliev, R. 2010, "V\&V and UQ of Next Generation System Code," Invited presentation, The 2nd Workshop on V\&V of Nuclear System Analysis, Myrtle Beach, South Carolina, USA, May 24-28, 2010. 
Berry, R. A., and R. Saurel, 2010, "Properties of the Discrete Equation Method (DEM) for Two-phase Nozzle Flows," The 18th International Conference on Nuclear Engineering (ICONE18), Xi'an, China, May 17-21, 2010.

Zhao, H., and V. A. Mousseau, 2010, "Use of Forward Sensitivity Analysis Method to Improve Code Scaling, Applicability, and Uncertainty (CSAU) Methodology," The 18th International Conference on Nuclear Engineering (ICONE18), Xi'an, China, May 17-21, 2010.

Luo, H., L. Luo, R. Nourgaliev, and V. A. Mousseau, 2010, “A Reconstructed Discontinuous Galerkin Method for the Compressible Navier-Stokes Equations on Arbitrary Grids," AIAA-2010-0364, The 48th AIAA Aerospace Sciences Meeting Including the New Horizons Forum and Aerospace Exposition, Orlando, Florida, USA, January 4-7, 2010.
Luo, H., A. Ali, R. Nourgaliev, and V. A. Mousseau, 2010, “A Parallel Reconstructed Discontinuous Galerkin Method for the Compressible Flows on Arbitrary Grids," AIAA-2010-0366, The 48th AIAA Aerospace Sciences Meeting Including the New Horizons Forum and Aerospace Exposition, Orlando, Florida, USA, January 4-7, 2010.

Luo, H., L. Luo, R. Nourgaliev, and V. A. Mousseau, 2009, "A Reconstructed Discontinuous Galerkin Method for the Compressible Euler Equations on Arbitrary Grids," AIAA-2009-3788, The 19th AIAA Computational Fluid Dynamics, San Antonio, Texas, USA, June 22-25, 2009.

Dinh, T.-N., V. A. Mousseau, R. R. Nourgaliev, and R.W. Youngblood, 2009, "A Next Generation of System Analysis Code to Support Risk-Informed Safety Margin Characterization," Keynote Lecture, The International Topical Meeting on Nuclear Reactor Thermal Hydraulics (NURETH-13), Kanazawa, Ishikawa, Japan,

September 27-October 2, 2009. 


\section{Nuclear Reactor Safety Case}

Vincent A. Mousseau, Haihua Zhao, Glen A. Hansen, Paul Bayless, Scott Lucas, Ling Zou, Hongbin Zhang

10-093

The major goal of this LDRD project is to quickly create a new generation production software foundation for reactor system analysis for different reactors based on new techniques in software engineering, advanced numerical methods, and physical models. Priority is being given to INL-developed tools (such as several MOOSE-based computer code applications like BISON and BIGHORN) and other advanced reactor physics codes, but the delivered product is intended to fill the gap between legacy system codes like RELAP5 and modern simulation tools like MOOSE-based applications, and meet the near-term needs of the Light Water Reactor Sustainability, Nuclear Energy Advanced Modeling and Simulation, Advanced Test Reactor, and Small Modular Reactor programs.

Our focus includes: (1) capturing the capabilities of current codes like RELAP5 to encapsulate the knowledge to validate, improve, and run the existing codes; (2) providing a flexible, extendable, scalable platform to test and implement advanced numerical methods and physical models at the system level; (3) having a modern system analysis code to couple with other advanced modern analysis tools that provide capabilities at different scales or higher dimensions for different physics; and (4) having a good foundation to quickly develop optimized system analysis codes for specific single fluid reactors, such as gas-cooled high-temperature reactors, sodium-cooled fast reactors, liquid-saltcooled reactors, or at the Advanced Test Reactor. Although RELAP5 can be used to simulate those reactors by adding new models or modifying existing models, its dated software structure and numerical algorithms make it difficult to add new models and have enough fidelity to simulate these reactors. It is much easier to implement those specific models directly in a new system framework and have better capabilities like multiple dimensional flow and heat transfer and strong multi-physics coupling.

\section{Project Summary}

During FY 2011, we developed a brand-new system analysis code pilot framework. Figure 1 (next page) shows the overview of the software structure design. In order to provide a flexible, extendable, scalable platform to test and implement matured and advanced numerical methods and physical models at the system level, modern software engineering principles have been strictly followed to develop this $\mathrm{C}++$ code.

Trilinos, a modern parallel, linear, and nonlinear solver package developed at Sandia, is used as the computational engine. R6 uses a Jacobian-free Newton-Krylov nonlinear solver provided by Trilinos to preserve and resolve the strong coupling between the physics components and improve the accuracy, robustness, and speed of the computer simulation. Backward compatibility with RELAP5 is preserved by having similar physics models and the same numerical method as one option. The extensive knowledge base within RELAP5 code and its validation database will be preserved.

At the end of FY 2011, this software framework has evolved to a relative stabile version that can solve 1D transient single fluid multi-physics problems with a modern numerical method. Major component models, such as a pipe, a junction, a heat exchanger, and a reactor core, have been implemented as well. Figure 2 (next page) provides the configuration of one test example to solve typical thermal hydraulics problems. The results match the RELAP5 simulation.

\section{Benefits to DOE}

This project will provide critical tools for creating science-based nuclear reactor safety cases that will benefit both the regulator and the nuclear energy industry. Increasing the percent of energy from nuclear power reduces the amount of greenhouse gas emissions. Nuclear power reduces our nation's dependence on foreign energy sources, which increases our national security. 


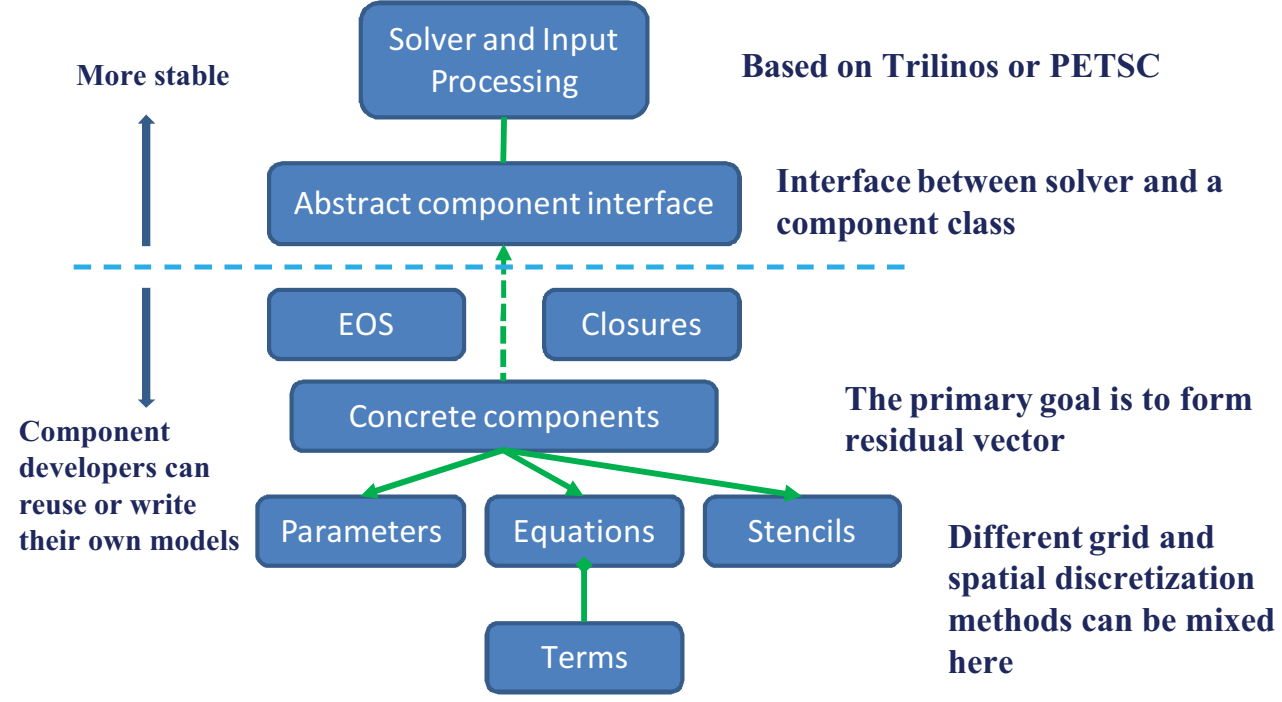

FIGURE 1. Overview of software design for the prototype code.
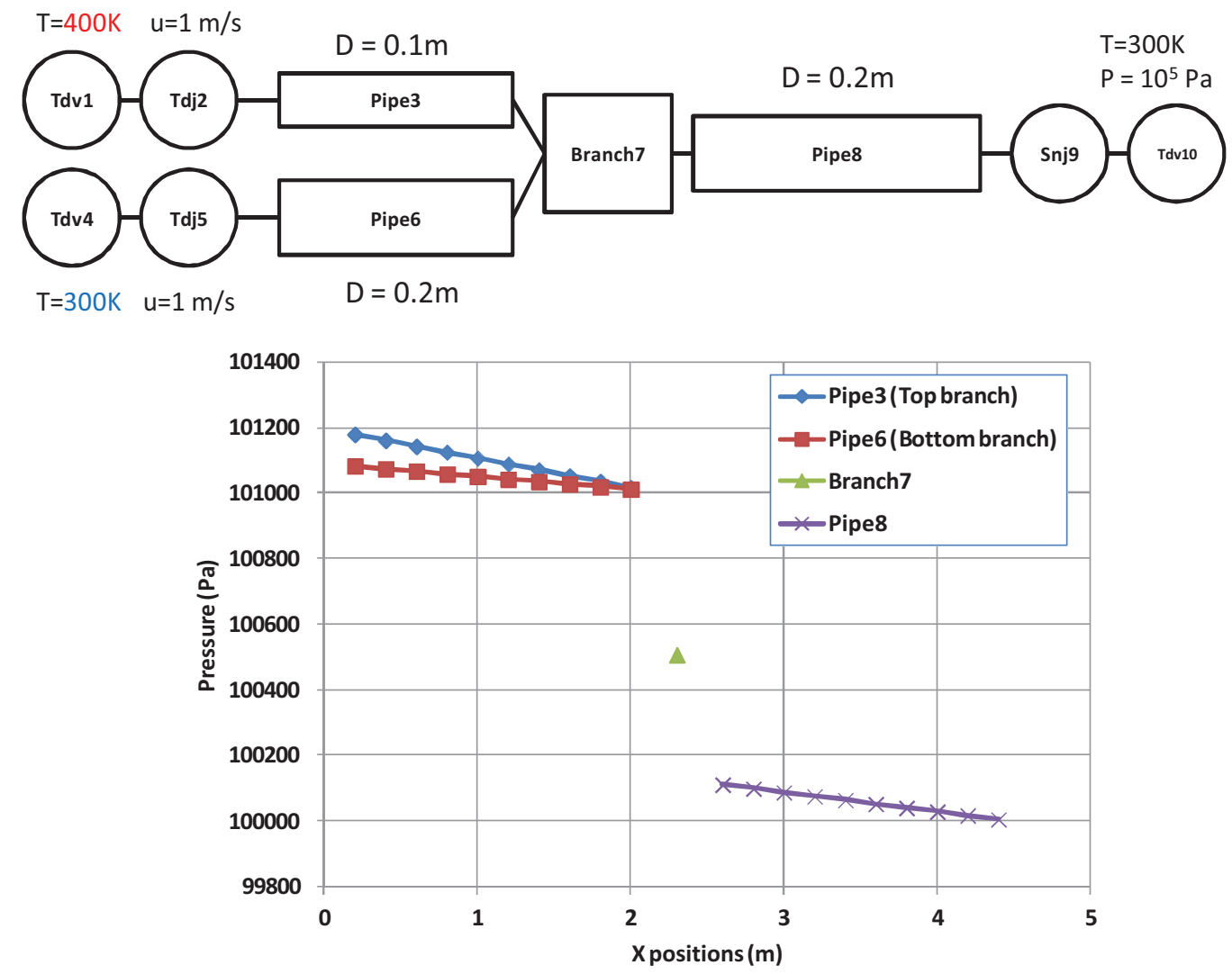

FIGURE 2. Test example - parallel flow and branch. 
Relevant Publications and Presentations

Zhao, H., V. A. Mousseau, 2012, "Use of Forward Sensitivity Analysis Method to Improve Code Scaling, Applicability, and Uncertainty (CSAU) Methodology," Nuclear Engineering and Design, in press.

Mahadevan, V. S., J. C. Ragusa, and V. A. Mousseau, 2012, "A Verification Exercise in Multiphysics Simulations for Coupled Reactor Physics Calculations," Progress in Nuclear Energy, in press.

Zhao, H., and V. Mousseau, 2011, "Extended Forward Sensitivity Analysis for Uncertainty Quantification," The 14th International Topical Meeting on Nuclear Reactor Thermal Hydraulics (NURETH-14), Toronto, Ontario, Canada, September 2530, 2011.

Wang, X., X. Sun, B. Doup, H. Zhao, 2011, "Dynamic Modeling Strategy for Flow Regime Transition in Gas-Liquid TwoPhase Flows," The 14th International Topical Meeting on Nuclear Reactor Thermal Hydraulics (NURETH-14), Toronto, Ontario, Canada, September 25-30, 2011.
Zhao, H., 2011, “Large Enclosure Thermal Mixing and Stratification Analysis for Reactor Safety," keynote address, 2nd Annual CAES Workshop on Modeling, Simulation, and Visualization, Boise, Idaho, USA, September 8-9, 2011.

Zhao, H., 2011, “A Pilot Framework for Modern Reactor System Analysis Codes," 2011 International RELAP5 User's Group Seminar, Salt Lake City, Utah, USA, July 25-28, 2011.

Zhao, H., P. F. Peterson, 2010, “An Overview of Modeling Methods for Thermal Mixing and Stratification in Large Enclosures for Reactor Safety Analysis," 8th International Topic Meeting on Nuclear ThermalHydraulics, Operation, and Safety (NUTHOS-8), Shanghai, China, October 1014, 2010.

Zhao, H., V. A. Mousseau, 2010, "Use of Forward Sensitivity Analysis Method to Improve Code Scaling, Applicability, and Uncertainty (CSAU) Methodology," 8th International Topic Meeting on Nuclear Thermal-Hydraulics, Operation, and Safety (NUTHOS-8), Shanghai, China, October 10-14, 2010. 


\title{
Spatially Resolved Positron Analysis of Hydride Formation in LWR Fuels with GE-Global Nuclear Fuels
}

\author{
Douglas Akers, Mark Drigert, Maria Okuniewski; Tony Hill (Idaho State University), \\ Paul Cantonwine (General Electric-Global Nuclear Fuels Division) \\ 11-055
}

Hydride formation in commercial nuclear fuel zircaloy cladding is one of the critical issues limiting the lifetime of commercial nuclear fuel designs to about $60 \mathrm{MWD} / \mathrm{kgU}$. INL and General Electric Company's Global Nuclear Fuels Division (GE-GNF) have entered into a cooperative research and development agreement to develop INL's Spatially Resolved Positron Annihilation (SRPA) technology to provide in situ, nondestructive measurements of hydride formation in nuclear fuel cladding and components following irradiation. The novel SRPA technology is the subject of an INL patent application and was nominated for an R\&D 100 award. This technology will provide an improved fundamental understanding of the atomic-level microstructure of GE-GNF reactor fuel cladding and the effects of hydride formation on the lifetime of the cladding when exposed to long-term irradiation.

Verification and testing of the SRPA method for use in the characterization of nuclear fuel cladding for hydride production is being performed using surrogate samples containing hydrogen at INL along with irradiated cladding measurements at the GE Vallecitos facility in FY 2012. The project includes a strong crosscorrelation component that will correlate the SRPA data, ex situ measurement data currently used for hydride characterization, and the effects of strain and irradiation damage on the cladding. These data will be used to develop the design of a pool-side SRPA system that can be used by GE-GNF to perform in situ characterization of fuel cladding for hydride content at GE fuel storage facilities. In addition to the direct application of the SRPA technology to the current and advanced light water reactor fuels programs at GE-GNF, the technology also has specific applications to other nuclear-fuel-related issues, such as fuel cladding interaction and stress corrosion cracking, as well as to a number of other INL projects.

\section{Project Summary}

Progress during FY 2011 includes: (1) an extensive series of SRPA measurements on a range of samples containing hydrogen; (2) the cross-correlation of SRPA data with ex situ measurement techniques, such as optical metallography and scanning electron microscopy; (3) the development and testing of a SRPA scanning system suitable for use in highradiation fields and on irradiated zircaloy specimens; and (4) the development of initial conceptual designs for both hot-cell and poolside basin scanning systems for use at GE-GNF facilities. GE-GNF technical experts have been involved in these efforts and have begun planning for implementation of the irradiated zircaloy cladding measurement system at their Vallecitos facility and for implementation of SRPA measurement systems for testing at GE-GNF's Wilmington hot-cell and fuel-pool facilities.

Extensive SRPA measurements have been performed on prepared specimens with known concentrations of hydrogen induced in zircaloy specimens provided by GE-GNF and the Electric Power Research Institute. Figures 1 and 2 (next page) show an SRPA scan for a 3000-ppm hydrogen Zircaloy-2 specimen and a polarized optical metallography measurement of a cross section of the same specimen. The SRPA response is significantly affected by the hydride effects relative to Zircaloy specimens with no hydrogen present. The data obtained demonstrate that the SRPA technology can nondestructively segregate specimens with different concentrations of hydrogen present.

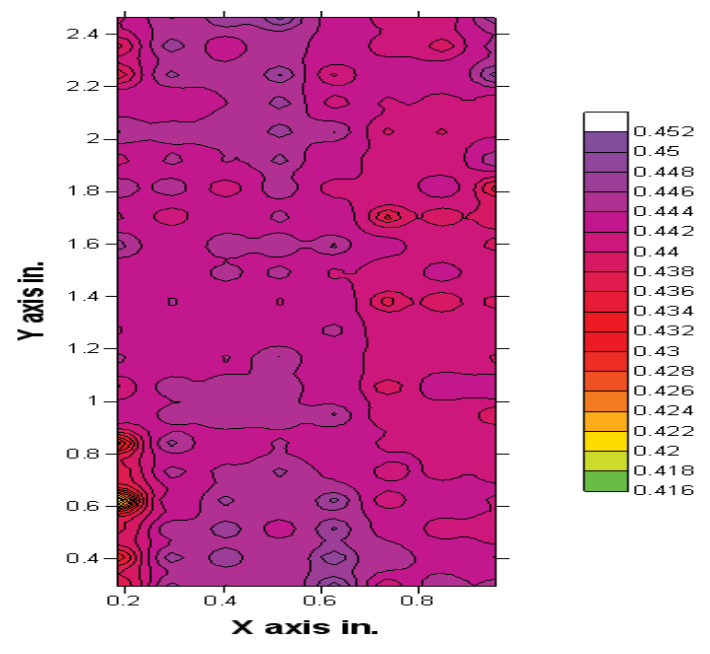

FIGURE 1. SRPA analysis (3000-ppm hydrogen). 


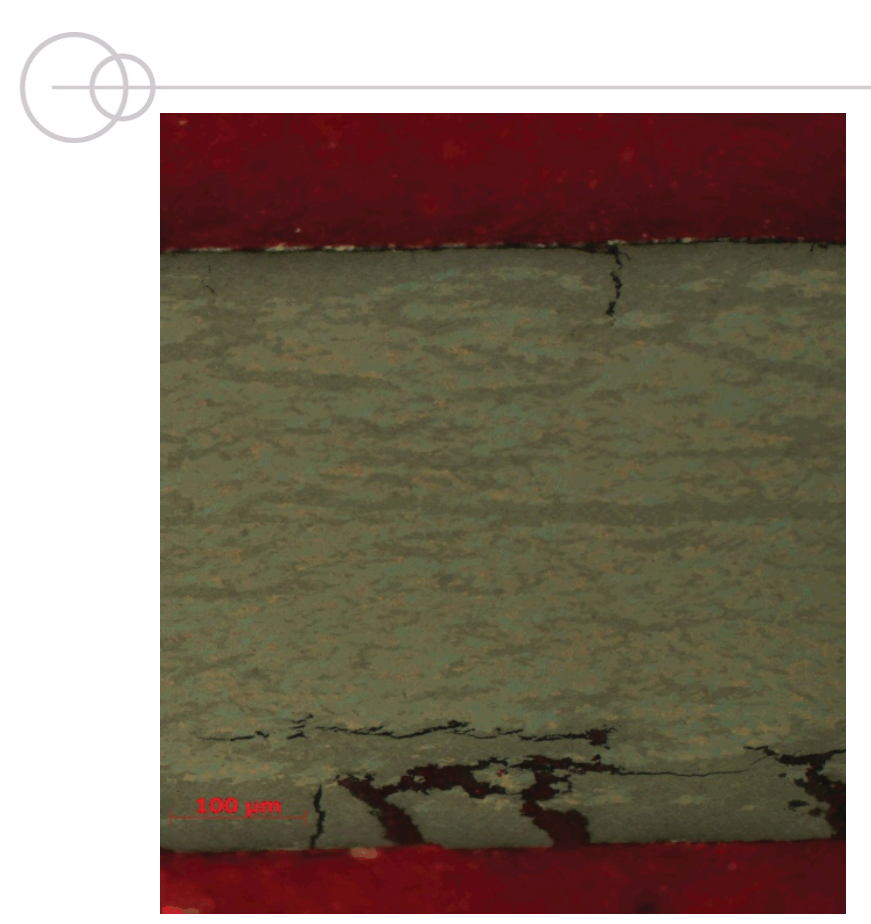

FIGURE 2. Optical metallography (3000-ppm cross section).

A specialized radiation-hardened SRPA system has been developed that is suitable for use in measuring irradiated zircaloy specimens and for use in a hot-cell environment. This system is designed to allow collimated detector SRPA measurements to be performed on highradiation field specimens.

\section{Benefits to DOE}

Implementation of the SRPA measurement system with GE-GNF through the INL cooperative research and development agreement process addresses one of the current critical issues for the U.S. nuclear power industry and demonstrates a good government/commercial partnership and the utilization of "science-based" research to address real-world problems. This project supports the INL mission of supporting the development of current and future reactor fuels optimized reactor systems. It will benefit other INL programs, such as the Light Water Reactor Sustainability program, the Consortium for Advanced Simulation of Light Water Reactors, the Advanced Test Reactor-National Scientific User Facility, and the characterization of advanced irradiated fuels for the Reduced Enrichment Research and Test Reactors and INSIGHTS programs.

\section{Relevant Publications and Presentations}

Akers, D. W., 2011, "SRPA Hydride Research Overview" (nonproprietary), EPRI and PNNL, September 7, 2011.

Akers, D. W., et al., 2011, "SRPA Hydride Research and Results" (proprietary), GE Wilmington, August 13, 2011.

Akers, D. W., et al., 2011, "SRPA Hydride Research and Results" (proprietary), $G E$ Vallecitos, July 26, 2011. 


\title{
Advanced Modeling and Simulation Concepts for ATR
}

\author{
Richard Martineau, Ray Berry, Samet Kadioglu; Richard Saurel (Ecole Polytechnique \\ Universitaire de Marseille, France), Frederick Gleicher, Barry Ganapol, Kord Smith, \\ David Nigg \\ 11-061
}

Accurately predicting the thermal and power behavior of INL's Advanced Test Reactor (ATR) will improve safety and help to extend the life of the reactor. To meet this goal, we are developing a multiphysics modeling and simulation methodology necessary to predict the behavior of the highly heterogeneous and complex threedimensional geometric configuration of the ATR. Our project includes the development and application of advanced, implicit numerical algorithms for nonlinearly coupled neutronic and multiphase conjugate heat transfer and will result in a prototype experimental software package and a plan for its further development into a production tool. Once the tool has been completed, we foresee a follow-on project directly funded by the ATR Life Extension Program to establish a fully verified and validated package useful for the support of ATR safety analysis and operational support for many years to come. The ability to accurately predict the thermal and power behavior of the ATR will greatly benefit the operation of the ATR by reducing the grossly conservative operating margins. This improved predictive capability will also benefit other reactor programs, such as the Light Water Reactor Sustainability Program.

The approach will examine the important coupling mechanisms of thermal feedback to the cross-sections in coupled neutronics and conjugate heat transfer between the multiphase coolant and the fuel of the ATR. A new multiscale radiation transport algorithm will be developed for higher-fidelity calculations unified with a multiscale multiphase flow capability to simultaneously resolve larger scale fluid dynamic interface problems along with the smaller scale multiphase mixtures of light water reactor systems. As an informal collaborative effort with Bettis Atomic Power Laboratory, the latter capability will also enable highly resolved fundamental flow studies for the important flow scenarios, such as departure from nucleate boiling and film boiling instability.

This LDRD project will result in advanced, mechanistic simulation tools for scientific expertise and methodologies on boiling flows and high-resolution, local scale two-phase flow: (1) for handling normal operation and accident conditions; (2) for covering multi-disciplinary coupled physics, including 3D multiphase flows; and (3) with in-depth physical and numerical validation. In addition to the departure from nucleate boiling, other examples where increased understanding of boiling flows presents a challenge include reactivity-initiated accidents, loss of coolant accidents, and severe accidents (sending water into a degrading core). Improved physics with more accurate and predictive models will provide enhanced safety and the reduction of excessive conservatisms. ATR is poised to play a major developmental role in this area. Indeed, one of the important ATR design basis accident scenarios is a loss of reflector water. In this design basis accident, some foreign materials can restrict the flow path in the reflector regions and introduce localized void. In order to accurately analyze such localized reactivity insertions, tightly coupled multiphaseflow, conjugate heat transfer and neutronic modeling is necessary.

\section{Project Summary}

The team made many advances toward modeling and simulation of ATR and achieved all the project objectives set for FY 2011. Most notable are the advancements made for flow modeling - including a complete rewrite of the single-phase Navier-Stokes formulation found in INL's ELK framework. This rewrite included Streamline-Upwind Petrov-Galerkin (SUPG) terms to allow for stable flows on coarser grids. Several advances were also made in advanced preconditioning methods such as the implementation of full Jacobian support, the Single Matrix Preconditioner (allowing a specified preconditioning matrix to be specified from the input file) and several fixes to the PETSc toolkit preconditioner interface for parallel physics-based preconditioning. Other notable improvements are: rewritten Discontinuous Galerkin support, custom quadrature rule selection, improved restart capability, many bug fixes, performance improvements and many more. All of these improvements are working toward high-fidelity modeling of ATR.

The Discrete Equation Method model/method for one-dimensional, variable area non-equilibrium two-phase flow [1] was modified to implicit time integration. The 
implicit time integration used was a second-order backward difference formula, selected for its stiff stability property. The Jacobian Free NewtonKrylov method was employed to solve the resulting nonlinear algebraic equation system. No preconditioner was employed; consequently, speedups were not realized. It was noted that a spatial convergence problem (cannot converge to the correct solution without an excessively large number of cells) was manifested in low-Mach compressible liquid flows that could not be corrected even by switching the scheme from an explicit to an implicit time integration. This probably occurs also for very low Mach number gas flows, but Mach numbers in gas are not as low as those in liquid corresponding to the same flow velocity.

Subsequently, we developed a modification for low-Mach liquid flows (not two-phase yet) that appears to solve the convergence issue, but which requires a significantly smaller Courant number (time step) if run explicitly (probably because the modification effectively makes the flow behave numerically like a flow with a higher Mach number). To be efficient, this lowMach modification needs, therefore, to be time integrated implicitly. This remains as an outstanding task for liquid flow only, followed subsequently by addressing the same issue for two-phase flow.

\section{Benefits to DOE}

Successful completion of this necessary, advanced proof-of-principal LDRD project will help extend the life of ATR - which is an important goal for both INL and DOE. This project is part of a long-term goal of using ATR as a platform for verification and validation of advanced modeling and simulation and as a tool for light water reactor performance enhancement. It will build upon the Phase 1 upgrade of ATR modeling and simulation to current state-of-the-art computational methods by jumping to proof of concept of Phase 3 nextgeneration coupled multiphysics computational methods for nuclear reactor design/analysis, which will use ATR as a test bed for applications and demonstrations of science-based methods for performance and safety analysis (Nuclear Energy Advanced Modeling and Simulation Program), which includes but is not limited to: (1) complex heterogeneous geometries; (2) use of flexible geometric configuration for nuclear heated core multiphase flow experiments as well as core physics benchmarks; and (3) extension of the validation database of Phase 1. 


\section{Characterization of a Consolidated Electrochemical Technique for Separation and Recovery of Actinides from Fission Products in Oxide Fuels}

S. D. Herrmann, S. X. Li, G. L. Fredrickson

09-059

Because the electrometallurgical treatment of sodium-bonded spent fuel from Experimental Breeder Reactor-II has shown pyroprocessing technology to be effective and well-suited for a metal fuel cycle, the extension of such technology to used oxide fuel has become the subject of much interest domestically and internationally. Recent efforts in the U.S. and abroad have focused primarily on converting oxide fuel to metal as a head-end step to existing electrorefining technology. Such an approach involves the management of two separate electrochemical molten salt systems that to date have been limited to bench-scale operations with used fuel. Another approach, pursued by this LDRD project, involves the adaption of existing industrial-scale technology (i.e., the Hall-Héroult process for aluminum metal production from aluminum oxide) to the production of refined uranium metal from spent oxide fuel.

Specifically, the basis of the Hall-Héroult process is dissolution and concurrent electrolysis of aluminum oxide in a molten fluoride salt (i.e., cryolite $-\mathrm{Na}_{3} \mathrm{AlF}_{6}$ ). Accordingly, the adequate dissolution of uranium oxide fuel in a select molten fluoride salt and the effective oxidation and reduction reactions at suitable anode and cathode structures could consolidate the electrochemical treatment of oxide fuel into a single-salt system with a single passing of current. The investigation of these characteristics for a uranium system was the primary objective of this LDRD.

\section{Project Summary}

A review of the literature identified previous attempts to effect electrolysis of dissolved uranium oxide in molten fluoride salts at $1200-1400^{\circ} \mathrm{C}$, producing a molten uranium metal product (m.p. $=1135^{\circ} \mathrm{C}$ ) and an oxygenbearing off-gas. The primary challenge in these previous attempts was process inefficiency due to the back reaction of uranium metal product with uranium fluoride in the molten salt (i.e., $\left.\mathrm{U}+3 \mathrm{UF}_{4} \rightarrow 4 \mathrm{UF}_{3}\right)$. To overcome this inefficiency, the approach in this LDRD was to form solid deposits of uranium at lower system temperatures that could be immediately removed upon cessation of applied electrical currents. Experiments involved the preparation and characterization of molten fluoride salt systems of $\mathrm{FLiNaK}^{-\mathrm{UF}_{4}, \mathrm{LiF}^{-\mathrm{UF}_{4}} \text {, and LiF-UF }}$ in contact with uranium oxide over a temperature range of $500-900^{\circ} \mathrm{C}$ using various anode and cathode materials and configurations. Cyclic voltammetry was applied to each of these systems and identified: (1) uranium reduction potentials on nickel and tantalum rods, and (2) oxidation reaction potentials on inert (platinum) and reactive (carbon) anodes relative to $\mathrm{Ni} / \mathrm{NiO}$ and glassy carbon reference electrodes. Uranium oxide $\left(\mathrm{UO}_{2}\right.$ and $\left.\mathrm{U}_{3} \mathrm{O}_{8}\right)$ particulate was loaded into permeable metal baskets and immersed in the salt to facilitate its dissolution. Samples of the salt were taken and analyzed for dissolved uranium oxide in the prescribed salt systems.

Observations from several experiments with the prescribed salt systems revealed inherent inefficiencies with the proposed process due to multivalent ion recycle. Specifically, on the cathode, uranium (IV) ions are first reduced to uranium (III) ions by a single electron transfer and then to uranium metal by a three-electron transfer. Thus, the production of uranium metal deposits on the cathode (which was achievable in each of the prescribed experiments) also produces uranium (III) ions in the salt, which are then available to be oxidized on the anode to uranium (IV) ions, creating a non-productive cyclical current path. Similarly, on the anode, uranium (IV) ions can be oxidized to uranium (V) ions in the absence of dissolved oxygen ions or to uranyl $\left(\mathrm{UO}_{2}{ }^{2+}\right)$ ions in the presence of dissolved oxygen ions. Each of these higher valence uranium ions can in turn be reduced back to uranium (IV) ions at the cathode, again creating a non-productive cyclical current path. To overcome these multivalent ion recycle mechanisms, an oxygen-ion diffusive membrane was used in the form of a closed-end yttriastabilized zirconia tube enveloping a carbon anode immersed in molten tin. The yttriastabilized zirconia anode configuration was tested at Boston University in support of this LDRD using a $\mathrm{LiF}-\mathrm{YbF}_{3}-\mathrm{Yb}_{2} \mathrm{O}_{3}$ system as a surrogate for LiF- $-\mathrm{UF}_{4}-\mathrm{UO}_{2}$. Boston University researchers successfully produced $\mathrm{Yb}$ metal cathode deposits and carbon dioxide gas at the anode in the prescribed system. When an identical anode configuration was applied in both LiF-UF $-\mathrm{UO}_{2}$ and LiF-UF $-\mathrm{UO}_{2}$ systems at INL, 
uranium metal deposits were readily produced, but no commensurate carbon anode consumption was observed. This observation indicated that the yttria-stabilized zirconia anode configuration failed to mitigate multivalent ion recycle in the prescribed salt systems, even when adequate uranium oxide dissolution was observed.

\section{Benefits to DOE}

This research addressed INL's fuel cycle research and separation needs by investigating the fundamental electrochemistry of a novel fuel cycle concept from an industrial perspective. This research also advanced INL's expertise in molten fluoride salt chemistry, which has other potential fuel cycle applications (e.g., fluoride volatility methods and thorium fuel cycles). The contracted work at Boston University also demonstrated the application of the prescribed molten salt chemistry to the production of rareearth metals.

Relevant Publications and Presentations

Yihong, J., P. A. Zink, and U. B. Pal, 2011, "Solid Oxide Membrane Process for the Reduction of Uranium Oxide Surrogate in Spent Nuclear Fuel," The $220^{\text {th }}$ ECS Meeting and Electrochemical Energy Summit, Boston, Massachusettes, USA, October 914, 2011. 


\title{
Neptunium Redox Chemistry in Irradiated Nitric Acid
}

\author{
Bruce J. Mincher, Stephen P. Pezyk, Alena Paulenova
}

10-001

Nuclear solvent extraction is performed in radioactive nitric acid solution. Irradiation has a number of chemical effects, such as ligand degradation, production of deleterious ligand degradation products, and changes in metal valence states. However, maintenance of preferred metal valence states is necessary for solvent extraction. Neptunium has long been known to be problematic in this regard, since $\mathrm{NpV}$ does not extract, while NpVI does extract in current processes. The $\mathrm{Np}$ valence changes during solvent extraction and $\mathrm{Np}$ partitions throughout the process. Several groups are studying this undesirable phenomenon worldwide. This LDRD program is examining the effect of irradiation.

\section{Project Summary}

We have installed Cary $6000 \mathrm{UV} / \mathrm{Vis}$ spectroscopic equipment adjacent to the ${ }^{60} \mathrm{Co}$ irradiator at the Materials and Fuels Complex to measure $\mathrm{Np}$ valence changes following irradiation. We created solutions of known oxidation states of $\mathrm{NpV}$ and $\mathrm{NpVI}$ by controlled potential electrolysis. These solutions were then irradiated to known absorbed gamma doses. We also irradiated solutions of mixed oxidation states. The UV/Vis absorbance of the irradiated solutions was collected, and plots of $\mathrm{NpV}$ and NpVI absorbance versus time were prepared.

Regardless of the starting point, $\mathrm{NpV}$ was oxidized to NpVI, meaning the extractable valence state was favored. However, after a short irradiation time, the trend reversed, and $\mathrm{Np}$ was reduced to inextractable $\mathrm{NpV}$. We believe that this chemistry is due to dissolved oxygen concentration changes in irradiated solution. Initially present, dissolved oxygen is consumed by reaction with radiolytically produced solvated electrons and hydrogen atoms, and $\mathrm{Np}$ is oxidized by produced hydroxyl radicals. Once oxygen is consumed, nitrous acid is produced, which reduces $\mathrm{Np}$. We are currently performing kinetic modeling of the experimental results to verify this hypothesis.

\section{Benefits to DOE}

Current and future plans for the reprocessing of used nuclear fuel will rely on solvent extraction. Neptunium speciation has plagued traditional Plutonium - URanium EXtraction (PUREX)-based processes and will remain important for future processes. Once the factors affecting Np valence state changes are understood, the process can be optimized to ensure predictable Np partitioning. Similar principals will also apply to other redox active metals in irradiated solution.

\section{Relevant Publications and Presentations}

Precek, M., B. J. Mincher, and A. Paulenova, 2011, "Interactions of Nitrous Acid with Neptunium in Nitric Acid Solutions," The 35th Actinide Separations Conference, Charlotte, North Carolina, USA, May 23-26, 2011.

Mincher, B. J., M. Precek, G. Elias, S. P. Mezyk, and A. Paulenova, 2011, "The Redox Chemistry of Neptunium in $\gamma$-irradiated Nitric Acid," in preparation for submission to Radiochim Acta.

Precek, M., A. Paulenova, P. Tkac, and N. Knall, 2010, "Effect of Gamma Irradiation on the Oxidation State on Neptunium in Nitric Acid in the Presence of Selected Scavengers," Sep Sci Technol, Vol. 45, pp. 1699-1705.

Precek, M., and A. Paulenova, 2010, "Kinetics of Reduction of Hexavalent Neptunium by Nitrous Acid in Solutions of Nitric Acid," J Radioanal Nucl Chem, Vol. 286, pp. 771-776.

Mincher, B. J., 2010, "Irradiation Effects on Metal Oxidation States," Actinide Recycling by Separation and Transmutation: ACSEPT, International Workshop, Lisbon, Portugal, March 31-April 2, 2010.

Paulenova, A., M. Precek, K. Hartig, and N. Knapp, 2010, "Redox Chemistry of Neptunium in Solutions of Nitric Acid," Actinide Recycling by Separation and Transmutation: ACSEPT, International Workshop, Lisbon, Portugal, March 31April 2, 2010. 


\section{Reversible Gas Phase Reactions for Recovery of Graphite from Recycled HTGR TRISO Fuel}

Scott Herbst, J. D. Christian, M. R. Greenhalgh

$10-026$

Advanced very high-temperature reactor (VHTR) concepts will utilize nuclear fuels containing tristructural isotropic (TRISO)-coated fuel particles imbedded in carbonaceous fuel compact material. The spherical TRISO particles are $0.5-1 \mathrm{~mm}$ in diameter and consist of a central kernel of the nuclear reactor fuel (uranium dioxide $\left[\mathrm{UO}_{2}\right]$ or uranium oxycarbide [UCO]), surrounded by successive layers of low-density porous carbon buffer, dense inner pyrolytic carbon $(\mathrm{PyC})$, silicon carbide $(\mathrm{SiC})$, and dense outer PyC, as depicted in the Figure 1. This project will examine recovering the carbon from these graphite fuels, including radioactive carbon-14 (C-14), as an alternative and improvement to burning the carbon to $\mathrm{CO}_{2}$ in air or oxygen with subsequent release to the environment. This carbon recovery step would form the basis for the headend processing of used VHTR fuels to recover valuable uranium and irradiation products. In this scenario, the carbonaceous layers would be removed and captured with recycle of the reactive gas while the exposed fuel kernel could be subsequently processed using conventional techniques. Reaction kinetics are being experimentally determined with surrogate TRISO particles and several reactive gases, including sulfur ( $\mathrm{S}$ ), fluorine $\left(\mathrm{F}_{2}\right)$, chlorine $\left(\mathrm{Cl}_{2}\right)$, and hydrogen $\left(\mathrm{H}_{2}\right)$, to establish the practicality of these processes. Each reactant gas is predicted to react with the different carbon-containing layers to form carbon-containing gaseous compounds that can be reversibly dissociated back into carbon and reactant gas. The results of the tests will be used to derive mass balance and throughput rates with the various gases for treatment of the VHTR fuels.

\section{Project Summary}

During the first year (FY 2010), procurement, set up, and operation of the thermal gravimetric analyzer, vacuum, and gas handling systems for the project were completed, including a sulfur vapor generator. Surrogate TRISO particles were also obtained from two sources: INL (Winston/Shaber) and Oak Ridge National Laboratory. The surrogate TRISO particles are important to the project since they differ from actual particles only in that they

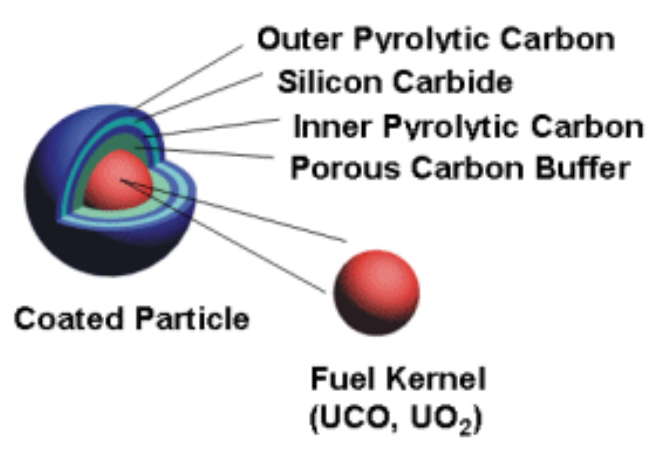

FIGURE 1. Structure of a typical TRISO fuel particle (www.nextgenerationnuclearplant.com/i/triso.gif).

contain a zirconium oxide fuel kernel as opposed to uranium compounds (important since this project is being conducted in non-radioactive laboratories). Initial experiments conducted at the outset of FY 2011 involved determination of the reaction rates of the different carboncontaining layers in the surrogate TRISO particle with sulfur gas. This turned out to be a very difficult task since elemental sulfur is nominally a solid and all portions of the system must be kept heated and well insulated to maintain the temperature around $430^{\circ} \mathrm{C}$ to keep sulfur in the gas phase. Although the reaction of sulfur gas with carbon is predicted to be feasible thermodynamically, reaction of sulfur gas was not observed with either the $\mathrm{PyC}$ or $\mathrm{SiC}$ layers of the TRISO particles at temperatures up to $1000^{\circ} \mathrm{C}$ (the upper temperature limit of the experimental equipment). Furthermore, during FY 2010, it was experimentally observed that the dissociation of carbon disulfide $\left(\mathrm{CS}_{2}\right.$, the reaction product of graphite with sulfur) will not reversibly decompose, as predicted thermodynamically, at a practical rate in the temperature range between $275^{\circ} \mathrm{C}$ and $400^{\circ} \mathrm{C}$. The alternative to recovering carbon from $\mathrm{CS}_{2}$ by reaction with $\mathrm{H}_{2}$ will be necessary if sulfur is reacted in other graphite systems - for example, for gentle selective removal of $\mathrm{C}-14$ from decommissioned reactor irradiated bulk graphite. We plan to investigate that (and other reactant gases) in FY 2012. Consequently, the emphasis of the program has focused on the reaction of fluorine $\left(\mathrm{F}_{2}\right)$ and nitrogen trifluoride $\left(\mathrm{NF}_{3}\right)$ gases with the different carbon-containing layers of the TRISO particle during the later portion of this 
year. The primary objective was to chemically remove the hard $\mathrm{SiC}$ layer that will not burn off in oxygen and thus avoid the need for difficult mechanical breakage (grinding) of the layer to expose inner carbon layers for burning in air.

The reaction of both $\mathrm{F}_{2}$ and $\mathrm{NF}_{3}$ with graphite would form carbon tetrafluoride (and potentially $\mathrm{SiF}_{\mathrm{x}}$ in the case of the $\mathrm{SiC}$ layer). Both gases provide a source of fluoride to react with the carbon layers; however, $\mathrm{NF}_{3}$ is considered less toxic, less corrosive, and less reactive at ambient temperature than $\mathrm{F}_{2}$ gas; consequently, it would be more easily handled during transport, storage, and processing operations than fluorine gas. It is therefore of interest to compare the reaction rates of these two gases with the different carbonaceous TRISO layers. Nickel and quartz glass were evaluated as materials of construction for both the sample holders and balance wires in the thermal gravimetric analyzer instrument with $\mathrm{F}_{2}$ as a function of reaction temperature. Nickel was found to be resilient to fluorine at temperatures at or below $600^{\circ} \mathrm{C}$ provided the sample holder and wire were passivated at elevated temperatures with fluorine gas prior to use. These results were consistent with literature reports regarding the compatibility of nickel with fluorine up to the $600^{\circ} \mathrm{C}$ temperature. ${ }^{1,2}$ Quartz glass was determined to be impractical for use as the sample holder and balance wire in the thermal gravimetric analyzer with either $\mathrm{F}_{2}$ or $\mathrm{NF}_{3}$ gases. Preliminary data indicate that the $\mathrm{SiC}$ layer is sufficiently reactive with either $\mathrm{F}_{2}$ at $400^{\circ} \mathrm{C}$ or $\mathrm{NF}_{3}$ at $500^{\circ} \mathrm{C}$ to be potentially useful for the reaction with the $\mathrm{SiC}$ layer. This reaction can be conducted with nickel materials of construction at these temperatures. The $\mathrm{NF}_{3}$ and $F_{2}$ are not reactive enough with the outer or inner pyrolytic carbon layers of the TRISO particles at temperatures below about $650^{\circ} \mathrm{C}$ to be practical. The PyC layers can be readily removed with oxygen (air) at temperatures around $775^{\circ} \mathrm{C}$ in quartz to expose the $\mathrm{SiC}$ layer for reaction with $\mathrm{NF}_{3}$ followed by subsequent burning in air to remove inner carbon layers and expose the fuel kernel.

\section{Benefits to DOE}

The ability to process used (irradiated) VHTR fuels and recover valuable uranium and fission products is an important aspect of future nuclear fuel cycles. Nuclear fuels based on TRISO particles contain a $\mathrm{SiC}$ layer that is inert (nonreactive) by design, purposely keeping the irradiation products and fuel contained inside the kernel of the fuel particles. An efficient, costeffective method of removing the $\mathrm{SiC}$ layer is important to the success of future fuel cycles based on the VHTR and TRISO particles. This need has also been expressed by cognizant industry experts. The reaction of PyC layers with $\mathrm{F}_{2}$ or $\mathrm{NF}_{3}$ is a potential method to fill this gap.

\section{Relevant Publications and Presentations}

A peer-reviewed publication comparing the reaction rates of pyrolytic carbon and $\mathrm{SiC}$ with $\mathrm{NF}_{3}$ and $\mathrm{F}_{2}$ gases is currently in preparation.

\section{References}

1. Kuriakose, A. K., and J. L. Margrave, 1964, "Kinetics of the Reactions of Elemental Fluorine with Zirconium Carbide and Zirconium Diboride at High Temperatures," J. Phys. Chem., Vol. 68, No. 2, August 1964.

2. Kuriakose, A. K., and J. L. Margrave, 1965, "Kinetics of the Reactions of Elemental Fluorine. IV. Fluorination of Graphite," $J$. Phys. Chem., Vol. 69, No. 8, August 1965. 


\section{Comprehensive Thermodynamic Models for the Aqueous Partitioning of Actinides from Used Nuclear Fuel}

Peter Zalupski; Simon Clegg (University of East Anglia), Laetitia Delmau (Oak Ridge

National Laboratory)

$10-027$

Quantitative description of chemical interactions in complex liquid phases may be realized through the process of thermodynamic parameterization of theoretically represented mixtures. The construction of such thermodynamic models allows for numerical representation of non-ideal solute behavior in aqueous and organic phase components of a liquid-liquid distribution. Ultimately, the construction of such theoretical models supports predictive modeling activities focused on the identification of correct equilibrium sets that control the liquid-liquid process. The primary objective of this project focuses on the proper consideration of non-ideal solution chemistry in aqueous electrolyte and non-aqueous solute mixtures to allow accurate characterization of liquid-liquid distribution equilibria involved in used nuclear fuel separations. A computational software package - SXFIT - is utilized in the modeling effort.

This year's objectives focused on developing the thermodynamic models for complex electrolyte mixtures containing trivalent metal ions, inorganic salts, carboxylates, and aminopolycarboxylate complexing reagents. Such aqueous systems, when equilibrated with nonaqueous solutions containing a phase transfer reagent, allow selective separation of actinides from lanthanides. The Pitzer ion-interaction model have been shown to accurately describe numerous thermodynamic properties of aqueous solutions and, as such, the Pitzer formalism was chosen to model the coefficients in aqueous solutions of interest to this project. The modeling activities populate Pitzer ion-interaction parameters as thermodynamic descriptors of solute behavior in solution. The specific aqueous system chosen for these activities corresponded to the aqueous buffer: $\mathrm{Eu}^{3+} / \mathrm{H}^{+} / \mathrm{Na}^{+} / \mathrm{NO}_{3}{ }^{-} /$ $\mathrm{H}_{2} \mathrm{Mal} / \mathrm{HMal}^{-} / \mathrm{H}_{2} \mathrm{O}$, where Mal describes malonate, based on Pitzer ion-interaction theory. On the SXFIT modeling front, liquid-liquid distribution datasets collected for the partitioning of trivalent metal ions between aqueous malonate buffering systems and organic mixtures of di 2 ethyl-hexyl phosphoric acid, in $n$-dodecane, were modeled to describe equilibrium sets that guide the separation.

\section{Project Summary}

This year, a tentative thermodynamic model, based on information collected in the literature, has been constructed and may be used to estimate $\mathrm{Eu}^{3+}$ complexation in a malonate buffered solution at all ionic strengths up to about $1 \mathrm{~mol}$ $\mathrm{kg}^{-1}$. Sensitivity analysis for the model has been performed to identify the contribution of each ion-ion interaction to the calculation of the activity coefficient of species of interest. This analysis identified the aqueous interactions of greatest influence on the activity of $\mathrm{Eu}^{3+}, \mathrm{H}^{+}$, $\mathrm{HMal}-$, and $\mathrm{H}_{2} \mathrm{Mal}$ species. The analysis identified the unknown interaction between Eu3+ and HMal- as the most significant one to the calculation of the activity coefficient of trivalent europium. The ternary mixture interactions " ++ , $\theta(\mathrm{Eu}, \mathrm{Na})$, , “++ - $\psi\left(\mathrm{Eu}, \mathrm{Na}, \mathrm{NO}_{3}\right)$," and “++ + , $\psi(\mathrm{Eu}, \mathrm{Na}, \mathrm{HMalo})$ " are also important to the model. Based on these calculations, experimental inquiries into fundamental physical properties of electrolyte mixtures, which will produce Pitzer parameters for identified interactions, have been initiated. Isopiestic equilibrations of ternary mixtures of $\mathrm{NaNO}_{3}$ and $\mathrm{Eu}\left(\mathrm{NO}_{3}\right)_{3}$ at five different molar ratios and $\mathrm{Eu}-154$ distributions in two-phase systems at different ionic strengths are in progress.

In addition, the effect of an uncertainty of 0.003 in water activity on the osmotic coefficient $(\phi)$ and the effect of mixture parameters in a $\mathrm{Na}^{+}$ $-\mathrm{Eu}^{3+}-\mathrm{NO}_{3}{ }^{-}$solution were investigated to identify whether hygrometry produces data of accuracy required to construct the model. It was concluded that direct measurements of water activity should not be used to determine mixture parameters in the Pitzer model. Their main use is likely to be determination of the properties of pure aqueous solutions and at very high concentrations only.

Expressions, based on calculations using the Pitzer model, to approximate stoichiometric dissociation constants for diethylenetriamine$\mathrm{N}, \mathrm{N}, \mathrm{N}^{1}, \mathrm{~N}^{11}, \mathrm{~N}^{111}$-pentaacetic acid $\left(\mathrm{H}_{5} \mathrm{DTPA}\right)$ complexing reagent in $\mathrm{NaNO}_{3}$ media have been developed. Binary interaction parameters for the cation-anion system of $\mathrm{Na}^{+} / \mathrm{DTPA}^{\mathrm{n}-}$ were also 
modeled based on the availability of literature data for the protonation of DTPA in solutions of various electrolyte compositions.

Pitzer interaction parameters $\beta^{(0)}$ (cation EuHR') and $\beta^{(1)}$ (cation - EuHR') were determined using the one-parameter SIT equation. Data for complexation of $\mathrm{Eu}^{3+}$ by $\mathrm{H}+$ and the DTPA anion $\left(\mathrm{R}^{5-}\right)$ in $\mathrm{Na}^{+}$electrolyte media, or any media, are sparse, and only tentative analyses could be carried out. New measurements of stoichiometric dissociation constants for $\mathrm{H}_{5}$ DTPA in $\mathrm{Na}^{+}$electrolyte media, over a range of concentrations, are needed to improve the model for this system.

The SXFIT calculations reproduced aciddependent analyses of liquid-liquid distribution of $\mathrm{Eu}^{3+}$ between non-aqueous solutions of HDEHP in $n$-dodecane and aqueous malonate media. The equilibrium set that governs the investigated system may be described by three mass-balance equations:

$\begin{array}{lr}2(\mathrm{HL})=(\mathrm{HL})_{2} & \text { dimerization of HDEHP } \\ \mathrm{Eu}^{3+}+3(\mathrm{HL})_{2}=\mathrm{Eu}(\mathrm{L})_{3}(\mathrm{HL})_{3}+3 \mathrm{H}^{+} & \text {extraction of europium } \\ \mathrm{Na}^{+}+2(\mathrm{HL})_{2}=\mathrm{Na}(\mathrm{L})(\mathrm{HL})_{3}+\mathrm{H}^{+} & \text {extraction of sodium }\end{array}$

The robustness and validity of the SXFIT prediction is being currently tested.

\section{Benefits to DOE}

The ongoing studies sponsored by this LDRD help establish predictive modeling capabilities for solvent extraction-based separations of metals. The project investigates how the complexities encountered in the solution phases that partake in actinides recycle from used nuclear fuel could impact modeling of such separations. The LDRD supports all four DOE national security missions in that a predictive modeling of separations would be a valuable asset to ensure the reliability of advanced nuclear fuel cycles.

\section{Relevant Publications and Presentations}

Zalupski, P., M. Gray, M. Nilsson, and L. Delmau, 2011, "Using SXFIT to Model Liquid-Liquid Distribution Systems: Initial Results for the Extraction of a Trivalent Metal by Dialkyl Phosphoric Acid," 2011 American Nuclear Society Annual Meeting, Hollywood, Florida, USA, June 26-30, 2011. 


\section{Speciation Behavior of Americium Oxidation States for the Separation of Americium from Curium in Nuclear Processing}

Catherine Riddle, Ken Czerwinski, Patricia Paviet-Hartmann

11-005

Many countries are currently expanding nuclear energy as a method for the safe generation of carbon-free energy. Closed fuel cycle technologies are key to waste reduction and a sustainable nuclear energy future. To support closing the nuclear fuel cycle, advanced separation technologies are being developed to effectively perform the difficult separation of the actinides, including Am, from the lanthanides as well as the separation of Am from $\mathrm{Cm}$. This will allow the actinides to be recycled to reactors, thus reducing the long-term heat load and radiotoxicity of the waste requiring geological disposal. Novel separation schemes are being investigated which use the higher oxidation states of Am in order to separate it from the lanthanides and $\mathrm{Cm}$ in used nuclear fuel reprocessing. Higher Am oxidation states have long been known to exist but are less well studied, and an investigation of the behavior of Am oxidation states would be an important step towards solving this difficult separation issue.

This project's focus is the examination of stereochemical arrangements of atoms in proximity to Am in different oxidation states using x-ray absorption fine structure spectroscopy (XAFS) analysis and single crystal $\mathrm{x}$-ray crystallography. XAFS analysis is being used to develop an atomic picture of americium at higher oxidation states in aqueous solution and use the data to better understand the processes taking place. Single crystals of $A m(V)$ and $\mathrm{Am}(\mathrm{VI})$ have not been previously produced using bismuthate. The synthesis of this actinide crystal as well as subsequent analysis will play an important role in the characterization of oxidized Am species. The data from both XAFS and the crystallography work will be developed using density functional theory as a computational characterization method adding further information on the behavior of oxidized Am. Ultimately, this work will result in an experimental data-based model to be used in the separation of species with very similar chemical properties.

\section{Project Summary}

First-year objectives included the preparation of Am samples for analysis by XAFS, baseline characterization of Am $+3,+5$, and +6 oxidation states using XAFS, an analysis of ground-state Am and oxidized Am by density functional theory. Beam time was procured from the Stanford Synchrotron Radiation Light Source for the XAFS analysis for the Am oxidation states, and the samples were prepared at our collaborator's facility at the University of Nevada-Las Vegas under the direction of the principal investigator. A key research development came with the analysis of baseline XAFS data from the Advanced Photon Source run at Argonne National Laboratory. This data showed a distinct increase in energy proving that characterization of $\mathrm{Am}(\mathrm{V}, \mathrm{VI})$ oxidation states with XAFS is possible. Refined experiments, which are the results of what was learned from the baseline experiments, have been designed for the next Advanced Photon Source run to take place in FY 2012 (October-December 2011). The new XAFS data will give definitive oxidation state information that will be analyzed through density functional theory.

Another key development in the project was the experimental design and subsequent production of a barium carbonate, sodium bismuthate, and neodymium oxide crystal. This crystal is a precursor to developing an oxidized $\mathrm{Am}(\mathrm{V}, \mathrm{VI})$ crystal through high-temperature fusion methods. The new high-temperature tube furnace system in which the oxidized Am crystals will be grown has been set up and preliminary testing is underway. The Am crystallography experiments are scheduled to begin in FY 2012. The research accomplished this fiscal year has allowed for the advancement of the principal investigator's doctoral work, which is currently on schedule with advancement to candidacy projected for FY 2012 and graduation from the Radiochemistry $\mathrm{PhD}$ program projected for FY 2013. Overall, the LDRD research is proceeding as planned, a successful collaboration has been established with the University of Nevada - Las Vegas and knowledge in XAFS measurement is being gained, resulting in increased capability within Nuclear Science and Technology.

\section{Benefits to DOE}

Separation of Am from lanthanides and $\mathrm{Cm}$ is a key focus of the DOE Office of Nuclear Energy Fuel Cycle Research and Development Program. This research will lead to a better 
understanding of the behavior of this actinide species in both complexed and uncomplexed forms in the stages prior to, during, and post separation as well as the separation of trivalent actinides from the lanthanides. The involvement of university partnering with INL will open more avenues for targeted funding agencies and enhance the training of the future workforce. This research will be the basis for the principal investigator's dissertation in partial fulfillment of a $\mathrm{PhD}$ in radiochemistry from the University of Nevada-Las Vegas and will ultimately benefit both INL and DOE by producing a new $\mathrm{PhD}$ in radiochemistry.

\section{Relevant Publications and Presentations}

Riddle, C., P. Paviet-Hartmann, K. Czerwinski, F. Poineau, P. Weck, and T. Hartmann, 2011, "Speciation Behavior of Americium Higher Oxidation States for the Separation of Americium from Curium," American Chemical Society $242^{\text {nd }}$ National Meeting \& Exposition, Division of Nuclear Chemistry \& Technology for the Session: Celebration of the 100th Anniversary of Madame Curie's Nobel Prize in Chemistry, Denver, Colorado, USA, August 28-September 1, 2011. 


\section{Development of a Simplified Soft Donor Technique for Trivalent Lanthanide Actinide Separations}

Leigh R. Martin, Peter R. Zalupski; Clint Sharrad, Louise Natrajan (University of

Manchester)

11-023

In this LDRD project, we explore novel extraction systems to complete the difficult separation of the minor actinides from the fission-product lanthanides in a more simplistic manner compared to current methodologies. The work performed will address actinide and lanthanide coordination behavior with amino acids in both aqueous and organic phases and may provide insight into the mechanisms for effective lanthanide/actinide separations. The main goal is to continue to develop an understanding of the various chemical processes that may lead to the successful separation of the trivalent lanthanides from the trivalent lanthanides using an amino acid buffer system and/or short-chain peptides. The effects of induced ligand conformation and counterions upon binding to a given metal ion will be studied in solution. Such effects have previously been overlooked in determining separation efficiencies. In addition, by exploring different amino acids with additional soft donor sites, experimentation will be performed to identify if, in these types of systems, the need for additional aqueous-phase complexants can be removed. Further, radiation chemistry will be used throughout the project to identify the most stable amino acids or peptides for this process.

\section{Project Summary}

We have conducted the initial feasibility study of a modified TALSPEAK process (an aqueous actinide-complexing medium forms the basis of the successful separation of americium and curium from lanthanides) replacing lactate with the amino acid aniline as a buffer. In an initial test, $\mathrm{Eu}$ (III) separation from Am(III) using L-alanine as a buffer/complexant at $\mathrm{pH}$ 1-3.6 with $50 \mathrm{mM}$ diethylene triamine pentaacetic acid (DTPA), showed that separation factors of $\mathrm{Eu}$ over Am up to 53 can be achieved. Even higher separation factors were established for the Ce/Am pair (up to 148).

Building on this, further solvent extraction separations work has been performed on the alanine system with the lanthanides relevant to used nuclear fuel $\mathrm{La}, \mathrm{Ce}, \mathrm{Pr}, \mathrm{Nd}, \mathrm{Sm}, \mathrm{Eu}, \mathrm{Gd}$, $\mathrm{Tb}, \mathrm{Dy}, \mathrm{Ho}$. The distribution coefficients of the minor actinides with a given lanthanide as a function of alanine concentration $(0.5,1.0$, and $1.5 \mathrm{M})$ at $\mathrm{pH} 2$ are shown in Figure 1. The

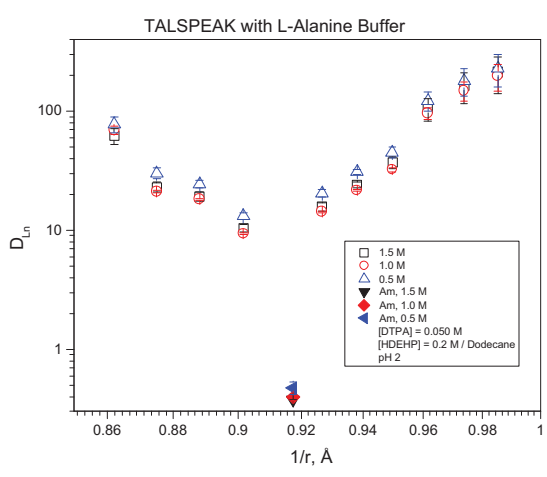

FIGURE 1. Final liquid-liquid distribution of actinides and lanthanides in L-alanine TALSPEAK.

extraction results show that TALSPEAK-type extraction behavior is observed for the lanthanides and actinides when DTPA is present. Without DTPA in the system, the separation breaks down. These results demonstrate that the TALSPEAK process has been significantly improved by the reduction in reagents required and can be operated at the industrially important $\mathrm{pH}$ 2. However, the use of DTPA in the extraction process is necessary to achieve higher SFs.

In order to investigate the origin of the selectivities and SFs observed in the extraction experiments, we have begun a comprehensive complexation investigation with the lanthanides. We have prepared and isolated the lanthanide DTPA complexes by reaction of $\mathrm{H}_{5}$ DTPA with $\mathrm{Ln}^{3+}$ salts $(\mathrm{Ln}=\mathrm{La}, \mathrm{Nd}, \mathrm{Pr}, \mathrm{Sm}, \mathrm{Eu}, \mathrm{Tb}, \mathrm{Dy}, \mathrm{Ho}$, $\mathrm{Er}, \mathrm{Yb}, \mathrm{Lu})$. The formation of the

$\left[\operatorname{Ln}(\mathrm{DTPA})\left(\mathrm{H}_{2} \mathrm{O}\right)\right]^{2-}$ complexes has been verified by paramagnetic nuclear magnetic resonance spectroscopy and luminescence spectroscopy. The number of inner-sphere water molecules (q) has been determined in these parent compounds from analysis of lifetime data in $\mathrm{H}_{2} \mathrm{O}$ and $\mathrm{D}_{2} \mathrm{O}$ $\left(\mathrm{Eu}^{3+} \mathrm{Tb}^{3+}\right)$ and are consistent with those reported in the literature, e.g., $\mathrm{q}=1.1$ for $\mathrm{Eu}^{3+}$. Titration experiments with L-phenylalanine and L-proline with SmDTPA and TbDTPA in a 2:1 ratio were performed at an initial $\mathrm{pH}$ of 4 to avoid partial decomplexation of the DTPA chelate. Upon the addition of L-proline, the relative intensity of the $\mathrm{Tb}^{3+}$ emission bands decreases when excited directly into the $f-f$ absorption bands at $366 \mathrm{~nm}$. However, when 
excited into the phenyl chromophore of Lproline, the emission intensity increases. This suggests that the aromatic groups in the amino acid are sensitizing the emission, thus more effectively populating the ${ }^{5} \mathrm{D}_{4}$ excited state of $\mathrm{Tb}^{3+}$, which in turn indicates that proline is interacting with the TbDTPA complex on the millisecond timescale. However, the excitation spectrum (recorded at the emission maximum of $545 \mathrm{~nm}$ ) shows the presence of both $f-f$ and aromatic $\pi \rightarrow \pi^{*}$ absorption bands, indicating that the L-proline is in fast exchange between bound and unbound forms on the timescale of the experiment. This may well lead to considerable vibrational quenching of the $\mathrm{Tb}^{3+}$ excited state, explaining why the emission intensity of solutions of DTPA and L-proline are attenuated with respect to TbDTPA itself. A similar situation is also met for solutions of TbDTPA and L-phenylalanine and for SmDTPA.

Time-resolved data was used to assess the inner-sphere hydration of the complexes. The lifetimes of lanthanide-centered emission in water and deuterium oxide can be used to calculate qLn, the number of inner-sphere water molecules bound to the lanthanide ion, using the equation:

$\mathrm{q}_{\mathrm{Ln}}=\mathrm{A}\left(1 / \tau_{\mathrm{H} 2 \mathrm{O}}-1 / \tau_{\mathrm{D} 2 \mathrm{O}}-\mathrm{B}\right)$

where $\mathrm{A}$ and $\mathrm{B}$ are constants for a given lanthanide ion. For europium $\mathrm{A}_{\mathrm{Eu}}=1.2 \mathrm{~ms}$ and $\mathrm{B}_{\mathrm{Eu}}=(0.25-0.075 \mathrm{x}) \mathrm{ms}^{-1}$, where $\mathrm{x}$ is the number of exchangeable $\mathrm{N}-\mathrm{H}$ oscillators. Effective calculations of $\mathrm{q}$ are also possible for terbium and ytterbium complexes (i.e., $\mathrm{A}_{\mathrm{Tb}}=5$ $\mathrm{ms}, \mathrm{B}_{\mathrm{Tb}}=0.06 \mathrm{~ms}^{-1}, \mathrm{~A}_{\mathrm{Yb}}=1 \mathrm{~ms}$, and $\mathrm{B}_{\mathrm{Yb}}=$ $0.1 \mathrm{~ms}^{-1}$ ), but the facility of $\mathrm{C}-\mathrm{H}$ quenching in other lanthanide complexes means that this approach cannot be applied to calculate $\mathrm{q}_{\mathrm{Ln}}$.
The luminescence lifetimes are affected by the presence of the amino acid added to the SmDTPA aqueous solution, indicative of bimolecular quenching as a result of fast exchange of bound and free amino acid and a weak interaction of the amino acid and the lanthanide complex. However, for the TbDTPA systems, the luminescence lifetimes, especially those recorded in D2O solutions, decrease, and the inner sphere hydration number $\mathrm{q}_{\mathrm{Tb}}$ decreases in the L-proline case and conversely increases in the L-phenylalanine case. These values and discrepancies suggest that calculated $q$ values are the only apparent ones, and other competitive decay processes are operative. In the case of TbDTPA, this is likely to be collisional quenching and a significant degree of back energy transfer from the $\mathrm{Tb}^{3+}$ excited state to the excited triplet state of the aromatic chromophores. This renders lifetime measurements and calculations of q unreliable. It is clear that adding L-proline and Lphenylalanine to solutions of LnDTPA results in complex kinetics on both the millisecond and microsecond timescales and/or the $\mathrm{pH}$ of the solutions has drastically changed upon addition of the amino acids. These experiments are currently being repeated in buffered solutions over a range of $\mathrm{pH}$ values (7.4 to 1$)$.

\section{Benefits to DOE}

The experimental program of study on the impact of utilizing amino acids in the separation of $\mathrm{An}^{3+}$ from $\mathrm{Ln}^{3+}$ is providing otherwise unavailable data on the long-term performance of viable actinide/lanthanide separations processes. This work is contributing to design of an advanced fuel cycle that is resistant to nuclear proliferation. 


\section{Development of a Small Sample Volume Mechanical Properties Testing Technique for Irradiated Fuels and Materials}

James I. Cole, Dennis Keiser, Jr.; David Bahr (Washington State University) 09-024

This project was targeted at developing a mechanical testing system and methodology to rapidly evaluate the strength, stiffness, and in some cases, toughness of nuclear fuels and materials prior to and following irradiation. A system was developed that combined conventional microhardness measurements with the ability to measure indenter deflection during the test. The goal was to deliver an instrument that can be placed within a limited volume controlled environment glove box so that the mechanical response of small irradiated samples can be assessed at a cost which is significantly lower than that using macroscopic tensile testing methodologies.

\section{Project Summary}

The indentation system developed for the project was designed and constructed by Professor Dave Bahr and graduate students at Washington State University in consultation with the INL principal investigators. During the first year of the project, a prototype system was developed, along with the initial instrument control software. The control software was based on the commercial program, Labview. The key development was to standardize the partial unload segment so that material properties could be evaluated at multiple depths. This development used a stepper motor driving system and a linear variable differential transducer for measuring displacement. Following testing of the prototype indenter system, a multi-tip indenter designed specifically for the glovebox environment was constructed incorporating several design changes to improve performance including optics and vibration isolation padding (Figure 1 shows the schematic and image of system).

The instrumented indentation system was calibrated and tested during the subsequent year. A full-featured control software for the system was written and the system was used to test a variety of unirradiated materials. Initial testing proved the system was working, the methodology for developing a quick and low cost screening instrument for assessing strength and hardening was valid, and the system was reproducible. Issues related to calibration and reproducibility across material types, however, became evident during testing and some minor redesign and modification became necessary. This extended the system development into the third year of the project and delayed delivery of the system to INL.

While the system was being developed, an environmentally controlled glove-box for conducting testing on lower activity level samples was established at INL. The indenter system was delivered to INL near the end of the project and will be used to support ongoing research on fuels and materials.
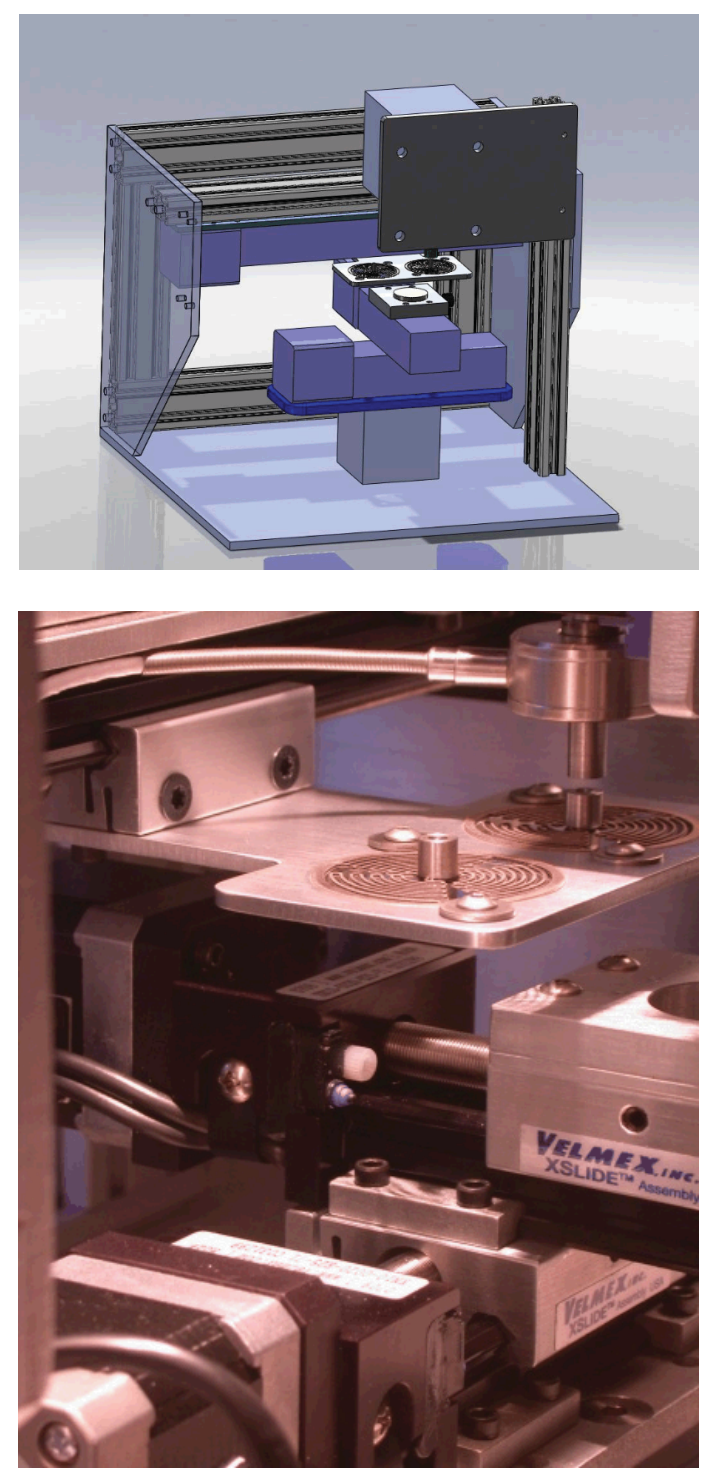

FIGURE 1. Schematic of indenter frame (top) and image of testing stage (bottom). The frame dimensions are such that it can easily fit through a glove-box port. 
Benefits to DOE

Rapid and lower cost screening of developmental fuels and materials is a consideration for next generation nuclear energy systems and can complement other testing techniques in providing data for predictive model development. A simple testing instrument and method that can provide initial guidance in down-selecting materials to a few for further, more extensive testing can greatly improve the options available for researchers. In addition, with many new developmental materials, the volume available is small; a micromechanical testing system allows limited experimental alloys to be assessed while providing statistically significant results. 


\section{Multiscale Modeling and Simulation of Nuclear Fuel Performance}

Rich Williamson, Derek Gaston, Michael Tonks, Cody Permann

10-017

Current state-of-the-art computational tools used to simulate nuclear fuel performance employ highly empirical models, which prohibit application to new fuel materials and designs, or to extended fuel burnup. These tools are further restricted to 1.5 - or $2 \mathrm{D}$ geometries, which prohibit analysis of important $3 \mathrm{D}$ behavior, and are limited to use on a single computer, which limits applications to simple problems. The purpose of this project is to develop a multiscale parallel 3D fuel performance capability that fully couples a mesoscale description of the nuclear fuel microstructural evolution, with an engineering-scale prediction of fuel behavior, resulting in a simulation capability that is truly predictive. Since 2008, the Multiphysics Object Oriented Simulation Environment, or MOOSE, framework has been under development at INL to facilitate creation of this capability. An engineering-scale fuel performance code, BISON, and a mesoscale fuels code, MARMOT, have also been under development. The coupling of these two fuel codes (within MOOSE) provides a multiscale, parallel, multidimensional fuel simulation capability. Specific FY 2011 objectives of this LDRD project included: (1) demonstrate coupled scale bridging between the mesoscale and engineering scale,

(2) demonstrate massively parallel computational capability ( $>10,000$ processors), and

(3) demonstrate an efficient rapidly converging nonlinear material model implementation within the MOOSE framework.

\section{Progress Summary}

All FY 2011 objectives were achieved. For the first, a coupled multiscale (BISON/MARMOT) model was developed to predict thermal conductivity evolution and its effects on fuel temperature. It was demonstrated that considering intergranular porosity, rather than uniformly distributed porosity, results in a significant rise in the fuel centerline temperature. This work has been extensively documented in the journal articles cited below. For the second objective, the parallel computational capability of MOOSE/BISON was demonstrated by simulating the behavior of a highly resolved fulllength discrete-pellet fuel rod. This fully coupled implicit simulation required over 234 million degrees of freedom and was run on the INL
FISSION high-performance computer, using up to 12,000 processors. Figure 1 shows the parallel scaling results obtained from this exercise. Very good strong and weak scaling was obtained for over 10,000 processors, demonstrating that MOOSE-based codes are applicable to and can be efficiently used to solve very large problems when needed. For the third objective, highly nonlinear material models for both time independent (plasticity) and time-dependent (creep) behavior were implemented within the

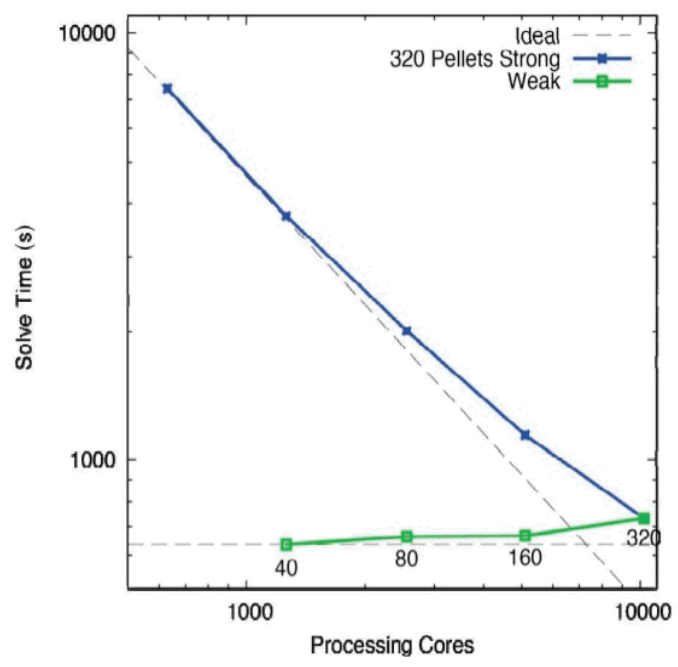

FIGURE 1. Parallel scaling results from MOOSE/BISON.

MOOSE framework. This is significant since such models have never been implemented within a Jacobian Free Newton Krylov numerical scheme. It was demonstrated that Jacobian Free Newton Krylov is well-suited for such analysis, as documented in a journal article cited below. An example of this nonlinear analysis capability is shown in Figure 2 (next page). Here, BISON was used to study the effects of a manufacturing fuel defect known as a missing pellet surface. The middle fuel pellet in the five-pellet rodlet is missing a portion of its outer surface, resulting in a large gas gap and much higher local fuel temperatures. Stress and deformation in the cladding adjacent to the missing pellet surface is significantly increased and can result in fuel-rod failure. This is the first time such behavior has been analyzed in 3D. 


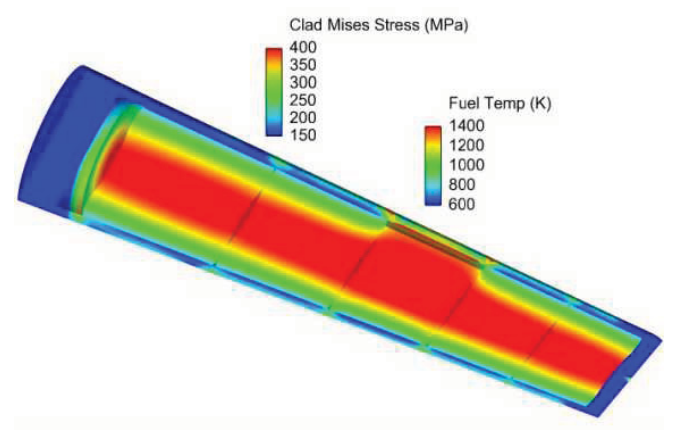

FIGURE 2. Temperature and stress results from a MOOSE/BISON nonlinear analysis of a fuel rod containing a defective fuel pellet.

Benefits to DOE

The MOOSE/BISON computational tools were recently selected both as the Nuclear Energy Advanced Modeling and Simulation fuel performance code and to support the Consortium for Advanced Simulation of Light Water

Reactors Materials Performance and Optimization focus area for pellet-clad interaction. Additionally, the code is supporting experimental design for the Fuel Cycle Research and Development campaign, whose technical leads recently agreed that MOOSE-BISONMARMOT is ready to play an active role in their fuel research efforts.

\section{Relevant Publications and Presentations}

Tonks, M. R., D. Gaston, P. C. Millett, D. Andrs, and P. Talbot, 2012, “An Object-Oriented Finite Element Framework for Multiphysics Phase Field Simulations," J. Comp. Material Science, Vol. 51, p. 20.

Gaston, D. L. Guo, G. Hansen, H. Huang, R. Johnson, H. Park, R. Podgorney, M. Tonks, and R. Williamson, 2011, "Parallel Algorithms and Software for Nuclear, Energy, and Environmental Applications. Part I: Multiphysics Algorithms," Communications in Computational Physics, in press.
Gaston, D., L. Guo, G. Hansen, H. Huang, R. Johnson, H. Park, R. Podgorney, M. Tonks, and R. Williamson, 2011, "Parallel Algorithms and Software for Nuclear, Energy, and Environmental Applications. Part II: Multiphysics Software," Communications in Computational Physics, in press.

Hales, J. D., S. R. Novascone, R. L. Williamson, D. R. Gaston, and M. R. Tonks, 2011, "Solving Nonlinear Solid Mechanics Problems with the Jacobian-Free Newton Krylov Method," submitted to Computational Mechanics.

Tonks, M. R., P. Millett, P. Nerikar, D. Andersson, C. Stanek, D. Gaston, D. Andrs, and R. Williamson, 2011, "Multiscale Simulation of Heat Conduction in $\mathrm{UO}_{2}$ Fuel," submitted to Journal of Nuclear Materials.

Millet, P. C., and M. R. Tonks, 2011, "Mesoscale Modeling of the Influence of Intergranular Gas Bubbles on Effective Thermal Conductivity," Journal of Nuclear Materials, Vol. 412, p. 281.

Hales, J. Hales, D. Andrs, D. Gaston, S. Novascone, C. Permann, M. Tonks, and R. Williamson, 2011, "Fully Coupled, 3D, Multi-physics for Analysis of Nuclear Fuel," 11th US National Congress on Computational Mechanics, Minneapolis, Minnesota, USA.

Tonks, M., D. Gaston, D. Andrs, C. Permann, R. Williamson, J. Hales, S. Novascone, P. Millett, B. Biner, C. Stanek, D. Andersson, and P. Nerikar, 2011, "Advanced Multiscale Fuel Performance Modeling," Materials Modeling and Simulation of Nuclear Fuel Workshop, Aix En Provence, France.

Tonks, M. R., D. Gaston, C. Permann, P. Millet, G. Hansen, and D. Wolf, 2010, "A Coupling Methodology for Mesoscale-informed Nuclear Fuel Performance Codes," Nuclear Engineering and Design, Vol. 240, p. 2877. 


\section{Irradiation Testing and Molecular Modeling of Irradiation- Assisted Diffusion and Microstructural Evolution (FCCI)}

Bulent H. Sencer; T. Hartmann, K. Czerwinski (University of Nevada-Las Vegas); L. Shao (Texas A\&M); Y. Sohn, K. R. Coffey (University of Central Florida); C. Deo

(Georgia Institute of Technology)

$10-068$

Irradiating nuclear fuel in reactors causes fuel swelling and production and transport of fission products to the cladding. The contact between fuel matrix fission products and fuel cladding will promote chemical and mechanical interactions with the cladding. The chemical compatibility between the fuel and cladding is imperative for safe operation of a reactor, and it is essential to limit the interdiffusion between the fuel and cladding. This study will investigate, with the use of ion irradiation created in an accelerator, the effects that different irradiation types might have on fuel-cladding interactions with prototypic material systems that have been traditionally difficult to study. Modeling efforts will be supported with this data to demonstrate the combination of testing simulated systems and modeling. The proposed study will contribute to a better understanding of fuel-cladding interaction by investigating model systems coupled with the fuel materials. Fuel-cladding diffusion experiments will be conducted at temperatures of up to $650^{\circ} \mathrm{C}$ under proton, helium, and other heavy ion irradiation if appropriate. The various diffusion couples will be analyzed by imaging, phase analytical, and chemical methods. We will use electron microscopy and other tools to investigate the interdiffusion kinetics, phase formation at the clad-fuel interface, diffusion coefficients, and the clad-fuel microstructural development.

The main purpose of the research is to measure activation energies that are thermal and radiation enhanced. We have about 40 diffusion couples (single and binary) for a single temperature. We will continue to prepare diffusion couples for thermal activation energies followed by irradiation to see if there is any enhancement in the interface.

\section{Project Summary}

University of Nevada-Las Vegas (UNLV), Texas A\&M, University of Central Florida (UCF), and Georgia Institute of Technology (GeTech) are the collaborators for this project. Contracts were set up with the new university partners (Texas A\&M and UCF) during the second year. Needed materials were cast or bought by INL and shipped to the universities. UNLV employed one postdoc and UCF, Texas $\mathrm{A} \& \mathrm{M}$, and GeTech each employed one graduate student. UNLV and UCF prepared diffusion couples for characterization. The couples are pure $\mathrm{Fe}, \mathrm{Mo}, \mathrm{Zr}, \mathrm{Ce}, \mathrm{Nd} / \mathrm{alloys}$ of $\mathrm{Ce}-50 \mathrm{Nd}, \mathrm{Fe}-$ $15 \mathrm{Cr}, \mathrm{Fe}-15 \mathrm{Cr}-15 \mathrm{Ni} /$ diffusion couples (bonded) of $\mathrm{Fe}$ versus $\mathrm{Zr}$ and Mo versus $\mathrm{Zr}$. These materials were irradiated with heavy ions $(\mathrm{Fe})$ to $100 \mathrm{dpa}$ at $450^{\circ} \mathrm{C}$ and $650^{\circ} \mathrm{C}$. Irradiations at $550^{\circ} \mathrm{C}$ will be carried out early next year by Texas A\&M. Characterization of $450^{\circ} \mathrm{C}$ and $650^{\circ} \mathrm{C}$ irradiation will start shortly. UNLV works with some non-U and $\mathrm{U}$-containing diffusion couples. UNLV is ready to send first non-U diffusion couples, and will follow by Ucontaining diffusion couples. UNLV samples will be irradiated by helium at three different irradiation temperatures. UNLV is helping UCF to characterize the diffusion couples with an electron probe micro-analyzer. Texas A\&M is also contributing to the characterization and used our focused ion beam to prepare transmission electron microscope samples from different regions of several diffusion couples $(\mathrm{Fe} / \mathrm{Zr}$, $\mathrm{Fe} / \mathrm{Mo}$ ), that are irradiated to 100 dpa with heavy ion $(\mathrm{Fe})$.

Several manuscripts are prepared for journal publication on microstructure. We also calculated a number of kinetic and diffusion properties (U/Mo, $\mathrm{U}-10 \mathrm{Zr} / \mathrm{Fe}, \mathrm{U} / \mathrm{Fe}$, etc). UCF also prepared several manuscripts for publication. The universities will refabricate any diffusion couples that did not properly bond and repeat the irradiations if necessary. GeTech is using models (current and new) for verification and validation. GeTech performed a series of single-point electronic structure calculations in the Vienna ab initio Simulation Package for migration barriers in body-centered cubic (bcc) Mo and bcc U. The phase diagram of the Fe-U system reveals two main intermetallic phases: the Fe rich $\mathrm{Fe}_{2} \mathrm{U}$ and uranium-rich $\mathrm{FeU}_{6}$. Not much is known about these phases, so we performed first-principles calculations using the Vienna ab initio Simulation Package to characterize these phases in more detail. We calculated the structural features, lattice positions, lattice parameters as well as elastic constants of these phases. GeTech is also preparing a manuscript for journal publication.

This research was carried out to learn about kinetic and diffusion coefficients of the diffusion 
couples, which has not been done before. We produced the missing data (kinetic, interdiffusion coefficients, etc.) for simple systems so that modelers can model starting from the simplest system and later move into a more complex system.

Next fiscal year, we will concentrate on the U-containing diffusion couples and heavy ion $(\mathrm{Fe})$ and $\mathrm{He}$ irradiations. If time allows, we also might use heavy fission gasses.

\section{Benefits to DOE}

Interdiffusion phenomena are very important to understanding the useful lifetime of nuclear reactor components and fuels. One of the goals of the materials and nuclear fuels science activity is to determine measurable material changes associated with service degradation that are indicative of incipient failure. For example, fast reactor fuels are often limited in operating characteristics or lifetime by interaction of the fuel or fission products with the fuel cladding materials. Detailed models for some of these effects, such as the fuel-cladding interactions, are just now being considered and data is needed to test the models (verification and validation). It is also difficult and costly to construct in-reactor experiments to study these effects. 


\section{Spatially Correlated Microstructural Characterization: From Centimeters to Nanometers}

Dawn E. Janney, Timothy R. McJunkin, Jill R. Scott

10-060

INL uses many scientific instruments to analyze spatially resolved data (i.e., data whose value can be expected to vary depending on where in the sample it was collected), on a continuum of spatial scales extending across seven orders of magnitude (from centimeters to nanometers). Each instrument analyzes different characteristics of the samples, so a thorough understanding of the material would require researchers to integrate information collected from the same area using different instruments. Integrating the information requires solving two major kinds of problems: (1) finding the same area with multiple instruments (and recognizing when you have done so) and (2) allowing researchers to identify all of the data from an area, no matter what instrument was used.

This LDRD addresses both of these problems by creating standardized sample holders and software that will make it possible to collect a wide variety of data (e.g., optical, chemical, microstructural, thermal, structural, and micro-hardness) from the same area and find data from each area collected using different instruments. Although the primary objective of the LDRD is to provide a tool that can be used to understand nuclear fuels and materials, the tools we develop can be used for a variety of applications.

\section{Project Summary}

This project has just completed its second year. The first year, we primarily developed a proof of principle in which we identified three widely disparate instruments, developed software that allowed us to find the same location and keep track of which instruments had been used to collect data at each location, and demonstrated that the software worked by finding the same location on a sample. We identified several problems requiring further work, including: (1) developing uniquely recognizable markers ("fiducials") that could be identified by all of the instruments with high spatial precision,

(2) developing a more user-friendly software interface, and (3) figuring out how to mount the same sample in a variety of instruments despite their different constraints on the sizes and shapes of samples they can accept.
Major accomplishments and activities during FY 2011 included:

- Developing a list of candidate instruments, which includes those currently at the Materials and Fuels Complex, INL Research Center, and Center for Advanced Energy Studies

- Designing, manufacturing, and testing new sample holders specifically intended for compatibility between all of the instruments that measure data on a spatial scale of a few tens of micrometers or larger, with instrument-specific adapters as required

- Experimenting with different sample materials to identify a possible candidate for a "round robin" demonstration involving all of the instruments in FY 2012

- Demonstrating that we could measure the coordinates of a location, remove the sample, replace the sample in the same instrument, and find the original location using various instruments (these instruments include, but are not limited to, a Bruker D8 Discover micro-focus X-ray diffractometer, a Cameca microprobe, a scanning thermal diffusivity microscope, several scanning electron microscopes, and two focused-ionbeam instruments)

- Demonstrating that we could measure the coordinates of a location, put the sample into a different instrument, and find the same location for several combinations of instruments

- Extensive software development, including:

- A new interface to make the software more user friendly by guiding the user through a dialog-box-driven interface

- Adding capabilities to make optimal use of new sample holders.

Benefits to DOE

The DOE is tasked with securing competitive and environmentally responsible nuclear technology to meet the energy needs of the United States. This requires designing and fabricating nuclear fuels and materials, which in turn requires the ability to use a variety of 
instruments to collect chemical, structural, and microstructural data from exactly the same locations on highly radioactive materials, and to be able to tell which instrument has been used to collect data from each location. This project will greatly enhance researchers' ability to locate the same area of a sample using a variety of instruments and correlate all of the data for each area. It will thus enable more comprehensive characterization of nuclear materials and fuels than is currently possible, providing new knowledge and understanding that can greatly enhance the DOE's ability to carry out its mission.

\section{Relevant Publications and Presentations}

Janney, D. E., T. R. McJunkin, T. L. Trowbridge, and J. R. Scott, 2011, "Spatially-correlated Microstructural Characterization: From Centimeters to Nanometers," 2011 TMS Annual Meeting \& Exhibition, sponsored by The Minerals, Metals \& Materials Society, San Diego, California, USA, February 27March 3, 2011. 


\title{
Alternative Processing for Improved $\mathrm{UO}_{2}$-Based Fuels
}

\author{
Paul A. Lessing, Pavel Medvedev, W. Roger Cannon (Rutgers University)
}

11-051

Developing a science-based understanding of alternative and advanced processing methods as they apply to depleted uranium oxide $\left(\mathrm{DUO}_{2}\right)$ will help improve the physical properties and hence in-reactor performance of sintered uranium-oxide-based fuels. To do this requires developing a fundamental understanding of the chemical and physical factors that affect the ceramic processing of $\mathrm{DUO}_{2}$. These factors include moisture content of powders, surface area and shape of crystallites, use of organic binders, and particle packing and granule formation. The final physical properties will be enhanced by using the fundamental knowledge to improve fabrication and sintering methods. Emphasis is placed upon dry processing methods suitable for inert atmosphere glove box operations. This includes the study, selection, and performance-quantification (e.g., pellet strength and fracture toughness) of radiation-resistant binders (e.g., for transuranicmixed oxide [TRU-MOX] fuels). The binders studied include both water alone and various classes of organic (polymer) binders. Rounded granules formed from powders plus binders will be homogeneously consolidated using isostatic pressure in a dry bag isostatic press and then pressureless-sintered to the desired final shape and density. Global Nuclear Power is very interested in our approach and would like to examine resulting pellets composed of natural $\mathrm{UO}_{2}$ and evaluate the performance of

INL-fabricated pellets in one of their reactors.

\section{Project Summary}

During this first year of the project, a radiological facility was equipped to enable processing of both depleted and natural $\mathrm{UO}_{2}$ (Fig. 1). Typically, binders are added to ceramics powder to improve green strength of a presintered part. It may be possible to avoid the use of binders altogether in nuclear fuel pellets if the adsorbed moisture content of the green part is adjusted properly). For instance, the short binderless route has been used to reduce the number of processing steps when MOX manufacturing is carried out within a glove box. Difficult pressing problems have been reported for TRU-MOX pellets that contain transuranic elements (Am, Np) that generate radiation that can rapidly degrade organic binders. This project

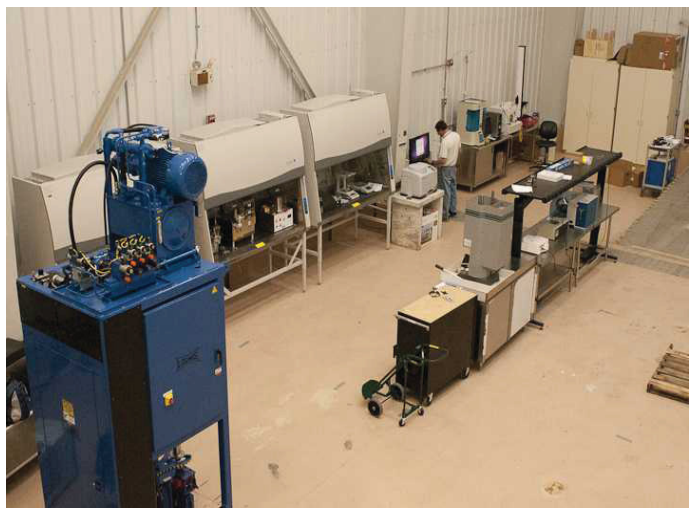

FIGURE 1. UO 2 processing lab at Intec-1634.

has documented that TRU-MOX green pellets, containing many organic binders, become extremely fragile (Fig. 2) within a few days after pressing. The strength values were measured using a special mechanical diametral fracture device designed and built at INL. To further define the degradation mechanism, films of binders were cast on glass slides and then irradiated at Florida State University using protons and helium nuclei. Various techniques (Fourier transform infrared spectroscopy, nuclear magnetic resonance, positron annihilation) are

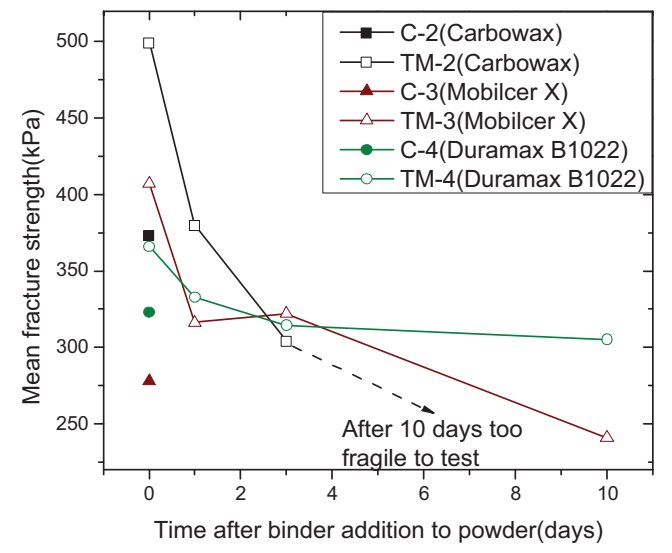

FIGURE 2. Fracture strength measured by diametral compression of pellets $v$ s. time after first mixing TRU-MOX with binder. Binders concentration are all $1.0 \mathrm{wt} \%$.

now being used at INL to characterize the accumulated damage to the polymer structures as a function of radiation flux. We also plan to develop non-organic binders that should be radiation resistant. A portable $\mathrm{DUO}_{2}$ sensor was designed and fabricated (Fig. 3, next page). This 
specialized monitor will help greatly in implementing ventilation and dust control procedures by providing a rapid assessment of the presence of DU. The monitor is composed of (1) a $2^{\prime \prime} \times 2$ " $\mathrm{NaI}(\mathrm{Tl})$ detector, (2) an integrated photomultiplier and multichannel analyzer system located in the base of the detector, and (3) integrated Labview software. This device provides a direct assessment of depleted uranium contamination and enrichment data with an easyto-use operator interface that provides real-time data on the quantities of depleted uranium present. This system is highly sensitive and automatically eliminates the effects of background on detector response.

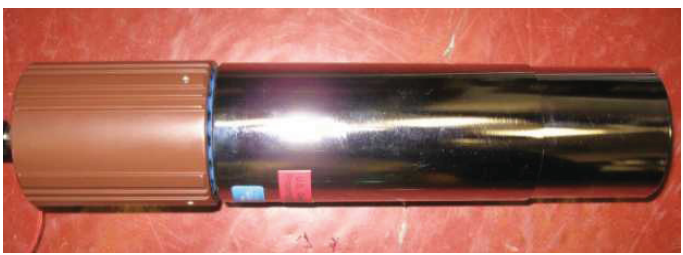

FIGURE 3. Developed special integrated NaI (Tl) detector for $\mathrm{DUO}_{2}$.

\section{Benefits to DOE}

INL will benefit from the work in three basic ways: (1) the results can be directly applied to the enhanced fabrication of $\mathrm{UO}_{2}$-based fuels of interest to DOE-Office of Nuclear Energy such as $\mathrm{MOX}\left(\mathrm{UO}_{2}+\mathrm{PuO}_{2}\right)$, TRU-MOX from recycled fuel $\left(\mathrm{UO}_{2}+\mathrm{PuO}_{2}+\right.$ actinides $)$ and enriched $\mathrm{UO}_{2},(2)$ the project builds INL's capability (knowledge and bench-top equipment) in fabricating $\mathrm{UO}_{2}$ that does not exist anywhere at INL, and (3) the knowledge can be applied to other non-oxide ceramic fuel compositions such as carbides and nitrides. Results benefit two DOE programs managed by INL: the Fuel Cycle
Research \& Development program and the Light Water Reactor program. The project will result in fuel pellets with the density (designed porosity) and homogeneity necessary to enable ceramic fuels to be used in a high-neutron-flux reactor (e.g., advanced recycling reactor) where the fuel is to be irradiated to high burn-up (beyond $100 \mathrm{GWd} / \mathrm{t}$ ). Variations on our new fabrication techniques will prove to be innovative and valuable to ceramic fuel pellets for industrial light water reactors, including MOX and inert matrix fuel for light water reactors.

\section{Relevant Publications and Presentations}

Lessing, P. A., W. R. Cannon, G. Egeland, L. Zuck, and P. Medvedev, 2012, "Strength Loss in TRU-MOX Green Pellets from Radiation Damage to Binders," In preparation for submission to Journal of Nuclear Materials.

Cannon, W. R., P. A. Lessing, and L. D. Zuck, 2011, "Crack Model for Toughness of Green Parts with Moisture or a Fluid Binder," Submitted to Journal of American Ceramic Society, September 27, 2011.

Lessing, P. A., 2011, "Alternative Processing of Improved UO2-based fuels," to INL outside peer review, June 23, 2011. 


\section{Instrumentation for In-pile Detection of Thermal Conductivity}

Darrell Knudson, Joy Rempe

09-007

Thermal conductivity is a key property that must be known for proper design, test, and application of new fuels and structural materials in nuclear reactors. Currently, changes in thermal conductivity of fuel or materials irradiated in the Advanced Test Reactor (ATR) are evaluated outof-pile, where properties of materials are measured after samples have been irradiated for a specified length of time. The labor and time to remove, examine, and return irradiated samples for each measurement makes out-of-pile approaches very expensive. In addition, data may not be obtained under prototypical conditions because they only capture the sample's endstate after it is removed from the reactor and removal and reinsertion into the reactor may disturb the phenomena of interest.

In this three-year project, INL and Utah State University, with assistance from the Institute for Energy Technology at the Halden Reactor Project, explored the benefits, limitations, and accuracy of two candidate in-pile thermal conductivity measurement approaches: (1) a multiple thermocouple steady-state approach, and (2) a transient hot wire method with the ultimate objective of providing ATR users a method for detecting changes in fuel or material thermal conductivity during irradiation.

\section{Project Summary}

During FY 2009 (the first year of this three-year project), efforts focused on evaluating the multiple thermocouple approach. Results show accuracies similar to those obtained with out-ofpile techniques could be achieved for a limited set of conditions. During FY 2010, investigations primarily focused on the transient hot wire method approach because of the increased accuracies possible with this technique. The transient hot wire method, or line heat source method, is applied by embedding a line heat source in the material whose thermal conductivity is to be measured (Fig. 1). When using this established technique for non-nuclear applications, the heat source is energized and heats the sample with constant power. The temperature response of the sample is a function of its thermal conductivity. In this project, a new hot wire probe design containing a resistance heater and a thermocouple embedded in a single probe was developed that was suitable for in-pile applications. During FY 2011, laboratory tests (Fig. 2, next page) were completed to evaluate accuracy of the probe for a range of operating conditions and stability of the probe during long-duration tests. Results show accuracy within $5 \%$ at room temperature and $7 \%$ at temperatures up to $600^{\circ} \mathrm{C}$ for materials with thermal conductivities between 0.2 and 23 $\mathrm{W} / \mathrm{m} \cdot \mathrm{K}$. Efforts were also completed to enhance measurement accuracies by using improved signal processing techniques. A prototype needle probe (Fig. 3, next page) was fabricated and shipped to the Massachusetts Institute of Technology for an upcoming irradiation of hydride fuel in the Massachusetts Institute of Technology Research Reactor. Hence, this research was able to go beyond its anticipated workscope by facilitating the irradiation of a prototype probe.

\section{Benefits to DOE}

Results from this project increase INL irradiation testing instrumentation capabilities, which support DOE's energy security mission by developing and demonstrating in-pile instrumentation needed to obtain data required for key DOE-Office of Nuclear Energy research programs, such as the Generation IV, Fuel Cycle Research and Development, and ATR National Scientific User Facility programs. Specifically, this research is enhancing INL's expertise for producing optimized, more reliable, and lowercost in-pile instrumentation. In addition, enhanced instrumentation from this research has the potential to increase testing capabilities of INL's ATR, which benefits various U.S.

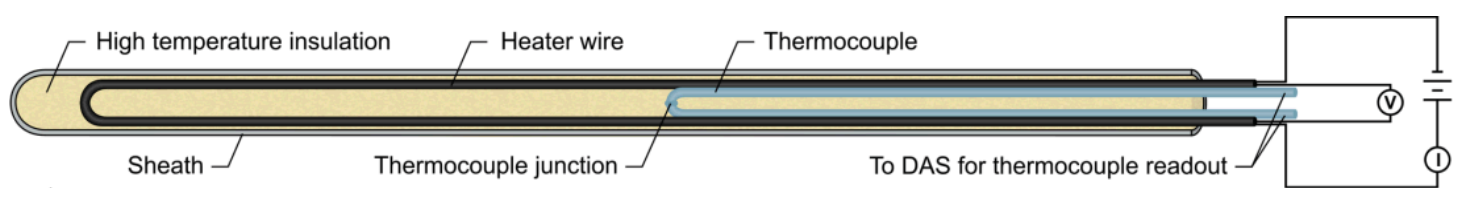

FIGURE 1. THWM needle probe design. 
programs that conduct testing at this unique facility. Finally, recognition (e.g., publications, patents, and awards) from this research promotes INL's instrumentation capabilities, furthering INL efforts to develop the Instrumentation, Control, and Intelligent Systems Distinctive Signature.
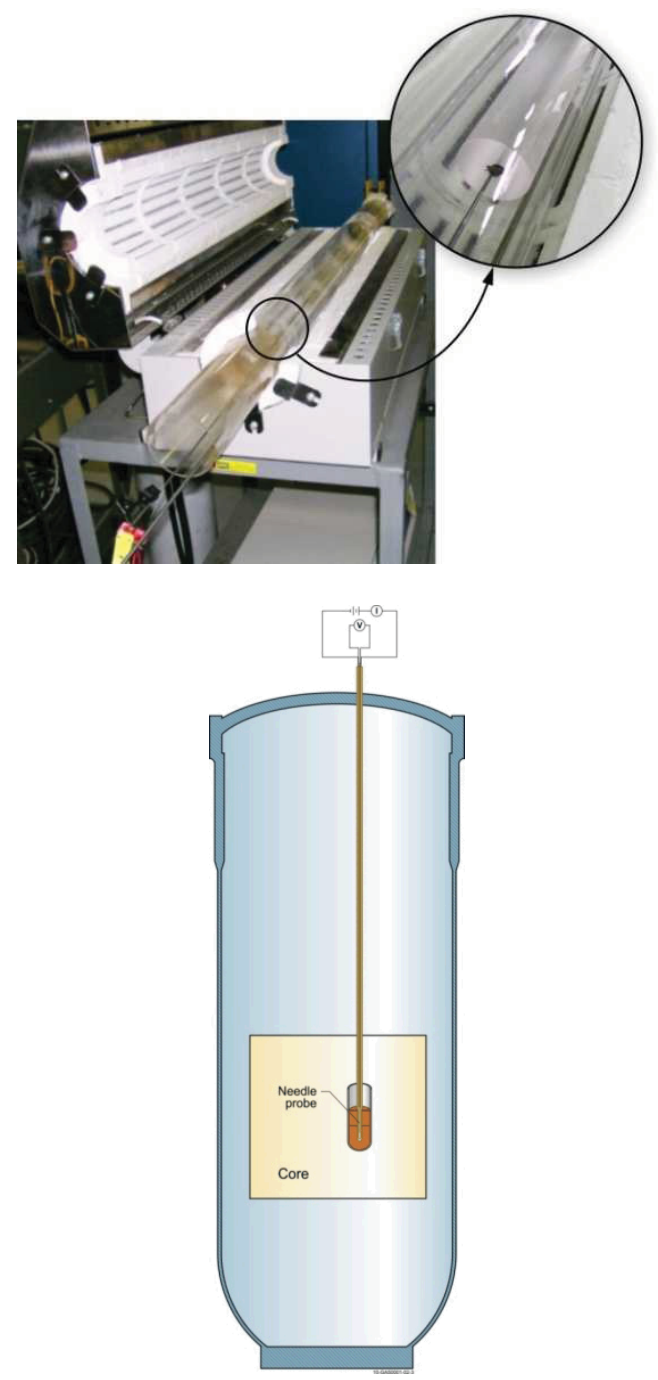

FIGURE 2. Laboratory testing setup for THWM needle probe (top) and conceptual design for in-pile testing (bottom).

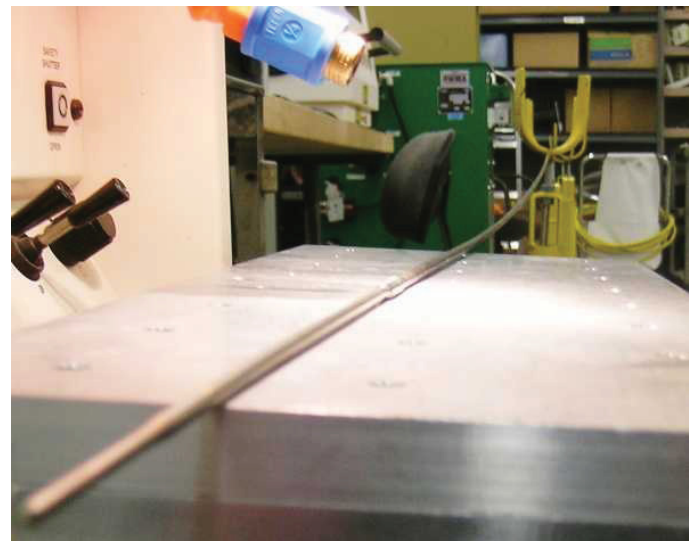

FIGURE 3. Transient hot wire method needle probe shipped to Massachusetts Institute of Technology.

\section{Relevant Publications and Presentations}

Kim, B. G., J. L. Rempe, J-F Villard, and S.

Solstad, 2011, "Review of Instrumentation for Irradiation Testing of Fuels and Materials," Invited review paper, Nuclear Technology, Vol. 176, November 2011, pp. $155-187$.

Rempe, J., D. Knudson, K. Condie, J. Daw, and S. C. Wilkins, 2011, "New Sensors for InPile Temperature Measurement at the Advanced Test Reactor National Scientific User Facility," Invited paper, NURETH13 Special Edition, Nuclear Technology, Vol. 175, September 2011.

Rempe J., D. Knudson, J. Daw, T. Unruh, B. Chase, and K. Davis, 2011, "Enhanced Inpile Instrumentation at the Advanced Test Reactor," Invited paper for ANIMMA 2011 Special Edition, IEEE Transactions on Nuclear Science, MS \# SCH-TNS-001962011, accepted September 27, 2011.

Rempe, J., D. Knudson, J. Daw, T. Unruh, B. Chase, K. Condie, J. Palmer, and K. Davis, 2011, "Enhanced In-pile Instrumentation at the Advanced Test Reactor," Proceedings of the Second International Conference on Advancements in Nuclear Instrumentation, Measurement Methods and their Applications (ANIMMA2011), Ghent, Belgium, June 2011. 
Daw, J., J. Rempe, K. Condie, D. Knudson, S. C. Wilkins, B. Fox, and H. Ban, 2010, "Hot Wire Needle Probe for In-Pile Thermal Conductivity Detection," 7th International Topical Meeting on Nuclear Plant Instrumentation, Control, and Human Machine Interface Technologies (NPIC\&HMIT 2010), Las Vegas, Nevada, USA, November 7-11, 2010.

Fox, B., H. Ban, J. Rempe, J. Daw, K. Condie, D. Knudson, 2010, "In-Pile Thermal Conductivity Measurement Method for Nuclear Fuels," Thermal Conductivity 30/Thermal Expansion 18, Daniela S. Gaal and Peter S. Gaal (Editors), DEStech Publications Inc., October 2010, p. 886.

Rempe, J., D. Knudson, K. Condie, J. Daw, and S. C. Wilkins, 2009, "New Sensors for InPile Temperature Detection at the ATR NSUF," Proceedings of the 13th International Topical Meeting on Nuclear Reactor Thermal-Hydraulics (NURETH13), Kanazawa, Japan, October 2009.

Fox, B., H. Ban, J. Rempe, J. Daw, K. Condie, and D. Knudson, 2009, "In-Pile Thermal Conductivity Measurement Method for Nuclear Fuels," $30^{\text {th }}$ International Thermal Conductivity Conference and $18^{\text {th }}$ International Thermal Expansion Symposium, Pittsburgh, Pennsylvania, USA, August 29-September 2, 2009.

Rempe, J., D. Knudson, K. Condie, J. Daw, H. Ban, B. Fox, G. E. Kohse, and S. C. Wilkins, 2009, "New Sensors for In-Pile Testing at the ATR NSUF," Proceedings of the First International Conference on Advancements in Nuclear Instrumentation, Measurement Methods and their Applications (ANIMMA), Marseilles, France, June 2009.

Rempe, J., and M. Meyer, 2009, “ATR NSUF Instrumentation Enhancement Efforts," Proceedings of the ANS NPIC HMIT 2009 Topical Meeting on Nuclear Plant Instrumentation, Controls, and Human Machine Interface Technology, Knoxville, Tennesee, USA, April 2009.
Rempe J., J. Daw, D. Knudson, R. Schley, and L. Bond, 2011, "FCRD In-Pile Instrumentation Development," Panel Discussion at the ANS 2011 Annual Meeting, Hollywood, Florida,USA, June 28, 2011.

Daw, Joshua E., Joy L. Rempe, Robert S. Schley, Kate B. Boudreau, and Gordon Kohse, 2011, "Instrumentation to Enhance ATR NSUF Irradiation Testing," Advanced Test Reactor National Scientific User Facility User's Week, June 9, 2011.

Daw J., and J. Rempe, 2010, "New Sensors for Advanced Test Reactor Irradiations," INL ATR NSUF ATR Workshop on Irradiation Testing and Planning, ANS Student Meeting, Georgia Institute of Technology, Atlanta, Georgia, USA, April 14, 2010.

Rempe, J. L., B. G. Kim, G. Kohse, S. Solstad, and J.-F. Villard, 2010, "Current Instrumentation to Support FC R\&D Irradiations," FCR\&D In-Pile Instrumentation Workshop, Las Vegas, Nevada, USA, November 10, 2010.

Rempe, J., 2010, “Challenges of In-Pile Sensing and Transmission," NanoNuclear Workshop, Washington, DC, September 13-15, 2010.

Rempe, J., 2010, “LDRDs to Enhance ATR NSUF Instrumentation," ATR NSUF Scientific Review Board Meeting, Idaho Falls, Idaho, USA, June 7, 2010.

Rempe, J., and G. Kohse, 2010, "In-core Instrumentation: Motivation, Approaches, and Examples," ATR NSUF 2010 User's Week, Experimenter's Course on Instrumentation, Idaho Falls, Idaho, USA, June 8, 2010.

Rempe, J., 2010, "In-pile Instrumentation for the ATR," Nuclear Engineering Colloquium, Pennsylvania State University, State College, Pennsylvania, USA, April 22, 2010.

Rempe, J., 2010, “In-pile Instrumentation for ATR NSUF Irradiations," INL ATR NSUF ATR Workshop on Irradiation Testing and Planning, ANS Student Meeting, University of Michigan, Ann Arbor, Michigan, USA, April 8, 2010. 
Rempe, J. L., 2010, "New Sensors for Advanced Test Reactor Irradiations," ATR NSUF

Industry Advisory Committee, Palo Alto, California, USA, February 9, 2010.

Rempe, J., 2009, "Instrumentation

Enhancements for the Advanced Test Reactor," Invited Presentation, Japan Atomic Energy Agency, Orai, Japan, September 25, 2009.

Rempe, J. L., 2009, “LDRDs to Improve ATR In-pile Instrumentation," Review of INL LDRD Program by DOE-NE, Washington, DC, April 9, 2009. 


\title{
Ultrasonic Transducer Sensors for In-pile Detection of Dimensional Changes
}

\author{
Steve Taylor
}

10-007

At INL's Advanced Test Reactor (ATR), deformation of specimens during irradiation is currently evaluated out-of-pile, where the properties of materials are measured after the specimens have been irradiated for a specified length of time. The labor and time to remove, examine, and return irradiated specimens for each measurement makes the current out-of-pile approach very expensive. In addition, the out-ofpile measurements only capture the specimen's end state after it is removed from the reactor. Therefore, data are not obtained under prototypic conditions, and removal and examination processes may disturb the phenomena of interest. Although linear variable differential transducers have been successfully deployed at other test reactors and are being investigated for ATR applications, ultrasonic transducers offer the potential for a more compact, higher temperature, more accurate, and multi-dimensional real-time sensor for detecting geometry changes of creep specimens during irradiation. This three-year project is investigating the feasibility of using ultrasonic transducers as an in-pile sensor for detecting geometry changes in creep specimens by completing laboratory demonstrations (including bench top, furnace, and autoclave tests). Experimental results are also compared to theoretical predictions to further validate measurement capabilities.

\section{Project Summary}

During FY 2010, efforts primarily focused on optimizing components required for using an in-pile ultrasonic transducer sensor and completing laboratory demonstrations. Efforts were initiated to identify and optimize key components of a candidate in-pile setup (Figure 1) that included a magnetostrictive transducer (e.g., a driver coil with magnetostrictive core), a Remendur guide, a coupling between the Remendur guide and a long stainless steel wave guide that allows the transducer to be located outside of the reactor, and a creep specimen with an acoustic horn to optimize the signal. A method was developed that allows the magnetostrictive transducer to be directly coupled to a 30 -foot-long stainless steel waveguide with minimal loss in signal. Welding methods were tested to determine a suitable way to attach the 30-foot-long waveguide to the creep specimens. Results indicate that tungsten inert gas welding provides both good mechanical strength and minimal loss in the ultrasonic signal. The electronics for performing the measurements were tested to optimize measurement variables. Computer programs were written to perform data collection and increase system automation.

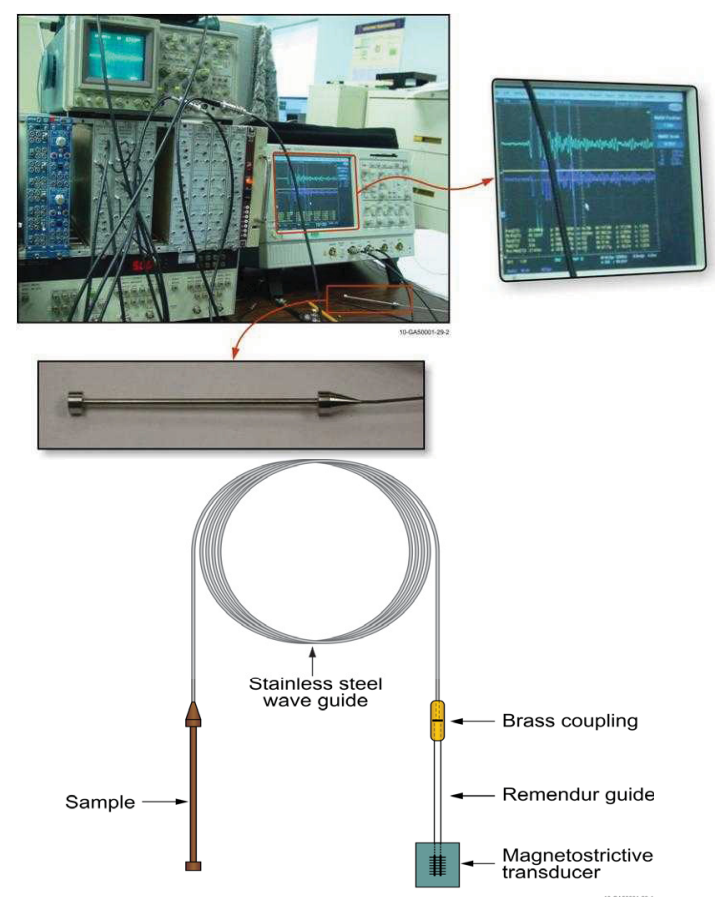

FIGURE 1. Key components required for an in-pile ultrasonic transducer demonstration.

After the necessary signal processing equipment was procured, high-temperature ultrasonic testing was initiated using a tube furnace at INL's High Temperature Test Laboratory to perform time-of-flight (TOF) measurements on stainless steel creep specimens at $300^{\circ} \mathrm{C}$. Figure 2 (next page) contains the recorded waveforms from 4- and 6-inch-long specimens. For both specimens, the data indicate the ultrasonic velocity in the gauge length is approximately $5 \mathrm{~mm} / \mu \mathrm{s}$, which is consistent with wave theory predictions for the cylindrical gauge sections at $300^{\circ} \mathrm{C}$. (Note: a $58.46-\mu \mathrm{s}$ TOF for the 4-inch gauge length specimen and a 78.72- $\mu \mathrm{s}$ TOF for the 6-inch gauge length specimen were actually measured.) 


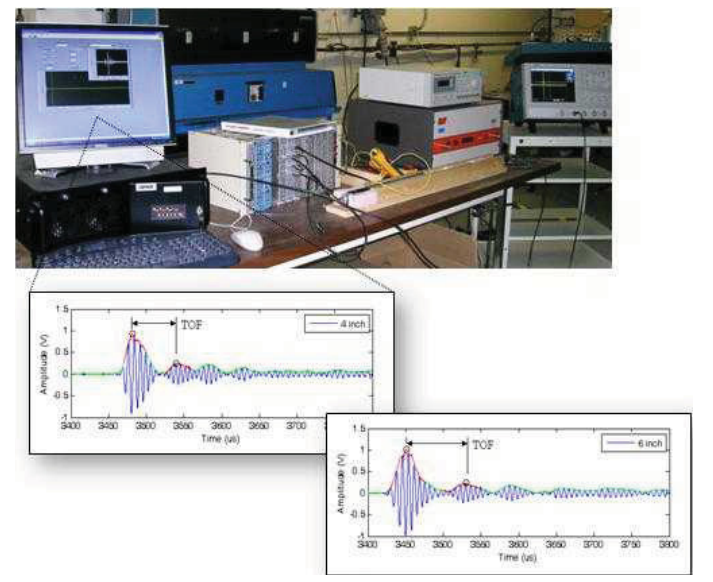

FIGURE 2. Setup and results from high-temperature UT testing.

During FY 2011, a second set of tests was completed in which the Lindberg furnace was heated to approximately $290^{\circ} \mathrm{C}$, and was then allowed to slowly cool down. TOF data were recorded as a function of temperature. Four signal-processing methods (analytic envelope, windowed analytic envelope, local maximum voltage, and local minimum voltage) were compared for calculating the TOF in a 6-inch stainless steel specimen. A discrete "jump" in the data was observed near $135^{\circ} \mathrm{C}$. Evaluations indicate that there is approximately a $7-\mu$ s shift in TOF between the two trend lines obtained with the data and that this 7- $\mu$ s TOF corresponds to one period at $143 \mathrm{kHz}$. Therefore, the "jumps" observed in the data are simply by-products of the signal-processing method. By accounting for this phase shift error, results indicate that the true trend versus temperature is linear as expected.

During FY 2011, a design was developed for testing this setup in an autoclave, and key system components have been ordered so that autoclave evaluations can be performed. As shown in Figure 3, this autoclave system can simultaneously accommodate ultrasonic testing of two specimens inside a 12-inch-tall pressure vessel. A positive displacement pump capable of a maximum of $17 \mathrm{gal} / \mathrm{h}$, with an independent pulse dampener and a simple back pressure valve, is used to develop the system pressure. Over-pressure protection for the system is provided by a relief valve set at $2800 \mathrm{psi}$, with backup protection provided by a 3000 -psi rupture disc. Heaters inside the autoclave can be controlled to develop system temperatures as high as $350^{\circ} \mathrm{C}$. The test rig is designed so that two specimen evaluations (e.g., one loaded with a bellows and one unloaded specimen) can be performed to provide direct compensation for temperature variations (given that TOF is a function of temperature and it is not possible to specifically control all temperatures potentially affecting sonic velocities during an ATR irradiation). The test rig has also been designed to accommodate a range of specimen gauge lengths. Insights gained from these laboratory evaluations will be used to recommend the design of a test rig for irradiation testing.

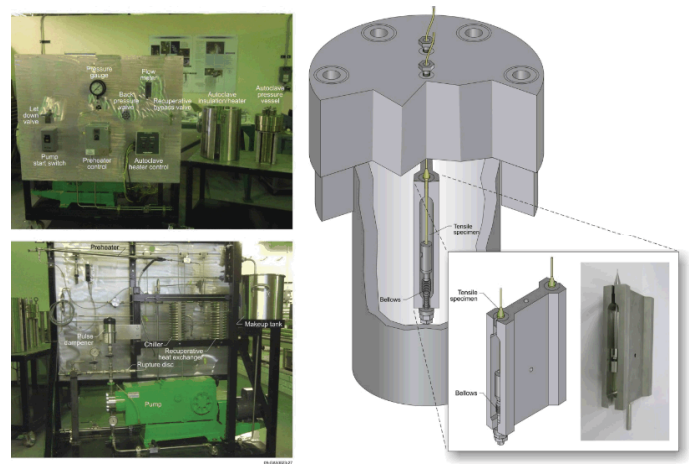

FIGURE 3. Setup for autoclave UT testing.

Benefits to DOE

Results from this project have the potential to increase INL instrumentation capabilities, which support DOE's energy security mission by developing and demonstrating high-temperature in-pile sensors needed to obtain key data required for key DOE-Office of Nuclear Energy research programs, such as the Generation IV, Fuel Cycle Research and Development, and ATR National Scientific User Facility programs. Specifically, this research is enhancing INL's expertise for producing optimized, more reliable, and more compact in-pile instrumentation. In addition, enhanced instrumentation from this research can increase testing capabilities of INL's ATR, which benefits various U.S. programs that conduct testing at this unique facility. Finally, recognition (e.g., publications, patents, and awards) from this research promotes INL's instrumentation capabilities, furthering INL's efforts to develop the Instrumentation, Control, and Intelligent Systems capabilities. 
Relevant Publications and Presentations

Kim, B. G., J. L. Rempe, J-F Villard, and S. Solstad, 2011, "Review of Instrumentation for Irradiation Testing of Fuels and Materials," Invited review paper, Nuclear Technology, MS-10-208, accepted October 2010, to be published November 2011.

Rempe, J., D. Knudson, K. Condie, J. Daw, and S. C. Wilkins, 2011, "New Sensors for InPile Temperature Measurement at the Advanced Test Reactor National Scientific User Facility," Invited paper, NURETH13 Special Edition, Nuclear Technology, Vol. 175, September 2011.

Rempe, J., J. Daw, D. Knudson, R. Schley, and L. Bond, 2011, "FCRD In-Pile Instrumentation Development," Panel Discussion at the ANS 2011 Annual Meeting, Hollywood, Florida, USA, June 28, 2011.

Rempe, J., D. Knudson, J. Daw, T. Unruh, B. Chase, and K. Davis, 2011, "Enhanced Inpile Instrumentation at the Advanced Test Reactor," Invited paper for ANIMMA 2011 Special Edition, IEEE Transactions on Nuclear Science, MS \# SCH-TNS-001962011, submitted June 16, 2011.

Daw, J. E., J. L. Rempe, R. S. Schley, K. B. Boudreau, and G. Kohse, 2011, "Instrumentation to Enhance ATR NSUF Irradiation Testing," Advanced Test Reactor National Scientific User Facility User's Week, June 9, 2011.

Rempe, J., D. Knudson, J. Daw, T. Unruh, B. Chase, K. Condie, J. Palmer, and K. Davis, 2011, "Enhanced In-pile Instrumentation at the Advanced Test Reactor," Proceedings of the Second International Conference on Advancements in Nuclear Instrumentation, Measurement Methods and their Applications (ANIMMA2011), Ghent, Belgium, June 2011.

Guers, M. J., 2011, In-situ Monitoring of Remote Specimens Using Ultrasonic Guided Waves, Pennsylvania State University, Ph.D. Dissertation, 2011.
Rempe, J. L., M. K. Meyer, D. L. Knudson, K. G. Condie, J. E. Daw, and S. C. Wilkins, 2011, "ATR NSUF Instrumentation Enhancement Efforts," Invited paper, NPIC HMIT Special Edition, Nuclear Technology, Vol. 173, pp. 66-77, January 2011.

Kim, B.-G., J. L. Rempe, D. L. Knudson, K. G. Condie, and B. H. Sencer, 2010, "Development of an In-situ Creep Testing Capability for the Advanced Test Reactor," 7th International Topical Meeting on Nuclear Plant Instrumentation, Control, and Human Machine Interface Technologies (NPIC \& HMIT 2010), Las Vegas, Nevada, USA, November 7-11, 2010.

Knudson, D., and J. Rempe, 2010, "Recommendations for use of LVDTs in ATR High Temperature Irradiation Testing," 7th International Topical Meeting on Nuclear Plant Instrumentation, Control, and Human Machine Interface Technologies (NPIC \& HMIT 2010), Las Vegas, Nevada, USA, November 7-11, 2010.

Rempe, J. L., B. G. Kim, G. Kohse, S. Solstad, and J.-F. Villard, 2010, "Current Instrumentation to Support FC R\&D Irradiations," FCR\&D In-Pile Instrumentation Workshop, Las Vegas, Nevada, USA, November 10, 2010.

Rempe, J. L., D. L. Knudson, K. G. Condie, J. E. Daw, H. Ban, B. S. Fox, and G. E. Kohse, 2010, "New Sensors for the Advanced Test Reactor National Scientific User Facility," Invited paper for ANIMMA Special Edition, IEEE Transactions on Nuclear Science, Vol. 57, No.5, pp. 2653-2661, October 2010.

Rempe, J., 2010, “Challenges of In-Pile Sensing and Transmission," NanoNuclear Workshop, Washington, DC, September 13-15, 2010.

Rempe, J., and G. Kohse, 2010, "In-core Instrumentation: Motivation, Approaches, and Examples," ATR NSUF 2010 User's Week, Experimenter's Course on Instrumentation, Idaho Falls, Idaho, USA, June 8, 2010. 
Rempe, J., 2010, "LDRDs to Enhance ATR NSUF Instrumentation," ATR NSUF Scientific Review Board Meeting, Idaho Falls, Idaho,USA, June 7, 2010.

Rempe, J., 2010, "In-pile Instrumentation for the ATR," Nuclear Engineering Colloquium, Pennsylvania State University, State College, Pennsylvania, USA, April 22, 2010.

Daw, J., and J. Rempe, 2010, "New Sensors for Advanced Test Reactor Irradiations," INL ATR NSUF ATR Workshop on Irradiation Testing and Planning, ANS Student Meeting, Georgia Institute of Technology, Atlanta, Georgia,USA, April 14, 2010.
Rempe, J., 2010, "In-pile Instrumentation for ATR NSUF Irradiations," INL ATR NSUF ATR Workshop on Irradiation Testing and Planning, ANS Student Meeting, University of Michigan, Ann Arbor, Michigan, USA, April 8, 2010.

Rempe, J. L., 2010, "New Sensors for Advanced Test Reactor Irradiations," ATR NSUF Industry Advisory Committee, Palo Alto, California, USA, February 9, 2010. 


\section{Haptic Tele-Operated End Effector for Hazardous Environments}

Anthony L. Crawford, Jeffrey D. Molitor, David R. Schipf

11-000

Natural movements and force feedback are important elements in using remote-handled equipment to perform complex, faster manipulation tasks in hazardous environments, such as hot cells, glove boxes, decommissioning, explosives disarmament, and space. We hypothesize that developing a system to provide users with the ability to fully use their natural dexterity and force sensations in their hands when performing remote manipulation tasks will enhance their remote-handling performance. To that end, our objective is to enhance the user's ability to perform tasks in hazardous environments via a radiation-hardened robotic end effector with the form and function of a human hand/arm and an adaptable haptic (force feedback) user interface.

If this system is successful, it will speed up the rate of performing in-cell tasks with the stateof-the-art manipulators by a factor of 5-10. This work is technically important because it revolutionizes the current remote manipulation technologies that are predominately based on a design patented in 1945 (Patent 2632574). The system would essentially combine the benefits of glove-box gloves and manipulator handling systems into one entity. Novel contributions of this work include the application of haptics to potentially radioactive environments, the design of a flexible haptic user interface mechanism (most current haptic systems use cumbersome gloves), investigating optimal techniques to scale a user interface's force workspace with that of its corresponding robotic counterpart, generating techniques to reduce the calculation overhead associated with haptic virtual environment interaction, and algorithms to identify the geometric properties of the user's hand and automatically adjust the hand controller to accommodate.

\section{Project Summary}

This LDRD project's first year has generated the means to test our theories regarding haptic interaction between a user, user interface, and a robotic device with the form and function of a human hand/arm. These means have come in the form of identifying and incorporating the human user's hand/arm attributes into a haptic user interface, control system, and robotic device. Such attributes include the human user's geometric dimensions, degrees of freedom, ranges of motion, common grasps, force execution during grasps, joint movement speed, force execution during manipulation, human ability to sense a just noticeable difference in force change, percentage of maximum force capability that will cause user fatigue, and the overall relationship between the number of active degrees of freedom and level of force throughout the entire execution of a grasping or manipulation task.

As a result of the acquired information, the user interface's kinematic structure has been designed such that users have uninhibited full range of motion of all their joints, thus providing them with the ability to achieve the grasps and manipulation movements that they have spent their entire life implementing and becoming familiar with. In addition, the 21 necessary motors have been sized such that their torque capacity will generate forces onto the user that are less than $15 \%$ of their maximum force capacity, ensuring that fatigue will not become a factor when using the device. The motors were also selected to achieve the joint movement speeds of the user, contributing to the transparency between the user and the user interface. Transparency is also reflected in the fact that the user only interfaces with the user interface at his or her fingertips and the hand's palm to control both the robotic hand and arm.

Fabrication of the user interface is near completion and the components (motors, bearings, gears, etc.) for the hand portion have been procured and received while the remaining motors will be procured next fiscal year. The robotic hand/arm device has been designed to mimic the human form and range of motion, and employs the full-force execution capabilities of the human hand. The prototype will be fabricated and the associated components will be procured next fiscal year.

The control system hardware and software to acquire the force data generated by the device and control the associated motors has been purchased, set up, and tested at scale. The initial motor drive electronics evaluated for the project were the recommended controllers specified by the motor manufacturer. The interface method to these controllers was through a serial link using 
CANbus. For one or two motors, it was possible to maintain the 1-ms control loop update rate desired for effective haptic interaction. However, further tests and calculations showed that the CANbus would not be able to handle the amount of communication required for the full-scale device.

Due to the findings from the CANbus-based controller, the new hardware is not based on a serial bus but rather a parallel arrangement of motor drivers where each is driven by its own set of control signals generated by a FieldProgrammable Gate Array board. By using this hardware arrangement, the system can be scaled up easily while maintaining the desired control loop rate. The new hardware has been tested and is successfully controlling a limited number of motors with the promise of having the capacity to control all of the motors that will be implemented in the device.

Within the control system, algorithms have also been developed to interpret the acquired information to accurately determine the position and speed of the user's joints despite the size of his or her hands and arms. Algorithms are also being developed to correlate the user's reduced force workspace ( $15 \%$ of maximum) to that of the full-force workspace of the robot. The most obvious and simple strategy being investigated is a linear correlation between the two workspaces.

However, based on the acquired data associated with the human hand's capabilities discussed above, this may not be the most effective technique, because forces at or below $35 \%$ of the finger's maximum force capability are utilized for complex manipulation tasks and $65 \%$ are utilized for simple yet strong grasps. This is because as the finger's force increases beyond $35 \%$ of its maximum capability, the tendon structure causes the primary flexor muscle to become dominant and essentially overrides the influence of the muscles utilized in manipulation-type tasks.

If the pure linear correlation strategy were imposed, only $5.25 \%$ of the finger's maximum force capability would be utilized for feedback during manipulation tasks. Because manipulation tasks place objects in orientations that are much more sensitive to slight changes in forces, grasps are less susceptible to changes in forces because they generally commence after an object has been firmly oriented in the hand. This research will investigate possible ways to correlate larger portions of the user interface force workspace to that of the manipulation workspace of the robot and use nonlinear relationships and possibly supplementary feedback (vibration) to correlate the smaller portions of the user interface force workspace to the grasp workspace of the robot.

A virtual reality environment is also being generated to assist in quantitatively evaluating the generated haptic user interface. The noteworthy scientific aspect for the method employed in this research is the reduction of overhead calculations through a localization technique. The technique operates by initially sorting through all the available data and determining a few nodes local to the virtual interaction tool. Well-structured matrices relating nodes to elements and vica versa are used to monitor only the local nodes/elements as the tool passes over the object, thus reducing the amount of associated computational calculations required per step.

\section{Benefits to DOE}

The discussed research benefits the DOE national security missions as an asset that increases the quality of conducting energy, nuclear, environmental, and science experiments that require remote handling of hazardous materials. In addition, it will increase the speed at which these activities can be conducted, thus reducing the time the contents of an experiment are exposed to theft or unapproved examination.

\section{Relevant Publications and Presentations}

An invention disclosure record has been submitted for this system and its novel aspects and publications will proceed once the prototype has been assembled and used to validate the generated algorithms. 


\title{
Development of In-Reactor Experiment Configuration for Laser-Based Material Properties Measurement
}

\author{
Robert S. Schley, Dave Hurley, Rory Kennedy
}

11-037

This project addresses the need for inreactor measurements of mechanical properties during irradiation. The objective is to develop and demonstrate a prototype test capsule incorporating laser ultrasonic measurement of mechanical properties in an experimental configuration suitable for use in the Advanced Test Reactor (ATR). This investigation will advance the state of the art for in-reactor measurements and provide significant benefit to ATR users by enabling real-time monitoring of material property evolution.

\section{Project Summary}

Project work began with the intent of using surface acoustic wave propagation parameters as a method of detecting changes in material properties. As detection of the surface acoustic waves in the intended environment proved elusive, a test configuration for measuring the natural frequency of small cantilever beams was developed. The beam oscillation frequency, which is a function of the beam material properties, was readily detected in a prototype test configuration.

The redistribution of porosity in ceramic nuclear fuels during irradiation is of great interest to the fuel development community. Initial work centered on the measurement of surface acoustic wave velocity as a means of monitoring the change in porosity of a material. Cerium oxide was selected as the test material due to its similarity to uranium oxide. For the initial measurements, a commercial optical-fiberbased interferometer was used to measure surface acoustic waves in the cerium oxide sample. A reflective foil placed on the surface of the cerium oxide was required to increase the reflectivity so that sufficient detection light could be collected. Although the surface acoustic waves were discernable in the resulting signal, the expected decrease in sensitivity without the reflective foil would make detection of these waves unlikely in a reactor environment. Based on these results, the measurement of a phenomena more likely to be successful in the reactor environment was sought.

The displacement of a small vibrating beam can be orders of magnitude larger than the displacement from a surface acoustic wave. The natural frequency of a vibrating beam is a function of the density and modulus of elasticity as well as the dimensions of the beam. Consequently, measuring changes in the natural frequency of vibration can be used to monitor changes in the elastic properties of the beam material. Based on this concept, a small vibrating beam design that could be incorporating into a test capsule was developed. The beam design consisted of a $13 \times 2 \times 2.5$-mm beam machined in the center of a 1" square copper plate. A top plate was designed to position the excitation and detection optical fibers relative to the beam. This top plate was attached to the beam plate using a shim to control the spacing between the optical fibers and the beam. Figure 1 shows a composite photo of the copper beam plate on the left and the assembled fixture with the optical fibers on the right. Two detection schemes based on fiber optic levers were investigated. Fiber optic lever detection uses an illumination fiber to deliver light to the sample. Light reflected from the

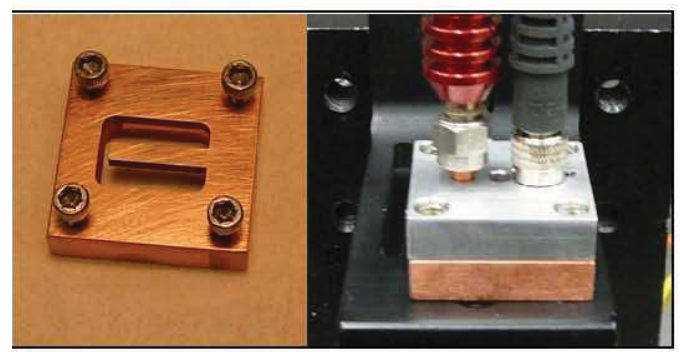

FIGURE 1. Left: Copper beam plate. Right: Assembled fixture with excitation and detection optical fibers.

sample is then collected by the illumination of other fibers and returned to a photo-detector. The amount of light collected is a function of the distance from the end of the fiber to the sample. Hence, as the beam vibrates, the light intensity returned to the photo-detector changes, and a signal proportional to the beam displacement is produced. This detection technique has the advantage that no additional optics or complicated alignment are required, which would simplify its use in a reactor test capsule. Two methods of exciting the beam were also investigated. First, a single laser pulse was used to excite the beam and the oscillations of the beam over time were recorded. Second, a chopped continuous laser source was used. In 
this case a network analyzer was used to sweep the chop frequency of the excitation beam while recording the signal from the detector. When the chop frequency of the laser matched the natural frequency of the beam, the amplitude of vibration was greatly magnified. Figure 2 shows the signals recorded using the two excitation methods. The top plot shows the beam oscillation induced from a short laser pulse, and the bottom plot shows the resonant frequency of the beam recorded by scanning the excitation laser chop frequency. The measured natural frequency of $6,500 \mathrm{~Hz}$ was in general agreement with the theoretical value.

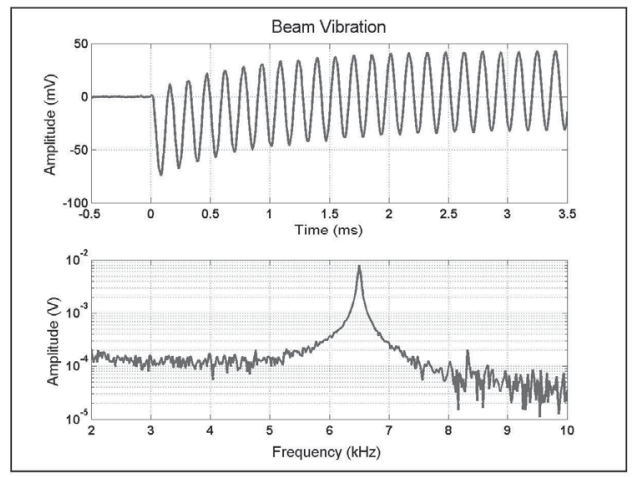

FIGURE 2. Top: Laser pulse induced beam oscillations. Bottom: Beam resonant peak induced by chopped laser.
Future work will focus on relating changes in the natural frequency of the beam to changes in material properties.

\section{Benefits to DOE}

The ability to make in-reactor measurements of material properties will provide new insights into the behavior of materials exposed to extreme radiation and temperature environments. This capability will also provide a powerful new tool for ATR National Scientific User Facility users to assess radiation effects on materials in real time. New in-reactor measurement capabilities will help INL fulfill its leadership role in nuclear technology and supports DOE-Office of Nuclear Energy's Advanced Nuclear Energy Initiative. 


\section{Human Simulation Laboratory for Nuclear Power Plant Human Performance Studies}

Jacques V. Hugo, David Gertman, Ronald Boring

11-062

Characterizing control room operator performance in the presence of changing technology is a significant challenge for DOE, the Nuclear Regulatory Commission, and the U.S. nuclear industry. In many ways, the advanced technology available today is driving the changes being proposed for existing and advanced reactor control room designs. However, the technical basis (i.e., the data for determining the efficacy of human performance under these conditions) is largely absent. Currently, the Human System Simulation Laboratory (HSSL) at INL is the only test bed facility in the DOE complex for the evaluation of various aspects of human performance and human system interaction for proposed reactor designs and upgrades, including staffing levels, situation awareness, and operator interface design. However, in its current state, the HSSL is still immature and its ability to support future DOE missions is severely limited, specifically in the assessment and improvement of human performance under normal as well as emergency situations in control rooms, remote shutdown stations, technical support centers, maintenance outage control centers, and other environments where humans interact with complex technology.

This project's focus is on the continual physical and functional refinement of the HSSL to make it a fully equipped and reconfigurable. This requires the implementation of the necessary plant-specific as well as generic nuclear power plant models from one or more approved industry partners and to use this capability to supply empirical research data on human performance with emerging humansystem interaction technologies. At present, the HSSL is able to perform experiments with one advanced small modular reactor model. To ensure that the results of the control room crew studies will be generalizable to the existing and evolving fleet of U.S. reactors, future expansion of the HSSL will also include other small modular reactor plant models, appropriate high temperature gas-cooled reactor models, and a generic plant model aligned to the current generation of pressurized water reactors. Leveraging existing and future nuclear power plant plant models in this way will make the reconfigurable HSSL a research platform that will provide the backbone for advanced human factors studies.

\section{Project Summary}

The first phase of the project focused on establishing a basic system architecture that enables human factors researchers to conduct human performance experiments with power plant simulations. To this end, the cooperative research and development agreement with industry partners NuScale and U.S. Governmentsponsored enterprise was renewed to allow the installation of state-of-the-art simulation software (i.e., the NuScale Phase III nuclear power plant model) and the development of prototype operator interfaces. This enabled the Human Factors team to configure the lab architecture to support multiple, simultaneous simulations. The physical configuration of the HSSL was also upgraded to achieve a better approximation of actual control room environments. This included upgrading the illumination of the HSSL to better comply with ergonomic requirements as described in NUREG-0700, "Human System Interface Design Review Guidelines." Workshops and meetings were held with international experts (i.e., the Organization for Economic Co-operation and Development [OECD] Halden Reactor Project) as well as industry partners (i.e., NuScale and U.S. Government-sponsored enterprise) to help establish a formal basis for future HSSL requirements and experiments that need to be conducted in support of DOE objectives. The training of Human Factors staff in the use of the Halden ProcSee software was completed as a part of the collaborative agreement with the OECD Halden Reactor Project. Other upgrades in the functional capability of the HSSL included the implementation of an updated version of the LabView software to allow advanced experimentation and data capture.

\section{Benefits to DOE}

The HSSL is a unique and vital resource in the INL's research arsenal. Without it, we cannot answer difficult questions such as, "Can you safely operate more than one power plant unit from a single control room? How many operators do you really need? How will operators 
maintain situational awareness with advanced technology and increased complexity?" and many more.

These questions can be addressed in a fullfeature, reconfigurable control room simulator that:

- Provides a much-needed facility for instrumentation and control and human factors research, including development of operator-centric design requirements, behavioral and performance testing, such as the role of operator limitations, mental models, perception, and cognition in human-system interaction.

- Satisfies not only INL's human factorsrelated research and development needs, but also directly or indirectly supports the objectives of the DOE and ultimately several sectors of the nuclear industry. This includes the evaluation of state-of-the-art humansystem interaction and interface technologies to support operator decisionmaking and situation awareness and also the development and evaluation methods for new concepts of operation of next generation power plant designs.
- Enables INL to provide all stakeholders with research-based, empirically determined guidance on the human contribution to system resilience and the operating performance of crews under various task and environmental conditions.

- Supports all current and anticipated projects, with special emphasis on the Light Water Reactor Sustainability and Small Modular Reactor programs.

- Establishes INL Human Factors and the DOE in a leadership role for advanced visualization, human-in-the-loop simulator studies, cognitive studies, and the empirical testing and validation of design approaches. 


\title{
Smart Grid Impact on Commercial Nuclear Plants
}

\author{
Magdy S. Tawfik, Thomas Baldwin, Binh Pham \\ 10-070
}

The proliferation of renewable energy resources on the U.S. electrical grid is already a reality. The renewable-energy portion of the complete energy portfolio is growing much faster than the overall projected growth of nuclear energy. As such, the generation of renewable energy and the development of the smart grid are challenges for utilities across the country. Interfacing the smart grid with traditional grid components can transform the way service is delivered and active components are monitored. However, with this unique systems integration, there are still many areas to be cautious about. Worries regarding technology compatibility continue, in addition to other complications as new communications capabilities emerge.

Historically, the power grid has been the dichotomy of a very large and complex grid topology of interconnected utilities using a relatively simple set of local controls coupled with hieratical controls for generation. With a dramatic increase in system complexity, new failure modes and vulnerabilities will occur, especially those of the wide-area disturbance category. This project researches the impact and potential vulnerabilities new grid elements could have on existing commercial nuclear power plants (NPPs). The study will also point out issues to be addressed by NPPs as their commercial offsite power quality and availability picture changes. Commercial nuclear plants are required by the Nuclear Regulatory Commission to shut down operations or take necessary actions when a plant deviates from the technical specifications outlined in its operating license. This should include all external factors. Therefore, another focus of this project will be to determine the integrity of the interface with the utility grid as well as the security and availability of external power sources for emergency operations.

\section{Project Summary}

During the first year of this project, we investigated the vulnerabilities of a NPP caused by electrical events on the power grid. Two NPP areas of vulnerability were addressed: (1) human factors and (2) plant components. It should be recognized that the NPP's passive plant components' apparent isolation from external events is not at all the same for the active electrical components and systems.

\section{Human Factors Vulnerabilities}

A key factor is the ability of reactor operators to respond accurately and quickly enough to the effect that grid instability is going to have on the NPP. Generally, increasing the frequency and severity of disturbances will increase the operator's workload. The operators' ability to handle such upsets will be severely compromised if insufficient information prevents them from anticipating the evolution of events and plan ahead. Studies conducted by the INL Human Factors group have shown that the increasing complexity of highly automated systems increases the potential for human error in NPP control rooms. This can usually be related to information overload and reduced time for decision-making. With the increasing task complexity introduced by variable sources of generation, the operator may become the weakest link in the advanced power delivery system unless this is factored into the design of grid systems, control rooms, and human-system interfaces. In order to support DOE and the energy industry in their efforts to maintain the integrity of the electric grid, it is vital that research be conducted to define the nature of the human factors challenges imposed by renewable energy sources. We concluded that a research strategy should be developed to include the development of appropriate metrics of human performance and criteria for the design of control rooms, human-system interfaces, and support functions that would enable operators to cope with increasing workload and complexity.

$\underline{\text { Plant Electrical Active Components and Systems }}$

NPPs are comprised of two distinct electrical power systems. The first is the power production system, consisting of the turbinedriven generator, the generator step-up transformer, and the high-voltage substation interface to the bulk power grid. The second is the industrial-house power system, which drives the many pumps and ancillary equipment that operate within the NPP. The electrical 1E safety system is part of this industrial power system. The second power system's electricity is supplied separately from the power production 
output of the first system. Generally, the power supply to the NPP comes from different parts of the utility power grid and at a different voltage level.

Outside the NPP, both power systems are subjected to disturbances. These disturbances can enter the NPP either directly across the power supply to critical pumps and loads, or indirectly through feedback as power is generated and delivered to the electrical grid. Electrical quantities that can be impacted include the voltage magnitude, grid frequency, power factor demand, amperage drawn, harmonic contents of the alternating current (AC) waveforms, and voltage transients.

One of the LDRD findings is that the system must operate with the growth of variable generation capacity from renewable energy production, such as wind and solar farms, which cannot be dispatched in the traditional utility sense. Variable generation causes the dynamics of the power grid to increase dramatically. Base loading generation, like nuclear, is being effected; large production levels from variable generation forces base generation to load-follow and do not operate in a constant state.

Additionally, many wind farms and all Photovoltaic solar farms use power electronic converters to produce $\mathrm{AC}$ power from $\mathrm{DC}$ sources. These converters generate harmonic distortion of the $\mathrm{AC}$ voltages and currents. The harmonic distortion propagates throughout the high-voltage grid and can reach the NPP.

Another LDRD finding is how the various chronic and acute grid disturbances can cause damage and accelerate aging of many electrical apparatus, which impacts safety and production. Even the loss of non-safety components has consequences to plant safety.

The LDRD has developed system modeling efforts of NPP components. For simplification, the focus has been directed to plants in the southeastern region of the U.S. The goal has been to identify the exact impact on selected components' remaining useful life. The following is a list of disturbances that researchers identified due to the presence of the renewable sources that can impact the NPP:
- Transient changes in the electric grid power balance between generation and consumption places stress on rotating machines such as pumps and valve motors as well as generators

- A sudden change in power production levels from larger wind or solar farms creates high stresses in turbine shafts and causes sheering forces

- Damage to bearings and seals also occur

- Harmonic distortion of the $\mathrm{AC}$ waveforms causes heating losses in power apparatus to increase

- Thermal insulation damage has been seen in motors, generators, and transformers due to harmonics

- Generators and their adjacent power apparatus are very susceptible as generators provide good sinks for absorbing harmonic components

- Harmonics also form unbalanced and pulsating power conditions that induce vibrations and transient torques in mechanical equipment

- Voltage variations also impact motors (pumps and valves) and cause excess heating to occur, which damages the insulation and accelerates equipment aging.

Mitigation solutions have existed for industrial power systems, but the existing technology is insufficient to filter out the magnitude of disturbances that a NPP may experience with large-scale renewable energy generation levels beyond a limit that are system dependent and can be identified accurately for specific grid configurations.

\section{Benefits to DOE}

This LDRD promotes America's energy security through generating reliable, clean, and affordable energy. It also meets DOE goals by creating a more flexible, reliable, and higher capacity U.S. energy infrastructure. This LDRD supports one of DOE's most important missions by maintaining existing and new nuclear generation's capacity to produce carbon-free 
electricity in the near term. This LDRD defines the limits in the mixture between traditional NPP outputs and renewable energy. For example, the Systems Analysis Program for Hands-on Integrated Reliability Evaluation program for probabilistic risk assessment does not account for ageing of active components. The risk calculation may vary if renewable energy's impact is considered. This research identifies the minimum instrumentation requirements of online monitoring for NPPs to combat possible harmful effects due to power quality considerations.

\section{Relevant Presentations}

Baldwin, T., S. Tawfik, and M. McQueen, 2011, "Contingency Analysis of Cascading Line Outage Events," IEEE Clemson Power Systems Conference, Clemson, South Carolina, USA, March 15-18, 2011. 


\title{
Characterization of Fluidized Beds via Pressure-Fluctuation
} Analysis

\author{
Douglas W. Marshall, David Drown (University of Idaho) \\ 09-042
}

Fluidized beds are used in a variety of industrial processes that produce or handle powders. Some of the industrial processes occur at very high temperatures or chemical environments that necessitate isolation, such as chemical vapor deposition of coatings on nuclear fuel particles, petroleum cracking, or handling of hazardous materials. The thermal and chemical environments can make direct observation of the processes occurring in the fluidized beds impractical or impossible. Instrumentation for indirect observation can also be limited by the harsh environment or stringent purity controls on a process. Ensuring that the powder remains fluidized and that it is circulating properly is essential for quality control. Fluidized beds exhibit different gas and solid circulation patterns depending on the configuration of the equipment and the nature of the gas and powdered solids. The fluidization industry has classified different gas-solid fluidization regimes based on the exhibited characteristic flow patterns. The fluidization regime can change in a process as gas properties, powder properties, and material flow rates change.

The purpose of the research was to obtain data from which correlations could be derived between the structure of pressure fluctuations, resulting from gas-bubble movement in fluidized particle beds, to measurable characteristics of the particles (density, diameter, shape, etc.) and fluidizing gas properties (density and viscosity). Furthermore, an effort was made to obtain data documenting how extreme temperatures (up to $1600 \mathrm{~K}$ ) influence the boundaries between differing fluidization regimes (i.e., flow and circulation patterns). Most data published in literature was collected at room temperature or slightly elevated temperatures.

A two-dimensional (2D) physical model was used to visualize the fluidization regimes, and a smaller conical-cylindrical model was used for high-temperature data collection.

\section{Project Summary}

A 2D model was constructed to be versatile enough to change the shape of the converging section and interchanging gas distributor nozzles. A high-temperature model was used to quantify the impacts of temperature on fluidization of particles, particularly when the gas is much colder than the bed media. A variety of bed media, with a wide range of particle densities, was obtained and tested, resulting in a collection of data from more than 4000 experiments. High-temperature data were collected in $200^{\circ} \mathrm{C}$ increments from $327^{\circ} \mathrm{C}(600 \mathrm{~K})$ to $1327^{\circ} \mathrm{C}$ $(1600 \mathrm{~K})$, several hundred degrees higher than previously published data. Work was sublet to the University of Idaho for support of this laboratory-directed research and development project, including the construction of the $2 \mathrm{D}$ models and the collection of data for mixed beds of spherical and rod-like particles to examine the influence of particle shape on particle movement.

Slow-motion cinematography was used to document gas-bubble and particle movements in the 2D models in addition to collecting high-frequency pressure data (up to $1000 \mathrm{Hertz}$ ) to map the structure of the pressure fluctuations. Data analysis will continue in the PI's effort to earn a $\mathrm{PhD}$ and should lead to enhanced correlations for identifying and/or forecasting fluidization regimes when direct observation is not possible.

\section{Benefits to DOE}

Spouted beds (fluidization where particles are transported through a central gas spout to the surface of the bed) are used in applying coatings on tri-structural isotropic fuel particles for hightemperature gas-cooled reactors and other coatings for proposed space nuclear propulsion research work. In these coating processes, no instruments or cameras can be inserted to identify the fluidization regime. Analysis of the pressure fluctuations should reveal information about the fluidization regimes, which will guide process adjustments for tri-structural isotropic and space nuclear fuel production.

\section{Relevant Publications and Presentations}

Several publications are planned, supporting the PI's effort to earn a Ph.D. A co-authored journal submission has been drafted with University of Idaho participants on the influence of rod-like particles on the movement of spherical particles in a spouted bed. 


\section{In-Pile Temperature Monitor and Control for ATR}

Jian Gan, James I. Cole; Jiming Bao (University of Houston)

10-022

It is well known that irradiation temperature is one of the most critical parameters to impact microstructural development under irradiation. INL's Advanced Test Reactor plays a significant role in nuclear materials and fuel development through in-pile irradiation experiments. The capability to record in-pile irradiation temperatures is essential to the success and the quality of these reactor irradiation tests. The objective of this LDRD project is to develop a prototype fiber-optic temperature sensor to measure and record the irradiation temperature for the materials and fuels irradiation test in a research and test reactor. The advantages of using a fiber-optic temperature sensor include its small size, no signal interference from electric and magnetic fields, high sensitivity, and reliability. The purpose is also to significantly improve the quality of irradiation test data by providing real-time temperature measurement and temperature history during irradiation.

\section{Project Summary}

The major components for the experimental system for the sensor test have been identified and implemented. We evaluated different sensor configurations and identified three key aspects of designs to proceed: (1) use an air-gap FabryPerot (F-P) for interferometer, (2) use silica fiber and an alumina thermal expansion tube for an air-gap F-P for sensor up to $700^{\circ} \mathrm{C}$, and (3) use sapphire fiber and an alumina thermal expansion tube for an air-gap F-P for high-temperature applications up to $1000^{\circ} \mathrm{C}$. The bench-top tests were performed for the design with great success in FY 2011. A prototype sensor has been fabricated and tested successfully up to $700^{\circ} \mathrm{C}$. The size of the sensor in the current design is about 5.0-mm long with a diameter of approximately $800 \mu \mathrm{m}$. With additional work for the current design, this sensor could potentially be used up to $800^{\circ} \mathrm{C}$. The great advantage for the current design is the simple configuration, low cost, and high resolution with sensitivity about $0.1^{\circ} \mathrm{C}$. Another major accomplishment in FY 2011 is the computer-automated spectrum analysis for real-time display and temperature recording. The current task is to develop a single measurement system with multiple sensors for multiple zone applications using a programmable optical switch. The prototype sensors with 20-meter-long fibers are ready for gamma irradiation testing at INL's Materials and Fuels Complex and, ultimately, the in-pile test in ATR.

\section{Benefits to DOE}

Research and development on this sensor is important to INL's nuclear fuel and materials irradiation testing mission. It will significantly improve the ATR National Scientific User Facility program for the nation by providing high-quality irradiation test data with reliable temperature measurement, which is currently unavailable due to high costs and space limitation in the core. Our first two years' results bring us one step closer to monitoring in-pile irradiation temperatures in real time. The experimental system developed by our collaborator at the University of Houston is critical to test and fabricate the sensor with different designs. Continued work on this project will ensure INL's leadership in nuclear research and development. At the completion of this project, this type of temperature sensor will be applicable in other federal agencies, such as the Department of Defense, where nuclear power is used. 


\section{Dissolution and Extraction Studies of Fission Products in Room Temperature Ionic Liquids (RTILs) and in Supercritical Fluid $\mathrm{CO}_{2}\left(\mathrm{ScCO}_{2}\right)$ and Determination of Radiolytic Stability}

Brian K. Harris, Bruce J. Mincher, William F. Bauer; Chien M. Wai (University of Idaho) 09-101

Liquid-liquid extractions have historically been favored in nuclear-fuel-cycle processes, using relatively conventional organic solvents, many of which have toxic, flammable, volatile, or radiolytic properties that impose certain limitations on the processes. The recovery and reuse of the organic extractants is often limiting due to radiolysis. The cost of disposing spent conventional solvents can be a significant issue, particularly when they are contaminated with radioactive and unknown radiolysis components. The design, then, of an environmentally more favorable extraction process that is also more resistant to radiolysis would be advantageous to processing nuclear fuel and waste materials. The primary goals were to optimize the extraction efficiencies for separating lanthanides from actinides in various matrices using a variety of ligands in two ionic liquids (ILs) and various acid concentrations. Irradiated IL was also investigated for radiolysis products.

\section{Project Summary}

In FY 2010 work was performed to assess which combination of a soft-donor ligand and acid range provided the most promising distribution ratio for separating lanthanides from americium. Unlike uranium and thorium, americium (and curium) are considered "minor" actinindes in the fuel process but are of paramount concern in the eventual scheme of reprocessing. The ligand studied was $\mathrm{N}, \mathrm{N}, \mathrm{N}^{\prime}, \mathrm{N}^{\prime}-$ Tetrakis(2-pyridylmethyl)ethylenediamine (TPEN). The ligand was mixed by sonication into an ionic liquid consisting of 1-butyl-3methylimidazoliumbis(trifluoromethanesulfonyl)-imide (BMIM$\mathrm{NTf}_{2}$ ) at a ratio of approximately five ligands per metal being extracted before beginning the extraction process. The extraction was carried out by contacting the IL:ligand mixture with $\mathrm{HNO}_{3}$ over a range of $0.05-10.0 \mathrm{M}$, which contained a standard reference material including the lanthanides plus americium from a stock solution on hand. Over the acid range, the best acid concentration for the IL:ligand combination was $0.05 \mathrm{M} \mathrm{HNO}_{3}$ (Fig. 1). Although both the actinides and lanthanides are generally classified as hard acids, there does seem to be some selectivity between the slightly softer nature of the minor actinide americium and the slightly harder lanthanides.

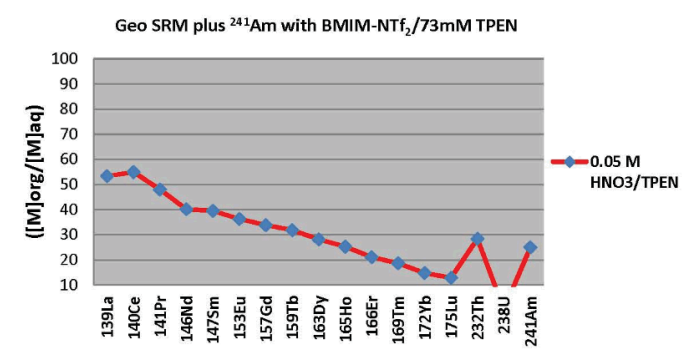

FIGURE 1. Distribution ratios for the extraction of ${ }^{241} \mathrm{Am}$ from lanthanides using BMIM NTf 2 :TPEN.

Radiolysis experiments were conducted by initially irradiating ionic liquids between $59 \mathrm{kGy}$ and $1080 \mathrm{kGy}$ doses using a ${ }^{60} \mathrm{Co}$ source. The darkening of the ILs increased with dose from light amber to a deep black color. This coloration was not as prominent when the ILs were irradiated when in contact with various acids. Figure 2 shows BMIM-NTf 2 irradiated at 1080 kGy as neat IL, IL in contact with $1 \mathrm{M} \mathrm{HNO}_{3}$, and IL in contact with 1M HCL. Although lesser doses did not cause a color change in the acids, here, both the $\mathrm{HCL}$ and $\mathrm{HNO}_{3}$ are discolored with the HCL color most prominent.

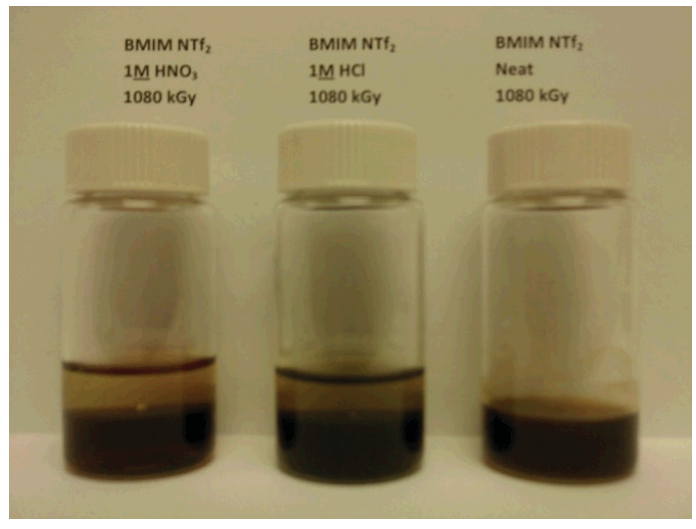

FIGURE 2. Post-irradiation (1080kGy) BMIM-NTf, in contact with $1 \mathrm{M} \mathrm{HNO}_{3}$ and $1 \mathrm{M} \mathrm{HCl}$.

Several analytical techniques are currently in use for identifying radiolysis products. Progress is being made using high-pressure liquid chromatography using various eluent mixtures, which serves as a guide for liquid chromatography-mass spectrometry analyses. 
Direct analysis of irradiated IL and un-irradiated IL with TPEN is being done by electrospray ionization mass spectrometry, and analysis of the irradiated acid fraction is being done by deabsorption electrospray ionization mass spectrometry. An alternate way to check the integrity of the IL towards radiolysis will be to compare the extraction of a lanthanide/actinide standard reference material using pre- and postirradiated ILs to verify if extraction efficiencies are altered at all by radiolysis.

This year also continued a subcontract for University of Idaho to provide the IL BMIM$\mathrm{NTf}_{2}$.

\section{Benefits to DOE}

In Nuclear Science and Technology, our initial radiolysis data contributes to a science-based approach to better understanding the behavior of a possible IL-based extraction scheme to improve on existing separation technologies, as well as providing alternate approaches to simplify or provide more efficient or cost-effective processes applicable to the nuclear fuel cycle, waste cleanup, etc. In the Energy and Environment Science and Technology Directorate, we have gained knowledge of the behavior of our ionic liquids in different matrices (soil, ores, and aqueous) and various conditions that will aid in understanding their potential application to understanding and dealing with environmental issues. An abstract of our results will be submitted to the Spring 2012 American Chemical Society conference in San Diego. 


\section{$\Rightarrow$ National and Homeland Security}

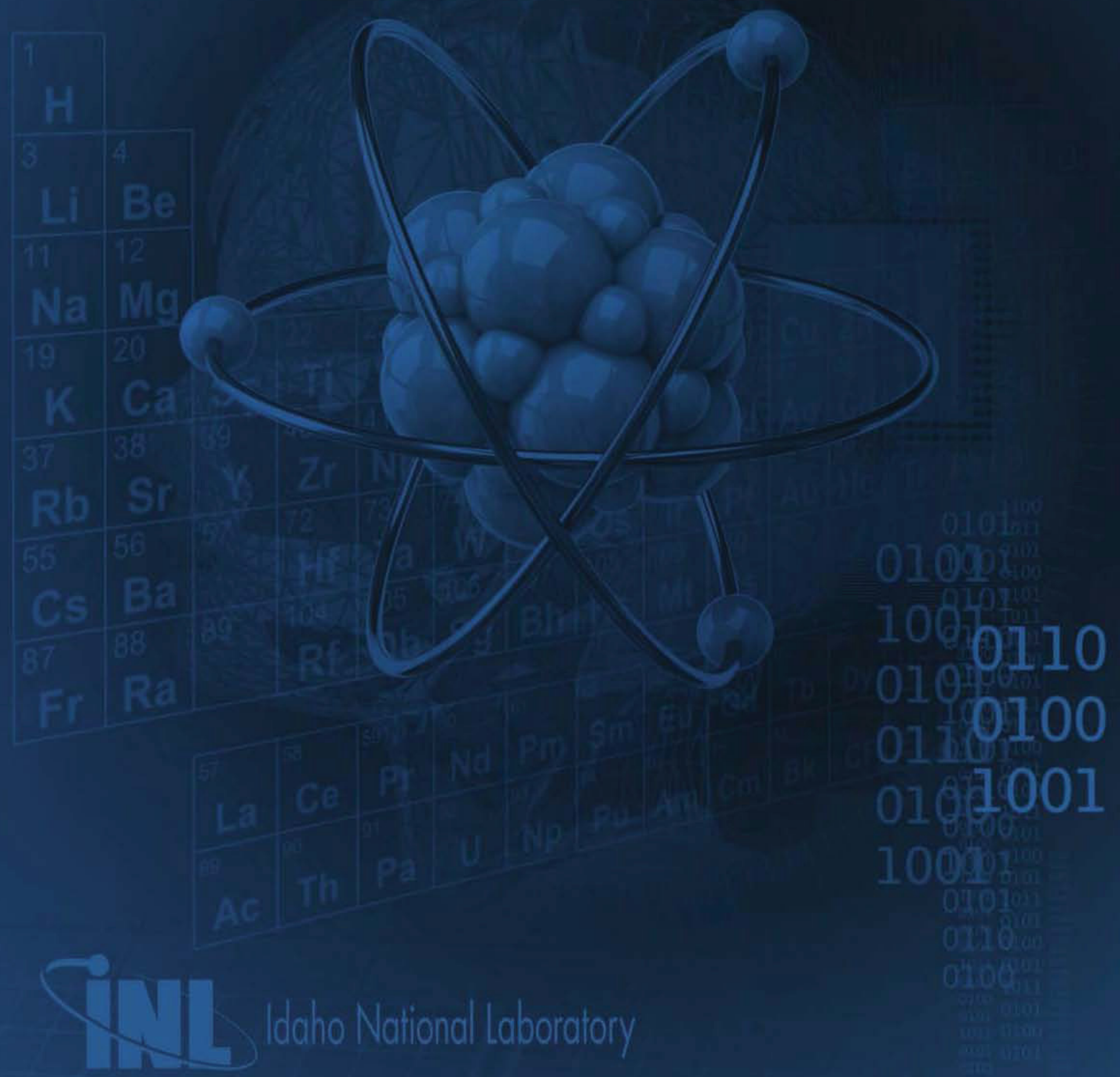




\section{Methodologies for the Design, Analysis, and Validation for Operation of Complex Resilient Networks}

Craig Rieger, Keith Daum; Venkat Venkatasubramanian (Purdue University)

09-096

Complex networks are highly organized sets of flows of entities such as material, energy, information, money, etc., whose is goal to deliver service levels that maximize some performance metric such as profitability, service availability, etc. Examples of such networks would be the electrical power grid, transportation networks, supply chains, advanced nuclear fuel cycles, and so on. Such networks are nationaleven global—in scope, highly regulated, require traceability, are integrated with power systems, and have to adjust to supply, demand, and technological disruptions to ensure competitive products, service, and prices on timely deliveries.

In such an uncertain environment, the ability to identify and monitor the complex interconnections and emergent properties, and to assess the current state, diagnose anomalous or abnormal events, and autonomously reconfigure the network is vital to guarantee service and manage profitability. When these disruptions occur, strategic, operational, and tactical decisions involving engineering, management, and information layers will have to be executed. In the design and analysis of such networks, efficiency, robustness, and cost are critical performance metrics. Efficiency measures how well the network performs under given constraints, robustness measures how well the network will perform when parts of it are taken down due to various disruptions, while cost considers not only the procurement cost of the system but also the cost to keep the system performing up to the requirements. Resilient networks are both robust and efficient.

The technical objectives and the project deliverables for this three-year project consist of developing and implementing:

- An understanding of how the topological features of a complex resilient network affect performance metrics such as efficiency, cost, and robustness

- A general mathematical and computational framework for the design of complex resilient decentralized control networks

- A demonstration of these concepts and systems by implementing them in a testbed that will simulate resilient network prototypes and scenarios

- Papers, presentations, reports, and software to facilitate technology transfer to INL.

\section{Project Summary}

Building on the foundations laid in the first year, we enhanced the graph theoretical mathematical framework for modeling and analyzing network topologies to also include a decision framework. We developed the Resilient Network Design Environment (RNEDE), a comprehensive framework for resilient network design. The RNEDE environment consists of an optimizer and a decision controller, as shown in Figure 1, which, when subject to a series of disruptions, determines a topology that achieves the functionality of the network and determines cost-efficient remedial measures. We developed a testbed prototype named RNEDESim, which includes capabilities for optimization, decision making, and visualization.

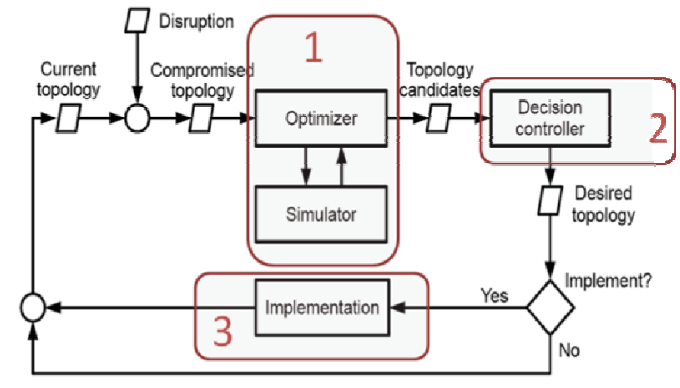

Objective 1: Optimizer problem

Objective 2: Decision control problem

FIGURE 1. Architecture of RNEDE.

Given a topology for a complex network and a sequence of incoming disruptions (resulting in a fault), RNEDE determines which remedial actions to take such that the network remains resilient to structural changes in the topology (due to a given disruption). In RNEDE a remedial action corresponds to determining the location and number of new nodes and/or edges that can be placed back on the network to make it resilient to the disruption. The problem of determining the number and location of the remedial action is solved in two parts. In the first 
part, RNEDE determines the set of optimal remedial actions that can be taken, i.e., how many nodes/edges to add and where to add them. This determination is based on several factors, such as the level to which the complex network has been compromised and the constraints associated in implementing the remedial actions on the network. In the second part, RNEDE decides whether it should choose to implement any of the suggested remedial actions. This decision-making is crucial, as a remedial action taken currently may not be valid in the future. This part of RNEDE is based on the sequence of incoming disruptions and the cost associated with the remedial actions.

We now describe the problem mathematically. Let the complex network be represented by a topology $\mathrm{T}=(V, E)$, with $V$ being the set of vertices and $E$ being the set of edges.

The topology specification $T$ satisfies a set of constraints $C=c_{1}, \ldots, c_{n}$. The function $S: T \rightarrow$ $R+$ determines the cost of maintaining a topology satisfying a set of constraints. Let $f_{i}$ be a disruption that arises in the network which "compromises" the topology of the network. Let $T$ ' be the compromised topology. $T$ ' may or may not satisfy the set of constraints $C$. Let $F: T \rightarrow$ $R+$ be a monotonic function that, given the original topology and the compromised topology, quantitatively measures the amount of the compromise. Let the system's knowledge base consist of a set of remedial actions, $A=$ $a_{1}, \ldots, a_{N}$ and a cost function $Q: A \rightarrow R+$. The two objectives in RNEDE, then, are to: (1) obtain that set of remedial actions such that when applied to $T^{\prime}$ result in a remedied topology $T$,' such that the compromise is minimized, i.e., $F\left(T^{\prime \prime} ; T\right)<=\epsilon$, and (2) if $\sigma=f_{1 \ldots} f_{n}$ is the sequence of disruptions that arise in the network and the knowledge about each disruption arrives sequentially at the system, then the cost of maintaining the compromised topology and the cost of making the change, i.e., $\min \sum \sigma\left(a_{i}\right.$ $\left.Q\left(a_{i}\right)+\mu S\left(T^{\prime}\right)\right)$, are minimized. $\mu$ is the weight parameter that equates the two costs. For the supply chain case study considered, the topology satisfies a degree constraint, i.e., $\mid\{e \mid e E,(V, E)$
$T\} \mid<=k$ for all $V T$ and for a given constant $k$. The function $F$ is equal to the diameter of the topology. The cost function is application dependent and can be represented in any of the network quantities such as bandwidth or latency.

In RNEDE the first part of the problem is solved by modeling it as an optimization problem, and the second part of the problem is solved by modeling it as a decision control problem. The framework is aided with a visualizer, which allows the system administrator managing the network to diagrammatically see which changes in topology are being suggested. RNEDE evaluates a disruption one at a time and suggests a remedial action. This suggestion is based on the already-seen threats and assumes minimal knowledge about the future sequence of disruptions that may arise.

RNEDESim has the following features: (1) able to visualize, create, edit, and analyze large complex networks/graphs; (2) has a modular design that makes it easy to add, remove, and extend functionality and new modules to the existing software; and (3) provides a dynamic simulation platform for development and evaluation of methods for control of networked systems. In RNEDESim, each node of the graph represents an active process that generates events and responds to external events. We envision that RNEDESim will be dynamic with respect to its analysis capabilities, which are (1) allows loading of existing connected and disconnected networks, which are as large as 5000 nodes and edges (completely connected) and (2) allows loading of a threat mode, i.e., a disruption and initiation schedule can be described. Also, nodes and edges are disabled for a given range of time; (1) on a large network, descriptions can be added for nodes, i.e., for producers/consumers/edge labels and (2) for the entire network, several metrics are available for computation and comparison, e.g., clustering coefficient, diameter, centrality, coloring, strongly connected components, etc. Figures 2 and 3 show an example of an optimized supply chain network. 


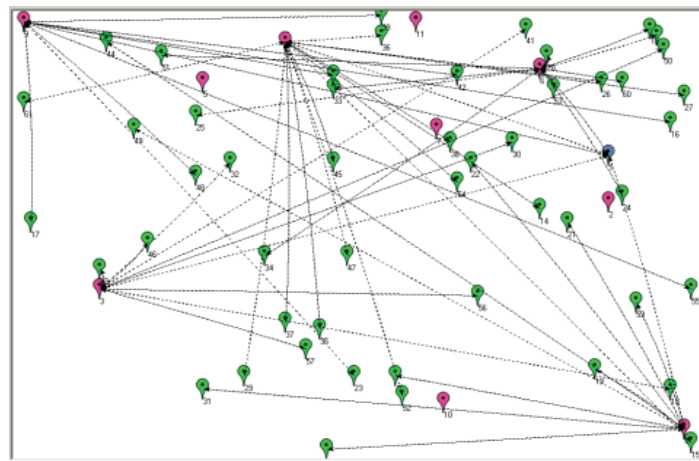

FIGURE 2. RNEDESim: optimized network.

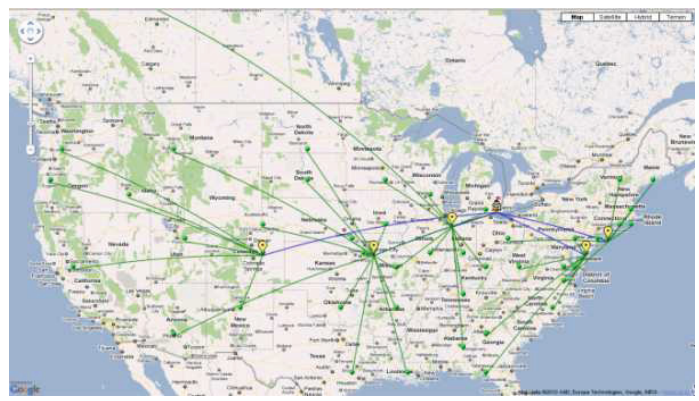

FIGURE 3. RNEDESim: view on the map.

In the third year, the RNEDE software was further extended for two applications. The first application relates to traffic management. The existing flow interception model for optimization of electric vehicle recharging stations, as studied earlier in the group, was modified to enable the evaluation of flow interception under uncertain scenarios, including the failure of roads (edges), junctions (nodes), and stations themselves. The back-end for this optimization is implemented in the General Algebraic Modeling System (GAMS).

The optimization with the facility interception model approach is based on a given and steady-state flow of agents (vehicles) in the network, thus assuming that individual agents do not adjust their route following the installation of recharging stations. To make a joint optimization of paths and facility interception possible, the approach is taken to the next level by optimizing the paths in the network. Currently, a working code is available that optimizes the vehicle paths in a greedy approach, i.e., vehicle per vehicle. This is a cost-effective approach, yet it is suboptimal. For this reason, an alternative
General Algebraic Modeling System formulation was based on Multi-Commodity Flow optimization. To adopt this approach, each set of vehicles with a distinct source and destination pair is considered a separate commodity. As of this writing, this code has been run successfully for small-scale examples (Fig. 4). For largerscale optimization, the existing Cplex solver fails to deliver. Since the Cplex solver defines the state-of-the-art in combinatorial optimization, heuristics will be developed and deployed at Purdue University for continued efforts to solve this problem.

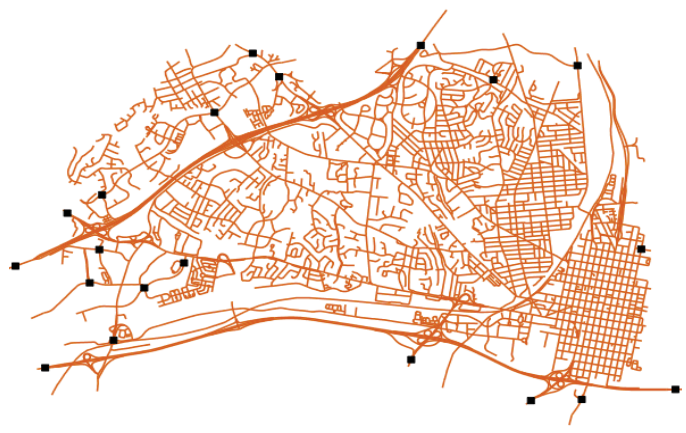

FIGURE 4. Optimized location of 20 service stations (node failures rate $5 \%$ ).

As a second application, a strategy to reroute information flow in a control network was also devised. To this end, a (control) function is defined over a network by means of sensing elements (source nodes), control and display elements (destination nodes), and logic nodes (where computations are done). Following this approach, multiple functions to be deployed on the network can be provided. With these function definitions, shortest-path algorithms are used to define the optimal flow of information over the network. In addition, when redundant elements are present in the network, they are selected optimally as well by means of an enumerative method, i.e., all possible choices are enumerated with respect to the nodal elements. The same implementation allows us to reroute the flow and/or re-select the nodal elements in case of identified failure or degradation of the network elements (sensing/actuating/computation nodes and data connections (edges). 
Benefits to DOE

The theoretical frameworks, dynamic simulations, and experimental campaigns are advancing the state-of-the-art in developing the next generation of highly automated resilient networks that can provide greater stability and efficiency while achieving graceful degradation by anticipating failures. Such an advance is required for a wide range of applications seen in energy, environment, and nuclear industries. The papers resulting from this project as well as the simulation environment are beneficial to core DOE mission objectives.

\section{Relevant Publications and Presentations}

Shukla, A., V. L. Agarwal, and V. Venkatasubramanian, 2011, "Optimizing Efficiency-Robustness Trade-offs in Supply Chain Design Under Uncertainty Due to Disruptions," International Journal of Physical Distribution \& Logistics Management, in press.
Shukla, A., J. Pekny, and V.

Venkatasubramanian, 2011, “An

Optimization Framework for Cost Effective Design of Refueling Station Infrastructure for Alternative Fuel Vehicles," Computers and Chemical Engineering, Vol. 35, pp. 1431-1438.

Villez, K., A. Gupta, V. Venkatasubramanian, and C. Rieger, 2011, "Resilient Design of Recharging Station Networks for Electric Transportation Vehicles," ISRCS 2011, Boise, Idaho, USA. 


\title{
Next Generation Control System "Smart Grid" Simulation Environment
}

\author{
Lyle Roybal, G. Shawn West \\ 10-073
}

The "Smart Grid" can be generally defined as an entity that merges or combines aspects of the telecommunications infrastructure with the management of the electric power grid. Parts of the electric power infrastructure are already "Smart" in that there is some level of automated centralized control over generation and large loads. However, the electric power industry is undergoing significant changes in operating philosophy as renewable generation technologies such as wind and photo-voltaic (PV) become more cost effective and mature. Also, big changes in communications and computational capabilities during the last decade have made it possible to monitor the overall health of the generation, transmission, and distribution networks simultaneously with a variety of new sensors such as phase measurement units. As these technologies mature and become more powerful, the entire electric power industry will evolve such that fundamental operations and design philosophies that have guided this industry are going to change.

This LDRD focused on two areas of Smart Grid development. The first area investigated and modeled was based on a domestic military installation connected to the larger national grid system, and the effects of local wind generation on the stability power system on the base when combined with large backup diesel generation. An installation of this size could be termed a Micro Grid. Here, the focus of this study was the interaction between the wind generation and the backup diesel generation including fast transients and strategies used to stabilize the system during fluctuations introduced by the intermittency of the wind generators. This study was done using the Real Time Digital Simulator (RTDS) hardware and software and calibrated with real data from a previous test conducted on a real system. The overall goal of this portion of the program was to validate the modeling concept of using RTDS for fast transient analysis on Micro Grids. This will allow better instrumentation and test designs for future Smart Grid experiments on INL power grid test facilities.

The second area of Smart Grid research completed this fiscal year is related to bigger picture issues with respect to grid stability and the increasing use of intermittent generation sources such as wind and PV. The primary issue with intermittent renewable generation is the time frames involved with which the generation profiles can change. Generation can decrease or increase over time frames of several minutes to potentially hours as storms come and go, or with diurnal variations in wind and solar energy profiles. A stochastic element involves wind gusts and intermittent cloud cover that can make for very choppy generation profiles over the 160 -second time frame. This is a very real issue as renewable generation percentages get larger than about $10 \%$ of the total generation, and a much more serious concern for utilities that are aggressively trying to reach $30-40 \%$ renewable generation.

\section{Project Summary}

Summaries for each of the major tasks accomplished this year are provided in two subsections below. Dr. Santiago Grijalva of Georgia Institute of Technology was a collaborative researcher in defining some of the important issues that the electric power industry is going to face as Smart Grid concepts are designed and implemented.

\section{$\underline{\text { RTDS Micro Grid Model }}$}

A model of a small power system including three induction wind generators, two diesel backup generators, and electrical loads commensurate with that of an islanded mediumsized military base was constructed using the RTDS modeling engine. Control algorithms were introduced for the wind turbines and diesel generators that closely mimicked the local control, response, and behavior of the power sources. Model response was compared to test data and used to verify that fast dynamic system response could be accurately modeled using the RTDS system. Once the proof-of-concept was verified, an INL electric grid test bed was modeled. The main purpose of this was to be able to predict grid behavior prior to actually running a test on the system. This capability will be used to help design tests and to examine potential control algorithms prior to actually installing them on real hardware, thereby saving considerable expense and time while protecting equipment in the test bed from potentially catastrophic combinations of events. 


\section{Smart Grid Multi-Time Scale Dynamic Model}

A multi-time scale model for predicting the generation and load management practices and parameters needed for a stable grid has been constructed and is based on an entirely new approach for looking at power grid dynamics over long periods of time. The model was developed using PowerSim, which is a systems dynamics modeling package. PowerSim is a commercial systems dynamics package and provides a modeling environment much like Microsoft Word provides a documentation environment. Customized calculations and concepts were developed based on first-principal physics to calculate power flow and frequency deviations of a nodal power grid. These included generation and individual generator management commonly referred as dispatch management, automated generation control schemes, some preliminary load management strategies based upon commercially available devices that can be installed at the residential level, power injection from non-traditional renewable generators like wind and PV, and the effects of load reduction at the residential level from solar based water heating. These capabilities were integrated into a user-friendly interface that allows adjustment of important parameters during model execution. The model has the ability to import load and dispatch data from actual utilities in spreadsheet or database form. The model can simulate an entire day of grid simulation in approximately ten seconds on a moderately fast personal computer and is designed to quickly investigate and compare competing Smart Grid operating schemes. Figure 1 shows the opening panel of the INL Smart Grid modeling environment.
Planned additions to the model include more generators with modifiable parameters, and electric vehicle submodel to simulate the increasing occurrence of all-electric and hybrid cars, more sophisticated automatic generation control to more closely model the coordination of multiple generators at the system level.

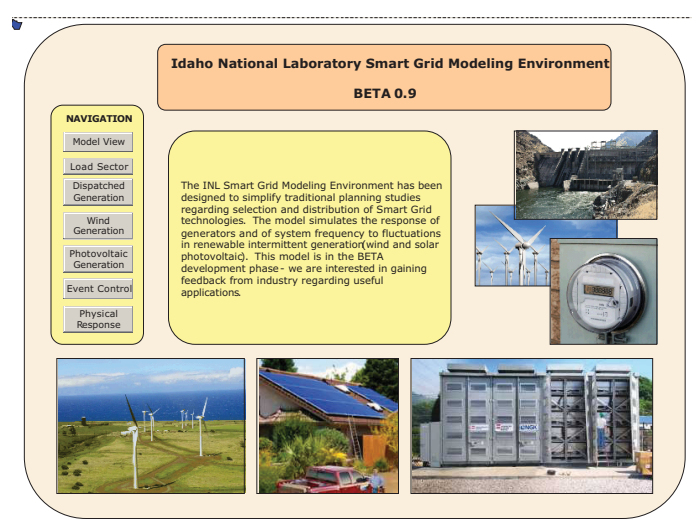

FIGURE 1. Opening panel/display for the Smart Grid modeling environment.

\section{Benefits to DOE}

This research benefits DOE by providing a planning tool and method to quickly evaluate Smart Grid technologies and control strategies to ascertain the degree to which the technology is effective from both a system reliability viewpoint and a cost to implement and operate viewpoint.

\section{Relevant Publications}

An invention disclosure record has been filed in the INL system for the multi-time scale dynamic grid model described above. 


\title{
Wireless Communications R\&D
}

\author{
Hussein Moradi, David Couch, Jose Loera \\ 10-075
}

The National and Homeland Security

directorate of INL, through its LDRD program, is building technology research, development, demonstration, and deployment capabilities to establish a national leadership role in wireless communications. For the past year, this LDRD has been able to bring focus to technical challenges facing the Spectrum Sharing initiative that is currently overseen by the Office of Science and Technology Policy, Public Safety Interoperability, and Smart Grid security. The objective of our research is to develop a communication scheme that is secure, reliable, and sustainable while utilizing the spectrum as efficiently as possible. Our hypothesis is based on Filter Bank (FB) technologies integrated with Multi-Carrier Spread Spectrum (FB-MC-SS) technology that produces more localized spectra for each subcarrier as compared to the conventional Multi-Carrier Spread Spectrum (MC-SS). This will enable reliable and secure communications within a congested radiofrequency environment.

\section{Project Summary}

MC-SS schemes such as those deployed by Orthogonal Frequency Division Multiplexing for signaling have been proposed as a robust spread spectrum (SS) technique to resist narrow-band and partial-band interference. Our goal is to research, design, and implement a FB-based MC-SS system. The proposed design is based on a filtered multitone (FMT) implementation. The proposed system has a number of possible applications. It may be used as a stand-alone SS system with robust performance where narrow/partial band interference channels are present. Many other applications of the proposed FB-MC-SS system are possible, especially in situations where low detection and low susceptibility to interference are required.

For FY 2011 we formed our initial concept and implemented a mathematical FB-MC-SS channel model. This model consists of a transmitter, a receiver, and over-the-air components.

A primary design goal of this system is to ensure reliable performance under harsh jamming conditions. To this end, we have chosen to transmit binary data symbols and (as noted above) to adopt the FMT for multicarrier transmission of SS signals. The properties of the proposed FMT waveform have been analyzed with emphasis on the properties that lead to carrier and timing synchronization schemes. We have also proposed a blind channel estimation algorithm. The following is a list of FB-MC-SS features:

- Enables simultaneous "underlay communication channel" in an occupied spectrum delivering low to medium datarates

- Operates under harsh/jamming radiofrequency environments

- Exhibits low probability of detection and interception

- Resists high-energy narrow and/or wide band interference

- Performs robustly in high-speed mobility environment

- Poses no taxation on occupied spectrum

- Can be deployed on any band of frequencies

- Establishes a secure communications link when augmented with "key" generation feature

- Enhances Orthogonal Frequency Division Multiplexing Access when orthogonality is lost (overlay channel)

- When used as an "underlay control channel," creates a foundation to building an adaptive/cognitive radio network that maximizes the use of the available white spaces, where high-data-rate "overlay channels" are dynamically negotiated.

To summarize, for FY 2011 proof of concept for FB implementation Phase 1, we completed and demonstrated the functionality of the following on a National Instrument SoftwareDefined Radio platform:

- Essential characteristics and system architecture that relate to FB coefficients with unity roll-off factor

- Design and implementation of the FB-MCSS “transmitter" 
- Design and implementation of the FB-MCSS "receiver" with the following components: (a) match filter, (b) carrier recovery, (c) timing recovery (acquisition and tracking), (d) maximum ratio combining methods and (e) a blind channel estimation method

- Develop the concept of using secret keys to further improve the security of the FB-MCSS when used as a stand-alone system for a super-secure communication link between two parties.

\section{Benefits to DOE}

This LDRD brings focus to technical challenges facing the national Spectrum Sharing initiative for re-purposing and sharing the radiofrequency spectrum that may affect many government agencies (DOE, Department of Defense, other federal agencies, etc.) that possess spectrums allocated to them by the National Telecommunications Information Administration. This LDRD lays a foundation for identifying spectrum availability when not in use.

\section{Relevant Publications and Presentations}

IEEE DySpan publication under review for Fall 2011 submission, September 29, 2011.

Moradi, H., 2010, "Spectrum Crisis and National Broadband Plan - Implications to National Security," Milcom 2010 Conference, November 01, 2010. 


\section{Electronic Warfare Digital Communications and Network Traffic Modeling}

Kurt Welker, Lance Murri, Tim Thompson

$11-022$

The "Electronic Warfare Digital

Communications and Network Traffic

Modeling" LDRD focused on being able to identify, extract, and assimilate communication network data from both static (fixed equipment sites) and dynamic (mobile, real-time equipment sites) data sources, codify the representative network information, and provide a missionplanning, engineering-level analysis (software modeling and simulation) of the network, including predictive traffic-flow modeling.

The overall process seeks to: (1) provide the ability to assemble network data from various static and dynamic sources such as government and public databases (such as the Modernized Integrated Database), graphic data sources (such as ESRI $^{\odot}$ shape files) and real-time data feeds, (2) assist the operator in resolving missing nodes and associations (i.e., the links between the nodes) and categorizing components of the network, (3) provide high-fidelity engineering analyses on the network such as radiofrequency analysis utilizing existing INL modeling and simulation capabilities, (4) develop and implement mission-planning level traffic-flow modeling that adheres to domain business rules, (5) provide a probable communication path from one network component to another, considering vulnerabilities and/or manipulations, and (6) develop and implement ad-hoc network analyses for equipment in unknown or moving/changing locations.

\section{Project Summary}

An overview of applicable intelligence sources for classified communications network data was created that documented the data content and specified how that data could be utilized in a network traffic model. A proof-ofconcept working software prototype titled "NetMuster" (Fig. 1) was designed and implemented. The NetMuster concept and design were externally reviewed by the United States Air Force $453^{\text {rd }}$ Electronic Warfare Squadron. The NetMuster software provides a mechanism for constructing networks with links designated as physical (copper, fiber, etc.) to represent good communication status (as opposed to those requiring engineering analysis) or as wireless links that require engineering analysis to determine effectiveness. Networks can be imported from geo-referenced shape files, from text-based flat files, or from a number of databases (such as the Modernized Integrated Database). NetMuster implements a methodology and algorithms necessary to implement logical, physical (reality-based or modeled), and business-rule-based network traffic-flow analyses (Fig. 2) for (a) logical only, and (b) multi-perspective, which includes highfidelity physics-based signals propagation analysis.

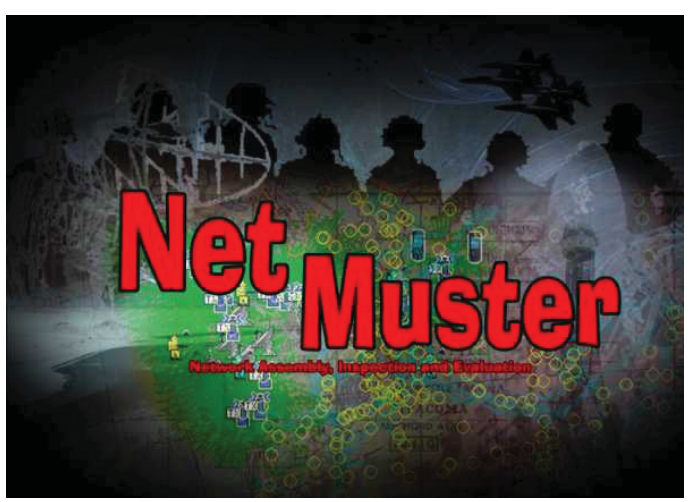

FIGURE 1. NetMuster prototype.

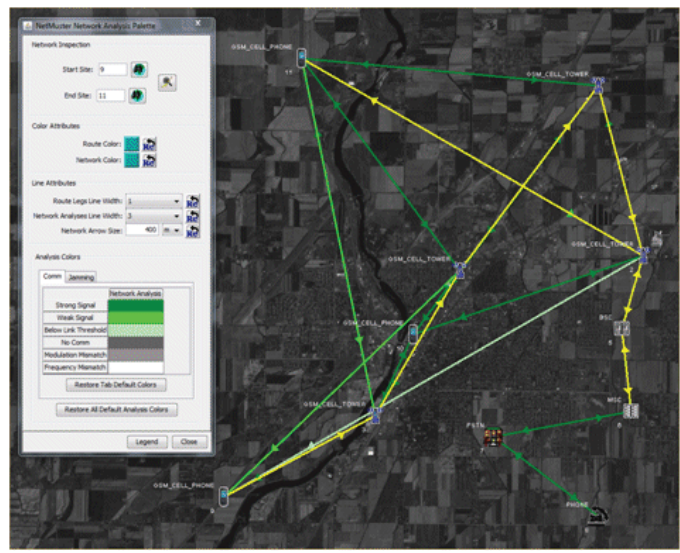

FIGURE 2. NetMuster network traffic analysis.

\section{Benefits to DOE}

The capabilities developed as part of this LDRD primarily benefit the National and Homeland Security focus at INL. This LDRD established a technical collaboration between INL National and Homeland Security Electronic Warfare and personnel involved with the Wireless Test Bed, modeling and simulation, cyber security, human behavior modeling, and 
software-defined radio technologies. These relationships will benefit the robustness of future work. For technology demonstration purposes, the LDRD developed Wireless Test Bed equipment parametric data and tests associated with communications network modeling. The methodology and algorithms for lashing together logical and physical network data with domainspecific mission-centric business rules to construct a mathematical graph that can be used to determine potential traffic/communications paths through the network have been developed and implemented as a software proof-of-concept prototype. This LDRD provides a new radiofrequency network modeling and simulation capability at INL that supports Wireless Test Bed activities and customers.

Relevant Publications and Presentations

Welker, K. and Murri, L. , 2011, "Electronic Warfare Network Traffic Modeling" Presented at Fiesta Crow '11, San Antonio, Texas, USA, April 13, 2011. 


\section{Experimental Cyber Security: Vulnerability Prediction, Discovery, and Mitigation \\ Miles McQueen, Jason Wright, Jason Larsen, Lawrence Wellman \\ $11-042$}

The quantity and diversity of an information technology or control system's vulnerabilities are related to the system's security. However, we currently have few effective ways of predicting the density, discovery, reporting, and patch development of software vulnerabilities (part of a system's attributes), and we are limited in understanding how effectively they are being mitigated. Consequently, we cannot easily determine or predict the degree to which the system is vulnerable to cyber attack. Critical infrastructure cyber security design, assessment, and measurement must take into account that vulnerabilities exist in the system and that the vulnerabilities require different levels of effort by the attacker to discover and exploit.

This research initiated the adoption of controlled experimentation and observational studies to INL's core cyber security capabilities for software vulnerability prediction, discovery, and mitigation. In conjunction with the goal of becoming recognized for experiments in security, two significant investigations were performed with the potential to be of significant interest to the general cyber security community and produce experimentally verifiable predictions about future vulnerabilities that will aid situational awareness and anticipation of attacks.

The first investigation extended and expanded research in estimating unpatched software vulnerabilities in deployed systems. Both the previous 0Day vulnerability estimations at INL and the evaluation of disclosed but unpatched vulnerabilities were advanced for predicting future, month-to-month occurrences in individual software packages related to control systems and critical infrastructure. The second investigation experimentally provided the initial assessment of whether many reported bugs that are not identified as vulnerabilities do in fact provide leverage for a violation of security.

\section{Project Summary}

Vulnerability research organizations Rapid7, Google Security team, and Zero Day Initiative recently imposed grace periods for public disclosure of vulnerabilities. The grace periods ranged from 45 to 182 days, after which disclosure might occur with or without an effective mitigation from the affected software vendor. At this time there is no direct evidence that the shorter grace periods of 45 and 60 days are justifiable. However, there is evidence that the recently announced Zero Day Initiative grace period of 182 days yields some benefit in speeding up the patch creation process, and may be practical for many software products (Fig. 1).

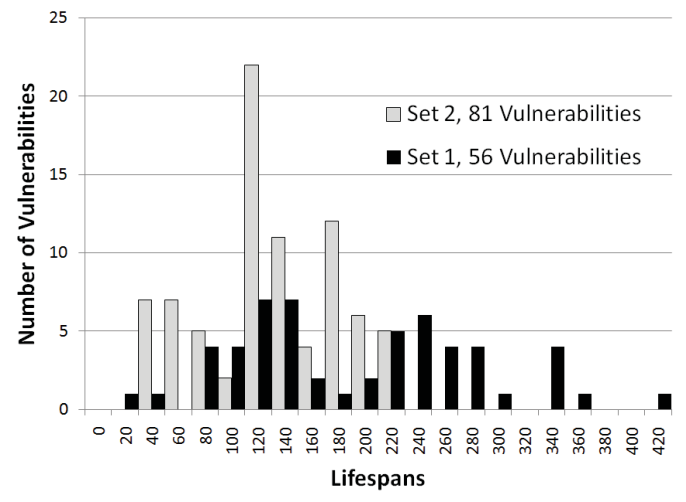

FIGURE 1. Vulnerability lifespans before 182-day grace period imposed (Set 1) and after grace period imposed (Set 2).

We proposed two new vulnerabilityexposure metrics with the end-user in mind. Both metrics, called vulnerability-free days and average active vulnerabilities per day, depend not just on vulnerability lifespans of a product but also on the rate of vulnerability reporting. We demonstrate these metrics in a case study using the four browsers: Safari, Chrome, Firefox, and Internet Explorer. Based on the derived metric values for each browser, there are large differences in vulnerability exposure, with Safari having the lowest exposure (Fig. 2). Firefox, which produces patches quickest, has one of the highest vulnerability exposures because so many vulnerabilities are discovered and reported.

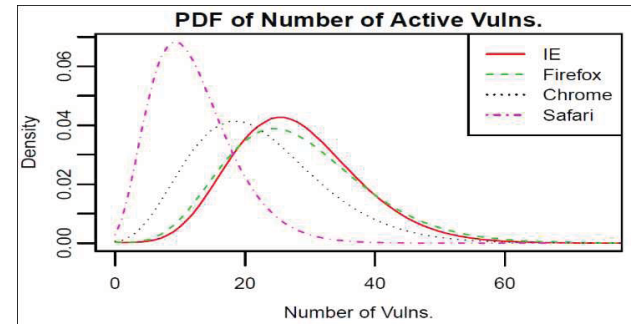

FIGURE 2. Average active vulnerabilities in four browsers. 
Our browser case study shows that even on products with roughly equivalent vulnerability exposures, imposed grace periods may not have equal effect on reducing exposure. If one of the product's average lifespan is already well below a newly specified grace period, its vulnerability exposure will not be reduced to any significant degree, while the other product may have a meaningful reduction. This indicates that there may be value in assigning different grace periods based on individual vendor products and the desired reductions in exposure levels.

In addition, we initiated experiments into software vulnerability density and conceptualization of classifiers to more effectively and accurately identify those bugs more likely to be vulnerabilities. Further experiments will be run next year, and development of classifiers will proceed.

We also held the first Experimental Security Panoramas workshop with a world-class contingent of speakers. This workshop brought together technical leaders to discuss their experiences and challenges in designing and executing credible cyber security experiments. The workshop is intended to provide a research forum and aid development of a research community for improved scientific experimentation related to all aspects of the cyber security eco-system. The workshop was very successful with the consensus that we should organize the workshop again next year.

\section{Benefits to DOE}

This research furthers the establishment of science and experimental bases for cyber security vulnerability prediction, discovery, and mitigation that is notably absent in many current evaluations and technologies that are coming into the market. These security evaluations and designs fall short in part due to lack of a strong, experimental basis of the underlying system security state and are often dependent upon personal experience, belief, and limited empirical evidence. With continued, scientifically based improvement in our understanding and prediction of software vulnerabilities, the risk management and the security of our critical infrastructure will be significantly enhanced.

\section{Relevant Publications and Presentations}

Wright, J., M. McQueen, and L. Wellman, 2012, "Two End-User Metrics: Estimating Individual Product Vulnerability Exposure," In preparation.

McQueen, M., J. Wright, and L. Wellman, 2011, "Are Vulnerability Disclosure Deadlines Justified?" 7th International Workshop on Security Measurements and Metrics, Banff, Canada, September 2011.

Zhu, Q., M. McQueen, C. Rieger, and T. Basar, 2011, "Management of Control System Information Security: Control System Patch Management," Workshop on Foundations of Dependable and Secure Cyber-Physical Systems, Chicago, Illinois, USA, April 2011.

McQueen, M., 2010, "Software and Human Vulnerabilities: Implications for Protection of Our Critical Infrastructures," IECON'10 Half-Day Tutorial, Phoenix, Arizona, USA, November 7, 2010.

McQueen, M., 2010, "Software, Human, and Physical Subsystems are Vulnerable to malicious Threats," TCIP-G, University of Illinois at Urbana-Champaign, USA, November 4, 2010.

McQueen, M., 2011, "Cyber Security and Critical Infrastructure Resilience," SANS SCADA Summit, Lake Buena Vista, Florida, USA, February 23, 2011.

McQueen, M., 2011, "Measurements from Vendor Notification to Public Disclosure," Mini-Metricon, San Francisco, California, USA, February 14, 2011. 


\section{Industrial Control Systems Threat Axis Analysis to Characterize and Quantify Malicious Intent from a Threat Actor}

Peter D. Gasper, Robert S. Anderson, Wayne F. Boyer, Rob Hoffman, Julio G. Rodriguez, Daniel T. Noyes

11-069

The objective of LDRD Project 11-069 is development of the Threat Axis Analysis (TAA) tool. The tool will consist of a set of technical knowledge bases and a specific and strictly defined analysis methodology, optimized to isolate, identify, and characterize Threat Actor capability and intent. The follow-on work for the TAA tool is intended to create analysis products and training of government agencies in the use of the TAA tool.

\section{Project Summary}

Concept of Operations: A preliminary draft of the analysis system configuration and operational flow concept of operations has been completed.

Safe Search System: During June and July, an initial site survey was conducted and design requirements were written for a testing network which is intended to be used for open source information acquisition. This network will be called the Safe Search System. An independent security assessment of the network will be completed during FY 2012.

Product Review: LDRD team analysts have established the preliminary Product Knowledge Base structure, isolated and cataloged data sources, and initiated data collection and knowledge base population.
Metrics Review: Five existing metric systems were assessed to support the development of an initial TAA metric base and the establishment of a reliable analysis regime. This assessment work indicated that none of the existing metric systems are fully adequate to support this LDRD effort. During FY 2012, our team will modify the systems that can be adapted and also develop new metrics to fill in the gaps.

\section{Benefits to DOE}

This LDRD project will benefit the Department of Energy in the characterization of threats to U.S. critical infrastructure.

\section{Relevant Publications and Presentations}

Our presentation Threat Axis Analysis Development of a Tool to Characterize Threat Actor Capability and Intent - Initial Findings was delivered at the Naval Postgraduate School Classified Advanced Technology Update course on July 28, 2011. In response to requests, the presentation was also delivered to various Department of Defense and other government agencies. 


\title{
Thermite Burn Characteristics in Oxygen-less Environment
}

\author{
Michael A. Daniels, Ronald S. Wallace, Reston A. Condit, Nikki Rasmussen
}

11-034

Thermite reactions are exothermic reactions between a fuel metal and a metal oxide. When ignited, the reaction rapidly achieves high temperatures $\left(>2,500^{\circ} \mathrm{C}\right)$ and is difficult to extinguish. These characteristics make thermites useful for multiple applications, including environments that do not contain any oxygen. Because thermites contain their own oxygen, their reaction with environmental oxygen is often neglected as trivial. However, empirical evidence from previous experiments conducted at INL suggested that some environmental oxygen may play a role in the reaction. The purpose of this research is to quantitatively and qualitatively compare the burn characteristics of four representative thermites reacting in open atmosphere and in an oxygen-free environment. Understanding this relationship between the thermite burn characteristics and the environmental oxygen is critical to future work involving these materials, especially in situations where the available environmental oxygen is variable.

\section{Project Summary}

To isolate environmental oxygen as a variable, test fixtures were built that did not affect the pressure surrounding the reaction. Each thermite was first tested in an open-air environment. A vented chamber was constructed and continually purged with argon to test the reactions in an oxygen-free environment.

To measure the quantitative property of burn rate, a digital fiber-optic sensor setup (Fig. 1) was used. The system consisted of 12 digital sensors and individual fibers at 1-inch intervals that measured when the reaction first produced $1 \mu \mathrm{W}$ of light within its field of view. The burn rate sensor system was validated with a Phantom high-speed camera. The Phantom was also used to evaluate the qualitative differences of the reactions in the oxygen and oxygen-less environments. Qualitative burn properties include flame bloom intensity, burn consistency, and secondary reactions.

Four micron-scale thermites were chosen based on their relative burn rates. Our hypothesis was that the slow-burning thermites would have the greatest burn rate difference between the two environments. However, this research showed that the fast-burning thermites burned at significantly slower rates when the availability of environmental oxygen was lowered, while the slow-burning thermites burned at relatively the same rate regardless of the availability of the environmental oxygen level. In contrast, the qualitative differences of the slow-burning thermites was pronounced due to the change in the oxygen environment, while the physical burn characteristics of the fast-burning thermites showed almost no difference between the two environments.

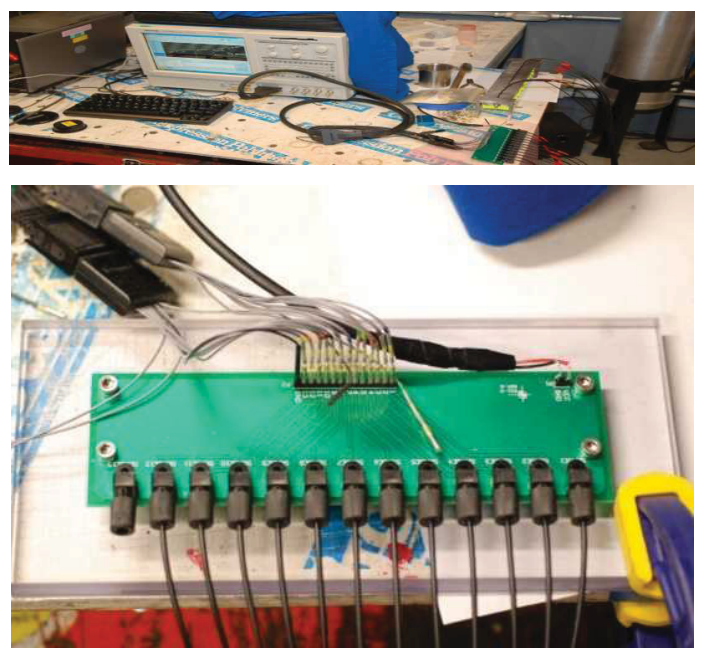

FIGURE 1. Fiber-optic measurement system.

Several differences other than burn rate were observed between burning the thermites in air and in an argon-purged chamber. These differences include the size and intensity of the bloom, the particle reaction at the perimeter of the bloom, and the secondary reactions presumably between the reaction effluent and the atmospheric oxygen.

From this research, we confirmed that atmospheric oxygen plays an important role in thermite reactions in both primary and secondary reactions. The role in the primary reactions was shown in the fiber optic burn rate differences, and the role in the secondary reactions was shown in the high-speed video of the reactions; the difference identified in the secondary reactions was qualitative in nature. The burn rates of the fast, less-fuel-rich reactions were more affected by environmental oxygen levels than the slow, fuel-rich mixtures. 
Benefits to DOE

This research directly supports the understanding of the critical relationship between environmental oxygen and the thermite reaction.

This research has identified techniques to measure reaction rate in various atmospheric environments, which will be important to meeting future requirements for enhanced energetic materials. 


\title{
Prototype INL Wildland Fire Early Warning and Situational Awareness System
}

\author{
Ryan Hruska, Kurt Derr, Matthew Anderson, Mike Snyder
}

11-077

Most fire detection systems rely on traditional human reporting, which means that valuable time is lost between when the fire starts and when it is reported. By developing a system to automatically monitor, detect, and model fires, the impact of the fire could be significantly decreased. The objective of this research project was to enhance INL wildfire suppression and emergency operation activities by developing and implementing a first proof-of-principle prototype of a Wildland Fire Early Warning and Situational Awareness System. There were two main objectives for this project: (1) the development of a prototype Wildland Fire Early Detection and Warning System and (2) the development of prototype Wildland Fire Situational Awareness and Fire Behavior Model for the INL Site.

\section{Project Summary}

The project has successfully developed a detection and a modeling system. The detection system completed an optical resolution and lineof-sight analysis to establish optimal plume detection coverage for standard high-definition cameras, while minimizing the number of cameras required to ensure complete coverage of the INL Site. The detection system also completed an initial evaluation of the capabilities of experimental commercial smoke plume and fire detection software to detect actual INL rangeland fires. The detection system software registered detects for all three range fires imaged by INL mountain top cameras, but there was insufficient data to validate the capability of the cameras and software to detect smoke plume and fire during the cameras' auto scanning mode. The detection system also developed software to automatically retrieve and convert external National Oceanic and Atmospheric Administration lightning-strike data for visualization on the commercial software's map display. INL is pursuing a technology transfer agreement to provide this software modification to the vendor.

The modeling system assessed performance specifications of U.S. Government fire behavior models for applicability to rangeland fire suppression activities. The modeling system also selected the U.S. Forest Service's Fire Area Simulator (FARSITE) for further fire propagation modeling. The modeling system configured FARSITE with INL Desert Sitespecific parameters for optimizing model performance. The system modeled the Jefferson (2010), Highway 33 (2011), and the T-17 (2011) fires (Fig. 1). The system determined that the model provides realistic representation of fire propagation on the INL Site with a modification of the rate-of-spread values.

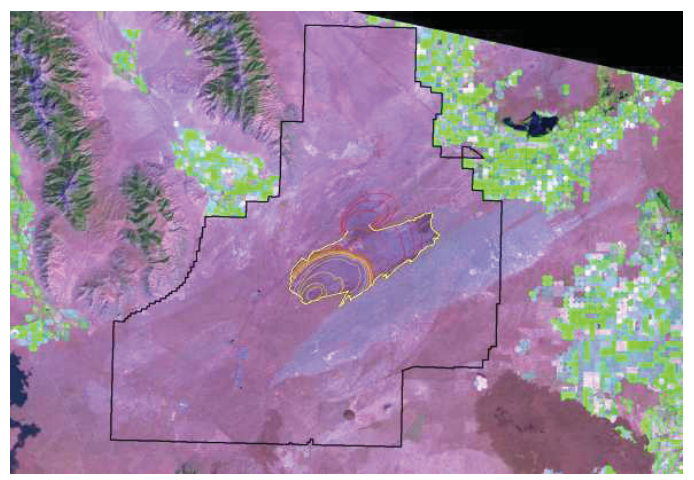

FIGURE 1. Orange to red: T-17 FARSITE Model run on August $27^{\text {th }}$ at 7:20 p.m. Yellow: Fire suppression lines. Yellow: Jefferson Fire Scar visible to the south; used as a fire break.

\section{Benefits to DOE}

This project has benefitted INL by developing a scientific-based understanding of how a sensor system can be configured for early detection and has demonstrated how fire propagation models could be effective in planning fire suppression activities.

\section{Relevant Publications and Presentations}

The LDRD results have been briefed to INL Emergency Response Organization (ERO) and INL Fire Department. Deployment of the LDRD technology includes: FARSITE fire propagation model has been installed in the INL ERO Emergency Operations Center. ERO is planning the procurement of the commercial smoke plume and fire detection software, with scheduled installation in the Emergency Operations Center during FY 2012. The ERO has been provided with procurement specifications for a future request for proposals to commercial providers of smoke plume fire detection systems. 


\title{
SMC Advanced Armor Materials and Systems R\&D
}

\author{
Henry Chu, Thomas Zagula, Michael Bakas, Benjamin Langhorst, Jeffrey Lacy
}

$11-060$

The intent of this LDRD was to explore high-risk technologies in the manufacture of novel and mass-efficient armor materials based on generically defined requirements provided by military customers.

In FY 2011, this LDRD program focused on three technological areas: (1) bainitic alternate armor steel, in which the FY 2011 objective was to identify the correlation between the as-cast chemistry, post heat-treatment and mechanical properties of the bainitic steel that can be employed as low-cost armor steel;

(2) ferroelectric (FE) crystal for defeat of shaped-charge (SC) device, in which the objective was to verify and study the effect of FE crystals on the reduction of penetration power of a SC jet; and (3) dual-hardness titanium, in which the objective was to perform a preliminary study in using a self-propagating, hightemperature synthesis (SHS) process to create a super-hard titanium metal matrix composite. The ultimate goal of this research program as directed by the Army Research Lab is to demonstrate that the SHS process is a viable technique in the fabrication of titanium laminate with dual hardness.

\section{Project Summary}

The determination and verification of the final chemistry of three variants of the large bainite ingots had been performed by commercial analytical labs. Based on reported chemistry, the time-temperature transformation plots were regenerated to determine the appropriate heat-treatment schedules. Metallurgical characterization of the final phases obtained was completed. Mechanical properties (hardness, Charpy impact, split Hopkinson pressure bar-dynamic strength) of the three bainite recipes were evaluated. The planned ballistic tests were not performed due to exhaustion of the allotted funds and safety concerns with the operation of the $30-\mathrm{mm}$ gun. The ballistic tests are expected to be resumed in FY 2012 once the inspection and re-assembly of the $30-\mathrm{mm}$ gun is completed to satisfaction and new funding is available.

A preliminary live-fire test in FY 2010 revealed that FE crystals had some effects on the penetration behavior of a 25 -mm-diameter SC jet. Based on recommendations from Tank Automotive Command and the Army Research Laboratory, the shaped-charge test devices were upgraded to 40-mm-diameter devices in FY 2011 in order to obtain more relevant ballistic data. Three iterations of testing with the 40-mm SC devices (a total of 44 shots) were planned to test three variants of FE in FY 2011: (1) to verify the behavior of the FE crystals with respect to standard armor materials such as armor steel, glass, and ceramics, and (2) to characterize the electrical discharge and/or electromagnetic field generated during the penetration by the SC jet. A total of fifty $40-\mathrm{mm}$ shaped-charge devices and eighteen 75-mm-diameter $\mathrm{LiNbO} 3$ and $\mathrm{LiTaO} 3$ crystals were procured. (The lead zirconate titanate manufacturer could not deliver the test material in the $75-\mathrm{mm}$ diameter configuration and hence dropped). The first two series of testing to calibrate the optimal standoff of the SC devices versus depth-of-penetration into plain armor steel witness blocks were completed. The final test into the specially prepared FE crystals and companion tests into silicon carbide ceramic, borosilicate glass, and standard armor steel at maximum penetration-depth standoff were not performed due to exhaustion of allotted funds. We expect this final series of tests to be completed and the complete set of test data assembled and reported to Tank Automotive Command points-of-contact within the first half of FY 2012 once funding resumes.

Two samples with a composition of $91 \mathrm{wt} \%$ titanium (Ti) and $9 \mathrm{wt} \%$ of amorphous boron (B) were fabricated and used to demonstrate the in situ formation of ultra-hard titanium boride (TiB) particles in a pure titanium matrix via the SHS process. Scanning electronic microscopy and $x$-ray diffraction examinations of the final samples confirmed the presence of TiB particles. Using a Vickers Diamond Pyramid Hardness micro-hardness test, the average Vickers hardness for the Ti/TiB samples was found to be 1015, approximately equivalent to a Rockwell hardness of 68+. Hence, we concluded that the SHS process was successful in creating a $\mathrm{TiB}$ hard phase in the relatively ductile Ti metal matrix. However, the method will need to be refined in order to obtain it in a sufficient quantity and quality for armor applications. The next phase of the research will address the above 
issues. Future experiments will need to be conducted to produce plates large enough for ballistic testing. The fact that SHS can be used as a means of obtaining TiB may be key in making dual hardness titanium a viable armor option.

Benefits to DOE

This project successfully reinforced INL's reputation in material science and engineering and process technology development. The materials being developed in this program will be applied to robust protection systems to mitigate advanced threats. This program benefits directly the security missions of INL National \& Homeland Security and the Specific Manufacturing Capability. 


\section{Cognitive Network Engine and Simulation Framework}

Juan Deaton

08-102

According to the President's advisors on science and technology, industrial wireless sensors could improve efficiency by 10 percent. Future wireless sensor networks will play a role in modernizing the electric grid and enhancing the security and reliability of energy infrastructure; cognitive network research will improve the capabilities of wireless sensor networks within industry. The primary goals of the program were to conduct research in critical areas to provide new wireless research capabilities for INL. The objectives of this year were to publish research in wireless cognitive networks. Research specifically focused on building cognitive capabilities into commercial wireless networks to overcome future challenges from the looming spectrum crisis.

\section{Project Summary}

In 2008, Juan Deaton began the PhD candidate program under Dr. Luiz DaSilva at Virginia Tech. In addition to Juan's accomplishments, one invention disclosure record and three publications were submitted this year. The invention disclosure record was recently elected and compiled into a patent that was filed in October 2011. This patent protects an invention that describes an architectural framework to support Dynamic Spectrum Access called the Spectrum Accountability Framework. This framework, filed under a provisional patent, was combined with additional academic analysis and was accepted for publication in the highly selective International Symposium on New Frontiers in Dynamic Spectrum Access Networks (DySPAN) conference. The paper was presented in May of 2011. In examining spectrum policies, Juan wrote another paper using decision theory to develop a method for evaluating the utility of different spectrum access rules. This decision theory paper was accepted into the 2011 Global Communications Conference. The conference was held December 2011 in Houston, TX. In the most recent publication, Juan extended his work from the Dynamic Spectrum Access Networks conference paper and created a mathematical model to determine the quantifiable benefits of using Dynamic Spectrum Access in a Long Term Evolution network. This paper was submitted to Elsevier's Journal on Physical Communications
Special Issue on Cognitive Radio for LTE Advanced \& Beyond, and the author is optimistically awaiting acceptance.

\section{Benefits to DOE}

In the transmission and distribution of electricity, the Department of Energy is partnering with industry to undertake research in developing cost-effective solutions in the areas of advanced sensors. Cognitive networking research will directly benefit these efforts by advancing concepts for reliable sensor networks. As reported by the President's advisors on science and technology, industrial wireless sensors could reduce emissions 25 percent or more. Because cognition will be a key attribute in the development of future wireless sensor networks, this research will indirectly benefit the environment.

\section{Relevant Publications and Presentations}

Deaton, J., C. Wernz, and L. A. DaSilva, "Decision Analysis of Dynamic Spectrum Access Rules," Will appear in GLOBECOM, December 2011.

Deaton, J., R. Irwin, and L. DaSilva, 2011, "Dynamic Spectrum Access in LTEAdvanced Networks," Awaiting acceptance, Elsevier Physical Communications: Special Issue on Cognitive Radio for LTE Advanced \& Beyond, November 2011.

Deaton, J., "The Effects of a Dynamic Spectrum Access Overlay in LTE-Advanced Networks," Poster presentation for Wireless@VT Symposium, June 2011.

Deaton, J., R. Irwin, and L. DaSilva, "The Effects of a Dynamic Spectrum Access Overlay in LTE-Advanced Networks," 6th IEEE International Symposium on New Frontiers in Dynamic Spectrum Access Networks (DySPAN), Aachen, Germany, May 2011.

Deaton, J., "Dynamic Channel and Power Assignment Techniques for Cognitive DSA Networks," Poster Presentation for White House Office of Science and Technology: Wireless Spectrum Research and Development Senior Steering Group, Idaho Falls, Idaho, USA, April 2011. 


\section{Enabling Dynamic Spectrum Access in Long-Term Evolution Networks \\ Juan Deaton \\ 11-078}

In the next decade, the use of smart phone devices and applications is expected to create a data demand sufficiently great that the Federal Communications Commission is predicting a 300-MHz spectrum deficit for mobile wireless broadband by 2014. One option for network operators is to increase spectral capacity by deploying a dynamic spectrum access (DSA) overlay in their Long Term Evolution-Advanced (LTE+ Networks). Additionally, network operators will also increase spectral capacity through cell-splitting by deploying Heterogeneous Network (HetNet). HetNet comprise a combination of macro-cells, femtocells, and relay nodes that manage spectrum coverage areas in multiple tiers of granularity.

\section{Project Summary}

Within the scope of this scenario, there are two research problems proposed. The first research problem proposes an analytical study to evaluate how to optimally assign DSA carriers when a cognitive Base Station reaches an over capacity peak period. In this first research problem, a mathematical analysis using optimization and utility theory will evaluate and quantify the tradeoffs between secondary interference to primary users and secondary user throughput. The second problem analyzes the operational effects of deploying DSA in a LTE+ HetNet. This second problem seeks to define protocol interfaces and also operational signaling required to deploy and manage a DSA spectrum in a HetNet. This analysis will include operational flow diagrams and architectural models. Results from the first research problem will provide an input into the Federal Communications Commission for developing appropriate DSA regulation, which protects primary users and simultaneously allows for additional wireless services to emerge.

Research from the second problem will serve as a direct input into standards bodies for developing regulation, which includes DSA for next-generation LTE networks.

Benefits to DOE

Future wireless networks will play a role in modernizing the electric grid and reliability of energy infrastructure. As a result, Alcatel-Lucent has announced new capabilities to support smart grid applications. Commercial networks and devices are being considered for use within public safety and the military. The development of new high data rate applications and media applications on smart phones can provide new capabilities to these agencies. With the growing number of wireless sensors, smart phones, and applications, more spectrum bandwidth and cognitive capabilities will be required. This research contributes to advancing nextgeneration commercial networks to overcome future challenges of spectrum use, which has benefits to future electric networks, public safety, and military applications. 


\title{
Multi-Rate Shock Physics Simulation of Blast and Penetration Events in Concrete
}

\author{
W. D. Richins, T. K. Larson, J. E. Blakeley
}

10-064

The primary purpose of this research is to experimentally establish and validate new methods and material parameters for simulating and enhancing the performance of critical concrete structures subjected to malevolent attacks and dynamic accident events. The project began in October 2009 with identifying concrete test sample designs that would likely be relatively straight forward to build and simulate. The project managers issued a subcontract to the University of Utah (UU) to design and fabricate the concrete test samples and provide assistance with testing. Since the concrete form work and materials were donated by Hanson Structural Precast, Salt Lake City, UT, we were able to increase the extent of our planned testing. We supported two graduate students at UU-Tim Garfield, who completed his Master of Science degree in Civil Engineering and Annie Weidner, who is just starting her research.

We hypothesize that experimental data and application methods derived from our proposed studies will extend the application of simulation results into regimes where large scale experiments are too costly or otherwise impossible to conduct. To test this hypothesis, we will:

- Model three benchmark problems to evaluate our ability to simulate dynamic concrete response: (1) high explosive blast loading, (2) high-speed penetrator, and (3) dynamic Brazilian test

- Conduct small-scale concrete sample testing to provide high quality data to compare, validate, and improve simulation results

- Design, construct, and test concrete samples to address the effects of concrete reinforcement and high temperatures expected in Next-Generation Nuclear Plant designs using standard steel rebar placement and three potential performance enhancing techniques: external fiber wrap, glass-based rebar, and synthetic fibers.

\section{Project Summary}

In FY 2011, we completed blast testing of 19 concrete test panels $4 \mathrm{ft} \times 4 \mathrm{ft} \times(6$-in, 10 -in, and 14-in) thick at the INL National Security Test Range. The reinforcement of the test panels varies to sample possible building material configurations: (1) plain concrete with steel rebar reinforcement typical of structural concrete, (2) synthetic fibers with no steel reinforcement, (3) glass fiber reinforced polymer reinforcement bars, (4) steel rebar reinforcement retrofitted with an external glass fiber reinforced polymer jacket to simulate the retrofit of existing buildings, and (5) combinations of the previous. We used 10 to $20 \mathrm{lb}$ of $\mathrm{C} 4$ explosive at a $1-\mathrm{m}$ standoff from the center of the panel front face to ensure adequate damage without destroying the panels. Several panels were also strength tested at UU to determine post-blast residual strength. Preliminary test results show significant reduction in concrete spalling and scabbing when using synthetic fibers in the mix or when the concrete test panels were retrofit with fiber wrap. Simulations of the response of the concrete panels to blast loading were undertaken using LS-DYNA (a multipurpose multi-physics simulation code).

An impact test machine was built at UU to conduct the dynamic Brazilian split-cylinder tests. Forty-four such tests were conducted in July 2011 with INL providing test oversight, data acquisition, and high-speed photography. We plan to conduct similar tests at high temperature in FY 2012 and have prepared a test plan. A Brazilian split-cylinder simulation model was developed to compare with the dynamic split-cylinder experiments at UU.

We have prepared an approved test plan, "Ballistic Penetration Resistance of Reinforced Concrete Test Plan for INL LDRD Project EN103." Five 0.9-kg prototype penetrators were machined of 300 Maraging steel and heat treated to test on sample concrete panels when the new National Security Test Range flash x-ray system is operational (expected in Nov. 2011). We have put on hold our plan to test and simulate the dynamic response of aged concrete. Our current budget does not allow us to explore the possibility of obtaining samples representative of 40-60-year-old DOE or commercial nuclear facilities. At the suggestion of Dr. Pantelides, we are using a glass fiber reinforced polymer wrapping material instead of carbon fiber. The use of carbon fiber to retrofit a large structure for blast protection is likely cost prohibitive. Initial 
simulations to model the behavior of our concrete panels to the high velocity penetrator were run with CTH (a shock physics code developed by Sandia National Laboratories).

\section{Benefits to DOE}

This project brings new analytical tools and capabilities to INL, as well as a defined, peer-reviewed method to validate dynamic simulation of concrete structures in future safety analyses of nuclear or other safety related systems. The fiber wrap process is likely to prove a viable method to retrofit buildings and increase blast resistance.

Exposure to difficult problems strongly enhances our capability to perform future research and development in simulating dynamic events related to both nuclear power safety and national and homeland security. INL

publications establish credibility in the technical community and contact with program managers who may need assistance in this area. Collaboration with the UU will lead to joint publications, proposals, and the potential for $\mathrm{PhD}$ opportunities for INL researchers.

\section{Relevant Publications and Presentations}

Pantelides, C. P., T. T. Garfield, W. D. Richins, T. K. Larson, and J. E. Blakeley, 2012, "Behavior of Concrete Panels Reinforced with Synthetic Fibers, Mild Steel and GFRP Composites Subjected to Blasts," 2012 ASCE Structures Congress, Chicago, Ilinois, USA, March 29-31, 2012, accepted.
Garfield, T. T., C. P. Pantelides, W. D. Richins, and T. K. Larson, 2012, "Post-blast Loading Response of Concrete Panels Reinforced with Synthetic Fibers, Mild Steel and GFRP Composites," 2012 ACI Spring Convention, Dallas, Texas, USA, March 18-22, 2012, submitted.

Garfield, T. T., W. D. Richins, T. K. Larson, C. P. Pantelides, and J. E. Blakeley, 2011, "Performance of RC and FRC Wall Panels Reinforced with Mild Steel and GFRP Composites in Blast Events," Procedia Engineering, Vol. 10, pp. 3534-3539.

Garfield, T. T., W. D. Richins, T. K. Larson, C. P. Pantelides, and J. E. Blakeley, 2011, "Performance of RC and FRC Wall Panels Reinforced with Mild Steel and GFRP Composites in Blast Events, ICM11-3709," 11th International Conference on the Mechanical Behavior of Materials, Milano, Italy, June 5-9, 2011. 
$\Rightarrow$ Energy and Environmental S\&T

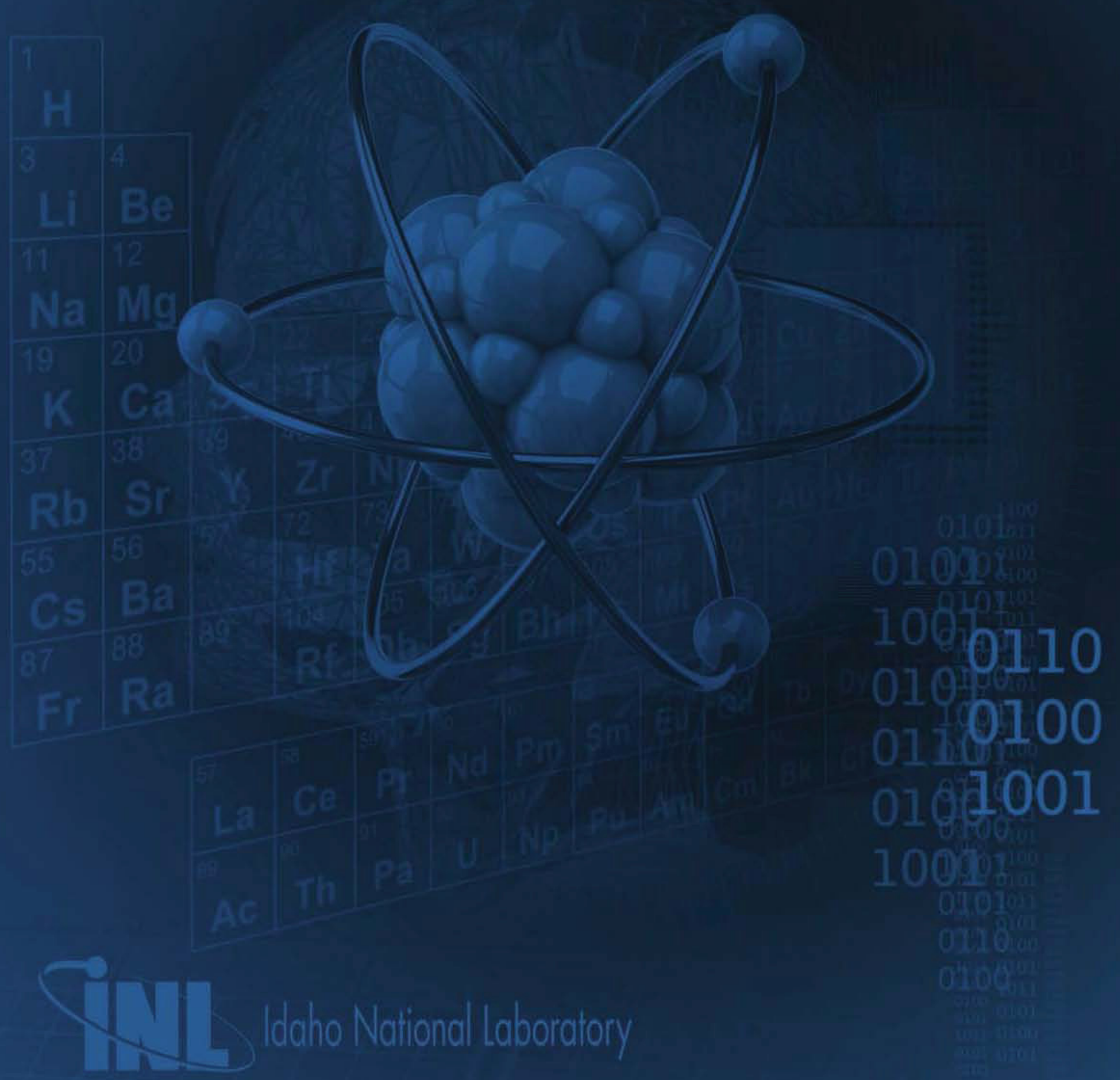




\title{
Advanced Remote Sensing for Energy and Environmental Applications using Unmanned Aerial Vehicles
}

\author{
Matthew O. Anderson, Ryan C. Hruska
}

09-019

INL has built a nationally recognized unmanned aerial vehicle (UAV) program through innovative command and control work and successful integration of various sensor technologies. Recently, this program was greatly enhanced by the development and integration of emerging miniature hyperspectral imaging sensor systems capable of acquiring data across 80 narrow bands in the visible and infrared portions of the electromagnetic spectrum (400$1700 \mathrm{~nm}$ ). Hyperspectral imagery can often be manipulated to identify, and in some cases quantify, materials or objects that would otherwise be unnoticeable. This research project's primary objective is targeted at advancing the state-of-the-art in environmental remote sensing, which can be applied directly to watershed and land-management applications.

\section{Project Summary}

The primary objective was to develop a UAV-based hyperspectral imaging system to support current and projected needs of landmanagement agencies and private industry by providing rapid, low-cost, effective environmental monitoring and mapping solutions. A secondary objective was to integrate intelligent autonomous path planning and collision-avoidance technologies into the UAVs to allow for optimized and safe operations throughout national airspace. Peer-reviewed research publications were aimed at enhancing INL's capability to work with programs and agencies focused on utilizing UAVs for safe and efficient data collection and processing. Figure 1 illustrates the UAV-based hyperspectral pushbroom sensor acquiring data over the INL UAV runway and research park. The following tasks were achieved under this LDRD project:

- Hyperspectral sensor selection, calibration, and ground testing (NovaSol vs. Resonon)

- Arcturus UAV T16 airframe characterization and flight readiness

- Sensor and subsystems integration into the UAV airframe

- Flight tests over control field using groundbased spectral measurements to validate the spectral quality of airborne image data
- Hyperspectral imagery of the UAV Test Area and control field

- Development of image processing methodology (radiometric corrections, spatial corrections, image classification, sensor fusion, etc.)

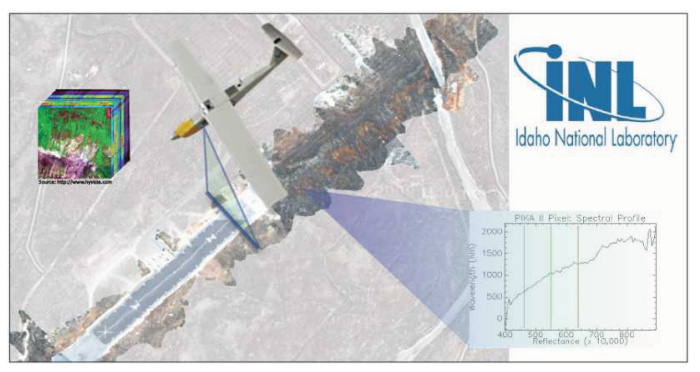

FIGURE 1. UAV-based hyperspectral imaging system.

- UAV hyperspectral data collection testing capability applied to real-world problem of nitrogen content in sagebrush

- Identification and development of Sense \& Avoid methods for UAV data collection including:

- Research of current Federal Aviation Administration directives on sensing requirements

- Development of sensor architecture

- Development of an on-board intelligent system

- Hardware in-the-loop simulation and ground-based testing with the goal to autonomously modify flight characteristics based upon environmental conditions

- Development of strategic partnerships with industry and university researchers.

During the past three years, INL and Idaho State University have performed sample missions to aid in initial characterization of the hyperspectral imaging system at an altitude of $340 \mathrm{~m}$ above ground level with a nominal pixel resolution of $0.25 \mathrm{~m}$. Mission elements included ground-truthing, image pre-processing and classification, and accuracy assessment. The spectral integrity of the imagery has been verified using field spectrometer measurements 
and cross-sensor validation using HyMap hyperspectral imagery acquired from a manned aircraft. Classification accuracy was assessed using a suite of data collected on the ground and from imagery obtained from the HyMap sensor and the INL RADAS system - an ultra-highresolution camera flown on board similar INL UAV platforms.

From May 9 to May 13, 2011, remote sensing data acquisition activities were conducted at the National Guard Orchard Training Area within the Department of Interior Bureau of Land Management (BLM) Birds of Prey National Conservation Area (NCA) illustrated in Figure 2. This was a collaborative effort between the INL/Idaho State University team, the BLM NCA manager, and the National Guard natural and cultural resource/geospatial information system managers. Both hyperspectral and high-resolution imagery were acquired, targeting vegetation classification for the BLM as well as unexploded ordinance needs of the National Guard. Figure 3 illustrates the resulting data and classification.

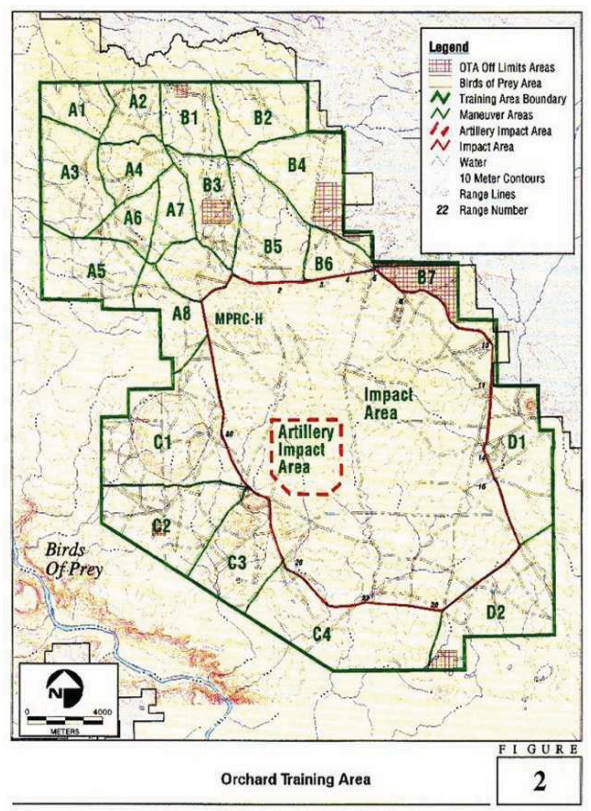

FIGURE 2. Deployment within the Department of Interior Birds of Prey National Conservation Area.

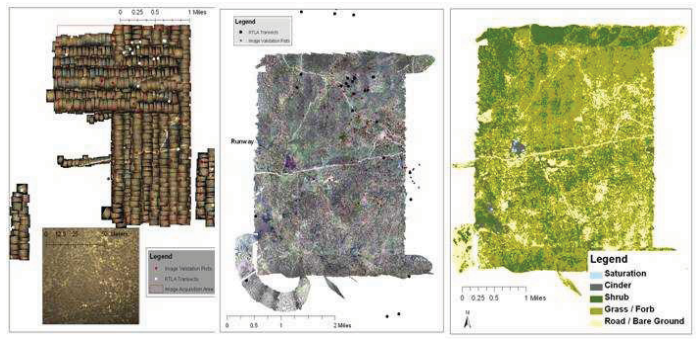

FIGURE 3. OTA RADAS and hyperspectral imagery with resulting classification.

\section{Benefits to DOE}

INL and its partners have successfully demonstrated an affordable and organic (personally owned; easier to acquire data compared to manned or satellite acquisition) remote sensing airborne solution and advanced obstacle avoidance capability. INL is becoming nationally recognized as a viable DOE UAV research center with subject matter experts and resources. Research studies performed within this LDRD have allowed INL to demonstrate how advanced research strategies can open the way for future remote-sensing business opportunities. Specifically, this work is instrumental in providing the foundation for INL's new Collaborative Remote Sensing Program. Additional examples include the following activities accomplished under this LDRD: collaborative efforts through a faculty staff exchange with Idaho State University, a post-doctorate position posting, identification of collaborative research opportunities resulting in a $\$ 1.3 \mathrm{M}$ National Aeronautics and Space Administration Research Opportunities in Space and Earth Sciences proposal and a Higher Education Research Council proposal. Additional examples include a Phase II Small Business Innovation Research project proposal to Resonon Inc. and a collaborative research opportunity with Guided Systems Technology and the Air Force Research Laboratory. 
Relevant Publications and Presentations

Hruska, R. C., M. O. Anderson, N. F. Glenn, and J. J. Mitchell, "In-flight Calibration Experiment for Unmanned Aerial Vehicle (UAV) Acquired Hyperspectral Imagery," IEEE Transactions on Geoscience and Remote Sensing, in preparation.

Mitchell, J., N. Glenn, T. Sankey, D. Derryberry, and M. Germino, "Hyperspectral Remote Sensing of Sagebrush Canopy Nitrogen," Remote Sensing of Environment, in review.

Mitchell, J. J., N. F. Glenn, T. T. Sankey, D. R. Derryberry, M. O. Anderson, and R. C. Hruska, 2012, "Spectroscopic Detection of Nitrogen Concentrations in Sagebrush: Implications for Hyperspectral Remote Sensing," Remote Sensing Letters, Vol. 3, No. 4, pp. 285-294.

Mitchell, J. J., N. F. Glenn, T. T. Sankey, D. R. Derryberry, M. O. Anderson, and R. C. Hruska, 2011, "Small-footprint LiDAR Estimations of Sagebrush Canopy Characteristics," Photogrammetric Engineering \& Remote Sensing, Vol. 77, No. 5, pp. 521-530.

Glenn, N. F., L. Spaete, T. T. Sankey, D. R. Derryberry, S. Hardegree, and J. J. Mitchell, 2011, "Errors in LiDAR-derived Shrub Height and Crown Area on Sloped Terrain," Journal of Arid Environments, Vol. 75, No. 4, pp. 377-382.

Mitchell, J. J., N. F. Glenn, T. T. Sankey, M. O. Anderson, and R. C. Hruska, 2011, "Hyperspectral Remote Sensing of Sagebrush Canopy," Proceedings of the 34th International Symposium of Remote Sensing, Sydney Australia, April 2011.
Mitchell, J. J., N. F. Glenn, M. O. Anderson, and R. C. Hruska, 2011, "HyspIRI Validation with Unmanned Aerial Vehicle Remote Sensing," 2011 HyspIRI NASA Workshop, Washington DC, August 23-25, 2011.

Mitchell, J. J., N. F. Glenn, T. T. Sankey, D. R. Derryberry, M. O. Anderson, and R. C. Hruska, 2010, "Spectroscopic Detection of Nitrogen Concentrations in Sagebrush: Applications in Hyperspectral Remote Sensing," Idaho Section of the Society of Rangeland Management, Winter Meeting, Idaho Falls, ID, November 10-12, 2010.

Anderson, M. O. , D. Wadsworth, J. J. Mitchell, N. F. Glenn, and R. C. Hruska, 2009, "Low Altitude Remote Sensing Program at the Idaho National Laboratory," IEEE NetRob09, Colorado School of Mines, CO, October 2009.

Mitchell, J. J., N. F. Glenn, T. T. Sankey, and D. R. Derryberry, 2009, "LiDAR Canopy Height Measurements in a Sagebrush Steppe Ecosystems," Silvilaser 2009, the 9th International Conference on LiDAR Applications for Assessing Forest Ecosystems, College Station, Texas, October 2009. 


\title{
Addressing the Spectrum of Nuclear-Related Non-Destructive Evaluation Needs
}

\author{
David Hurley, Dennis Kunerth
}

09-090

The development of on-line health monitoring will be critical to extending the lifetime of existing nuclear plants and integral to the design of advanced nuclear reactors. Given that nuclear energy is reemerging as a key alternative to fossil fuel, it is vital that INL develop enhanced capability in this key research area. Accordingly, the aim of this three-year LDRD project was to develop enhanced laser ultrasound and eddy current inspection techniques for eventual on-line health monitoring of nuclear power plants. A large portion of the effort in Years 1 and 2 involved the development of novel eddy current inspection and time domain laser ultrasonic techniques. Work in the third and final year has centered on development of frequency domain laser-based resonant ultrasound spectroscopy (LRUS) for characterizing microstructure-mediated mechanical properties. The enhanced characterization tools developed under this LDRD were geared towards thermophysical property measurement of materials in harsh environments.

\section{Project Summary}

A laser ultrasonic technique to monitor the onset of melting and ablation in metallic materials was demonstrated. This approach relates the changing character of the ultrasonic source with increasing laser energy to the time domain waveform and has diverse applications ranging from localized determination of melting point to laser cleaning of surfaces. For nondestructive evaluation applications, identification of the ablation threshold is important for determination of the onset of material damage.

It was demonstrated that LRUS has the potential to measure the polycrystal average elastic stiffness tensor of materials in harsh environments. This information can be directly related to x-ray measurements of microstructure. We have identified certain instances where LRUS produces incorrect values for the elastic constants. Specifically, it was found that large elastic anisotropy associated with texture gives rise to uniqueness issues. To overcome these difficulties we propose to constrain the inverse problem utilizing additional measurements of the elastic constants using time domain laser ultrasound. These observations are discussed in detail in a manuscript that is being prepared for submission to the Journal of Applied Physics.

It was demonstrated that LRUS can be used to measure the crystallographic orientation of single crystal samples. The crystal orientation is reflected in the symmetry of the LRUS mode shape as shown in Figure 1. The experimental mode shape shown on the right lacks symmetry in the $\mathrm{x}_{2}$ and $\mathrm{x}_{1}$ directions. This mode is compared with two theoretical modes shown on the right of Figure 1. The theoretical mode corresponding to a rotation of the crystal axes relative to the specimen axes shows close agreement with the experimental mode shape. We have executed the inverse problem to find the crystal orientation using the difference in mode shape as the figure of merit. Our results are in close agreement to results obtained using electron backscatter diffraction.

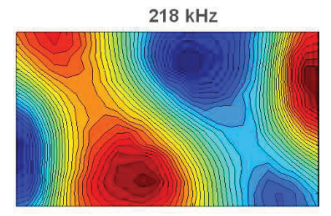

EBSD ( 15 degrees rotation)

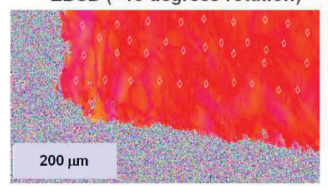

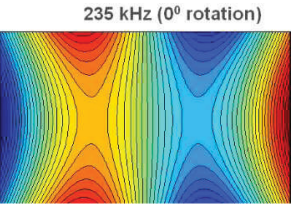

$225 \mathrm{kHz}\left(15^{\circ}\right.$ rotation $)$

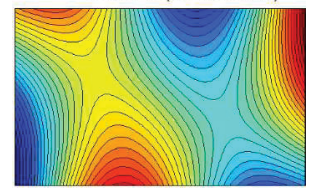

FIGURE 1. Measurement of crystal orientation using laserbased resonant ultrasound spectroscopy. Experimental mode shape (top left) is compared with theoretical mode shape using crystal orientation as a parameter (left). Results agree closely with electron backscatter diffraction measurements (bottom left).

\section{Benefit to DOE}

Nuclear security: This work, involving the development of new nondestructive evaluation tools to monitor material property evolution in harsh environments, directly supports the nuclear energy research mission of the Laboratory. The development of new nondestructive evaluation capability will be an integral part of light water reactor sustainability.

Science: Grain microstructure plays a fundamental role in determining mechanical property evolution of structural metals used in 
advanced nuclear energy systems. The ability to remotely characterize microstructure-mediated mechanical properties of highly radioactive materials using LRUS is crucial to the development of a unified model of deformationdriven mechanical properties.

\section{Relevant Publications and Presentations}

Hurley, D. H., F. Farzbod, and S. J. Reese, 2011, "Application of Laser-based Resonant Ultrasound Spectroscopy to Study Texture in Rolled Copper," in preparation for submission to Journal of Applied Physics.

Farzbod, F., D. H. Hurley, 2011, "Determination of Crystallographic Orientation of Single Crystals using Laser Resonant Ultrasound Spectroscopy," in preparation of submission to IEEE-UFFC.

Utegulov, Z., F. Farzbod, and D. Hurley, 2011, "Localized Determination of Melting Point using Laser Ultrasonics," in preparation for submission to Ultrasonics.
Park, Seung-Kyu, Sung-Hoon Baik, Hyung-Ki Cha, S. J. Reese, and D. H. Hurley, 2010, "Characteristics of a Laser Resonant Ultrasonic Spectroscopy System for Measuring the Elastic Constants of Materials," Journal of the Korean Physical Society, Vol. 57, pp 375.

Hurley, D. H., S. J. Reese, S. Park, Z. Utegulov, J. R. Kennedy, K. Telschow, 2010, “In Situ Laser-Based Resonant Ultrasound Measurements of Microstructure-Mediated Mechanical Property Evolution, Journal of Applied Physics, Vol. 107, pp. 063510.

\section{Intellectual Property}

An invention disclosure record titled "A New Method to Measure Crystallographic Orientation and Texture using Laser Resonant Ultrasound Spectroscopy" was filed with the Technology Deployment Department at INL (tracking \# - BA-639-2059). This new measurement technique compliments $\mathrm{x}$-ray diffraction and electron backscatter diffraction measurements by looking below the surface. 


\section{Water and Energy System Interdependency Modeling for Multi-criteria Decision Analysis}

Robert F. Jeffers, Jacob J. Jacobson

10-065

When planning regional energy

development, complex feedback between various water uses is often inadequately represented. The overall objective of this work is to improve the holistic value of energy development strategies by integrating management criteria for water availability, water quality, and ecosystem health into the energy system planning process. The Snake River Basin in southern Idaho is used as a case study to show options for improving full economic utilization of aquatic resources given multiple scenarios such as changing climate, additional regulations, and increasing population. Through the incorporation of multiple management criteria, potential crosscutting solutions to broad energy and water issues can be developed. The final result of this work will be a simulation environment - usable by policy makers and researchers alike - that will give insight into the behavior of management criteria over time and will allow users to experiment with potential solutions.

\section{Project Summary}

This LDRD has developed a modeling framework for Water and Energy Scenario
Testing (WEST). A proof-of-concept model using the WEST framework has been developed with a system dynamics methodology, which lends itself to collaborative discussions about system behavior and assumptions. WEST is being linked to a web-deployable geographical information system interface, which represents state-of-the-art in the field of system dynamics. The combination of system dynamics and the geographical information system interface will enhance stakeholder group discussion of energy and water issues within a basin.

The proof-of-concept WEST case study focuses on the Snake River Basin, highlighting the effect of variable climate and the feedback between electricity price and agricultural water consumption on power system planning criteria. WEST is built using an object-oriented approach that links sub-models to reflect the dynamic interaction of water and energy components in a basin such as the Snake River Basin. A graphical representation of this system is shown in Figure 1. When linked together, the sub-models form the WEST framework.

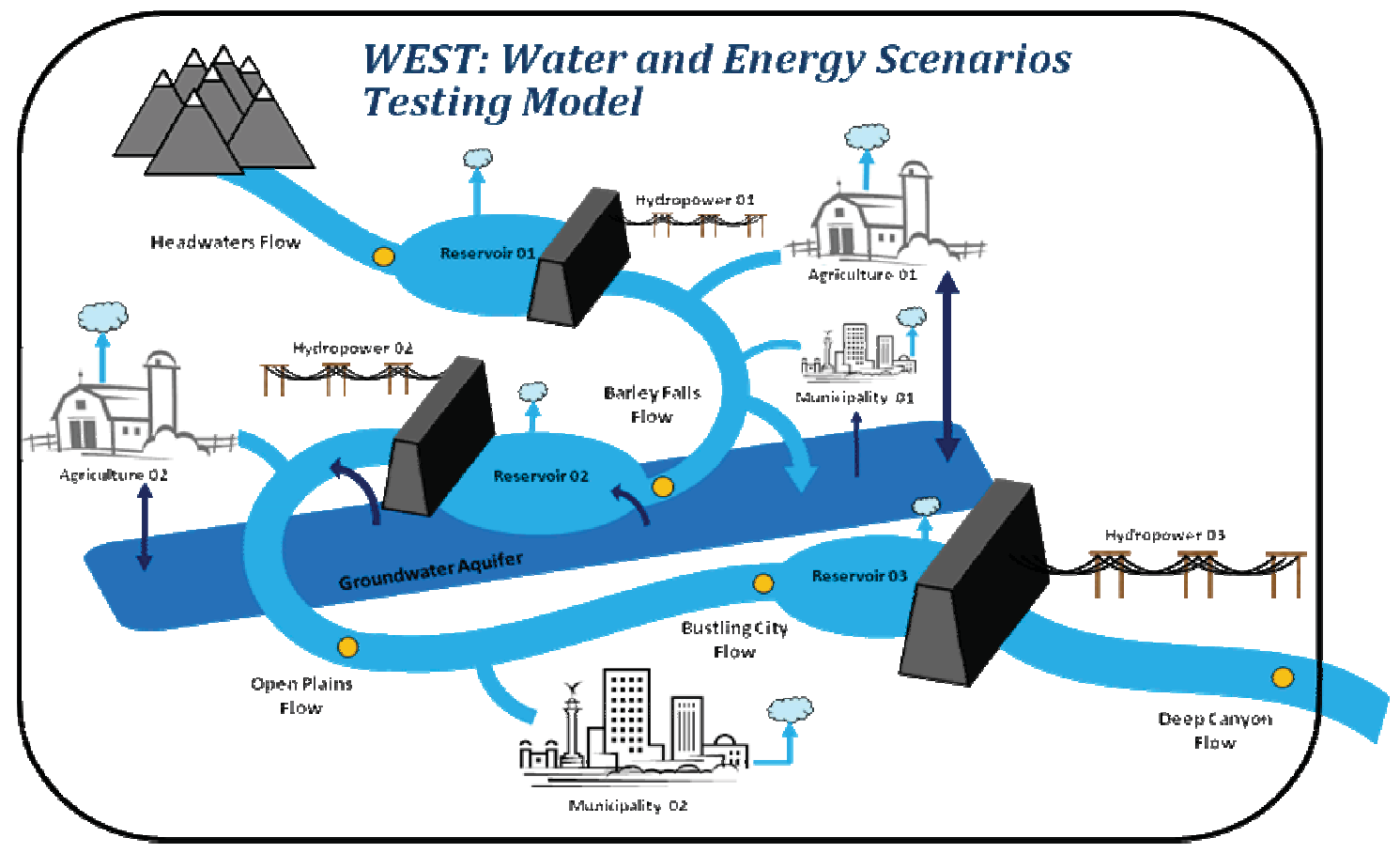

FIGURE 1. Graphical representation of components in the WEST framework. 
While WEST has been designed to simulate the dynamics of water availability, diversion, and consumption, work with Dr. Andrew Ford at Washington State University is linking WEST with the University's electricity market model. This model simulates the response of electricity pricing to changes in water availability and resource prices. Based on modeling scenarios, it is hypothesized that lower prices for electricity will induce higher groundwater pumping in certain months, and eventually decrease surface water available for downstream hydropower, thereby increasing operating costs to the utility and partially negating the lower prices. The strength of this feedback loop and the inherent delays in seeing these effects will be investigated through multiple scenarios. Additional scenarios will test further utilization of the connection between groundwater and surface water to augment flows at opportunistic times for multiple stakeholders.

\section{Benefits to DOE}

A collaborative modeling environment such as WEST, that analyzes and presents complex interactions in a straightforward manner, has numerous benefits to the energy security and environmental responsibility missions of DOE. WEST brings analysts, policy makers, and stakeholders together to create clear and sciencebased energy and water policy for geographically and politically diverse regions. Currently, the project investigators are concentrating on supplementing the integrated resource planning process that private utilities and public utility commissions engage in for holistic projections of electricity resource additions. This will help ensure that regional plans for water and energy development are cohesive instead of conflicting. Eventually, the WEST framework can go beyond the interaction between water and electricity to examine all types of energy development and the status of the resources and ecosystems on which they depend.

\section{Relevant Publications and Presentations}

Jeffers, R. F., J. J. Jacobson, and K. Scott, 2011, "A Framework for Analysis of EnergyWater Interdependency Problems," Proceedings of the $29^{\text {th }}$ International System Dynamics Conference, Washington, DC, July 24-28, 2011.

Jeffers, R. F., and J. J. Jacobson, 2011, “A Framework for Analysis of Energy-Water Interdependency Problems," Western Energy Policy Research Conference, Boise, Idaho, USA, August 25-26, 2011. 


\title{
Degradation and Conversion of Lignin using Extremophilic Systems
}

\author{
W. A. Apel, J. Lacey, B. Lee, D. Newby, D. Reed, D. Thompson, V. Thompson
}

$10-011$

Lignins represent a significant underutilized waste product in the processing of biomass and as such represents a largely untapped resource. The goal of this project is to assess the use of extremophilic systems for the bioconversion of lignin to feedstocks and value-added products. This research seeks to characterize the presence and activity of bacterial lignin degradation systems in acidic and alkaline hot springs by (1) isolating and characterizing microbial isolates from alkaline hot springs in the Alvord Desert (AD) of Oregon and Yellowstone National Park (YNP); (2) characterizing lignin degradation activity by the thermoacidophile Alicyclobacillus acidocaldarius, isolated from a hot spring in YNP; and (3) mining metagenomic data collected from hot springs in the AD and YNP. This research has required an interdisciplinary approach integrating microbiology, genetics, biochemistry, and metabolomics. A combination of these methods is necessary to fully characterize the lignin degradation potential of these extremophilic systems. Completion of this work will lead to the identification and isolation of industrially important thermo-stable bacteria and/or enzymes with applications in converting lignocellulosic compounds to sugars and other chemicals for downstream processes, such as fermentation.

\section{Project Summary}

Isolation and characterization of isolates. Sixteen species have been isolated in the presence of lignin from $\mathrm{AD}$ and $\mathrm{YNP}$ hot springs $\left(60-80^{\circ} \mathrm{C}\right.$, and $\mathrm{pH}$ 3.5-9.0). Screening experiments showed that these isolates can oxidize remazol brilliant blue $\mathrm{R}$ dye, a lignin analog, to varying extents, and three of the isolates were able to grow in medium with kraft lignin as the sole source of organic carbon. Further experiments have characterized the effects of phenolic acids on the growth of four of these strains. To varying degrees, these compounds either inhibited or increased the growth of these strains. Because these phenolic compounds are the building blocks and common degradation products of lignin, positive effects on growth suggest these strains would not be inhibited by the secondary production of these compounds during bioprocesses.
Characterization of A. acidocaldarius. Experimental results suggest that $A$. acidocaldarius may affect lignin to some extent (as measured by optical density at $280 \mathrm{~nm}$ after various levels of filtration). These effects increase significantly in the presence of $100 \mu \mathrm{M}$ copper, suggesting that putative copper oxidases identified in the genome of $A$. acidocaldarius may exhibit activity on lignin. Similarly, the presence of copper enabled $A$. acidocaldarius to degrade inhibitory concentrations of phenolic acids $(1 \mathrm{~g} / \mathrm{L})$ to between 100 and $500 \mathrm{mg} / \mathrm{L}$, allowing more rapid growth of $A$.

acidocaldarius.

Metagenomic Analyses. Samples from AD and YNP hot springs were collected, and metagenomic DNA was extracted and amplified. This DNA was then sequenced by Eurofins MWG|Operon. Bioinformatic tools have been applied to compare this metagenomic data against known databases for enzymes relevant to lignin degradation. These comparisons suggest the presence of several enzymes related to the oxidation of lignin and lignocellulosic compounds, including laccases, amylases, celluloses, hemicelluloses, oxidases, and peroxidases. In addition, a database has been constructed from the metagenomic data against which specific enzymes of interest may be compared.

\section{Benefits to DOE}

This project will continue to develop systems biology expertise at INL. This LDRD project will also expand our expertise in extremophilic enzymes related to the processing of biomass for fuel and chemical feedstocks, which supports the DOE's energy security mission. In addition, the research is supporting a Master of Science student at Montana State University. 


\title{
Innovative Mathematical Morphology Approach to Optical Color Image Analysis and Pattern Recognition
}

\author{
Michael V. Glazoff, John E. Garnier, Sergey N. Rashkeev \\ 11-001
}

Two classes of problems exist in image analysis: (1) extracting useful information from images obtained under continuously varying conditions (weather, illumination), and (2) monitoring known objects/terrain in order to automatically identify the appearance of new "targets" (which could be distorted very strongly as weather and illumination change). The principal goal of this project was to develop a general-purpose platform that would utilize innovative "projectional" morphological algorithms based on filtering with simple geometrical figures for image analysis and feature extraction. The principal advantage of such algorithms is their ability to solve the two types of problems mentioned above. This approach to image and signal analysis is based on the analysis of "scenes of observation," extracting their shapes (i.e., information that inherently belongs to a given image), and analyzing ("projecting") the changed scenes upon such shapes in order to make conclusions about their nature with clearly formulated decision criteria. In such a formulation, our approach, originally developed by Prof. Yuri Pyt'ev, represents a new step in the general field of image analysis (Pyt'ev 1975).

One of the principal objectives of the project was not only to develop such new algorithms, but also to create a designated platform (MorphoHawk) that could be used later for solving many classes of image analysis problems. As such, it could be patented, or trademarked, and licensed to the interested industry and Government customers. Again, the emphasis is upon facilitation of solving two classes of problems - extracting useful information from images obtained under continuously varying conditions (weather, illumination) and monitoring known objects/terrain in order to automatically identify the appearance of new "targets."

"Projectional" mathematical morphology is different from linear integral transforms. It operates with the fundamental concept of image "shape," making it uniquely suitable for analysis of color images acquired under different illumination/weather conditions. Seamless integration of information from images obtained in different spectral ranges can be achieved. Our estimates give processing speed consistently about one order of magnitude faster than Fast Fourier Transform.

Another goal of the project was to expand the development of a morphological approach to pattern recognition for color images using filtering with simple geometrical figures and collapsing all operations of conventional morphological analysis in a single sweep with clearly defined criteria used to drive the algorithms to successful completion. This goes well beyond limitations of point-wise morphological operations introduced by Serra (year), leading to innovative solutions of image analysis problems.

\section{Project Summary}

While elementary morphological operations of erosion, dilation, opening, and closing have been developed for quite some time now (Serra, Maugeron, 1978), the principal problem with such algorithms is in selecting and justifying criteria for feature extraction, classification, pattern recognition, etc. The "projectional" variant of mathematical morphology developed by Prof. Pyt'ev (since 1975) provides researchers with powerful tools for designing criteria according to which a given algorithm must execute and achieve a certain goal. These algorithms are remarkably robust with respect to changes in illumination, angles of observation, time of day/year, and the like. An example of identification of a new object against the backdrop of a well-known scene under different illumination conditions, are presented in Figure 1 (next page). 


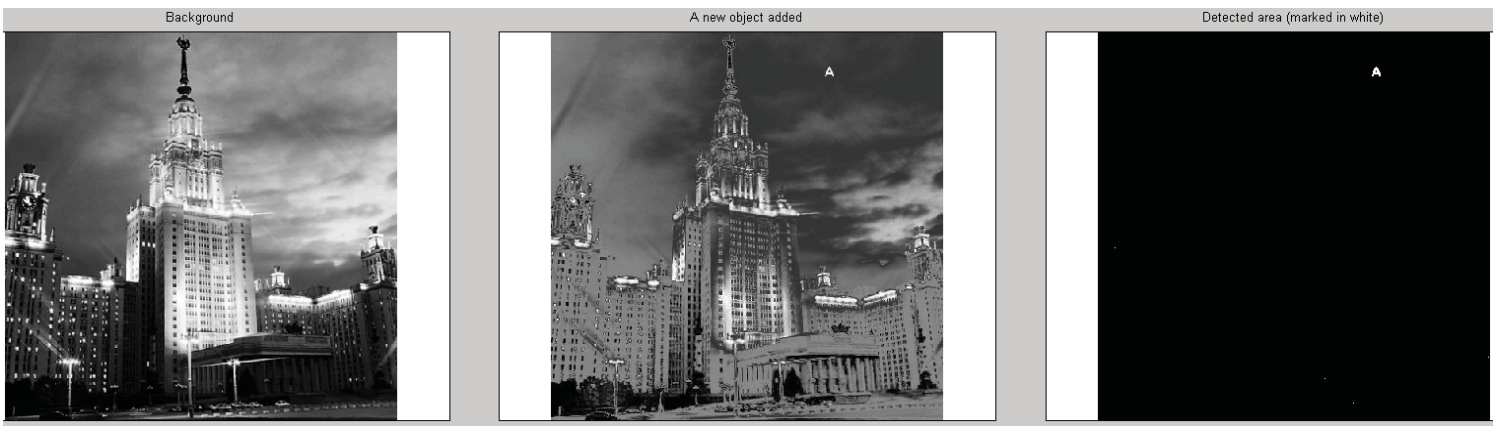

FIGURE 1. Left: photograph of a building; center: same building under different illumination conditions, also note a new object (right top corner); right: extraction of new feature (letter " $A$ ").

\section{Benefits to DOE}

The developed algorithms and a computational platform for projectional morphological analysis could help in solving important problems of extracting information from aerial observations, observations from space, situation monitoring, etc. Additionally, these algorithms are universally applicable in many areas of materials science, geology, chemical and nuclear engineering for extracting and classifying information relevant to properties, for enhanced feature extraction that is remarkably robust with respect to different kinds of noise present in images.

\section{Relevant Publications and Presentations}

Garnier, J. E., M. V. Glazoff, H. Moradi, and S. N. Rashkeev, 2010, "Method for Analysis of Sequential Digitized Electromagnetic Signals," Patent BA-517, IDR \# 1750, June 14, 2010.
Garnier, J. E., M. V. Glazoff, and S. N. Rashkeev, 2010, "Method for Analysis of Objects within Digital Optical Images," Patent BA-503, IDR \# 1820, April 26, 2010.

Garnier, J. E., M. V. Glazoff, and S. N. Rashkeev, 2010, "Method for Analysis of Non-Linear Digitized Electromagnetic Signals," Patent BA-481, IDR \# 1752, March 10, 2010.

Glazoff, M. V., S. N. Rashkeev, Yu.P. Pyt'ev, J.W. Yoon, and S. Sheu, 2009, "Interplay Between Plastic Deformations and Optical Properties of Metal Surfaces: A Multiscale Study," Applied Physics Letters, Vol. 95, No. 8, Article 084106.

Pyt'ev, Yu.P., 1975, "Morphological Notions in Problems of Image Analysis," Reports of the Russian Academy of Sciences, Vol. 224, pp. 1283-1286. 


\section{Network Interaction in the Thermoacidophile Alicyclobacillus Acidocaldarius in Response to Nano Metal Oxides}

Brady Lee

09-025

The physiological response of a microbe to its growth environment is the product of complex interactions between networks of genes, gene operons, proteins, and metabolites. These interactions are especially important during growth on different carbon sources, since the carbon source will govern the physiological response of the microbe to the growth environment. An important process that controls this physiological response is the expression of transcriptional regulators that control operons encoding proteins that catalyze interactions with available carbohydrates in the growth environment. Metabolites or the carbon source themselves will act as regulators or as signals for regulation of expression of many of these operons, a process called carbon catabolite repression (CCR). Of specific interest to this research is CCR of a Gram-positive thermoacidophile capable of growth on carbon sources ranging from simple sugars, such as xylose and glucose, to complex polysaccharides such as cellulose and hemicellulose. Growth on these substrates requires expression and regulation of different genes and operons involved in carbon metabolism. The goal of the proposed research will be to understand carbohydrate-induced differential gene expression patterns by this Gram-positive thermoacidophile during growth on a range of carbon and energy sources. Understanding the effect of CCR on regulation of genes during growth on different carbon sources, and the ability to control this regulation, represents the first step in optimizing growth and/or enzyme production. The research approach will be targeted toward carbohydrate active proteins to explore issues related to utilization of sugar monomers and poly- and oligo-saccharides by this bacterium, with a focus on regulation of gene expression.

\section{Project Summary}

Over the duration of the project, three primary research tasks were performed: (1) bioinformatic analysis of the Gram-positive thermoacidophiles genome for genetic elements associated with CCR; (2) analysis of differential gene expression during growth on a variety of mono-, oligo- and poly-saccharides; and (3) analysis of protein-DNA interactions of CCR elements with genes and operons associated with carbohydrate metabolism by the Gram-positive thermoacidophile.

\section{Genome Analysis:}

An in silico approach was initiated to begin looking at genes and operons related to carbohydrate processing by the Gram-positive thermoacidophile. As part of this task, additional curation and annotation of the genome information was performed. Of specific interest were genes and operons related to metabolisms of mono-, poly-, and oligo-saccharides. When present as part of operons, these genes, as is typical with other Gram-positive bacteria, are present with regulatory, sugar transport, and sugar utilization genes. Numerous glycosyl hydrolase enzymes for the metabolism of polyand oligosaccharides were identified in the genome and are present in operons or as individual genes. Numerous genes were identified within the genome that are common to CCR in other Gram-positive bacteria, specifically repressor proteins such as catabolite control protein A (CcpA), and catabolite responsive elements (cres) for binding of CcpA. Interestingly, many sugar transporters encoded in the genome of the Gram-positive

thermoacidophile, specifically those for glucose and xylose, are different than the types of sugar transporters found in other Gram-positive bacteria that exhibit classical CCR. These results may indicate that the Gram-positive thermoacidophile may be able to grow metabolizing multiple sugars at the same time.

\section{Experimental Analysis of CCR:}

Chemostat experiments were run to determine CCR in the Gram-positive thermoacidophile being studied when grown on mixtures of sugars and on model cellulose and hemicellulose compounds and sugars. Continuous-flow chemostats were operated at steady-state to monitor differential gene expression when the Gram-positive thermoacidophile was grown on mixtures of xylose/glucose, xylose/arabinose, or fructose/glucose. Chemostat cultures were grown to steady-state on the first sugar, and then the second sugar was added to potentially stimulate CCR. Results from these experiments indicate 
that upon addition of the second sugar, use of the first sugar dropped off initially, but then both sugars were used simultaneously as the culture returned to steady-state operation. While CCR was apparent initially, biomass in the reactors increased, indicating the culture was using the sugars simultaneously for growth. Samples for RNA extraction, cDNA synthesis, and microarray analysis were taken upon steady-state growth on the first sugar, after the addition of the second sugar, and then once the culture returned to steady-state growth on the mixture of two sugars.

Batch chemostats were used to determine the effect of xylose and glucose on induction of CCR in the Gram-positive thermoacidophile when grown on the model cellulose and hemicellulose compounds, wheat starch and wheat arabinoxylan. Cultures were grown to mid-log phase and the inducer sugar was added. Growth rates increased substantially when the inducer sugar was added, indicating that the metabolism shifted for growth on the monomer sugar, which in turn indicated that CCR may have been in effect. Samples were taken for RNA extraction, cDNA synthesis and microarray analysis just before and just after addition of the inducer sugar.

Forty-eight microarray experiments were performed using the cDNA generated from the chemostat experiments described above. cDNA was sent to Roche NimbleGen, labeled with a fluorescent dye and hybridized to microarrays manufactured with probes for each gene from the Gram-positive thermoacidophiles genome, and analyzed. Data from these analyses are currently being analyzed using a microarray bioinformatic pipeline. Results from the bioinformatic analysis are expected to produce two publications: one for the monomer sugar research and one for CCR related to cellulose and hemicellulose model compounds.

\section{Network Interaction:}

An additional task to look at protein-nucleic acid interactions related to CCR was added to the project during the year. The protein-nucleic acid interactions will be determined using electrophoretic mobility shift assays and DNase footprinting studies, for which materials have been purchased. Genome analysis has revealed two copies of many genes encoding many of the elements related to CCR. A Brevibacillus expression system has been chosen to express the recombinant proteins that will be used during these studies. Results from this research are expected to produce one additional publication.

\section{Benefits to DOE}

The proposed research will develop expertise in systems biology at INL. Specifically, the project will expand our expertise in transcriptome analysis through the use of highdensity microarrays and also develop expertise in the area of protein-DNA interaction analysis. This expertise will then be available for projects relating to all aspects of microbiology, including bioenergy, environmental, homeland security, and even medical microbiology. The project has also lead to interactions with local and regional universities, specifically Idaho State University, as well as the Institute for Systems Biology, which is affiliated with the University of Washington. This LDRD project represents the basis for a doctoral dissertation for the principal investigator. 


\section{Specific Biological Responses to Nano Metal Oxides}

James R. Henriksen, Robert Fox, Yoshiko Fujita, Kevin Feris

10-076

Nanoparticles (NPs) are researched, developed, and manufactured by several DOE national laboratories, and industrial use of NPs is growing rapidly. Along with this increased use comes the potential for accidental or weathering release to the environment. A diverse array of synthetic NPs exists, but little is known about their environmental impacts. There is a need for both monitoring and screening technology and an improved understanding of the potential toxicity, transport, and fate of NPs in natural environments. The purpose of this project is to evaluate biological responses to and interactions with engineered $\mathrm{TiO}_{2} \mathrm{NPs}\left(\mathrm{nTiO}_{2}\right)$. We hypothesize that the formation of intracellular reactive oxygen species (ROS) is a common mechanism underlying the response of biological systems to metal oxide NPs. The white-rot fungus Phanerochaete chrysosporium and the soil bacterium Pseudomonas putida are model systems with environmental relevance that provide molecular tools, known responses to ROS and metals, initial evidence of NP effects, and unique biological characteristics to test these responses. The comparison of these responses will provide the scientific underpinnings for novel methods to detect bio-available NPs in complex environmental backgrounds, and identify characteristics and concentrations that indicate potential (eco)-toxicity. The characteristics of the NP following interaction with the biological cultures will also be evaluated in order to better understand the potential impacts on their subsequent environmental fate and transport.

\section{Project Summary}

Experiments with the white-rot fungus Phanerochaete chrysosporium indicated spore germination and growth occur even at high NP loadings $(5 \mathrm{mg} / \mathrm{ml})$. The lignolytic activity of white-rot fungi is important in many environments. In the lignolytic state, $P$. chrysosporium produces the enzyme lignin peroxidase. Experiments measuring lignin peroxidase activity over a range of NP concentrations show that this extracellular activity is inactivated by high NP loadings. The inactivation of this enzyme has implications for the environmental impact of $\mathrm{nTiO}_{2}$ and $\mathrm{nTiO}_{2}$ treated wood products.
Fungi are highly useful in biosorption applications because of the surface charge and microstructural characteristics of their chitin exteriors, their coatings of extracellular polymers, and their filter-like hyphae macrostructure. Spectral, dynamic light scattering sizing and scanning electron microscopy data indicates that sorption of NPs by fungal biomass completely removes up to $0.05 \mathrm{mg} / \mathrm{ml} \mathrm{nTiO}_{2}$ agglomerates from growth media within 3 days and up to $5 \mathrm{mg} / \mathrm{ml}$ removal over a period of weeks. In addition, dynamic light scattering sizing and spectral measurements indicate that this sorption material can be released into media that stabilizes NP, but that they are also released in an aggregated form, even into media where NP are usually partially dispersed. This has implications on the fate and transport of NP in the environment, and applications in the use of inexpensive fungal biomass in sorbtion applications.

A series of experiments have been performed to quantify genetic and physiological responses in $P$. putida across a range of nanoscale (35-nm diameter) anatase $\mathrm{nTiO}_{2}$ concentrations ( 0 to $5 \mathrm{mg} / \mathrm{ml}$ ) with concomitant exposure to a full spectrum light source to stimulate ROS production by the $\mathrm{nTiO}_{2}$ under conditions reflective of a natural environment. Genetic and physiological responses were assessed after a 20 -hour exposure regime under controlled temperature and light conditions. Results suggest a quantifiable and integrated response between cell physiology and gene expression.

Specifically, viability of the metal-resistant P. putida KT2440 was not affected by any of the NP exposure concentrations, but cell size and gene expression patterns were significantly, differentially, and non-linearly affected by high and low concentrations of NPs. Cell size (e.g., length, diameter, surface area, and volume) was significantly different with NP exposure relative to controls $(\mathrm{p}<0.05)$. A dose-response dependent production of a yellow compound with fluorescence spectra similar to that published for pyoverdine was observed. Pyoverdine is a Pseudomonad siderophore that binds many metals and is associated with iron limitation and heavy metal exposure, and has a 
possible link to ROS. Effects of NP and light exposure on gene expression were determined for a subset of genes previously described in $P$. putida to commonly be up-regulated during exposure to ROS and/or heavy metals that can induce ROS production. Significant levels of up-regulation were observed for a small set of the gene screened; these include $\operatorname{sod} B$ (i.e., superoxide dismutase $\mathrm{B}$ ) and $z n t A$. $\operatorname{sod} B$ is one of two superoxide dismutase encoding genes in $P$. putida and zntA encodes a well-described heavy metal translocating type II ATPase. $f t z K$ and fin $R$ were also up-regulated representing a cell division response and enhanced expression of a global response regulator putatively associated with a global ROS-stress response in $P$. putida, respectively. These data support the rapidly accumulating body of data that suggest nanoscale NPs induce toxic cellular effects via ROS production. They also demonstrate the variability in toxicity of $\mathrm{nTiO}_{2}$ to microorganisms and indicate the potential resistance of environmentally relevant organisms to this class of novel environmental toxins. The specific response to NPs indicates a potential for a biodetector of active, bioavailable NPs.

\section{Benefits to DOE}

We have provided several benefits to DOE by establishing a research program that will provide valuable information to industry and government for an improved understanding of the potential toxicity, transport, and fate of NPs to the environment. This work supports the
"Environmental Impacts of Energy" goal of the Energy Security theme. To speed adoption of nanomaterials developed for energy uses (such as fuel cells, catalysts, in unconventional oil recovery, or in hydrogen storage), their safety must be ascertained. This work supports the environmental quality goal by increasing the understanding of the toxicology, fate, and transport of materials by providing information and tools to guide research and regulation in the developing field of the environmental impacts of nanomaterials. This work supports the BER objective to increase understanding of environmental contaminant fate and transport, the BES Materials Sciences and Engineering Division focus on nano- and biomolecular materials. DOE benefits from the establishment of research in this area of environmental, health, and safety impacts of NP, as these NP impacts are a funding priority of the cabinet-level National Science and Technology Council.

\section{Relevant Publications and Presentations}

Henriksen, J. R., 2010, "Nano-Chemical \& Micro-Biological Diversity," Invited presentation, Center for the Environmental Implications of NanoTechnology, Duke University, Raleigh-Durham, North Carolina, USA, March 12, 2010. 


\section{Development of Non-Lethal Methods for Enhanced Lipid Recovery from Microalgae}

Deborah T. Newby, Brady Lee, Lance Seefeldt

10-078

This LDRD was designed to serve as the nucleation point to add an algal component to INL's renewable energy/alternative fuels portfolio. The focus is on the development of an array of non-lethal methods for enhanced lipid recovery from algae. Physicochemical milking and molecular/systems biology approaches to understanding algal lipid metabolism and excretion are being developed and employed. The end objective of this work is to exploit physiological responses of microalgae to stress/shock conditions and/or biocompatible solvents in order to improve the economic feasibility of production and recovery of lipids from microalgae.

\section{Project Summary}

During FY 2011, we developed additional expertise in the growth of a variety of algal
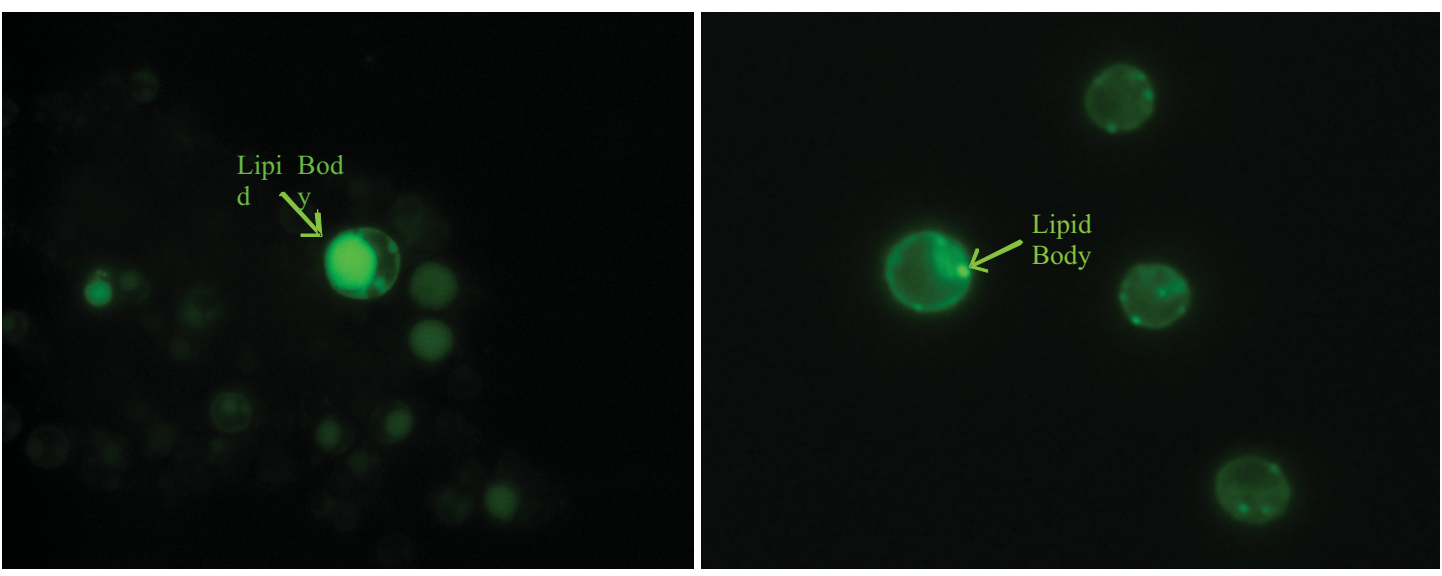

FIGURE 1. Algal cells stained with Nile Red, showing increased amounts of lipid on Day 3 (left) vs. Day 10 (right).

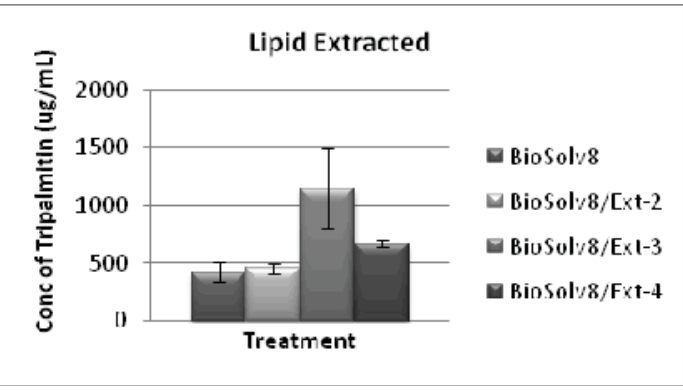

FIGURE 2. Quantity of a representative lipid (tripalmitin) extracted from C. gracilis with sonication and gas chromatography/flame ionization analysis.

This year, the investment from Year 1 in the metagenome sequence of a culture enriched for strains, in understanding lipid development within algal cells, and in analytical techniques for lipid analysis including fluorescence staining (Nile Red) and gas chromatography/flame ionization detection. Figure 1 shows increasing amounts of lipid over time. In addition, we tested a variety of shock (e.g., salt, temperature, light), and biocompatible solvents $(>10)$ were assessed to determine their ability to non-lethally remove lipids. Results obtained from the studies with the biocompatible solvents showed the greatest promise. A two-phase system to recover lipids from actively growing algal cultures using biocompatible solvents and both with and without a sonication step was developed. Figure 2 shows the amount of lipid extracted from a lipid-producing diatom, Chaetoceros gracilis, using a biocompatible solvent used in combination with different extractants.

Chaetoceros gracilis, a high-lipid-producing alga, that also contained sequences from the alga's bacterial consortia has produced a data resource, provided a foundation for transcriptomic studies, established expertise in cutting-edge metagenomic analysis, and has lead to important insights in algal biofuels applications. We have achieved a targeted genome annotation, and built capabilities for future work. This metagenomic information enables genetic and expression analysis of Chaetoceros gracilis and is a significant contribution to the algal biofuels field.

Significant effort this fiscal year has been spent developing and executing tools to utilize 
this valuable resource for a genomic approach that will support this and future algal biofuels projects. A metagenomic analysis pipeline to identify and annotate the algal sequence was developed for this analysis, utilizing INL's High Performance Computing resources. The pipeline includes metagenome quality analysis, taxonomic classification and binning by homology and nucleotide composition, extraction of sequences for annotation pipelines, and merging and displaying these various sources of information. This bioinformatics infrastructure is a valuable new capability for all biological research projects at INL and collaborating institutions. The annotation of this binned metagenome was performed using the DOE Joint Genome Institute's Integrated Microbial Genomes/Microbiome Samples Expert Review system as well as advanced bioinformatic methods for target gene annotation and identification implemented at INL, further developing our capabilities. This enriched metagenomic approach has provided information that will be essential in the characterization of algal biofuel ponds.

The associated bacteria and their capabilities were identified by RNA and other phylogenetic genes, combined with homology and binning information. Three bacterial genera dominated the algae-associated consortia: Algoriphagus/Hongiella, Sulfitobacter, and Porphyrobacter. Similar organisms have been found in association with other natural algal assemblages. Approximately 3,000 functional genes were identified for each genera, and initial physiological predictions based on phylogeny and these gene functions indicate mechanisms by which these organisms could effect, both positively and negatively, the growth of algae and the production of biofuels.

Algal sequences were overrepresented in long scaffolds, many over a megabase, for a total of 39 megabases of algal genomic information. Over 41,000 protein coding genes were identified in the scaffolds of 45 million base pairs. The sequence of genes of interest, including hundreds involved in algal lipid synthesis pathways, were identified, and will be used in subsequent transcriptomic analyses. In preparation for transcriptomic experiments, an
RNA extraction protocol using a RNeasy Mini Kit and a QIAshredder (Qiagen) with the plant protocol has been optimized, and pilot differential display growth experiments have been conducted. Due to the analysis of the metagenomic sequence, we are able to carry out either the original microarray-based transcriptomics or use high-throughput sequencing for a new RNA-seq transcriptomics that provides deeper resolution, expanded detection, and cheaper analysis of differential expression experiments.

Benefits to DOE

Algae have great promise as a platform for economical, domestic, low-carbon, low-landimpact, low-water-impact liquid biofuel. However, to be viable in the energy marketplace, algae will need to be grown in open-air pools, a major challenge to realizing theoretical yields and economics. By increasing our understanding of biofuel relevant algae and their natural consortia, we have enabled the DOE's mission to secure a sustainable energy future. In addition, data we have collected may support mission involving water (both related to remediation and to toxic algal blooms) and $\mathrm{CO}_{2}$ capture in algal dominated ecosystems.

Additional algal projects stemming from this work include two projects funded from DOE's Office of Biomass Programs, one looking at algal feedstock logistics and the other at algal dewatering using cross-flow filtration. It is also anticipated that a cooperative research and development agreement focused on addressing technical issues related to algal biofuels will be executed. We also continue to build strong regional and national laboratory partners in the algal biofuels arena.

The focus on separation of algal lipids has combined with another LDRD investment and lead to the filing of nine inventions disclosure records for applications involving both switchable solvents and algal lipid extraction.

\section{Relevant Publications and Presentations}

At least two publications are expected to be generated from this LDRD. It is anticipated that one will be focused on the use of biocompatible 
solvents for lipid milking and the other on genomic/transcriptomic analysis of C. gracilis.

Henriksen, J. R., 2011, "Metagenomic Analysis:

From Reading DNA to Environmental Understanding," Invited presentation, Second Annual CAES Workshop on Modeling, Simulation, and Visualization, Boise, Idaho, USA, September 9, 2011.

Newby, D. T., B. D. Lee, J. R. Henriksen, J. Ellis, and R. Simmons, 2011, "Targeted Genomic Analysis of Chaetoceros gracilis," Poster presentation, Algae Biomass Summit of the Algal Biomass Organization, Minneapolis, Minnesota, USA, October 25-27, 2011. 


\section{Development of Thermally Generated In Situ Precipitation Barriers due to Subsurface Heat Injection}

Earl Mattson, Mitch Plummer, Carl D. Palmer, Jeff Sondrup

10-090

The primary technical objective of this project is to understand how barriers may develop in fractured and porous media where anthropogenic heating is taking place. In the context of energy-water studies, this project focuses on in situ oil shale retorting, where establishment of barriers could effectively limit water ingress into an oil shale retort. Such barriers can be formed by the injection of polymers/gels or may form as a result of mineral precipitation due to concentration of solutes from evaporation of groundwater. The feasibility of forming polymer barriers is explored by determining the thermal stability of several potential compounds that have been identified. To evaluate thermally induced in situ precipitation, we evaluated conditions necessary to create mineral deposition events through geochemical simulations of evaporation and laboratory validation experiments. While the proposed study focuses on in situ oil shale retorting, the capabilities developed through this study would also be applicable to engineered geothermal systems, water injection into steam geothermal reservoirs, nuclear heat storage in the subsurface, nuclear waste depository storage, and environmental remediation methods that require large inputs of energy to the subsurface. Better understanding of other heat/water evaporation systems (i.e., scaling systems, near surface evaporation of ground water) could also benefit from capabilities developed through this study.

\section{Project Summary}

We have conducted polymer stability tests over a range of temperatures expected near the edge of an in situ retort. The use of polymers as conformance control agents at low temperature and low salinity is quite common and many polymers are commercially available for this purpose. However, the temperature profile developed from an in situ oil shale retort will have a very steep gradient and will move outward with time. Little data exists to judge polymer performance for this moving boundary and its effect on injected polymer long-term thermal stability. Five water control agents were selected for thermal stability tests. Two of these samples are commercial polymer products, one is a lab-synthesized polymer, and the other two are commercial sodium silicate products. Four temperature settings will be used for long-term evaluation: $25^{\circ} \mathrm{C}, 60^{\circ} \mathrm{C}, 120^{\circ} \mathrm{C}$, and $180^{\circ} \mathrm{C}$, which represent conditions of expected stability to that of decomposition. These samples were prepared in an anaerobic glove box with a nitrogen atmosphere, sealed in small bottles, and stored in an oven for two months. Stability of the conformance control agents was observed by measuring the viscosity weekly to determine if degradation had occurred.

We have conducted geochemical modeling of groundwater in the Piceance Basin to better understand how the groundwater chemistry will evolve during heating. The PHREEQC interactive code with the llnl.dat thermodynamic database was used to make the calculations. The initial water chemistries are based on reported values found in available reports and water quality databases. Since no mention is made in published reports concerning $\mathrm{pH}$ measurement, we assume that they are either laboratory values or were measured in the field without the use of flow-thru cells. This assumption would suggest that the $\mathrm{pH}$ values are high relative to the actual in situ values due to $\mathrm{CO}_{2}$ loss. We therefore calculated the $\mathrm{pH}$ based on assumption of equilibrium with calcite, a mineral commonly reported throughout the stratigraphic section. We calculated the mineral saturation states of this groundwater and how its chemistry might evolve upon in situ heating.

An experimental apparatus was designed and built to evaluate the development of a precipitation barrier as a function of the temperature gradient and flux. A fracture was created between two blocks of oil shale, instrumented with thermocouples, strain measurement devices, and pressure transducers. A flow was established through the fracture and the system was heated to $80-120^{\circ} \mathrm{C}$ under stress. Permeability changes, determined by the changes in the pressure heads, will be correlated to temperature measurements.

Benefits to DOE

This project directly addresses issues associated with the development of clean energy resources for the nation. Results of this study are useful not only in evaluating barriers to reduce water ingress into oil shale retort zones, thereby 
decreasing heating costs and minimizing environmental impact to groundwater, but also for evaluation of barriers in engineered geothermal systems, nuclear heat subsurface storage, injection into steam geothermal systems, and in situ coal gasification. Experimental capabilities developed through this proposal would enhance our high-temperature/highpressure permeability measurement abilities that we are currently using for oil shale and shale gas. The development of both of these capabilities would help in expanding collaborations with our colleagues in the Western Energy Corridor and the Mountain West Water Institute.

\section{Relevant Publications and Presentations}

Mattson, E. D., C. D. Palmer, R. W. Perkins, 2010, "Potential Geochemical Releases to Groundwater from an in-situ Oil Shale Retort," Groundwater Protection Council, Pittsburgh, PA, September 29, 2010.
Mattson, E., E. Kara, J. Boak, C. D. Palmer, 2010, "Water Consumption Analysis for an In-Situ Oil Shale Retort," Geological Society of America Annual Meeting, Denver, CO, October 31-November 3, 2010.

Palmer, C. D., J. Little, E. D. Mattson, 2011, "Geochemical Evolution of Piceance Basin Groundwater During Heating," 2011 Oil Shale Symposium, Colorado School of Mines, Golden, CO, Oct. 17-21, 2011.

Mattson, E. D., 2011, “Conformance Control for In-Situ Oil Shale Retorting," Oil Shale Symposium, Colorado School of Mines, Golden, CO, October 17-21, 2011. 


\section{Hybrid Osmosis Water Purification Systems Research}

Frederick F. Stewart, Mark Stone, Aaron Wilson

10-092

Considering the decreasing supplies of available fresh water and the ever-increasing demand on those limited resources, there is a need to create a high-efficiency water purification system that is adaptable to varying feeds, such that nearly any water-containing stream can be dewatered. In traditional single-pass membrane water purification systems, feed quality and content plays a significant role in the practicality of the system. What is proposed in this work is a dual system that uses a combination of forward osmosis and ultrafiltration to create a system that is relatively insensitive to the applied feed stream. Thus, it is envisioned that a system can be constructed that will dewater varied feed streams including saline or organics that tend to foul current technologies. Forward osmosis systems will be integrated with ultrafiltration technology to determine the practicality of the concept. If successful, this technology will represent a significant advance in the state-of-the-art in that the system will be specifically engineered to avoid many of the downfalls of current membrane technology, most specifically fouling and concentration polarization. The novel aspects include the integration of mature technologies (i.e., reverse osmosis and ultrafiltration) with an exciting newer technology - forward osmosis, which itself will be enabled by this project.

\section{Project Summary}

Forward osmosis has two enabling aspects for successful operation: membranes and draw solutions. Forward osmosis is a liquid/liquid separation process that is facilitated through an osmotic pressure gradient that provides the driving force for water transport. Membranes are typically microporous reverse osmosis membranes. Draw solutions, to which water is drawn from the feed, is usually some type of salt solution. Numerous studies are reported using either sodium or magnesium chloride. The disadvantage of these un-engineered salts is that they readily back diffuse from the draw through the membrane and into the feed, which results in a loss of efficiency needed for a continuous process. Additionally, the salts must be removed from the water in a second step using reverse osmosis, which can be costly.
New salt solutions have been developed during this project. These materials were designed using a dendrimeric motif. To a central core structure was appended anionic groups that yielded fully water-soluble materials. In solution, these salts create significant ionic pressure. However, unlike simple sodium and magnesium chloride, these engineered salts, because of their relatively large size exhibit little to no back diffusion. Further, their size will facilitate liberation of water from the draw solution. All new salts have been studied against commercial membranes obtained from Hydration Technology, Inc. (HTI). HTI membranes consist of porous cellulose acetate, which has limited chemical stability. The new salts have demonstrated good stability against HTI membranes where little membrane degradation has been detected. These new salts and their use as forward osmosis draw solutes are the subject of a U.S. patent application that will be filed in the first quarter of FY 2012.

\section{Benefits to DOE}

A new capability for water purification has been developed at INL, which has the promise of being valuable to DOE and other government agencies for a variety of water purification applications, including agriculture, industrial manufacturing, energy production, and domestic use. DOE is tasked with safeguarding energy security, which is known to be inextricably linked to water security. Water security addresses supply concerns and this project directly addresses the utilization and recycling of water. Another potential application for this technology includes produced water, which is both an energy and environmental concern. Thus, this project is of great benefit to DOE and other governmental agencies that are involved in water resource management. 


\section{Biological Fixation of $\mathrm{CO}_{2}$ for Fuel and Chemical Production using Acetogen Intermediary Metabolism}

William A. Apel, Brady Lee, Hope Lee

10-087

Acetogenic bacteria possess flexible metabolisms allowing the use of many different substrates for catabolic and anabolic processes. Of particular interest to this project is their ability to convert $\mathrm{CO}_{2}$ to acetyl-CoA via the Wood/Ljungdahl pathway. Acetyl-CoA is a versatile intermediate metabolite that may serve as a precursor for the synthesis of different biomolecules, or may be oxidized for energy - $\mathrm{a}$ process that produces acetate. Other potential compounds produced by acetogens are pyruvate, butyrate, propionate, formate, syn-gases, ethanol, and butanol. As such, they are excellent targets for development of a biological system that can be optimized for the conversion of atmospheric $\mathrm{CO}_{2}$ into value-added products. Laying the groundwork for the development of this system is the overall goal of this effort. Specifically, this project consists of three synergistic efforts to: (a) analyze metagenomic data from thermal hot springs from the Alvord Desert of Oregon (AD) and Yellowstone National Park (YNP);

(b) develop and apply a metabolic model for the known acetogen, Moorella thermoacetica; and (c) isolate and characterize novel species or strains from hot spring environments from the $\mathrm{AD}$ and $\mathrm{YNP}$.

\section{Project Summary}

Metagenomic Analyses-Metagenomic data from five hot springs in YNP were obtained through a joint collaboration with the Thermal Biology Institute at Montana State University. In addition, metagenomic data from an $\mathrm{AD}$ hot spring and two additional YNP hot springs were made available. These sequence data were trimmed and assembled into contigs. This data has been assembled into a unique database, against which known enzymes related to the fixation of $\mathrm{CO}_{2}$ may be compared. In this way, we will develop a profile of carbon fixation genes and enzymes from multiple hot springs environments. This information will identify enzymes and genes of commercial interest and inform future research with respect to geochemical and environmental controls of carbon fixation.
Metabolic Modeling-A metabolic flux analysis model has been developed for the metabolism of M. thermoacetica. This model is designed to predict the flow of material and energy through this acetogenic metabolism as a function of substrate and pathway availability. Comparisons with experimental data show that this model can successfully predict carbon distribution between biomass and multiple metabolites under different nutrient conditions. In addition, the model was applied to predict nutrient conditions that optimized ethanol production by $M$. thermoacetica.

Isolation and Characterization of Novel Organisms - Environmental samples were collected from three hot springs (A, B, and M) from the $\mathrm{AD}$ and four hot springs (GS, $\mathrm{EG}, \mathrm{BC}$, and EA) from the Norris Geyser Basin in YNP. The temperatures of these environments range from $40-80^{\circ} \mathrm{C}$, and the pHs vary between 3.5 and 9.0. Aliquots from these samples have been enriched under acetogenic conditions, and metabolomic analyses have shown a shift towards acetate and ethanol production correlated with $\mathrm{CO}_{2}$ and $\mathrm{H}_{2}$ consumption. Isolation efforts have yielded two species, and efforts are continuing to elucidate additional species. The 16S genes from these species will be sequenced and compared against NCBI databases to either identify them or indicate them to be novel organisms. Any novel organisms will be characterized with respect to their ability to convert $\mathrm{CO}_{2}$ to value-added products, and a "best candidate" will be sequenced. This new sequence will represent a source of information and a template for further model development.

\section{Benefits to DOE}

This research further expanded INL's expertise in the area of stable enzymes for use in extreme industrial and environmental conditions. The work also supported the DOE theme "promoting America's energy security through reliable, clean, and affordable energy" via the development of unique, stable enzymes for converting biomass to fuels and value-added 
compounds that could displace petroleum usage. Furthermore, this research is directly applicable to the goals of the Office of Science, one of which is to better understand biological conversion processes at a fundamental level, and to the Office of Energy Efficiency and

Renewable Energy, which is developing the use of enzymatic/biological processes for the conversion of biomass to fuels and value-added chemicals. 


\title{
Electrochemical Production of Synthesis Gas from $\mathrm{CO}_{2}$
}

\author{
Tedd E. Lister, Eric J. Dufek
}

11-019

Electrochemical reduction of $\mathrm{CO}_{2}$ is a technology that could integrate intermittent alternative energy sources into the electric grid by using excess energy to create a storable energy product. Electrochemical reduction of $\mathrm{CO}_{2}$ has been demonstrated for many years and is capable of generating high product yields of reduced carbon species using moderate conditions. This LDRD project involves developing the technology for electrochemical reduction of $\mathrm{CO}_{2}$ to syn-gas or simple hydrocarbon products. This project is positioned within the carbon-to-chemicals thrust of the Science and Technology directorate. It hopes to serve as a source of reduced feedstock for follow-on synthetic pathways to commodity chemicals. The INL effort is focused on developing systems reported in lab-scale studies into working bench-scale and eventually pilotscale processes. The challenge for this work is to perform the electrolysis with the minimum energy input and produce a useful product stream at high throughput. The project has added a postdoctoral candidate to help the effort. The eventual goal of this project is to develop a cohesive system that, if successful, could become a pilot-scale operation for the Hybrid Energy Systems Testing facility.

\section{Project Summary}

The completion of ongoing experiments using the ambient pressure flow cell was completed. This activity was initiated the previous year. This work resulted in three accepted peer-reviewed publications and a fourth in preparation. Two well-received presentations were made at the $220^{\text {th }}$ Electrochemical Society Meeting in Boston. The papers investigated the use of Ag gas diffusion electrodes to perform $\mathrm{CO}_{2}$ reduction at throughput densities higher than previously reported. The effect of sulfur contamination was investigated due to the possibility of S-contamination in $\mathrm{CO}_{2}$ feedstock streams. This resulted in a paper that described exaggerated poisoning as being mostly reversible. The effect of different membrane and electrolyte combinations was also investigated where use of anion exchange membranes increased electrolyte longevity. A paper describing this work was recently accepted for publication. A novel process that unites $\mathrm{CO}_{2}$ reduction with an anodic process has resulted in the submission of a joint invention disclosure record with Olin Corporation. A full proposal with Olin as an industrial partner has been submitted to the 2012 Energy Efficiency and Renewable Energy Innovative Manufacturing Funding Opportunity Announcement. To further increase throughput, a high-pressure electrolysis system was built using a cell procured from Giner Electrochemical Systems (GES). The system has been successfully operated with current densities up to $350 \mathrm{~mA} / \mathrm{cm}^{2}$ with up to $80 \%$ CO production. We have also partnered with GES in a proposal to develop a syn-gas generation system for the Air Force through a small business technology transfer. The eventual goal is to develop an efficient method path to synthetic aviation fuel. This was recently awarded to Giner, and INL will support GES through testing and evaluation. Should the initial work show progress, additional development funding is anticipated.

\section{Benefits to DOE}

This project has initiated an effort at INL in developing electricity-based routes to production of hydrocarbon fuels and products from $\mathrm{CO}_{2}$. This project fits into a larger effort to develop technologies that use low value energy to transform $\mathrm{CO}_{2}$ into hydrocarbon products. This project addresses issues of integrating the vast renewable energy resources of the Western Inland Energy Corridor, which possesses many natural energy resources but has limited grid connections to transport electricity to larger population areas. Generation of hydrocarbonbased fuels during periods of low-cost power could act as a load level during periods of high power output or low customer usage. Thus this project could be a part of a group of technologies to reduce U.S. dependence on foreign oil imports. Early results have been presented at an international conference and a paper describing novel results is in preparation.

\section{Relevant Publications and Presentations}

Dufek, E. J., T. E. Lister, and M. E. McIlwain, 2012, "Influence of Electrolytes and Membranes on Cell Operation for Syn-Gas Production," Electrochem. Solid-State Lett, in press. 
Dufek, E. J., T. E. Lister, and M. E. McIlwain, 2011, "Influence of S Contamination on $\mathrm{CO}_{2}$, Reduction at Ag Electrodes," J Electrochem Soc, Vol. 158, B1384.

Dufek, E. J., T. E. Lister, and M. E. McIlwain, 2011, "Bench-Scale Electrochemical System for Generation of CO and Syn-Gas," J Appl Electrochem, Vol. 41, p. 623.

Dufek, E. J., T. E. Lister, and M. E. McIlwain, 2011, "Investigation of $\mathrm{CO}_{2}-\mathrm{H}_{2} \mathrm{O}$ Reduction Scale-up: Production of Syn-Gas Mixtures," Poster at the Electrochemical Energy Summit, $220^{\text {th }}$ Electrochemical Society Meeting, Boston, MA, Oct 9-11, 2011.
Dufek, E. J., T. E. Lister, and M. E. McIlwain, 2011, "Implications Associated with S Contamination for the Production of SynGas from $\mathrm{CO}_{2}$ Reduction," Electrochemical Society Meeting, Boston, MA, October 11, 2011.

Dufek, E. J., T. E. Lister, and M. E. McIlwain, 2010, "Bench-Scale System for Electrochemical Reduction of $\mathrm{CO}_{2}$," Future Direction in $\mathrm{CO}_{2}$ Conversion Chemistry Workshop, Princeton University, Princeton, NJ, October 31, 2010. 


\section{Multiscale Coupled Hydrogeophysics Modeling: Advancing Understanding of Reactive Transport and Effective Subsurface Environmental Monitoring}

Hai Huang, Chuan Lu; Tim Johnson (Pacific Northwest National Laboratory)

11-045

An increased understanding of complex resistivity signals, in terms of the fundamental properties of geochemical and geophysical processes, would improve study and monitoring of reactive transport. To that end, this project is developing a parallel, fully coupled, multiscale, reactive transport and complex resistivity model that works at both pore and continuum scales by unifying a reactive transport simulator called RAT (Reactive Transport), a pore-scale complex conductivity predictor, and a $3 \mathrm{D}$ complex resistivity code, SIP3D, into the MOOSE framework. Specifically, we seek to (1) develop a finite-element-based 3D pore-scale complex resistivity model and implement the model within the MOOSE framework for a better understanding of the relationships between volume-averaged complex resistivity signals and pore-scale biogeochemical reactions, (2) couple INL's reactive transport modeling code RAT with INL's complex resistivity tomography code for field-scale, effective monitoring of contaminant plume migration, and (3) prepare manuscripts for peer-reviewed journal publications.

\section{Project Summary}

At the beginning of FY 2011, co-principal investigator Tim Johnson moved to Pacific Northwest National Laboratory; thus, we changed the research focus during FY 2011 for this two-year project to couple INL's reactive transport code RAT with the complex resistivity code written by Tim Johnson. We plan to focus on Objective 1 (above) during FY 2012. During FY 2011, the main research accomplishment was to meet Objective 2 .

Within FY 2011, we developed a methodology for time-elapse electric resistivity tomograph (ERT) data inversion that provides real-time effective monitoring of contaminant plume migration. This method utilizes the inversion results for the previous time step as the initial guess and model constraint for the next time step inversion. The continuum scale 3D ERT inversion code FERM3D (developed by Timothy Johnson) has been modified accordingly. According to our numerical experiments, the new methodology greatly enhances the convergence, performance, and stability of FERM3D.
Besides methodology and code development, we simultaneously applied the coupled ERT/RAT codes to investigate the possibility of using ERT as a monitoring tool for $\mathrm{CO}_{2}$ geological sequestration, as an example application of the coupled hydrogeophysics model. Figures 1-3 (next page) show some simulation results.
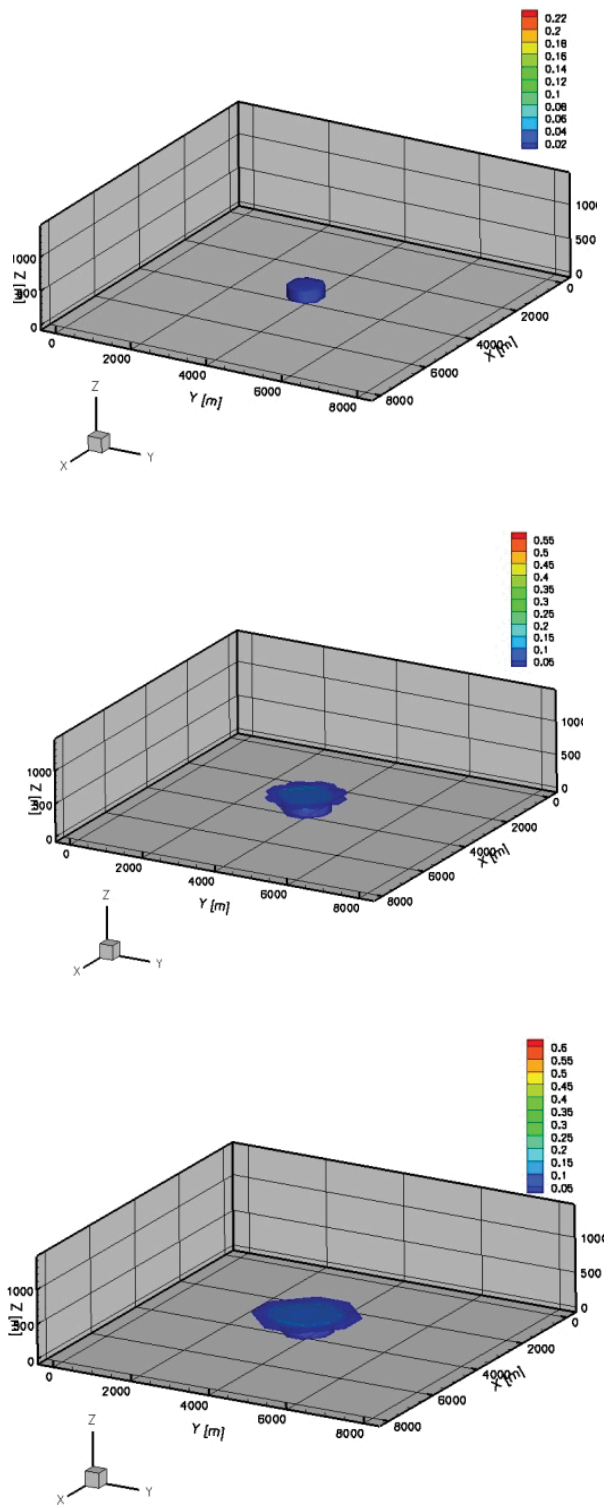

FIGURE 1. Simulated $\mathrm{CO}_{2}$ plume migration in a deep saline reservoir at 1, 3, and 10 yrs. 


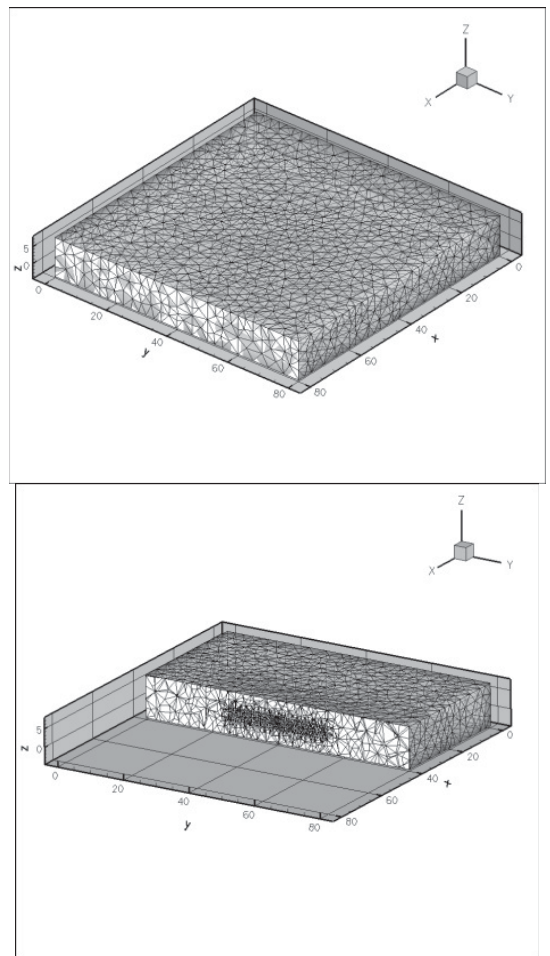

FIGURE 2. Finite-element grid used in ERT forward and inversion calculation using TETGEN.

ERT inversion calculations were calculated with FERM3D. The number and locations of electrodes were adjusted to obtain optimal results. The results in Figure 3 showed quantitative agreements. They validate the methodology and implementation.

The ERT inversions on this synthetic case study demonstrate the potential capability of time-lapse ERT as an effective monitoring tool for $\mathrm{CO}_{2}$ geological storage. Besides the widely used seismic method, another geophysical method will be introduced and recognized by the $\mathrm{CO}_{2}$ capture and storage community. One manuscript is in preparation for publication in International Journal of Greenhouse Gas Control.

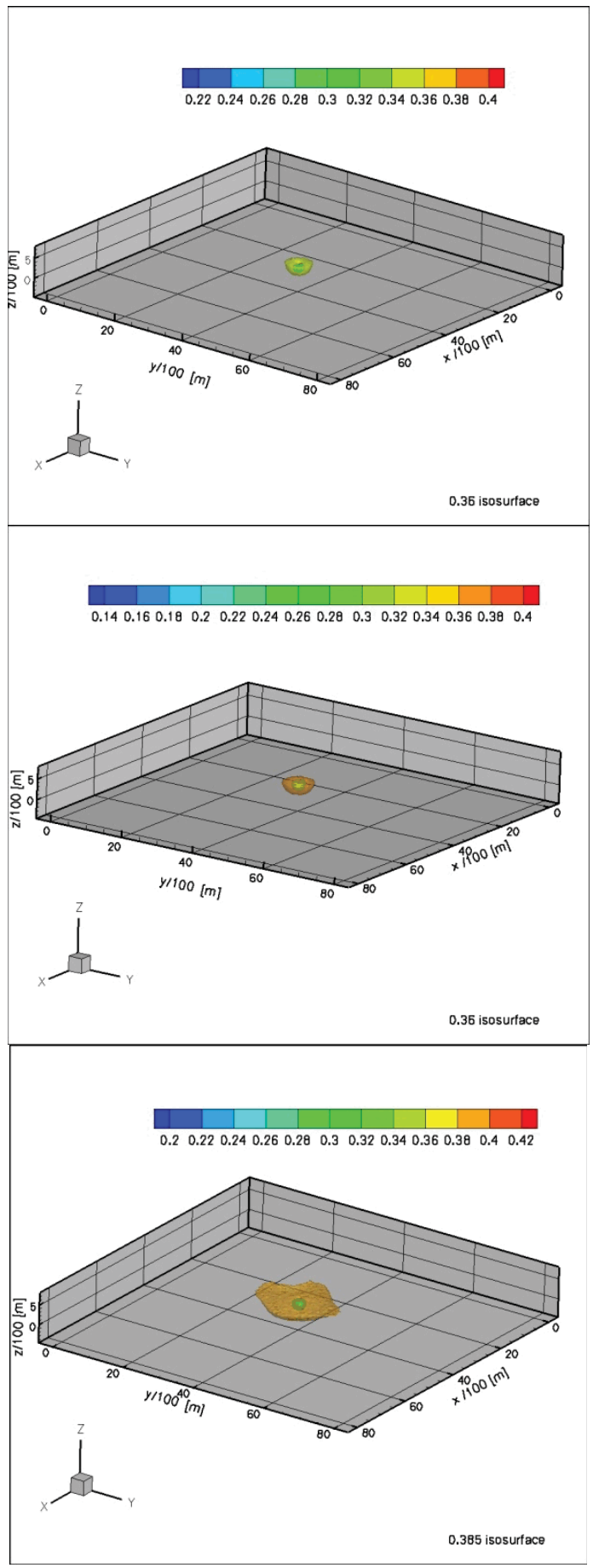

FIGURE 3. Inverted electric conductivity fields from ERT inversion showing the growth and migration of the $\mathrm{CO}_{2}$ plume. 
Benefits to DOE

This project will increase DOE's understanding of reactive transport by introducing a novel modeling capability. We have coupled a reactive transport model with ERT/SIP (spectral induced polarization) inversion model, developed the capabilities to perform time-lapse ERT inversion, and numerically assessed using time-lapse ERT inversion as monitoring method, using $\mathrm{CO}_{2}$ capture and storage as an example. This novel introduction of ERT inversion to the $\mathrm{CO}_{2}$ capture and storage community could guide INL's subsurface modeling group into this frontline research field. Work in FY 2012 will be more focused on the pore-scale reactive transport process and volume-averaged complex resistivity; we will perform coupled continuumscale reactive transport and complex resistivity simulations under conditions similar to INL's Science Focus Area program experiments for model validation calibration. 


\section{Mechanisms of Self Inhibition of Catalytic Conversion of $\mathrm{CO}_{2}$ to Methanol \\ Daniel M. Ginosar, Lucia M. Petkovic, Harry W. Rollins, Rao Kondamudi, Nicholas E. McGuire \\ 11-031}

The ability to convert significant quantities of carbon dioxide $\left(\mathrm{CO}_{2}\right)$ directly to methanol and higher-chain hydrocarbons is limited by $\mathrm{CO}_{2}$ self inhibition on catalyst kinetics. To realize the capability of the Carbon to Chemicals program, new catalysts need to be developed that are not inhibited by high concentrations of $\mathrm{CO}_{2}$. The significance of this proposed research project is to develop the theory that identifies the nanoscale morphology/self inhibition of $\mathrm{CO}_{2}$ on methanol/hydrocarbon production and to use this theory to enable a significant increase in $\mathrm{CO}_{2}$ conversion to methanol/hydrocarbons.

\section{Project Summary}

In FY 2010, to achieve the above goals, numerous catalysts were synthesized. To understand the effect of $\mathrm{CO}_{2}$ on methanol synthesis, two different morphologies of the same catalyst material were synthesized (Fig. 1).

The physicochemical properties of the materials were characterized using various techniques available at INL. To understand the $\mathrm{CO}_{2}$ effect on hydrocarbon synthesis a series of zirconium-based catalysts were synthesized using a surfactant-assisted method, as shown in Figure 2.
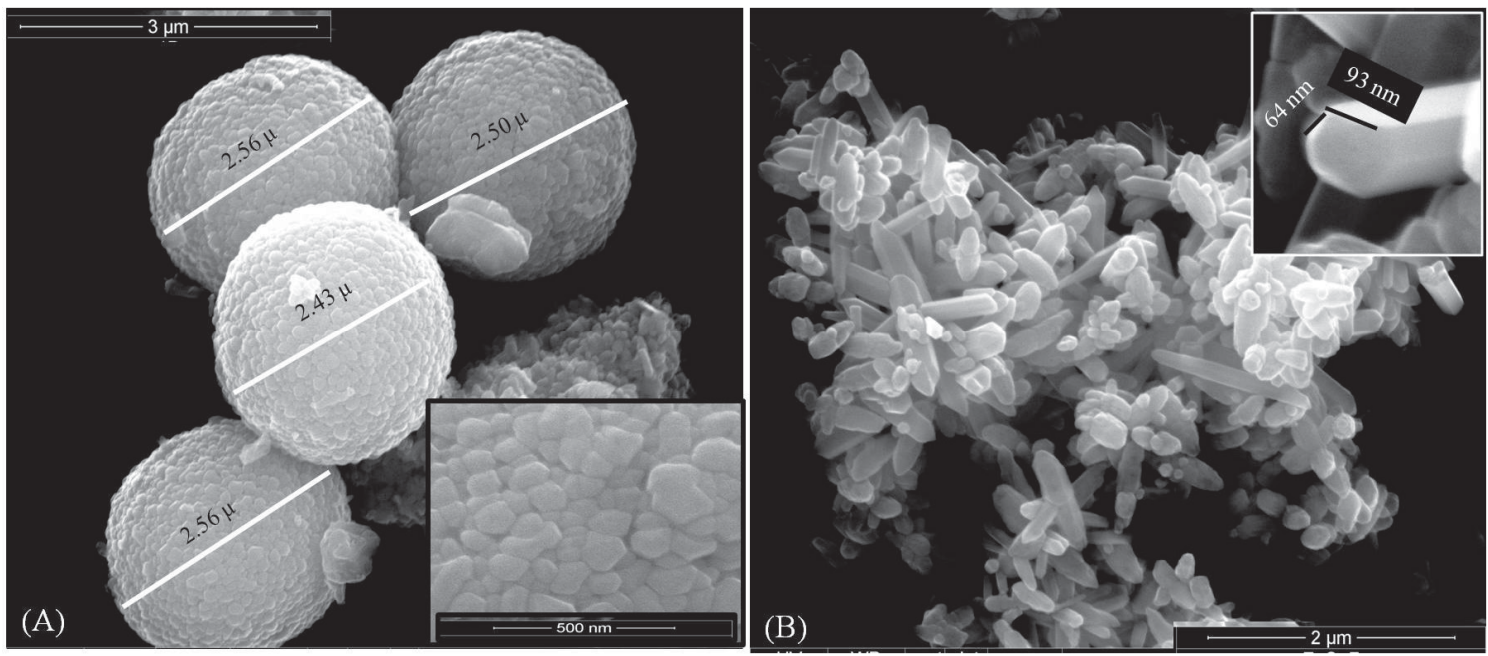

FIGURE 1. Spherical (A) and rod-like (B) morphologies of methanol synthesis catalysts. in the catalysis research team as an important step in building a sustainable critical mass of catalysis researchers. The successful deployment of the project outcome will reduce the carbon footprint and help reduce the nation's foreign-oil dependency.

\section{Relevant Publications and Presentations}

McGuire, N. E., N. Kondamudi, L. M. Petkovic, and D. M. Ginosar, 2012, "Effect of Lanthanide Promoters on Zirconia-Based Isosynthesis Catalysts Prepared by Surfactant-Assisted Coprecipitation," in preparation for submission to Applied Catalysis $A$.

McGuire, N. E., N. Kondamudi, L. M. Petkovic, and D. M. Ginosar, 2011, "Effect of Modifiers on Zirconia-Based Catalysts for Isosynthesis," American Institute of Chemical Engineers Annual Meeting, Minneapolis, Minnesota, USA, October 1621, 2011.

\section{Benefits to DOE}

Successful completion of this project will INL 

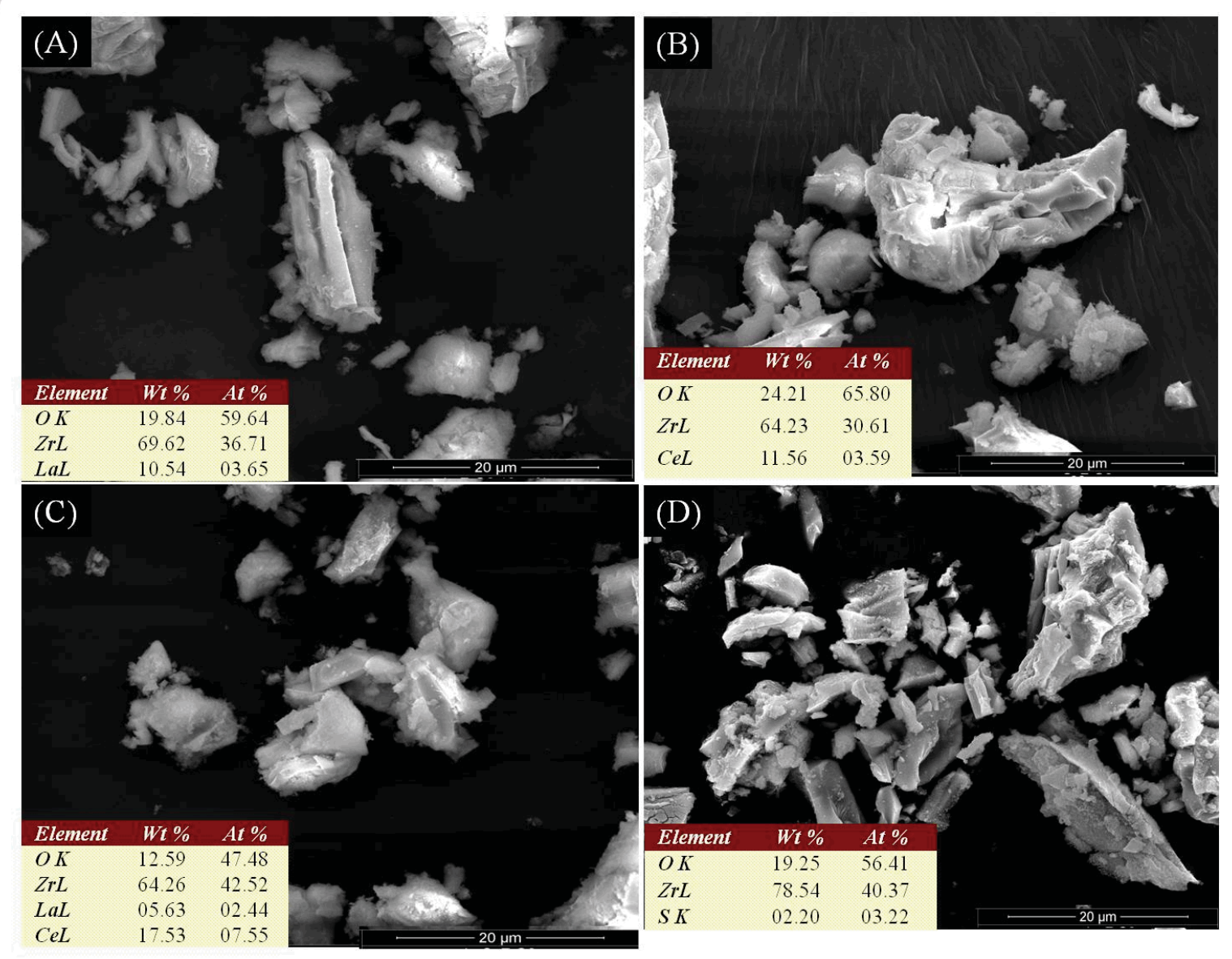

FIGURE 2. Scanning electron microscope images of isosynthesis (carbon to hydrocarbon) catalysts. (Energy-dispersive x-ray analyses are given in the inset for each catalyst) 


\section{Dynamic Thermo-Mechanical Energy Conversion Simulation Diana K. Grauer, Michael E. Reed 11-064}

This project creates a new type of model by hybridizing and integrating previously developed modeling tools. The project is a joint effort between the Energy Efficiency \& Industrial Technology and the Advanced Processes \& Decision Systems departments in support of the INL Hybrid Energy Systems(HES) program under the auspices of the INL Energy Security Initiative. The intent of this research is to further the understanding of hybrid energy system dynamics through development of multi-physics dynamic modeling tools, including reduced order models for controls integration.

These tools can then be integrated into cocurrently developed dynamic fundamental process and economic evaluation tools to inform energy system-wide dynamic interaction and decision making. This work will create a dynamic prime mover engine model assimilated into an Aspen Custom Modeler interface, allowing for the detailed analysis of unit and sub-unit operations. The research team will first create a 1D fluid-dynamic model to capture the spatial and temporal distribution of temperature, pressure, and density in the air delivery and exhaust removal systems created by the incylinder combustion event. This 1D model will create a framework and provide boundary conditions for a 3D multi-physics computational fluid dynamics in-cylinder combustion model. These two models will then be coupled into a hybrid 1D/3D prime mover engine model.

Concurrently, an Aspen Custom Modeler interface will be created to provide a mechanism for integrating the dynamic information created by these models into co-currently developed conceptual design dynamic Aspen Custom Modeler models. The discovery of operating conditions and anomalies that contribute to declining reliability and catastrophic failure of prime mover engines is one application of this hybrid model. Applications of this model will include a variety of prime mover operating conditions and industrial applications to comprehensively investigate the integration of this technology into highly coupled, interdependent unit and sub-unit operations. Portions of this work are fundamental research conducted for the purpose of publishing in the open literature.
The objective of the research is to create a dynamic multi-physics prime mover engine model capable of interfacing with reduced order models and other tools that have been previously developed as part of the HES toolbox. These tools are identified in the HES Systems Analysis, Integration, Monitoring and Control Platform including economic evaluation and life-cycle assessments, and dynamic and steady state models for fundamental chemical and thermal processes.

\section{Project Summary}

In FY 2011, the research team successfully completed the framework for the geometrically flexible 1D fluid-dynamic fuel design model in order to capture the spatial and temporal distribution of temperature, pressure, and density in the air delivery and exhaust removal systems created by the in-cylinder combustion event. Concurrently, an Aspen Custom Modeler conceptual design interface was also completed to provide a mechanism for integrating the dynamic information created by this model into co-currently developed conceptual design dynamic Aspen Custom Modeler models. The discovery of operating conditions and anomalies that contribute to declining reliability and catastrophic failure of prime mover engines is one application of this hybrid model.

At the conclusion of the project, the dynamic prime mover engine model will also have the capability to implement informed changes in technology operation and management by:

1. Interfacing directly with previously developed steady-state chemical/thermal process models and economic evaluations.

2. Building a baseline for reliable operation of prime mover engines and identify changes in operation to ensure continued reliable operation for extended life-times.

3. Identifying needed retrofit technology (e.g., sensors and controls) that must to be developed.

4. Focusing and identifying next steps for the HES Systems Analysis, Integration, Monitoring and Control Platform. 
Finally, the prime mover engine model will be validated with engine operating data collected by operators of this industrial equipment. This information has been made available in the literature as operating companies have struggled to understand the dynamic operation of their engines while retrofitting for environmental compliance.

\section{Benefits to DOE}

This project directly contributes to the DOE Energy Security theme by creating a simulation framework and set of tools to "address domestic energy security through...increasing reliability and productivity," "strengthen the systems that transmit and distribute electricity and fuels to consumers," and "enhance the security and reliability of the energy infrastructure." Our primary goal is to create a means to interface directly with previously developed steady-state chemical/thermal process models, concurrently developed dynamic conceptual models, and economic evaluations; build a baseline for reliable operation of prime mover engines and identify changes in operation to ensure continued reliable operation for extended life-times; and identify needed retrofit technology (e.g., sensors and controls) that must to be developed to continue to reliably operate prime mover engines.

Within the DOE Energy Security theme, interfacing with the (EPA) to "accelerate deployment of energy efficient technologies" is explicitly identified. The Environmental Protection Agency has recently targeted prime mover engines as major sources of pollutant emissions through the promulgation of National Ambient Air Quality Standards and National Emission Standards for Hazardous Air Pollutants. These regulatory changes have forced prime mover engines to operate outside their design envelope to maintain compliance, thereby decreasing reliability. The prime mover engine modeling tool will allow for the analysis of controlled operation and identification of needed technologies to resolve this conflict between reliability and environmental compliance.

The DOE Scientific Discovery and Innovation theme overtly emphasizes Research Integration as a mechanism to "accelerate innovation and create transformational solutions for energy." The process of creating the prime mover engine modeling tool will create an environment for hybridization and integration of existing technologies and modeling tools at INL. This will allow INL to accelerate its analysis of energy-system dynamics and develop leadership in the area of dynamic thermo-mechanical energy conversion.

As discussed previously with regard to the DOE Energy Mission and Energy Security theme, it is important that we continue to develop technologies that maintain and "enhance the security and reliability of the energy infrastructure." One goal is to create a tool to evaluate the security and reliability of prime mover engines used in electricity and fuels transmission as a part of the larger U.S. energy infrastructure.

INL will directly benefit from this research by increased modeling and simulation capability for general application to the Hybrid Energy Systems Modeling and Simulation platform. Specifically, the research team will create a new class of fused models for the assessment of dynamic highly coupled, interdependent unit and sub-unit operations identified by the HES program. This research places INL in a position to become a world leader in the areas of dynamic thermo-mechanical energy conversion and internal combustion engine simulation. Finally, this work places the research team in a position to compete for follow on direct funded research. Future proposals for continuation of this research would be directed at the Department of Defense, who have recently published calls for advanced soldier portable power technologies (Broad Agency Announcement, U.S. Army Research, Development and Engineering Command), and waste to energy converters for overseas contingency operations (Strategic Environmental Research and Development Program, FY 2012), Energy Conservation Applications for the U.S. Navy (Broad Agency Announcement, Naval Surface Warfare Center, Carderock Division). Also, the research will facilitate proposals to and collaborations with industry research consortiums such as the Pipeline Research Council International, Inc. and Gas Machinery Research Council; various private and industrial 
firms (e.g., ADAGE, Cameron, Caterpillar); and DOE's Office of Science and Industrial Technologies Program.

\section{Relevant Publications and Presentations}

Grauer, D. K., and M. E. Reed, “Operational Energy for Domestic Installation Energy Islanding: Using Prime Mover Engines to Balance Dynamic Wind Turbine Output," Energy, submitted.

Grauer, D. K., and M. E. Reed, "Electrical Power Grid Delivery Dynamic Analysis: Using Prime Mover Engines to Balance Dynamic Wind Turbine Output," American Society of Mechanical Engineers Journal of Engineering for Gas Turbines and Power, submitted.

Grauer, D. K., and M. E. Reed. 2011, "Electrical Power Grid Delivery Dynamic Analysis: Using Prime Mover Engines to Balance Dynamic Wind Turbine Output," Proceedings of the American Society of Mechanical Engineers International Mechanical Engineering Congress and Expo, IMECE2011-62232, Denver, CO, November 11-17, 2011, accepted.

Grauer, D. K., and M. E. Reed, 2011, “Dynamic Analysis of Electrical Power Grid Delivery: Using Prime Mover Engines to Balance Dynamic Wind Turbine Output," Proceedings of the American Society of Mechanical Engineers International Mechanical Engineering Congress and Expo, IMECE2011-62232, October 2-5, 2011, accepted.
Reed, M. E., and D. K. Grauer, 2011, "Dynamic Analysis of Prime Mover Combined Heat and Power Delivery to Chemical Processing," Proceedings of the American Society of Mechanical Engineers Internal Combustion Engine Division Conference, ICEF2011-60169, October 2-5, 2011, accepted.

Grauer, D. K., and M. E. Reed, 2011, "Electrical Power Grid Delivery Dynamic Analysis: Using Prime Mover Engines to Balance Dynamic Wind Turbine Output," American Society of Mechanical Engineers International Mechanical Engineering Congress and Expo, IMECE2011-62232, Denver, CO, November 11-17, 2011, accepted.

Grauer, D. K., and M.E. Reed, 2011, “Dynamic Analysis of Electrical Power Grid Delivery: Using Prime Mover Engines to Balance Dynamic Wind Turbine Output," American Society of Mechanical Engineers Internal Combustion Engine Division Conference, ICEF2011-60170, October 2-5, 2011, accepted.

Reed, M. E., and D. K. Grauer, 2011, “Dynamic Analysis of Prime Mover Combined Heat and Power Delivery to Chemical Processing," American Society of Mechanical Engineers Internal Combustion Engine Division Conference, ICEF201160169, October 2-5, 2011, accepted. 


\section{Reduction and Retention of Technetium Using Fe(II) Hydroxides}

Sarah Pepper, Dean Peterman

11-033

This LDRD project builds on a substantial amount of prior research performed by the principal investigator concerning the reactivity and oxidation/reduction (redox) chemistry of ferric $\left(\mathrm{Fe}^{2+}\right)$ hydroxides. The objective of this project is to control the transport of technetium in soils and ground water using the redox chemistry of ferric hydroxides. Since technetium 99 is a long lived radioisotope produced by nuclear fission and found in high concentrations in used fuel $(\sim 6 \%)$ long term management of used fuel requires immobilization of the technetium to retard release to the environment. Once technetium is released, the $\mathrm{TcO}_{4}^{-2}$ species is highly stable and soluble in water under most conditions. This project seeks to determine if a mixture of ferric and ferrous hydroxide containing a variety of anionic species, collectively called green rust, has the reactivity to covert $\mathrm{TcO}_{4}^{-2}$ to $\mathrm{TcO}_{2}$ (an insoluble species). Once converted, the other components contained in the green rust will combine with the $\mathrm{TcO}_{2}$ so that it cannot reenter the environment. To achieve this objective, the research explores and attempts to define a range of chemical variables that control the conversion and subsequent capture of technetium.

\section{Project Summary}

To effectively study green rust chemistry, the combination of iron hydroxides must be prepared in a controlled environment where oxygen is excluded, since the slight presence of oxygen will oxidize $\mathrm{Fe}^{2+}$ to $\mathrm{Fe}^{3+}$ and destroys the redox chemistry of the green rust. Tc 99 is a long lived beta emitting radioisotope and must be controlled according to accepted radiological practices. The initial approach was to prepare the green rust in a controlled atmosphere glove box located in one of the radiochemistry labs at CFA 625. Prior to this project, the glove box had been extensively modified from its original design to accommodate various spectroscopic and electrochemical measurement associated with an actinide chemistry project. Repeated attempts to maintain an inert environment in this glove box failed due to the numerous modifications made to the glove box. Given the limited functionality of the glove box, experiments were conducted to measure the short term reactivity and capture of technetium by green rust. Although some reactivity was observed, the eventual oxidation of the green rust did not permit analyzable materials and samples to be recovered and analyzed.

Since green rust is oxidized to goethite by contact with air, the reactivity of technetium with goethite and magnetite were investigated in place of the green rust. Experiments were conducted using either goethite or magnetite, various concentrations of sodium chloride, and $\mathrm{pH}$. A variety of concentrations of technetium were tested with sodium chloride concentration varying from $0.25 \mathrm{M}$ to $1.0 \mathrm{M}$ and $\mathrm{pH}$ varied between 4 and 9 . Over all these variables, no capture or conversion of Tc was observed indicating that the reduced iron species was necessary.

Unfortunately, an inert environment able to maintain green rust and employ the radioactive Tc species was not achieved. Other efforts to obtain an inert environment were deemed to be unacceptable so the work had to be terminated without conclusive results to prove or disprove the hypothesis that green rust was effective in reducing $\mathrm{TcO}_{4}{ }^{2-}$ to $\mathrm{TcO}_{2}$ and capturing the $\mathrm{TcO}_{2}$ in an insoluble matrix.

\section{Benefits to DOE}

Investigating the environmental impacts of energy generation is part of Strategic Theme 1 of the DOE's Strategic Plan. The strategies for meeting this goal include creation of new nuclear generation capacity, which must include managing nuclear waste. Long term disposal of used nuclear fuel and wastes associated with reprocessing must be concerned with limiting the release of mobile technetium species. Green rust offers an approach to restrict the mobility of technetium species and could serve as a tool in controlling the release of radionuclides from interim and long-term storage of used nuclear fuel.

Investigations into methods for the safe sequestration of technetium are, therefore, critical to dealing the nation's legacy and future nuclear waste. 


\section{Ammonia-based Extraction Methods for the Separation and Purification of Metals}

Harry W. Rollins, Mark D. Ogden

$11-032$

The separation and purification of metals from complex mixtures, such as electronic waste and low-grade mineral ores, is becoming increasingly important as global demand increases and viable reserves decrease. Currently identified "critical" metals include gallium, indium, platinum group metals, and rare earths. Presently, liquid-liquid extraction is the preferred technology for production of these metals. However, this results in the generation of large quantities of liquid waste streams with associated hazards and disposal problems.

The objective of this project was to develop a supercritical fluid extraction methodology using ammonia as the basis of the supercritical phase $\left(\mathrm{SC} \mathrm{NH}_{3}\right)$. The initial developmental focus was to extract and separate electronically relevant transition metals: cobalt, nickel, and copper. Once the methodology was developed, it was applied to the extraction of "critical" metals.

\section{Project Summary}

Supercritical fluid extraction of transition metal nitrates was found to be highly effective. Copper, nickel, and cobalt nitrates were found to be particularly soluble in ammonia at moderate temperatures and pressures. When the supercritical fluid extraction method was applied to the rare earth elements (such as europium and lanthanum), we found that the corresponding anions were extracted and the corresponding metal oxide and oxy-hydroxide species were formed. The following equation shows the reaction for ammonia extraction of lanthanum phosphate:

$\mathrm{Ln}\left(\mathrm{PO}_{4}\right)+3 \mathrm{H}_{2} \mathrm{O}+3 \mathrm{NH}_{3} \rightleftharpoons \mathrm{Ln}(\mathrm{OH})_{3} \cdot x \mathrm{H}_{2} \mathrm{O}+\left(\mathrm{NH}_{4}\right)_{3} \mathrm{PO}_{3}$
The method resulted in nearly quantitative extraction of nitrate, $\sim 15 \%$ extraction chloride, but very low extraction efficiencies for the phosphates.

\section{Benefits to DOE}

This project resulted in the initial development of an extraction and purification method for the rare earth elements. Developing an extraction methodology that can be applied to "critical" metal sequestration from complex matrices will allow U.S. energy markets to be less reliant on international supply for materials critical to $\mathrm{CO}_{2}$-emissions-free energy technologies.

\section{Relevant Publications and Presentations}

Ogden, M. D., H. W. Rollins, B. J. Mincher, and

W. H. Bauer, 2011, "Ammonia-based

Extraction Methods for the Separation and Purification of Critical Metals from Problematic Matrices," presented to DARPA, May 18, 2011.

Ogden, M. D., H. W. Rollins, and R. V. Fox, 2010, "Extraction and Separation of Rare Earths Using Supercritical Fluids: $\mathrm{scCO}_{2}$, Ionic Liquids, and $\mathrm{scNH}_{3}$," 26th Rare Earth Research Conference (RERC11) Santa Fe, NM, June 19-23, 2010. 


\section{Scaling of Welding Processes}

Herschel B. Smartt, Charles R. Tolle, (South Dakota School of Mines and Technology)

09-006

This work addressed cost and energy reductions in manufacturing of aluminum components normally requiring foundry operations by developing a hybrid process based on combining an additive manufacturing process, friction stir welding (FSW) with a subtractive manufacturing process, computer numerically controlled (CNC)-machining. This process may significantly reduce the facility cost needed to manufacture such components while simultaneously reducing the energy content of the components below those associated with conventional manufacturing methods.

\section{Project Summary}

We started from the perspective of threedimensional (3D) printing, a class of processes used to build three-dimensional components or models on a layer-by-layer basis. Most 3D printing processes deposit drops of polymer using a deposition head rastered by a gantry mechanism. Our original objective was to produce small molten metal drops by determining the scaling laws for droplet size and melting rate in gas metal arc welding. Our hypothesis was that it would be possible to scale (up or down) metal transfer from the process by independently controlling (a) growth of a molten metal drop on the end of a welding electrode and (b) detachment of a molten metal drop. It became apparent that we needed a greater mass deposition rate than provided by small metal drops. To obtain fine-scale dimensional resolution we have combined a high deposition rate process, FSW, to deposit metal (contradictory to the conventional approach of achieving fine-scale dimensional resolution in the deposited material by use of small droplets) with a fine resolution subtractive process, CNC-machining, to remove excess metal.

FSW uses a spinning metal tool to heat materials by friction while causing the materials to flow plastically so as to mix the materials into a solid, continuous weld zone. FSW allows metal or polymer plates to be welded together on a layer-by-layer basis; CNC-machining allows the metal or polymer plates to be dimensionally configured both before and after welding.

As part of our work, we also aim to quantify the energy consumption associated with production of aluminum components. Comparing the energy required to fabricate aluminum plate products with that required for castings made a simple analysis. Data provided in Appendix A of U.S. Energy Requirements for Aluminum Production, Historical Perspective, Theoretical Limits and Current Practices (prepared for Industrial Technologies Program, Energy Efficiency and Renewable Energy, U. S. Department of Energy by BCS, February 2007) states that the gross energy required for rolled aluminum products is $1.26 \mathrm{kWh} / \mathrm{kg}$ and that for shaped castings is $2.64 \mathrm{kWh} / \mathrm{kg}$. This implies that our process may save considerable energy given that the total (2007) market for shaped aluminum castings is 2,000,000 metric tons. (To this we need to add the process energy per unit mass required by FSW and machining.) Assuming a savings of $1 \mathrm{kWh} / \mathrm{kg}$ and $10 \%$ market penetration, this gives an annual energy savings of approximately $2 \times 10 * * 8 \mathrm{kWh}$.

We conducted process development with conceptual drawings and fabricated billets of aluminum without CNC-machining, followed by CNC-machining of a solid bar from a billet. We then fabricated a simple model component (a closed box containing two cavities) as shown in Figures 1 and 2 (next page). We also evaluated a Python-based process modeling framework incorporating Python, wxPython, and VPython (including NumPy) using IDLE and VIDLE development tools. This provided a means for coding a user interface with 3D graphical output and a (simplistic) spherical particle dynamics model. This was found to be compatible with this framework although limitations exist regarding the number of particles that are practical. Also, wxPython and VPython have their own event loops, which presents an interesting challenge; we used the Grab application that comes with Mac OS-X as a workaround to capture graphical images, but this was not optimal. In future work we will use $\mathrm{C}++$ or Java for process modeling. 

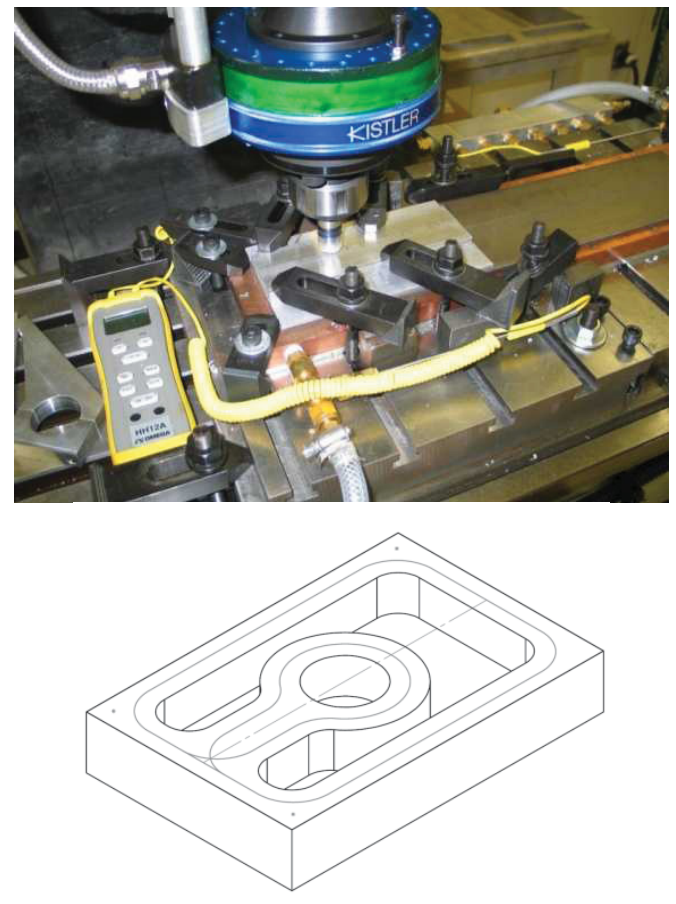

FIGURE 1. FSW process joining a metal plate on top of another shown on left. Drawing of model component containing cavities shown on right, without top and bottom plates shown.

\section{Benefits to DOE}

This work has developed experimental capabilities to fabricate aluminum components having complex shapes, including internal cavities in hollow components. This capability is targeted at industrial and/or government manufacturing of large, complex aluminum components, such as engine cylinder heads and blocks, without needing a foundry. This new capability and expertise will significantly increase our credibility to industrial partners and make our industrial research more relevant to DOE-Energy Efficiency and Renewable Energy program needs.

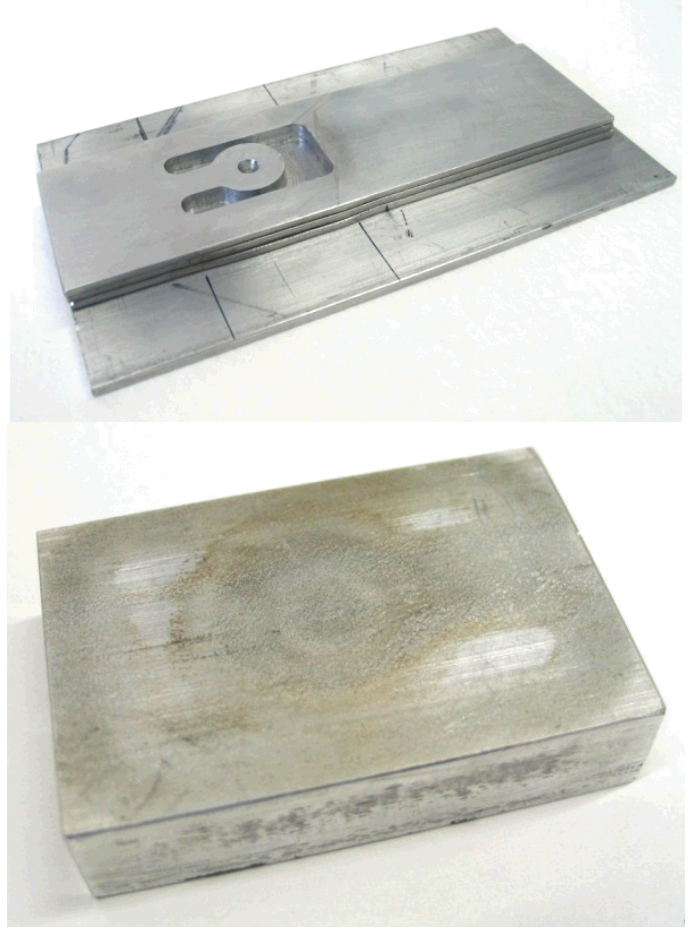

FIGURE 2. Aluminum fabrication on left showing details corresponding to drawing in Figure 1. A completed, sealed box containing the internal cavities shown on right.

We have authored a concept paper on this work titled "Combined Additive/Subtractive Manufacturing of Metal Components" as the basis for proposals we are writing for submittal to the National Science Foundation and Defense Advanced Research Projects Agency. The concept paper is also the basis for a manuscript to be submitted to ASM International during FY 2012. 


\section{Enhanced Metal Ion Analysis}

Mark Stone, James Delmore, Darin Snyder, Matthew Watrous

09-009

Thermal ionization mass spectrometry (TIMS) is a standard method used for the analysis of geologic materials, isotope fingerprinting of pollutants, trace metals, medical measurements, groundwater, and chemical metrology. Sample preparation is a tedious process that routinely requires days to complete.

We have developed a new technology for enhancing the ionization efficiency for TIMS analysis. It uses a microporous ion emitter (PIE), which is a mixture of metal powders that are scintered onto the filament. Using this new method, the detection limit has been reduced by orders of magnitude for praseodymium. This new approach reduces the loss of sample and allows for the analyte to be directly applied to the PIE.

Our goal this year was to improve ion production efficiency and simplify sample preparation by looking at current TIMS analysis methods to see if we could apply the PIE-type approach to other methods for easier sample preparation and improved analysis, accuracy, and sensitivity.

\section{Project Summary}

One analysis of interest is that of iodine. Currently, a thin layer of a compound called lanthanum hexaboride $\left(\mathrm{LaB}_{6}\right)$, which is a purplish colored solid, is electroplated onto the surface of a piece of rhenium. Our first attempt was to simply electroplate it onto a rheniumplatinum (Re/Pt) PIE. Figure 1 shows that the compound did coat the PIE surface, but it did not deposit deep down into the PIE structure.

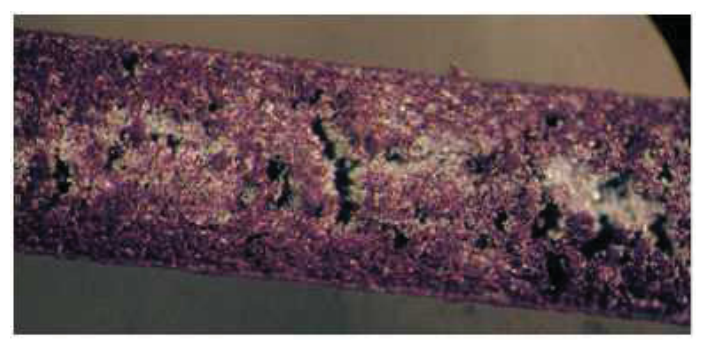

FIGURE 1. Electroplated LaB L $_{6}$ onto a PIE.
Next, we mixed the $\mathrm{LaB}_{6}$ with the components of the PIEs; and heated, mixed, and spread out a film composed of that mixture on a glass plate. Then, using a sharp blade, we cut out small strips, simply laid them on the filaments, and then heated them to melt the incorporated adhesive. The filaments were then heated in a vacuum to just above the operating temperature to form the filament containing the $\mathrm{LaB}_{6}$.

Figure 2 shows the film and a small sample strip that was removed from the upper portion of the film. The strip was then placed onto a filament, as shown in Figure 3.

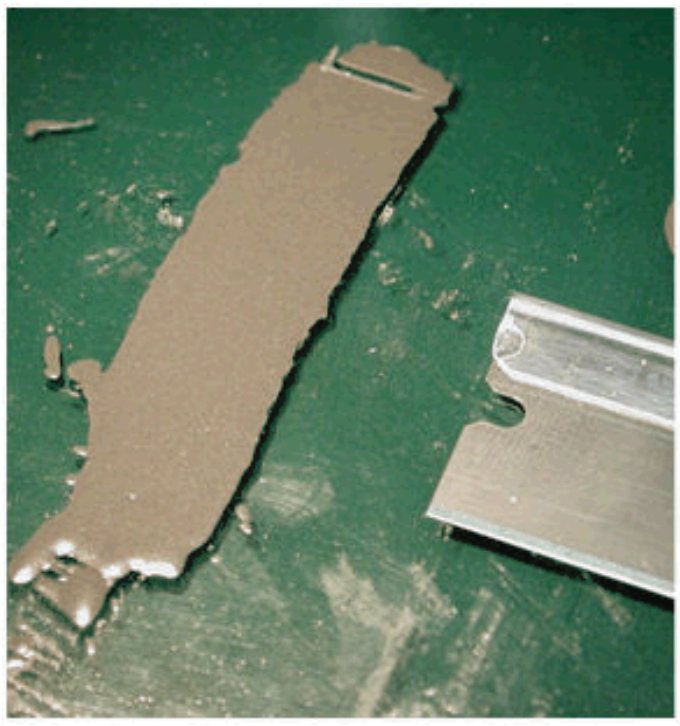

FIGURE 2. LaB 6 /PIE mixture spread on a glass plate.

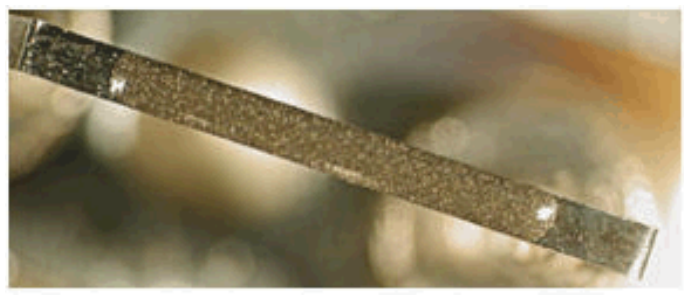

FIGURE 3. LaB 6 /PIE mixture affixed to a filament.

This filament was then heated, which resulted in the completed filament shown in Figure 4 (next page). The structure's porosity is visible from end to end, and the purple $\mathrm{LaB}_{6}$ can be seen distributed deeply within the structure. 


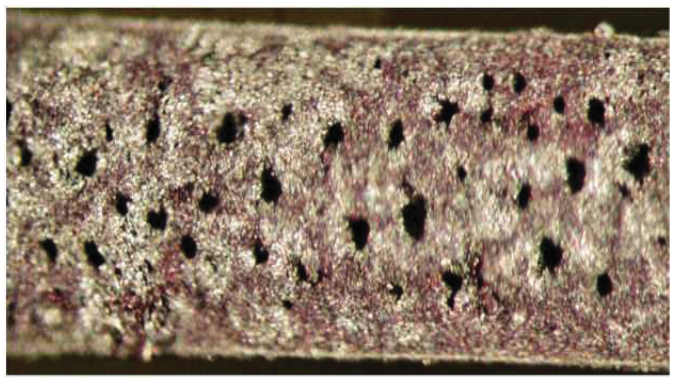

FIGURE 4. Completed $\mathrm{LaB}_{6} / \mathrm{PIE}$.

Finally, Figure 5 provides a close up scanning electron microscope image of the porous type of structure that is created when a PIE is produced. It gives very high surface areas for the samples to reside on, which leads to more sensitivity than can be achieved by simply applying a sample onto a filament or by using an ion exchange resin bead. One of the unique and powerful aspects of using PIEs is that we can examine a variety of mixtures easily and make useful structures repeatedly with the same composition.

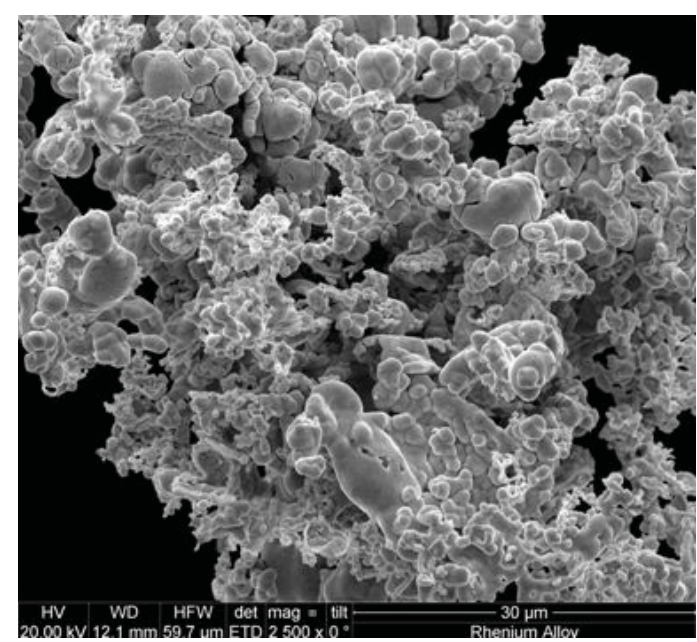

FIGURE 5. Scanning electron microscope photomicrograph showing the porous nature of the resulting PIE structure.

This is an excellent example of the way these new PIE types of structures can improve and simplify TIMS analysis. Both of the goals set out for this program have been achieved.

\section{Benefits to DOE}

A porous TIMS emitter constructed from a $\mathrm{Re} / \mathrm{Pt}$ alloy tightly bonded to a rhenium filament has been developed. This type of emitter is more reliable and shows sensitivity superior to the established resin bead method for uranium. It is rugged; none of the $100+$ samples tested were lost. The overall efficiency ranged between $1 \%$ and $2.2 \%$ for uranium, an improvement over the resin bead method of $0.58 \%$. In a related research and development project, the ionization efficiency of Sb by the traditional Si gel loading technique was compared with the Si gel method combined with the Re/Pt PIEs technology. The PIE technology provided an enhancement by a factor of $\sim 4$. Finally, some interesting differences were observed when using different shapes and sizes of PIEs, work that will continue.

This research directly relates to national security because it allows for more sensitive detection of ions related to nuclear nonproliferation. It also relates to environmental quality and science by permitting researchers to more easily and accurately detect and identify ions of interest in the environment and to permit isotope fingerprinting of pollutants. Other geological and trace metal analysis could benefit from this work as well. 


\section{Effect of Glycosylation on the Activity and Stability of Bacterial Enzymes}

William A. Apel, Brady D. Lee, Vicki S. Thompson

09-041

Literature surveys have recently shown that many bacterial enzymes are post translationally modified by glycosylation (i.e., the decoration of certain amino acids on the protein backbone with sugar groups). This was unexpected since it was believed until very recently that bacteria were incapable of glycosylation. As an incidental finding from a previous project, we identified a glycosylated extremophilic cellulase active at high temperatures and low $\mathrm{pH}$. Based upon this finding we hypothesized that these posttranslation modifications contribute to extremophilic enzyme stability. Initial studies were directed at examining a thermal alkaline stable catalase that we had previously isolated and characterized from a bacterium found in Yellowstone National Park. In the last year of the project we examined a post-translationally modified thermal acid stable pectinase from a different Yellowstone National Park bacterium.

\section{Project Summary}

Initial efforts with the catalase were described in previous annual reports. During the last year, our work concentrated on a thermal acid stable pectinase that was directed at three primary tasks: (1) adaptation of a zymography technique for identification of pectinase activity; (2) determination of the cellular location of the pectinase; and (3) purification of the pectinase with liquid chromatography techniques.

\section{Zymography}

A previously published technique for visualizing pectinases in protein gels was adapted for use at high temperatures and acidic $\mathrm{pH}$. Briefly, proteins from the Gram-positive thermoacidophile were separated in denaturing gradient polyacrylamide gels containing polygalacturonic acid. After electrophoresis, proteins were renatured and incubated at $60^{\circ} \mathrm{C}$ overnight. Proteins with pectinase activity were visible as a zone of clearing after polygalaturonic acid was stained with ruthenium red.

\section{Cellular Location of Pectinase}

To determine enzyme activity, cells were grown in ATCC 573 medium containing one of three separate carbon sources. Of these, the supernatant, cytoplasmic supernatant, and cellular membrane fractions were screened for enzyme activity. Cells grown on polygalacturonic acid exhibited most prominent enzyme activity, with the majority of activity in the cell membrane fraction. Samples showing enzyme activity were further analyzed with zymography and enzyme assays.

\section{Protein Purification}

Purification of the pectinase enzyme from the cell membrane fraction was attempted using fast protein liquid chromatography. However, both cation exchange and hydrophobic interaction columns were not successful under the conditions tested.

\section{Benefits to DOE}

This research further expanded INL's expertise in the area of stable enzymes for use in extreme industrial and environmental conditions. The work also supported the DOE theme "promoting America's energy security through reliable, clean, and affordable energy" via the development of unique, stable enzymes for converting biomass to fuels and value-added compounds that could displace petroleum usage. Furthermore, this research is directly applicable to the goals of the Office of Science, one of which is to better understand biological conversion processes at a fundamental level, and to the Office of Energy Efficiency and Renewable Energy, which is developing the use of enzymatic/biological processes for the conversion of biomass to fuels and value-added chemicals. 


\title{
Kolbe Electrolysis of Bio-Oils for the Production of Diesel and Aviation Fuels
}

\author{
Aaron D. Wilson, Tedd E. Lister \\ 11-036
}

The functional conversion of biomass to a useful liquid fuel is important to our efforts to move away from fossil-fuel-based energy systems. However, many of the potential biomass-derived fuels contain notably less energy than pure hydrocarbons. Conventional diesel and gasoline have an energy density of $46 \mathrm{MJ} / \mathrm{Kg}$, significantly higher than bio-fuels such as trans-esterified biodiesel at $42 \mathrm{MJ} / \mathrm{Kg}$, butanol at $37 \mathrm{MJ} / \mathrm{Kg}$, and ethanol at $30 \mathrm{MJ} / \mathrm{Kg}$. Not only do these fuels have lower energy densities, they also have less desirable physiochemical properties in terms of viscosities, vapor pressures, boiling points, freezing points, and flash points, as well as solvation properties producing negative interactions with conventional seals and hosing when compared with a pure hydrocarbon. Biomass can be completely broken down, gasified into synthesis gas, and reconstructed as a pure hydrocarbon. While this pathway can transform virtually any biomass, it lacks efficiency, breaking $\mathrm{C}-\mathrm{C}$ and $\mathrm{C}-\mathrm{H}$ bonds that must be later reformed, and requires a massive centralized reforming infrastructure.

The fatty acid is the form of biomass closest to our desired liquid fuel types. The conventional method of processing fatty acids is to transesterify them from a triglyceride form to a liquid "biodiesel" with reasonable physio-chemical properties for a fuel. It would be more ideal to produce a pure hydrocarbon through decarboxylation of the fatty acid. Currently, there are limited methods for decarboxylate, most of which have a very poor atom economy or that replace the carboxylic group with an undesirable functional group.

The heterogenous Kolbe electrolysis of a fatty acid with a shorter carboxylic acid is a decarboxylation reaction that leads to a pure hydrocarbon with the co-production of hydrogen. Relevant reports concerning the Kolbe electrolysis of fatty acids exist in the peerreviewed and patent literature, but these methods have significant shortcomings, preventing the transition from academic curiosity to applied technology.

Prior to this work, the best-reported conditions for the heterolytic Kolbe electrolysis of long-chain fatty acids was the application of

nearly equal parts methanol and non-polar solvent with alkali hydroxide or alkoxide. The strong base reacts with carboxylic acids to form alkali carboxylates, which act as the electrolyte. The major drawback to this protocol is the delicate balance between solvation and precipitation of many of the reaction mixture components. During the electrolysis, general and localized shifts occur in chemical concentrations and $\mathrm{pH}$. Under these dynamic conditions, the organic salts readily precipitate, tying up reagents and coating electrodes, increasing solution resistance, and reducing current densities. In addition, there is the constant threat of phase separation into polar and non-polar phases. The challenge was to find an electrolyte/solvent/surfactant that could facilitate the electrolysis process while solubilizing the reactants and products as a single phase throughout the entire process.

Our specific objectives are: (1) to advance the state-of-the-art conditions for the heterolytic Kolbe electrolysis of long-chain fatty acids; and (2) to evaluate the economic viability of heterolytic Kolbe electrolysis of long-chain fatty acids.

\section{Project Summary}

We have demonstrated the use of the tertiary amine dimethylcyclohexylamine as a surfactant and pro-electrolyte to facilitate the heterolytic Kolbe electrolysis of linoleic acid $\left(\mathrm{C}_{18} \mathrm{H}_{32} \mathrm{O}_{2}\right)$ and acetic acid $\left(\mathrm{C}_{2} \mathrm{H}_{4} \mathrm{O}_{2}\right)$ to linear diunstaturated hydrocarbons $\left(\mathrm{C}_{18} \mathrm{H}_{34}\right)$ with minimal amounts of homolytic side product $\left(\mathrm{C}_{34} \mathrm{H}_{62}\right)$. The linear diunstaturated hydrocarbons product has potential uses as a fine chemical or fuel with better energy density $(46 \mathrm{MJ} / \mathrm{Kg})$ and physiochemical properties than trans-esterified biodiesel (42 MJ/Kg). The use of dimethylcyclohexylamine compared to conventional Kolbe electrolysis conditions allows for high concentrations of reactants ( $>80 \mathrm{wt} \%$ ), improved product selectivity, absence of alkyl salt precipitants, and negation of phase-separation issues. The electrical cost for this process is less than $\$ 0.22$ per gallon at $\$ 0.03$ per $\mathrm{kWh}$ with a system running at $10 \mathrm{~V}$ and $120 \mathrm{~mA} / \mathrm{cm} 2$. These results demonstrate the viability of converting fatty acids to pure 
hydrocarbons under scalable whole-cell conditions.

In this project, we quantitatively addressed the effects of cell potential, tertiary amine concentration, and solvent concentration on current density (Fig. 1). This characterization of the cell is a requisite step before the economic viability of a system can be considered. To optimize a total system, many variables will need to be dynamically adjusted with the intention of minimizing the operating potential, solvent volumes, and electrolyte concentrations while maximizing product rations, current densities, and cell stability, all in light of each outcome's relative economic impact on the entire system. This sort of fundamental analysis is also a prerequisite before attempting more unusual modifications, such as the inclusion of a catalyst or electron chain to obtain the oxidative potential from atmospheric oxygen.

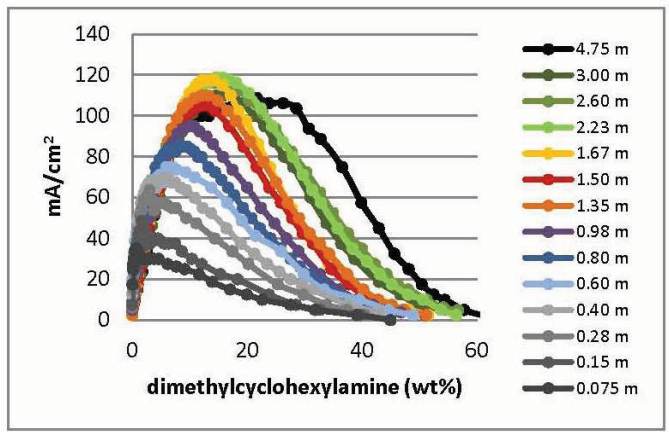

FIGURE 1. Flow cell $\left(26 \mathrm{ml} / \mathrm{min}\right.$.) with $4.0 \mathrm{~cm}^{2}$ platinum cathode and anode polarized at a constant $10.0 \mathrm{~V}$. Each line correlates to an experiment with a different initial total carbolic acid concentration with a 1:4 molar ratio of linoleic acid and acetic acid. During individual experiments, dimethylcyclohexylamine was incrementally added and the total current observed.

\section{Benefits to DOE}

INL has a vested interest in a number of biomass to fuel projects. Biomass conversion to fuels generally follows two paths. Firstgeneration biofuels are obtained from an established bio-reforming process or a process with a minimal amount of chemical modification; often these processes are inefficient and they produce low-grade fuels. Second-generation fuels are achieved through "deep" chemical transformation (e.g., gasification, pyrolysisis, thermocracking, etc.), which dramatically alter the chemical composition of the materials, breaking them down and reconstructing in a more desirable form. These "deep" chemical transformations lack molecular efficiency breaking many $\mathrm{C}-\mathrm{C}$ and $\mathrm{C}-\mathrm{H}$ bonds, which must be later reformed. More importantly, they require a large, centralized, and capital-intensive reforming infrastructure. Through this LDRD project, we were able to pursue a third option - an efficient and limited chemical transformation that converted fatty acids directly to pure hydrocarbons. We advanced the state-of-the-art conditions for the heterolytic Kolbe electrolysis of long-chain fatty acids, thereby creating an avenue to a better bio-diesel than conventional methods. We also established general economics parameters of heterolytic Kolbe electrolysis of long-chain fatty acids.

\section{Relevant Publications and Presentations}

Wilson, A. D., C. Rae, and T. E. Lister, "Use of Tertiary Amines as Surfactants and ProElectrolytes in Kolbe Electrolysis of Fatty Acids for the Production of Fuels and Fine Chemicals," In preparation to be submitted to RCS Energy \& Environmental Science.

Wilson, A. D., and T. E. Lister, 2011, "Use of Tertiary Amines as Surfactants and ProElectrolytes in Kolbe Electrolysis of Fatty Acids for the Production of Fuels and Fine Chemicals," INL IRC Professional Exchange, Idaho Falls, ID, August 2011.

Wilson, A. D., C. Rae, and T. E. Lister, 2011, "Kolbe Electrolysis of Bio-Oils for the Production of Diesel and Aviation Fuels Abstract 1537," $220^{\text {th }}$ Electrochemical Society Meeting Boston, MA, October 2011. 


\section{Dissolution, Extraction, and Separation of Rare Earth Elements using Soft Donor Ligands in Conjunction with Ionic Liquids and Supercritical Carbon Dioxide}

Brian Harris, Bruce Mincher, William Bauer, Chien Wai (University of Idaho)

11-038

Rare earth elements (REEs), or lanthanides, form the largest chemically coherent group in the periodic table. Though generally unfamiliar, REEs are essential for many hundreds of applications. The versatility and specificity of REEs has given them a level of technological, environmental, and economic importance considerably greater than might be expected from their relative obscurity. The United States once was largely self-sufficient in the production of these critical elements, but over the past decade has become highly dependent upon imports. By 1999-2000, more than 90\% of REEs required by U.S. industry came from deposits in China, which brings the future supply of these elements at reasonable cost into question. Therefore, the United States has endeavored to utilize their strategic continental deposits of rare earth ores. The approach was to improve current methods and investigate novel methods for recovery of REEs from ores and various matrices. This included optimizing the extraction efficiencies for separating lanthanides and actinides using various ligands in conjunction with ionic liquids ((BMIM)+ [PF6]- or [Tf2N]-) over a range of acid concentrations followed by back extraction into supercritical carbon dioxide $\left(\mathrm{sc}-\mathrm{CO}_{2}\right.$ ).

\section{Project Summary}

In FY-11, four ligands were studied tributylphosphate (TBP); 4,4,4-trifluoro-1-(2thienyl)-1,3-butanedione (TTA); 1,1,1,5,5,5hexafluoro-2,4-pentanedione (HFA); and $\mathrm{N}, \mathrm{N}, \mathrm{N}^{\prime}, \mathrm{N}^{\prime}$-Tetrakis $(2-$

pyridylmethyl)ethylenediamine (TPEN)to assess which combinations of ligands and matrices provided the most promising distribution ratios for separating REEs from uranium and thorium. Each ligand was mixed by sonication into either of two ionic liquids (ILs) at a ratio of approximately five ligands per metal being extracted before beginning the extraction process. The extraction was carried out by contacting each IL:ligand mixture with HCL or $\mathrm{HNO}_{3}$ over a range of 0.05 to $10.0 \mathrm{M}$, which contained a "geo" standard reference material including the lanthanides plus thorium and uranium. Of the sixty resulting combinations, the best IL:ligand combination was BMIM
$\mathrm{NTf}_{2}$ :TPEN in $0.05 \mathrm{M} \mathrm{HNO}_{3}$, as shown in Figure 1.

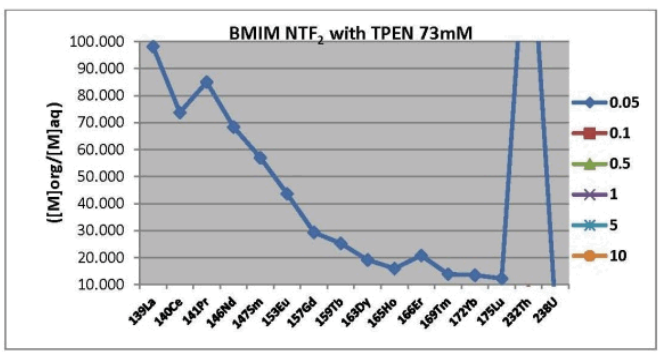

FIGURE 1. Distribution ratio for the extraction of REEs and actinides using BMIM NTf2:TPEN.

Once these conditions were established, this IL:ligand combination was used to investigate if the metals could be further back extracted from the IL using $\mathrm{sc}-\mathrm{CO}_{2}$. By varying the temperature and pressure, the density of the sc- $\mathrm{CO}_{2}$ can be changed or "tuned" for extractions. Initial extractions used temperature-pressure combinations of 1100 psi: $40^{\circ} \mathrm{C}$ and 2300 psi: $40^{\circ} \mathrm{C}$ that showed less than $1 \%$ recovery of the metals back-extracted from the ionic liquid into the sc- $\mathrm{CO}_{2}$. Figure 2 shows the comparison between extractions using TPEN and an ionophore ligand N,N,N,N-

tetra[cyclohexyl]diglycolic acid diamide, across the lanthanides.

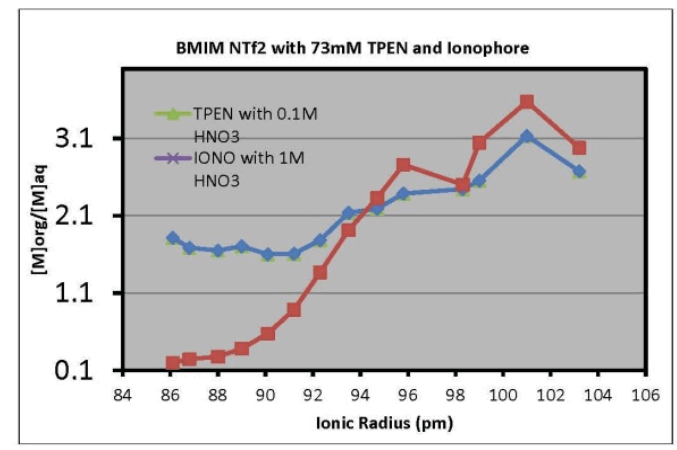

FIGURE 2. Distribution ratio for the extraction of REEs using TPEN and ionophore.

Other extractions on bastnesite ore using the IL BMIM-PF 6 and the TBP $\left(\mathrm{HNO}_{3}\right)_{1.8}\left(\mathrm{H}_{2} \mathrm{O}\right)_{0.6}$ complex showed an almost exclusive separation of thorium from other REEs. This year also continued a subcontract for the University of Idaho to provide the IL BMIM-NTf 2 . 
Benefits to DOE

Rare earths play a key role in the expected growth of many emerging energy technologies. Developing technologies for efficient extraction of REEs is of national importance for the entire core of DOE Strategic Themes, which are designed to deliver their strategic goals of energy security, scientific innovation, and environmental cleanup. Also, the nature of room- temperature ionic liquids affords a wide range of "tunability" in their design. As such, this research can serve as a basis for further studies in electrodeposition for fuels research, electrochemistry elements for advanced energy cells, and biocatalysis studies with roomtemperature ionic liquids as surfactants in novel biofuels. 


\section{Aleatory vs. Epistemic Uncertainty in Seismic Hazard Analyses}

Suzette Payne, Annette Schaffer

$10-038$

We address issues related to defining and reducing aleatory and epistemic uncertainty in probabilistic seismic hazard analyses (PSHAs). We focus on two areas that are significant contributors to predicted ground motion levels where aleatory variability can be replaced by epistemic uncertainty: (1) estimation of earthquake recurrence (maximum magnitude, $\mathrm{M}_{\max }$, and rate), and (2) parameterization of sitespecific ground motion attenuation models. The first effort is focused on performing analyses with multivariate relationships between magnitude (M) and fault dimensions (surface and subsurface displacement, rupture width, surface and subsurface rupture length, and rupture area) using the well-known "Wells and Coppersmith" (1994) dataset. Because their analysis assumed independence, when all five relationships are applied on a single fault, there is a resultant nonphysical multi-modal $M_{\max }$ distribution, which leads to greater uncertainty about mean seismic hazard levels. The second effort is focused on taking a new approach for how to incorporate direct or inferred subsurface data and their epistemic uncertainty into site-specific and empirical ground motion models rather than accounting for uncertainty through inter-model (irreducible) aleatory variability.

\section{Project Summary}

The first year focused on multivariate analyses using magnitude-fault dimension data. For the multivariate analyses, the 1994 data set was obtained from Don Wells (Wells and Coppersmith, 1994) and univariate regression analyses authenticated and validated the original 1994 dataset. Subsequent multivariate analyses in the second year demonstrated that the discrepancy between $\mathrm{M}_{\max }$ estimated using fault displacement (D) and surface rupture length (SRL) parameters were in part a result of assuming independence in the regression analyses. The primary contributor is inconsistent use of fault data where fault segment length $\left(\mathrm{L}_{\mathrm{seg}}\right)$ is equated with SRL. Many large historical, multi-segment-rupturing earthquakes yielded secondary and/or sympathetic faulting (e.g., 1983 Borah Peak, Idaho, earthquake), which are included in the measurements of SRL and used to derive empirical relationships. Because SRL is greater than $\mathrm{L}_{\mathrm{seg}}$, these relationships underestimate $\mathrm{M}_{\max }$ when Lseg is used and is apparent when compared with $\mathrm{M}_{\max }$ estimated from fault displacement (D). We developed an alternative empirical relationship using $\mathrm{L}_{\text {seg }}$ (instead of SRL) for historical, surface-rupturing, strike-slip, and normal faulting earthquakes. We verified the new relationship for a separate set of faults. We found that the $\mathrm{M}_{\max }$ estimated using $\mathrm{L}_{\text {seg }}$ are strikingly consistent with $\mathrm{M}_{\max }$ estimated using displacement data for Idaho and Utah faults, demonstrating self-similarity and implying that the new relationship should supplant the previous relationships currently employed in seismic hazard analyses.

Seismic velocity distributions are typically derived from single-well stratigraphic data and functional velocity-depth relationships for specific geological material types. Individual realizations of the velocity profiles are then used in ground motion models to derive a probabilistic distribution of motions by perturbing the base realization by the variance in velocity. This assumes that the stratigraphic column can achieve juxtaposition in a random manner, without regard to depositional or geomorphic processes. We took an alternative approach based on the philosophy that geologic processes are spatially correlated, not random. Spatial correlation can be imposed through the application of geostatistics. To evaluate the difference in aleatory uncertainty introduced through the two different approaches, in the first year we collected and analyzed stratigraphic data relevant to depth intervals of interest $(<600 \mathrm{~m})$ from wells near the Advanced Test Reactor. This involved reviewing well logs, performing variography, and kriging the resultant sedimentary-basalt structure. In the second year, we first obtained an older version of the proprietary code RASCAL that has been used in INL PSHAs, and the current version of the U.S. Geological Survey ground motion code SMSIM. Secondly, the 3D geostratigraphy was mapped to velocity based on material type and depth. This resulted in an array of velocity profiles that were lithologically and geologically correlated in a realistic manner. In contrast to the approach used in previous PSHAs, this process constrains the complete random-variability inherent in the random velocity perturbation approach 
commonly in use today. The resultant 3D "block-model" of seismic velocities was then used to predict ground motions using the automated SMSIM model. The resultant probability distribution is much better constrained, exhibiting lower variability because of the reduced uncertainty in geologic distribution.

\section{Benefits to DOE}

A recent sensitivity analysis indicated that different seismic design levels may result from an update of the INL seismic hazard, primarily arising from incorporation of aleatory variability in ground motion models. Results of this research will directly impact approaches that can be taken in future hazard analyses to reduce uncertainties in parameters and models, as demonstrated by developing an alternative magnitude-fault dimension relationship that reduces the span of predicted magnitudes. Reducing such uncertainties can lead to costeffective data collection and more accurate hazard analysis.

\section{Relevant Publications and Presentations}

Carpenter, S., S. J. Payne, and A. Schafer, 2012, "Toward Reconciling Magnitude Discrepancies Estimated from Paleoearthquake Data," Seismological Research Letters, Vol. 83, No. 2, in press.

Carpenter, S., S. J. Payne, and A. Schafer, 2011, "Toward Reconciling Magnitude Discrepancies Estimated from Paleoearthquake Data: A New Approach for Predicting Earthquake Magnitudes from Fault Segment Lengths," Abstract S21A2149, presented at 2011 Fall Meeting, American Geophysical Union, San Francisco, CA, December 5-9, 2011.

Coryell, B. D., A. Schafer, and S. J. Payne, 2011, "Toward Constraining Seismic Velocity Models using Geostatistical Representations of Stratigraphy in the Prediction of Probabilistic Ground Motions," Submitted to Seismological Research Letters in December 2011. 


\section{Particle-Discrete Element Model Simulation of the Coupling between Material Failure/Deformation and Fluid Generation/Flow}

Paul Meakin, Chuan Liu

09-003

Unconventional fossil energy, particularly shale gas and shale oil, is greatly improving the energy security of the United States. Oil shale is potentially even more important since the amount of oil that could be recovered from U.S. oil shale is greater than all the oil that has been produced worldwide. The objective of this project is to develop a better understanding of the coupling between fluid generating processes fluid flow and the deformation and fracturing of very low permeability rocks with an emphasis on applications for unconventional fossil energy recovery. The initial focus was on computational methods, but much of the research was experimental work performed in collaboration with the Center for the Physics of Geological Processes at the University of Oslo in Norway. INL's focus was on oil shale while the work at Center for the Physics of Geological Processes focused on primary migration. From a scientific point of view, the recovery of oil from oil shale via in situ retorting is virtually identical to primary migration, except for the greatly different rate. At the University of Oslo, we have been using Mahogany Formation Green River Shale, potentially the world's largest oil shale resource, as a model system for primary migration.

\section{Project Summary}

The main accomplishment was the application of 4-dimensional (3D + time) synchrotron $\mathrm{x}$-ray tomography coupled with chemical analysis to investigate the coupling between decomposition of kerogen, fracture nucleation and growth, hydrocarbon expulsion, and volume change as Green River Shale was heated in a synchrotron $\mathrm{x}$-ray tomography beam line.

The computational work was less successful. We were unable to develop a coupled smoothed particle hydrodynamics/discrete element model to simulate the coupling between fluid generation fracturing and fluid expulsion in very low permeability rocks. However, work towards this goal is continuing under the direction of INL's Dr. Hai Huang. Follow-on work will include cooperation with Prof. Moubin Liu at the Key Laboratory for Hydrodynamics and Ocean Engineering, Institute of Mechanics, Chinese Academy of Sciences. Prof. Liu has extensive experience with smoothed particle hydrodynamics, and we worked with him previously when he was a postdoctoral associate at INL.

\section{Benefits to DOE}

The project increased the experience and expertise of Paul Meakin, the principal investigator, in the area of unconventional fossil energy. This contributed to his ability to help establish the INL Carbon Resource Management Department. Development of computational geoscience capabilities and experience with integrated computational and experimental research are important to a variety of INL and DOE research areas, including unconventional fossil energy recovery, $\mathrm{CO}_{2}$ sequestration, and geothermal energy. In addition, Dr. Lu gained experience with smoothed particle hydrodynamics, which will be valuable in future computational geomechanics work at INL.

\section{Relevant Publications and Presentations}

Panahi, H., M. Kobchenko, F. Renard, A. Mazzini, J. Scheibert, D. K. Dysthe, B. Jamtveit, A. Malthe-Sorenssen, and P. Meakin, 2011, "4D Synchrotron X-ray Tomography Imaging of Source Rocks and Investigation of Hydrocarbon Escape Pathway Formation in Heated Organic-Rich Shales - A Proxy for Primary Migration?" SPE Journal, submitted.

Kobchenko, M., H. Panahi, F. Renard, D. K. Dysthe, A. Malthe-Sorenssen, A, Mazzini, J. Scheibert, B. Jamtveit, and P. Meakin, 2011, "4D Imaging of Fracturing in Organic Rich Shales during Heating," Journal of Geophysical Research, Vol. 116, B12201.

Meakin, P., H. Huang, and A. Malthe-Sorenssen, 2011, "Fracturing: From Paint Films and Microsphere Monolayers to Geoscience and Energy Applications," Braided Ways of Physics Seminar to Honor Arne T. Skjeltorp on the Occasion of his 70th birthday, Norwegian Academy of Science and Letters, May 20, 2011. 
Meakin, P., H. Huang, A. Malthe-Sorenssen, D. K. Dysthe, F. Renard, A. Mazzini, H.

Panahi, and M. Kobchenko, 2010,

"Coupling Between Fluid Generation, Deformation, Fracturing, and Fluid Flow in Low Permeability Porous Media," Gordon Research Conference on Flow and Transport in Permeable Media, Bates College, Maine, July 11-16, 2010.

Meakin, P., H. Huang, and A. Malthe-Sorenssen, 2008, "Coupling between Fluid Generation, Fluid Flow, Deformation, and Fracturing in Subsurface Porous Media: Discrete Element, Particle, and Continuum Methods,"

American Geophysical Union Meeting, San Francisco, CA, December 17, 2008. 
$\Rightarrow$ Energy Security Initiative

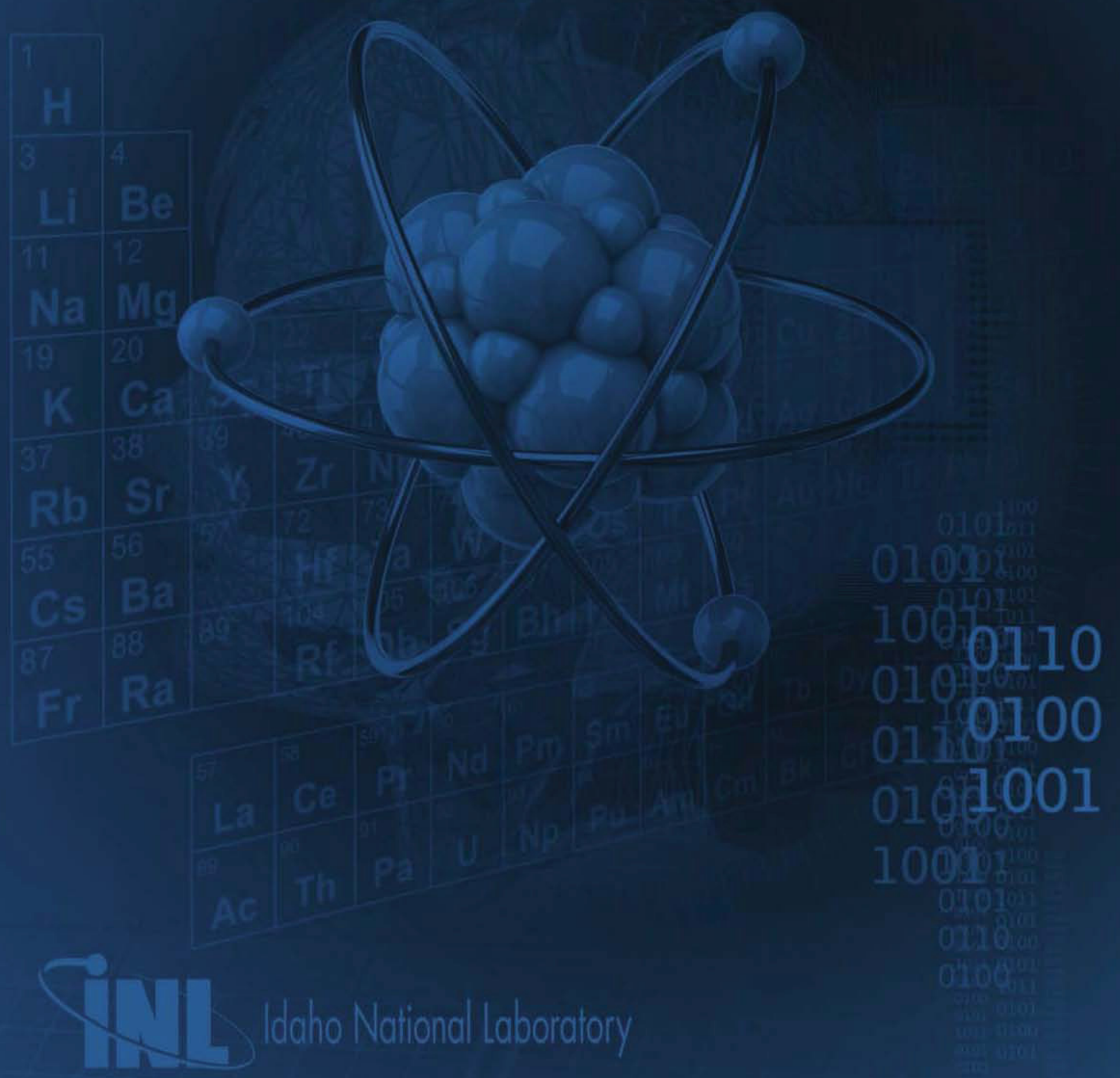




\section{Adaptive Process Modeling for Hybrid Energy Systems}

\section{Lee Shunn}

09-105

Much of the current energy infrastructure is built on single-resource/single-product processes (e.g., nuclear to electricity, coal to electricity, petroleum to gasoline, etc.) and relies heavily on fossil resources with unsustainable environmental implications. Hybridization of energy systems - that is, combining two or more energy processes into a single system - can increase overall system efficiency, improve the energy supply/demand balance, and reduce the environmental impacts of the hybrid system relative to its component subsystems.

Functional hybrid energy systems are vastly more complex than their stand-alone counterparts in terms of both design and operation. Balancing and exploiting the dynamics between system components is a challenge to the wide-scale deployment of hybrid energy systems. Research is needed to characterize how dynamic operations impact process stability and longevity. Engineering solutions to optimally dispatch material and energy within a hybrid system must also be developed.

The objective of this project is to use physics-based modeling and lab-scale experiments to characterize the transient response of hybrid systems to disturbances and changes in operating conditions. The transient data collected in the experiments are used to validate the models and to provide guidance for model development. The computational models are used for prognostics in model-based control systems for hybrid energy processes.

\section{Project Summary}

A laboratory-scale methanation experiment was constructed and operated during the first year of this project. The methanation system was operated for more than 40 hours of substitute natural gas production. This experiment demonstrated coupled operations with the hightemperature steam electrolysis system for nuclear hydrogen production. A commercial membrane system was used to demonstrate $\mathrm{CO}_{2}$ separation from flue gas, and the $\mathrm{CO}_{2}$ was recycled to produce a useful product (natural gas).

During Year 2, dynamic models for chemical reactors and process units were developed and validated. This lead to the development of the Hybrid Energy Dynamic Simulator (HyEnDSim) as a virtual test bed for investigating hybrid systems. HyEnDSim is currently being used by two LDRD projects at INL and two university collaborations.

An industrial collaboration was also initiated with a manufacturer of Raman spectroscopy equipment to test an early prototype probe for in situ measurement of gas composition at high pressure and temperature. INL tested prototype probes beginning in Fall 2010, and the resulting "WetHead" probe was recently added to Kaiser Optical Systems' commercial product line.

In Year 3, a liquid fuel synthesis reactor was constructed and operated for over 100 hours of testing. Both steady and transient operating conditions were investigated, and the system produced over 6 liters of methanol fuel. Hydrogen feed gas was supplied to the experiments using a high-pressure electrolysis unit that was operated simultaneously. The Raman spectroscopy system was used to provide real-time measurements of the chemical composition at several locations within the system. The experimental data is being used for model validation and development.

\section{Benefits to DOE}

Current and next-generation nuclear reactors (including small modular reactors) can be coupled with renewable and fossil resources in nuclear hybrid energy systems (NHESs). NHESs present a pathway to expand nuclear energy to applications beyond baseload electrical power generation by using nuclear-produced electricity and process heat to enhance the integration and stability of intermittent renewables and to produce clean, synthetic transportation fuels from domestic carbon resources. In this way, NHESs are exceptionally positioned to contribute to sustainable and secure energy production and establish U.S. leadership in clean-energy technologies for the coming century.

The hybrid energy systems at the core of this effort demonstrate the successful integration of nuclear energy (production of hydrogen via hightemperature steam electrolysis) with conventional fossil- and bio-energy systems (coal/biomass gasification and synthetic fuels 
production). These unconventional applications of nuclear energy directly contribute to the nation's sustainability goals for energy supply. This project has helped identify and address technological gaps and research needs in the field of instrumentation, monitoring, and control of advanced hybrid energy systems.

\section{Relevant Publications and Presentations}

Stoots, C. M., L. Shunn, and J. E. O’Brien, 2011, "Integrated Operation of the INL HYTEST System and High-Temperature Steam Electrolysis for Synthetic Natural Gas Production," Nuclear Technology, accepted.
Linda, O., M. Manic, and T. R. McJunkin, 2011, "Anomaly Detection for Resilient Control Systems using a Fuzzy-Neural Data Fusion Engine," 4th International Symposium on Resilient Control Systems (ISRCS), pp. 3541.

McJunkin, T. R., R. L. Boring, M. A. McQueen, L. Shunn, J. L. Wright, D. I. Gertman, O. Linda, K. McCarty, and M. Manic, 2011, "Concept of Operations for Data Fusion Visualization," European Safety and Reliability Conference, Troyes, France, September 2011. 


\section{Feedstock Processing and Energy Storage}

Daniel M. Ginosar, Lucia M. Petkovic, Nicholas E. McGuire, Rao Kondamudi

10-059

In order to reduce foreign dependence on crude oil, it is crucial to develop processes that convert hydrogen and carbon oxides to liquid fuels. This project is exploring and developing catalysts for economic processes to convert gaseous feeds of hydrogen and carbon oxides to liquid fuels. The main liquid fuel synthesis processes examined through the course of this project include dimethyl ether (DME) synthesis and isobutylene synthesis (isosynthesis). Both processes provide valuable chemicals that can be utilized directly as transportation fuels or as intermediates to other chemicals normally derived from crude oil.

\section{Project Summary}

\section{Single-step DME Synthesis}

DME has attracted attention recently as a viable "drop-in" alternative fuel to conventional diesel. DME is typically produced in a two-step process (methanol synthesis followed by dehydration) but it can be produced in a single step from synthesis gas by physical mixing of two catalyst components. In FY 2011, the catalysis research team at INL experimented with modifying the dehydration component of such a two-part catalyst in an effort to achieve increased yields of DME. Table 1 highlights some of the DME synthesis data collected during FY 2011. It was found that by both increasing the silicon-to-aluminum ratio and adding a small percentage of $\mathrm{MgO}$ to the zeolite dehydration component, increased yields of DME were obtained. Furthermore, longer-term tests of the single-step DME catalysts indicated excellent stability for all cases.

\section{Isobutylene Synthesis}

More recent efforts in the synthesis of liquid fuels have focused on the production of isobutylene from synthesis gas (isosynthesis). Isobutylene is a valuable chemical intermediate that has attracted much attention recently because it can be oligomerized into octane enhancers that are not detrimental to the environment. In FY 2011, the catalyst research team at INL explored using high surface area, lanthanide-promoted zirconia catalysts for isosynthesis. Addition of metal promoters to zirconia is known to have an effect on the

\begin{tabular}{|c|c|c|c|}
\hline Catalyst & $\begin{array}{c}\text { CO } \\
\text { Conversion } \\
\text { after } 24 \mathrm{~h} \\
(\%)\end{array}$ & $\begin{array}{c}\text { DME/ } \\
\text { organic } \\
\text { Products } \\
(\%)\end{array}$ & $\begin{array}{c}\text { DME } \\
\text { Yield } \\
\text { (kg h-1 } \\
\text { kgcat-1) }\end{array}$ \\
\hline $\begin{array}{l}\text { HZSM-5 (Si/Al } \\
=30)\end{array}$ & 35.2 & 98.3 & 0.75 \\
\hline $\begin{array}{l}1 \% \mathrm{MgO} / \mathrm{HZSM}- \\
5(30)\end{array}$ & 28.7 & 98.3 & 0.71 \\
\hline HZSM-5 (80) & 34.3 & 98.8 & 0.76 \\
\hline $\begin{array}{l}1 \% \mathrm{MgO} / \mathrm{HZSM}- \\
5(80)\end{array}$ & 38.5 & 98.1 & 0.85 \\
\hline
\end{tabular}

physicochemical properties of the catalyst which in turn affects catalytic performance.

Isosynthesis activity is evaluated in a packed bed reactor configuration and the results are analyzed in terms of the physicochemical characteristics of the catalysts.

Preliminary isosynthesis data shown in Figure 1 (next page) suggests that high-surfacearea zirconias have high initial levels of carbon monoxide conversions that exhibit rapid deactivation. Addition of the lanthanide promoters results in a lower initial conversion but more stability over the life of the test. Table 2 lists the instantaneous isobutene yield for the various lanthanide promoted catalysts after 10 hours on stream. It is found that the species of

TABLE 2. Isobutene yield for isosynthesis catalysts.

\begin{tabular}{lc} 
Catalyst & $\begin{array}{c}\text { Isobutene yield } \\
\mu \mathrm{mol} \mathrm{s}^{-1} \mathrm{~g}_{\text {cat }}^{-1}\end{array}$ \\
$\mathrm{ZrO}_{2}$ & 17.65 \\
$\mathrm{La}-\mathrm{ZrO}_{2}$ & 3.76 \\
$\mathrm{Ce}-\mathrm{ZrO}_{2}$ & 7.42 \\
$\mathrm{LaCe}^{-} \mathrm{ZrO}_{2}$ & 3.15 \\
\hline
\end{tabular}

the promoters has an effect on the isosynthesis activity. Clearly, the unmodified zirconia has the highest isobutene yield, correlating with the greater conversion levels shown in Figurel (next page). The addition of any promoters reduces the yield substantially. It is interesting to observe that the addition of lanthanum has a far greater impact on the isobutene yield than ceria. Diffuse reflectance inferred spectroscopy spectra of the 
catalysts that indicate relative acidity reveal that the acidity is closely related to the isosynthesis activity. Research is currently underway on modifiying catalyst acidity to maintain high levels of conversion and isobutene yield.

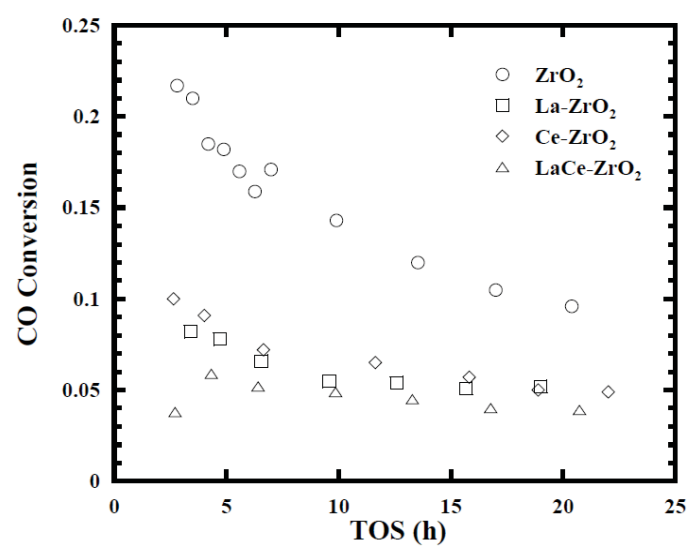

FIGURE 1. Isosynthesis stability.

Benefits to DOE

Development of alternative gas-to-liquids process such as isosynthesis and single-step dimethyl ether synthesis enables more efficient use of natural gas reserves and reduces the nation's dependence on foreign sources of crude oil.

\section{Relevant Publications and Presentations}

McGuire, N. E., N. Kondamudi, L. M. Petkovic, D. M. Ginosar, 2011, "Effect of Lanthanide Promoters on Zirconia-Based Isosynthesis Catalysts Prepared by Surfactant-Assisted Coprecipitation," in preparation.

McGuire, N. E., N. Kondamudi, L. M. Petkovic, and Daniel M. Ginosar, 2011, "Effect of modifiers on zirconia-based catalysts for isosynthesis," American Institute of Chemical Engineers Annual Meeting, Minneapolis, MN, October 16-21, 2011. 


\title{
Mesosilicate Supported $\mathrm{CO}_{2}$ Capture Material
}

\author{
Michael T. Benson, Frederick F. Stewart, John R. Klaehn \\ $10-035$
}

Currently used $\mathrm{CO}_{2}$ capture agents are liquid amines, such as monoethanolamine. These agents have several drawbacks, such as corrosiveness, volatility, and cost. Due to the weak acid $\left(\mathrm{CO}_{2}\right)$-weak base (amine) interaction, allowing capture and subsequent release during regeneration, amines are still the best candidate for $\mathrm{CO}_{2}$ capture. The objective of this work was to synthesize and test solid $\mathrm{CO}_{2}$ capture materials based on substituted triazines tethered to a high-surface-area solid support. The materials proposed were based on substituted 1,3,5triazine. The materials are solid, so volatility will not be an issue, are noncorrosive, very versatile, and inexpensive; thus these materials will be an improvement in the state of the art. This work was broken down into four tasks: (1) synthesis of substituted triazines, (2) synthesis of zeolites and attachment of triazines, (3) $\mathrm{CO}_{2}$ uptake/release characterization, and (4) construction of a working $\mathrm{CO}_{2}$ capture device. A tether was attached to the substituted triazines, allowing the triazine to be attached to the mesosilicate support. In addition to synthesis, the material was tested for $\mathrm{CO}_{2}$ uptake and release.

\section{Project Summary}

An apparatus was constructed for measuring $\mathrm{CO}_{2}$ uptake. This is a benchtop system, with a maximum sorbent capacity of roughly $50 \mathrm{~g}$ in a column. The flexibility built into the system allows a variety of gases to be investigated. Currently, only nitrogen and carbon dioxide are connected, but sorbent poisons, such as $\mathrm{SO}_{\mathrm{x}}$, $\mathrm{NO}_{\mathrm{x}}$, and $\mathrm{H}_{2} \mathrm{~S}$, could also be included.

Supported materials have been generated using incipient wetness to attach the sorbent to the support. Initial loading has been low, but the results are promising. The most promising INL material (as a non-porous powder) captures 1.90 mol CO $2 / \mathrm{Kg}$ sorbent. After loading onto an alumina support, capture increased to $1.99 \mathrm{~mol}$ $\mathrm{CO}_{2} / \mathrm{Kg}$ sorbent, even with low loading. As with any sorbent system, surface area is crucial. The exposure of more functional groups will increase the capacity. Incipient wetness is not the best method, since the functional groups could be laying down on the surface, or covered by other triazines, thus decreasing the exposure of the functional groups. Tethering the triazine to the surface through a single tether is a better method for exposure of the functional groups, increasing the surface area.

Several methods to tether the triazine to the surface were unsuccessful. Attaching a siloxyl group to the triazine did not yield any reaction. The nucleophilicity of the amine attachment point was likely too low for nucleophilic attachment. Substituting one of the triazine arms with p-methoxyphenol did yield the desired product. Removal of the methyl group, to expose the alcohol for attachment to the surface, was not successful, though. The harsh conditions needed to convert the methoxy group to an alcohol were too aggressive, destroying the compound. Substituting one of the arms with diethyl iminodiacetate was successful, as shown in Figure 1 below, as was removing the ethyl groups, producing carboxylic acids, and hydrogenating the nitrile groups to primary amines. The yield of the reaction was very low.

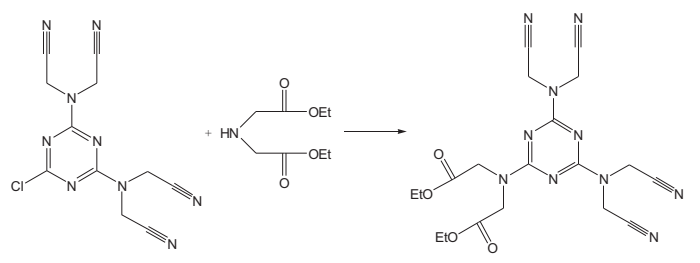

FIGURE 1. Substitution of diethyl iminodiacetate proved successful in experiments.

\section{Benefits to DOE}

There is an ongoing effort for cleaner energy production, with part of that being lower $\mathrm{CO}_{2}$ emissions. The development of new, more efficient $\mathrm{CO}_{2}$ capture agents will benefit energy resources and environmental quality. More efficient $\mathrm{CO}_{2}$ capture agents will also keep the cost of electricity down. Current technology (ethanolamines) is expected to raise the cost of electricity by at least $40 \%$.

\section{Relevant Publications and Presentations}

Benson, M. T., F. F. Stewart, and J. R. Klaehn, 2011, "Substituted Triazine Materials as $\mathrm{CO}_{2}$ Sorbents," $36^{\text {th }}$ International Technical Conference on Clean Coal \& Fuel Systems, Clearwater, FL, June 5-9, 2011. 
Benson, M. T., F. F. Stewart, J. R. Klaehn, M. Christenson, and N. Sing, 2011, "Novel

Triazine Materials as $\mathrm{CO}_{2}$ Sorbents," $241^{s t}$

National ACS Meeting, Anaheim, CA,

March 27-31, 2011.

Benson, M. T., 2010, "Methods of Removing Carbon Dioxide and Sorbents for Same,"

Patent application submitted September 9, 2010, Serial \# 12/878,853. 


\title{
Membrane Separation System Research for HYTEST
}

\author{
Frederick F. Stewart, Mark L. Stone, John R. Klaehn, Christopher J. Orme
}

$10-043$

Chemical separation systems can be considered enabling technologies for various applications. During FY 2011, the research team demonstrated a membrane-based separations capability for hybrid energy systems (HES) to affect both product purification and reactant recycle. The project has two main thrusts. First, membrane materials have been selected and characterized for their ability to address the separations requirements for various HES configurations. These separations include $\mathrm{CO}_{2}$ from hydrogen and $\mathrm{CO}, \mathrm{CO}_{2}$ from light hydrocarbons, and dimethyl ether (DME) from $\mathrm{CO}, \mathrm{CO}_{2}$ and $\mathrm{H}_{2}$. Second, the hardware necessary for a "bolt-on" membrane separator that can handle gas flow rates of $0.25 \mathrm{ft}^{3} / \mathrm{min}$ has been demonstrated. To facilitate these two thrusts, previous LDRD, DOE, and industrial investments in INL have been leveraged. Many promising materials have been developed in our labs and have been characterized at a fundamental level. This project has served to advance the state of development of membrane technology more towards deployment.

\section{Project Summary}

Development of separations systems for Hybrid Energy Systems Testing (HYTEST) focused on four central tasks: (1) developing a research needs plan for the inclusion of membrane systems into HES; (2) developing hardware for characterization of membrane modules against syn-gas at temperatures up to $400^{\circ} \mathrm{C}$ and pressures as high as $400 \mathrm{psi}$; (3) selecting and characterizing suitable membrane materials for syn-gas separations; and (4) characterizing and demonstrating gas separations relevant to HYTEST at pilot scale. The technical needs document, which outlined specific gas separations, was submitted to the HES project manager in FY 2010. In this document, membrane capabilities were reviewed and clear technological gaps, which were addressed in this LDRD project during FY 2011, were identified.

To meet the separation challenges of HES, a new membrane test system was designed and constructed. This system is capable of meeting the physical and chemical requirements of the HES concepts.
The study of available technologies revealed that the hollow fiber geometry offered the most facile pathway for the development of high-flux modules. A technological gap was the lack of knowledge concerning the permeability and selectivity of membranes towards DME, which may be considered a liquid fuel. This gap is significant because in hollow fiber modules, a dense polymer layer is applied that provides gas selectivity. Without empirical knowledge about DME behavior, it was unknown which polymer would perform the desired separation. We have completed studies into characterizing polymer membrane performance against the analyte gases $\left(\mathrm{CH}_{4}, \mathrm{H}_{2}, \mathrm{CO}, \mathrm{CO}_{2}\right.$, and DME). We have determined that DME is a highly condensable gas, meaning that its permeability is driven by solubility. Separation of DME from lesser condensable gases, such as $\mathrm{CH}_{4}$ and $\mathrm{CO}$, appears to be practical. Another technological gap was the need to separate and recycle a gas stream that included $\mathrm{H}_{2}, \mathrm{CO}, \mathrm{CO}_{2}$, and light hydrocarbons.

Due to the hazardous nature of $\mathrm{CO}$ and $\mathrm{H}_{2}$ at high flow rates and volumes, the separation was simulated successfully with $\mathrm{N}_{2}$ and Ar.

Commercial high-surface-area modules were found to be effective in the separation of $\mathrm{CO}_{2}$ from $\mathrm{N}_{2}$ and Ar at pilot-scale flow rates.

\section{Benefits to DOE}

During the course of this project, we have determined that membranes are an enabling technology for the HES concept, thus supporting the energy resource and science thrusts within DOE. The separations needs in this project are not unique to this HES application. Equipment and processes developed under this LDRD project have been and will continue to be a basis for follow-on support. As an example, the DOE Office of Fossil Energy awarded a new project to a team in which INL is a part for the development of novel membranes for carbon capture, which was enabled by this LDRD project. Thus, this LDRD improved INL's position to provide technical solutions in gas separation and carbon capture. 
Relevant Publications and Presentations

Orme, C. J., and F. F. Stewart, 2011, "Separation of Dimethyl Ether from Syn-Gas Components by Poly(dimethylsiloxane) and Poly(4-methyl-1-pentene) Membranes," Chemical Engineering Journal, Vol. 170, pp. 178-183.

Ogden, M. D., C. J. Orme, and F. F. Stewart, 2011, "Effects of Alkyl Substitution on the Physical Properties and Gas Transport Behavior in Selected Poly(Rphenoxyphosphazenes)," Polymer, Vol. 52, pp. 3879-3886.
Stewart, F. F., and C. J. Orme, 2011, "Dimethyl Ether and Syn-Gas Permeability and Selectivity Through Poly[dimethyl siloxane] (PDMS) and Poly[4-methyl-1-pentene] (TPX) Membranes," Presented at the North American Membrane Society Meeting, Las Vegas, NV, June 2011. 


\section{Hybrid Systems Process Integration and Dynamics Studies}

Robert S. Cherry, Humberto Garcia; Charles Forsbert (Massachusetts Institute of

Technology)

10-067

The major problem facing the alternative energy market is based on the ability to store energy at large scales (days to months) to facilitate the incorporation of intermittent wind and solar energy into hybrid energy supply systems. However, such technology does not exist in more than basic, small, or conceptual forms. This work focused on the dynamic analysis of hybrid energy systems (HES) when they are used to mitigate power-system variability arising from the integration of renewable energy. This is done by configuring systems to produce power during periods of high demand, and synthetic fuels when power demand is lower (or if renewable generation is available). Time-average analyses of the dynamics identify the overall energy security incentives to develop and implement these systems, while dynamic analysis of these highly coupled energy configurations identify their key dynamical properties and limitations in mitigating the high variability introduced from incorporating renewable energy into the energy matrix. Comparative dynamic cost analyses are also conducted to determine which level of renewable penetration advanced HES solutions may be more economical to pursue than traditional ones.

Massachusetts Institute of Technology (MIT) proposed running a nuclear power plant at constant output with electricity to grid when required and storing large amounts of nuclear heat underground for production of peak electricity on demand or to provide heat for industrial purposes as required. The goal is to create a system with up to a gigawatt-year of heat storage capacity that would: (1) enable management of seasonal swings in renewable energy production and thus enable nuclear and renewable systems to operate at full capacity at all times, and (2) allow coupling nuclear reactors to industrial uses of heat without having to address challenges such as reactor shutdowns for maintenance or refueling. The proposed work is to analyze this concept in order to understand the potential capabilities, limits, and economics.

\section{Project Summary}

One line of work demonstrated that hybrid systems can provide - from intermittently available power generation capacity—enough energy to make, in the form of methanol, the energy equivalent of 5 million barrels per day of gasoline. If renewable biomass rather than natural gas is the carbon feed, about 4.3 million barrels per day can be made, about equal to the amount of petroleum currently imported from OPEC.

Dynamic models of various unit operations were developed and integrated within two different HES options. One HES option, termed "traditional," produces electricity only and consists of a primary heat generator, a steam turbine generator, a wind farm, and battery storage. The other HES option, termed "advanced," includes not only the components present in the traditional solution, but also a chemical plant complex to repurpose excess energy for non-electricity services, such as for the production of chemical goods. Dynamic analysis of these highly coupled energy configurations were performed, as well as comparative dynamic cost analyses. The functional cost includes a set of metrics for computing not only fixed costs (such as fixed operations and maintenance and overnight capital costs), but also variable operational costs (such as the cost of variability, variable operations and maintenance cost, and revenues). Preliminary results identified at which level of renewable penetration a given advanced HES option becomes increasingly more economical than a traditional electricity-generation-only solution.

An initial analysis of nuclear geothermal storage was conducted that indicated the technology is potentially feasible and potentially economic in the role of providing intermediateload electricity demand. The analysis assumed the reactor was a pressurized water reactor. The dynamic analysis defined both the potential and the constraints in engineering such a system. The analysis also indicated the possibility to use the technology in a modified form to provide peak electricity production and provide a method for efficient recovery of heavy oil.

\section{Benefits to DOE}

This work evaluated a clean, ubiquitous, large energy resource, and excess power generation capacity. If hybrid systems can be developed to provide intermediate 
load-following power generation, their concomitant ability to produce synthetic fuels using clean nuclear energy will address both $\mathrm{CO}_{2}$ emission reduction goals, as well as those related to securing domestic energy supplies.

The Massachusetts Institute of Technology work potentially creates a new large-scale seasonal energy storage option to match energy production with energy demand for a low-carbon world. Large-scale energy storage is an alternative to burning fossil fuels to meet variations in electricity demand.

\section{Relevant Publications and Presentations}

Forsberg, C. W., 2011, Nuclear Energy for

Variable Electricity and Liquid Fuels

Production: IntegratingNuclear with

Renewables, Fossil Fuels, and Biomass for a

Low-Carbon World, CANES Report Series, Massachusetts Institute of Technology.

Garcia, H. E., A. Mohanty, R. Cherry, and W.-C. Lin, 2012, "Dynamic analysis of hybrid energy systems under variable renewable generation," Journal of Energy, in preparation.

Aumeier, S., R. Cherry, R. Boardman, and J. Smith, 2011, "Nuclear Hybrid Energy Systems: Imperatives, Prospects and Challenges," Energy Procedia, in press.

Forsberg, C. W., 2011, Nuclear with Renewables, Fossil Fuels, and Biomass for a Low Carbon World, MIT-NES-TR-015, Massachusetts Institute of Technology, September 2011.

Lee, Y. H. and C. W. Forsberg, 2011, Conceptual Design of Nuclear-Geothermal Energy Storage Systems for Variable Electricity Production, MIT-NES-TR-014, Massachusetts Institute of Technology, June 2011.

Lee, Y. H., 2011, Conceptual Design of NuclearGeothermal Energy Storage System for Variable Electricity Production, MS Thesis (C. Forsberg Thesis Advisor), Massachusetts Institute of Technology, June 2011.

Cherry, R. S. and R. D. Boardman, 2011, "Using Excess Electric Generation Capacity to
Make Synthetic Fuels at the National Scale," Energy Policy, submitted.

Cherry, R. S. and H. Garcia, 2011, "Multiple Hybrid Energy Systems for Load-Following and Offsetting Renewable Generation's Variability," Energy, submitted.

Forsberg, C. W., R. Krentz-Wee, Y. H. Lee, and I. O. Oloyede, 2010, Nuclear Energy for Simultaneous Low-Carbon Heavy-Oil Recovery and Gigawatt-Year Heat Storage for Peak Electricity Production, MIT-NESTR-011, Massachusetts Institute of Technology, December 2010.

Cherry, R. S., S. Aumeier, and R. Boardman, 2010, "The Role of Dynamic Large-Scale Hybrid Energy Systems in Providing Energy Security," Energy and Environmental Science, revision submitted.

Forsberg, C. W., Y. Lee, M. Kulhanek, and M. J. Driscoll, 2012, "Gigawatt-Year NuclearGeothermal Energy Storage for Light-Water and High-Temperature Reactors," Paper \#12009, 2012 International Congress on the Advances in Nuclear Power Plants, June 24-28, 2012, Chicago, IL, submitted.

Cherry, R. S., 2011, "Performance of Cooperating SMR-Based Hybrid Energy Systems used to Offset Fluctuations in Wind Energy Supply," ASME 2011 Small Modular Reactors Symposium, September 30, 2011, Washington D.C.

Cherry, R. S., 2011, "Hybrid Energy Systems to Address Renewable Energy's Variability," Western Wind and Transmission Leadership Symposium, Big Sky, MT, September 27, 2011.

Aumeier, S., T. Baldwin, R. Boardman, R. Cherry, H. E. Garcia, and D. Grauer, 2011, "Grid-Scale Hybrid Energy Systems," Nuclear and Renewable Energy Synergies Workshop, Golden, CO, September 12-13, 2011. 
Garcia, H. E., S. Aumeier, R. Boardman, R. Cherry, and A. Mohanty, 2011, "Optimal Energy Management via Hybrid Energy Systems," Conventional Generator Cycling and Variable Generation Workshop, Washington, DC, June 14, 2011.

Cherry, R. S., 2011, "Hybrid Energy Systems in the Oil Sands," Invited Presentation, International Society for Automation, Oil Sands Automation Conference, Fort McMurray, Alberta, Canada, March 9, 2011.

Aumeier, S., R. Boardman, and J. Smith, 2010, "Nuclear Hybrid Energy Systems: Imperatives, Prospects, and Challenges," Invited presentation, Second International Conference on Asian Nuclear Prospects 2010, New Delhi, India, October 2010.

Cherry, R. S., 2010, "Hybrid Energy Systems for Energy Security," Invited presentation, Edison Electric Institute Conference on Transmission, Distribution, and Metering, Denver, CO, October 2010.
Cherry, R. S., S. E. Aumeier, R. D. Boardman, and J. D. Smith, 2010, "Hybrid Energy Systems: Improving Energy Security by CoProducing Variable Ratios of Power and Synthetic Fuels," $60^{\text {th }}$ Canadian Chemical Engineering Conference, Saskatoon, Saskatchewan, Canada, October 2010.

Lee, Y. H., C. W. Forsberg, M. Driscoll, and B. Sapiie, 2010, "Options for NuclearGeothermal Gigawatt-Year Peak Electricity Storage Systems," Paper \#10212, 2010 International Congress on Advances in Nuclear Power Plants, San Diego, CA, June 13-17, 2010.

Cherry, R. S., 2010, "Transforming North American Energy Systems," Invited Plenary Panel Discussion, $103^{\text {rd }}$ Annual Air and Waste Management Association Conference, Calgary, Alberta, Canada, June 2010. 


\section{Conversion of Light Hydrocarbons to Fuels and Chemicals}

\section{Lucia Petkovic, Harry Rollins}

10-049

Hybrid Energy Systems Testing (HYTEST) is a complex of facilities and resources built at INL to support hybrid energy systems research and development. This project is aimed at obtaining the data necessary for integration into HYTEST. To do this, we aim to study the conversion of light hydrocarbons to fuels and chemicals via catalytic dehydro-aromatization reactions. This research project started in FY 2010 and was planned for three years. The first year was dedicated to obtaining the reactionrate data on a molybdenum-zeolite catalyst. Different temperatures, flow rates, and concentrations were studied, and spent catalysts were characterized by surface area and coke oxidation to obtain an estimate of the optimum parameters for the process. Experiments during the second year were aimed at analyzing the effects of catalyst preparation methods, catalyst particle size, and gas feedstock composition on product selectivity, conversion, and catalyst deactivation.

\section{Project Summary}

A series of catalyst characterization analyses and reaction-rate measurements was performed on molybdenum/zeolite catalysts synthesized by vaporization-deposition of molybdenum oxide and by impregnation with a molybdenum complex salt. The effect of catalyst preparation method, particle size, and feedstock composition was studied. The results were presented at four peer-reviewed professional meetings including two publications in meeting proceedings (see relevant presentations below).

\section{Benefits to DOE}

This work directly supports the future success of HYTEST. Development of hybrid energy systems helps attain the DOE's mission of working to assure clean, affordable, and dependable energy supplies for our nation. For the particular case of conversion of light hydrocarbons to fuels and chemicals, methane dehydroaromatization can be considered as a potential alternative to steam reforming of methane and also as a way to convert by-product methane streams, which are usually flared, into valuable aromatic compounds and hydrogen, without production of carbon dioxide. This project provides knowledge that may be utilized in the production of fuels and chemicals, starting from biomass, a carbon-neutral and renewable source. It also supports science and technology interests by increasing the knowledge about the behavior under different reaction conditions of heterogeneous catalysts. In addition, successful realization and testing of hybrid energy systems will reduce our country's reliance on imported fuels. This project supports those interests by providing crucial parameters for scaling up and analyzing a technology that so far has not been deployed beyond laboratory scale.

\section{Relevant Presentations and Publications}

Petkovic, L. M., D. M. Ginosar, K. C. Burch, and H. W. Rollins, 2011, "Effect of Preparation Method on Catalytic Activity of Molybdenum-Zeolite for Methane Dehydroaromatization," 22nd NAM (North American Meeting) of the North American Catalysis Society, Detroit, MI, June 5-10, 2011. Published in meeting proceedings.

Petkovic, L. M., D. M. Ginosar, N. Kondamudi, N. E. McGuire, K. C. Burch, H. W. Rollins, S. N. Rashkeev, and H. H. Farrell, 2011, "Heterogeneous Catalysis for Energy and Environment Applications," ACS Idaho Local Section Annual Meeting, Idaho Falls, ID, April 28, 2011.

Petkovic, L. M., D. M. Ginosar, K. C. Burch, and H. W. Rollins, 2011, "Effect of Preparation Method on Catalytic Activity of Molybdenum-Zeolite for Methane Dehydroaromatization," Annual Symposium of the Western States Catalysis Club, Albuquerque, NM, February 18, 2011. Published in meeting proceedings.

Petkovic, L. M., D. M. Ginosar, K. C. Burch, and H. W. Rollins, 2010, "MolybdenumZeolite Catalysts for Conversion of Light Hydrocarbons to Fuels and Chemicals," 2010 AIChE (American Institute of Chemical Engineers) Annual Meeting, Salt Lake City, UT, November 7-12, 2010. 


\section{Integration of Renewable Energy Resources through Energy Storage and SuperGrid}

Thomas Baldwin, Humberto Garcia, William Phoenix, Robert Cherry

11-007

As research and development efforts in hybrid energy systems containing renewable and nuclear resources mature, integration problems with the electrical power grid have come to the forefront. Several key issues have been plaguing the energy industries, including: having appropriate transmission grid resources in proper locations to move electrical energy from optimal generation resource locations to load center locations, being able to put energy into the electrical grid at the time the energy is ready and available from energy sources and not being constrained by the loading demand, and controlling the flow of energy within the grid to achieve reliable service, meet stability requirements, and maximize efficiency while addressing the marketing and business objectives of the energy industry.

This LDRD aims to: (1) develop the modeling and analysis tools to understand the interfacing issues of renewable energy generation to the electrical power grid, (2) determine the impact of placing large-scale energy storage in the power grid to support integration of renewable energy generation, and (3) determine the impact of operating a SuperGrid on the penetration of renewable energy generation. The LDRD begins with tools and model development, leading to analysis and the development of best engineering practices. At the end of the LDRD, the conceptual plans for a follow-on demonstration project are developed.

The developed computational tools characterize the dynamic interactions of grid systems where energy storage is used to stabilize the grid. The computational tools support the engineering expertise to analyze and integrate renewable and hybrid energy systems to the electrical grid. The LDRD builds on INL's capabilities to address electrical power storage where electrical power may not always be dispatchable and where grid stability and reliability depends on managed load following and supply.

\section{Project Summary}

Technical Review of Bulk Energy Storage $\underline{\text { Systems }}$

Energy storage serves the important function of dampening the variations in energy flows of power and energy systems. Energy production systems, like many manufacturing processes, operate most efficiently and economically when the systems have a constant or steady-state behavior in energy levels and power flow rates. However, natural variations occur throughout the energy production chain, including source supply quality and quantity, and cyclic consumption levels. Many of the energy-level variations have daily and weekly cycles, while others have weather and political influences. Transient variations with significant power flow changes over a very short period (with respect to the system time constants) are most problematic and can cause system instabilities.

Classical energy storage is found in the fuel storage of power plants. Slow energy variations are addressed by adjusting the rates of fuel consumption during energy conversion into heat, steam, and other forms of energy. Modern energy storage consists of specialized systems that can easily absorb excess energy or deliver stored energy quickly on demand. They are based on unique physical principles of materials and simple machines to temporarily hold energy. However, many energy storage systems are costly from an economic standpoint or physically (weight or volume per unit energy).

A review of advanced energy storage systems (superconducting magnetic energy storage, SuperCaps, compressed air energy storage, high-speed flywheels, etc.) shows that they serve best in niche applications that take full advantage of the unique storage characteristics. It is possible to avoid the use of large-bulk energy storage systems by selectively placing smaller amounts of energy storage at locations in the energy supply system where energy variations can best be managed.

\section{$\underline{\text { Renewable Energy and SuperGrid Systems }}$}

SuperGrids are energy networks that span large geographic regions. They differ from power networks, which require that the balance of source and sink powers be maintained. A SuperGrid for renewable energies that covers large sections of the North American continent may provide stability of supply from a mixture of many forms of renewable energy resources while taking advantage of spatial and temporal 
diversities. Solar and wind power are particularly problematic as variable generation resources. Weather patterns and daily cycles cause large swings in power output levels over short periods of time, forcing conventional fuel power plants to operate and fill in the gaps.

A study of wind generation diversity has been conducted using a three-year set of wind data. The data was collected by the National Renewable Energy Laboratory. The analysis of power production levels integrated across the Western U.S. reveals that weather patterns introduce correlations when wind farms are located less than 500 miles. There is a stronger correlation pattern in the east-west direction than in the north-south direction. By considering the power production levels over greater geographic regions, the variations in wind power production output levels stabilizes and is less prone to periods of large power generation drops.

\section{Dynamic Modeling of Energy Systems}

The impact of dynamic power levels caused by variable generation of renewable resources can be problematic in the operation and maintenance of conventional power plants, including nuclear power plants. A dynamic model has been developed for the power train and primary control loops of a light-waterreactor nuclear power plant. The model includes the electrical generation, low- and high-power turbines, the steam generator and throttle valve, and the reactor core. Dynamic loading of the power plant can be simulated with data from wind farms and utility time-domain demand plots. The transient and power variations can be observed flowing back towards the core. The model also permits the placement of a two-way valve to permit modeling of hybrid energy systems with various energy product production lines.

\section{Benefits to DOE}

The project aims to support the energy infrastructure goal of creating a more flexible, more reliable, and higher-capacity energy infrastructure. Specifically, the work aligns with the strategy of integrating advanced technologies by addressing technical issues with the integration of renewable and green energy resources to the electrical power grid. The work focuses on the development of study methods and analysis tools necessary for the study of energy storage systems for the electrical power grid, controls to maintain a balance between loading and generation variability of renewables (particularly solar and wind), and the impact of transmission SuperGrids to take advantage of the geographic diversity of renewables.

\section{Relevant Publications and Presentations}

Baldwin, T., M. West, and M. Reed, 2011, "Fault Locating in Distribution Networks with the Aid of Advanced Metering Infrastructure," IEEE Transaction on Industry Applications, submitted September 2011.

Baldwin, T., 2011, "Grid Integration of and Nuclear and Renewable Power," Conventional Generator Cycling and Variable Generation Workshop, Arlington, VA, June 14, 2011.

Phoenix, W., 2011, "Current Reactor Fleet Load Following - Overview," Conventional Generator Cycling and Variable Generation Workshop, Arlington, VA, June 14, 2011.

Garcia, H., 2011, "Hybrid Energy Systems with Nuclear Power," Conventional Generator Cycling and Variable Generation Workshop, Arlington, VA, June 14, 2011.

Patent Activities

The following patent disclosure has been submitted:

Baldwin, T., "Fault Location using Smart Meters," June 2011. 
$\Rightarrow$ Nuclear Nonproliferation Initiative

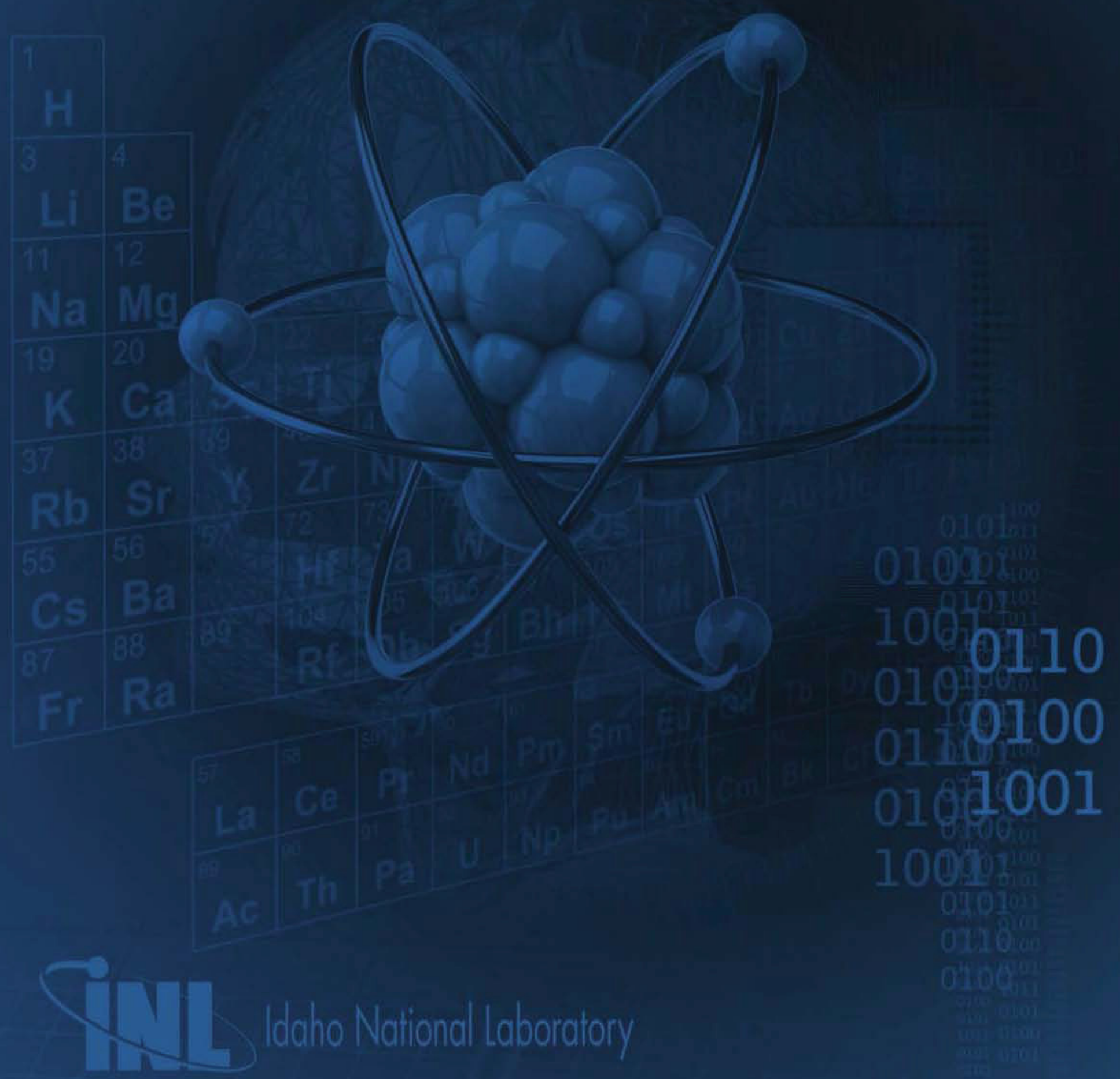




\section{Developing a Next-Generation, Risk-Informed Approach for Robust and Resilient Design Development}

Trond Bjornard, Curtis Smith, Gregory English, Jerry Phillips, Richard Metcalf, Jerry Weber, David Schwieder

08-100

The traditional approach to physical protection is based on the concept of designing to protect against a design basis threat (DBT), and the technique used to assess a system's performance against the DBT is called vulnerability assessment (VA). VA is performed according to highly prescriptive methodology and assumptions, including that the probability of attack is fixed (1.0) and consequences are not explicitly taken into account. Thus, VA considers explicitly only one of the three terms in the classic risk equation (risk $=$ frequency $\times$ probability of adversary success $\times$ consequences) and largely ignores the other two.

The fundamental premise of this project is that elements of risk-informed techniques from probabilistic risk assessment and safety can be applied usefully to the problem of physical protection and would have the potential to ameliorate some of the shortcomings of the DBT approach that has been shown to be inadequate in the safety world. A risk-informed approach to physical protection may consider a much broader variety of attack scenarios, threats, and consequences along with varying frequencies of attack. A significant challenge for riskassessment security is adequately addressing adversary behavior, traditionally regarded to be a showstopper for quantitative risk-assessment of security. A risk-informed approach should be useful in risk-managing security and informing security design and operational decisions.

\section{Project Summary}

The project team included a mix of security experts and probabilistic risk assessment/safety experts. The team collaborated on analysis of a sample physical protection problem performed two different ways: (1) traditional VA approach and (2) application of risk-informed elements where feasible.

The team concluded that elements of risk-informed techniques can indeed be (and in the case of some details, already are) usefully applied to security problems, though not as simply as a straight-forward application of probabilistic risk assessment as it currently exists, because of two factors: (1) the importance of timeline considerations in security analysis, where they are handled in much greater detail and somewhat differently than in PRA, and (2) the difficulty of quantitatively predicting the absolute frequency of attack. The team did conclude that it is reasonable to predict the relative frequency of attack by modeling the adversary's decision process for target selection.

The LDRD team members wrote three white papers describing each of three products produced by the project. Based on one of these papers, a PowerPoint presentation was developed for a meeting with the Nuclear Regulatory Commission to discuss a proposal to upgrade INL's existing probabilistic risk assessment models of the operating U.S. nuclear power plants to include security modeling. The meeting with NRC is expected to take place in the first quarter of FY 2012.

\section{Benefits to DOE}

The methodology produced by this project can be used for risk-managed security for nuclear and other complex installations, provided that the regulatory environment provides the flexibility to do so. In addition, the adversary target-selection model has the potential for broad use. The authors believe the concept to be a substantial improvement over known existing models and an important research area for DOE and other nuclear-energy stakeholders.

\section{Relevant Publications and Presentations}

Smith, C., D. Schwieder and T. Bjornard, 2011, "Augmenting Probabilistic Risk Assessment with Malevolent Initiators," IMECE201162556, Proceedings of the 14th International Mechanical Engineering Congress \& Exposition, IMECE 2011, Denver, CO, November 11-17, 2011. 


\section{Radionuclide Collection-Detection Device for the In Situ Remote Monitoring of ${ }^{99} \mathrm{Tc}$ as a Proliferation Indicator Mitchell Greenhalgh \\ 09-114}

This project is intended to produce the experimental data necessary for the development of an integrated radioisotope-collection-detection device capable of concentrating and detecting specific beta or alpha-emitting radioisotopes in situ and transmitting the data to offsite locations via telemetry and satellite link. The device will enable the target radioisotope to be concentrated inside the actual detector, providing very high counting efficiencies and the ability to passively collect the radionuclide while the device is in sleep mode. Specifically, the work under this proposal will be focused on a device for concentrating and measuring the fissionproduced radionuclide technetium- $99\left({ }^{99} \mathrm{Tc}\right)$, which can be used as a key identifier of nuclear fuel reprocessing to recover ${ }^{239} \mathrm{Pu}$ or uranium enrichment to produce highly enriched ${ }^{235} \mathrm{U}$. The ability to accurately detect and quantify low levels of ${ }^{99} \mathrm{Tc}$ is of primary importance in determining, monitoring, and assessing declared or undeclared nuclear proliferation activities.

\section{Project Summary}

Technetium-selective ion-exchange resins were prepared on three different polystyrene support materials with varying surface areas (900, 750, and $500 \mathrm{~m}^{2} / \mathrm{g}$ ) by impregnating the support material with a quaternary amine extractant. The amount of extractant added to the support material was varied to evaluate the extraction capacities of each support material. The surface areas of the newly prepared resins were measured to monitor the surface area effect of the resin preparation. Equilibrium batch contacts were performed with the developed ion-exchange resins with technetium-99m $\left({ }^{99 \mathrm{~m}} \mathrm{Tc}\right)$, technetium-99 $\left({ }^{99} \mathrm{Tc}\right)$, and rhenium $(\mathrm{Re})$ in southeastern Idaho well water with a $\mathrm{pH} \approx 8$. The data from these experiments was used to create isotherms of the quantity of ${ }^{99} \mathrm{Tc}$ absorbed versus the quantity remaining in the aqueous phase at equilibrium. The results indicated that a minimum extractant loading of $10 \mathrm{wt} \%$ is required to achieve concentration factors of $>10^{5}$ regardless of the support materials being used.

Based on these preliminary results, scintillating ion-exchange resins were prepared with extractant loadings $>10 \%$, various scintillating fluors, and wavelength shifters, and were evaluated for detection efficiency and extraction capacity. Extraction capacities of the scintillating resins were compared with the observed uptake capacities of the nonscintillating resins. The data indicated the detection efficiency of ${ }^{99} \mathrm{Tc}$ in the prepared resins ranged from 50 to $70 \%$, and the extraction capacity was only mildly affected by the additional loading with scintillating fluors and wavelength shifters. The results also showed that one support material obtained higher detection efficiencies than the other support materials independent of the fluor or wavelength shifter used.

Two candidate scintillating resins were prepared utilizing different fluors and extractant loadings on the superior support material. The resins were evaluated for equilibrium capacities and detection efficiencies. The data collected was used to calculate a theoretical minimum detectable activity and was fit to a Langmuir equation. The results indicate that a detection limit of 0.017 disintegrations per second can be achieved assuming a background count rate of 0.016 counts per second, a $70 \%$ detection efficiency and a seven-hour count time.

The necessary data has been collected, and a candidate extractive scintillating resin has been prepared and tested. A conceptual design of a ${ }^{99} \mathrm{Tc}$ collection-detection device has been completed, and it is anticipated that the candidate resin will be incorporated into the device. A presentation of the experimental results and conceptual device design was provided to National Nuclear Security Administration NA22. United States Patent No. 7,923,692 was awarded for this device.

\section{Benefits to DOE}

This work will fill a niche in proliferation detection for detecting undeclared activities and monitoring declared activities. Science and engineering advances gleaned from this project will benefit the National Nuclear Security Administration and the International Atomic Energy Agency in future research and development work. 


\title{
Neutron Spectrometer Development
}

\author{
David L. Chichester, James T. Johnson, Edward H. Seabury, Scott M. Watson
}

09-066

Research in the area of radiation instrumentation for nuclear nonproliferation and counterproliferation primarily focuses on gamma-ray spectrometers and gross thermal-neutron counting detectors; together these present a useful suite of tools for passive screening and active interrogation. One area that has received comparatively less attention has been instrumentation for neutron spectrometry. Neutron spectra contain valuable information about the source material from which they are emitted. For example, $(\alpha, n)$ neutron spectra tend to be highly structured; this structure can provide insight into the material absorbing the alpha particles and generating the neutrons (e.g., Be, B, $\mathrm{O}, \mathrm{F}$, etc.). Spontaneous and induced fission produce a smooth continuum of neutron energies that is distinctive from $(\alpha, n)$ spectra. For both types of sources the presence of intervening materials that scatter neutrons can be investigated using fast-neutron spectrometry by assessing the perturbation of the source neutron spectra. For example, neutron spectrometry may be used as a tool for nonproliferation transparency and verification measurements by providing data to determine if plutonium in an inspection object is in a metallic or an oxide form.

One method to determine the energy distribution of a fast-neutron radiation field is to use a ${ }^{3} \mathrm{He}$ ionization chamber and measure the amplitude of signal pulses from the device. In these detectors, neutrons are absorbed by ${ }^{3} \mathrm{He}$ in the ${ }^{3} \mathrm{He}(\mathrm{n}, \mathrm{p})^{3} \mathrm{H}$ reaction; the resulting proton and tritium atoms then proceed to ionize the gas in the ionization chamber. An electronic signal is registered as these electrons and ions are collected. Neutron capture in ${ }^{3} \mathrm{He}$ is exothermic with a $\mathrm{Q}$ value of $0.764 \mathrm{MeV}$; the amplitude of signal pulses resulting from neutron capture corresponds to the sum of this $\mathrm{Q}$ value plus the incident neutron's energy. If proper care is taken to eliminate spurious noise signals and to amplify the small current pulses without adding noise, a neutron energy resolution of better than $20 \mathrm{keV}$ can be achieved using this signal. Specialized ${ }^{3} \mathrm{He}$ ionization chamber designs have been developed to achieve this level of performance and are described in the literature; these instruments are occasionally referred to as Cuttler-Shalev (C-S) spectrometers, after two early researchers in the field. More sophisticated detectors have been designed that incorporate ${ }^{3} \mathrm{He}$ between opposing surface barrier detectors using solid-state electronics to record the coincident energy deposition from protons and tritium atoms directly, but they are generally limited to small sizes.

This LDRD project is directed towards the study and development of high-resolution fast-neutron spectrometry capabilities at INL exploiting the kinematic behavior of the ${ }^{3} \mathrm{He}(\mathrm{n}, \mathrm{p})^{3} \mathrm{H}$ reaction. The ultimate goal is to develop techniques for performing fast-neutron detection and spectrometry in the $0.05-5 \mathrm{MeV}$ range. The primary method for performing these measurements is to use the C-S style detector. Our focus this year has been towards improving the performance of the technique and performing additional demonstration experiments to illustrate performance capabilities and limitations.

\section{Project Summary}

Previous measurements with the neutron spectrometer revealed that the energy resolution of the system was poorer than the manufacturer's specifications. It was believed that the resolution could be improved by employing a higherresolution digitizer. This digitizer, with a higher bit depth, came with a couple of consequences, namely, a slower digitization rate and a lower gain amplifier. This resulted in the need for a preamplifier to boost the signal amplitude.

Energy resolution evaluation: The manufacturer specified that the detector's energy resolution for the thermal peak is less than 20 $\mathrm{keV}$; they had measured the resolution of the detector to be $16.6 \mathrm{keV}$. This resolution was not achieved with the 10-bit system. The 12-bit system was able to achieve the manufacturer's specification, but a correlation was noticed between the rise-time and the peak amplitude (Fig. 1, next page). A more sophisticated energy calibration method was developed that used the rise-time of the signal to compensate for what would otherwise be discarded events due to slower collection times. This improvement is seen in Figure 2 (next page), which shows the spectrum of a $2.5-\mathrm{MeV}$ (DD) neutron source as recorded with the 10-bit system and with the 12- 
bit system. The enhanced energy resolution of the 12-bit system is very apparent while the reduction of the unwanted features, such as recoil, is just as good as with the 10-bit system.

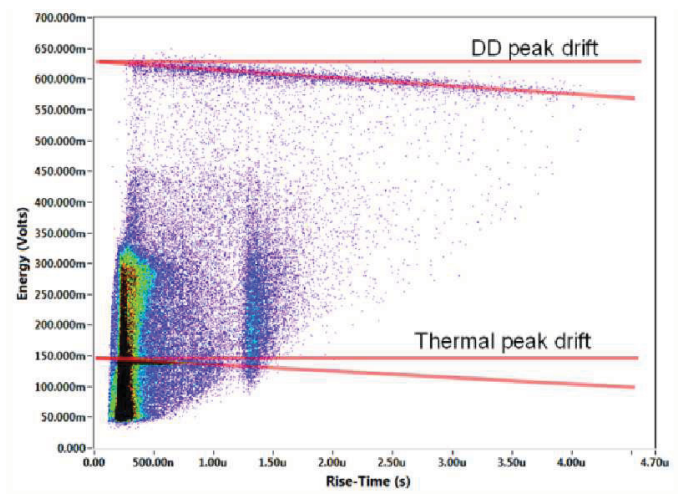

FIGURE 1. The C-S spectrometer peak-height drift with rise-time, using the new 12-bit digitizer.

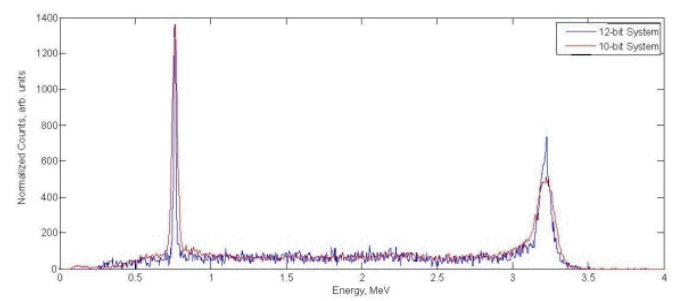

FIGURE 2. Comparison of the energy resolution of the thermal peak and the 2.5-MeV peak with the 10-bit and 12-bit digitizers.

The DD peak energy resolution of the 12-bit system was measured to be $80 \mathrm{keV}$. This is a marked improvement over the 10-bit system that was measured to be $140 \mathrm{keV}$. There was also a $20 \%$ improvement in the efficiency of the new process method with the 12-bit system. The improved efficiency is due to the higher fidelity of the 12-bit system as well as improved processing methods such as the rise-time compensation.
Transition measurement for process method validation: To validate the new equipment and processing algorithm, four transmission measurements were performed using water, graphite, a beryllium alloy, and an elemental simulant of the explosive Compound-4 (C-4). These materials were chosen because they contain elements that have distinctive neutron attenuation cross sections, and they were readily available. Californium-252 was used as the neutron source, and the target material was placed in between the detector and the source. Source-target-detector distances were adjusted in each case to maximize the solid angle but keep count rates low enough so that the data acquisition system was not overwhelmed. A no-target case was also recorded to determine an overall system efficiency curve that would be used to scale the processed spectrum and attempt to obtain an attenuation spectrum. This spectrum is compared to the neutron resonance cross sections of the primary elemental content of each target. Normalization of the data makes it possible to compare the data directly to the neutron resonant cross sections of the targets. Spectra for the four experiments are shown in Figure 3. The attenuation measurements show good agreement to the primary element's neutron resonance cross section for each target.

Deviations are attributed to other trace elements present but not included in the cross section and also in variation in neutron-scattering effects from the changing geometry in the experimental setups. 


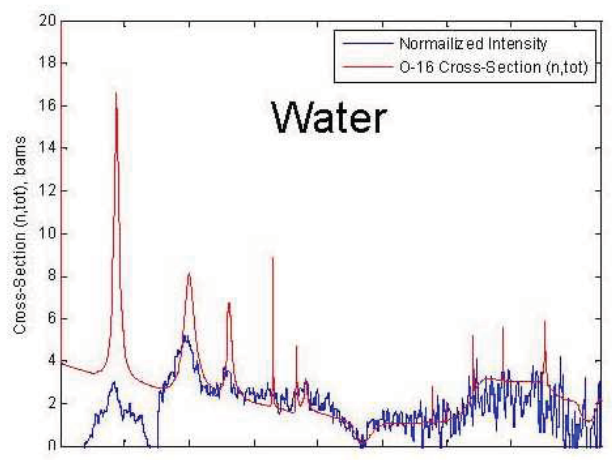

\section{Benefits to DOE}

Fast-neutron spectrometry may be used as a complementary analytical method to support nuclear nonproliferation and nuclear safeguards measurements. Neutron spectrometry may provide forensic-level evidence concerning the mean particle size of the subcomponents in some neutron sources. For safeguards or arms control applications, neutron spectrometry may be capable of discriminating metallic and oxide forms of plutonium.
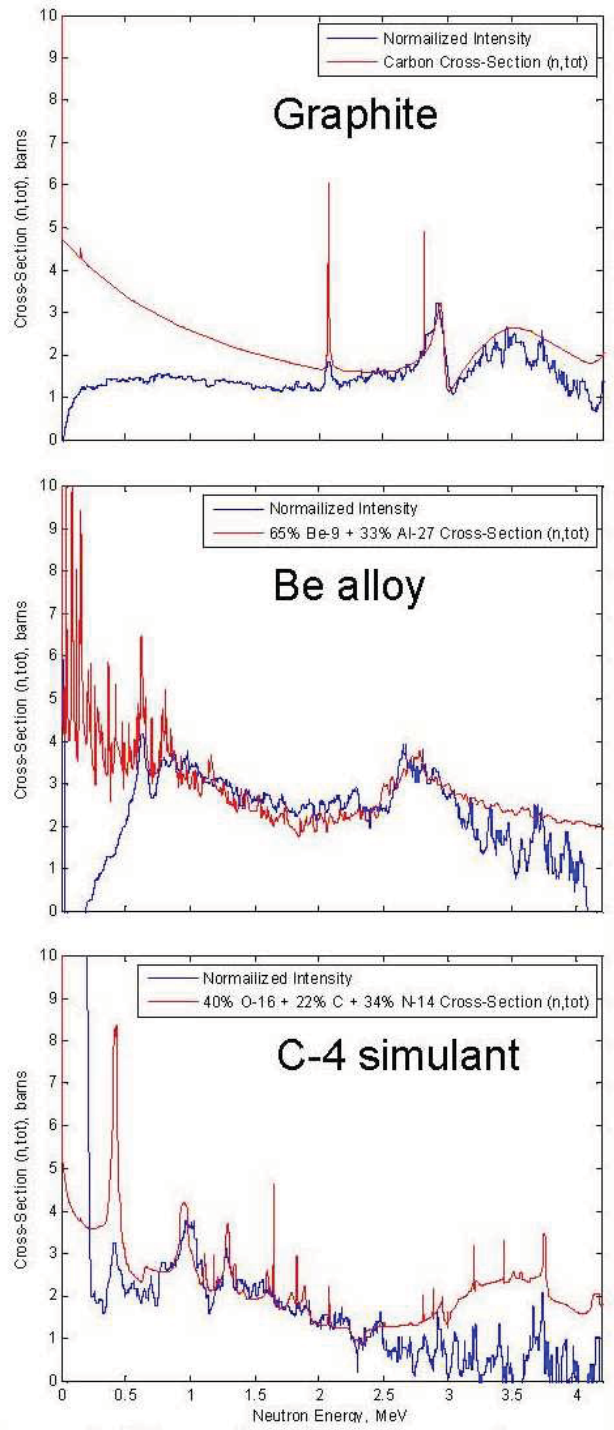

FIGURE 3. Normalized spectra and neutron cross sections for four cases. 


\title{
On-line Monitoring of Actinide Concentrations for Advanced Aqueous Separations
}

\author{
Jan-Fong Jue, Shelly X. Li, Steven Herrmann, Scott Herbst \\ 09-068
}

The reprocessing of spent nuclear fuels is an integral part of the next-generation nuclear fuel cycle. Real-time information on the actinide concentrations in the processing streams can enhance the transparency and accountability of special nuclear materials for these processes. Monitoring actinide concentrations in the process streams is very challenging due to the highly corrosive and radioactive environment involved. The main objective of this LDRD project is to develop a sensor-based actinide concentration monitoring technology for aqueous reprocessing systems. The advantages of this technology are simplicity, reliability, and high in situ sensitivity. Even though the basic theory for the potentiometric sensor is well known, the electrolyte materials for actinide monitoring sensor applications are not currently available. The ideal actinide sensor materials for the aqueous separation processes should have high trivalent ionic conductivity at room temperature, good corrosion resistance in acidic aqueous solutions, good radiation damage resistance, and quick response to concentration changes in the solutions. Identification, synthesis, and characterization of candidate trivalent ion conducting sensor materials for such applications were the initial tasks to be accomplished, followed by design of sensor assemblies and evaluation of their performance. In this LDRD project, cerium was selected as a surrogate for actinides.

\section{Project Summary}

The initial work of this project focused on developing a strategy for actinide sensor development. Two groups of ionic conducting materials were selected for more detailed study: (1) PVC-based polymer and (2) NASICONbased ceramic. After careful evaluation, NASICON-structured oxides $\left(\mathrm{Ce}_{0.1} \mathrm{Zr}_{0.9}\right)_{40 / 39} \mathrm{Nb}\left(\mathrm{PO}_{4}\right)_{3}$ were selected as the composition for a solid electrolyte due to its moderate trivalent ion conductivity and good stability in a high-radiation environment. Extensive effort was then devoted to synthesis of the material. A dense and nearly single-phase material was produced by improved fabrication techniques. The room temperature conductivity of $\left(\mathrm{Ce}_{0.1} \mathrm{Zr}_{0.9}\right)_{40 / 39} \mathrm{Nb}\left(\mathrm{PO}_{4}\right)_{3}$ measured by alternating current (AC) impedance spectroscopy met the conductivity requirements for the sensor applications $\left(>10^{-6} \mathrm{~S}^{-\mathrm{cm}^{-1}}\right)$. Before the LDRD project, only a Japanese research group had the capability to synthesize a similar trivalent conducting NASICON.

Sensor performance testing was the main focus in FY 2011. A custom setup was designed and manufactured. This setup consisted of two glass beakers coupled together on both sides of a solid electrolyte disc to form a concentration cell. Measurements of a range of test concentrations against a reference solution of one molar cerium nitrate were performed. The recorded signals agreed well with the potentials predicted by Nernst potential. This proved the concept of using trivalent conducting NASICON for aqueous sensor applications. The results of testing performed in acidic aqueous solutions are inconclusive at this time. However, no adverse corrosion effects on a NASICON solid electrolyte were observed after testing in acidic solutions for several hours.

Computer simulation was also carried out at Boston University in FY 2011 on the selected Ce-NASICON structure. The possible Ce conducting pathways were determined. This information is beneficial in explanation/ prediction of the selectivity of Ce-NASICON in complex aqueous solutions.

Benefits to DOE

A safe and proliferation-resistant fuel cycle is critical for U.S. nuclear industry and national energy security. Aqueous separation processes developed for treating spent nuclear fuels is a key component in this advanced fuel cycle. Successfully developing sensor-based actinide monitoring technology will provide a reliable and cost-effective way to monitor and safeguard spent-fuel treatment processing facilities. The near-term applications of this technology will be monitoring actinide concentrations during nuclear waste separation processes at INL and other DOE facilities. This sensor technology may also find applications in environmental monitoring for heavy metals. 
Relevant Publications and Presentations

Jue, J.-F., S. Herrmann, S. Herbst, S. Li, and S. Gopalan, 2011, "Development of Sensor Materials for On-line Process Monitoring," The 35th International Conference \& Exposition on Advanced Ceramics \& Composites (ICACC), Daytona Beach, FL, January 23-28,2011.

Jue, J.-F., S. Li, T. P. O'Holleran, S. M. Frank, and B. F. Cowan, 2010, "Development of Ceramic Sensors for On-line Actinide Monitoring," The 34th International Conference \& Exposition on Advanced Ceramics \& Composites (ICACC), Daytona Beach, FL, January 25-29, 2010. 


\title{
Develop a Safeguards Approach for INL Pyroprocessing Lines
}

\author{
Richard Metcalf, Robert Bean
}

09-117

Electrochemical refinement

(pyroprocessing) technology is being scaled up to industrial-scale facilities. This nuclear reprocessing technology can generate metallic uranium, metallic plutonium, and a metallic mix of transuranics. Unlike wet-chemistry reprocessing, pyroprocessing has not been widely deployed globally and, to date, the techniques to secure and safeguard this material have not been developed. Fundamentally, this LDRD was to assess an assumption that pyroprocessing can be safeguarded similarly to aqueous facilities.

Pyroprocessing is needed for rapid expansion of fast-reactor technology or other metal fuel and will only become acceptable if a comprehensive safeguards approach that can meet the International Atomic Energy Agency goal quantity and timeliness criteria (detection of $8 \mathrm{~kg} \mathrm{Pu}$ within one month) can be demonstrated. Otherwise, proliferation concerns will impede this technology. The assumptions in the approaches in previous work had not been tested. It is hypothesized, and now confirmed, by the authors that some of these assumptions, e.g., salt homogeneity or strict $\mathrm{Cm} / \mathrm{Pu}$ ratios, do not represent the actual conditions in a pyroprocessing facility. Furthermore, previous work did not address the unique attributes of pyroprocessing facilities, such as process variables (e.g., salt composition) tightly correlated to the final product, which can be turned into new safeguards methods that support the Goal Driven Safeguards and Safeguards by Design efforts at INL. This project has initiated further assessments of all aspects of security and safeguards for this technology, including additional diversion analysis, Materials Control and Accountability complication resolution, Signature and Observables studies, and technology development for a comprehensive safeguards approach.

\section{Project Summary}

The major assumption that the $\mathrm{Cm} / \mathrm{Pu}$ ratio was constant and easily measured was refuted, which removes the cornerstone assumption from all prior work on the safeguards methods of these facilities. The homogeneity assumption, the second most important assumption of the prior work, was also refuted. Voltage and other process-monitoring techniques that had been hypothesized as approaches for material accountability (dendrite analysis, for instance) were analyzed. The results confirmed that voltage can be used to measure and secure the process.

The LDRD generated an expansion to the Goal Driven Safeguards approach developed in prior years as applicable to pyroprocessing. Furthermore, individual promising technology areas (such as voltage monitoring) were expanded to recommend future development paths.

\section{Benefits to DOE}

This research has resulted in future research paths of interest to National Nuclear Security Administration and DOE- Office of Nuclear Energy Materials Control and Accountability campaign. 


\section{Laser-Induced Breakdown Spectroscopy: Development and Application of In Situ Elemental Analysis for Process Streams in Spent-Fuel Reprocessing Facilities}

Jill R. Scott, Jeremy Hatch, Cynthia Hanson, Timothy McJunkin, Andrew Effenberger, Jr. $07-052$

Improved monitoring of nuclear fuel processing will enable DOE and other interested parties (i.e., the International Atomic Energy Agency) to better control and monitor nuclear fuels and materials and ensure the security of the USA. The current state-of-the-art for monitoring such materials requires time-consuming sample preparation. Therefore, this research aims to develop a technology to remotely measure and analyze the real-time concentrations of special nuclear materials in process streams in spent-fuel reprocessing facilities. If successful, it would enhance the transparency of reprocessing facility operations and improve material control and accountability. Specifically, a state-of-the-art analytical technique, laser-induced breakdown spectroscopy (LIBS), will be investigated in spent fuel reprocessing facilities (e.g., pyroprocessing and aqueous processing). LIBS is an elemental analysis method based on the emission from a plasma generated by focusing a laser beam at the interface of the medium to be sampled. This technology is reportedly applicable to solids, liquids (including molten metals), and gases. The advantages of applying LIBS technology for reprocessing facilities are:

- Rapid real-time elemental analysis-one measurement/laser pulse, or average spectra from multiple laser pulses for greater accuracy in $<1$ minute

- Direct detection of elements and impurities in the system with low detection limitselement specific, ranging from $2-1000 \mathrm{ppm}$ for most elements

- Nearly non-destructive elemental analysis (about $1 \mu \mathrm{g}$ material).

The following are the two major challenges in applying this technology: (1) to obtain quantitative measurements in a complex matrix both from an elemental composition and spectral perspective and (2) to invent engineering designs for the analysis to be performed remotely via optical fiber, allowing measurements to be taken in highly radioactive reprocessing facilities.

\section{Project Summary}

During the first year of this project (FY 2007), the experimental apparatus for conducting LIBS on molten salts was developed using equipment and facilities at the University of California San Diego. LIBS experiments on molten salts containing $\mathrm{Cr}, \mathrm{Mn}, \mathrm{Co}, \mathrm{K}$, and $\mathrm{Li}$ were performed to investigate the best methods for performing LIBS on molten salts and developing calibration curves. In the second year (FY 2011), components were acquired to produce bench-scale mockups of a LIBS system at INL. A portable LIBS systems with fiber-optic laser and light collection was designed that should be amenable for conducting LIBS analyses on radioactive samples (e.g., $\mathrm{U}$ and $\mathrm{Pu}$ ). The performance of the INL LIBS system has been evaluated and optimized during the mockup stages.

\section{Benefits to DOE}

This research has provided INL with a plausible methodology for rapid, real-time elemental analysis of metals in molten salts for monitoring processes related to spent nuclear fuels. Successful completion of this project will help DOE reduce the risk of nuclear materials proliferation and provide technology to improve nuclear safeguards. In addition, these activities may prove useful to INL and DOE for developing more efficient actinide recycling under the Global Nuclear Energy Partnership or to support Next Generation Nuclear Plant activities. The LIBS system, especially if coupled with other INL LIBS technology, could allow for smaller, portable analysis instruments that could be used to monitor levels of $U$ and/or $\mathrm{Pu}$, including isotopic measurements to help ensure that these elements are accounted for and not diverted.

\section{Relevant Publications and Presentations}

Hatch, J. J., T. R. McJunkin, C. Hanson, and J. R. Scott, 2011, "Automated Interpretation of LIBS Spectra using Fuzzy Logic Inference," Applied Optics, in press.

Effenberger Jr., A. J., and J. R. Scott, 2011, "Effect of Atmosphere on Collinear Double Pulse Laser Induced Breakdown Spectroscopy," Analytical and Bioanalytical Chemistry, Vol. 400, pp. 3217-3227. 
Effenberger Jr,. A. J., J. R. Scott, and T. R. McJunkin, 2011, "Method and Apparatus for High Resolution Laser-induced Breakdown Spectroscopy (LIBS)," INL/BEA Docket No.BA-560. US Patent Pending-Application 13/183,228 filed on July 14, 2011.

Scott, J. R., 2011, “Technologies for Improved Sensitivity for Chemical Forensics," FIU Department of Chemistry Seminar and IFRI Frontiers in Forensic Science Summer Seminar Series, Florida International University (FIU) and International Forensic Research Institute (IFRI), Miami, FL, July 27, 2011 (invited).

Hatch, J. J., T. R. McJunkin, and J. R. Scott, 2011, "Automated Interpretation of LIBS Spectra using Fuzzy Logic Inference," $3^{\text {rd }}$ North American Symposium on LaserInduced Breakdown Spectroscopy (NASLIBS 2011), Clearwater, FL, July 18-20, 2011.
Scott, J. R., A. J. Effenberger Jr., and T. R. McJunkin, 2011, "Economical HighResolution Detection Method for LaserInduced Breakdown Spectroscopy (LIBS)," $3^{\text {rd }}$ North American Symposium on LaserInduced Breakdown Spectroscopy (NASLIBS 2011), Clearwater, FL, July 18-20, 2011.

Effenberger, A., and S. Buckley, 2007, "Detection of Common and Trace Elements in Molten Salts using LIBS," $2^{\text {nd }}$ North American Symposium on Laser-Induced Breakdown Spectroscopy (NASLIBS 2007), New Orleans, LA, October 8-10, 2007. 


\section{Object Reconstruction Technique for Use in Radiography}

Clinton DeW. Van Siclen

10-096

Techniques to produce 3D images from one or more 2D radiographs constitute a national security need. This need is driven by first-responder situations in which a suspicious package is found in a public space and x-ray radiographs are taken to ascertain the contents. Thus we must answer the following questions: (1) how many radiographs are needed to obtain a "useful" 3D reconstruction, (2) how do we know when that number is reached, and (3) how do we obtain a $3 \mathrm{D}$ reconstruction from a few $2 \mathrm{D}$ radiographs?

The $\mathrm{x}$-ray imaging exercise produces a set of linear equations that has to be solved for the $3 \mathrm{D}$ reconstruction (that is, for the x-ray attenuation coefficients associated with the voxels comprising the suspicious package). In the situation of interest here, where only a minimal number of radiographs are available, the set of equations will always be under-determined, meaning that there are an infinite number of 3D reconstructions that reproduce the radiographs. Our hope, then, is that there are features common to all or most of those reconstructions, such that a "useful" reconstruction can be determined.

Project Summary

An iterative method was derived for solving the under-determined set of linear equations. Of particular relevance to this project, the method can (1) generate all (infinite in number) 3D reconstructions, and (2) utilize additional equations not necessarily related to the imaging exercise to constrain the (infinite) set of solutions. Further, by regarding all solutions as points in a multi-dimensional space (the number of dimensions equals the number of voxels), the variation in the infinite set of reconstructions can be quantified and a criterion for deciding whether the number of radiographs is sufficient to give a useful 3D reconstruction can be found.

\section{Benefits to DOE}

This project benefits DOE's national security mission, which is accomplished in large part by radiation (neutron, gamma-ray, $\mathrm{x}$-ray) imaging.

\section{Relevant Publications and Presentations}

The technical paper "Iterative Method for Solution of Radiation Emission/Transmission Matrix Equations" is available at http://arxiv.org/abs/1101.0819. 


\section{High-Energy Photofission Signatures Study}

Mathew T. Kinlaw, Scott J. Thompson; Alan W. Hunt (Idaho Accelerator Center)

11-053

Prompt fission neutrons, delayed neutrons, and delayed gamma-rays have all been shown to be reliable signatures for detecting the presence of fissionable material and continue to be utilized in the many current active inspection technologies. As interests continue in technology that can detect and/or identify fissionable material at increasingly large standoff distances $(>100 \mathrm{~m})$, increasingly high beam energies $(\sim 50$ $60 \mathrm{MeV}$ ) are being sought in an effort to extend the distance at which targets of interest can be effectively stimulated. Past policy and logistical considerations, however, have minimized experimental studies of many of these signatures for inspection beam energies above $\sim 20 \mathrm{MeV}$. Despite considerable funding for modeling of these signatures and the current design and construction of standoff detection systems, few measured data sets on photofission emissions from commonly inspected materials exist for beam energies greater than $40 \mathrm{MeV}$. Further, with this increase in inspection beam energies comes an increase in the available photonuclear reaction channels in both fissionable and non-fissionable materials. Hence, this LDRD was directed at assessing gamma-ray and neutron emission from fissionable and non-fissionable materials when inspected with bremsstrahlung beam energies up to $\sim 44 \mathrm{MeV}$. Because interfering reactions will decrease the fissionable material sensitivity for standoff detection, particular attention was given to understanding and predicting the most probable interfering reactions.

\section{Project Summary}

Initially, experimental measurements were performed to assess two commonly utilized neutron signatures at beam energies of $\sim 40 \mathrm{MeV}$. Data collected for both the prompt and delayed neutron signatures suggested potential difficulties in discriminating fissionable and non-fissionable material. Typically, when prompt fission neutrons are sought, beam energies are limited to $\sim 10-12 \mathrm{MeV}$. At these lower beam energies, neutrons emitted from non-fissionable material are limited to energies well below those available from fission emissions. In contrast, absorption of a $40-\mathrm{MeV}$ photon by ${ }^{207} \mathrm{~Pb}$ allows neutrons to be emitted with energies exceeding $33 \mathrm{MeV}$. Overall, results of the prompt neutron data at $40 \mathrm{MeV}$ showed essentially no measurable differences in the prompt neutron signatures from ${ }^{238} \mathrm{U}$ and non-fissionable materials such as $\mathrm{Pb}$ and $\mathrm{Fe}$. There did appear to be a significant difference in the spectral shape generated from a Be target, perhaps suggesting further examination as a possible alternative signature. The delayed neutron signatures generated modestly improved results, with fissionable material count rates well above background. Delayed measurements with non-fissionable materials did suggest, however, that the potential exists for further interferences. When the measurements were finished, it was concluded that the possible interferences were generated by a substantial increase in prompt neutron production resulting from the increased beam energies. That production led to a marked swell in the number of neutrons interacting with the surrounding environment and reaching the detector systems at times indicative of delayed fission neutrons.

Once the preliminary measurements were completed, efforts were shifted to an empirical assessment of potential photonuclear reactions in non-fissionable materials. Isotopes with atomic masses from 1 to 120 were examined for those that could potentially emit delayed neutrons and/or gamma-rays with characteristics similar to those emitted from fissionable materials. Seventy-six possible photonuclear reactions were identified as producing daughter products capable of emitting signatures that could interfere with fissionable material detection. Of these 76 reactions, $72 \%$ have photonuclear thresholds above $20 \mathrm{MeV}$, and $75 \%$ have no experimentally validated cross-section data. Each of these reactions was recognized as potentially causing interferences, although further vetting based on the natural abundance of the target nuclei, branching ratio, and daughter product half-life resulted in nine reactions of interest. Only one of the nine identified reactions has available experimental cross-section data, and four do not have calculated cross-section data in the Evaluated Nuclear Data Files. For those reactions that have some available experimental and/or calculated cross-section data, the majority of the cross sections are relatively small; however, they are comparable to the ${ }^{18} \mathrm{O}(\gamma, \mathrm{p})^{17} \mathrm{~N}$ and the ${ }^{9} \mathrm{Be}(\gamma, \mathrm{p})^{8} \mathrm{Li}$ cross sections. Members of 
the research team have previously identified the latter two reactions experimentally as reactions that generate signatures comparable to fissionable material.

\section{Benefits to DOE}

Within the most recent Special Nuclear Materials Movement Detection Program Roadmap, provided by DOE's National Nuclear Security Administration (NA-22), detecting special nuclear material at standoff distances was presented as one of the three high-level program requirements. Further, this work readily supports the DOE strategy of providing and developing technologies to detect the proliferation of nuclear materials. By furthering the understanding of how characteristic signatures from fissionable isotopes are emitted and how best to utilize and integrate these signatures and mitigate potential interferences, this LDRD was aimed at enhancing the nation's ability to detect and interdict the illegal trafficking, or diversion, of nuclear materials.

\section{Relevant Publications and Presentations}

Hunt, A. W., et al., 2011, “Two Dimensional Signature Phase Space in Active Interrogation for the Detection of Fissionable Materials," Presented to John Hopkins Applied Physics Laboratory, John Hopkins University, September 2011.

Kinlaw, M. T., 2011, "Photo-Induced Signatures," Presented to the Strategic Advisory Committee, Idaho National Laboratory, July 2011.

Kinlaw, M. T., 2011, "Photo-Induced Signatures," Presented to the BEA S\&T Board of Directors, Idaho National Laboratory, July 2011. 


\section{Treaty Verification with Photo-Induced Prompt Signatures}

Mathew T. Kinlaw, Scott J. Thompson; Heather A. Seipel (Idaho State University);

Alan W. Hunt, Mike Smith (Idaho Accelerator Center)

11-054

A continued interest exists within DOE, as well as other U.S. Government agencies, for technologies that can reliably detect and locate special nuclear material for multiple applications. Within the last several years, the renewed focus on strategic arms reduction has further contributed to interests in nuclear material detection technologies for treaty verification and dismantlement applications. Hence, the current LDRD research examined the use of actively induced, prompt signatures for detecting and counting multiple sources of nuclear material. The principal methodology utilizes an inspection beam to generate prompt signatures for detecting and localizing high- $Z$ targets. Fusing multiple prompt signatures enables further discrimination of targets composed of nuclear material from those consisting of high-Z, non-nuclear material.

\section{Project Summary}

Initial efforts conducted under the LDRD focused on proof-of-concept measurements to assess the efficacy of combining gamma-ray signatures with a more conventional detection technique to produce 3D images of targets of interest. One-dimensional target localization was demonstrated with multiple targets positioned at various locations along the inspection beam axis. The 1D localization was then extended to produce 2D and 3D images. The top plot in Figure 1 shows a $2 \mathrm{D}$ image using only the time-domain signature; three discernible high-Z targets can be identified. The bottom plot in Figure 2 illustrates the advantage of enhancing the time-domain signature with an additional, conventional signature. These data, which have been normalized to account for the different target positions along the beam axis, indicate the two upper targets consist of nuclear material, while the bottom target, although high-Z, is benign. Figure 2 shows the 3D representation, with a $2 \mathrm{D}$ projection on the rear plane (this projection is the same data shown in the bottom plot of Figure 2).
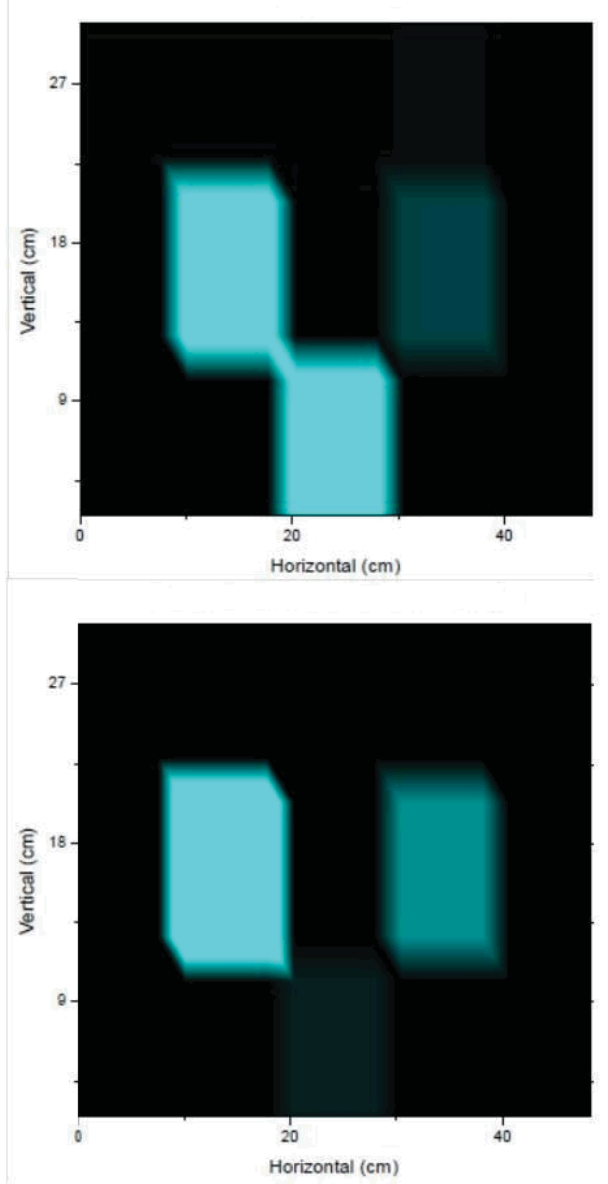

FIGURE 1. 2D time-domain imaging data (top). 2D timedomain imaging data fused with additional signature (bottom).

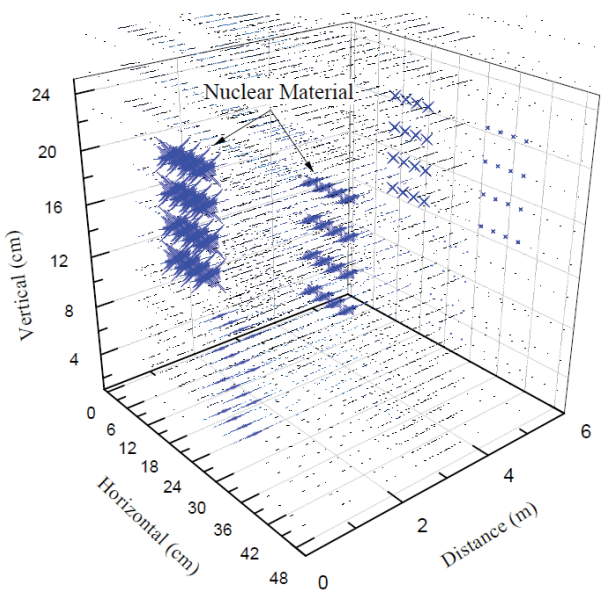

FIGURE 2. 3D representation of the data shown in the bottom plot in Figure 2. The data on the rear plane are a $2 D$ projection of the $3 D$ data. 
The second phase of the research focused on empirical calculations and modeling to more fully characterize the signature-generating mechanisms. Comparing simulation results provided an assessment of the dependence of the return signals on secondary particle production within the targets. Further, an examination of fluence on the front and rear faces of various thicknesses of detection filters showed a substantial decrease in the signature yield at the rear face of the filter when the lower-energy components of the secondary particles were restricted from further interacting within the target.

\section{Benefits to DOE}

This research demonstrated strong potential for application to treaty-verification scenarios, particularly for those scenarios in which technology for detecting and counting multiple sources of nuclear material is sought. Because the technique can be performed as a one-sided, end-on approach, its application space is not restricted by inspection scenarios where only limited physical access is available. The use of a sufficiently energetic photon beam allows materials of interest to be stimulated, even when those materials are located within considerable thicknesses of low-Z matrices (magazines, wood, etc.). Further, the primary return signature appears to be dominated by higher-energy, secondary particle production, allowing a greater level of penetration of the return signal compared with alternative techniques. Similarly, the additional available signatures are also inherently higher-energy, which enables greater penetration of these return signals. This technology could directly contribute to multiple additional nonproliferation applications. Finally, with the potential to generate both high- and lowresolution, 3D images, the technique could potentially provide characterization of suspect items for emergency response and non-nuclear illicit material applications (e.g., improvised explosive device detection/characterization).

\section{Relevant Publications and Presentations}

Kinlaw, M. T., 2011, "Photo-Induced Signatures," Presented to the Strategic Advisory Committee, Idaho National Laboratory, July 2011.

Kinlaw, M. T., 2011, "Photo-Induced Signatures," Presented to the BEA S\&T Board of Directors, Idaho National Laboratory, July 2011. 


\title{
Unconventional Radiation Detection Methods for Nuclear Nonproliferation, Safeguards, and Treaty Verification
}

\author{
David L. Chichester, Scott M. Watson, Edward H. Seabury, James T. Johnson
}

11-059

The detection, identification, and assay of special nuclear materials for nuclear nonproliferation, safeguards, and treaty verification applications has reached a relatively high level of technical maturity following over fifty years of direct and indirect research, development, and engineering in the field. High levels of sophistication have been reached for gamma-ray spectrometry; neutron detection, coincidence and multiplicity counting; radiation imaging, and health physics measurements for contamination detection and quantification. However, new measurement challenges have arisen over the past decade that require yet newer and more innovative technical solutions. The wide distribution and expansion of gaseous enrichment technology, and subsequent construction of enrichment facilities, has created significant nonproliferation measurement challenges for safeguarding world fissile material production capacity. The multinational embrace of pyroprocessing as a method for treating spent nuclear fuel has raised challenges for plutonium inventory safeguards and handling, especially on the Korean peninsula. And of course, the international threat of world terrorism networks has become of paramount concern for U.S. domestics and international security.

Some of the technical challenges that are not currently met by today's nuclear instrumentation technology include the need for wide-area, continuous radiation monitoring for safeguards monitoring inside centrifuge enrichment halls, wide-area radiation monitoring in higherthroughput spent nuclear fuel handling and processing facilities, noncontact sensing methods capable of working within the high-radiation fields of pyroprocessing hot cells, plutonium assay methods for advanced transuranic fuel forms, and high-data-rate signal processing for active interrogation of cargo and in stand-off screening systems. This project consists of a series of small concept developments aimed at trying advanced unconventional radiation detection methods to address these topics and other shortfalls. In some cases these approaches are unconventional simply because they branch out beyond traditional sensor reliance on neutron and gamma rays. In other cases they are unconventional because they seek to adapt measurement approaches developed in one field for use in a different way for very different applications.

The objective of this project is to examine unconventional radiation detection methods that have not been previously examined for nonproliferation, safeguards, and treaty verification applications and to assess their potential usefulness in these fields. The candidate technologies selected for the first year of this study are listed below. In most cases the equipment needed for these small projects was already available at INL; this LDRD project continues to leverage prior technology investments for counterproliferation and emergency-response applications to assess measurement techniques for nuclear nonproliferation missions:

- Ultraviolet imaging of low-activity alpha-particle contamination

- Infrared thermographic detection of radiation sources

- Whole-area radiation-field monitoring using scintillating-fiber detectors.

Project Summary

Ultraviolet imaging of low-activity alphaparticle contamination: Ionizing radiation passing through air leads to the ionization of nitrogen in the air; as this nitrogen de-excites, it is accompanied by the emission of nearultraviolet (NUV) light. NUV-sensitive optical imaging may be used to analyze this light to infer the spatial location and distribution of ionizing radiation fields in air. Due to their high linear energy transfer, alpha particles are the most readily located and detected using this technique. Overlaying visible light images onto ultraviolet light images of air fluorescence provides a novel, selective method for assessing spatially inhomogeneous ionizing radiation fields around alpha-emitting sources. The air ionization dose fields near alpha sources are quite high. Near a $0.185-\mathrm{MBq}^{241} \mathrm{Am}$ source, the air dose rate can be as high as $1.57 \mathrm{~Gy} /$ hour (157 Rad/hr). Experiments were performed to evaluate the detection of UV air fluorescence. The range of the high-energy alpha particles from ${ }^{241} \mathrm{Am}$ is clearly seen with the technique, as shown in Figures 1 and 2. 


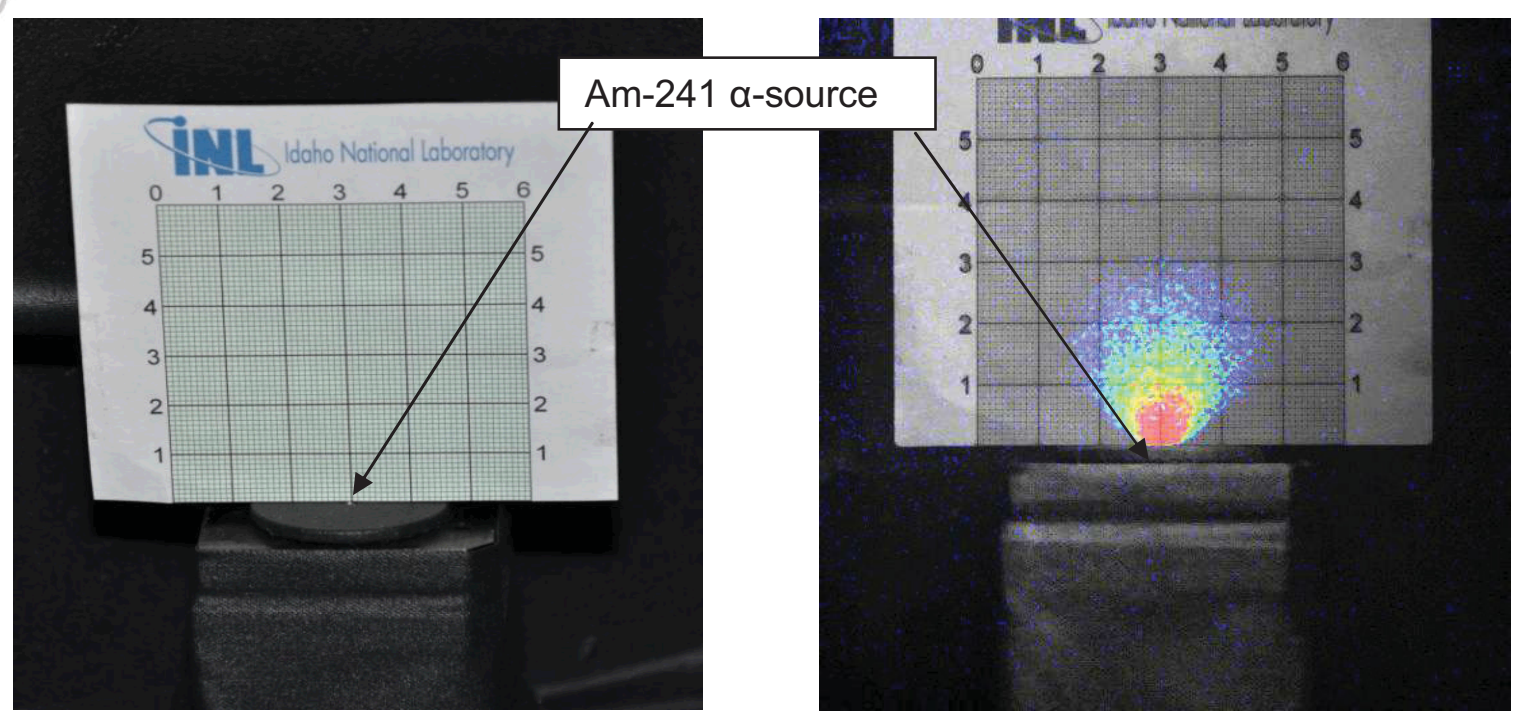

FIGURE 1. A close-up view of the source holder and the grid screen. The black lines in the grid screen are spaced $1 \mathrm{~cm}$ apart while the green lines in the screen are $0.1 \mathrm{~cm}$ apart. The paper grid was removed for the long duration UV exposure.

Multispectral imaging, combining ultraviolet and visible light data, can be used to locate and assess ionizing radiation in air. One particularly interesting application for this approach is the assessment of alpha-emitting radionuclides, but the capability also has the potential for investigating fluorescence induced via lower linear energy transfer quanta as well. The conversion from radiation quanta to electron-ion pairs in air to fluorescence light is inefficient in terms of energy conservation but does multiply the number of signal carriers. For alpha particles in air over 20 NUV photons, and possibly up to 200 NUV photons, are created from each alpha particle in the 4-5 MeV range. This increase in the number of signal carriers from decay events might be useful for noise reduction in some cases.

For alpha-particle measurements it is interesting to consider the radiation dose-in-air as a scaling factor for interpreting the NUV signals and for assessing the potential capability of this imaging technique for other radiation sources. For the small-scale alpha source studied here, air ionization near the source corresponded to a local dose rate of $157 \mathrm{Rad} / \mathrm{hr}$. This is much higher than might be generally expected because
FIGURE 2. A composite image with a black and white, visiblelight image of the grid screen in the background and the UV-light image generated from $\alpha$-particle-induced air fluorescence in the foreground. The UV-light image is a false color image, with red corresponding to high-intensity areas and blue corresponding to low-intensity areas.

conventional thinking from a health-physics perspective does not usually address the dose field near an alpha source unless it is ingested or inhaled. Misunderstanding of this aspect of alpha radiation sources could lead to confusion when extrapolating laboratory alpha-particle imagery measurements to other cases. Combining information about the efficiency for producing NUV light from air ionization, a proposed optical system's quantum performance, and its separation from the source location may all be used to make reasonable assessments of measurements for some applications by also considering the expected dose rates of those fields and comparing them with the radiation fields of the measurements described here and elsewhere.

Infrared thermographic radiation detection: Thermographic imagery has been suggested as a method for detecting and assessing shielded radiation sources, exploiting radiative decay heat as a detection signature. With this specific application in mind, a proof-of-principal experiment was conducted using a midrange (7.5-13 $\mu \mathrm{m})$ infrared thermal imaging camera (FLIR Systems AB, Model SC325) that was purchased for this project. Two small cast-iron 
pipe sections were placed in the field of view of our infrared camera. One pipe, our laboratory control item, was left alone. A small, filament light bulb was inserted into the second pipe; end caps were placed on the pipes. The electrical voltage and current used to power the light bulb was monitored and varied. The heated pipe was operated at varying power levels up to $1 \mathrm{~W}$; this level represents power levels encountered in traditional safeguards calorimetry measurements. The heated pipe was also monitored with a thermocouple. Images were collected at each power level once thermal equilibrium was observed in the thermocouple readings. Power levels of $0.02 \mathrm{~W}$ and above were clearly discernable over the background level of the control object.

Whole-area radiation field monitoring using scintillating-fiber gamma-ray detectors: Looking forward to future arms control treaties (the treaty after the New Strategic Arms Reduction Treaty [START], the Fissile Material Cut-Off Treaty, etc.), a new need will exist for monitoring fissionable materials in storage. Facility portal monitors can provide a coarse tool for material control and accountancy in these areas, but more comprehensive measurements will be needed for transparency and verification. To this end, we are studying the efficacy of using long-length scintillating fiber bundle detectors for monitoring fissile material storage areas for security and verification applications. SFB sensors may be able to provide lower cost, higher-coverage-area radiation sensors with continuous coverage over larger areas; they will also allow continuity of knowledge and are easier to install and maintain than discrete sensor instruments. These detectors may also be suitable for use in secure areas while leaving the data acquisition systems outside the facility (e.g., feeding the fibers through a port but leaving the photomultiplier tubes and data acquisition systems in a non-sensitive area).

\section{Benefits to DOE}

Unconventional radiation detection methods contribute to a number of DOE missions including advanced nuclear technology for the next-generation of nuclear power through the development of new diagnostic tools and spectroscopic capabilities (a) for use in basic nuclear science and engineering research and development, and (b) for practical use in advance nuclear system instrumentation and control, transformational nuclear safeguards, radiological safety and dosimetry, and environmental radiation monitoring and compliance verification. This work is applicable to DOE's environmental mission through development of advanced instrumentation capable of providing spectroscopic analyses of legacy waste packages from nuclear weapons production and nuclear fuel reprocessing, allowing more accurate and efficient material assays to be performed. It is also applicable to the DOE science and technology mission by providing a new technology base for scientific discovery and innovation, perhaps finding utility at DOE facilities including the Spallation Neutron Source (Oak Ridge National Laboratory), the National Ignition Facility (Lawrence Livermore National Laboratory), the Los Alamos Neutron Science Center (Los Alamos National Laboratory), the ZR-machine (Sandia National Laboratory), and the Advanced Test Reactor (INL). Finally, this work applies to DOE's national security mission through development of new measurement techniques for nuclear nonproliferation measurements related to advanced safeguards, nuclear forensics, emergency response, and nuclear material detection. 


\section{Pyro Processing Facility Nuclear Material \& Environmental Radiological Signature Characterization in the Hot Cell}

James D. West, Richard Metcalf, Brian Castle

11-067

Measurement and verification of current argon hot-cell radiological activity levels and in particular the radiological activity levels of the materials in situ in the Fuel Conditioning Facility (FCF) hot cell processing instrumentation and nuclear material movements is crucial to the development of new Safeguards regimes and instrumentation. Quantitative measurement of the current material in situ as well as qualitative measurement of the production processes must be performed to acquire real-time process and environmental data that support operator declarations concerning nuclear material processing and throughput. Acquisition of a real-time radioactivity thumbprint of FCF combined with additional Monte Carlo NParticle (MCNP) transport code modeling will provide a more accurate empirically based scalable model with which a baseline for Pyroprocessing Safeguards can be developed. Currently the International Atomic Energy Agency (IAEA) lacks proven safeguards regimes for application in pyroprocessing facilities. INL is uniquely positioned to analyze processes and day-to-day operations of pyroprocessing for safeguards applications. A number of countries under IAEA safeguards (South Korea, Japan, and India) are pursuing the use of electrochemical processes in support of fast-burner reactors.

Specifically, the installation of precisely shielded, moderated, and collimated neutron and gamma detectors combined with process monitoring and surveillance instrumentation to determine current radioactivity levels and identify the related processing steps within the FCF argon hot cell are required. Currently a high percentage of safeguards instrumentation is believed to be unable to survive the extreme environment of the hot processing areas in spent-fuel reprocessing facilities (e.g., pyroprocessing and aqueous processing). The major challenges in applying current technologies in spent-fuel reprocessing facilities are to obtain quantitative measurements in a complex matrix both from an elemental composition and spectral perspective and to acquire operational data for analysis in an extreme environment. Successful data acquisition will enable facility modeling. Accurate modeling will provide a foundation to begin to design a safeguards approach for implementing instrumentation capable of surviving the highly radioactive and extreme reprocessing facility environment.

\section{Project Summary}

The project team installed four DCM-14 IAEA Safeguards cameras into the observation room in the center of the FCF hot cell. The cameras are configured to trigger on four different event configurations. The cameras store all collected data on an on-board Personal Computer Memory Card International Association Type 2 flash memory card configured with Advanced Encryption Standard encryption. The cameras are sealed and capable of nearly 30 frames per second recording speed. The current configuration of the cameras records $3-5$ pre- and post- event pictures. The team also acquired significant historical data from the facility which provides previous hot-cell radiation survey results for FY 2001 and FY 2007. The team also completed a set of hotcell neutron radiation measurements and is awaiting the data reports on the measurements. This is the first known measurement of the neutron flux in the FCF hot cell.

MCNP models provided theoretical estimates of the expected dose, given the known parameters of the facility and previous fuel processing information. MCNP model uncertainty in the generated models is a result of previous incomplete data regarding the actual neutron fields and neutron energies within the hot cells. Calculations were performed using measured material mass and activity levels prior to introduction to the hot cell to build as accurate as possible neutron flux, activity, and energy-level based MCNP models.

Overall the data was acquired to build an accurate radiation characterization map of the hot cell to use for future instrument MCNP analyses for improved simulation of instrument performance and survivability while fielded in hot cells.

\section{Benefits to DOE}

The invested research areas of the "INL Nuclear Nonproliferation Initiative" are (1) transformational safeguards, (2) proliferationresistant and secure nuclear materials and 
processes, and (3) signatures and detection. The proposed research is specifically targeted for Area 2 in support of DOE's national security priorities to promote international nuclear safety and advance nuclear non-proliferation.

Relevant Publications and Presentations

Castle, B., R. Metcalf, and J. West, 2011, "Neutron Measurements in FCF Hot Cells, Technical Evaluation Study," Document ID TEV-1369, REV 0, September 30, 2011. 
$\Rightarrow$ Institute for Nuclear Energy S\&T

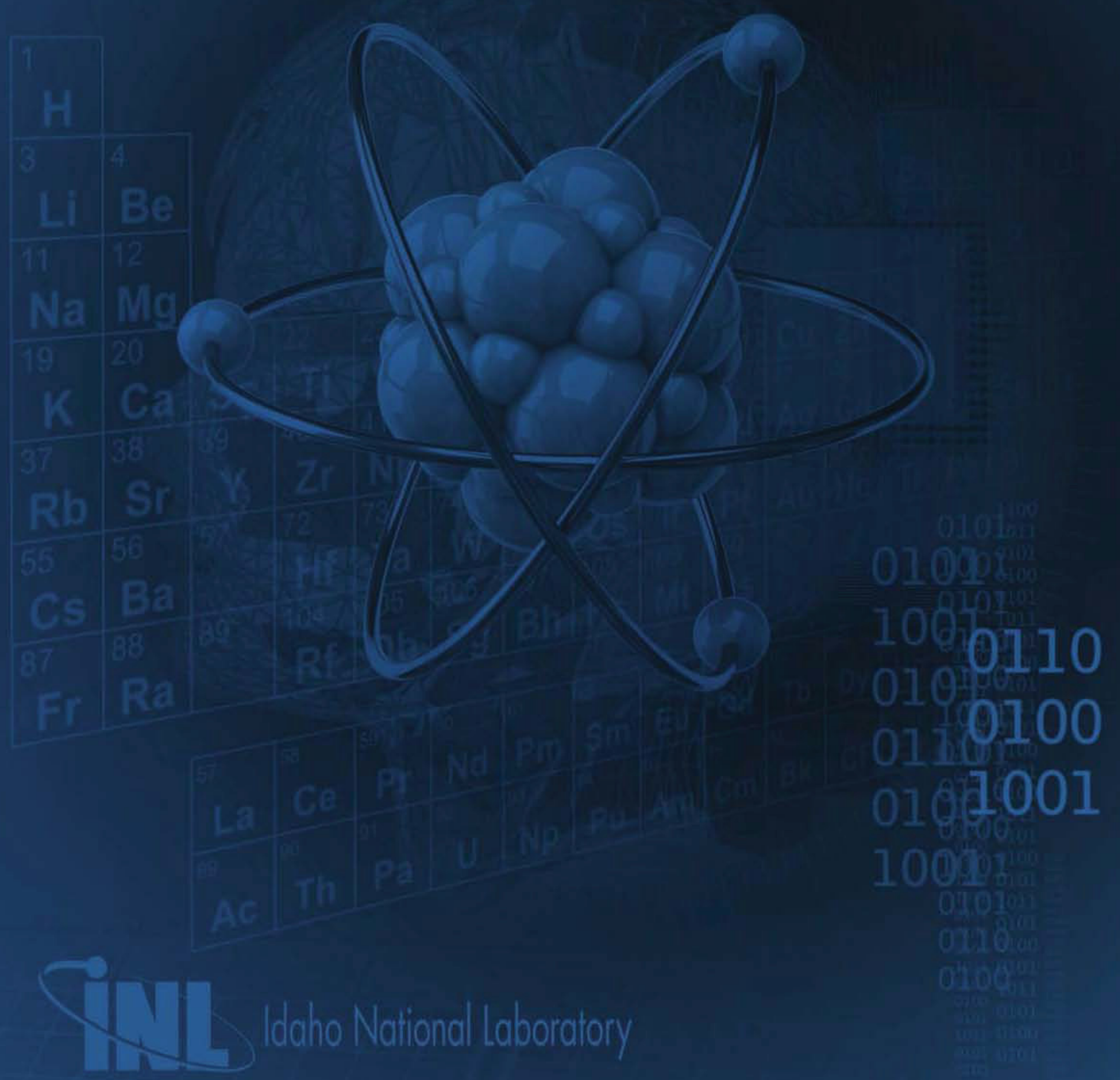




\section{Evaluation of Covalent Interactions in Actinide-Coordination Compounds}

Dean R. Peterman; Dale D. Russell (Boise State University)

09-071

Complexing agents containing soft-base (e.g., $\mathrm{N}$ or $\mathrm{S}$ ) donors such as dithiophosphinic acids have significant potential for the efficient separation of trivalent actinides, An(III), from lanthanides, $\mathrm{Ln}(\mathrm{III})$. A family of dithiophosphinic acids (DPAH) developed at INL has shown unprecedented selectivity for americium and curium over lanthanides in acidic solution. We propose to study the coordination and separations chemistry of the INL DPAH compounds with U(III), Np(III), Pu(III), Am(III), and $\mathrm{Cm}$ (III). As the actinide series is crossed from $\mathrm{U}$ to $\mathrm{Cm}$, the extent of covalency observed in the metal ligand bond is expected to decrease due to the decrease in the spatial extent of the $5 \mathrm{f}$ orbitals. UV-vis, infrared, and Raman spectroscopies can be used to quantify the extent of covalency in the An(III)-DPAH coordination compounds. By systematically studying variations in An(III)-DPAH coordination complexes, we plan to develop a detailed understanding of the interactions of $\operatorname{Ln}(\mathrm{III})$ and An(III) ions with soft donor ligand systems. This detailed understanding holds the promise of providing great insight into the nature of actinide interactions with soft-donor bases.

\section{Project Summary}

Collaboration with Prof. Dale Russell of the Chemistry Department at Boise State University continued during FY 2011. The goal of this collaboration was the continued development of a detailed understanding of the electrochemical conditions necessary for the production of trivalent actinide species in aqueous and organic media.

A master's student, Jonathan Scaggs, working in Prof. Russell's Boise State University laboratories has demonstrated the electrochemical reduction of $\mathrm{U}(\mathrm{VI})$ to $\mathrm{U}(\mathrm{IV})$ using a Pt working electrode. Due to the very high reduction potential required, this electrode material was not suitable for the electrochemical production of U(III). A boron-doped diamond electrode material was subsequently investigated for the U(IV) to U(III) reduction. During FY 2011, the electrochemical preparation of $\mathrm{U}(\mathrm{III})$ using the boron-doped diamond electrode material was demonstrated in aqueous solution using several electrolytes.
The glove box, which was installed during FY 2009, was used to conduct experiments with macroscopic quantities of uranium, plutonium, and americium. Unfortunately, attempts to maintain an inert atmosphere in this glove box were not successful. The presence of oxygen in the atmosphere of the glove box prevented the preparation of U(III).

\section{Benefits to DOE}

By investigating potentially superior technology for the selective separation of minor actinides from used fuel, the project:

1. Addresses the need for improved nuclear waste clean-up processes that mitigate the risks and hazards posed by the legacy of nuclear weapons production and research

2. Supports the United States' and DOE's leadership in science and technology of the nuclear fuel cycle

3. Promotes international nuclear safety and nonproliferation.

\section{Relevant Publications and Presentations}

Peterman, D. R., M. R. Greenhalgh, R. Tillotson, J. R. Klaehn, M. Harrup, T. Luther, and J. D. Law, 2011, "Evaluation of Covalent Interactions in Actinide Coordination Compounds," Invited presentation, Boise State University, Boise, Idaho, March 4, 2011.

Peterman, D. R., M. R. Greenhalgh, R. D. Tillotson, J. R. Klaehn, M. K. Harrup, T. A. Luther, and J. D. Law, 2011, "Evaluation of Covalent Interactions in Actinide Dithiophosphinate Coordination Compounds," Invited presentation, Tennessee Technological University, Cookeville, TN, April 15, 2011.

Ogden, M. D., D. R. Peterman, R. Tillotson, and J. R. Klaehn, 2010, "Aggregation and Separation Behavior of Symmetrical Dithiophosphinic Acids for 'Minor Actinide' Partitioning," NORM 2010, Pullman, WA, June 30, 2010. 
Peterman, D. R, L. Martin, J. R. Klaehn, M.

Harrup, M. R. Greenhalgh, and T. Luther, 2009, "Selective Separation of Minor

Actinides and Lanthanides using Aromatic

Dithiophosphinic and Phosphinic Acid

Derivatives," Journal of Radioanalytical

and Nuclear Chemistry, Vol. 282, pp. 527-

531. 


\section{Measurement of Degradation Products of Irradiated Ligands Used in Separations Measuring Actinide Speciation in High-pH Solutions}

Gary S. Groenewold, Gracy Elias, Bruce Mincher

09-084

The separation of metals from solutions derived from used nuclear fuel is important for minimizing quantities of nuclear waste and for recycling heavy elements. The most widely used approach for achieving separations is liquid extraction, in which metals are selectively partitioned from a nitric acid aqueous phase into an organic phase. To accomplish this, metals are complexed with organic ligand molecules that are selective for specific metals but not for others. The complexes that are formed are hydrophobic and are transferred to the organic phase, thus achieving separation from metals left behind in the nitric acid. The PlutoniumURanium EXtraction (PUREX) separation of $U$ and $\mathrm{Pu}$ from fission products relies on this approach, with the two $5 \mathrm{f}$ elements forming hydrophobic complexes with tri-n-butyl phosphate.

One potential issue with the solvent extraction approach is that radiation can produce different complexing molecules from the organic ligands. These new species can form alternative coordination complexes and have a deleterious effect on separation efficiency. Hence, understanding changes occurring to the organic ligands under high-radiation conditions has motivated research to characterize and measure the radiolytically formed complexing molecules. Such measurements are challenging because the new compounds tend to strongly adhere to many surfaces, and the background of the intact ligands tends to overwhelm detection devices.

One approach that has potential to overcome these difficulties is electrospray ionization-mass spectrometry (ESI-MS), which functions by spraying charged droplets of analyte solutions at a sampling aperture of a mass spectrometer. The droplets undergo evaporation and Rayleigh discharge events that have the effect of moving the solutes into the gas phase. The objective of this LDRD was to determine whether new measurement methods could be tailored for compounds produced by irradiation of the organic ligand molecules. This objective represents a Year 3 re-direction of the LDRD; the first two years of the project were focused on development of ESI-MS and desorption ESI-MS for measuring composition and structure of metal coordination complexes.

\section{Project Summary}

An ESI-MS capability was developed and applied to several different ligands that had been subjected to irradiation. The capability includes both quadrupole ion trap and high mass resolution time-of-flight ESI-MS instruments that provide both multiple-stage dissociation reactions and accurate mass measurement. This combination addresses both composition and structure queries. Ligand systems investigated included N,N-di-isobutyl carbamoylmethyl (octyl,phenyl)phosphine oxide, lactic acid, Cyanex 301 and related dithiophosphinates, and N,N'-dimethyl-N,N'-dioctyl-hexyl-ethoxymalonamide. In each case, significant radiolysis pathways were determined based on identification of newly formed organic degradation products.

In addition, one ESI-MS instrument was modified to conduct desorption electrospray experiments. These involved orienting the ESI capillary in a co-linear fashion with the MS ion aperture. A target capillary supplied the analyte solution and was placed in the pathway of the primary ESI droplets. Impact of the ESI droplets with the liquid from the target capillary produced high-intensity mass spectra from as little as $10^{-11}$ moles of cerium-ligand coordination complexes, demonstrating the potential for measuring tiny volumes of radionuclide-containing solutions.

\section{Benefits to DOE}

This research project has produced a new capability for INL - enabling mass spectrometric analyses of radiolysis products and metal coordination complexes. This is important for improving understanding and control of nuclear separations and metal speciation in a wide variety of environments.

\section{Relevant Publications and Presentations}

Groenewold, G. S., and J.-J. Gaumet, 2011, " $\mathrm{Ce}^{3+}$-Tributyl Phosphate Coordination Complexes Produced by Desorption Electrospray Ionization of Solutions," Journal of Mass Spectrometry, submitted. 
Groenewold, G. S., M. J. van Stipdonk, J. Oomens, W. A. de Jong, and M. E. McIlwain, 2011, "The Gas-Phase BisUranyl Nitrate Complex [(UO2)2(NO3)5]-: Infrared Spectrum and Structure," International Journal of Mass Spectrometry, in press.

Dain R. P., G. Gresham, G. S. Groenewold, J. D. Steill, J. Oomens, and M. J. van Stipdonk, 2011, "IRMPD Spectroscopy of Group II Metal Complexes with Salicylate," Rapid Communications in Mass Spectrometry, Vol. 25, pp. 1837-1846.

Gresham, G. L., A. Dinescu, M. T. Benson, M. J. van Stipdonk, and G. S. Groenewold, 2011, "Investigation of Uranyl Nitrate Ion Pairs Complexed with Amide Ligands using Electrospray Ionization Ion Trap Mass Spectrometry and Density Functional Theory," Journal of Physical Chemistry A, Vol. 115, pp. 3947-3508.

Groenewold, G. S., A. D. Appelhans, M. E. McIlwain, and G. L. Gresham, 2011, "Characterization of Coordination Complexes by Desorption Electrospray Mass Spectrometry," International Journal of Mass Spectrometry, Vol. 301, pp. 136142.

Groenewold, G. S., 2011, “Gas-Phase Investigations of Uranyl Coordination Chemistry," Invited talk, Glenn T. Seaborg Center Seminar Series, Lawrence Berkeley National Laboratory, Berkeley, CA, May 11, 2011.

Elias, G., G. S. Groenwold, B. J. Mincher, and S. P. Mezyk, 2011, "Analytical Techniques to Detect and Quantify CMPO and its Radiolysis Products," Fall 2011 National Meeting \& Exposition of the American Chemical Society, Denver, CO, Aug. 28Sept. 1, 2011.

Groenewold, G. S., M. J. van Stipdonk, J. Oomens, W. A. de Jong, G. L. Gresham, and M. E. McIlwain, 2010, "Vibrational Spectra of Discrete UO22+ Halide Complexes in the Gas Phase," International Journal of Mass Spectrometry, Vol. 297, pp. 67-75.
"Electrospray Ionization of Metal Species," Groenewold, G. S., In Beauchemin D., and Matthews D. E., editors, Elemental and Isotope Ratio Mass Spectrometry (Gross, M. L. and Caprioli, R.M., editors. The Encyclopedia of Mass Spectrometry, Vol. 5.) Elsevier Science, Inc., Amsterdam, 1088p, pp. 361-368.

Groenewold, G. S., W. A. de Jong, J. Oomens, and M. J. van Stipdonk, 2010, "Variable Denticity in Carboxylate Binding to Uranyl Coordination Complexes," Journal of the American Society for Mass Spectrometry, Vol. 21, pp. 719-727.

Dain, R. P., C. M. Leavitt, J. Oomens, J. D. Steill, G. S. Groenewold, and M. J. van Stipdonk, 2011, "IRMPD Spectroscopy of Sodium and Potassium Chlorate Anions," Rapid Communications in Mass Spectrometry, Vol. 24, pp. 232-238.

Groenewold, G. S., C. M. Leavitt, R. P. Dain, J. Oomens, J. D. Steill, and M. J. van Stipdonk, 2009, "Infrared Spectrum of Potassium-Cationized Triethylphosphate Generated Using Tandem Mass Spectrometry and Infrared Multiple Photon Dissociation," Rapid Communications in Mass Spectrometry, Vol. 23, pp. 2706-2710.

Aubriet, F., J.-J. Gaumet, W. A. de Jong, G. S. Groenewold, A. K. Gianotto, M. E. McIlwain, M. J. van Stipdonk, and C. M. Leavitt, 2009, "Cerium Oxyhydroxide Clusters: Formation, Structure and Reactivity," Journal of Physical Chemistry A, Vol. 113, pp. 6239-6252.

Leavitt, C. M., V. S. Bryantsev, W. A. de Jong, M. S. Diallo, W. A. Goddard III, G. S. Groenewold, M. J. Van Stipdonk, 2009, "Addition of $\mathrm{H} 2 \mathrm{O}$ and $\mathrm{O} 2$ to Acetone and Dimethylsulfoxide Ligated Uranyl(V) Dioxocations," Journal of Physical Chemistry A, Vol. 113, pp. 2350-2358.

Leavitt, C. M., J. Oomens, R. P. Dain, J. Steill, G. S. Groenewold, and M. J. Van Stipdonk, 2009, "IRMPD of Anionic Group II Metal Nitrate Cluster Ions," Journal of the American Society for Mass Spectrometry, Vol. 20, pp. 772-782. 
Groenewold, G. S., M. J. van Stipdonk, W. A. de Jong, and J. Oomens, 2009, "IRMPD of Uranyl Coordination Complexes," 25th Asilomar Conference on Mass Spectrometry: Ion Spectroscopy, Pacific Grove, CA, October 16-20, 2009.

Groenewold, G. S., M. J. van Stipdonk, W. A. de Jong, L. Visscher, and B. Siboulet, 2009, "IR and DFT Studies of Dioxo U(V,VI) Complexes," Invited talk, 238th American Chemical Society National Meeting and Exposition, Presentation NUCL 145, Washington, DC, August 16-20, 2009.

de Jong, W. A., G. S. Groenewold, M. J. van Stipdonk, and F. Aubriet, 2009, "Computational Chemistry and Gas-Phase Experiments Working Together to Gain Insights in Actinide Chemistry," 238th American Chemical Society National Meeting and Exposition, Presentation NUCL 52, Washington, DC, August 16-20, 2009.

van Stipdonk, M. J., S. S. Curtice, R. P. Dain, G. S. Groenewold, G. L. Gresham, J. D. Steill, and J. Oomens, 2009, "IRMPD Spectroscopy of Metal Complexes with Hydroxamides and Salicylic Acid," International Mass Spectrometry Conference, Presentation 1248, Bremen, Germany, August 30-September 4, 2009.
Groenewold, G. S., M. Kullman, R. P. Dain, J. Oomens, J.D. Steill, and M. J. van Stipdonk, 2009, "Structural Characterization of Gasphase Uranyl Trihalide Anions Using IRMPD Spectroscopy," 57th ASMS Conference on Mass Spectrometry and Allied Topics, Philadelphia, PA, May 31June 4, 2009.

Dain, R. P., C. M. Leavitt, J. Oomens, J. D. Steill, G. S. Groenewold, and M. J. van Stipdonk, 2009, "IRMPD Spectroscopy Investigation of Gas-Phase Sodium and Potassium Chlorate Anions," 57th ASMS Conference on Mass Spectrometry and Allied Topics, Philadelphia, PA, May 31June 4, 2009.

Groenewold, G. S., 2009, "Infrared Spectroscopy of Uranyl Coordination Complexes," Invited lecture, Very Heavy Metals 2009

Conference, Canet-Plage, France, May 27June 1, 2009. 


\section{Isotope Ratio Measurements Methods for Direct Analysis of Samples}

Jill R. Scott, Andrew J. Effenberger, Jr., Timothy R. McJunkin

09-087

Both the Nuclear Nonproliferation Initiative and the Separations and Actinide Science Distinctive Signature have identified the need to develop advanced detection technologies for identifying nuclear signatures (i.e., $\mathrm{Pu}$ and $\mathrm{U}$ isotope ratios) from a variety of sample types. The current workhorse for determining isotope ratios is thermal ionization mass spectrometry (TIMS). Two of the limiting factors for application of TIMS are: (1) it requires extensive time-consuming sample preparation, and (2) its ionization efficiency is reported to be only 0.2 to $10 \%$. Both of these factors contribute to its incompatibility with the analysis of small particles and heterogeneous environmental samples. Therefore, we are developing two laser-desorption-based methods for the analysis of isotope ratios from heterogeneous samples with little or no sample preparation: (1) laserinduced breakdown spectroscopy (LIBS) and (2) laser desorption/ionization Fourier transform ion cyclotron resonance mass spectrometry. Both of these techniques will be coupled with our highly accurate laser positioning system associated with the laser-based optical and chemical imager (LOCI) that allows surfaces of heterogeneous samples to be mapped.

\section{Project Summary}

During the first year (FY 2009), we focused on incorporating a new single-photon ionization source and improvements to cell design and electronics used for laser desorption/ ionization Fourier transform ion cyclotron resonance mass spectrometry. In Year 1, we also conducted a preliminary investigation on the best methods to improve LOCI's optical detection for detecting isotope ratios via LIBS. The keys to improvement required understanding the best vacuum or low-pressure environment conditions and how the high magnetic field effects the LIBS plume. To this end, we constructed a test chamber to explore the vacuum/pressure conditions separate from the magnetic field effects. During FY 2010, we (1) conducted studies on the effect that different atmospheric conditions have on LIBS plume and spectra, especially vacuum and gases (e.g., Ar and He); (2) explored improved sensitivity using doublepulsed LIBS at the various conditions, including the variations in experimental arrangements; and
(3) designed and tested an optical detection methodology that should allow observation of high-resolution spectra for desired isotopes more economically and in a more compact form factor compared to the traditional, expensive 1-2-m long spectrographs. In FY 2011, a Fabry-Perot etalon was incorporated with a Czerny-Turner spectrograph and intensified charge coupled device camera to enhance the resolution to $\sim 45,000(\lambda / \Delta \lambda)$ or $\sim 10 \mathrm{pm}$, which is sufficient to separate some isotopic lines of elements of interest. A method to improve the resolution by another factor of three was also conceived that would allow this concept to apply to almost all isotopes of interest with a LIBS instrument with a small, compact footprint.

\section{Benefits to DOE}

While the technologies being developed in this project are not yet fully realized, they have attracted interest from DOE and other agencies (i.e., the International Atomic Energy Agency) because of the potential to eliminate the time-consuming and costly sample preparation associated with isotope ratio analysis by TIMS. These new capabilities, coupled with the imaging capability of LOCI, would allow heterogeneous samples to be analyzed via the imaging capability of LOCI as opposed to the bulk analysis provided by TIMS. This type of capability would be useful for nonproliferation analyses as well as analyzing nuclear fuels to determine burn-up rates, which could lead to better, more efficient designs of nuclear fuel. The new high-resolution LIBS capability has the potential to allow essentially real-time monitoring of isotope ratios with an inexpensive, compact instrument, which would be of great value for the International Atomic Energy Agency. Thus, this work can help DOE and the nation in the area of nuclear energy, in the arena of both national security and energy efficiency.

\section{Relevant Publications and Presentations}

Effenberger Jr., A. J., T. R. McJunkin, and J. R. Scott, 2011, "Practical High Resolution Detection Method for Laser-Induced Breakdown Spectroscopy (LIBS)," Applied Optics, submitted. 
Effenberger Jr., A. J. and J. R. Scott, 2011, "Effect of Atmosphere on Collinear Double Pulse Laser Induced Breakdown Spectroscopy," Analytical and Bioanalytical Chemistry Vol. 400, pp. 3217-3227.

Scott, J. R., 2011, "Technologies for Improved Sensitivity for Chemical Forensics," FIU Department of Chemistry Seminar and IFRI Frontiers in Forensic Science Summer Seminar Series, Florida International University (FIU) and International Forensic Research Institute (IFRI), Miami, FL, July 27, 2011.

Scott, J. R., A. J. Effenberger Jr., and T. R. McJunkin, 2011, "Economical HighResolution Detection Method for LaserInduced Breakdown Spectroscopy (LIBS)," $3^{\text {rd }}$ North American Symposium on LaserInduced Breakdown Spectroscopy (NASLIBS 2011), Clearwater, FL, July 18-20, 2011.

Effenberger Jr., A. J., J. R. Scott, and T. R. McJunkin, 2011, "Method and Apparatus for High Resolution Laser-induced Breakdown Spectroscopy (LIBS)," Patent Pending-Application 13/183,228 filed on July 14, 2011.

Scott, J. R., P. L. Tremblay, and T. R. McJunkin, 2011, "Apparatuses and Methods for Forming Electromagnetic Fields," Patent Pending_PCT/US11/24644 filed on April 29, 2011.

Scott, J. R., P. L. Tremblay, and T. R. McJunkin, 2011, "Apparatuses and Methods for Forming Electromagnetic Fields," Patent Pending-Application 13/096,823 filed on April 28, 2011.
Effenberger Jr., A. J. and J. R. Scott, 2010, "Effect of Atmospheric Conditions on LIBS Spectra," Sensors, Vol. 10, pp. 4907-4925.

Effenberger Jr., A. J. and J. R. Scott, 2010, "Effect of Atmosphere on Collinear Double Pulse Laser Induced Breakdown Spectroscopy," LIBS 2010, Memphis, TN, September 13-17, 2010.

Dahl, D. A., J. R. Scott, and T. R. McJunkin, 2010, "Method and Apparatus for Ion Cyclotron Resonance Spectrometry," US Patent \#7,777,182(B2), August 17, 2010.

Scott, J. R., and T. R. McJunkin, 2009, "Exploring Integrated Analytical Techniques for Characterizing Nuclear Materials with No Sample Preparation," $2^{\text {nd }}$ Japan-IAEA Workshop on Advanced SGs Technology for Future Nuclear Fuel Cycle, Tokai-mura, Ibaraki, Japan, November 1013, 2009.

Tremblay, P. L., T. R. McJunkin, and J. R. Scott, 2009, "Incorporating Laser-induced Breakdown Spectroscopy (LIBS) into the Laser-based Optical and Chemical Imager (LOCI)," NORM 2009 (64 ${ }^{\text {th }}$ Northwest Regional Meeting of the American Chemical Society, Tacoma, WA, June 28-July 1, 2009.

McJunkin, T. R., and J. R. Scott, 2009, "FT-ICRMS Cell with Linear Excitation/Detection and No Net Magnetron Motion," $237^{\text {th }}$ American Chemical Society National Meeting \& Exposition, Salt Lake City, UT, March 22-27, 2009. 


\section{Irradiation-Induced Evolution of Defect and Microstructure in Nanocrystalline Body-Centered-Cubic Mo}

Paul Millett; Yuntian Zhu (North Carolina State University)

10-008

Body-centered-cubic metals, such as ferritic steels and alloys, are used extensively as structural materials in reactor cores. This research project aims to fill a knowledge gap pertaining to the underlying deformation mechanisms associated with body-centered-cubic metals in nuclear reactor conditions. We are developing an atomistic-based knowledge of their deformation behavior for varying conditions: with and without irradiation damage, and with and without helium gas. This research is breaking new ground in this respect and will better enable other scientists and engineers to predict crack initiation and propagation and ductile-to-brittle transitions, and understand how irradiation and inert gases may accelerate these failure processes. Such a capability will improve the safety and performance of existing nuclear power plants, as well as allow improved material designs for future plants.

To achieve the above objectives, we are using both atomistic simulations (molecular dynamics) and experimental techniques (high-resolution transmission-electron microscopy of helium-ion irradiated samples) to characterize helium bubble formation and its effect on deformation.

This LDRD project initiated at the beginning of FY 2010 with a projected budget for three years. The majority of the budget ( $\$ 273 \mathrm{~K}$ for 2010) goes toward supporting two postdoctoral associates (one at INL and one at North Carolina State University [NCSU]) to carry out the computational and experimental research described below. The award was announced in October 2009, and the subcontract to NCSU was approved and sent out in March 2010. At INL, Dr. Yongfeng Zhang was hired as a postdoc and began work on May 10, 2010. At NCSU, Dr. Quangming Cheng was hired as a postdoc and began work on April 7, 2010.

\section{Project Summary}

We have made significant progress during the project's first two years. For the computational investigation, Dr. Zhang and Dr. Millett have performed an extensive amount of molecular-dynamics simulations to elucidate three primary issues: (1) deformation of un-irradiated nanocrystalline Mo, (2) helium bubble nucleation and growth in the nanocrystalline Mo, and (3) dislocation pinning due to helium bubbles. Our deformation studies have revealed that plastic straining predominantly occurs by twinning, and we have found an interesting microcracking mechanism relating to twin-grain boundary intersections (Fig. 1). Our helium-bubble formation studies have yielded very interesting results on the heterogeneous nucleation of helium bubbles and its dependency on grain boundary type (Fig. 2). Finally, our dislocation/bubble interaction study has characterized the dependency of heliumbubble pinning force on the bubble's size and gas pressure.

The experimental component of this research has made important progress, although some initial difficulties have delayed this work getting off the ground as quickly as expected. These difficulties primarily involved an initial inability to fabricate samples with good grain size distributions below $50 \mathrm{~nm}$. However, with the purchase of a magnetron sputtering apparatus, this has been overcome, and good samples are reliably being produced (Fig. 3). Next fiscal year, we will irradiate these samples with $\mathrm{He}$ ions at Los Alamos National Laboratory and use tensile testing of both irradiated and non-irradiated samples to understand the effect of He bubbles on deformation. 

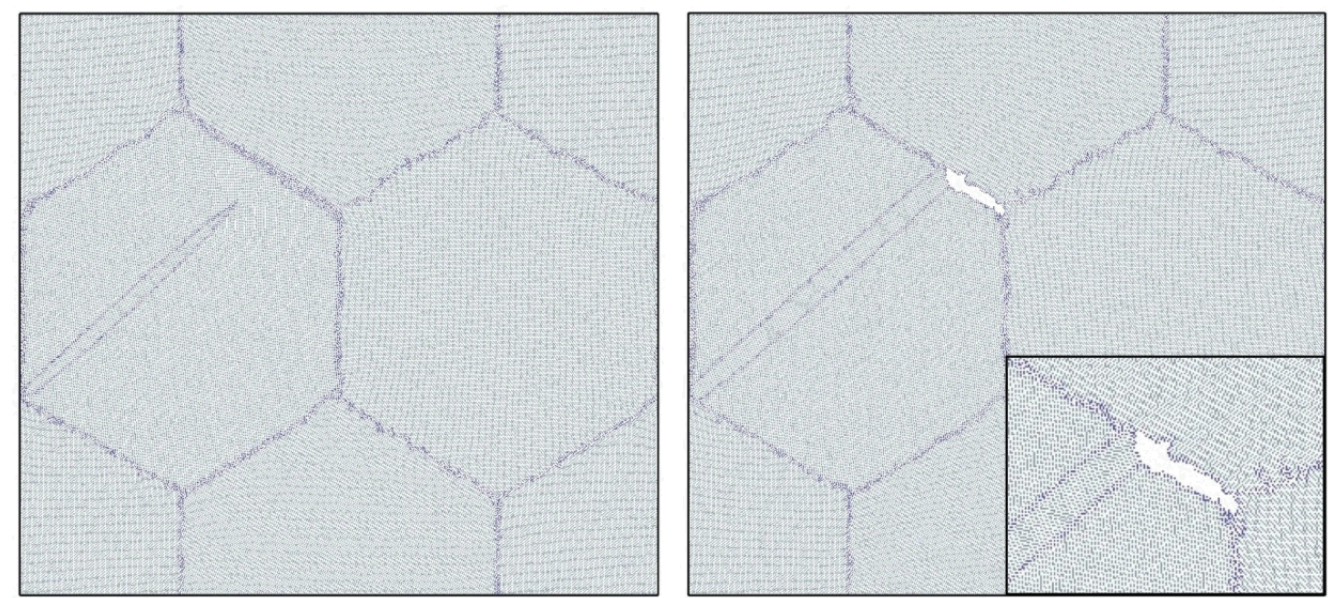

FIGURE 1. Twinning deformation and micro-cracking in nanocrystalline Mo using molecular dynamics simulation.
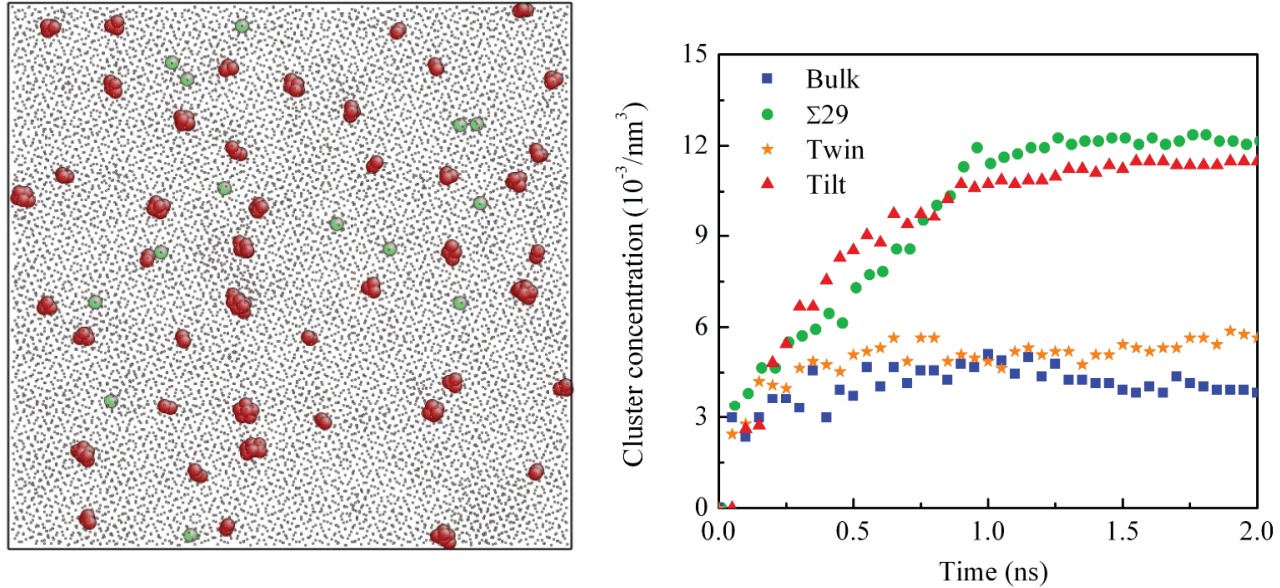

FIGURE 2. (left) Molecular dynamics simulation result showing helium bubble nucleation on a grain boundary plane. (right) Helium cluster concentration evolution versus grain boundary type.
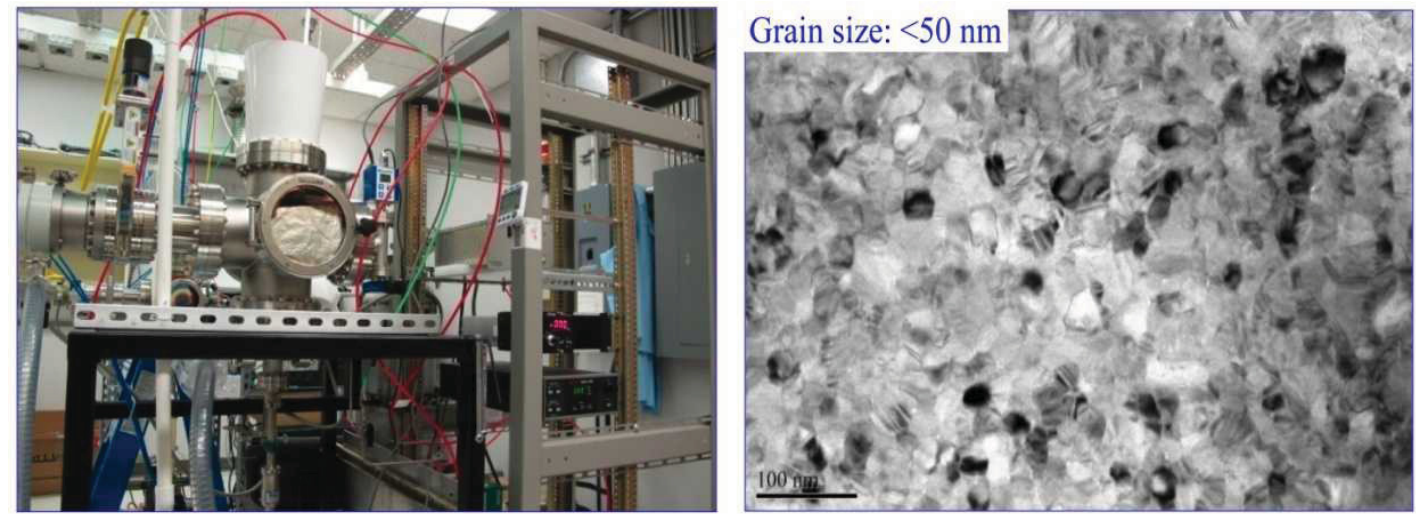

FIGURE 3. (left) Magnetron sputtering apparatus purchased at NCSU. (right) Prepared nanocrystalline Mo with grain sizes below $50 \mathrm{~nm}$. 


\section{Benefits to DOE}

This research further supports INL as the leading nuclear energy laboratory within the DOE complex. Furthermore, this research contributes to our ongoing ambition to develop a goal-oriented, science-based approach to understanding and developing nuclear fuels and materials. We are developing an atomistic-based knowledge of metallic deformation behavior for varying conditions: with and without irradiation damage and with and without helium gas. This research is breaking new ground in this respect, and will better enable other scientists and engineers to predict crack initiation and propagation and ductile-to-brittle transitions, and understand how irradiation and inert gases may accelerate these failure processes. Insights that will be developed in this project will feed into higher-level modeling and simulation capabilities currently developed at INL, thus providing higher-accuracy, physics-based modeling that will uniquely distinguish INL from other laboratories. As these modeling capabilities improve, INL will uniquely be able to validate them with an experimental comparison enabled by the Advanced Test Reactor (which we have already begun planning), thus solidifying INL's role as the leader in science-based discoveries relating to nuclear materials.

\section{Relevant Presentations and Publications}

Zhang, Y., H. Huang, P. C. Millett, M. R. Tonks, D. Wolf, and D. Phillpot, 2011, "Do Grain Boundaries Saturate under Radiation Damage?" Physical Review B, in review.
Zhang, Y., P. C. Millett, M. R. Tonks, and S. B. Biner, 2011, "Deformation-Twin-Induced Grain Boundary Failure: An Atomistic Study," Scripta Materialia, in review.

Zhang, Y., P. C. Millett, and M. R. Tonks, 2011, "Energetics and Diffusional Properties of $\mathrm{He}$ in BCC Mo: An Empirical Potential for Molecular Dynamics Simulations," Computational Materials Science, Vol. 50, pp. 3224-3229.

Zhang, Y., H. Huang, P. C. Millett, M. R. Tonks, D. Wolf, and S. Phillpot, 2010, "NonSaturable Sinks at Grain Boundaries in Nanocrystalline Mo under Electron Irradiation," Materials Research Society Fall Meeting, Boston, MA, November 29December 3, 2010.

Zhang, Y., H. Huang, P. C. Millett, M. R. Tonks, D. Wolf, and S. Phillpot, 2011, "Do Grain Boundaries Saturate in Nanocrystalline Materials-Molecular Dynamics Simulations," The Minerals, Metals \& Materials Society Annual Meeting 2011, San Diego, CA, February 27-March 3, 2011. 


\title{
Microstructural Evolution of $\mathrm{UO}_{\mathbf{2}}$ and $\mathrm{U}$ under Irradiation
}

\author{
Maria Okuniewski, Doug Akers, Craig Shull, Mark Drigert, and Lyle Roybal; \\ Kelvin Lynn, Marc Weber (Washington State University) \\ $10-058$
}

Uranium oxide $\left(\mathrm{UO}_{2}\right)$ is a model oxide fuel for nuclear reactors, and uranium $(\mathrm{U})$ is a model metallic fuel for fast reactors. In order to obtain unique data and a better understanding of the impact of microstructure on the thermal and mechanical properties of fuels and provide crucial input and validation data for multi-scale, multi-physics models, our objective is to elucidate the microstructural evolution in irradiated $\mathrm{UO}_{2}$ and $\mathrm{U}$ with positron annihilation spectroscopy (PAS) and transmission electron microscopy (TEM). The results from the PAS and TEM will be used to understand the microstructural evolution in $\mathrm{UO}_{2}$ and $\mathrm{U}$ as a function of various irradiation parameters, such as dose, type of irradiation particle, and temperature. PAS and TEM will be utilized to analyze defects and determine defect energies and diffusion lengths in $\mathrm{UO}_{2}$ and $\mathrm{U}$. Since PAS is relatively insensitive to defects greater than $1 \mathrm{~nm}$, TEM will be utilized to qualify and quantify defects greater than $1 \mathrm{~nm}$.

\section{Project Summary}

During Year 1, we focused on three major areas: (1) acquiring and fabricating specimens, (2) accelerator operations, and (3) designing a PAS system to analyze irradiated samples. Polycrystalline metallic alloy specimens (pure U, $\mathrm{U}-\mathrm{Zr}$, and U-Mo) were acquired, fabricated, and used for the PAS and TEM analyses. A singlecrystal $\mathrm{UO}_{2}$ fabrication system was designed by a collaborator to fabricate specimens for this research. The accelerator that is located at the university collaborator's lab was upgraded to allow for implantation ions, current, and temperatures of interest to this project. A system was also designed to utilize PAS to examine specimens that have been irradiated.

Year 2 objectives focused on: (1) specimen characterization, (2) testing of the newly designed PAS system, (3) data acquisition through PAS, (4) conducting irradiations, and (5) the design of a reconfigurable PAS system. The polycrystalline metallic alloy specimens (i.e., pure U, U-Zr, and U-Mo), which were fabricated during Year 1, were characterized via electron microscopy. The PAS system went through initial testing and was successfully used for data acquisition. Samples of U-Zr are currently ready for insertion into the High Flux Isotope Reactor at Oak Ridge National Laboratory for low-dose neutron irradiations. This test was originally slated for June 2011 but has been delayed until March 2012. These specimens will enable us to test our first neutron-irradiated fuels in FY 2012. Finally, an additional reconfigurable PAS system that will allow for the capability of conducting Doppler broadening, coincident Doppler broadening, and lifetime studies of low-activity specimens were designed, and most of the components were received this year. This system will allow for numerous researchers to be trained on the system, which will have user-friendly software and an easy-to-use operating system, thereby increasing the Laboratory's experimental infrastructure and accessibility.

\section{Benefits to DOE}

This research has benefited DOE's national security mission of science through the design of a unique positron annihilation system that has been specifically designed to analyze radioactive materials. In addition, Dr. Okuniewski gave a detailed presentation on the microstructural evolution of nuclear fuels to DOE Energy Secretary Steven Chu during his September 2010 visit to INL.

\section{Relevant Publications and Presentations}

Okuniewski, M. A., 2010, "Microstructural Evolution in Nuclear Fuels: Connecting Experiments and Simulations," Idaho National Laboratory for Secretary of Energy Dr. Steven Chu, Idaho Falls, ID, September 2010.

Okuniewski, M. A., M. H. Weber, K. G. Lynn, B. W. Beeler, B. J. Good, C. S. Deo, M. I. Baskes, S. A. Maloy, and J. F. Stubbins, 2010, "Positron Annihilation Spectroscopy in Nuclear Structural Materials and Fuels," The Twelfth International Workshop on Slow Positron Beam Techniques, Magnetic Island, Australia, August 2010. 


\section{In Situ Characterization of an Oxide Film for Prediction of Stress Corrosion Cracking Susceptibility}

Sebastien Teysseyre, David Hurley, Tedd Lister, Zhandos Utegulov; Brian Gorman

(Colorado School of Mines)

10-083

Stress corrosion cracking and irradiation stress corrosion cracking are phenomena that challenge the integrity of our nuclear power plants (energy resources) and the long-term storage of nuclear waste (environmental quality). These phenomena challenge the U.S.' effort to extend the lifetime of the current nuclear reactor fleet, increase the cost of energy production, and raise concern for the design of the next generation of nuclear reactors. Working to solve these issues will help achieve energy independence.

This work, and the equipment developed, will study stress corrosion cracking mitigation techniques and predict the deleterious effect of chemistry changes in the operation of a nuclear power plant. Specifically, we will develop the capability and expertise to characterize, in situ, the response of an oxide film to water chemistry changes and stress by performing Raman spectroscopy and electrochemical and laser acoustics measurements. It is believed that changes in the oxide layer composition are linked to stress corrosion cracking initiation and that the efficiency of the mitigation techniques and the long-term influence of a temporary loss of water chemistry control on stress corrosion cracking initiation can be determined by in situ characterization of such changes.

\section{Project Summary}

During FY 2010, two Raman spectrometers, a micro-Raman LabRam HR and a macroRaman spectrometer iHR550 from Horiba, and one laser (Verdi V2) were received and installed. The various optics and instruments have been installed on an optical table in IRC-B3, and the equipment has been tested. Oxide powders $\left(\mathrm{Fe}_{3} \mathrm{O}_{4}, \mathrm{NiCrO}_{4}, \mathrm{NiFe}_{2} \mathrm{O}_{4}\right)$, relevant for future in situ analyses, were used to acquire data and analyze spectra, which allowed personnel training and preparation of a database for analysis of complex oxide layers. Similar work was performed on the pre-oxidized sample made of nickel alloy X750.
During FY 2011, a high-purity corrosion loop has been designed, built, and validated. For maximum water chemistry control, the loop was mainly built of titanium. A high-temperature, high-pressure titanium autoclave, rated up to pressurized water reactor testing conditions, has been designed and procured. This system has a sapphire window to achieve optical transparency for analysis while containing the high-pressure liquid. The sample loading procedure has been determined and a special holder has been designed. This autoclave is to be used for testing in boiling water reactor/pressurized water reactor conditions. Another electrochemical cell, made of glass, has been designed and built for testing at low pressure. Raman spectroscopy analysis was performed on surface oxide of X750 and zircaloy (Zirc 4), and the internal oxidation along corrosion fatigue cracks was analyzed with micro Raman.

\section{Benefits to DOE}

In situ characterization of the response of an oxide film to water chemistry changes and stress will help us understand how changes in the oxide layer composition are linked to stress corrosion cracking initiation and determine the efficiency of the mitigation techniques and the long-term influence of a temporary loss of water chemistry control on stress corrosion cracking initiation. Stress corrosion cracking and irradiation stress corrosion cracking are phenomena that hinder the integrity of nuclear power plants and the long-term storage of nuclear waste. Therefore, these phenomena inhibit lifetime extension of the current U.S. fleet, impose down times that increase the cost of energy production, and raise concern for the design of next generation of nuclear reactors. This work, and the equipment developed, contribute to the study of stress corrosion cracking mitigation techniques and predict the deleterious effect of chemistry changes in the operation of a nuclear power plant. It will also permit an increase in fundamental knowledge about a phenomenon present in many industries. 


\section{Investigation of Molten Bromide Salt Systems for Separation and Recovery of Actinides from Fission Products}

Tedd E. Lister, Guy L. Fredrickson, Steven D. Herrmann

$10-032$

Separation and recovery of the actinides from other fission fragments is an important nuclear technology that will not only reduce waste volume but also increase utilization of resources. The use of bromide-based salts may introduce three technical benefits-a lower operating temperature, improved separation of actinides from fission products, and the potential to improve recovery of the actinides by employing bromide electrolysis at the anode. The objective of this research is to investigate the applicability of a bromide-based molten salt system, such as $\mathrm{LiBr}-\mathrm{KBr}$ and $\mathrm{CsBr}-\mathrm{LiBr}-\mathrm{KBr}$, to improve the separation and recovery of actinides from fission products in spent nuclear fuel.

\section{Project Summary}

In the initial year of work, an experienced post-doctoral candidate from the Massachusetts Institute of Technology, Dr. Prabhat K. Tripathy, was hired to design and perform experiments. His initial work involved performing a literature search on bromide-based molten salt systems as it pertained to electrorefining of used nuclear fuel. This literature search, captured in a report, demonstrated that while some knowledge on the application of bromide salts existed, there was very little on the electrochemistry of elements of interest (actinides and rare earth elements). To perform the experimental work, an argon atmosphere-controlled glove box had to be procured and modified to perform molten-salt electrochemistry. This involved installation of a molten-salt furnace to perform electrochemical experiments, under a very low moisture and oxygen atmosphere, at elevated temperatures. The molten-salt furnace has the provisions for placing the electrochemical cell with electrodes and other attachments, including heat shields to keep the inside temperature of the glove box well below the maximum permissible temperature $\left(40^{\circ} \mathrm{C}\right)$, for performing the experiments.

After the installation was complete, a series of experiments were performed to determine the melting points of three potential molten-salt systems: (i) $\mathrm{LiBr}-\mathrm{GdBr}_{3}$ (ii) $\mathrm{LiBr}-\mathrm{KBr}-\mathrm{GdBr}_{3}$ and (iii) $\mathrm{LiBr}-\mathrm{KBr}-\mathrm{CsBr}-\mathrm{GdBr}_{3}$. We intend to use these electrolyte systems for performing the electrorefining (of gadolinium) experiments. Gadolinium was chosen as a surrogate to mimic uranium and plutonium in the electrorefiner. Specific quantities of $\mathrm{GdBr}_{3}$ were added to (i) $\mathrm{LiBr}$, (ii) $\mathrm{LiBr}-\mathrm{KBr}$ (eutectic composition), and (iii) $\mathrm{LiBr}-\mathrm{KBr}-\mathrm{CsBr}$ (eutectic composition) to determine the eutectic melting points of the salt compositions. With increased addition of $\mathrm{GdBr}_{3}$, a corresponding drop in the melting points of the salt compositions was observed. It was found that the melting points were lower than the corresponding chloride salt compositions. Such an observation appears to be beneficial from a technical standpoint. Based on the liquidus data obtained for eutectic $\mathrm{LiBr}-\mathrm{KBr}$ compositions with different amounts of $\mathrm{GdBr}_{3}$, it was decided to carry out further electrochemical investigations in $\mathrm{LiBr}-\mathrm{KBr}-\mathrm{GdBr}_{3}$ electrolyte at $400^{\circ} \mathrm{C}$. Cyclic voltammetric experiments were carried out to record the current versus voltage characteristics of pure eutectic melt at scan rates between 10-500 $\mathrm{mVs}^{-1}$. In pure eutectic melts, both lithium deposition and bromine evolutions reactions were recorded on three electrodes. The CVs were also recorded with addition of $\mathrm{GdBr}_{3}$ (in the range of 1-2 wt.\%) where the gadolinium deposition reaction was recorded during cathodic sweep. Further experiments are in progress. It is expected that these experiments shall produce novel data for comparison with the existing data in chloride-based salts.

\section{Benefits to DOE}

The proposed research shall expand expertise in the electrochemical treatment of spent nuclear fuels and further establish INL as a world leader in innovative fuel-cycle technology. This work currently supports a hired postdoctoral researcher and directly supports advancement in the spent nuclear fuel treatment and spent nuclear fuel cycle research programs. Advances in the separation of actinides could lead to the development of proliferation-friendly technologies for peaceful recycling of spent nuclear fuels. By way of providing insights into the electrochemical behavior of lanthanide and actinide fission-product metals (in hitherto unstudied electrolyte systems), this research directly supports efforts in the direction of achieving a reduction as well as minimization of radioactive wastes being generated by the spent nuclear fuel treatment and spent nuclear fuel cycle programs. 


\section{In Situ Micro-Raman Spectroscopy and Modeling of Breakaway Oxidation of Zircaloy Cladding}

Marat Khafizov, Zhandos Utegulov; Haitham El Kadiri (Mississippi State University)

11-041

Zirconium alloys are widely used as nuclear fuel cladding materials in nuclear reactors. Corrosion of these materials limits the operational lifetime of nuclear fuel and affects its environmentally safe storage opportunities. The initial oxide layer acts as a protection layer and slows further corrosion. As the oxide layer becomes thicker, the stresses associated with growth and phase transformations become strong. These large stresses can lead to crack development, spallation of the oxide layer, and overall loss of protection from rapid deterioration leading to mechanical failure of the cladding. Therefore, development of experimentally validated computational models for this breakaway oxidation process in zirconium alloys is important for more efficient, economical, and safer utilization of nuclear energy.

This project aims to identify and predict the process of oxide-layer deterioration due to different mechanisms related to the generation of internal stresses. To achieve this goal, microRaman spectroscopy and phase-field (PF) modeling were utilized by a research team consisting of members from INL and Mississippi State University. In FY 2011, the project had three major objectives: (1) develop an ex situ micro-Raman measurement methodology to determine phase composition and residual stresses in the oxidized layer of Zircaloy-4 at high temperature, (2) develop a PF model for capturing the evolution of precipitates during solid-state phase transformation, and (3) develop a stress-dependent PF model for oxidation of zirconium to predict oxidation-layer thickness and compositional growth stresses.

\section{Project Summary}

An experimental methodology was developed to identify phase composition of oxide layer and measure residual stresses with micron resolution using micro-Raman spectroscopy. Atomic force microscopy was used to characterize the surface morphology of the oxide layer. Both techniques suggested the presence of tetragonal phase precipitates in an otherwise monoclinic oxide layer (Fig. 1).
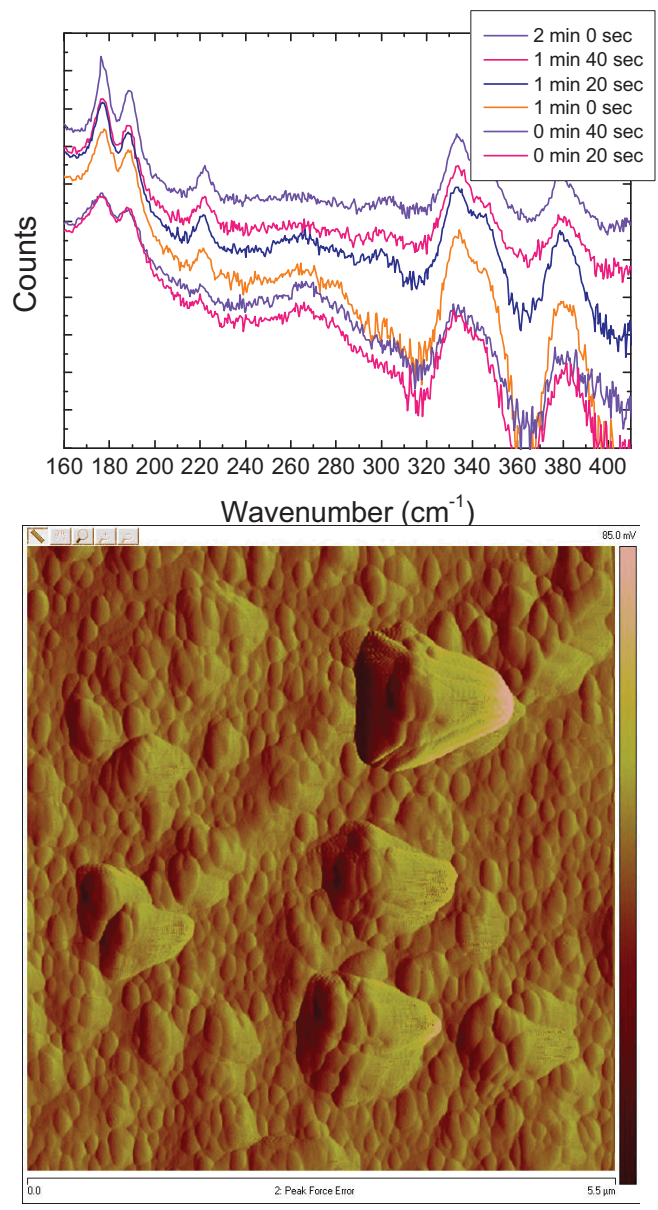

FIGURE 1. Raman spectra of oxide layer grown at $900^{\circ} \mathrm{C}$ (top) and an atomic force microscopy image of tetragonal phase precipitates (bottom).

An elastic PF model capturing the stability and coarsening of coherent precipitates was developed to incorporate the simultaneous actions of precipitate size and volume fraction, precipitate mechanical strength, compositional strain, and growth of an intermediate phase (Fig. 2). The model was formulated with nondimensional groups to increase its applicability. 

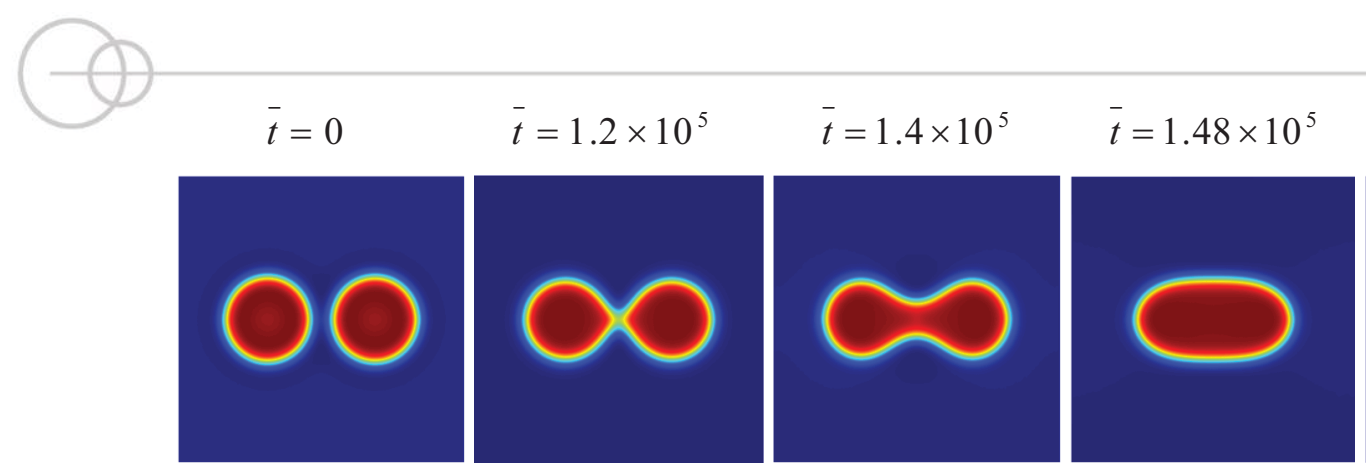

$\bar{t}=1.6 \times 10^{5}$

FIGURE 2. PF simulation results showing necking mechanisms of closely spaced particles.

A PF model was identified for oxidation of pure metals in which the diffusion-controlled phase transformation is the mode of the oxide growth (Fig. 3). The governing evolution equations of $\mathrm{PF}$ variables were coupled to the mechanical equilibrium equations to investigate the evolution of stresses in the oxide and the metal substrate. This model captured the composition depth profile of the oxygen in the oxide layer and in the metallic substrate.
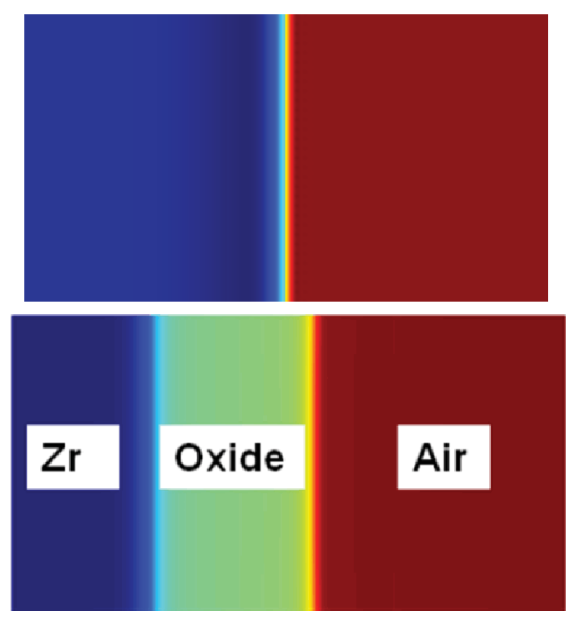

FIGURE 3. PF simulations of Zr oxidation at $900^{\circ} \mathrm{C}$.

\section{Benefits to DOE}

The developed micro-Raman validated PF model will help to enable the extension of the lifetime of the current fleet of nuclear reactors, impose down times that increase the cost of energy production, and raise concern for the design of the next generation of nuclear reactors. In addition, the failure of nuclear materials presents a serious concern for our environment. This LDRD would benefit the work of those in charge by ensuring safe and secure management of nuclear materials and radioactive waste. Finally, models developed in this research also form solid foundations for future development of experimentally validated predictive tools to characterize solid-state phase transformation in different material alloys in a variety of environments.

\section{Relevant Publications and Presentations}

Zaeem, M. A., H. El Kadiri, M. Khafizov, and Z. Utegulov, 2012, "Experimental and PhaseField Studies of Oxidation Mechanisms of Zirconium Alloys," in preparation for Journal of Nuclear Energy.

Zaeem, M. A., H. El Kadiri, M.F. Horstemeyer, M. Khafizov, and Z. Utegulov, 2011, "Effects of Internal Stresses and Intermediate Phases on the Coarsening of Coherent Precipitates: A Phase-Field Study," Current Applied Physics, Vol. 12, p. 570.

Zaeem, M. A., H. El Kadiri, M. F. Horstemeyer, and P. T. Wang, 2011, "A Finite ElementPhase Field Study of Solid State Phase Transformation: Coarsening of Coherent Precipitates," TMS 2011, San Diego, CA, February 28, 2011. 


\section{Characterization of Nanomechanical Response of Material to Determine Key Variables in Stress Corrosion Cracking}

John H. Jackson, William Gerberich

11-043

Although stress corrosion cracking (SCC) has been a known issue for many decades, the mechanisms responsible for stress corrosion crack initiation and growth are complex and not well understood. The goal of this project is to investigate broadly the primary factors contributing to SCC in reactor systems through the use of nanomechanical testing. Much of the background data on cold work, fatigue, creep and SCC of austenitic alloys suggests that nearsurface nucleation of dislocations is critical to the initiation of damage leading to SCC. The idea presented here is to use nanopillars fabricated using a focused ion beam to provide a test bed for chemo-mechanical mapping of crack initiation and propagation mechanisms. This project is a proof-of-principle study using alloy 304L stainless steel and Fe-Ni thin films to demonstrate the sensitivity of material response in nanopillar deformation experiments to the measurement of fundamental parameters that may affect SCC. For example, in one set of studies, the effect of surface oxidation on dislocation velocities can be measured. Ultimately, through the use of this type of testing, we hope to reduce the number of experimental timesteps required to reach a conclusion about which mechanistic phenomena are most logical to pursue in detail to provide a better mechanistic understanding of SCC. Understanding of the mechanistic basis by which SCC occurs may lead to development of SCC-resistant replacement materials for light water reactors or better SCC mitigation processes.

\section{Project Summary}

Refinement of the ion milling technique for production of nano-pillars has consumed a large part of the first year of this project. This work included milling an analog material (silicon) to refine system parameters; this was followed by milling of 304 stainless-steel pillars. A triboindenter was used to obtain baseline material properties for the 304 stainless-steel nano-pillars and to determine the correct aspect ratio and geometry for the nano-pillars that gives optimally consistent data. A material of interest to the nuclear industry (Nitronic 50) was obtained and cold worked to nominally $20 \%$. The cold work is known to increase the material's susceptibility to SCC; this material will be used to study the fundamental differences between materials that lead to SCC. Additional recent work has resulted in production of thin films for transmission electron microscopy studies to determine grain boundary characteristics of the 304 stainless steel and the cold worked Nitronic 50.

\section{Benefits to DOE}

The need for a quantitative predictive SCC model is well understood by the nuclear community and by the wider materials community. This project directly addresses this need if successful and will contribute to the fundamental understanding of material failure in operating nuclear reactors. This will allow decisions to be made on re-licensing of the current nuclear reactor fleet as well as planning for future nuclear reactor design. Work on fundamental issues in crack growth has recently been funded by DOE's Office of Science, and further interest is anticipated.

\section{Relevant Publications and Presentations}

Gerberich, W. W., and D. D. Stauffer, 2012, "Damage Mechanisms, Historical," Gaseous Hydrogen Embrittlement of High

Performance Materials, an encyclopedic volume, Woodhead Publishing, U.K. Cambridge, in press.

Gerberich, W. W., D. D. Stauffer, A. R. Beaber, and N. I. Tymiak, 2011, "The Brittleness Transition Due to Scale," Journal of Materials Research, submitted, August 2011. 


\section{Supercritical Fluid Extraction of Actinides from TRISO Reactor Fuels}

Bruce J. Mincher; Chien M. Wai (University of Idaho)

11-006

DOE has selected very high temperature reactors (VHTRs) as a high-priority concept for Generation IV reactor development. The treatment and/or reprocessing of the used TRISO (tristructural isotropic) fuels from VHTRs have not been addressed in these programs.

Conventional acid dissolution and extraction is inefficient for this fuel matrix. This proposal is to develop a supercritical carbon-dioxide-based $\left(\mathrm{SF}-\mathrm{CO}_{2}\right.$ ) technology for extracting actinides from the used TRISO fuels from gas-cooled VHTRs. SF- $\mathrm{CO}_{2}$ is effective for removing lanthanides and actinides directly from solid and densely packed materials such as crushed TRISO fuel, because it possesses both gas-like and liquid-like properties; therefore, it is capable of penetrating into porous materials and dissolving constituents in a solid matrix. SF- $\mathrm{CO}_{2}$ is also a tunable solvent because its density can be varied by changing temperature and pressure, having a significant effect on the solubility of selected metals, including actinides. This affords the possibility for actinide/lanthanide separations, options for which are to be explored in this project.

\section{Project Summary}

In this first year of the project, the supercritical fluid extraction apparatus and ultraviolet/visible spectroscopy equipment was set up at the Center for Advanced Energy Studies lab. At this lab, $\mathrm{SF}-\mathrm{CO}_{2}$ extractions were performed by University of Idaho students on intact and crushed TRISO fuels, with extracted uranium measured by ultraviolet/visible spectroscopy. Optimization of the amount of uranium extracted was achieved by varying process parameters. Conventional acid leaching experiments were also performed for comparison to $\mathrm{SF}-\mathrm{CO}_{2}$ extractions. The collected data is qualitative in nature but suggests that direct application of the ligand to the fuel prior to ramping up to supercritical conditions greatly enhances extraction, and that the efficiency of $\mathrm{SF}-\mathrm{CO}_{2}$ extraction is much higher than for acid leaching. Samples of pre- and post-extraction material for quantitative interpretation are currently being analyzed.

\section{Benefits to DOE}

Reprocessing of used nuclear fuel will extend the uranium supply and mitigate environmental issues to enhance the sustainability of nuclear power generation. The research represents a pioneering study using a non-traditional extraction technique to explore the feasibility of extracting and separating actinides from the TRISO fuels. The research may lead to a new extraction technique, which could have a big impact on the development of environmentally sustainable processes for managing spent fuels from VHTRs. 


\section{Mars Hopper Project \\ James O'Brien, Robert O'Brien, Nathan Jerred, Spencer Cooley, Steven Howe \\ 10-081}

The Center for Space Nuclear Research (CSNR) has proposed a design for the Mars Hopper, which is an instrumented platform to be placed on the surface of Mars designed to acquire highly detailed data from the surface and subsurface, travel large distances to multiple sites in short periods of time, and perform this task repeatedly. The overall goal of this project is to design, develop, and eventually build a prototype Mars Hopper, which requires a radioisotope thermal rocket (RTR). The RTR concept utilizes the decay energy from a radioisotope, which is thermally stored. The energy is then transferred to a gas that is expanded out a nozzle, producing thrust. Ultimately, the Hopper concept is based on using beryllium as a thermal capacitance material for the RTR core. For the prototype, Hastelloy C276 will instead be employed due to its similar coefficient of thermal expansion to beryllium, which will test the integrity of the prototype at temperature. The RTR core (Fig. 1) will be heated by a radioisotope source in the flight version, but with an electrical heat source in the prototype. Liquid $\mathrm{CO}_{2}$ will be used as the propellant due to its abundance in the Martian atmosphere. The optimized storage pressure and temperature for application in the Martian environment have already been determined, and a point design of the Hopper has been completed.

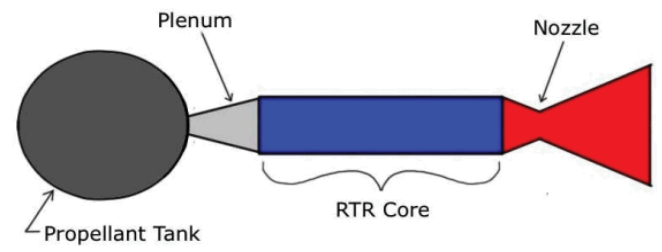

FIGURE 1. Simple schematic of the Mars Hopper rocket engine.

\section{Project Summary}

This fiscal year's goals were to perform design and evaluation studies of the sub-systems necessary for the Mars Hopper and perform both heat transfer calculations using the multi-physics codes COMSOL and STAR-CCM+ to predict the Hopper's performance. In addition, a major portion of the CSNR effort went into acquiring parts and components to build a "Hopper" prototype. The prototype, as stated, will be electrically heated and used to validate the computational models of heat transfer for the non-conventional flow channels being proposed in the Hopper.

Within the point design of the Hopper, subsystems necessary for operation have been identified. To complete this project, a team has formed from INL, the CSNR, and three universities: Utah State University, Oregon State University, and the University of Idaho. This year, Utah State continued to design and assess the performance of the $\mathrm{CO}_{2}$ liquefaction subsystem and was responsible for condensing the $\mathrm{CO}_{2}$-rich atmosphere into a storable and usable form. The University of Idaho designed and built a test rig to compare the performance of an opencycle and closed-cycle Brayton (with and without a regeneration loop) for the power conversion sub-system and was responsible for supplying electricity for station keeping and operating the liquefaction sub-system. Oregon State performed heat transfer calculations, in conjunction with the CSNR, focusing mostly on the STAR-CCM+ code.

A major undertaking this year was the modeling of the heat transfer for a set of flow-channel shapes and design of a prototype rig and testing facility to validate computational models due the proposed flow-channel shapes and the uncertainty in modeling turbulent $\mathrm{CO}_{2}$ flow. Two prototypes will be developed, each based on a specific flow-channel design; one will contain a complex four-point star shape (Fig. 2) and the other will utilize a standard 2-mm-dia. circular channel. Figure 3 shows a profile view of each prototype's core. In addition, computational modeling will also extend to optimizing the flow-channel size and shape by looking at flow channels having a 1-mm, 1.5$\mathrm{mm}, 2.5-\mathrm{mm}$, and $3-\mathrm{mm}$ diameter for a circle, as well as the unique flow-channel shapes of a square and equilateral triangle. 


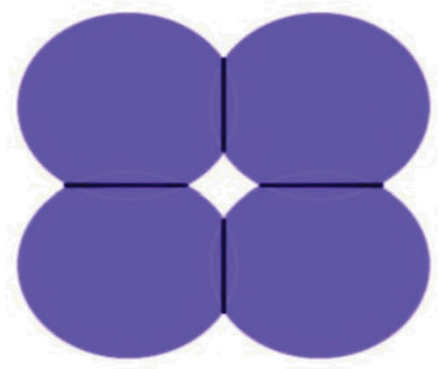

FIGURE 2. The 4-point star flow-channel shape is created by stacking four rods together after special flats have been shaved into their sides.

The prototypes were designed in conjunction with an entire test facility, geared towards safely heating the core and handling the injection of cold $\mathrm{CO}_{2}$ gas and removal of hot $\mathrm{CO}_{2}$ gas. The test facility will utilize a large pressure vessel to house the prototypes, where the ambient pressure of $0.01 \mathrm{~atm}$ and a $\mathrm{CO}_{2}$ atmosphere, simulating the Martian surface, can be applied (Figure 4 shows the prototype that will be used within the pressure vessel). The test facility will utilize data acquisition software to both monitor in real-time the temperatures at various locations throughout the prototype, gather gas pressures and temperatures at both the inlet and exit and other pertinent data.

Information gathered will be used for code validation and to determine a more accurate performance potential of a flyer version on the Martian surface.

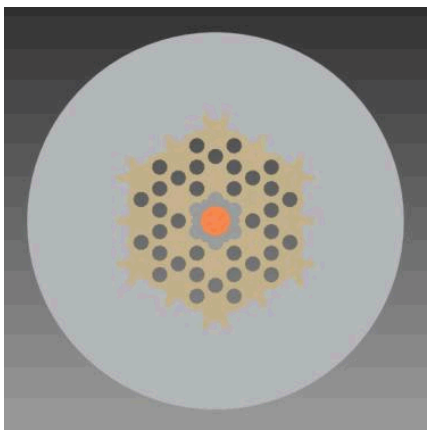

(a)

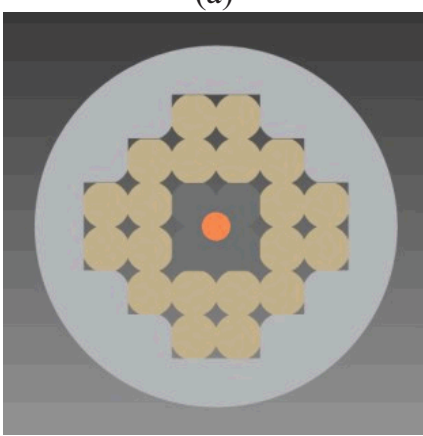

(b)

FIGURE 3. Prototype core based around the (a) hexagonal rod bundle, producing circular flow channels and the (b) circular rod bundle, producing the four-point-star shaped flow channels.

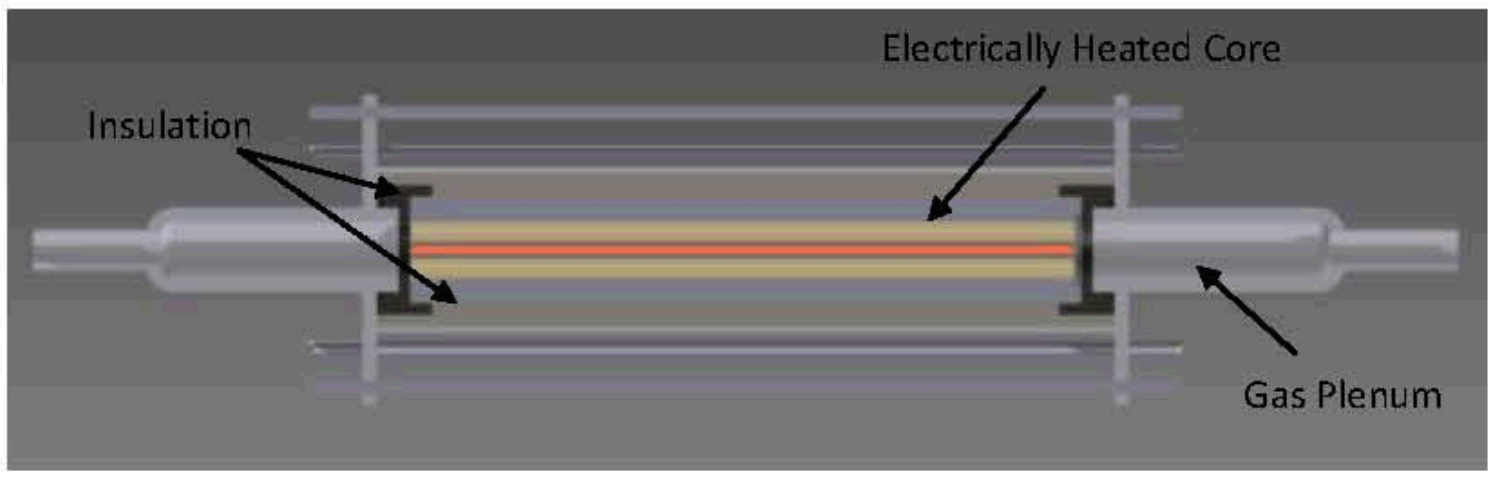

FIGURE 4. Axial-based view of the Mars Hopper prototype, used for code validation. 


\section{Benefits to DOE}

This research has enhanced the capabilities of INL and the CSNR to computationally model heat transfer in turbulent flow conditions, specifically in $\mathrm{CO}_{2}$, and to use complex multiphysics codes to model coupled systems. In addition, the prototypes and test facilities will add to the infrastructure of the INL laboratories. Because of the university participation, twelve students have benefited through experience in subsystem design activities for the Mars Hopper. Nationally and internationally, the Mars Hopper concept could revolutionize planetary exploration and will maximize the amount of science that can be conducted per launch, greatly reducing the overall cost to explore the Martian surface. The DOE benefits from the visibility of developing innovative concepts for space exploration utilizing nuclear technologies, and this work highlights its expertise and capabilities in handling radioisotopic fuels. Finally, proficiency gained in using the multi-physics codes enables the lab researchers to work on a number of different national programs, including next-generation ground-based nuclear systems.

\section{Relevant Publications and Presentations}

O'Brien, R. C., A. C. Klein, W. T. Taitano, J. Gibson, B. Myers, and S. D. Howe, 2011, "Thermal-hydraulics of a Radioisotopepowered Mars Hopper Propulsion System," Proc. of Nuclear and Emerging

Technologies for Space, Albuquerque, New Mexico, September 30, 2011.
O'Brien, R. C., N. D. Jerred, and S. D. Howe, 2011, "Development of a Propulsion System and Component Test Facility for Advanced Radioisotope Powered Mars Hopper Platforms," Proc. of Nuclear and Emerging Technologies for Space, Albuquerque New Mexico, September 30, 2011.

Howe, S. D., R. C. O'Brien, W. T. Taitano, D. Crawford, N. Jerred, S. Cooley, J. Crapeau, S. Hansen, A. Klein, and J. Werner, 2011, "The Mars Hopper: A Radioisotope Powered, Impulse Driven, Long-Range, Mobile Platform for Exploration of Mars," Proc. of Nuclear and Emerging Technologies for Space, Albuquerque, New Mexico, September 30, 2011. 


\section{Science-Based Simulation Model of Human Performance for Human Reliability Analysis}

Dana Kelly, Ron Boring; Carol Smidts (Ohio State); Ali Mosleh (University of Maryland)

11-068

The objective of this work is to begin exploring a dynamic simulation approach to Human Reliability Analysis (HRA), one whose models have a basis in psychological theories of human performance and whose quantitative estimates have an empirical basis. In FY 2011, the project leaders will formalize collaboration between INL, University of Maryland, and Ohio State University (OSU) to continue development of a simulation model initially formulated at the University of Maryland. Work in FY 2011 will focus on enhancing the underlying human performance models with the most recent psychological research and on planning follow-on studies to establish an empirical basis for the model, based on simulator experiments to be carried out at INL and OSU.

\section{Project Summary}

The INL investigators met with Professor Carol Smidts at OSU to plan the infrastructure needs for the OSU simulator lab to be funded in FY 2012 and to discuss the IDAC simulation framework. Ron Boring performed a review of the psychological literature on individual differences to identify potential enhancements to the IDAC framework. Meetings were held at INL with the team developing the NextGeneration Safety Analysis Code (NGSAC) to discuss code architecture requirements. The goal is to ensure that tools developed with LDRD funding can be adapted by NGSAC in out years. Discussion was held with Professor Ali Mosleh about the IDAC source code and arrangements were made to obtain the source code and documentation.

\section{Benefits to DOE}

DOE has benefitted in several ways from this work. A solid collaboration has been established with two universities at the forefront of research into dynamic HRA models. This can be expected to create opportunities for student internships at INL, leading to post-doctoral research and regular employment. The INL researchers have gained increased visibility by being part of cutting-edge research with gamechanging possibilities for the field of HRA. Follow-on funding is anticipated from Institute for Nuclear Energy Science and Technology for FY 2012. If the research is successful, within 23 years, there is opportunity for DOE-Office of Nuclear Energy funding to integrate the approach in the NGSAC, supporting the Light Water Reactor Sustainability Program, and perhaps other DOE-Office of Nuclear Energy programs as well. The model of research developed further opens the doors to new funding sources in domains where the lack of a solid empirical basis for HRA has hindered its adoption. The Nuclear Regulatory Commission has expressed interest in this research as well.

\section{Relevant Publications and Presentations}

Kelly, D. L., R. L. Boring, R. Youngblood, C. Smidts, and A. Mosleh, 2012, "ScienceBased Model of Human Performance for Human Reliability Analysis," $11^{\text {th }}$ International Probabilistic Safety Assessment and Management (PSAM) Conference, Helsinki, Finland, June 2012.

Kelly, D. L., R. L. Boring, A. Mosleh, and C. Smidts, 2011, "Science-Based Simulation Model of Human Performance for Human Reliability Analysis," Enlarged Halden Programme Group Meeting, Sandefjord, Norway, October 2-7, 2011. 
$\Rightarrow$ Center for Advanced Energy Studies

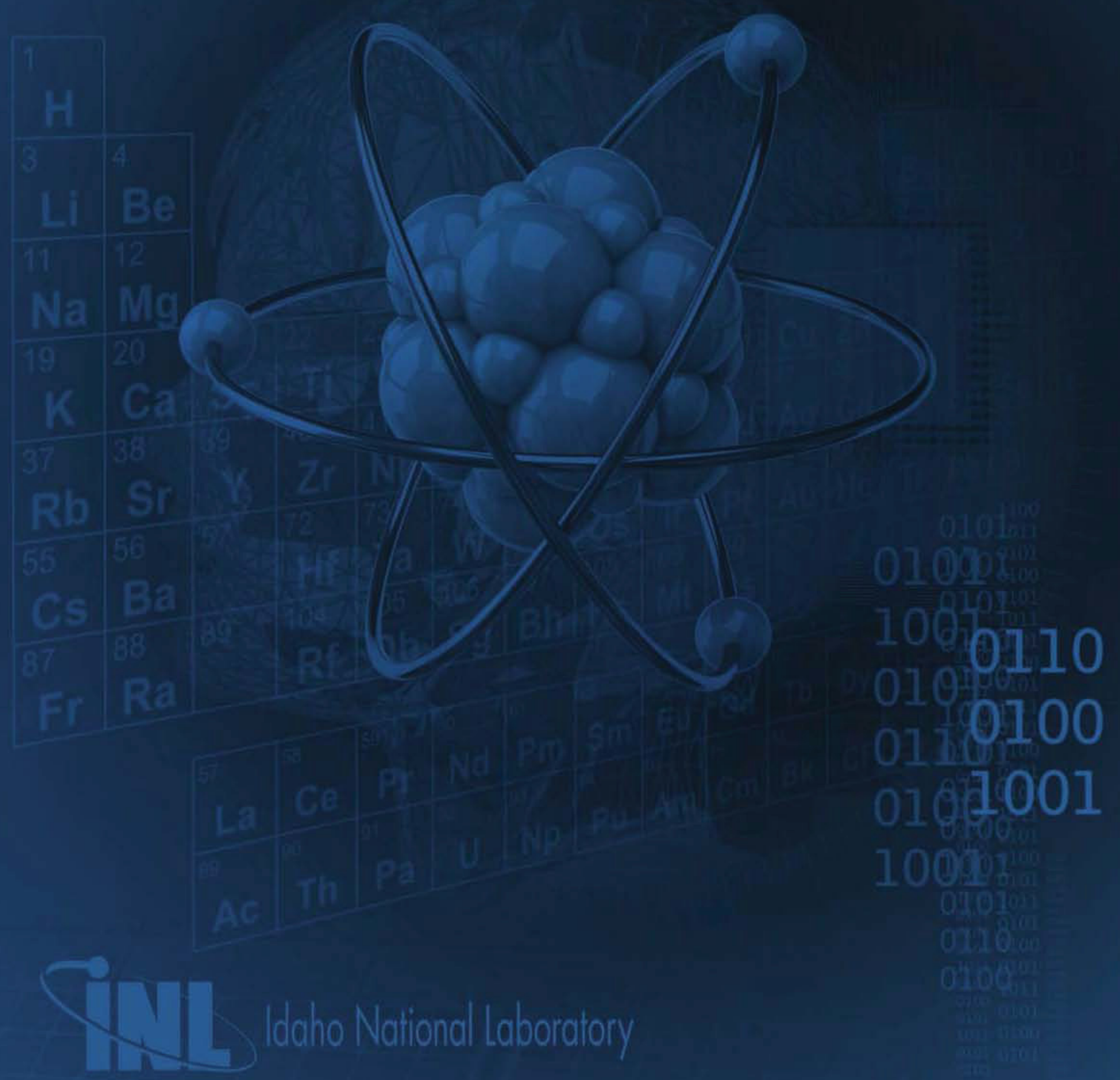




\title{
Development of Lignocellulosic Ethanol Production Potential in Idaho
}

\author{
Greg Bala; Kevin Feris (Boise State University); Tim Magnuson (Idaho State \\ University); Jon Van Gerpen (University of Idaho) \\ 09-056
}

There is a compelling need for new sources of clean, inexpensive, and renewable energy to replace/augment petroleum-based energy systems. One promising route of exploration includes discovery and development of new bioenergy strategies to enhance the efficiency of biomass conversion to chemicals and fuels and improving our ability to harness biofuelproducing capabilities of biological catalysts. Such bioenergy strategies have significant potential for expanding our current regime of energy-producing technologies and therefore may play a significant role in transitioning our petroleum-dependent energy system to one based on clean, carbon-neutral renewable energy sources. Advancements in biofuels research and technology have the potential to provide huge economic benefits for the State of Idaho. Idaho's agricultural activities result in large amounts of potential feedstocks for biofuel production, feedstocks that are often viewed as waste to the producer (i.e., forest residue, potato processing waste, sugar beet processing waste, etc.). Exploiting such "waste" feedstocks via the coupling of new biotechnologies and biofuels production facilities can create a new industry base for Idaho.

Our goal is to use expertise and collaboration among scientists at INL, Boise State University, Idaho State University, and the University of Idaho to provide solutions for the problem of high feedstock cost for biofuels production. Novel feedstocks available within the State of Idaho (e.g., lignocellulosic) agricultural wastes or products may allow ethanol producers within the state and region to increase production capacity and improve profitability.

This project forms an important foundation for the focus area of Idaho materials as it strives to widen the applicable envelope of operations for ethanol production within the state. Additionally, it fully supports an existing, tangible connection between the state research institutions and the Center for Advanced Energy Studies that is already focused on and engaged in bioenergy research.

\section{Project Summary}

Significant findings and accomplishments have included (1) detailed characterization of important microbial isolates, (2) discovery of novel lineages of lignocellulolytic strains that have not been previously described (a major discovery was the identification of genera not previously known to degrade lignocelluloses), (3) metaproteomic analysis of a lab-scale photobioreactor, (4) immobilization of microbial isolates in support media to enhance enzymatic activity and facilitate improved reactor operations, (5) investigations of novel additives to immobilization support media to enhance growth and activity, (6) modeling of the rate equations for the mass transfer in and out of encapsulated enzymes to understand the factors responsible for maximum enzyme production, and (7) design and fabrication of a pilot-scale bioreactor to produce fermentable sugars.

Collectively, these accomplishments summed to a successful demonstration of technology. We optimized operational parameters for a novel two-stage semicontinuous bio-reactor consisting of a nursery reactor and a hydrolysis reactor. This design spatially separates enzyme production and hydrolysis activities, allowing for simultaneous process optimization and operation for extended periods. Optimal encapsulation matrix parameters (e.g., size, encapsulation matrix concentration, $\mathrm{pH}$, etc.), reactor dimensions, and agitation rates identified in the first stage of this work were employed in the bench- and 70-L pilot-scale two-stage reactor test. Hydrolysis reactor products (e.g., soluble sugars) were tested as enzyme inducers for continual operation of the nursery reactor. Hydrolysis activity of the encapsulated enzyme solution was $14-40 \%$ higher than the unencapsulated counterparts. Feeding hydrolysis reactor products to the nursery reactor yielded high levels of enzyme activity. Initial studies suggest that the onsite catalyst production and utilization system developed by this pilot project is feasible and economically viable when driven solely by ballmilled forest residue. 


\section{Benefits to DOE}

This research has provided numerous benefits including: (1) developing a long-term collaborative relationship between researchers at the University of Idaho, Boise State University, Idaho State University, and INL; (2) developing expertise and research capacity at the three state universities in a field of interest to INL, the region, the nation, and DOE; (3) educating students, including a Ph.D. student at the University of Idaho and several graduate and undergraduate students at Boise State University and Idaho State University; and (4) providing scientific data for the feasibility of encapsulated enzymes as a tool for conversion of lignoceullulosic biomass to ethanol.

The work directly supports the DOE strategic goal for Energy Efficiency and Renewable Energy "to protect our national and economic security by promoting a diverse supply of reliable, affordable and environmentally sound energy." This is implemented by two Energy Efficiency and Renewable Energy strategic goals: (1) "Dramatically reduce or even end dependence on foreign oil," and (2) "Create the new, domestic bioindustry." Supporting goals of the Biomass Program include the topical areas for feedstocks, sugar platforms, products, and integrated biorefineries. As a composite, these topical areas all address the production of fuels and materials, albeit through different pathways or intermediates.

\section{Relevant Publications and Presentations}

Deis, B., G. Bala, and K. P. Feris, 2011, "Defining Optimal Culture Parameters of Calcium Alginate Encapsulated Trichoderma reesei RUT C-30 for Cellulase and Xylanase Production," Biores Tech, In preparation, targeted submission date October 2011.

Deis, B., T. Jain, J. Van Gerpen, G. Bala, and K. P. Feris, 2011, "Optimization of a Novel Two-Stage Lignocellose Saccharification Reactor Design Employing On-Site Catalyst Production by Calcium Alginate Encapsulated Trichoderma reesei RUT C30," Biores Tech, In preparation, targeted submission date November 2011.
Ellis, J. T., M. W. Swenson, and T. S. Magnuson, 2011, "Thermostable and Alkalistable Xylanases Produced by the Thermophilic Bacterium Anoxybacillus flavithermus TWXLY3 Isolated from the Alvord Basin Hydrothermal System, Oregon," Appl Environ Microbiol, in preparation.

Ellis, J. T., M. W. Swenson, and T. S. Magnuson, 2011, "Alkali-Thermostable Xylanases Produced by the Mesophilic Bacterium Bacillus pumilus DSX6, Isolated from Lignocellulosic Dairy Waste," World J Microbiol Biotechnol, submitted.

Deis, B., and K. P. Feris, 2011, "Optimization of a Novel Two-Stage Lignocellose Saccharification Reactor Design," 111th American Society for Microbiology General Meeting, New Orleans, LA, May 21-24, 2011.

Magnuson, T. S., 2011, "Microbial Bioenergy: From Electrons to Complex Carbon," University of Minnesota Biotechnology Institute Seminar Series, Minneapolis, MN, September 15, 2011.

Magnuson, T. S., 2011, “The Search for Biocatalysts: From Pure Culture to Complex Ecosystems," Idaho National Laboratory Seminar Series, Idaho Falls, ID, July 8, 2011.

Jain, T., J. Van Gerpen, and A. McDonald, 2010, "Production of Fuel Ethanol from Woody Biomass," Journal of Biofuels, Vol. 1-1, ISSN: 0976-4763.

Magnuson, T. S., 2010, “Animal Waste Conversion Research at Idaho State University," Center for Advanced Energy Studies Bioenergy Summit, Idaho Falls, ID, 2010.

Magnuson, T. S., and M. W. Swenson, 2010, "Collaborative Research Between ISU and the PNNL," Pacific Northwest National Laboratory, Richland WA.

Jain, T., and J. Van Gerpen, 2010, "Criteria for Locating a Lignocellulosic Ethanol Plant in Idaho," ASABE Annual Meeting 2010, Pittsburgh, PA, June 20-23, 2010. 
Jain, T., B. Deis, M. R. Erdomnez, J. Van Gerpen, and K. Feris, 2010, "Enzymatic

Conversion of Lignocellulosic Biomass to

Sugars," ASABE Meeting, Lethbridge,

Canada, September 9-12, 2010. 


\section{Optimization of Ceramic Waste Forms Used for Electrochemical Processing of Spent Nuclear Fuel}

Michael Simpson; Supathorn Phongikaroon (University of Idaho)

10-015

Electrochemical spent fuel technology, also known as pyroprocessing, has been developed by INL and other labs to separate actinides from fission products and other nuclear waste components. It is currently being used at INL to condition spent fuel from Experimental Breeder Reactor-II for permanent disposal. Other countries including Japan, South Korea, India, and Russia are currently developing pyroprocessing as a candidate for recycling commercial spent fuel and eventually using it to fabricate fuel for fast reactors. Unlike aqueous processes such as Plutonium-URanium EXtraction (PUREX), pyroprocessing is not capable of separating pure $\mathrm{Pu}$ from the used fuel and thus has inherent non-proliferation benefits. It is also attractive due to its compact space requirements and compatibility with a metal fuel cycle, which is a popular option for fast reactors. This project aims to develop technology for minimizing the volume of high-level nuclear waste generated from pyroprocessing of spent fuel. The approach being investigated includes zone freeze refining of salt to recycle the majority of useful LiCl-KCl back to the electrorefiners. Zone freezing concentrates fission products into approximately $20 \%$ of the original salt volume. To then immobilize the concentrated salt phase into a waste form, sorption of that salt into zeolite-A is being studied. Ultimately, it is expected that the volume of high-level waste can be reduced by a factor of 5 or more compared to current technology.

\section{Project Summary}

The overall strategy of this project is to use experiments with zone freezing and salt sorption to develop models for these two processes. Those models can then be used to design and optimize an integrated salt treatment/waste fabrication process to be coupled with spent fuel electrorefining. Over the first two years, extensive experimentation was performed on both zone freezing and salt sorption. A zonefreezing furnace was installed in the Center for Advanced Energy Studies Radiochemistry Laboratory and has been used to collect transient temperature and salt-phase segregation data using $\mathrm{LiCl}-\mathrm{KCl}-\mathrm{CsCl}$ and $\mathrm{LiCl}-\mathrm{KCl}-\mathrm{CeCl}_{3}$ salt mixtures. The effect of sample movement speed, temperature gradient, salt composition, and salt mass have all been examined and compared to models that are still in development. Phase segregation has been observed for both salt systems with the temperature gradient having the greatest effect on degree of separation. Work continues to optimize the process parameters and develop a predictive model. In the area of salt sorption in zeolite, a variety of experiments have been performed involving salt and zeolite in contact at temperatures ranging from 300 to $500^{\circ} \mathrm{C}$. Single-component salts such as $\mathrm{CsCl}$, $\mathrm{SrCl}_{2}$, etc., have been demonstrated to diffuse into the zeolite in the solid state via sorption tests run below their melting point. To understand the mechanism and rate limiting processes involved with this sorption, contacts between zeolite and molten LiCl-KCl-MClx (where M is Cs or Sr) were run at various zeolite particle sizes. Uptake measurements were fit to several models with a diffusion-limited one demonstrating the best fit to the data. Insight gained into the fundamental process for sorption led to the discovery of a zeolite modification that could be made in order to speed up sorption of Cs, a key fission product in electrorefiner salt. Optimally engineered zeolite samples will be prepared in the last year of the project and tested for application to sorption of both electrorefiner salt and oxide reduction salt. Though not originally part of the project's scope, application of this technology to treating oxide reduction salt could turn out to be a valuable development. No such process has yet been discovered for this purpose, which has represented a major technical deficiency for pyroprocessing of spent oxide fuel.

\section{Benefits to DOE}

This project benefits DOE's energy security mission by supporting the closing of the nuclear fuel cycle, potentially making nuclear energy sustainable with minimal environmental impact. If successful, the project will lead to development of improved spent fuel pyroprocessing technology that generates significantly less waste than current systems. Such a development would be expected to lower processing costs and minimize or even eliminate the need for a long-term repository for spent fuel. 
Relevant Publications and Presentations

Williams, A. N., S. Phongikaroon, and M. Simpson, 2010, "Zone Freezing Model for Pyrochemical Process Waste Minimization," 2010 Annual Meeting of the American Institute of Chemical Engineers, Salt Lake City, Utah, November 7-12, 2010.

Simpson, M. F., J. Allensworth, M. S. Yim, M. Shaltry, S. Phongikaroon, C. Ferguson, and M. L. Dunzik-Gougar, 2010, "Immobilization of Salt from Zone Freezing Process in Zeolite-A," 2010 International Pyroprocessing Research Conference, Dimitrovgrad, Russia; November 29 December 3, 2010.
Williams, A. N., S. Phongikaroon, and M. Simpson, 2011, "Zone Freezing Study for Pyrochemical Process Waste Minimization," Center for Advanced Energy Studies Colloquium Series, Idaho Falls, Idaho, April 2011.

Simpson, M. F., M. S. Yim, M. Lineberry, M. L. Dunzik-Gougar, A. Williams, M. Shaltry, D. LaBrier, J. Allensworth, R. Bezzant, C. Ferguson, and J. Wallis, 2011, "Developing Chemical Separations Methods to Minimize High Level Waste from Pyroprocessing of Spent Nuclear Fuel," Center for Advanced Energy Studies Colloquium Series, Idaho Falls, Idaho, August 31, 2011. 


\title{
Prediction and Monitoring of $\mathrm{CO}_{2}$ Behavior in Deep Reactive Geologic Formations
}

\author{
Travis McLing, Robert Podgorney; Jerry Fairley (University of Idaho), Kasper van Wijk \\ (Boise State University) \\ $10-053$
}

This LDRD project is a cooperative research effort between INL, Boise State University (BSU), and University of Idaho (UI), with each of the organizations bringing a very specific skill set needed to help bridge the large knowledge gap associated with the characterization of reactive rocks (specifically the mafic rocks of Idaho and the Pacific Northwest) for potential $\mathrm{CO}_{2}$ storage. UI and INL have lead the field characterization and modeling activities, while BSU has lead the geophysics modeling and experiments. The project is on budget and on scope, with INL, BSU, and UI working together to accomplish its goals. Two students are working on degrees supported by this project: Ryan Polleya, a PhD student at UI (expected to graduate May 2012), and Thomas Otheim, a MS student at BSU (expected graduation August 2012). Both students have made significant progress in their research during this past year.

\section{Project Summary}

$\underline{\text { Strata characterization and modeling }}$

UI and INL have worked closely to develop characterization and modeling strategies for both the thoelitic basalt provinces of the Pacific Northwest as well as the basalts found on ocean spreading ridges (Juan De Fuca Plate). UI's Computational Hydrology Group focused on numerical simulation of carbon sequestration in the fractured basalts of the Eastern Snake River Plain (ESRP). Data collection during the previous years of the study (see below) focused on obtaining sufficient data on the spatial distribution of fractures in the ESRP basalts to condition numerical models. With the spatial distribution analyses largely completed, the Computational Hydrology Group focused on using the inferred property distributions to populate numerical simulations in an effort to answer the following questions: Do the fractured ESRP basalts demonstrate sufficient conductivity and connectivity to allow sustained multiphase injection of supercritical carbon dioxide? What level of containment of injected $\mathrm{CO}_{2}$ can be expected from these low-volume basalt flows? Might it be possible to evaluate the potential of a given injection zone for geological carbon sequestration on the basis of a simple algorithm, rather than relying on complex and expensive multi-well studies?
During FY 2011, the team made significant progress in these areas. It collected data on the spatial distribution of high-permeability zones (zones of fracturing) in the ESRP basalts; this was partially done "by hand" at Box Canyon, Idaho, and partially done using terrestrial laser scanning (also known as ground-based LiDAR). As described in our previous annual report, these data were analyzed using spatial-statistical methods; our analysis of the spatial distribution of fracturing was based on the method of indicator variograms, which indicate the probability of material continuity at a point a given distance from a known point. These models of spatial distribution were tested against ESRP data found in the literature and were examined for error and bias using cross validation (a kriging method of sampling with replacement). We used a sequential indicator simulation (sisim) to generate multiple, statistically equivalent realizations of the distributions of ESRP basalts that could be used as synthetic ESRP aquifers for hypothesis testing. The data collection and spatial analysis of fracture data were critical to the success of this year's modeling and simulation work. In addition to the data analysis tools developed in previous years, the spatial distribution model fed directly to the models used to investigate the feasibility of geological carbon sequestration on the ESRP.

\section{Sub Seafloor Modeling}

As part of this LDRD project a collaborative modeling effort was started in 2010 and continues with Lamont Doherty Earth Observatory to examine the injection potential and evaluate the capacity of deep-sea basalt formations (specifically Juan De Fuca). Subseafloor carbon sequestration is another potential option for geologic storage of $\mathrm{CO}_{2}$. A number of reservoir characteristics, namely mafic-rockhosted injection horizons, extensive sediment caps, and deep, cold overlying seawater make these reservoirs particularly attractive. However, there are significant science and engineering challenges that must be examined relative to subseafloor storage. 


\section{Geophysics experimentation and modeling}

$\mathrm{BSU}$ is conducting the experimental work associated with selected samples of basalt that have been analyzed so that changes in seismic and ultrasonic velocities resulting from $\mathrm{CO}_{2}$ sequestration in basalt rocks can be measured, the goal of which is to describe the process that would increase the elastic modulus and velocity of the rock when exposed to $\mathrm{CO}_{2}$. Typically, reservoirs where the rock properties change as a result of fluid or pressure changes are commonly monitored with seismic methods. However, in our research, BSU is conducting a set of experiments to study the feasibility of monitoring $\mathrm{CO}_{2}$ migration in non-typical basalt formations, which are notoriously difficult to monitor using geophysical techniques. Our goal is to measure the rock's elastic response to mineralization with non-contacting ultrasonic lasers and the effect of fluid substitution at reservoir conditions at seismic and ultrasonic frequencies.

In the early stages of this study, BSU collected and prepared specific basalt samples that INL had previously chemically and petrographically characterized. These samples were prepared so that the elastic rock properties at seismic and ultrasonic frequencies and at reservoir conditions (pressure, temperature, and chemistry) could be measured. Samples prepared were for the fluid substitution experiments that were conducted at the Colorado School of Mines. The $\mathrm{CO}_{2}$ mineralization dissolution experiments are being conducted at BSU. Results of this task are reported in our extended abstract for the annual meeting of the Society of Exploration Geophysicists:

http://earth.boisestate.edu/pal/files/2011/04/Final SEG2011Basalts.pdf. The MS student funded on this project, L. Thomas Otheim, spent two months this summer in The Center for Rock Abuse at Colorado School of Mines to utilize the school's unique facilities to measure the seismic and ultrasonic velocities of basalt samples dry, $\mathrm{CO}_{2}$ - and water-saturated at seismic frequencies and reservoir pressures, as well as mixtures of water and $\mathrm{CO}_{2}$. In Colorado, Thomas also collected numerous CT scans, where the gray scale in the resulting images is directly related to density (Figure 3 in
http://earth.boisestate.edu/pal/files/2011/04/Final SEG2011Basalts.pdf). A part of our objectives is to determine the impact of mineralization on the seismic properties. We are reproducing these results in our lab at BSU. Ultrasonic wave speeds were measured before placing the samples in a $\mathrm{CO}_{2} /$ water mixture for 15 weeks, and we are now exposing the samples to the $\mathrm{CO}_{2}$ /water mixture for another 15 weeks. A $3-$ $12 \%$ increase was observed in the P-wave speed due to the first 15 -week mineralization.

\section{Benefits to DOE}

The expense of proposed geological carbon sequestration schemes is the single most important factor limiting their practical application to date. The ability to inexpensively evaluate the injectivity of a single well would be a major step forward for geological sequestration efforts. The two-dimensional simulations completed in this fiscal year show that such an evaluation is possible and can realistically be achieved. During the up-coming fiscal year, the follow-on three-dimensional simulations will provide the required information for the design of field tests.

Beyond the benefits of (relatively) inexpensive geological carbon sequestration to the nation and DOE, the development of the ability to inexpensively characterize the deep aquifer of the ESRP carries with it benefits for INL, the region, and DOE in terms of potential exploitation of the geothermal resources of the Snake River Plain. The modeling developed in this study offers a means for making the required estimates and hence provides a possible way forward for developing the enhanced geothermal systems potential of the Snake River Plain.

We have positioned ourselves as national experts on the analysis of large datasets through the publication of papers on terrestrial laser scanning data collection and analysis in refereed journals and have enhanced our reputation for multiphase, non-isothermal modeling of highresolution Monte Carlo domains. This work has been carried out thanks to our collaboration with the INL's High Performance Computing Center and the Center for Advanced Energy Studies Center for Advanced Modeling and Simulation, which have made it possible to run large 
numbers (order of 100) of extremely large (up to 3.2M gridblocks) multiphase simulations.

\section{Relevant Publications and Presentations}

Pollyea, R. M., and J. P. Fairley, 2011, "Experimental Investigations of LiDARbased Surface Roughness Estimates," Geosphere, accepted (in revision).

Pollyea, R. M., J. P. Fairley, R. K. Podgorney, and T. L. McLing, 2011, "Developing a Field Sampling Strategy for Continuum Representation of Fractured Rock using Outcrop Analogs," Ground Water Monitoring and Remediation, in review.

Pollyea, R. M., and J. P. Fairley, 2011, "Implications of Reservoir Uncertainty for $\mathrm{CO}_{2}$ Sequestration in Low-Volume Basalt," Hydrogeology Journal, in review.

Pollyea, R. M., and J. P. Fairley, 2011, "Estimating Surface Roughness of Terrestrial Laser Scan Data using Orthogonal Distance Regression," Geology, Vol. 39, No. 7, pp. 623-626, doi:10.1130/G32078.1.

Podgorney, R. K., and J. P. Fairley, 2011, “A Hypothetical Test of Sub-Seafloor Storage of Carbon Dioxide on the Juan de Fuca Plate, Western North America," $A G U$ Annual Meeting, OS31A-1610 (poster).

Pollyea, R. M., and J. P. Fairley, 2011, "Implications of Reservoir Uncertainty for Geologic Carbon Sequestration in LowVolume Basalt Flows," AGU Annual Meeting, H24B-06 (oral).

Pollyea, R. M., and J. P. Fairley, 2011, "Evaluating Errors in Least-Squares Based Surface Roughness Models for Analyzing Terrestrial LiDAR Data," GSA Annual Meeting, Paper 48-10 (oral).
Otheim T., L. Adam, K. van Wijk, T. L. McLing, and R. K. Podgorney, 2011, " $\mathrm{CO}_{2}$ Sequestration in Basalt: Laboratory Measurements," AGU National Meeting.

Otheim T., L. Adam, K. van Wijk, T. L. McLing, and R. K. Podgorney, 2011, " $\mathrm{CO}_{2}$ Sequestration in Basalt: Carbonate Mineralization and Fluid Substitution," Society of Exploration Geophysicist 2011.

Pollyea, R. M., J. P. Fairley, and R. K. Podgorney, 2011, "Characterizing Analog Mid-Ocean Ridge Basalts for $\mathrm{CO}_{2}$ Injection Modeling, Geological Carbon Capture and Storage in Mafic and Ultramafic Rocks," 2011 IODP/ICDP Workshop on the Role of Oceanic and Continental Scientific Drilling, Muscat, Oman, January 8-10, 2011.

Podgorney, R. K., A. Slagle, T. L. McLing, and D. Goldberg, 2011, "Numerical Simulation of Carbon Dioxide Sequestration in DeepSea Basalts, Geological Carbon Capture and Storage in Mafic and Ultramafic Rocks," 2011 IODP/ICDP Workshop on the Role of Oceanic and Continental Scientific Drilling, Muscat, Oman, January 8-10, 2011.

McLing, T. L., and W. Smith, 2011, "The Characterization of $\mathrm{CO}_{2}$ Mineralization in Basalt Formations from a Natural Analogue Site, Soda Springs Idaho," Western Energy Policy Research Conference Boise Idaho.

Fairley, J. P., S. E. Ingebritsen, and R. K. Podgorney, 2010, "Challenges for Numerical Modeling of Enhanced Geothermal Systems," Ground Water, doi:10.1111/j.1745-6584.2010.00716.x.

McLing, T. L., 2010, “ $\mathrm{CO}_{2}$ Near Surface Interactions," Presentation at the IEA-GHG Natural Analogues Workshop, Maria Laach, Germany, November 2010. 


\section{Fabrication of Advanced ODS Alloys using Field-Assisted Sintering \\ James I. Cole, Larry Zirker; Indrajit Charit, Darryl Butt (University of Idaho) \\ 10-056}

Oxide dispersion-strengthened (ODS) steels show promise as future fuel cladding materials for advanced fast reactors due to their excellent high-temperature strength, creep resistance, and radiation damage resistance. These alloys are generally made via high-energy ball milling of constituent powders followed by a series of relatively complex and expensive consolidation and forming processes. The purpose of this research has been to evaluate the spark plasma sintering (SPS) method, a process using high current to supplement temperature and pressure during sintering, to reduce the number of subsequent processing steps needed to fabricate the final useable product. The project fosters active collaboration between INL, Boise State University, and the University of Idaho through the Center for Advanced Energy Studies.

\section{Project Summary}

Within the scope of the overall project, several principle subtasks are distributed among the institutions. University of Idaho is responsible for fabrication development of lanthana $\left(\mathrm{La}_{2} \mathrm{O}_{3}\right)$-based alloys, Boise State University is focusing yttria $\left(\mathrm{Y}_{2} \mathrm{O}_{3}\right)$-based alloy development, and INL has focused primarily on development of a diffusion bonding or joining process for ODS tubes - specifically using the pressure resistance welding process.

During 2011, $\mathrm{La}_{2} \mathrm{O}_{3}$ and $\mathrm{Y}_{2} \mathrm{O}_{3}$ powders with particles smaller than $100 \mathrm{~nm}$ were ball milled with alloyed or constituent powders to form the feedstock for the subsequent SPS studies. Optimized ball milling conditions were obtained through parametric studies involving milling time. Similarly, studies varying sintering temperature and pressure were performed to optimize the SPS fabrication parameters. Interestingly, the hardness of ODS alloys containing $\mathrm{La}_{2} \mathrm{O}_{3}$ was found to be higher than that of ODS alloys containing $\mathrm{Y}_{2} \mathrm{O}_{3}$, while the density was less. The maximum hardness was achieved at $950^{\circ} \mathrm{C}$ for both the alloys. The relative density and hardness results associated with the samples recently sintered are shown in Figure 1 as functions of the sintering temperature. The sintering time was 30 minutes, and the applied pressure was $80 \mathrm{MPa}$. The $1000^{\circ} \mathrm{C}$ sintering temperature was associated with the optimal hardness and relative density. Microstructural characterization of these samples is presently being undertaken and will continue into the next fiscal year.

At the Center for Advanced Energy Studies, pressure resistance welding was used to join HT-9 end plugs to ODS cladding tubes using a resistance welding system manufactured at Centerline LTD in Windsor, Canada. Excellent metallurgical bonding was obtained during these tests. New electrode collets were designed and fabricated in 2011 to achieve consistent tube welds. Scanning electron microscopy in combination with electron backscatter diffraction and transmission emission microscopy analysis methods were used to investigate the welding microstructure and evaluate joining integrity in 2011.

\section{Benefits to DOE}

Research using the field-activated sintering for ODS steels is relatively novel. INL and its university partners are well positioned to lead this effort. ODS alloys can potentially improve safety margins in current commercial reactors and dramatically improve fuel cladding performance of more advanced reactor systems envisioned for future deployment. An example of the uniqueness or flexibility of the SPS process is presented in Figure 1, which also shows that hardness of alloys can be adjusted by the sintering temperature used.

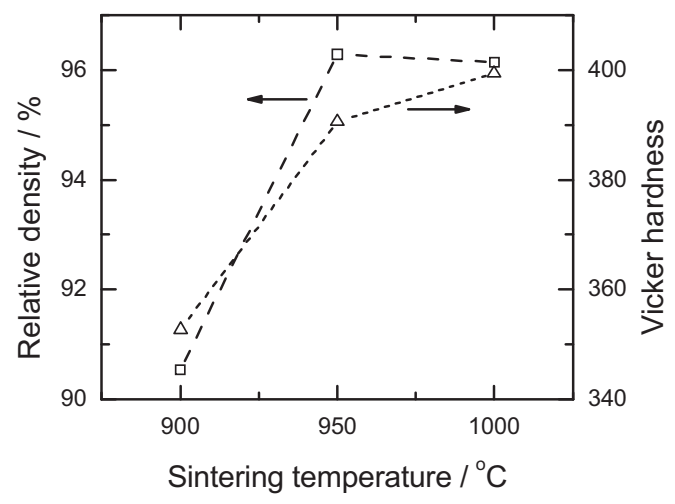

FIGURE 1. Relative density and Vicker hardness as functions of sintering temperature for samples sintered by SPS.

This research directly facilitates development of state-of-the-art research capabilities and technical expertise at the Center 
for Advanced Energy Studies, engaging two Idaho universities, creating opportunities for graduate students and other research personnel in their educational/training activities, and supporting advanced fuel cladding development.

Relevant Publications and Presentations

Pasebani, S., I. Charit, K. Allahar, B. Jaques, D. Butt, and J. Cole, 2011, "Development of Oxide Dispersion Strengthened Steels via Mechanical Alloying and Spark Plasma Sintering," Presented at THERMEC, Quebec, Canada, August 2011.

Allahar, K. N., J. Burns, B. Jaques, I. Charit, D. Butt, and J. Cole, 2011, "Spark Plasma Sintering of Ferritic Oxide Dispersion Strengthened Alloys," Processing and Properties of Powder-Based Materials, 2011 TMS Annual Meeting \& Exhibition, San Diego, CA, March 2011.
Pasebani, S., I. Charit, K. Allahar, B. Jaques, D. Butt, and J. Cole, 2011, "Microstructural Characterization of Mechanically Alloyed Lanthana-Bearing Oxide Dispersion Strengthened Steels," Processing and Properties of Powder-Based Materials, 2011 TMS Annual Meeting \& Exhibition, San Diego, CA, March 2011.

Jerred, N., L. Zirker, B. Jaques, T. Bradshaw, J. Carrillo, E. Young, I. Charit, J. Cole, M. Frary, D. P. Butt, M. Meyer, and K. L. Murty, 2010, "Pressure Resistance Welding of High Temperature Metallic Materials," Joining of Advanced and Specialty Materials XII, Materials Science and Technology 2010, Houston, TX, Oct. 17-21, 2010. (Presented at conference; manuscript accepted for proceeding publication). 


\title{
Small Specimen Test Techniques for Evaluating Radiation- Induced Changes in Mechanical Properties
}

\author{
Bulent H. Sencer, Mitchell K. Meyer; Darryl Butt, Frank A. Garner (Boise State \\ University) \\ 10-024
}

Development of a flexible test station for testing of small specimens in the Center for Advanced Energy Studies (CAES) Materials Laboratory will allow a broader spectrum of university faculty and students to conduct scientific and engineering studies on the physical and mechanical properties of nuclear material without having to use a hot cell. This project develops improved small sample testing techniques and uses them to provide an outstanding opportunity to train the next generation of radiation materials scientists.

The project will focus initially on two mechanical property techniques that are exceptionally well suited for testing of very small specimens either in a fume hood or behind light shielding: the horizontal tensile testing and shear-punch techniques pioneered at various national laboratories. Although very useful, further development is required to ensure that these techniques can provide reliable engineering data. A test station will be designed to incorporate tensile and shear-punch equipment and include provisions to perform other nondestructive (electro-resistive and ultrasonic) test equipment to study void swelling, transmutation, and other radiation-induced property changes of small specimens and staging for examination by electron microscopy before and after testing. Provisions for installation of a commercial hardness tester will also be made. Additions to the test station could include mechanical testing equipment, the primary candidate technique being the Charpy/Dynamic fracture toughness test.

This proposal team's seasoned national laboratory researchers, with university students and faculty, will design and build equipment and conduct experiments. With national lab and consultant guidance, the university faculty and students will design the two test fixtures and test station, incorporating the best design features of previous systems; conduct the necessary feasibility and safety studies; build the shielded facility and the test machines; and validate the improved performance of the equipment. This proposal relies on graduate students to perform the research and provides an excellent opportunity to link the CAES research with the
Advanced Test Reactor National Scientific User Facility.

\section{Project Summary}

Recent equipment purchases were the Instron tester, high-temperature grips, furnace, two anvils, linear variable differential transformer, and drop tower. We installed the Instron in the Materials Lab at CAES. Currently, we can perform shear-punch and tensile tests.

We are working on the modification for the drop tower that will do the Charpy test. Instron is enclosed to reduce any kind of hazard (from furnace and/or radioactivity) and is connected to the ventilation system to create negative pressure. We obtained a permit to ship radioactive materials and test them at CAES. In addition, a micro-density measurement cell was manufactured and assembled during the summer. This is in addition to the small sample testing techniques. We scheduled ATR-irradiated oxide dispersion-strengthened steel of five shear-punch discs from the Materials and Fuels Complex and a number of shear-punch discs from

Westinghouse. The graduate student started 304 austenitic stainless steel shear punch and tensile tests. Currently, the graduate student is studying deformation effect and strain effect on 304. We recently tested Experimental Breeder Reactor (EBR)-II irradiated 304 stainless steel and oxide dispersion-strengthened steel.

\section{Benefits to DOE}

This project research supports life extension of the current fleet of light water reactors and development of new materials for the next generation of reactors. This project comes at a time when it is critical to provide opportunities and training for a new generation of radiation materials scientists to enable continued use of nuclear energy in the U.S. as a clean, reliable energy source. 
Relevant Publications and Presentations

Rabenberg, E., K. Knori, B. Jaques, B. Sencer, D. Butt, and F. A. Garner, 2012, "Small Specimen Testing for Evaluating RadiationInduced Changes in Mechanical Properties of Structural Reactor Materials at High Irradiation Doses," 2012 TMS Annual Meeting \& Exhibition, Orlando, FL, March 11, 2012. 


\section{Design and Operational Improvements and LCA in Anaerobic Digestion of Fermented Dairy Manure using a Two-Stage Process}

Erin Searcy, A. Briones, E. Coats, K. Feris, D. Keiser, T. Magnuson, A. McDonald, D. Shrestha

10-080

Current dairy manure management strategies such as anaerobic digestion (AD) or lagoon storage/land application do not fully recover valuable organic matter and are ineffective in handling greenhouse-gas emissions or removing nutrients. While $\mathrm{AD}$ is a mature technology that handles multiple substrates, cost and operational challenges have minimized use. Common operational challenges are variable $\mathrm{pH}$ and ammonia inhibition of organisms. Conventional two-stage $\mathrm{AD}$ involves manure fermentation in a tank followed by AD of the supernatant in another tank. This operational configuration may address the $\mathrm{pH}$ issue; however, valuable organic matter remains unrecovered, and ammonia inhibition is not necessarily remedied. If we conversely focus on maximizing resource recovery from the manure, we could simultaneously produce multiple high-value commodities while addressing environmental concerns. This project will focus on the production of two high-value commodities, methane $\left(\mathrm{CH}_{4}\right)$ and biofuels, and treatment of nutrients in the $\mathrm{AD}$ effluent.

Our objective is to design and study two novel processes for maximizing recovery and conversion of Idaho dairy manure into energy products. The first process will maximize conversion of pre-fermented manure to $\left(\mathrm{CH}_{4}\right)$, while the second sequesters $\mathrm{AD}$ emissions $\left(\mathrm{CO}_{2}\right.$, nitrogen $[\mathrm{N}]$, phosphorus $[\mathrm{P}]$ ) to produce lipid-rich algae that can be recovered and converted to biofuels. A life cycle analysis (LCA) and economic study will be conducted to characterize the system. A two-stage fermentation and $\mathrm{AD}$ test apparatus will be assembled to test system impact on $\mathrm{CH}_{4}$ production from manure. The supernatant will be separated from the solids, and the effectiveness of nutrient removal using encapsulated algae (which may limit shear stress and increase lipid production) will be studied and compared to lipid and biomass production by unencapsulated algal cultures.

\section{Project Summary}

Improvements were made to the two-stage AD system based on results from FY 2011 (Year 1), and performance measurements were made that support successful operation of the system (i.e., methane production from the two- stage systems is slightly better than a standard AD system, and the two-stage system also allows for the extraction of higher-value components from the manure).

\section{$\underline{\text { The Two-Stage AD system }}$}

We operated and comprehensively evaluated two mesophilic AD systems: AD1 was fed raw manure (control system), while AD2 was fed pre-fermented manure. AD2 was shown to generate quantities of biogas comparable to that of AD1 (Fig. 1), and biogas production over the 24-hr operational cycle was relatively constant and similar between both ADs (Fig. 2). Further, methane content was improved in $\mathrm{AD} 2$, thereby yielding a higher value end product. Overall AD process stability using pre-fermented manure was enhanced over that processing raw manure. Nutrient removal $(\mathrm{P}, \mathrm{N})$ was comparable between the two ADs. Key results are highlighted in Figures 1-3 (next pages) and summarized in Table 1.

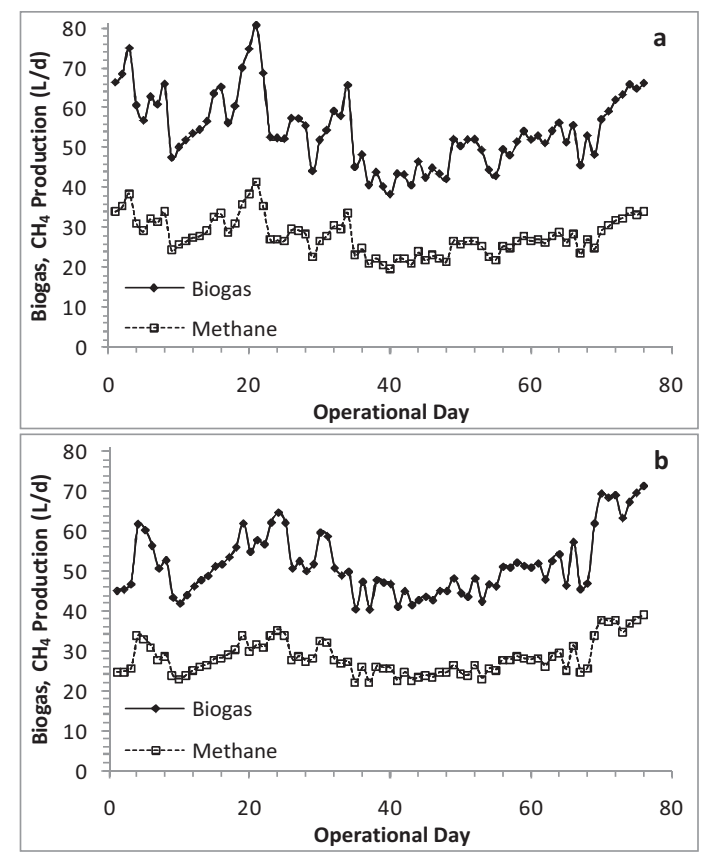

FIGURE 1. Daily biogas/methane production over the operational analysis period for (a) AD1 and (b) AD2. 


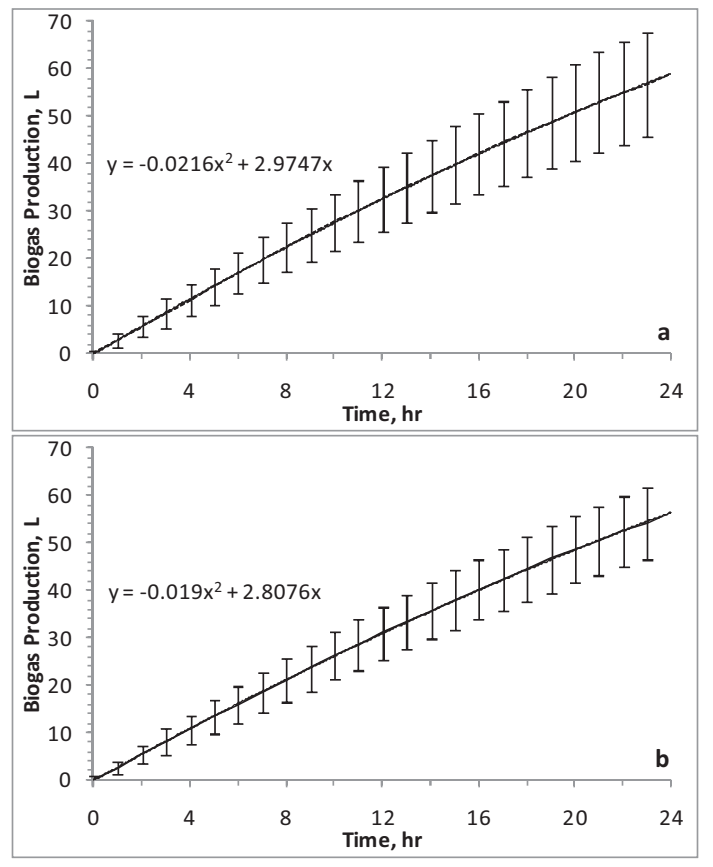

FIGURE 2. Typical biogas production over a 24-hour operational cycle (average and standard deviation $(n=10)$ for (a) AD1 and (b) AD2.

The two-stage $\mathrm{AD}$ system is designed to ultimately produce bioplastics from the fermenter supernatant and biogas from the digestion of residual fermented substrate. The group is also interested in improving the efficiency of the $\mathrm{AD}$ by utilizing a wider range of microbes known to be involved in fiber degradation in the cow rumen. This ecosystem is considered superior in terms of solids breakdown compared to conventional AD systems.

Anaerobic fungi of the order Neocallimastigales are capable of colonizing the plant materials in the early stage of rumen fermentation. These microbes play a major role in the initial breakdown of lignocellulosic materials. We aim to detect and identify anaerobic fungi originating from cow manure and to test our hypothesis that stage separation will allow for a greater degree of biodegradation of lignocellulolytic material by anaerobic fungi. Currently, we are concentrating on anaerobic fungal enrichment and identification based on ribosomal RNA gene sequences derived from cow manure. We are in the process of detecting and identifying anaerobic fungi in the cow manure feed, the fermenter, and the digester. Knowledge of anaerobic fungal communities and populations in these sources will be used to evaluate the contribution of fungi during two-stage anaerobic digestion of the cow manure.

Our key results indicate that anaerobic fungi (AF) are clearly present in cow manure. A polymerase chain reaction (PCR) designed to amplify portions of the AF rRNA gene could consistently amplify AF DNA from freshly extruded feces. The confirmation of the presence of AF in cow manure consisted, firstly, of enrichment of the fungi from manure samples. After optimizing conditions for growing AF, we were able to visualize AF zoospores with morphologies consistent with known AF. However, it is difficult to identify the fungi by relying on microscopy. So far, we have not yet been able to establish pure cultures.

Identification was accomplished by sequencing the ribosomal RNA gene segment amplified during PCR.

A clone library of AF rRNA gene fragments amplified from freshly extruded manure from a cow at the University of Idaho dairy farm was constructed. A total of 96 clones were submitted for bidirectional sequencing. Analysis of the sequences obtained is on-going. Preliminary analysis of 18 selected sequences suggests that there are at least three main groups of $\mathrm{AF}$ associated with our cow manure: these groups are related to the genera Cyllamyces, Orpinomyces, and Neocallimastix, which is the largest group of clones grouped with known Cyllamyces sequences. This may represent the most abundant group of AF in our manure sample.

Phylogenetic analysis also suggests that the AF populations could be distinguished based on variations in sizes of the PCR amplicons. PCR to detect $\mathrm{AF}$ from samples collected from feed, fermenter, anaerobic digester 1 (AD1, traditional single-stage $\mathrm{AD}$ fed raw manure), and $\mathrm{AD} 2$ (fed by pre-fermented manure) has consistently shown higher levels of detection in the fermenter samples (using a standard amount of template DNA for the PCRs). 
TABLE 1. Summary of operational and performance characteristics for both anaerobic digesters.

\begin{tabular}{|c|c|c|c|}
\hline & & $\mathrm{AD} 1$ & $\mathrm{AD} 2$ \\
\hline Average Reactor TS ${ }^{1}$ & $\%(\mathrm{w} / \mathrm{w})$ & 4.8 & 4.6 (AD2); 4.5 (fermenter) \\
\hline Average Influent $\mathrm{AD} \mathrm{VS}^{2}$ & $\mathrm{~g} \mathrm{VS} / \mathrm{d}$ & $148 \pm 16 \quad(n=25)$ & $168 \pm 18.3(n=25)$ \\
\hline Average VS destruction & $\%$ & $43.7 \pm 8.1 \quad(n=25)$ & $\begin{array}{l}40.6 \pm 6.7(\mathrm{n}=24 ; \text { AD only }) \\
51.6 \pm 7.4(\mathrm{n}=24 ; \text { fermenter }+ \\
\mathrm{AD})\end{array}$ \\
\hline Average Biogas produced & $\mathrm{L} / \mathrm{d}$ & $54.5 \pm 9.1 \quad(\mathrm{n}=76)$ & $51.8 \pm 7.9(\mathrm{n}=76)$ \\
\hline Average Methane content & $\%$ & $51.4 \pm 1.1 \quad(\mathrm{n}=28)$ & $54.3 \pm 1.2(\mathrm{n}=28)$ \\
\hline \multirow[t]{4}{*}{ Yield } & L biogas/gVS destroyed & 0.84 & 0.76 \\
\hline & L biogas/gVS applied & 0.37 & 0.31 \\
\hline & L biogas/L-d & 1.36 & 1.30 \\
\hline & $\mathrm{L} \mathrm{CH}_{4} / \mathrm{L}-\mathrm{d}$ & 0.70 & 0.71 \\
\hline \multirow[t]{2}{*}{ Influent VFAs ${ }^{3}$} & $\mathrm{mg} / \mathrm{L}$ as $\mathrm{COD}^{4}$ & $2,625 \pm 1,042(n=63)$ & $5,667 \pm 2,576(\mathrm{n}=68)$ \\
\hline & $\mathrm{mg} / \mathrm{L}$ as VFAs & $1,876 \pm 731 \quad(n=63)$ & $3,283 \pm 1,379(n=68)$ \\
\hline \multirow[t]{2}{*}{ Effluent VFAs } & $\mathrm{mg} / \mathrm{L}$ as $\mathrm{COD}$ & $907 \pm 564 \quad(n=63)$ & $1,116 \pm 809(n=69)$ \\
\hline & $\mathrm{mg} / \mathrm{L}$ as VFAs & $697 \pm 448 \quad(n=63)$ & $846 \pm 670(n=69)$ \\
\hline
\end{tabular}

$\mathrm{TS}=$ total solids

$\mathrm{VS}=$ volatile solids

VFAs $=$ volatile fatty acids

$\mathrm{COD}=$ chemical oxygen demand

These results suggest that the stageseparated bioreactors (fermenter and AD2) mimic the natural distribution of $\mathrm{AF}$ in ruminants, i.e., elevated levels are encountered in the rumen (fermentation compartment) compared to the large intestine (predominantly methanogenic). PCR analysis of samples collected within one batch of manure fed to the bioreactors suggests that there may be shifts of AF populations through time. One-day-old versus eight-day-old manure appears to select for different populations of $\mathrm{AF}$ in the fermenter, as seen by the shift in size of the main amplicon.

Reactor Monitoring via Proteomics. We analyzed raw reactor samples as well as electrophoretically detected lignocellulose degrading enzymes using proteomic methods. In spite of this, a variety of polysaccharide hydrolases were detected in bioreactor (dairy waste) supernatants. These results were confirmed by electrophoretic detection of enzymes in samples. Customized databases are key to accurate detection, and we have made progress in designing and building databases for proteomics queries. Example: Assemble all known bacterial xylanase sequences, or subsets thereof (e.g., Clostridium enzymes, actinomycete enzymes). Currently we are assembling databases for "nutrient"-related enzymes, e.g., nitrate conversion, sulfur conversion, and phosphorus conversion. This approach should yield similar results.

Functional Gene Detection in Reactors. We have had initial success with PCR detection of genes involved in nitrate reduction (nar) and sulfate reduction $(d s r)$. Dairy waste bioreactor samples were taken over a time course and analyzed using specific primers. Presence of these genes seems to be time-dependent; however, this needs to be confirmed by quantitative PCR. Additional primers specific for genes involved in sulfur oxidation, methane oxidation, and $\mathrm{CO}_{2}$ fixation will be evaluated. 
Future work will quantify and monitor the changes in specific populations of AF in the two-stage system and measure their contributions to the fermentation and AD processes. Alternatively, the interactions between AF, bacteria, and archaea during two-stage anaerobic digestion will be investigated.

\section{Biofuels Production}

Nutrient removal (total $\mathrm{N}$, total $\mathrm{P}$, chemical oxygen demand, $\mathrm{NH}_{4}+$ ) from the liquid manure effluent at various concentrations using algae is ongoing, and results have shown significant removal of nutrients (Figs. 3 and 4).

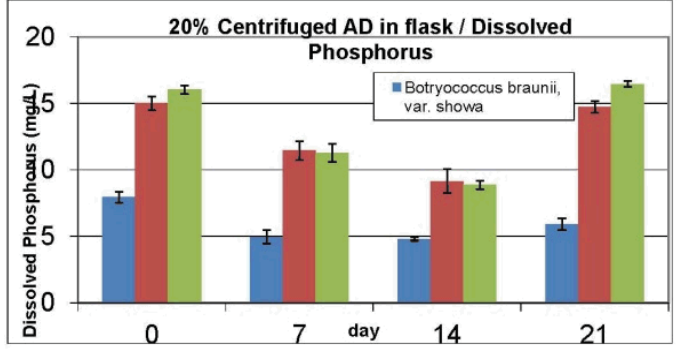

FIGURE 3. Removal of dissolved phosphorous by various algal species growing in 20\% AD effluent.

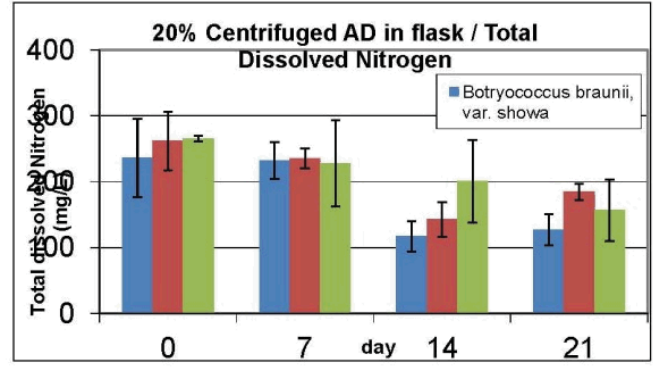

FIGURE 4. Removal of dissolved nitrogen by various algal species growing in $20 \%$ AD effluent.

The level of oil production and quality of oil is currently being evaluated, which will impact the economics of the process. Initial results are promising (see Table 2). The team is looking at the growth potential of additional algal species as well as a range of effluent concentrations. We have and continue to evaluate the growth potentials of additional algal species, including Chlorella sp UTEX 2168, Chlorella zofingiensis UTEX B32, and compare the results to
Botrycoccus braunii var. showa. On-going experiments are also assessing the nutrient removal and biomass production rates of mixed algal communities recovered from local wastewater treatment systems. High AD effluent concentrations show variable biomass yields across strain types and niche partitioning. The B. braunii tends to populate surface layers of the substrate, while the Chlorella strains tend to populate bottom layers and sides of flasks. On going experiments are monitoring algal growth rates, nutrient removal, and lipid production in small-scale systems fed differing concentrations of AD effluent).

TABLE 2. Lipid yields for various algal cultivars grown under standardized media conditions. On-going experiments are quantifying lipid yield when grown on diluted $A D$ effluent.

\begin{tabular}{|l|c|c|}
\hline \multicolumn{1}{|c|}{$\begin{array}{c}\text { Algae } \\
\text { Species }\end{array}$} & $\begin{array}{c}\text { Biomass Yield } \\
(\mathrm{mg} / \mathrm{ml})\end{array}$ & $\begin{array}{c}\text { Lipid Yield } \\
(\mathrm{mg} / \mathrm{g})\end{array}$ \\
\hline $\begin{array}{l}\text { Botryococcus } \\
\text { braunii-2 }\end{array}$ & 2.60 & 411 \\
\hline $\begin{array}{l}\text { Chlorella sp. } \\
\text { UTEX-2168 }\end{array}$ & 1.28 & 35 \\
\hline $\begin{array}{l}\text { C. Zotiniesis } \\
\text { UTEX-B32 }\end{array}$ & 1.21 & 56 \\
\hline $\begin{array}{l}\text { C. vulgaris } \\
\text { UTEX-2714 }\end{array}$ & 1.53 & 33 \\
\hline
\end{tabular}

Future work will involve optimization of $\mathrm{CO}_{2}$ sequestration, nutrient removal, and lipid production in the $10 \mathrm{~L}$ reactor when fed $\mathrm{AD}$ effluent and artificial AD biogas.

\section{Life Cycle Analysis}

The life cycle analysis is progressing, and a non-disclosure agreement has been signed with Cargill to obtain data for the analyses, which included several visits to two AD facilities. Dairy industry partners have been consulted to help us understand real challenges encountered in AD facilities. Other data has been collected through an extensive literature review, and a graduate student is working with Cargill to acquire baseline data to which the two-stage AD system will be compared. The next steps will involve comparing the conventional AD performance to the two-stage AD performance. 
Future Funding Opportunities Explored

Several opportunities for future funding have been explored through this project. Some examples include a submission for a DOEEPSCoR (Experimental Program to Stimulate Competitive Research) grant (declined), and discussion with the U.S. dairy industry through the United States Department of Agriculture's (USDA's) Innovation Center. A submission was made to the USDA-Agriculture and Food Research Initiative Foundational Program on a proposal titled "Investigating the Microbiology and C Cycling During the Integrated Processes Of Composting, Biochar Application and Anaerobic Digestion." Other collaborations are being pursued with Caterpillar on value-added products from manure digestion, Simplot on research-directed art converting waste to biofuels, and with Amalgamated Sugar on research to convert sugar-beet waste into biofuels as well as digestion of energy crops. Potato Growers of Idaho, Ag Power, AlgaePower, Northwest Food Processors Association, South Central Washington Dairymen, and C-3 Holdings are among some other potential collaborators.

\section{Benefits to DOE}

This project has benefited DOE national security missions by helping develop safe, competitive, and sustainable energy systems using U.S. resources. The project is also helping meet DOE goals by developing partnerships with local universities, which not only develops DOE's capabilities but also introduces new future researchers to DOE work. Several students have been supported to various degrees through this project, including:

- Linda Shen, summer intern, Idaho State University

- Pritee Pradhan, graduate student, Idaho State University

- Maxine Prior, graduate student, Boise State University

- Chirawan Thana, graduate student (partially supported), University of Idaho

- Alex Rheault, undergraduate student intern (partially supported), University of Idaho

- Kyle Artrip, graduate student, University of Idaho

Relevant Publications and Presentations

Coats, E. R., I. Ibrahim, A. Briones, and C. K. Brinkman, 2012, "Methane Production on Thickened, Pre-Fermented Manure," Bioresource Technology, Vol. 107, pp. 205212.

Artrip, K. G., D. Shrestha, and E. Coats, 2011, "GHG Emissions Reduction from an Anaerobic Digester in a Dairy Farm: Theory and Practice," Journal of Applied Engineering in Agriculture, submitted.

Searcy, E., 2011, "Investigating the Role of Anaerobic Fungi during the Anaerobic Digestion of Cow Manure," Poster presentation, Regional Approaches to Climate Change, Pacific Northwest Area Meeting, Moscow, Idaho, May 9, 2011. 


\section{Transmission Site Suitability Decision Support Technology}

Jeffrey Joe; David Solan, Michael Louis, David Koehler (Boise State University);

Juliet Carlisle, Daniel Ames, Robert Beazer (Idaho State University)

10-079

New electricity transmission lines must be built in Idaho and across the western region to meet increasing demand. Developers currently submit proposals for transmission line corridors that are least-cost based on technical and geospatial considerations. Initial proposals of where transmission lines should be placed miss the mark or create severe public opposition due to companies' acknowledged lack of understanding regarding public opinion and participation in siting decisions. The goal of this project is to develop a prototype geographic information system (GIS)-based decision support tool that can be used to facilitate electricity transmission line route selections. It will assess proposed alternatives and/or perform least-cost path analyses to identify feasible and potentially optimal transmission corridors by integrating geospatial data with socio-political constraints and provide a visualization of the results. The socio-political constraints will be collected through a survey that identifies and measures levels of opposition, and this information will be fed into a newly constructed GIS-based tool. The methodology and the tool will be demonstrated by analyzing potential routes for a future transmission development project.

\section{Project Summary}

Our main goals in the first year of the project (FY 2010) were to develop detailed objectives and questions, formulate a detailed research plan, and draft questions/items for the survey. In the process of accomplishing these goals, the research team had many different perspectives and ideas and discussed what the objectives and research questions should be. Over time, however, each team member's understanding evolved and the team came to a consensus on what some of the key research questions are and how the hypotheses will be tested. The research team has met their FY 2010 deliverable goals by producing the draft research plan and draft survey questions for this project.

In FY 2011, our main goals were to develop and issue the survey instrument, conduct preliminary analyses of the survey results, develop a theoretical GIS model, and begin writing software code. We accomplished all of these goals. The survey was completed in March 2011 and issued in April 2011. The analyses of the survey results were completed in May 2011. The theoretical GIS model was completed in the October- December 2010 timeframe. During the remainder of FY 2011, efforts were spent on writing software code to instantiate the theoretical concept, including working on the integration of the survey data with the GIS model.

\section{Benefits to DOE}

The strategic value of this research project is that the resulting GIS tool will aid electrical infrastructure siting decisions by providing cost savings to public and private entities, minimizing impacts to the environment, minimizing the risk of project failure due to technical and sociopolitical factors, and strengthening our national security through enhancing our energy infrastructure. The results of the project will also provide insights into needs and prioritization of future siting decisions in other domains. In fact, the research team submitted a proposal to the DOE SunShot initiative, which is a collaborative national initiative and funding grant to make solar energy technologies cost-competitive with other forms of energy, and in 2011 was awarded \$2.8 million to develop a GIS decision support tool to identify utility-scale solar facility sites based on quantifiable natural resource and physical restraints, as well as a scientific measure of social risk and public acceptance of screened sites. This SunShot project is scheduled to run for three years.

This research has also benefitted INL and state universities by providing funding to INL staff and university faculty and students as they collaborate on this research and development project. Specifically, this research has increased the ability of the four main institutions to collaborate effectively and is leading to Robert Beazer earning a Ph.D. in Geosciences from Idaho State University. Furthermore, this LDRD research has and will continue to improve INL's eminence as an institution that has considerable subject matter expertise in the area of energy research. 
Relevant Publications and Presentations

Carlisle, J., J. C. Joe, D. Solan, D. Ames, and R. Beazer, 2011, "Public Reactions to Energy Transmission Siting: The Role of Nimbyism," Poster presented at the Annual Meeting of the American Political Science Association, Seattle, Washington, September 2011.

Beazer, R., and D. Ames, 2011, "Integrating Social Attitudes and Spatial Data in Siting Power Lines using the DotSpatial Framework: Introducing the LineSiter Application," Poster presented at the Free and Open Source Software for Geospatial Conference, Denver, Colorado, September 2011.

Beazer, R., D. Ames, D. Solan, J. Carlisle, and J. C. Joe, 2011, "Integrating Social Attitudes and Spatial Data in Siting Power Lines," Presented at the 2011 Western Energy Policy Research Conference, Boise, Idaho, August 2011.

Carlisle, J., J. C. Joe, D. Solan, D. Ames, and R. Beazer, 2011, "Energy Transmission Siting. The Social Bases of Support," Presented at the 2011 Western Energy Policy Research Conference, Boise, Idaho, August 2011.
Joe, J., and S. Piet, 2011, "Better Public Engagement and Management of Technical Challenges: Keys to Addressing Wicked Energy Policy Problems," Paper presented at the 2011 Western Energy Policy Research Conference, Boise, Idaho, August 2011.

Solan, D., 2011, “Transmission Siting Decision Support Tool: Integrating GIS and Social Risk," Invited presentation to the Pacific Northwest Economic Region 21st Annual Summit, Portland, Oregon, July 2011.

Ames, D., and R. Beazer, 2011, "Integrating Social Attitudes and Spatial Data in Siting Power Lines Using the DotSpatial Framework," Presentation at the $2^{\text {nd }}$ International MapWindow GIS and Dotspatial Conference 2011, San Diego, California, June 2011.

Solan, D., 2011, "Transmission Siting and Options for Improvement: Incorporating GIS Tools and Social Risk," Invited presentation at the Northwest Wind and Wildlife Workshop, Portland, Oregon, June 2011.

Solan, D., 2010, “Transmission Siting Decision Support Tool," Invited presentation to the National Wind Coordinating Collaborative, Quarterly Meeting, Boise, Idaho, November 2010. 


\section{CAES Vertical Axis Wind Turbine Project}

Kurt Myers; John Gardner, Damon Woods (Boise State University)

10-088

The Blackhawk vertical-axis wind turbine (VAWT) is unique in that it incorporates simple mechanisms to perform complex physical functions - rotating, tipping, and teetering based on helicopter rotor design theory. Blackhawk has produced ten VAWTs of the current design that have withstood over two years of field testing mainly for robustness and safety. This project is focused on continued research and development to bring about performance gains in the design and implementation of this type of wind turbine. Our technical objective is to optimize a small (1.5 KW) VAWT with a unique articulating rotor by developing a comprehensive dynamic model, focusing on airfoil angle of attack to increase energy capture and reduce structural loading for minimal cost.

To achieve this main objective, Boise State University (BSU)/INL worked on the following tasks in FY 2011: (1) calculate theoretical lift and drag as function of angle of attack (continuation from FY 2010), (2) develop dynamic model of turbine system (continuation from FY 2010), (3) begin wind tunnel tests (strobing simulation) to determine airfoil lift and drag characteristics, (4) calculate theoretical torque for single and multiple blades, (5) compare theoretical to actual (measured) values, (6) add additional instrumentation to measure key parameters as determined by evaluating the system model versus observed performance, (7) begin design of candidate mechanical/electrical upgrades based on performance data, and (8) report results at an appropriate conference.

\section{Project Summary}

During FY 2011, BSU met Objectives 1, 2, 4, and 8. Partial progress was made on Objectives 5 and 7. Parts of Objectives 3 and 6 were progressing to happen as part of field testing on the turbine installed at the Center for Advanced Energy Studies (CAES). New instrumentation, inverter and communication wiring and programming, and troubleshooting were being discussed and were slated to start in early summer 2011. The generator and electronics for the turbine did not work very well during the first year, and efforts were underway to start troubleshooting to improve the programming and operation of the system. Upwind anemometers and inverter and powercurve reprogramming were installed, and changes/improvements were in planning. In late FY-11, discussions were started with CAES, $\mathrm{BSU}$, and the INL principal investigator to transition the mechanical engineering research, models, and results to a hydroturbine design that has similar characteristics to the Blackhawk (although it does not tilt/cant and operates in water instead of air).

Other accomplishments and research findings include:

- Found and digitized lift and drag data for the National Advisory Committee for Aeronautics (NACA) 0015 airfoil

- Developed a coordinate transformation system from the end of the blades to the rotor disc

- Wrote a function to describe the torque on the rotor given any wind speed and orientation

- Verified torque function using hand calculations and large volumes of geometry

- Developed a SimMechanics model of the non-tilting turbine using the coordinate transformations

- Verified these results with a simulink model based on integration principles instead of the mechanics blocks

- Met with Kurt Myers in Idaho Falls and discussed the current status of the turbine model TR-10 in operation at INL and talked about possible instrumentation to be installed this summer

- Added induction factor to model to account for power extraction from wind

- Charted basic power curve of the turbine for a range of wind speeds

- Researched ideal turbine pitch function

- Presented results at American Wind Energy Association Windpower 2011 Conference in Anaheim, CA. 
Other features of the current VAWT

simulation/model include:

- Vertical axis, 3-bladed non-tilting turbine with no "cant"

- Overall geometry comparable to TR-10

- Simulation uses NACA 0015 data (NACA 0014 data not readily available)

- Loading turbine with simple direct-current generator model

- Pitch angle can follow a user-defined function of rotor angle

- "SimMechanics" module in Matlab can easily accommodate forces and rotations in other planes (i.e., will accommodate tilting rotor).

Currently, BSU has successfully modeled a three-bladed non-tilting VAWT with articulating foils. They have demonstrated self-starting as well as other behaviors consistent with the literature and with the scant data available so far from the Blackhawk devices. The original plan was to continue this model to take into account the cant of the foils, the tilt of the rotor, and the kinematic connection between the tilt and the articulation of the foils. Many interesting research questions could be explored in this area, but without a test turbine and industry partner, a transition to a similar available technology appears applicable. A potential transition of the project to similar research on a similar hydroturbine design is currently in discussion and planning.

\section{Benefits to DOE}

VAWT design and small hydro and wind turbines will ultimately benefit INL, BSU, and DOE in that capabilities will be developed to support development and deployment of new small wind turbines and add to the large-scale deployment possibilities of wind and hydro energy around the nation. Deployment of renewable energy technologies is currently a major focus of DOE and the Department of Defense and projects of all sizes are needed to meet the goals of the U.S. government. Not all government facilities have the land availability or mission compatibilities to deploy large renewable energy projects, so all types of technologies and electrical grid/smart grid integration strategies need to be considered. The renewable energy work at CAES has led to the potential for testing many types of small renewable energy systems and grid integration strategies with the infrastructure being implemented at the site. All of these developments can help reduce the environmental impacts of power generation and increase the nation's energy security.

\section{Relevant Publications and Presentations}

Gardner, J., and D. Woods, 2011, "Dynamic Modeling of an Articulating Rotor VAWT," Poster presentation at the AWEA Windpower 2011 Conference, Anaheim, CA, May 22-25, 2011. 


\section{Development and Demonstration for National Fuel Efficiency}

David I. Gertman; Milos Manic, Graduate Internn Ondrej Linda (University of Idaho); David Bruemmer, Scott Hartley (5D Robotics)

$11-065$

Increasing national fuel efficiency for DOE bus fleets such as the INL Integrated Transportation Services (ITS) fleet is a significant challenge for DOE and the U.S. transportation industry. Increases in engine efficiency are possible through recent advances in technology; however, considerable variability between drivers ranging from 12 to $30 \%$ is known to exist. Reducing this variability profoundly reduces operating costs. The application of learning algorithms and real-time data management of sensor information can result in GPS-informed models that can aid bus drivers by cueing changes in planned accelerations that take into account windage and slope factors. To date, much of the industry's current emphasis has been on improving cab design aerodynamics, identifying proper maintenance schedules based on on-line monitoring of truck or bus subsystems, and stateof-the-art smart systems directed toward selftuning and optimized transmission shifting. However, largely absent is the complementary development of methods, models, and data that can be used not only to inform drivers in realtime, but to identify a best-driver model for known route applications. Driver interaction is a key component of vehicle efficiency and needs to be an integrated part of current approaches. In order to develop a sound technical basis, expertise from multiple domains is required, including: model development from historical and real-time data sources, real-time sensor data management, human system interface development, and usability lab and field tests. Additionally, as part of human factors and interface design, it is paramount that driver situational awareness and workload do not detract from driver safety once additional system information is provided to drivers. Finally, it is important that real drivers, fleet mechanics, and vendors providing vehicle sensor information work as an integrated team in development of an approach to increasing driver and fleet efficiency.

\section{Project Summary}

This LDRD focuses on three phases: (1) development of the initial route model including use of algorithms to develop a necessary and sufficient set of sensor information; (2) development of the real-time data handling and data management system, means to update a GPS and sensory-informed graphic user interface, measurement approach to defining driver-system efficiency, and a continual physical and functional refinement during the course of testing and validation studies; and (3) application to additional vehicle types such as hybrid vehicles. This requires implementation of the necessary sensors available from either the INL ITS fleet or one or more approved industry partners and to use this capability to drive the displays and gather empirical research data on human performance using the advanced driver graphical user interface system. At present, the ITS fleet uses a number of monitoring systems (DDEC \#5-7, and PowerDriver) for its 103 buses consisting of a number of engine and transmission types. The result of cueing drivers to methods for efficiency is believed to be generic, however, to ensure that results of studies will be generalizable to the existing and evolving bus fleet. Limited field studies to be conducted in Years 2 and 3 will also include bus models and information integraters such as Fleet Solutions who monitor performance factors across diverse vehicle types.

We developed a computational intelligence model that uses a MATLAB fuzzy logic controller to implement optimal driver action based upon current speed and slope indication while taking into account slope and speed variables. This model makes use of synthetic or surrogate data of driver behavior for model building and algorithm development. Real bus data was received in late FY 2011. As part of further model development, MATLAB feedforward artificial neural network architecture has been implemented to predict the near future of the vehicle. The neural network is trained in a supervised manner using a combination of the Error-Back Propagation and the LevenbergMarquardt training algorithm based on the recorded data.

Also, industry contacts were made with Detroit Diesel, Network Fleet, and Daimler Benz, all of whom are working to improve engine and fleet efficiency. During April 2011, we prepared a poster presentation at Engineers' Day documenting our project objectives, 
progress, and benefit to DOE. We also met with INL bus drivers to gather human performance insights regarding bus-driving efficiency and conducted a tabletop task analysis, each in the first quarter of FY 2011. A meeting with FLEET mechanics and management helped us extract parameter data from bus fleet operations. Finally, using surrogate data sets, we tested software architecture for high-bandwidth data streaming and verification.

\section{Benefits to DOE}

This research provides access to bus performance data on a more detailed level that can be used to develop predictive displays for bus driver(s) and also provides human factorsrelated research and field study methods for DOE transportation systems. These insights regarding the development and evaluation of methods for driver efficiency, workload, and situational awareness can be applicable to complex-wide transportation systems.
This work establishes empirically determined guidance on a best-driver model that can be used to support driver training. It also places INL, Hartley, and DOE in a leadership role for advanced visualization, human in-theloop simulator studies, cognitive studies, and the empirical testing and validation of design approaches to driver efficiency. Issues critical to the design and testing of human-centered technologies for the DOE and Department of Transportation were identified. Findings from this LDRD on driver-system efficiency are relevant to the Department of Defense where fuel costs and maintaining driver awareness are highly significant factors contributing to operational success. 


\title{
Targeted Energy Management Toolset Support
}

\author{
Criag Rieger, John Gardner; Milos Manic, Kevin Van Den Wymelenberg (University of \\ Idaho) \\ 11-066
}

Energy management systems (EMSs) in

buildings incorporate advances in microprocessor technology and implement standard communication protocols to retrieve sensor data and correspondingly adjust heating, ventilation, air conditioning (HVAC) and lighting systems to maintain human comfort levels while managing energy consumption. EMSs can also provide raw data back to building managers to assess the performance of HVAC and lighting systems and apply any applicable energy-efficiency measures and/or improve human comfort levels within the building. However, obtaining useful information from raw data poses a challenge because features and capabilities of EMS controls are often underutilized due to inexperienced users and time needed to gain familiarity. This project is developing a user-friendly integrated toolset that analyzes and fuses disparate data sets (lighting, HVAC, and human comfort measures) and presents the information in an actionable visualization platform for building operators. Thus, it enables multiple stakeholders (building operators, owners, users, and service providers) to more effectively manage energy use while minimizing negative human comfort consequences and promoting improved occupant satisfaction. The developed toolset will be tailored to cover the needs for a diverse set of building types with a variety of existing EMSs through a Bacnet open protocol and wireless mesh network. The analysis techniques will use advanced computational intelligence (neural networks and fuzzy arithmetic) to accomplish these goals.

This project is planned for three years. During the first year, building operators and owners were surveyed to gain additional knowledge about how they use EMSs to maintain the buildings' HVAC and lighting systems and to gather additional ideas for improved data analytics and visualization. This has included gaining information about how current EMSs present data back to the operators and owners and what combinations of sensors and synthetic data are combined on individual graphics. Also, in Year 1, initial data were analyzed to determine what additional sensors and acquisition devices would benefit the project. Finally, several institutional partners were explored for collaboration.

In Year 2, identification and selection of at least two building types will be identified to demonstrate the toolset, and its functionality will be initially reviewed with building operators for the selected buildings. This will include the planning and coordination to purchase and install supplementary sensors and acquisition devices for use in selected buildings. The data collected from EMSs and supplementary systems will be made available to Professor Manic. In addition, Professor Van Den Wymelenberg shall provide any information that correlates available sensors to synthetic data needs based on building operator and owner surveys. In the end, Professor Manic will lead the development of a paper on "concepts of reducing complexity and tailoring presentation" for a peer-reviewed conference or journal.

In Year 3, the demonstration of the final analysis and visualization system with the building operators of the selected buildings and with the industrial partner(s) will be given, and Professor Van Den Wymelenberg will lead the development and submission of a peer-reviewed paper on the "concepts of achieving human comfort and improved efficiency through enhanced visualization and supplemental sensors" from an architectural standpoint.

\section{Project Summary}

In the life of this project, Idaho Power Company has been established as an industrial partner and other industrial partners have been explored including a large commercial building developer, an institution of higher education, a military complex, and a national energy services provider. An extensive literature review was completed regarding the capabilities of EMSs in new research applications. For example, EMSs have been shown to provide monitoring-based commissioning where experienced building managers use EMS data to diagnose known problems that arise in HVAC and lighting equipment. Furthermore, electrical demand respond programs have been shown to reduce customers' peak demand and manage utility load profiles. More can be done with EMS to reduce the time invested to resolve known problems as 
well as identify further optimization by building operates. More can be done to improve the effectiveness of demand response programs to deliver load management while maintaining occupant comfort and productivity.

These findings were used to inform the building operator and owner surveys in order to gauge their interest and expectations of these EMS improvements. Seven organizations, collectively responsible for over $2,000,000$ square feet of commercial office buildings, academic buildings, research labs, maintenance shops, and warehouses, were interviewed. Most organizations use EMS to trend data only as problems arise. Operators would like improved visual representation of the data collected and improved analytics on comfort parameters, where energy savings opportunities exist as well as guidance on how to achieve these savings. Owners would like to understand where, how, and when energy is being used in their facilities and campuses. To date, 1-15-minute raw data from two buildings has been collected for a period of several months for initial analysis. A plan for a supplemental wireless mesh sensor data collection network was developed. This equipment is in procurement and will be installed in three buildings. These data, along with the minute data from the existing EMS will be fed to a sequel database and transmitted via cellular network to the research team on an ongoing basis over the next three months.

\section{Benefits to DOE}

This effort seeks to improve the effective usage of available energy within buildings to shrink the nation's dependence on foreign fuel supplies by reducing the energy intensity of America's economy and in turn improve the quality of the environment, energy and economic performance of buildings. Furthermore, this project will promote improved comfort and productivity of building occupants.

\section{Relevant Publications and Presentations}

Duarte, C., B. Acker, R. Grosshans, M. Manic, K. Van Den Wymelenberg, and C. Rieger, 2011, "Prioritizing and Visualizing Energy Management and Control System Data to Provide Actionable Information for Building Operators," Western Energy Policy Research Conference, Boise, ID, August 2526, 2011. 
$\Rightarrow$ Center for Advanced Modeling \& Simulation

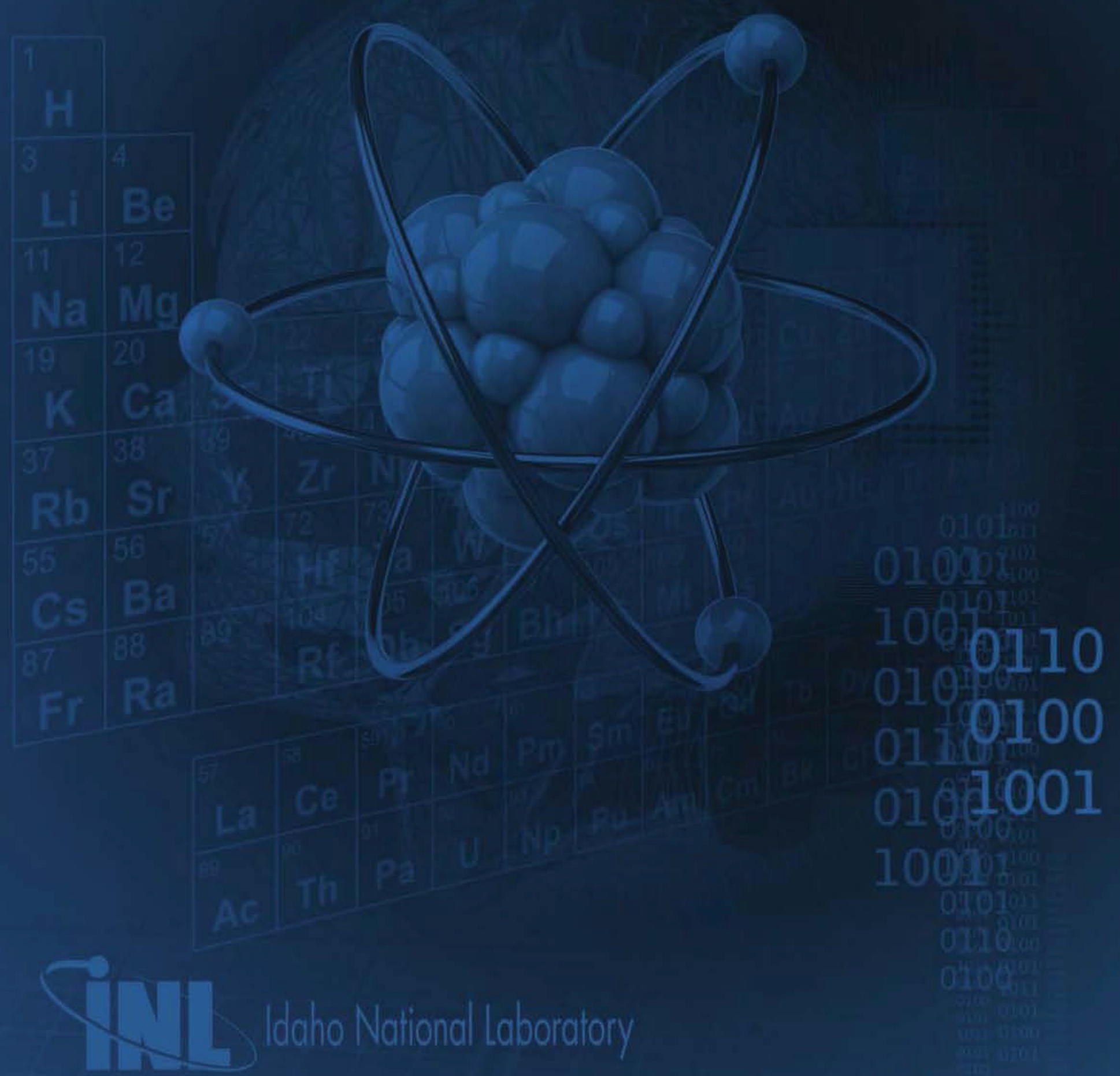




\title{
Preconditioning Methods for Hybrid Neutronics Calculations
}

\author{
Cristian Rabiti; Jim E. Morel, Sergiy Manolov (Texas A\&M University)
}

10-023

Our objective is to develop preconditioning methods for use in conjunction with Krylov methods to perform neutronics analyses using different angular discretizations in different reactor regions. Calculations in which multiple discretizations are used are defined as "hybrid calculations." Examples of these different angular discretizations include the even-parity discrete-ordinates equations, the sphericalharmonics equations, and the method of characteristics. In FY 2010, we demonstrated effective local and global preconditioners for a mixed discrete-ordinates/diffusion model in one dimension (1D). Our plan for FY 2011 and FY 2012 has been and will continue to be to generalize our $1 \mathrm{D}$ approach to $2 \mathrm{D}$, combining two high-order angular discretizations. Our local preconditioners will be based upon block-Jacobi iteration schemes that are commonly referred to as response matrix methods. Our initial global preconditioner will be based on a diffusion approximation. Very little research relating to preconditioned Krylov methods for hybrid calculations has been accomplished. This is the first time an overall global iteration approach will be tested. Even with large improvements in computing power, the challenge of performing a high-fidelity neutronics simulation of a full reactor seems unrealistic for several years to come. On the other hand, strong local heterogeneities arising from control rods, transmutation targets, and reflectors pose challenges with respect to the simulation accuracy that can be optimally met with a combination of different transport discretizations in a single calculation. If successful, this research will enable us to tailor computational discretizations to the needs of high-fidelity simulations and to maximize efficiency.

Texas A\&M University, under a subcontract funded by this LDRD project, will develop preconditioners for hybrid Sn-diffusion in 1D and 2D and for hybrid Sn-Pn in 2D. The resulting algorithms will be implemented in an INL neutronics code, and the lessons learned in developing these preconditioners will impact the development of more general preconditioners developed within the INL code.

\section{Project Summary}

During the first part of FY 2011, we completed a preconditioning scheme for onedimensional, two-region problems using the $\mathrm{Sn}$ approximation in one region and the diffusion approximation in the other. Our iterative algorithm consisted of a Sn sweep in the transport region followed by a global diffusion solve. In the transport region, the diffusion solution provided an additive correction to the Sn iterate for the scalar flux and thus played the role of diffusion-synthetic acceleration in that region. In the diffusion region, the diffusion solution did not provide any sort of correction, but rather constituted the complete solution. Furthermore, Sn-consistent vertex diffusion differencing was used in the transport regions while standard cell-centered diffusion differencing was used in the diffusion region. The disparate nature of both the purposes of the diffusion solutions and their discretizations made it challenging to couple the diffusion equations at the interface between the two regions. We were able derive conditions that ensured continuity of current but not continuity of the scalar flux. We also wrapped our iteration scheme in a Krylov solver as well, obtaining improved performance. A benefit of our approach was that only the transport scalar fluxes, the interface diffusion scalar flux, and the interface diffusion current were in the Krylov vector rather than the entire vector of unknowns.

After completing the above study (which is documented in our 2010 progress report), we turned our attention to two-region problems with $\mathrm{Sn}$ in one region and $\mathrm{Pn}$ in the other. Note that the Sn order is assumed to be higher than the Pn order. The first task was to determine a reasonable iteration scheme for the Pn equations, preferably one that bounds the spectral radius away from unity. We settled upon a combination of two types of iterations. To describe these iterations, we consider the $\mathrm{P} 3$ equations with isotropic scattering in even-parity form:

$$
-\frac{2}{3 \beta_{1}} \frac{\partial^{2} \phi_{2}}{\partial x^{2}}-\frac{1}{3 \beta_{1}} \frac{\partial^{2} \phi_{0}}{\partial x^{2}}+\beta_{0} \phi_{0}=0 \text {, }
$$




$$
-\frac{2}{15 \beta_{1}} \frac{\partial^{2} \phi_{0}}{\partial x^{2}}-\frac{11}{21 \beta_{1}} \frac{\partial^{2} \phi_{2}}{\partial x^{2}}+\beta_{0} \phi_{0}=0
$$

where $\phi_{n}$ is the n'th Legendre moment of the angular flux, $\beta_{n}=\Sigma_{t}-\Sigma_{n}, \Sigma_{n}$ is the n'th Legendre moment of the differential scattering

$$
\begin{aligned}
& \frac{2}{3 \beta_{1} h}\left[-\phi_{2, i-1}+2 \phi_{2, i}-\phi_{2, i+1}\right]+\frac{1}{3 \beta_{1}}\left[-\phi_{0, i-1}+2 \phi_{0, i}-\phi_{0, i+1}\right]+\beta_{0} \phi_{0, i} h=0 \\
& \frac{2}{15 \beta_{1} h}\left[-\phi_{0, i-1}+2 \phi_{0, i}-\phi_{0, i+1}\right]+\frac{11}{21 \beta_{1}}\left[-\phi_{2, i-1}+2 \phi_{2, i}-\phi_{2, i+1}\right]+\beta_{1} \phi_{2, i} h=0,
\end{aligned}
$$

where $i$ is the spatial index and $h$ is the cell width. The first scheme we consider is a block scheme in which all the spatial coupling between cells is lagged:

$$
\begin{aligned}
& \frac{2}{3 \beta_{1} h}\left[-\phi_{2, i-1}^{\ell}+2 \phi_{2, i}^{\ell+1}-\phi_{2 i+1}^{\ell}\right]+\frac{1}{3 \beta_{1}}\left[-\phi_{0, i-1}^{\ell}+2 \phi_{0, i}^{\ell+1}-\phi_{0, i+1}^{\ell}\right]+\beta_{0} \phi_{0, i}^{\ell+1} h=0, \\
& \frac{2}{15 \beta_{1} h}\left[-\phi_{0, i-1}^{\ell}+2 \phi_{0, i}^{\ell+1}-\phi_{0 i+1}^{\ell}\right]+\frac{11}{21 \beta_{1}}\left[-\phi_{2, i-1}^{\ell}+2 \phi_{2, i}^{\ell+1}-\phi_{2, i+1}^{\ell}\right]+\beta_{1} \phi_{2, i}^{\ell+1} h=0,
\end{aligned}
$$

where $\ell$ is the iteration index. The second is a Gauss-Seidel scheme in $\phi_{2}$ is lagged in the $\phi_{0}$ equation but the latest value of $\phi_{0}$ is used in the $\phi_{2}$ equation:

$$
\begin{aligned}
& \frac{2}{3 \beta_{1} h}\left[-\phi_{2, i-1}^{\ell}+2 \phi_{2, i}^{\ell}-\phi_{2 i+1}^{\ell}\right]+\frac{1}{3 \beta_{1}}\left[-\phi_{0, i-1}^{\ell+1}+2 \phi_{0, i}^{\ell+1}-\phi_{0, i+1}^{\ell+1}\right]+\beta_{0} \phi_{0, i}^{\ell+1} h=0 \\
& \frac{2}{15 \beta_{1} h}\left[-\phi_{0, i-1}^{\ell+1}+2 \phi_{0, i}^{\ell+1}-\phi_{0 i+1}^{\ell+1}\right]+\frac{11}{21 \beta_{1}}\left[-\phi_{2, i-1}^{\ell+1}+2 \phi_{2, i}^{\ell+1}-\phi_{2, i+1}^{\ell+1}\right]+\beta_{1} \phi_{2, i}^{\ell+1} h=0
\end{aligned} .
$$

The overall scheme we use can be described as follows:

1. Given the solution iterates from step $\ell$, perform a block iteration to obtain the iterates at step $\ell+1 / 3$.

2. Average the iterates from steps $\ell$ and $\ell+1 / 3$ to obtain the iterates at step $\ell+2 / 3$.
3. Given the iterates at step $\ell+2 / 3$, perform a Gauss-Seidel iteration to obtain the iterate at step $\ell+1$.

Using Fourier analysis, we can show that Steps 1 and 2 eliminate the lowest frequency error, while Step 3 eliminates the highestfrequency error. The basic idea behind this overall scheme is that completely eliminating the errors at the two bounding frequencies should lead to good attenuation of the intermediate 
frequencies. These iterations are trivially extended to the case of general Pn order. We refer to this overall scheme as the diffusion relaxation iteration.

The coupling between the $\mathrm{Sn}$ and $\mathrm{Pn}$ regions is achieved as follows:

1. A Marshak-type boundary condition is used for the Pn equations such that the incoming half-range currents defined by the $\mathrm{Sn}$ solution are preserved by the Pn solution. These incoming currents are computed using the Sn quadrature formula in both the Sn and Pn equations. For instance, assuming that the left region is $\mathrm{Sn}$ and the right region is $\mathrm{Pn}$, the Sn currents the statement of halfrange current preservation is as follows:

$$
\sum_{\mu_{m}>0} \psi_{m} \mu_{m} P_{n}\left(\mu_{m}\right) w_{m}=\sum_{\mu_{m>0}}\left[\sum_{k=0}^{N} \frac{2 k+1}{4 \pi} \phi_{k} P_{k}\left(\mu_{m}\right)\right] \mu_{m} P_{n}\left(\mu_{m}\right) w_{m} \quad, n=0,2, N-1 .
$$

where $\psi$ denotes the $\mathrm{Sn}$ angular flux, $\mu$ denotes the Sn direction cosines, $P_{n}(\mu)$ denotes the Legendre polynomial of degree $n, w$ denotes the Sn weights, and $N$ denotes the degree of the Pn approximation.

2. The incoming $\mathrm{Sn}$ angular flux values are set equal to the corresponding Pn angular flux values:

$\psi_{m}=\sum_{k=0}^{N} \frac{2 k+1}{4 \pi} \phi_{k} P_{k}\left(\mu_{m}\right) \quad, \mu_{m}<0$.

The iteration scheme for the two-region SnPn problem can be described as follows:

1. Perform a sweep in the Sn region using the initial Pn solution at the interface.

2. Perform a relaxation iteration in the Pn region using the initial $\mathrm{Sn}$ solution at the interface.

3. Compute a residual for the $\mathrm{Sn}$ iterate.

4. Compute a residual for the Pn iterate.

5. Solve a global diffusion equation to provide an additive correction to the scalar flux iterates in both the $\mathrm{Sn}$ and Pn regions.

We are now completing implementation of this scheme. We will also wrap it in a Krylov method, i.e., use the iteration scheme as a preconditioner and solve the preconditioned system using a Krylov method. We intend to test this scheme and include results in our final FY 2011 report.

\section{Benefits to DOE}

Our overall goal is to achieve a highperformance scheme for the integration of different algorithms for the solution of the transport equation. Success will eventually enhance accuracy and speed for nuclear reactor simulation. The approach under investigation, if it leads to a positive outcome, will be implemented in the reactor analysis suite of tools under development at INL (PHISICS). Once implemented, this new approach should enhance INL's capability to perform fast and accurate neutronic core simulation. Moreover, this approach pioneers a new philosophy to solve computationally intensive problems that could be used in other applications of interest for the simulation of nuclear reactors throughout the DOE complex. 


\section{Fickian and Thermal Diffusion in Nuclear Materials from Linear Response Theory and Multiscale Simulations Michael Pernice; Jacob Eapen (North Carolina State University) 10-031}

Properties of materials in nuclear reactors, in particular nuclear fuels, are strongly affected by radiation. Detailed understanding of how fuel properties such as Fickian and thermal diffusivities evolve under irradiation is essential to enable plant optimizations such as extended fuel burnup or to develop new fuel forms. The changes in fuel microstructure caused by radiation are slow thermo-diffusion processes, which occur on time scales of microseconds and above. This regime is inaccessible for traditional methods of molecular dynamics (MD), which are impractical beyond the nanosecond time scale. This project focuses on developing new statistical-mechanical models and long-time atomistic simulation methods to determine complex diffusive properties in metallic alloy systems and $\mathrm{UO}_{2}$ under different ambient and irradiation conditions.

\section{Project Summary}

A review of the molecular simulation methods on thermo-diffusion has been completed. Following this, we have established that thermo-diffusion can be computed through activated dynamics - also known as slow dynamics, transition state dynamics, state-tostate dynamics, inherent structure dynamics, or dynamics of rare events. First, we start from the basic thermo-diffusion equations and then derive a form based on activation energy.

We reviewed candidates developed by the Voter group, including hyperdynamics (which introduces an additional bias potential) and temperature accelerated dynamics (TAD) (which evolves the system at a higher temperature to rapidly find transitions, followed by filtering out the incorrect events). We also reviewed accelerated MD methods developed outside the Voter group, including metadynamics, in particular the Autonomous Basin Climbing (ABC) method. Among the methods, we have implemented $\mathrm{ABC}$ for our work. $\mathrm{ABC}$ is similar in construction to hyperdynamics and metadynamics, employing an interatomic bias potential used to accelerate the slow dynamical processes.
While $\mathrm{ABC}$ is not originally mentioned in the proposal, it functions like an accelerated molecular dynamics technique, and our review found that it could potentially offer some advantages over hyperdynamics and TAD. ABC shares the same theoretical underpinning as hyperdynamics (as in employing a bias potential). Unlike hyperdynamics or TAD, ABC has been applied successfully to deeply supercooled (disordered) states as well as crystalline states, suggesting a wider applicability. ABC also works well with systems with small energy barriers, which indicates a potential advantage for thermo-diffusion processes at high temperatures.

\section{Benefits to DOE}

Performance optimization and lifetime extension of the current fleet of light water reactors requires understanding nuclear fuel performance and material degradation in operating regimes where there is little to no observational data. Modeling and simulation based on first principles can help to accelerate this understanding. Techniques for firstprinciples determination of thermal properties of metallic alloys and uranium oxide fuels will benefit the Light Water Reactors Sustainability Program, the Consortium for Advanced Simulation of Light Water Reactors, and the fuels program element of the Nuclear Energy Advanced Modeling and Simulation program.

\section{Relevant Publications and Presentations}

Kushima, A., J. Eapen, Ju Li, S. Yip, and T. Zhu, 2011, "Time Scale Bridging in Atomistic Simulation of Slow Dynamics: Viscous Relaxation and Defect Activation," Eur. Phys. J. B, Vol. 82, pp. 271-293. 


\section{Error Estimation for Stochastic Uncertainty Quantification}

Michael Pernice, Jacob Eapen (North Carolina State University/INL Joint Appointment)

10-095

Our central objective is to develop new statistical mechanical models and long-time atomistic simulation methods to determine complex diffusive properties in nuclear materials. Specifically, we want to develop stateof-the-art methods to compute Fickian and thermal diffusivities in metallic alloy systems and $\mathrm{UO}_{2}$ under different ambient and irradiation conditions.

\section{Project Summary}

We have completed our review of the molecular simulation methods on thermodiffusion and have established that thermodiffusion can be computed through activated dynamics, also known as slow dynamics, transition state dynamics, state-to-state dynamics, inherent structure dynamics, or dynamics of rare events. First, we start from the basic thermo-diffusion equations and then derive a form which is based on activation energy. Traditional molecular dynamics (MD) method is impractical beyond the nanosecond time scale and have limitations in accessing the slow thermo-diffusion processes in nuclear materials, which occur on time scales of microseconds and above. Accelerated molecular dynamics (a term introduced by Voter and coworkers at Los Alamos National Laboratory) is a technique for extending traditional molecular dynamics approaches to access these longer time scales. We reviewed candidates developed by the Voter group, including hyperdynamics (which introduces an additional bias potential) and temperature accelerated dynamics (TAD), which evolves the system at a higher temperature to rapidly find transitions, followed by filtering out the incorrect events. We also reviewed accelerated MD methods that were developed outside the Voter group, including metadynamics, in particular the Autonomous Basin Climbing (ABC) method. Among the methods, we have implemented $\mathrm{ABC}$ for our work. ABC is similar in construction to hyperdynamics and metadynamics, employing an interatomic bias potential used to accelerate the slow dynamical processes.
While $\mathrm{ABC}$ is not originally mentioned in the proposal, it functions like an accelerated MD technique, and our review found that it could potentially offer some advantages over hyperdynamics and TAD. ABC shares the same theoretical underpinning as hyperdynamics (as in employing a bias potential). Unlike

hyperdynamics or TAD, ABC has been applied successfully to deeply supercooled (disordered) states as well as crystalline states, suggesting a wider applicability. ABC also works well with systems with small energy barriers, which indicates a potential advantage for thermo-diffusion processes at high temperatures.

\section{Benefits to DOE}

Performance optimization and lifetime extension of the current fleet of light water reactors requires understanding nuclear fuel performance and material degradation in operating regimes where there is little to no observational data. Modeling and simulation based on first principles can help to accelerate this understanding. Techniques for firstprinciples determination of thermal properties of metallic alloys and uranium oxide fuels will benefit the Light Water Reactors Sustainability Program, the Consortium for Advanced Simulation of Light Water Reactors (CASL), and the Fuels program element of the Nuclear Energy Advanced Modeling and Simulation program.

\section{Relevant Publications}

Kushima, A., J. Eapen, Ju Li, S. Yip and T. Zhu, 2011, "Time Scale Bridging in Atomistic Simulation of Slow Dynamics: Viscous Relaxation and Defect Activation," Eur. Phys. J. B, Vol. 82, pp. 271-293. 


\section{Advanced Visualization using Immersive Environments \\ Eric Whiting; William Sherman, Eric Werner (Indiana University); Amy Ulinski \\ (University of Wyoming); Patrick O'Leary (VisualIdeation); Kitware Inc. \\ 10-094}

Immersive environments have demonstrated to be useful tools to support and enhance scientific and engineering visualization workflows. However, broad adoption of immersive environments in the day-to-day scientific and engineering workflow is limited by the following: the lack of differentiation between desktop and immersive environment user interfaces and the limited control, navigation, and interaction offered by currently available input and feedback devices for immersive interfaces. To overcome these problems, we will perform two integrated activities. First, we will expose, in-depth, immersive interfaces for enhanced visualization, exploration, and analysis of both scientific and engineering workflows. Second, we will design an immersive interface toolkit that takes advantage of Apple's newly released $\mathrm{iPad}^{\mathrm{TM}}$, as well as other portable touchscreeen devices, as an input and feedback device.

Through this system, it will be possible to change visualization parameters, navigate within the environment, annotate discoveries, and visually analyze presented data. Instead of all user interactions performed as immersive interfaces, we will develop a natural division of work. User interactions predisposed to clicking or typing buttons, sliders, text input, and menus may migrate to a portable touchscreen user interface, whereas actions that involve grabbing, touching, moving, probing, or measuring in three-space should be assigned to the immersive interface.

The proposed activity has three distinct phases: (1) the preliminary technical analysis and development of a new input and feedback device for immersive environments; (2) the proof-ofprinciple experimental analysis based on separating immersive (three-dimensional) interfaces from user (two-dimensional or desktop) interfaces to demonstrate enhanced usability in immersive environments by utilizing the new input and feedback device; and (3) innovative research and development (new data structures and algorithms) in scientific visualization, utilizing immersive environments on a variety of strategic INL and DOE Office of Nuclear Energy operational and scientific data.
Finally, the focus of this work is not on inconsequential advances in computer graphics or computer gaming, but rather on innovative and novel approaches in scientific visualization, utilizing an immersive environment of relevant and important data at INL, DOE Office of Nuclear Energy, and beyond.

\section{Project Summary}

ParaViews's core code base has been modified to support immersive environments. To accomplish this, we: (1) successfully implemented "off axis asymmetric stereo" for virtual reality (VR) and desktop environment in Visualization Toolkit (VTK), an open-source, freely available software system for three-dimensional computer graphics, image processing, and visualization; (2) exposed "off axis asymmetric stereo" feature of VTK to CAVE like environments via ParaView, an open-source, multi-platform data analysis and visualization application; (3) added the ability to use a wand (tracked input device) for interactions in ParaView; (4) achieved significant performance improvement for tracking in ParaView by complete redesign of the existing code and usage of threads for non-blocking track data read from track server; (5) designed and implemented framework for interactions, one of the key components of VR; (6) added ability to navigate within ParaView scene using VR input devices; and (7) added basic but important ability to analyze the data by allowing usage of clip planes as part of interactions, using inputs from a VR input device in ParaView.

This project also includes work by Amy Ulinski from the University of Wyoming. Her expertise is in the area of three-dimensional human computer interaction. Her students are working to quantify the user experience and to design a natural user interface for these immersive applications.

Activities for this project included:

1. On November 29, 2011, in Las Vegas, Nevada, Eric T. Whiting (INL) and William R. Sherman (Indiana University) presented a paper detailing technologies of the IQStation and discussed immersive interfaces. They also met with many interested collaborators. 
2. From August 21-24, 2011, at Indiana University in Indianapolis, IN, Eric Whiting (INL), William Sherman (Indiana University), Aashish nmi Chaudhary (Kitware, Inc.), Nikhil Jyothi Shetty (Kitware, Inc.), and Eric Wernert (Indiana University) held a two-day code-camp where the immersive features of ParaView were further enhanced and refined. Work related to the user interaction also began with programming of $3 \mathrm{D}$ interaction. Indiana University's CAVE as well as their IQ-Station were both used in this work.

3. From May 9-12, 2011, Eric Whiting (INL), William Sherman (Indiana University), Amy Ulinski (University of Wyoming), Aashish nmi Chaudhary (Kitware, Inc.), Nikhil Jyothi Shetty (Kitware, Inc.), and Berk Geveci (Kitware, Inc.) met at Kitware headquarters in Albany, NY, for a project review and code camp. As part of this meeting, Amy Ulinski's role as the 3D human-computer interaction researcher was defined, the internals of 3D stereo rendering in ParaView were re-written adhering to best practices, and the research plan moving forward was laid out.

4. From April 26-29, 2011, in Asheville, NC, Eric Whiting (INL) and William Sherman (Indiana University) presented the Immersive ParaView poster and discussed applicability to DOE projects complex-wide at the annual DOE Computer Graphics Forum.

5. From March 19-23, 2011, William Sherman (Indiana University), Dan Coming (Desert Research Institute), and Amy Uliniski attended the Institute of Electrical and Electronics Engineers VR conference in Singapore and presented a poster detailing the work on immersive ParaView. They met and discussed future direction and opportunities.

\section{Benefits to DOE}

A large number of disseminations and proposals were developed, and the work was more than well received by our intellectual community, INL staff, peer DOE national laboratories, and collaborating university partners. This work is of high interest to INL in that it opens the door to new scientific visualization work in the CAVE. Without the immersive ParaView, researchers would be required to write custom code for scientific immersive visualization. Given immersive ParaView, almost any DOE researcher can have access to his or her data in a fully immersive display environment.

This work is beneficial to both the Nuclear Energy Advanced Modeling and Simulation program as well to the Consortium for Advanced Simulation of Light Water Reactors Energy Innovation Hub. Results from INL multiphysics codes can be displayed in the CAVE system using this new version of ParaView. In addition, researchers in biology, chemistry, and remote sensing have all expressed interest in these new tools.

\section{Relevant Publications and Presentations}

Sherman, W. R., P. O'Leary, E. T. Whiting, S. Grover, and E. A. Wernert, 2011, "IQStation: A Low Cost Portable Immersive Environment," International Symposium on Visual Computing, Las Vegas, Nevada, November 29, 2011.

O'Leary, P., 2010, “Employing Immersive Environments for Exploration and Analysis in Virtual Nuclear Engineering," Poster Session, SciDAC 2010, Chattanooga, Tennessee, July 11-15, 2010.

Sherman, W. R., 2011, "Immersive ParaView: A Community-based, Immersive, Universal Scientific Visualization Application," IEEE VR, Singapore, March 19-23, 2011. Abstract prepared. Paper Submitted.

Shetty, N., A. Chaudhary, D. Coming, W. R. Sherman, P. O'Leary, E. T. Whiting, and S. Su, 2011, "Immersive ParaView: A Community-based, Immersive, Universal Scientific Visualization Application," IEEE VR, Singapore, March 19-23, 2011. Poster presented. 


\section{Uncertainty Analysis for Multiscale Models of Nuclear Fuel Performance}

Michael Pernice; Don Estep, Simon Tavener (Colorado State University)

11-074

Developing nuclear fuel cycles that optimize uranium utilization and energy generation, minimize waste and proliferation risk, and increase safety is a principal priority of the DOE Office of Nuclear Energy. Modeling and simulation can help reduce the cost and time required to develop new nuclear fuel forms and to understand the behavior of fuel at high burnup. There is scant experimental data for the new fuel forms and performance regimes under consideration, which brings the validity of existing empirical models into question. Consequently, a first-principles approach to determining properties of materials under radiation is being pursued. This approach uses atomistic calculations to construct a mesoscale description of microstructural evolution of irradiated materials and then upscaling that description to determine material properties that can then be used in engineering-scale models of nuclear fuel performance.

We focus on reliable uncertainty analysis of these multiscale simulations of nuclear fuel performance. Essential elements of our approach include consistent mathematical formulation of the coupled model, a sampling-based approach to coupling the mesoscale and engineering scale, and a posteriori error analysis of both the stochastic and deterministic aspects. In addition to accounting for the stochastic behavior of uncertain parameters typical of current approaches to uncertainty analyses, we will also account for numerical error incurred in formulation of the deterministic problem as well as approximation and sampling errors in the stochastic problem.

\section{Project Summary}

This is a late-start project that began in July 2011. The initial goals of this project are to devise a rigorous mathematical foundation for the coupling of the macroscale and mesoscale components of multiscale models of fuel performance and then to devise and implement efficient numerical methods for numerical solution and sensitivity analysis for the coupled multiscale model. The The Colorado State University team has focused on acquiring necessary background in fuel performance models and INL codes and implementing and testing the proposed macro-mesoscale coupling method in a test bed code in Matlab. Specifically, all three team members (Estep, Newton, Tavener) have visited INL during this period. In addition to attending the International Summer School on the Evolution of Microstructural Defects on In-Reactor Material Response at INL, the postdoc Newton has carried out a systematic study of background materials related to the modeling of fuel performance. Estep and Tavener have developed and implemented a meso-macroscale modeling method using an intermediate numerical transfer scale, devised a fixed point iteration for the coupling, and studied the convergence. This was done in the context of a simplified stationary model with nonlinear elliptic problems for each scale. The results are very promising. Newton has now acquired the test bed code and has begun implementing it in $\mathrm{C}++$. At INL, we are studying the target mesoscale model application code, Marmot, in preparation for an initial set of uncertainty analyses and to develop the expertise needed to make modifications to suit our methods.

\section{Benefits to DOE}

Multiscale simulation of the performance of nuclear fuel supports several programmatic objectives of the DOE Office of Nuclear Energy, in areas such as light-water reactor sustainability and alternative fuel cycles. The new analysis capabilities that we develop in this project will lead to improved predictive capabilities for multiscale models of nuclear fuels. The framework that we develop will also be applicable to other research areas of interest at INL, including carbon sequestration and unconventional fossil fuel recovery, as well as the Advanced Simulation Capability for Environmental Management program in the DOE Office of Environmental Management. 


\title{
Uncertainty Analysis of Mesoscale Models of Radiation Effects
}

\author{
Michael Pernice; Anter El-Azab (Florida State University)
}

11-075

Improved understanding of the behavior of nuclear fuel and other materials in the harsh environment of a nuclear reactor is essential for extending the lifetime of light water reactors and developing new fuel forms for advanced reactor design concepts. Phase field models are used to simulate the evolution of microstructure in nuclear fuel. These models are based on a coupled set of Cahn-Hilliard and Allen-Cahn equations; the former governs the dynamics of the concentration fields of relevant defects while the latter defines the interfaces between the changing microstructure and the surrounding matrix. Phase field models are parameterized by lower scale atomistic calculations that provide a fundamental description of the defect generation due to interaction between neutrons and the fuel material, as well as the behavior of these defects. Significant uncertainty is associated with these phase field models, which arise from the lower scale input. This project establishes a foundation for a comprehensive uncertainty analysis of phase field models of microstructure changes in fuels due to irradiation.

We focus on the void growth model in a single-component material, where voids grow as part of the dynamics of vacancy and interstitial generation, diffusion, and reactions. We will examine two sources of uncertainty in this model. The first source is the result of using a diffuse interface description, which is a defining characteristic of phase field models, instead of a sharp interface representation. This source of uncertainty is parameterized by the interface width, and it will be analyzed through asymptotic matching with the sharp interface model via an asymptotic expansion in powers of the interface width. The second type of uncertainty arises from the atomistically determined model parameters that define the void growth model. Little is known about the probability distributions of these parameters. We will characterize these probability distributions and their dependence on the interface width and conduct a comprehensive uncertainty analysis of the phase field model to improve our understanding of how uncertainty propagates in this model.

\section{Project Summary}

This is a late-start project that began in late July 2011. As a part of preparing for the uncertainty quantification modeling effort, we completed the necessary tests of the phase field model, with a preliminary screening of sources of uncertainties. Among these sources are the material parameters and the model used to introduce the radiation damage into the phase field model. We have also reviewed two methods for matching phase field models with their sharp interface counterparts. These are the asymptotic matching method and the numerical matching method. A preliminary application of the asymptotic matching method has been initiated to try to reduce the phase field equations to the corresponding sharp interface ones to prove consistency and determine the model parameters. A previously developed, thermodynamically consistent sharp interface model is now being modified to include gradient terms characteristic of generalized diffusion equations. This step is necessary in order to match this model with the phase field model.

\section{Benefits to DOE}

Extending the life of today's light water reactors is a primary research and development objective of the Office of Nuclear Energy. Alternative fuel cycles have the potential to reduce the amount of waste that must be stored in a repository. Modeling and simulation based on first principles can help to improve our understanding of the fundamental processes that drive irradiation-driven microstructural evolution, reducing the cost and time needed to understand the behavior of fuel at high burn-up and to develop new fuel forms. The Nuclear Energy Advanced Modeling and Simulation program is exploring the use of phase field models in comprehensive atomistic-tocontinuum models of nuclear fuel performance.

\section{Relevant Presentations}

El-Azab, A., N. Wang, and M. Pernice, 2012, "On a Class of Phase Field Models for Irradiated Materials,"15th SIAM Conference on Parallel Processing for Scientific Computing, Savannah, GA, February 15-17, 2012. 


\title{
Exploratory Nuclear Reactor Safety Analysis and Visualization via Integrated Topological and Geometric Techniques
}

\author{
Michael Pernice; Valerio Pascucci (University of Utah)
}

11-076

Nuclear energy is a clean, affordable, domestic energy source that has been identified as a key component of the current U.S. energy portfolio. To better understand the safety performance of the aging reactor fleet, advanced modeling and simulation tools are being used to reduce the need for large, expensive integrated experiments. With the increasing sophistication and accuracy of these simulations, their predictive capabilities are increasingly called upon to inform national policy. In this context, the ability to estimate the likelihood of a given prediction and a quantification of potential uncertainties are crucial. Our approach maps this uncertainty quantification problem to the analysis of functions in high-dimensional spaces. We will develop and demonstrate a new set of mathematical and computational tools for analyzing, visualizing, and interacting with large, high-dimensional data sets that arise in samplebased uncertainty analysis of reactor safety analyses in order to provide better understanding of their inherent structure and how such structure relates to the choice of input parameters of largescale nuclear simulations.

\section{Project Summary}

This is a late-start project that began in July 2011. The focus of this project is uncertainty analysis of the $\mathrm{R} 7$ reactor safety analysis code as generated by DAKOTA (Design Analysis Kit for Optimization and Terascale Applications, developed at Sandia National Laboratories). An initial understanding of how DAKOTA works and requirements for improving its functionality have been identified. A specification of the file formats needed to achieve the data exchange from R7 was provided, and the corresponding routines have been implemented. An initial lowdimensional dataset of a preliminary uncertainty analysis of a "VR2+" virtual reactor has been provided, and the prototype visual analysis tool has been tested on this data, with a delivery of a preliminary report. A set of synthetic data and proxy test simulations represented as highdimensional scalar functions have been explored. The integration of topological and geometric analysis among proxy test cases has been validated. We have yet to compare these application-dependent objective functions and to identify possible differences and similarities in order to project possibilities of applying to nuclear simulations. Following this evaluation, a preliminary evaluation of the performance bottlenecks related to nuclear simulation data sets will be provided. Possible extensions to our tool for analyzing time series data and observing how geometry and topology evolve during the simulation are being considered, and the possibility of using stochastic collocation and how it would influence the analysis has been discussed.

Prototype interfaces that provide exploratory analysis capabilities for users are being considered. Progress toward providing the means to analyze topological features at different scales has been made. These interfaces have been tested on the recently obtained nuclear simulation data.

Access to the R7 source code to perform analyses using the simulation output is being arranged.

From the uncertainty quantification aspect, three new topological scoring functions have been proposed, and they have been shown to outperform traditional techniques in adaptive sampling for complicated functions and higher dimensions, also with a delivery of a preliminary report.

\section{Benefits to DOE}

New techniques for visual analysis of uncertainty will provide a unique capability that will help analysts to more fully explore the highdimensional landscape of uncertainties in a simulation. This will facilitate risk-informed safety margin characterization of reactor safety and will directly impact light water reactor sustainability. Projects directly impacted include the Consortium for Advanced Simulation of Light Water Reactors (CASL), the Center for Exascale Simulation of Advanced Reactors, and the Reactors program element of the Nuclear Energy Advanced Modeling and Simulation program. 
Relevant Publications and Presentations

Maljovec, D., B. Wang, A. Kupresanin, G.

Johannesson, V. Pascucci, and P.-T. Bremer, 2011, "Adaptive Sampling with Topological

Scores," Working with Uncertainty

Workshop at IEEE VisWeek 2011,

Providence, RI, October 24, 2011.

Wang, B., B. Summa, V. Pascucci, and M.-V. Johansson, 2011, "Branching and Circular Features in High Dimensional Data," IEEE Visualization 2011 Conference, Providence, RI, October 23-October 28, 2011. 
$\Rightarrow$ Instrumentation, Control, and Intelligent Systems

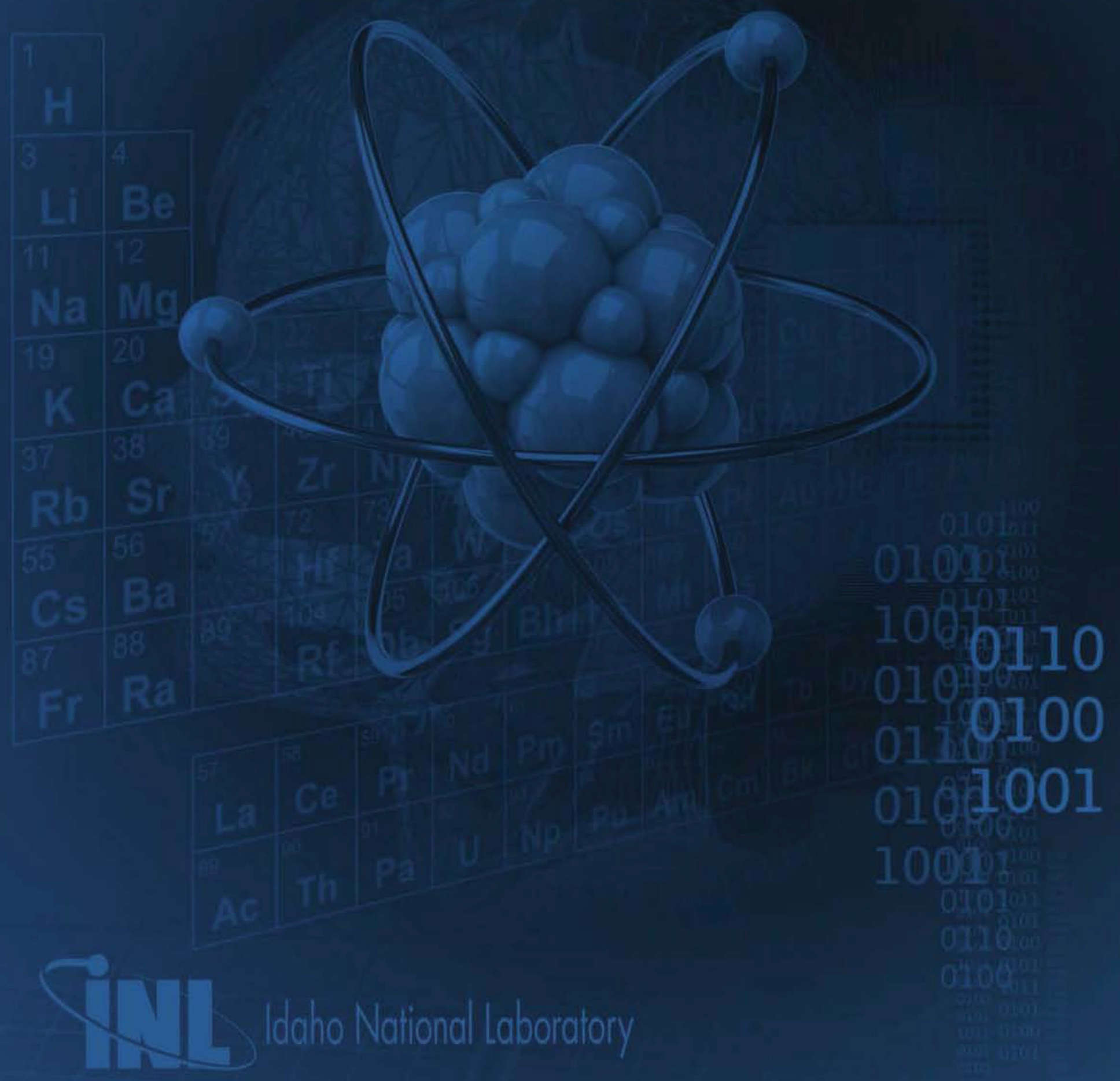




\section{Integrated Control Systems Data Fusion}

Tim McJunkin, Miles McQueen, Milos Manic, Lee Shunn, Ronald Boring, David Gertman

09-070

Modern critical infrastructure control and security systems have the capability to provide facility managers, operators, and security personnel with an abundance of monitoring data. This data comes from multiple sources, including process and control status as well as network and physical security information that are deployed at different levels within the system to provide situational awareness. The hypothesis of this research is that a holistic assessment and prioritization of a system will provide a basis for fusing data from various sources and in a specific manner to draw the relationships among them. Year 3 (FY 2011) continued on the efforts of Year 2 by refining the MATLAB's Simulinkbased Hybrid Energy Systems Testing (HYTEST) model into a highly usable form. With the model at a satisfactory quiescent initial condition, step functions were applied to the control inputs (e.g., heaters, flows, etc.) of $\pm 10 \%$ of the values that achieved the steady-state starting point to characterize the boundaries of the model and produce initial baseline data for data-mining methods. An initial framework for data fusion to both identify anomalies and assist the human-machine interface visualization is emphasized this year.

\section{Project Summary}

The project has fulfilled prerequisite needs of a faster and highly functional plant model and secured the initial useful data. The initial data has been applied to computational methods developed during the previous year with a brief description and example of promising results presented here.

The project has continued to invest in simulation capabilities to establish a common toolset and benchmark applications that can be used across the Instrumentation, Control, and Intelligent Systems signature. To this end, project personnel have contributed to the development of HyEnDSim, a software package written for the MATLAB Simulink environment, for dynamic simulations of chemical processes and hybrid energy systems. This package includes transient models for packed bed catalytic reactors, compressors, valves, storage tanks, heaters, coolers, and their controllers. HyEnDSim is being used to model an application from INL's HYTEST program, in which gasified coal is converted into synthetic fuel. Contributions from this project to HyEnDSim include visualization and datalogging capabilities and various "disturbance" models such as controller and sensor malfunctions. The HYTEST model is the current platform for development and demonstration of data fusion concepts and techniques.

A three-layer computational intelligence algorithm concept is being implemented. The first layer consists of conventional min-max bounds on the measurements for normal operating conditions set by specification or procedure. The second layer consists of a selforganizing map, which was trained to model the previously observed normal behavior patterns. The set of self-organizing map-based fuzzy rules determines the similarity of the current plant behavior with the previously observed patterns. The third layer consists of an artificial neural network-based behavior predictor, which processes the intermediate preceding history of plants' behavior and predicts near-future patterns. These predicted patterns are then compared to the real observations, and significant deviations are reported as prediction error (Fig. 1, next page). The prediction error from multiple sensors is fused into a robust anomaly indicator by a dedicated fuzzy-logic controller. 

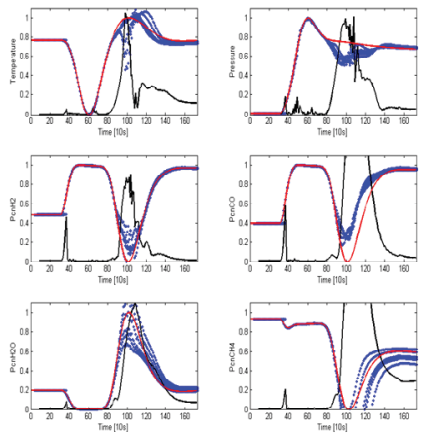
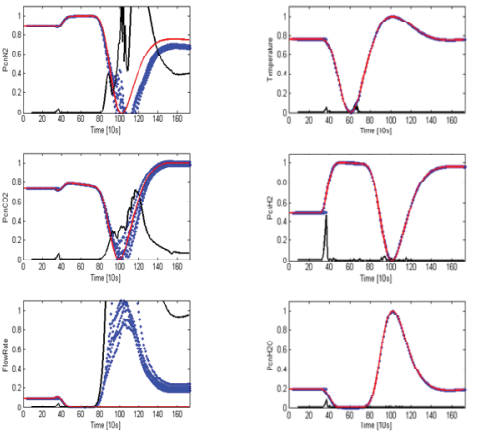
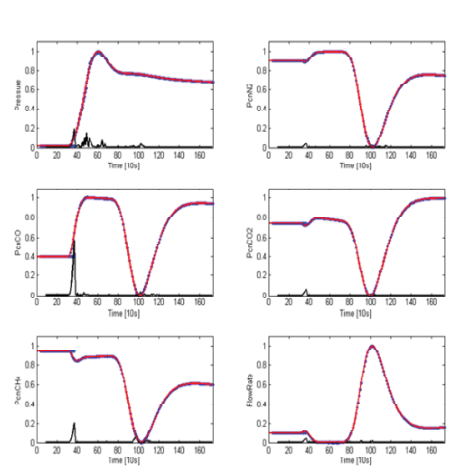
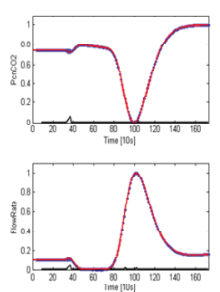

FIGURE 1. Detection of a valve stuck in a module of the model by a brief look behind, brief predict ahead transient artificial neural network. Stuck valve case is shown on the left. Normal case is shown on the right.

\section{Benefits to DOE}

The research benefits INL directly through better understanding of the emerging plethora of data from numerous sources, and the capability to draw actionable information together has benefitted the INL team but will be further exploited as the results are extended and published in the coming year. Applications of this research extend to energy, national security, and other areas where control of critical infrastructure is of concern.

\section{Relevant Publications and Presentations}

Linda, O., M. Manic, T. R. McJunkin, 2011,

"Anomaly Detection for Resilient Control Systems using Fuzzy-Neural Data Fusion Engine," $4^{\text {th }}$ International Symposium on Resilient Control Systems (ISRCS), 4th International Symposium, doi: 10.1109/ISRCS.2011.6016085, pp.35-41, August 9-11, 2011.
Boring, R., et. al., 2011, "Concept of Operations for Data Fusion Visualization," ESREL 2011, September 2011.

McJunkin, T., and D. Grauer, 2011, "Situational Awareness for Prime Mover Facilities," White paper to CORE Natural Gas Industry, CORE 2011 Symposium, Houston, Texas, May 2011.

McCarty, K., et. al., 2010, “A Temporal-Spatial Data Fusion Architecture For Monitoring Complex Systems," IEEE HSI, May 2010.

McQueen, M. A., and Wayne F. Boyer, 2009, "Deception Used for Cyber Defense of Control Systems," IEEE HSI 2009, May 2009.

Gertman, D. I., 2009, "Human Factors and Data Fusion as Part of Control Systems Resilience," IEEE HSI, May 2009. 


\section{Wireless Sensor Testing}

John Buttles, Scott Peterson, Jason Wright

09-021

Wireless sensor network (WSN)

technologies have become a focus of development for instrumentation and control applications in many industries. The promise of lower installation cost, while achieving reliable monitoring of process parameters at virtually any location when needed, has driven many existing instrumentation vendors as well as new startup companies to fill a need in wireless technology. These technologies are in their infancy and have not been fully tested for security, reliability, compatibility, or cooperation. It is proposed that radiofrequency $(\mathrm{RF})$ and/or cyber interference to a WSN can lead to destabilization of the control system by introducing latency or modifying data, which can undermine the control system design and amplify traditional concerns where impact is limited to the target of the interference. The result is a domino effect that, while it may have originated as loss of an individual sensor's data or bad data, will lead to changes on facility-level impacts. Distributed control networks and complex network theory, though current topics of research, have not considered how multiple interference regimens can lead to latencies that create destabilization. Therefore, to address this knowledge gap, the research has lead to design and analysis of full-scale WSN control systems that can be stressed by multiple interference types in order to develop approaches and solutions to resolve the expected system degradation and instability.

\section{Project Summary}

In the third and final year of this LDRD, tests were completed to measure the effects of interference with multiple WSNs simultaneously operating in a challenging RF environment. This testing confirmed the hypothesis that RF interference to a WSN can introduce latency, which may undermine the control system design. Figure 1 shows that the latency increases with increasing system interference from many WSNs, as shown by the red trace, when compared to a WSN operating without any interference, as shown by the blue trace.
Limitations of current WSNs and recommended improvements are as follows:

- Limitations:

- Multiple WSNs can affect each other, so users must be aware of their presence when setting up a WSN

- When used in feedback loops, the results may not be what is expected or consistent during operation

- Proper design, placement, and coordination with other wireless (information technology) services can help avoid overlaps of coverage, thereby reducing interference.

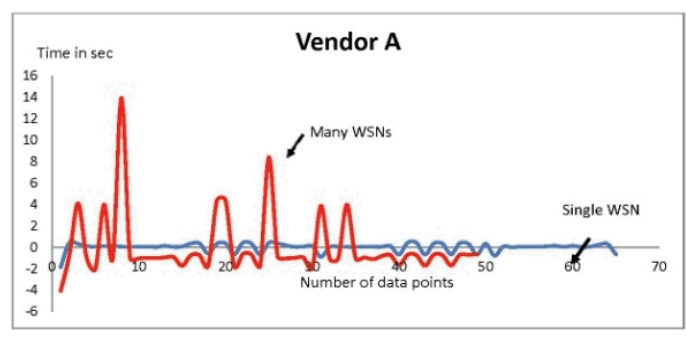

FIGURE 1. Increase in latency of WSN with the increase of operating WSNs.

- Improvements to be made:

- Smarter WSNs that have a sense of the state of the network and can use multiple communication channels at the same time

- Better or new metrics to track data flow between sensors and/or gateway to show increase in retransmissions, path changes, and RF congestion

- Better synchronizing methods using a background channel to keep updated with the gateway for timing and security changes

- Create methods to alter the communication path to prevent a sensor node from becoming a single point of failure. 
Benefits to DOE

With the establishment of WSN research expertise, INL now has the capabilities to test different wireless sensor systems and have a better awareness of WSNs along with the problems they face when used in critical infrastructure industries. The work under this LDRD will expand our capabilities in this field and become a valuable asset of the DOE

National SCADA (supervisory control and data acquisition) Testbed program at INL.

Relevant Presentations and Publications

Buttles, J., 2011, "Wireless Sensor Testing," ICIS Signature Review, Boise, ID, August 8, 2011. 


\section{Anomaly Detection, Diagnosis, and Resilient Control in Complex Engineered Systems}

Craig Rieger, Humberto Garcia; Venkat Venkatasubramanian (Purdue University)

09-097

One of the most important challenges for control-system engineers today is how to design and implement intelligent, resilient control systems that will be robust to accidental and intentional abnormal events and assist operators in making supervisory control decisions for such abnormal situations as well as startup and shutdown, performing controller assessments, and so on. Operators' failure to exercise the appropriate supervisory control decisions in abnormal conditions often has an adverse effect on product quality, process safety, occupational health, and environmental impact. The economic impact of such abnormal situations is enormous - about $\$ 20$ billion/year in losses in the petrochemical industries alone in the U.S. The cost is much more when one includes similar situations in other industries such as pharmaceuticals, specialty chemicals, power, and desalination. Thus, considerable incentive exists for developing intelligent resilient control systems that can provide automated operator assistance for supervisory control in complex process plants.

The objective is to develop a model-based hybrid framework for anomaly detection, diagnosis, and resilient control in complex process systems. This hybrid framework will integrate signed digraphs-based causal models of the process, dynamic trend models, statistical techniques for state awareness, and model-based supervisory control. Such an approach overcomes the shortcomings of the individual components and is a significant advancement over the state-of-the-art. This framework will be tested and evaluated on both dynamic simulations and experimental testbeds. The lessons from this research can be generalized for other such process systems.

\section{Project Summary}

In the first year, mathematical frameworks for principal component analysis, signed digraphs, qualitative trend analysis, and modelbased Kalman filtering were implemented. In the second year, the developments for full Bayesian integration using these methods were continued and initial tests were extended on a continuous stirred-tank reactor system as well as the Machine Condition Monitoring (MCM) "big loop" system dynamic simulations. Additionally, an extensive measurement campaign in the second year by means of automated experiments was carried out at INL. Sensor bias, actuator faults, valve drift, valve stiction, and noise were simulated to test our framework with successful detection and diagnostic outcomes. A LabView program was written at Purdue University to facilitate the testing of the Machine Condition Monitoring testbed for such faults. Figure 1 shows the likelihood function evaluated for four faults in the continuous stirred-tank reactor testbed system when the correct fault is identified (Fault \#4, sensor bias) by the pronounced peak in the likelihood function.

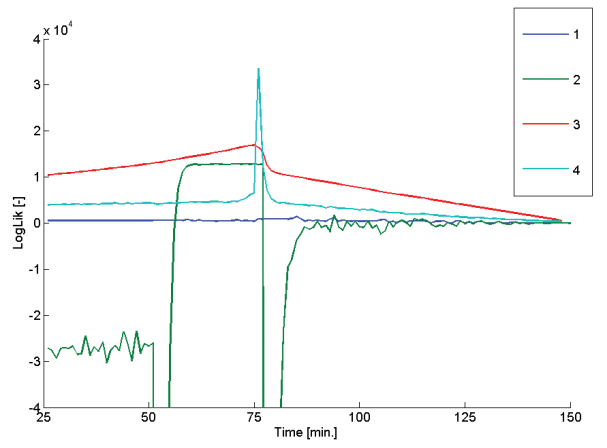

FIGURE 1. Kalman-based likelihoods for four faults (1: valve stiction, 2: valve stuck, 3: valve bias, 4: sensor bias).

To integrate the methods developed, a Bayesian framework is developed to simultaneously use all information that becomes available through the different methods. To this end, each method delivers a likelihood to observe the available data, Y, in each fault scenario in the library of faults, written as: $\mathrm{L}(\mathrm{Y} \mid$ $\mathrm{M}, \mathrm{S}$ ), where $\mathrm{M}$ stands for the method and $\mathrm{S}$ for the evaluated scenario.

Then, the likelihood for the data set for a given scenario is based on the integration over the four available methods: $\mathrm{L}(\mathrm{Y} \mid \mathrm{S})=\Sigma$ $\mathrm{L}(\mathrm{Y} \mid \mathrm{S}, \mathrm{M})$. $\mathrm{L}(\mathrm{S} \mid \mathrm{M})$. Here, $\mathrm{L}(\mathrm{M})$ is the prior for each likelihood, conditional to the method used. Using these priors as weights in the integration scheme, one can account for the fact that one or the other method is better at identifying certain types of faults. Figure 2 (next page) shows the MCM Big Loop testbed for simulating a cooling loop for a nuclear reactor system. 


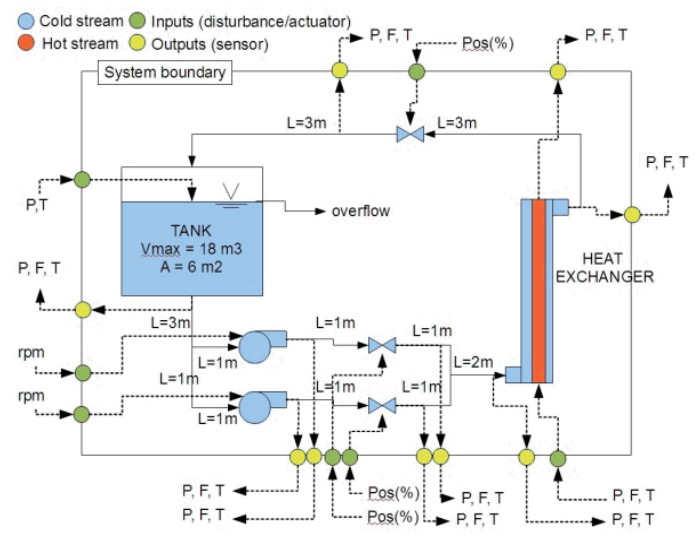

FIGURE 2. MCM big loop.

Our differential algebraic equation model is sufficiently complex to simulate realistic challenges in fault detection and diagnosis for this testbed. Our first campaign, aimed primarily at the identification of the big loop in the MCM test bed, was executed in July 2010. During this three-day measurement campaign, on-line measurements were taken related to the hydraulics of the system. On-line measurements included the position of the butterfly valve (BFV), pressures in the system, and the flow rate. The gate valve position was noted down manually. Different faults were also induced to identify the system's behavior qualitatively and quantitatively in such conditions. Induced faults were introduced manually (e.g. leak flow, cavitation) or semi-automatically (BFV bias, drift, stiction and noise, flow-rate bias, drift, stiction, and noise). Faults were introduced while the BFV position was controlled (inner loop in cascade controller). Different scenarios were created by switching the controller for the flow rate on or off (outer loop in cascade controller).

By means of the measurement campaign, an extensive data set has been produced including data from nominal and anomalous operational conditions. The nominal operation data will be used for full characterization of the system. Figure 3 shows a short time segment of the nominal data in which the valve position was increased and decreased in steps to characterize its steady-state performance. In this experiment, the flow rate was not controlled. An initial result of this effort is the dynamic characterization of the relationship between the BFV and its discrete inputs (open/close digital channels). In Figure 3, the filtered values for the BFV measurement are also shown using the resulting model and the Kalman filter.

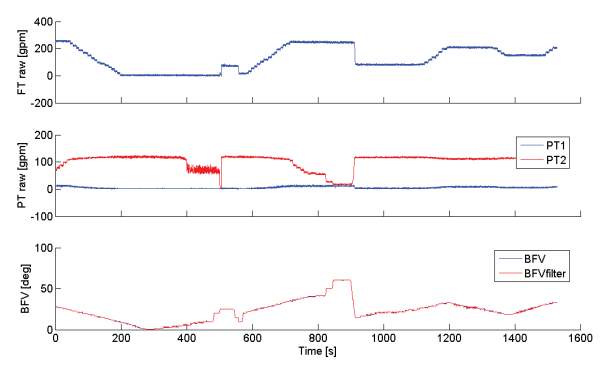

FIGURE 3. Charts for BFV, pressure transmitter, and flowrate variables.

Further simulations allowed us to evaluate the Kalman filter based on different conditions prior to on-line testing. An interesting result was that under certain conditions it may be beneficial for diagnostic purposes to introduce dynamics. Specifically, for the MCM test bed, it was observed that a drift and stuck problem can only be told apart effectively if the valve is moved during the time window of data collection for diagnosis. This is because under the stuck scenario, the valve sensor appears to be drifting with the exact speed of the valve. To avoid such confounding effects in diagnosis, a strategy was devised in which the valves are opened as soon as a fault is confirmed. This generates the necessary dynamics in the model prediction residuals to clearly discern a drift from a stuck valve problem. Figure 4 shows the dynamics that result in such a scenario. Following the success of this approach in simulation, it was also included for on-line implementation on the MCM test bed.

In the third year, an additional valve, the socalled glove valve (GLV), was installed by INL upon request. This installation allowed for a redundant valve in the big loop, enabling the development of a reconfigurable controller. Three configurations are then indeed possible to manipulate the flow: (1) BFV and GLV, (2) BFV only, and (3) GLV only. A measurement campaign was executed (May 2011) in which the initial form of the resilient control system was tested. This system included a single Kalman-filter-based module for diagnosis. 

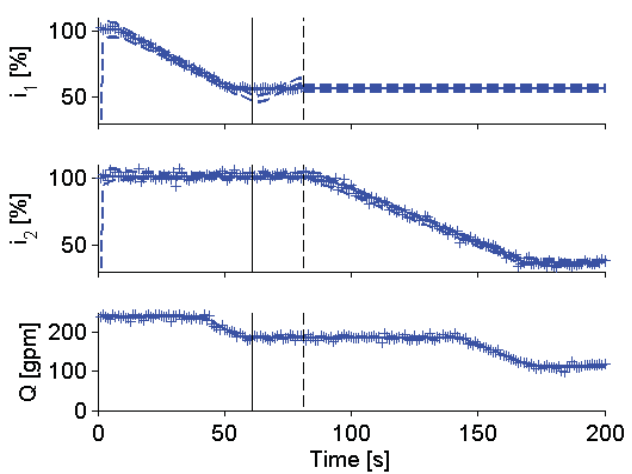

FIGURE 4. Scenario in which Valve 1 gets stuck at 50 seconds. Top: Valve 1 axle; middle: Valve 2 axle; bottom: flow rate. Full lines indicate true value and dashed lines indicate 5-sigma confidence bounds of the Kalman state estimate. Vertical lines indicate the times of fault confirmation (full) and fault diagnosis (dashed). Following the introduction of the fault, the fault is confirmed after 10 seconds. Subsequently, the controller tries to open the valves, leading to successful identification after another 20 seconds. Following that, the controller is switched to one that uses

Valve 2, thereby regaining effective control of the flow rate.

Results indicated that fault type identification is generally correct. At this time, the LabView implementation of the artificial faults (sensor bias, drift) did not permit multiple drift to be accumulated in the system over time. Also, the estimated drift parameter following positive identification of drift was observed to be severely affected by noise. Because such fault parameter estimates are fed back into the control system via signal correction, a reduction of this noise was targeted.

Modeling developments permitted to calibrate a working principal component analysis model specifically aimed at the identification of a stuck (i.e., blocked) valve. A regression-based module allows us to identify drift as well as to obtain an improved estimate of the drift parameter. A probabilistic integration of the results (likelihoods) for each fault by each module was based on a Bayesian Belief Network architecture.

Following these modeling efforts, the LabView/Matlab program set was extended to incorporate these modules as well as the probabilistic integration. The whole methodology and its implementation were successfully tested in the last measurement campaign of September 2011. Initial

observations were made qualitatively:

- The complete system was functioning well following tuning of the weights given to each module, in turn based on initial test runs.

- In particular, the system was able to detect and identify faults of a diverse nature in the valve system.

- Furthermore, the information given by the controller permits the effective switching to an alternate, better controller when necessary (e.g., valve blocking).

- The strategy with which the valves are opened is demonstrably effective for proper discrimination between a stuck and drift problem in the same valve.

- The additional drift module allows better drift parameter identification, though it is not necessarily better than the Kalman-filterbased module at detection and identifying its type. In addition, current interpretation of the system's working is that the Kalmanfilter-based module fares better in dynamic situations (valves moving) and that the specific drift module does better when valves are not moving. This is strong evidence in support of a multiple-module approach to diagnosis.

- Additional stress tests were executed by means of randomized setpoint changes, additional noise addition to sensors, and by making deliberate changes in model parameters (valve hysteresis parameter), thus establishing severe model-reality mismatch. The system was fairly robust to setpoint changes and noise addition. However, the Kalman-filter-based module turned out to be rather sensitive to parameter changes.

\section{Benefits to DOE}

The theoretical frameworks, dynamic simulations, and experimental campaigns are advancing the state-of-the-art in developing the next generation of highly automated resilient control systems that can provide greater stability and efficiency while achieving graceful 
degradation by anticipating failures. Such an advance is required for a wide range of applications seen in the energy, environment, and nuclear industries. The Several papers have resulted as well as the development of a simulation environment beneficial to core DOE mission objectives.

\section{Relevant Publications and Presentations}

Villez, K., B. Srinivasan, R. Rengaswamy, S. Narasimhan, and V. Venkatasubramanian, 2011, "Kalman-Based Strategies for Fault Detection and Identification (FDI):

Extensions and Critical Evaluation for a Buffer Tank System," Computers and Chemical Eng., Vol. 35, pp. 806-816.

Villez, K., V. Venkatasubramanian, and C. Rieger, 2011, "Supervisory Control of a Pilot-Scale Cooling Loop," ISRCS 2011, Boise, ID. 


\title{
3D Spatial Representation in Support of Design Inspection \& Verification
}

\author{
Douglas A. Few, David Gertman, Richard Metcalf
}

10-029

Nuclear facilities are subject to safeguards via the International Atomic Energy Agency (IAEA). As a part of these safeguards, the IAEA performs design information verification (DIV) inspections to verify that facilities of interest have not been altered in any safeguards-relevant manner that would allow misuse or diversion of special nuclear material. The practice of DIV relies heavily on inspector knowledge and experience and is performed within a highly information-intensive environment.

There are two fundamental difficulties when inspecting nuclear facilities. The first is sifting through facility documentation in search of relevant information (i.e. safeguards information), and the second is spatially correlating relevant information to the facility physical design. We propose creating a facility information system (FIS) to address data storage and spatial registration of facility information, coupled with extraction of the relevant information on demand. Inspectors will interact with the FIS through a 3D digital replication of the facility.

3D virtual environments (VEs) provide rich visual cues, but evidence suggests visual information alone is not sufficient to adequately navigate a VE effectively. Therefore, we will investigate positioning solutions for accurately tracking human movement in global positioning system (GPS)-denied areas and use this position information to auto-register the VE perspective to that of the human operator in the physical world.

Solving challenges associated with GPSdenied positioning and creating a useful FIS will also require novel contributions in:

- Task analysis of inspector roles, responsibilities, and information requirements

- Rapid VE modeling toolsets

- Position correlation from physical to virtual environment

- Human-computer interaction research.

The proposed work provides the platform proof-of-principle for a facility inspector toolset and advances INL's non-proliferation, nuclear security, and advanced instrumentation and control core capabilities.

\section{Project Summary}

In FY 2011 we completed several tasks with the help of two interns from the University of New Mexico and Montana State University. We: (1) evaluated the human task analysis of a DIV inspector by soliciting a former IAEA DIV inspector, (2) designed a DIV database to contain information pertinent to the DIV task, (3) updated the virtual model of the INL Fuel Conditioning Facility to be spatially accurate, (4) modeled the North Boulevard Annex facility for a program testbed, (5) coupled the intertial position tracking system to the VE camera perspective, (6) published a conference paper outlining our progress to date at the Institute of Nuclear Materials Management, and (7) developed rapid modeling prototyping tools utilizing robot systems and laser range finders to collect facility data into a sub-centimeter accurate point cloud that is viewable through a web browser or the INL Center for Advanced Energy Studies CAVE.

\section{Benefits to DOE}

The "3D Spatial Representation in Support of Design Inspection \& Verification" LDRD project has benefitted the DOE and national security missions by developing a set of tools that may be used to address non-proliferation DIV. Additionally, the infrastructure being created for the nonproliferation domain can be extended to a variety of other domains of interest to the Departments of Energy, State, and Defense. Particularly, the DIV system can be modified to be an electronic procedure system for leading plant operators through nuclear power plant evolutions.

\section{Relevant Publications and Presentations}

Few, D. A., D. I. Gertman, K. LeBlanc, K. Michel, B. Hayes, 2010, “Augmented Reality: Don't we all wish we lived in one?" In Proc. of the Institute of Nuclear Materials Management $51^{\text {st }}$ INMM Annual Meeting, Baltimore, MD, July 2010. 


\section{Resilient Condition Assessment Monitoring (ReCAM) System}

Humberto E. Garcia, Wen-Chiao Lin; Semyon Meerkov (University of Michigan)

10-037

In today's world, systems used to monitor infrastructure operations must be able to adapt to changing conditions while maintaining their reliability. To meet this need, we are developing an innovative architecture for implementing condition assessment monitoring systems that are both resilient and adaptive.

\section{Project Summary}

During this past fiscal year, overall functional and mathematical architectures of Resilient Condition Assessment Monitoring (ReCAM) were developed and implemented, as illustrated in Figure 1. In this architecture, process variables of monitored plants are observed by sensors. These observations, along with assessed data qualities of sensors, are then used to calculate probability distributions of observed signals. Combination rules, such as the Dempster-Shafer combination rule, were used to combine probability distributions if more than one sensor is active for a process variable. The combined process variable probability distributions are then fed via a first-order filter in order not to abruptly change the information to the assessment algorithm. Models of the monitored plant, namely the conditional probability tables in the belief network-based assessment algorithm, are modified based on aggregated data qualities calculated from the filtered probability distributions. The filtered probability distributions are then entered as soft evidence into an assessment algorithm using an iterative procedure. The assessment algorithm then outputs the assessment of the monitored plant conditions in the form of a probability distribution of plant status. The entropy of the probability distribution in the plant assessment is then calculated. If this assessment entropy is lower than a user-selected threshold, a decision on the monitored plant condition is made, and the assessment algorithm resets. If the assessment entropy is higher than the threshold, a calculation is made to estimate the time for the assessment entropy to decrease and meet the threshold. Penalties are generated

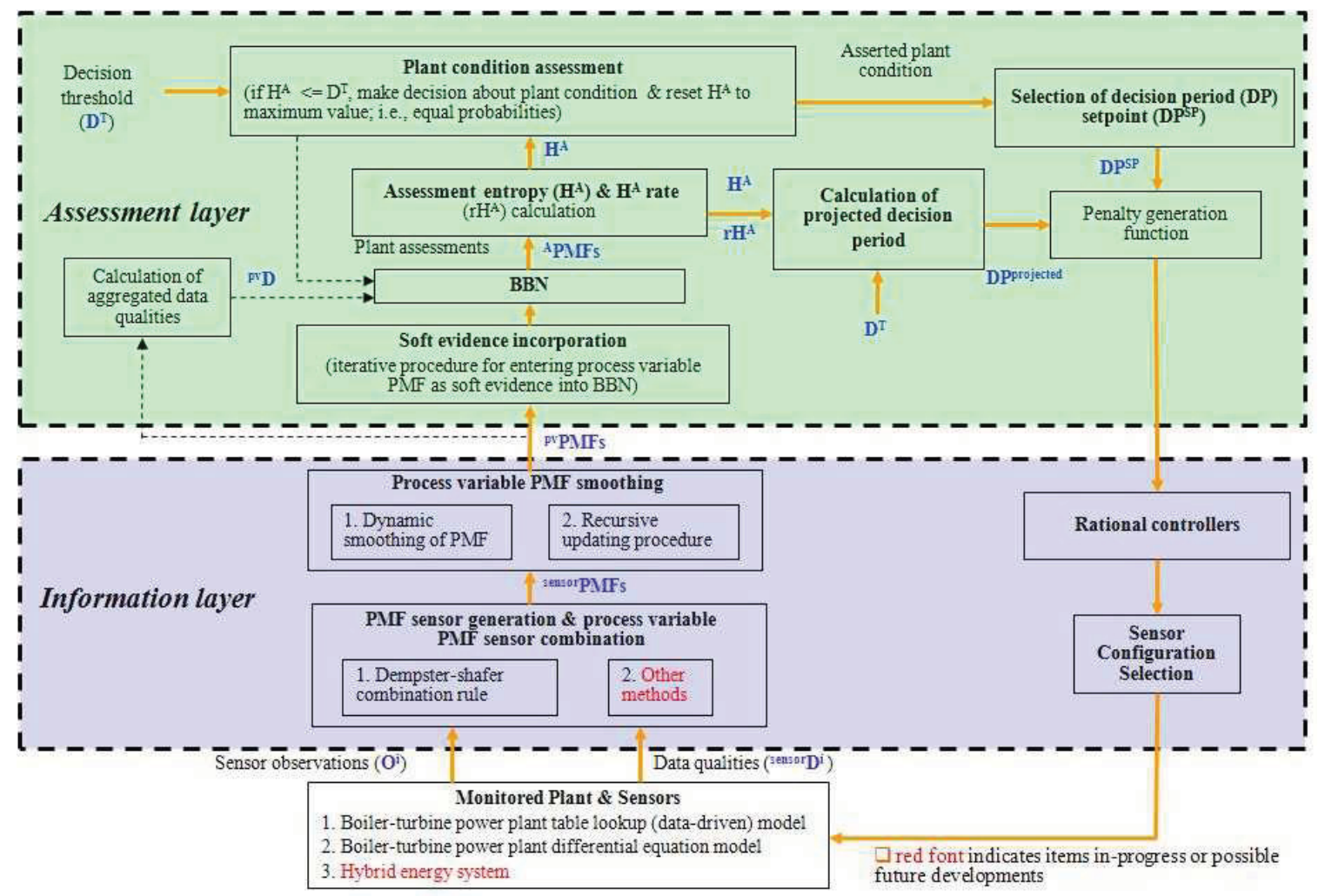

FIGURE 1. ReCAM mathematical architecture. 
by comparing this estimated time with a desired decision period (selected based on the previous decision on monitored plant conditions) and fed to rational controllers that select sensors monitoring the plant to meet the desired decision period.

The mathematical architecture was also completed, implemented, and demonstrated using a simplified steam power plant consisting of six unit operations and 16 process variables:

- The steam power plant consists of a boiler (with main and reheat steam generators) low- and high-pressure turbines, a condenser, and a feedwater pump.

- $\quad$ Sensors considered for the process variables are shown in yellow in Figure 2. Two sensors may be assigned to each process variable, which amounts to a total of 32 sensors. This arrangement is deemed complex enough to demonstrate the developed ReCAM system.

- Four types of anomalies are considered for the steam power plant: (1) low heat transfer in main steam generator, (2) pipeline rupture in the reheat steam generator, (3) improper heat insulation in the high-pressure turbine, and (4) decreased efficiency in the lowpressure turbine. Characteristics of these anomalies have also been identified.

- Bayesian network modeling relations among process variables and power-plant anomalies and assessments were developed.

Numerous simulations were also completed. For example, we tested the performance of ReCAM under a given scenario. In particular, at the start of the simulation, it is assumed that the monitored plant is in normal conditions, while half of the sensors are being attacked. The sensor readings of attacked sensors are biased. At time 750 , anomalies were introduced. These results showed that ReCAM is able to make correct plant assessments within (or close to) the desired decision period despite a significant number of sensors being attacked.
Another operational scenario compared the performance of the developed ReCAM system to that of a non-resilient monitoring system. At the beginning of the simulations, it was assumed that the monitored plant conditions and all sensors are normal. At time 750, an anomaly-a pipeline rupture in the reheat steam generatorwas introduced. A set of sensors was attacked from time 1,500 to 2,500 , while another set was attacked from time 2,500 to 4,000. Results showed that ReCAM is able to make correct plant condition assessments despite the sensor attacked.

In this fiscal year, one peer-reviewed conference paper was published and two more papers are in progress. We continued to collaborate with the University of Michigan. Progress was made in developing an enhanced approach to a ReCAM system, and a peerreviewed conference paper was published based on this line of work.

\section{Benefits to DOE}

The primary benefit of this project is the improved protection of key infrastructure operations by employing mathematical architecture and methods for implementing monitoring systems that are both resilient and adaptive. Such protection leads to a more secure national energy system and a more secure nation.

\section{Relevant Publications and Presentations}

Lin, W.-C., H. E. Garcia, and T.-S. Yoo, 2011, "Selecting Observation Platforms for Optimized Anomaly Detectability Under Unreliable Partial Observations," INL/CON11-21677, 2011 American Control Conference (ACC2011), San Francisco, CA, June 29 - July 1, 2011.

Garcia, H. E., 2011, "Integrated Process Monitoring based on Systems of Sensors for Enhanced Nuclear Safeguards Sensitivity and Robustness," Institute of Nuclear Materials Management (INMM) $52^{\text {nd }}$ Annual Meeting, Palm Desert, CA, July 17-21, 2011. 
Garcia, H. E., N. Jhamaria, H. Kuang, W.-C.

Lin, and S.M. Meerkov, 2011, "Resilient

Monitoring System: Design and

Performance Analysis," Institute of

Electrical and Electronics (IEEE)

$4^{\text {th }}$ International Symposium on Resilient

Control Systems, Boise, ID, August 9-11,

2011. 


\section{Automated Differential-Equation-Based Identification}

Lee Shunn, Charles Tolle

10-014

System identification (sys-ID) relates to building mathematical models of dynamical systems based on observed input-output data. For the case of linear models, there are wellstructured theories, methodologies, and algorithms to construct the relationships. For complex nonlinear models, however, general techniques are not as established or robust. The main objective of this project is to develop and demonstrate a new class of algorithms for nonlinear sys-ID based on the trajectory method of Perona et al. (Nonlinear Dynamics, Vol. 23, pp. 13-33, 2000).

There are several differences between the current method and other available sys-ID techniques. Discrete-time autoregressive models (e.g., AR, ARMA, ARX, ARMAX) use past values from a time series to predict future values. The resulting relationships are typically linear, although some nonlinear variants exist. Transfer function models use linear dynamics and time delays to approximate nonlinear systems. This approach can result in cumbersome expressions in the Laplace domain whose physical interpretation can be problematic. HammersteinWiener models offer a convenient framework to parameterize some nonlinear features; however, all such models have a linear transfer function at their core and use a nonlinear transformation on the input and output to describe the nonlinearities. While all of these methods can provide predictions of the system's outputs $y$, none offer a description of the internal state variables $x$. Discrete-time state-space models offer a description of both the state variables and outputs. However, current implementations only allow for linear analysis. The present approach provides a complete state-space description of nonlinear systems (including the outputs and state variables) in the time domain.

The main objectives for this project are:

- Extend the sys-ID method and tools (developed under previous Instrumentation, Controls, and Intelligent Systems LDRD funding) to support high-dimensional systems

- Incorporate forcing terms into the sys-ID method
- Automate basis function selection using a genetic algorithm (or other suitable approach)

- Explore mapping functions that can cleanly transform embedded dynamics to the original phase space

- Apply sys-ID tools for proof-of-principle to problems of interest at INL.

Project Summary

A new $\mathrm{C}++$ code has been written to include arbitrarily high-dimensional systems (restricted only by the physical limits of the computer hardware). Collaborators at the South Dakota School of Mines and Technology (SDSMT) have completed the coding, debugging, and validation of the code. The new implementation "frees" this work from commercial software packages such as MATLAB and creates a faster stand-alone tool with a high degree of portability.

Previous implementations of this (and similar) methods have related the system dynamics only to the internal state variables, i.e., $\mathrm{dx} / \mathrm{dt}=\mathrm{A}(\mathrm{x}(\mathrm{t}))$. This is of limited utility in control systems, as it does not permit a straightforward description of the interaction of the system with the control actions. The new sys-ID code includes explicit forcing terms and a unique "cross-term" contribution, i.e., $\mathrm{dx} / \mathrm{dt}=$ $\mathrm{A}(\mathrm{x}(\mathrm{t}))+\mathrm{B}(\mathrm{u}(\mathrm{t}))+\mathrm{C}(\mathrm{x}(\mathrm{t}), \mathrm{u}(\mathrm{t}))$, where $\mathrm{u}(\mathrm{t})$ represents external forcing by the controller.

Recent work by the team at SDSMT has identified the Moore-Penrose pseudo-inverse as a promising transformation between the original manifold (attractor) and the manifold defined by a time-delay embedding. Coefficients in the transformation have been optimized using a random walk algorithm to produce a "best-fit" linear mapping. Work to optimize simple nonlinear mappings and numerically demonstrate Taken's embedding theorem is in progress, and a journal paper detailing this work is being drafted.

The current method requires the analyst to supply a model expression that includes all of the possible functional terms that might appear in the system. This limits the method to "grey-box" problems where the basic form of the model is understood before the analysis or necessitates the 
inclusion of an overly general set of basis functions as the candidate model. An approach to fully automate the selection of basic functions using a genetic algorithm and a novel grammarbased function evolution process is being developed. This has obvious value for analyzing systems that are poorly understood. The code for grammatical evolution has been written and tested and will be coupled to the sys-ID code in FY 2012.

Several applications have been identified as targets for the sys-ID method. The project has continued to fund construction of two demonstration experiments at SDSMT as test beds for method validation - a radially driven inverted pendulum and a propeller test stand for autonomous submarines. These experiments are simple enough to generate clean, manageable data, yet complicated enough to be interesting and relevant to "real" systems. The SDSMT collaboration has also opened the door for potential sys-ID demonstrations using data from a Society for Automotive Engineers Baja racer and flight data from a quad-rotor helicopter. Data from the INL Hybrid Energy Testing facility will feed this effort as they become available in FY 2012.

\section{Benefits to DOE}

This work will enable the deployment of highly adaptable systems for prognostics and control, providing potential benefit to a variety of DOE and Department of Defense application areas. Developments will be communicated through peer-reviewed journal articles and conference presentations, enhancing INL's technical standing in the instrumentation and controls community. 


\section{Known Secure Sensor Measurements for Detecting Unauthorized Process Manipulation and Falsification of State}

Miles McQueen; Milos Manic, Ondrej Linda (University of Idaho); Kameshwar Poolla, Annarita Giani (University of California at Berkeley)

$11-046$

This research is an investigation of a low-cost, low false-alarm, reliable mechanism for detecting manipulation of critical physical processes and falsification of system state. We call this novel mechanism "Known Secure Sensor Measurements" (KSSM). The method moves beyond analysis of network traffic and host-based state information and uses physical measurements of the process being controlled to detect falsification of state. KSSM is intended to be incorporated into the design of new, resilient, cost-effective critical infrastructure control systems. Perhaps more importantly, it can also be included in incremental upgrades of already installed systems for enhanced resilience. KSSM is based on "known secure" physical measurements for assessing the likelihood of a cyber attack and is intended to demonstrate a practical approach to creating, transmitting, and using the known secure measurements for detection.

\section{Project Summary}

We assume that the attacker can compromise any of the components in the information layer of a control system without being detected as long as the attacker does not modify the sensor signals being transmitted back to the controller and the control room. KSSM is not designed to detect the system process exceeding its operational performance envelope; normal system monitoring is expected to detect that situation. Rather, KSSM is designed to reliably detect any attempt to falsify system state through manipulation of one or more of the sensor measurements being reported back to the control room.

We have conceptualized a design of a KSSM system for general systems and identified the most important components. The KSSM module residing in the control room is represented in Figure 1. It is responsible for modifying the subset of KSSM-enabled sensors that perform encryption and is also responsible for detecting attacks. Many functions are needed to provide these capabilities, and a few of the highest level functions will now be briefly described.

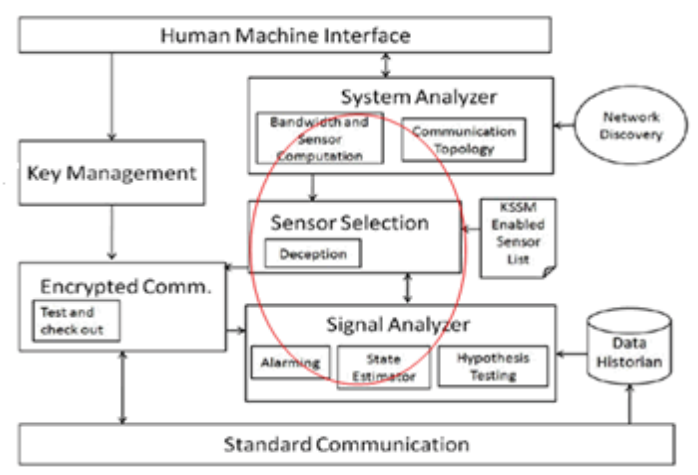

FIGURE 1. Core components within the control-room KSSM module.

The system analyzer receives input from network discovery tools, which can both reside on the system and operate in real time or can be one-time-only devices used during a phase such as system acceptance testing. It develops simplified models of the communication network to aid the sensor selection function in choosing smart subsets of sensors.

The signal analyzer is responsible for analyzing the sensor measurements that are provided to the control room and alarming when appropriate. When encrypted and associated unencrypted values do not match, an alarm will be set; if some number of requested encrypted values do not arrive in a timely fashion and are distributed over a variety of communication paths, then it may be appropriate to raise an alarm based on probabilistic assessment of likely communication and sensor failures. Other conditions and analyses for alarming need to be explored.

The sensor selection algorithm will incorporate what is known about the communication topology and the failure rates of all components within the system. The failure rates may be based on empirical data or models built into the algorithm. Further, some understanding of the limits on computation cycles available, sensor power restrictions, limitations in communication bandwidth, and cost will be incorporated to aid not only the selection of a new subset of sensors for KSSM, but also the selection for each chosen sensor of the $\mathrm{k}_{\mathrm{i}}$ of measurements that will be encrypted and forwarded. Sensor selection and the percent 
of measurements for which dual-encrypted values are required may also be made selectable by the operators so that they have control in limiting the sensor processor cycles, sensor power consumption, and communication bandwidth utilized by KSSM.

The cryptographic functions will be adopted from currently well-understood cryptographic components and systems. The KSSM-enabled sensor list is needed so that sensor selection can accommodate systems that are slowly being upgraded with KSSM-enabled sensors.

In evaluating these various functions, we then decided to focus on sensor selection algorithms since we believed it was the most significant issue that might impede the viability of the KSSM concept.

As the first attempt, we decided to look at placement of a small set of $p$ phasor measurement units (PMUs) within the power grid recognizing that it is the equivalent of selecting an optimal set of $p$ PMUs if a much larger set of $\mathrm{N}$ were available. We first introduced and characterized irreducible cyber attacks on the power grid, represented as a general graph in Figure 2. We developed an efficient algorithm to find all irreducible attacks that involve the compromise of exactly two power injection meters. We also derived canonical forms for all 3-,4-, and 5-sparse attacks under the assumption that all lines are metered. We then developed countermeasures against arbitrary unobservable attacks using knownsecure PMUs and showed that $p$ PMUs are sufficient to disable p-1 attacks.

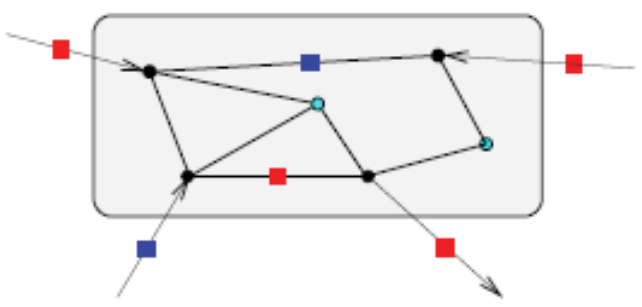

FIGURE 2. General power system represented as a graph with edges representing lines and nodes representing buses. Injection buses are black circles, null buses cyan circles, meters blue squares, and compromised meters red squares.
In parallel with PMU placement, we developed simulations to study sensor selection algorithms for more general physical processes. Initial and relatively simplistic network models were developed and used to test a few techniques for sensor selection and alarming. The results were positive and reflect the feasibility of applying KSSM concepts to systems beyond the power grid.

\section{Benefits to DOE}

This research is establishing scientific and engineering-based techniques for improved and reliable detection of cyber attacks against control-system processes. The techniques are based on control-system-specific "known secure" physical measurements, which are not available in information technology-based detection mechanisms and have not been incorporated into control-system cyber-securityattack detection tools. The research was focused on optimal placement of PMUs within the power grid in order to detect an attacker's falsification of system state. Initial algorithms for PMU placement have been created and validated with simulations on small- and medium-sized grids. More general infrastructure control systems and their corresponding infrastructures have also been investigated and the feasibility of the core KSSM concepts evaluated using initial simulations.

\section{Relevant Publications and Presentations}

Giani, A., E. Bitar, M. McQueen, P.

Khargonekar, and K. Poolla, 2011, "Smart

Grid Data Integrity Attacks:

Characterizations and Countermeasures,"

IEEE SmartGridComm, Brussels, Belgium,

Oct 2011.

McQueen, M., and A. Giani, 2011, "Known Secure Sensor Measurements for Critical Infrastructure Systems: Detecting Falsification of System State," SERENE 2011: 3rd International Workshop on Software Engineering for Resilient Systems, Geneva, Switzerland, September 2011.

Poolla, K., 2011, "Smart Grid Data Integrity," EKC2+ACCESS Workshop, Stockholm, Sweden, May 2011. 
Giani, A., 2011, "Known Secure Measurements for Critical Infrastructure Protection," Systems and Optimization Aspects of Smart Grid Challenges Workshop, Gainesville, Florida, April 2011.

Giani, A., 2011, "Cyber Security for Smart Grid Devices," TCIP-G Seminar Series, University of Illinois Urbana-Champaign, February 2011.
McQueen, M., 2011, "Cyber Security and Critical Infrastructure Resilience," SANS SCADA Summit, Lake Buena Vista, Florida, February 23, 2011.

McQueen, M., 2011, "Software, Human, and Physical Subsystems are Vulnerable to Malicious Threats," TCIP-G Review, University of Illinois Urbana-Champaign, November 4, 2011. 
$\Rightarrow$ Appendices

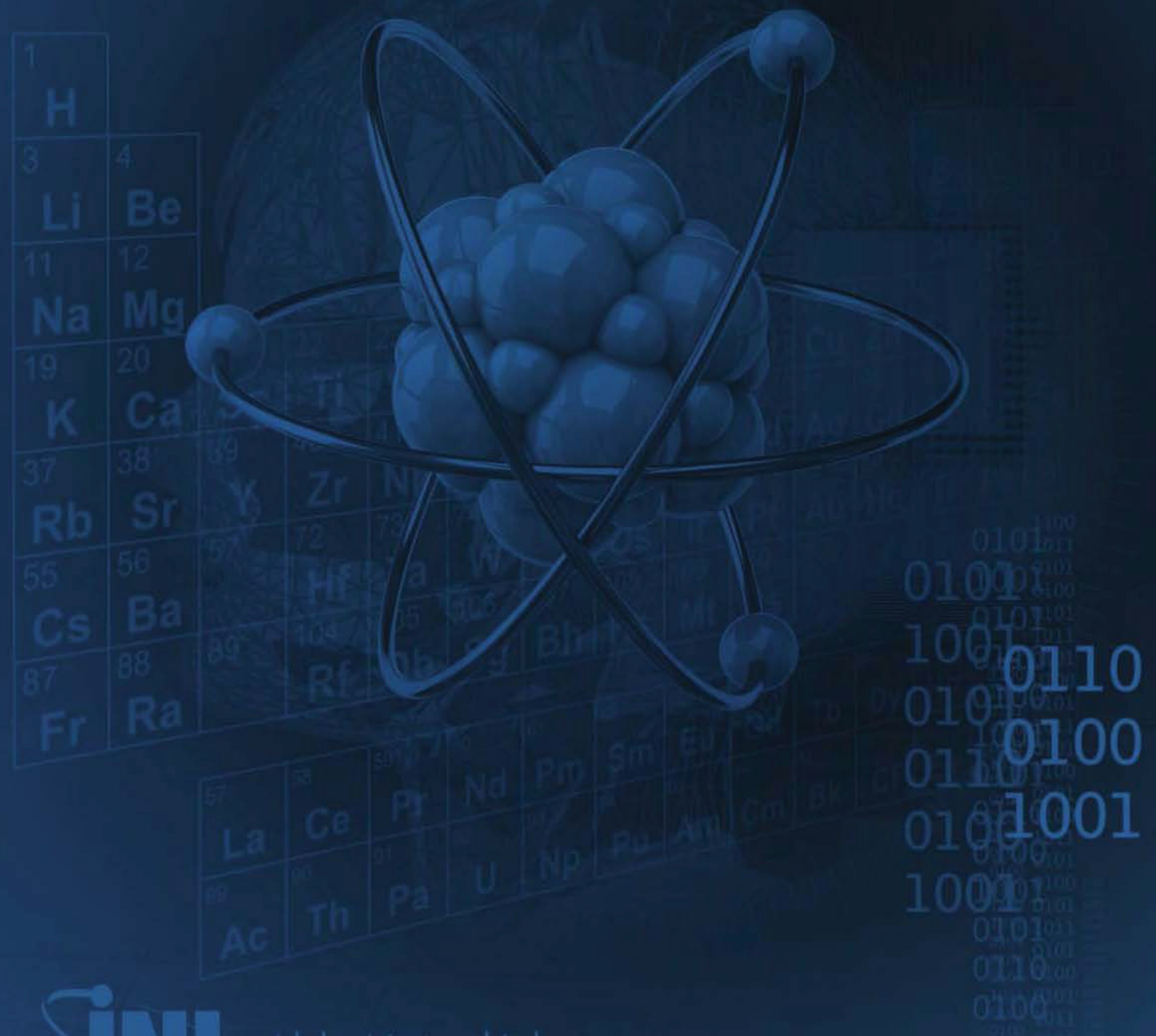

Idaho National Laboratory 
Appendix A -

Authors 


\section{Appendix A - Authors}

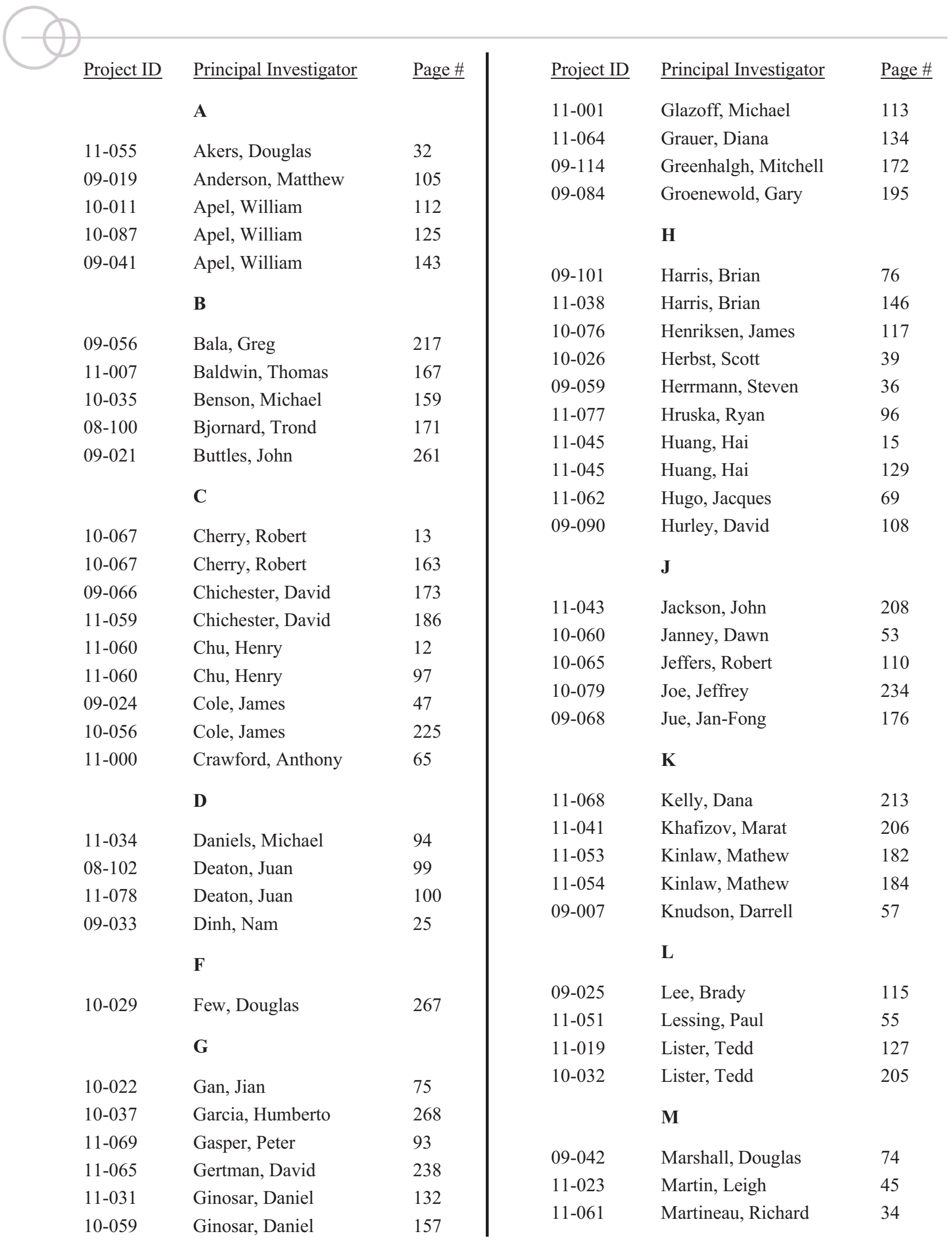




\section{Appendix A - Authors}

\begin{tabular}{|c|c|c|c|c|c|}
\hline Project ID & $\underline{\text { Principal Investigator }}$ & $\underline{\text { Page \# }}$ & $\underline{\text { Project ID }}$ & $\underline{\text { Principal Investigator }}$ & $\underline{\text { Page \# }}$ \\
\hline $10-090$ & Mattson, Earl & 122 & $11-032$ & Rollins, Harry & 138 \\
\hline 09-070 & McJunkin, Timothy & 259 & $10-073$ & Roybal, Lyle & 85 \\
\hline $10-053$ & McLing, Travis & 222 & & $\mathbf{S}$ & \\
\hline $11-042$ & McQueen, Miles & 91 & & $\mathrm{~S}$ & \\
\hline $11-046$ & McQueen, Miles & 273 & $11-037$ & Schley, Robert & 67 \\
\hline 09-003 & Meakin, Paul & 150 & $07-052$ & Scott, Jill & 11 \\
\hline $09-117$ & Metcalf, Richard & 178 & $07-052$ & Scott, Jill & 179 \\
\hline $10-008$ & Millett, Paul & 200 & 09-087 & Scott, Jill & 198 \\
\hline $10-001$ & Mincher, Bruce & 38 & $10-080$ & Searcy, Erin & 229 \\
\hline $11-006$ & Mincher, Bruce & 209 & $10-024$ & Sencer, Bulent & 7 \\
\hline $10-075$ & Moradi, Hussein & 87 & $10-068$ & Sencer, Bulent & 51 \\
\hline $10-093$ & Mousseau, Vincent & 29 & $10-024$ & Sencer, Bulent & 227 \\
\hline \multirow[t]{3}{*}{$10-088$} & Myers, Kurt & 236 & 09-105 & Shunn, Lee & 155 \\
\hline & & & $10-014$ & Shunn, Lee & 271 \\
\hline & $\mathbf{N}$ & & $10-015$ & Simpson, Michael & 220 \\
\hline \multirow[t]{3}{*}{$10-078$} & Newby, Deborah & 119 & 09-006 & Smartt, Herschel & 139 \\
\hline & $\Omega$ & & $10-092$ & Stewart, Frederick & 124 \\
\hline & 0 & & $10-043$ & Stewart, Frederick & 161 \\
\hline $10-081$ & O'Brien, James & 210 & 09-009 & Stone, Mark & 141 \\
\hline \multirow[t]{2}{*}{$10-058$} & Okuniewski, Maria & 203 & & $\mathbf{T}$ & \\
\hline & $\mathbf{P}$ & & $10-070$ & Tawfik, Magdy & 71 \\
\hline $10-038$ & Payne, Suzette & 148 & $10-007$ & Taylor, Steve & 61 \\
\hline $11-033$ & Pepper, Sarah & 137 & $10-083$ & Teysseyre, Sebastien & 204 \\
\hline $10-031$ & Pernice, Michael & 248 & & & \\
\hline $10-095$ & Pernice, Michael & 249 & & V & \\
\hline $11-074$ & Pernice, Michael & 252 & $10-096$ & Van Siclen, Clinton & 181 \\
\hline $11-075$ & Pernice, Michael & 253 & & $\mathbf{W}$ & \\
\hline $11-076$ & Pernice, Michael & 254 & & $\mathbf{W}$ & \\
\hline 09-071 & Peterman, Dean & 193 & $11-022$ & Welker, Kurt & 9 \\
\hline \multirow[t]{3}{*}{$10-049$} & Petkovic, Lucia & 166 & $11-022$ & Welker, Kurt & 89 \\
\hline & & & $11-067$ & West, James & 189 \\
\hline & $\mathbf{R}$ & & $10-094$ & Whiting, Eric & 250 \\
\hline 09-013 & Rabiti, Cristian & 23 & $10-017$ & Williamson, Rich & 49 \\
\hline $10-023$ & Rabiti, Cristian & 245 & $11-036$ & Wilson, Aaron & 144 \\
\hline $10-064$ & Richins, William & 101 & & 7 & \\
\hline $11-005$ & Riddle, Catherine & 43 & & $\mathbf{Z}$ & \\
\hline 09-096 & Rieger, Craig & 81 & $10-027$ & Zalupski, Peter & 18 \\
\hline $11-066$ & Rieger, Craig & 240 & $10-027$ & Zalupski, Peter & 41 \\
\hline 09-097 & Rieger, Craig & 263 & & & \\
\hline
\end{tabular}


Appendix B -

Relevance to Major National Programs

Idaho National Laboratory 


\section{Appendix B - Relevance to Major National Programs}

\begin{tabular}{|c|c|c|c|c|c|c|c|c|c|c|c|c|c|c|}
\hline $\begin{array}{l}\text { Project } \\
\text { ID }\end{array}$ & PI & Title & $\begin{array}{l}\text { 兄 } \\
\text { 允 }\end{array}$ & 牙 & $\sum_{I I}$ & 닏 & u & Z & 学 & Z & $\sum_{\substack{\infty \\
0}}$ & 됭 & $\begin{array}{l}\mathbb{I} \\
\text { 至 } \\
\end{array}$ & $\sum_{J}$ \\
\hline $07-052$ & $\begin{array}{l}\text { Scott, } \\
\text { Jill }\end{array}$ & $\begin{array}{l}\text { Laser Induced } \\
\text { Breakdown } \\
\text { Spectroscopy: } \\
\text { Development and } \\
\text { Application of in-situ } \\
\text { Elemental Analysis for } \\
\text { Process Streams in } \\
\text { Spent Fuel } \\
\text { Reprocessing Facilities }\end{array}$ & $\mathrm{P}$ & & & & & $\mathrm{S}$ & & & & & & \\
\hline 08-100 & $\begin{array}{l}\text { Bjornard, } \\
\text { Trond }\end{array}$ & $\begin{array}{l}\text { Developing a Next } \\
\text { Generation, Risk- } \\
\text { Informed Approach for } \\
\text { Robust and Resilient } \\
\text { Design Development }\end{array}$ & $\mathrm{S}$ & & & & & $\mathrm{P}$ & & & & & & \\
\hline 08-102 & $\begin{array}{l}\text { Deaton, } \\
\text { Juan }\end{array}$ & $\begin{array}{l}\text { Cognitive Network } \\
\text { Engine and Simulation } \\
\text { Framework }\end{array}$ & $\mathrm{P}$ & $\mathrm{S}$ & & & & & & & & $\mathrm{S}$ & & \\
\hline 09-003 & $\begin{array}{l}\text { Meakin, } \\
\text { Paul }\end{array}$ & $\begin{array}{l}\text { Particle-discrete } \\
\text { Element Model } \\
\text { Simulation of the } \\
\text { Coupling between } \\
\text { Material Failure/ } \\
\text { Deformation and Fluid } \\
\text { Generation/Flow } \\
\end{array}$ & & & $\mathrm{S}$ & $\mathrm{P}$ & $\mathrm{S}$ & & & & & & & \\
\hline 09-006 & $\begin{array}{l}\text { Smartt, } \\
\text { Herschel }\end{array}$ & $\begin{array}{l}\text { Scaling of Welding } \\
\text { Processes }\end{array}$ & & $\mathrm{P}$ & & $\mathrm{S}$ & $\mathrm{S}$ & $\mathrm{S}$ & & & & & & \\
\hline 09-007 & $\begin{array}{l}\text { Knudson, } \\
\text { Darrell }\end{array}$ & $\begin{array}{l}\text { Instrumentation for in- } \\
\text { pile detection of } \\
\text { thermal conductivity }\end{array}$ & & & & & & $\mathrm{P}$ & $\mathrm{S}$ & & & & & \\
\hline 09-009 & $\begin{array}{l}\text { Stone, } \\
\text { Mark }\end{array}$ & $\begin{array}{l}\text { Enhanced Metal Ion } \\
\text { Analysis }\end{array}$ & $\mathrm{P}$ & & $\mathrm{S}$ & & $\mathrm{S}$ & $\mathrm{S}$ & & & & & $\mathrm{S}$ & \\
\hline 09-013 & $\begin{array}{l}\text { Rabiti, } \\
\text { Cristian }\end{array}$ & $\begin{array}{l}\text { Development of } \\
\text { Reactor Physics } \\
\text { Sensitivity Analysis, } \\
\text { Uncertainty } \\
\text { Quantification, and } \\
\text { Data Assimilation } \\
\text { Capability at INL for } \\
\text { Validation Applications } \\
\end{array}$ & & & & & & $\mathrm{P}$ & & & & & & \\
\hline 09-019 & $\begin{array}{l}\text { Anderson, } \\
\text { Matthew }\end{array}$ & $\begin{array}{l}\text { Advanced Remote } \\
\text { Sensing for Energy and } \\
\text { Environmental } \\
\text { Applications using } \\
\text { Unmanned Aerial } \\
\text { Vehicles }\end{array}$ & $\mathrm{S}$ & & $\mathrm{P}$ & $\mathrm{S}$ & $\mathrm{S}$ & $\mathrm{S}$ & & & & & & $\mathrm{S}$ \\
\hline 09-021 & $\begin{array}{l}\text { Buttles, } \\
\text { John }\end{array}$ & $\begin{array}{l}\text { Wireless Sensor } \\
\text { Testing }\end{array}$ & $\mathrm{S}$ & & $\mathrm{S}$ & & & $\mathrm{P}$ & & & $\mathrm{S}$ & & $\mathrm{S}$ & \\
\hline
\end{tabular}




\section{Appendix B - Relevance to Major National Programs}

\begin{tabular}{|c|c|c|c|c|c|c|c|c|c|c|c|c|c|c|}
\hline $\begin{array}{l}\text { Project } \\
\text { ID }\end{array}$ & PI & Title & $\begin{array}{l}\text { 出 } \\
Z \\
Z\end{array}$ & 됟 & $\sum_{I I}$ & 던 & U & 至 & 点 & Z & $\sum_{\substack{0 \\
0}}^{\substack{0 \\
0}}$ & 띵 & $\begin{array}{c}\mathbb{v} \\
\text { 工1 } \\
\end{array}$ & $\sum_{-}$ \\
\hline 09-024 & $\begin{array}{l}\text { Cole, } \\
\text { James }\end{array}$ & $\begin{array}{l}\text { Development of a } \\
\text { Small Sample Volume } \\
\text { Mechanical Properties } \\
\text { Testing Technique for } \\
\text { Irradiated Fuels and } \\
\text { Materials }\end{array}$ & & & & & $\mathrm{S}$ & $\mathrm{P}$ & $\mathrm{S}$ & & & & & \\
\hline 09-025 & $\begin{array}{l}\text { Lee, } \\
\text { Brady }\end{array}$ & $\begin{array}{l}\text { Network Interaction In } \\
\text { the Thermoacidophile } \\
\text { Alicyclobacillus } \\
\text { acidocaldarius In } \\
\text { Response to Different } \\
\text { Complex Carbon } \\
\text { Sources }\end{array}$ & & $\mathrm{P}$ & & & $\mathrm{S}$ & & & & & & & \\
\hline 09-033 & $\begin{array}{l}\text { Dinh, } \\
\text { Nam }\end{array}$ & $\begin{array}{l}\text { Next-Generation Code } \\
\text { for Nuclear Systems }\end{array}$ & & & & & & $\mathrm{P}$ & S & & & & & \\
\hline 09-041 & $\begin{array}{l}\text { Apel, } \\
\text { William }\end{array}$ & $\begin{array}{l}\text { Effect of Glycosylation } \\
\text { on the Activity and } \\
\text { Stability of Bacterial } \\
\text { Enzymes }\end{array}$ & & $\mathrm{S}$ & & & $\mathrm{P}$ & & & & & & & \\
\hline 09-042 & $\begin{array}{l}\text { Marshall, } \\
\text { Doug }\end{array}$ & $\begin{array}{l}\text { Characterization of } \\
\text { Fluidized Beds via } \\
\text { Pressure-Fluctuation } \\
\text { Analysis }\end{array}$ & & $\mathrm{S}$ & & $\mathrm{S}$ & & $\mathrm{P}$ & & & & & & \\
\hline 09-056 & $\begin{array}{l}\text { Bala, } \\
\text { Greg }\end{array}$ & $\begin{array}{l}\text { Development of } \\
\text { Lignocellulosic Ethanol } \\
\text { Production Potential in } \\
\text { Idaho }\end{array}$ & & $\mathrm{P}$ & & & & & & & & & & \\
\hline 09-059 & $\begin{array}{l}\text { Herrmann, } \\
\text { Steve }\end{array}$ & $\begin{array}{l}\text { Characterization of a } \\
\text { Consolidated } \\
\text { Electrochemical } \\
\text { Technique for } \\
\text { Separation and } \\
\text { Recovery of Actinides } \\
\text { from Fission Products } \\
\text { in Oxide Fuels }\end{array}$ & & & & & & $\mathrm{P}$ & & & & & & \\
\hline 09-066 & $\begin{array}{l}\text { Chichester, } \\
\text { David }\end{array}$ & $\begin{array}{l}\text { Neutron Spectrometer } \\
\text { Development }\end{array}$ & $\mathrm{P}$ & & $\mathrm{S}$ & & $\mathrm{S}$ & $\mathrm{S}$ & S & & $\mathrm{S}$ & & $\mathrm{S}$ & \\
\hline 09-068 & $\begin{array}{l}\mathrm{Li} \text {, } \\
\text { Shelly }\end{array}$ & $\begin{array}{l}\text { On-line Monitoring of } \\
\text { Actinide } \\
\text { Concentrations for } \\
\text { Advanced Aqueous } \\
\text { Separation Processes }\end{array}$ & $\mathrm{P}$ & & & & & $\mathrm{S}$ & & & & & & \\
\hline 09-070 & $\begin{array}{l}\text { McJunkin, } \\
\text { Tim }\end{array}$ & $\begin{array}{l}\text { Integrated Control } \\
\text { System Data Fusion }\end{array}$ & & & & & $\mathrm{S}$ & $\mathrm{S}$ & & & & $\mathrm{P}$ & & \\
\hline 09-071 & $\begin{array}{l}\text { Peterman, } \\
\text { Dean }\end{array}$ & $\begin{array}{l}\text { Evaluation of Covalent } \\
\text { Interactions in Actinide } \\
\text { Coordination } \\
\text { Compounds }\end{array}$ & $\mathrm{S}$ & & S & & $\mathrm{S}$ & $\mathrm{P}$ & & & S & & & \\
\hline
\end{tabular}




\section{Appendix B - Relevance to Major National Programs}

\begin{tabular}{|c|c|c|c|c|c|c|c|c|c|c|c|c|c|c|}
\hline $\begin{array}{l}\text { Project } \\
\text { ID }\end{array}$ & PI & Title & $\begin{array}{l}\text { 岕 } \\
\text { 允 }\end{array}$ & 壵 & $\sum_{I I}$ & 聓 & U & Z & 光 & 玄 & $\sum_{\substack{\infty \\
0}}^{\substack{0 \\
0}}$ & 딩 & $\begin{array}{l}\mathbb{I} \\
\text { 工 } \\
\text { 포 }\end{array}$ & $\sum_{S}$ \\
\hline 09-084 & $\begin{array}{l}\text { Groenewold, } \\
\text { Gary }\end{array}$ & $\begin{array}{l}\text { Measuring Actinide } \\
\text { Speciation in High } \mathrm{pH} \\
\text { Solutions }\end{array}$ & & & $\mathrm{S}$ & & $\mathrm{P}$ & $\mathrm{S}$ & & & & & & \\
\hline 09-087 & Scott, Jill & $\begin{array}{l}\text { Isotope Ratio } \\
\text { Measurements Methods } \\
\text { for Direct Analysis of } \\
\text { Samples }\end{array}$ & $\mathrm{P}$ & $\mathrm{S}$ & $\mathrm{S}$ & & $\mathrm{S}$ & $\mathrm{S}$ & $\mathrm{S}$ & & & & & $\mathrm{S}$ \\
\hline 09-090 & $\begin{array}{l}\text { Hurley, } \\
\text { David }\end{array}$ & $\begin{array}{l}\text { Addressing the } \\
\text { Spectrum of Nuclear } \\
\text { Related NDE Needs: A } \\
\text { Hybrid Laser } \\
\text { Ultrasonic and Eddy } \\
\text { Current Approach }\end{array}$ & & & & $\mathrm{S}$ & & $\mathrm{P}$ & $\mathrm{S}$ & & & & & \\
\hline 09-096 & $\begin{array}{l}\text { Rieger, } \\
\text { Craig }\end{array}$ & $\begin{array}{l}\text { Methodologies for the } \\
\text { Design, Analysis, and } \\
\text { Validation for } \\
\text { Operation of Complex } \\
\text { Resilient Networks }\end{array}$ & & $\mathrm{S}$ & & $\mathrm{S}$ & $\mathrm{S}$ & $\mathrm{S}$ & & & & $\mathrm{P}$ & $\mathrm{S}$ & \\
\hline 09-097 & $\begin{array}{l}\text { Rieger, } \\
\text { Craig }\end{array}$ & $\begin{array}{l}\text { Anomaly Detection, } \\
\text { Diagnosis, and } \\
\text { Resilient Control in } \\
\text { Complex Engineered } \\
\text { Systems }\end{array}$ & & $\mathrm{S}$ & & $\mathrm{S}$ & $\mathrm{S}$ & $\mathrm{P}$ & & & & $\mathrm{S}$ & $\mathrm{S}$ & \\
\hline 09-101 & Harris, Brian & $\begin{array}{l}\text { Dissolution and } \\
\text { Extraction Studies of } \\
\text { Fission Products in } \\
\text { Room Temperature } \\
\text { Ionic Liquids and in } \\
\text { Supercritical Fluid CO2 } \\
\text { and Determination of } \\
\text { Radiolytic Stability } \\
\end{array}$ & $\mathrm{S}$ & & $\mathrm{P}$ & & & $\mathrm{S}$ & & & & & & \\
\hline 09-105 & Shunn, Lee & $\begin{array}{l}\text { Adaptive Process } \\
\text { Modeling Using } \\
\text { Parameter Estimation } \\
\text { and Mechanism } \\
\text { Sensitivity Analysis. }\end{array}$ & & & & $\mathrm{S}$ & & $\mathrm{P}$ & & & & & & \\
\hline $09-114$ & $\begin{array}{l}\text { Greenhalgh, } \\
\text { Mitch }\end{array}$ & $\begin{array}{l}\text { Radionuclide } \\
\text { Collection-Detection } \\
\text { Device for the in situ } \\
\text { Remote Monitoring of } \\
\text { 99Tc as a Proliferation } \\
\text { Indicator }\end{array}$ & $\mathrm{P}$ & & $\mathrm{S}$ & & $\mathrm{S}$ & $\mathrm{S}$ & & & $\mathrm{S}$ & & $\mathrm{S}$ & \\
\hline $09-117$ & $\begin{array}{l}\text { Metcalf, } \\
\text { Richard }\end{array}$ & $\begin{array}{l}\text { Develop a Safeguards } \\
\text { Approach for INL } \\
\text { Pyroprocessing lines } \\
\text { for Demonstration to } \\
\text { IAEA }\end{array}$ & $\mathrm{P}$ & & & & & S & & & & & & \\
\hline
\end{tabular}




\section{Appendix B - Relevance to Major National Programs}

\begin{tabular}{|c|c|c|c|c|c|c|c|c|c|c|c|c|c|c|}
\hline $\begin{array}{l}\text { Project } \\
\text { ID }\end{array}$ & PI & Title & 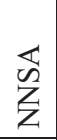 & 牙 & $\sum_{I I}$ & 峑 & U & 工 & 点 & Z & 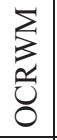 & 띵 & $\begin{array}{l}\mathbb{I} \\
\text { 工ు } \\
\text { In }\end{array}$ & $\sum$ \\
\hline $10-001$ & $\begin{array}{l}\text { Mincher, } \\
\text { Bruce }\end{array}$ & $\begin{array}{l}\text { Neptunium Redox } \\
\text { Chemistry in Irradiated } \\
\text { Aqueous Nitric Acid }\end{array}$ & & & $\mathrm{S}$ & & & $\mathrm{P}$ & & & & & & \\
\hline $10-007$ & $\begin{array}{l}\text { Taylor, } \\
\text { Steven }\end{array}$ & $\begin{array}{l}\text { Ultrasonic Transducer } \\
\text { Sensors for In-Pile } \\
\text { Detection of } \\
\text { Dimensional changes }\end{array}$ & $\mathrm{S}$ & & & & & $\mathrm{P}$ & S & & & & & \\
\hline 10-008 & Millet, Paul & $\begin{array}{l}\text { Irradiation-Induced } \\
\text { Evolution of Defects } \\
\text { and Microstructures in } \\
\text { Nanocrystalline BCC } \\
\text { Mo }\end{array}$ & & & & & $\mathrm{S}$ & $\mathrm{P}$ & & & & & & \\
\hline $10-011$ & $\begin{array}{l}\text { Apel, } \\
\text { William }\end{array}$ & $\begin{array}{l}\text { Degradation and } \\
\text { Conversion of Lignin } \\
\text { using Extremophilic } \\
\text { Systems }\end{array}$ & & $\mathrm{P}$ & & & $\mathrm{S}$ & & & & & & & \\
\hline $10-014$ & Shunn, Lee & $\begin{array}{l}\text { Automated Differential } \\
\text { Equation-Based } \\
\text { Identification }\end{array}$ & $\mathrm{S}$ & $\mathrm{S}$ & & & $\mathrm{P}$ & $\mathrm{S}$ & & & & & & \\
\hline $10-015$ & $\begin{array}{l}\text { Simpson, } \\
\text { Michael }\end{array}$ & $\begin{array}{l}\text { Optimization of } \\
\text { Ceramic Waste Forms } \\
\text { Used for } \\
\text { Electrochemical } \\
\text { Processing of Spent } \\
\text { Nuclear Fuel }\end{array}$ & & & & & & $\mathrm{P}$ & & & & & & \\
\hline $10-017$ & $\begin{array}{l}\text { Williamson, } \\
\text { Richard }\end{array}$ & $\begin{array}{l}\text { Multiscale Modeling } \\
\text { and Simulation of } \\
\text { Nuclear Fuel } \\
\text { Performance }\end{array}$ & & & & & $\mathrm{S}$ & $\mathrm{P}$ & & & & & & \\
\hline $10-022$ & Gan, Jian & $\begin{array}{l}\text { In-Pile Temperature } \\
\text { Monitor and Control for } \\
\text { ATR }\end{array}$ & & & & & & $\mathrm{P}$ & & & & & & \\
\hline $10-023$ & $\begin{array}{l}\text { Rabiti, } \\
\text { Cristian }\end{array}$ & $\begin{array}{l}\text { Study of } \\
\text { Preconditioning } \\
\text { Techniques for Krylov } \\
\text { Solvers Applied to } \\
\text { Hybrid Neutron } \\
\text { Transport Calculations }\end{array}$ & & & & & & $\mathrm{P}$ & & & & & & \\
\hline $10-024$ & $\begin{array}{l}\text { Sencer, } \\
\text { Bulent }\end{array}$ & $\begin{array}{l}\text { Small Specimen Test } \\
\text { Techniques for } \\
\text { Evaluating Radiation- } \\
\text { induced Changes in } \\
\text { Mechanical Properties }\end{array}$ & & & & & & $\mathrm{P}$ & & & & & & \\
\hline $10-026$ & Herbst, Scott & $\begin{array}{l}\text { Reversible Gas Phase } \\
\text { Reactions for Recovery } \\
\text { of Graphite from } \\
\text { Recycled HTGR } \\
\text { TRISO Fuel }\end{array}$ & & $\mathrm{S}$ & & & & $\mathrm{P}$ & & & & & & \\
\hline
\end{tabular}




\section{Appendix B - Relevance to Major National Programs}

\begin{tabular}{|c|c|c|c|c|c|c|c|c|c|c|c|c|c|c|}
\hline $\begin{array}{l}\text { Project } \\
\text { ID }\end{array}$ & PI & Title & 苋 & 空 & $\sum_{I=1}$ & 떧 & Un & 岂 & 号 & Z & $\sum_{\substack{0 \\
0}}$ & 덩 & $\begin{array}{l}\mathbb{U} \\
\text { 工 } \\
\text {. }\end{array}$ & $\sum$ \\
\hline $10-027$ & $\begin{array}{l}\text { Zalupski, } \\
\text { Peter }\end{array}$ & $\begin{array}{l}\text { Comprehensive } \\
\text { Thermodynamic } \\
\text { Models for Aqueous } \\
\text { Partitioning of } \\
\text { Actinides from Used } \\
\text { Nuclear Fuel. }\end{array}$ & $\mathrm{S}$ & & $\mathrm{S}$ & & $\mathrm{S}$ & $\mathrm{P}$ & & & $\mathrm{S}$ & & & $\mathrm{S}$ \\
\hline $10-029$ & Few, Doug & $\begin{array}{l}\text { 3D Spatial } \\
\text { Representation in } \\
\text { Support of Design } \\
\text { Inspection and } \\
\text { Verification }\end{array}$ & $\mathrm{P}$ & & & & & $\mathrm{S}$ & & & & & & \\
\hline $10-031$ & $\begin{array}{l}\text { Pernice, } \\
\text { Michael }\end{array}$ & $\begin{array}{l}\text { Fickian and Thermal } \\
\text { Diffusion in Nuclear } \\
\text { Materials from Linear } \\
\text { Response Theory and } \\
\text { Multiscale Simulations }\end{array}$ & & & & & $\mathrm{S}$ & $\mathrm{P}$ & & & $\mathrm{S}$ & & & \\
\hline $10-032$ & Lister, Tedd & $\begin{array}{l}\text { Investigation of Molten } \\
\text { Bromide Salt Systems } \\
\text { for Separation and } \\
\text { Recovery of Actinides } \\
\text { from Fission Products }\end{array}$ & & & $\mathrm{S}$ & & & $\mathrm{P}$ & & & $\mathrm{S}$ & & & \\
\hline $10-035$ & $\begin{array}{l}\text { Benson, } \\
\text { Michael }\end{array}$ & $\begin{array}{l}\text { Mesocilicate Supported } \\
\text { CO2 Capture Material }\end{array}$ & & $\mathrm{P}$ & & $\mathrm{S}$ & & & & & & & & \\
\hline $10-037$ & $\begin{array}{l}\text { Garcia, } \\
\text { Humberto }\end{array}$ & $\begin{array}{l}\text { Resilient Condition } \\
\text { Assessment Monitoring } \\
\text { (ReCAM) System }\end{array}$ & $\mathrm{S}$ & $\mathrm{P}$ & & $\mathrm{S}$ & $\mathrm{S}$ & $\mathrm{S}$ & & & & S & $\mathrm{S}$ & \\
\hline $10-038$ & $\begin{array}{l}\text { Payne, } \\
\text { Suzette }\end{array}$ & $\begin{array}{l}\text { Aleatory vs Epistemic } \\
\text { Uncertainty in Seismic } \\
\text { Hazard Analyses }\end{array}$ & $\mathrm{S}$ & & $\mathrm{S}$ & & & $\mathrm{P}$ & $\mathrm{S}$ & & & & & S \\
\hline $10-043$ & $\begin{array}{l}\text { Stewart, } \\
\text { Frederick }\end{array}$ & $\begin{array}{l}\text { Membrane Separation } \\
\text { System Research for } \\
\text { the HYTEST Facility }\end{array}$ & & $\mathrm{P}$ & & $\mathrm{S}$ & & & & & & & & \\
\hline $10-049$ & $\begin{array}{l}\text { Petkovic, } \\
\text { Lucia }\end{array}$ & $\begin{array}{l}\text { Conversion of Light } \\
\text { Hydrocarbons to Fuels } \\
\text { and Chemicals }\end{array}$ & & $\mathrm{P}$ & & $\mathrm{S}$ & $\mathrm{S}$ & $\mathrm{S}$ & & & & $\mathrm{S}$ & & \\
\hline $10-053$ & $\begin{array}{l}\text { McLing, } \\
\text { Travis }\end{array}$ & $\begin{array}{l}\text { Prediction and } \\
\text { Monitoring of } \mathrm{CO} 2 \\
\text { Behavior in Deep } \\
\text { Reactive Geologic } \\
\text { Formations }\end{array}$ & & & & $\mathrm{P}$ & & & & & & S & $\mathrm{S}$ & \\
\hline $10-056$ & Cole, James & $\begin{array}{l}\text { Fabrication of } \\
\text { Advanced ODS Alloys } \\
\text { using Field Assisted } \\
\text { Sintering }\end{array}$ & & & & & $\mathrm{S}$ & $\mathrm{P}$ & & & & & & \\
\hline $10-058$ & $\begin{array}{l}\text { Okuniewski, } \\
\text { Maria }\end{array}$ & $\begin{array}{l}\text { Microstructural } \\
\text { Evolution of UO2 and } \\
\text { U Under Irradiation }\end{array}$ & & & & & P & S & & & & & & \\
\hline
\end{tabular}




\section{Appendix B - Relevance to Major National Programs}

\begin{tabular}{|c|c|c|c|c|c|c|c|c|c|c|c|c|c|c|}
\hline $\begin{array}{l}\text { Project } \\
\text { ID }\end{array}$ & PI & Title & 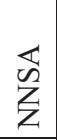 & 牙 & $\sum_{I I}$ & 屸 & U & 工 & 点 & Z & $\sum_{\substack{\text { s. } \\
0}}$ & 띵 & $\begin{array}{l}\mathbb{I} \\
\text { 工ు } \\
\text { In }\end{array}$ & $\sum_{\exists}$ \\
\hline $10-059$ & $\begin{array}{l}\text { Ginosar, } \\
\text { Daniel }\end{array}$ & $\begin{array}{l}\text { Feedstock Processing } \\
\text { and Energy Storage }\end{array}$ & & $\mathrm{P}$ & & $\mathrm{S}$ & & $\mathrm{S}$ & & & & $\mathrm{S}$ & & \\
\hline $10-060$ & $\begin{array}{l}\text { Janney, } \\
\text { Dawn }\end{array}$ & $\begin{array}{l}\text { Spatially-correlated } \\
\text { Microstructural } \\
\text { Characterization: From } \\
\text { Centimeters to } \\
\text { Nanometers }\end{array}$ & $\mathrm{S}$ & & $\mathrm{S}$ & & $\mathrm{S}$ & $\mathrm{P}$ & & & $\mathrm{S}$ & & & $S$ \\
\hline $10-064$ & $\begin{array}{l}\text { Richins, } \\
\text { William }\end{array}$ & $\begin{array}{l}\text { Multi-Rate Shock } \\
\text { Physics Simulation of } \\
\text { Blast and Penetration } \\
\text { Events in Concrete }\end{array}$ & $\mathrm{S}$ & & & & $\mathrm{S}$ & $\mathrm{P}$ & & & $\mathrm{S}$ & & & \\
\hline $10-065$ & $\begin{array}{l}\text { Jeffers, } \\
\text { Robert }\end{array}$ & $\begin{array}{l}\text { Water and Energy } \\
\text { System } \\
\text { Interdependency } \\
\text { Modeling for } \\
\text { Multicriteria Decision } \\
\text { Analysis }\end{array}$ & & $\mathrm{P}$ & $\mathrm{S}$ & $\mathrm{S}$ & $\mathrm{S}$ & $\mathrm{S}$ & & & & $\mathrm{S}$ & & \\
\hline $10-067$ & $\begin{array}{l}\text { Cherry, } \\
\text { Robert }\end{array}$ & $\begin{array}{l}\text { Hybrid Systems process } \\
\text { integration and } \\
\text { dynamics studies }\end{array}$ & & $\mathrm{S}$ & & $\mathrm{S}$ & & $\mathrm{P}$ & & & & & & \\
\hline $10-068$ & $\begin{array}{l}\text { Sencer, } \\
\text { Bulent }\end{array}$ & $\begin{array}{l}\text { Irradiation Testing and } \\
\text { Molecular Modeling of } \\
\text { Irradiation-Assisted } \\
\text { Diffusion and } \\
\text { Microstructural } \\
\text { Evolution (FCCI) }\end{array}$ & & & & & & $\mathrm{P}$ & & & & & & \\
\hline $10-070$ & $\begin{array}{l}\text { Tawfik, } \\
\text { Magdy }\end{array}$ & $\begin{array}{l}\text { Smart Grid Impact on } \\
\text { Commercial Nuclear } \\
\text { Plants }\end{array}$ & & $\mathrm{S}$ & & & & $\mathrm{P}$ & & & & & & \\
\hline $10-073$ & Roybal, Lyle & $\begin{array}{l}\text { Next Generation } \\
\text { Control System "Smart } \\
\text { Grid" Simulation } \\
\text { Environment }\end{array}$ & & $\mathrm{S}$ & & & & & & & & $\mathrm{P}$ & & \\
\hline $10-075$ & $\begin{array}{l}\text { Moradi, } \\
\text { Hussein }\end{array}$ & $\begin{array}{l}\text { Wireless } \\
\text { Communications R\&D }\end{array}$ & $\mathrm{S}$ & & & & $\mathrm{S}$ & $\mathrm{S}$ & & & $\mathrm{P}$ & & & \\
\hline $10-076$ & $\begin{array}{l}\text { Henriksen, } \\
\text { James }\end{array}$ & $\begin{array}{l}\text { Specific Biological } \\
\text { Responses to Nano } \\
\text { Metal Oxides }\end{array}$ & & & $\mathrm{S}$ & & $\mathrm{P}$ & & & & & & $\mathrm{S}$ & \\
\hline 10-078 & $\begin{array}{l}\text { Newby, } \\
\text { Deborah }\end{array}$ & $\begin{array}{l}\text { Development of Non- } \\
\text { Lethal Methods for } \\
\text { Enhanced Lipid } \\
\text { Recovery from } \\
\text { Microalgae }\end{array}$ & & $\mathrm{P}$ & & & $\mathrm{S}$ & & & & & & & \\
\hline $10-079$ & Joe, Jeffrey & $\begin{array}{l}\text { Transmission Site } \\
\text { Suitability Decision } \\
\text { Support Technology }\end{array}$ & & $\mathrm{S}$ & $\mathrm{S}$ & & $\mathrm{S}$ & & & & & P & & \\
\hline
\end{tabular}




\section{Appendix B - Relevance to Major National Programs}

\begin{tabular}{|c|c|c|c|c|c|c|c|c|c|c|c|c|c|c|}
\hline $\begin{array}{l}\text { Project } \\
\text { ID }\end{array}$ & PI & Title & $\begin{array}{l}\text { 兄 } \\
\text { 允 }\end{array}$ & 덛 & $\sum_{I I}$ & 㭊 & U & Z & 学 & Z & $\sum_{\substack{\infty \\
0}}^{\substack{0 \\
0}}$ & 딩 & 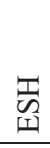 & $\sum$ \\
\hline $10-080$ & Searcy, Erin & $\begin{array}{l}\text { Design and Operational } \\
\text { Improvements and } \\
\text { LCA in Anaerobic } \\
\text { Digestion of Fermented } \\
\text { Dairy Manure using a } \\
\text { 2-Stage Process }\end{array}$ & & $\mathrm{P}$ & $\mathrm{S}$ & & & & & & & & & \\
\hline $10-081$ & $\begin{array}{l}\text { O'Brien, } \\
\text { James }\end{array}$ & $\begin{array}{l}\text { Design and Testing of a } \\
\text { Mars Hopper }\end{array}$ & & & & & $\mathrm{S}$ & $\mathrm{P}$ & & & & & & \\
\hline $10-083$ & $\begin{array}{l}\text { Teysseyre, } \\
\text { Sebastian }\end{array}$ & $\begin{array}{l}\text { In situ characterization } \\
\text { of an oxide film for } \\
\text { prediction of stress } \\
\text { corrosion cracking } \\
\text { susceptibility }\end{array}$ & & & & & $\mathrm{S}$ & $\mathrm{P}$ & & & & & & \\
\hline $10-087$ & $\begin{array}{l}\text { Apel, } \\
\text { William }\end{array}$ & $\begin{array}{l}\text { Biological Fixation of } \\
\mathrm{CO} 2 \text { for Fuel and } \\
\text { Chemical Production } \\
\text { Using Acetogen } \\
\text { Intermediary } \\
\text { Metabolism }\end{array}$ & & $\mathrm{P}$ & & & $\mathrm{S}$ & & & & & & & \\
\hline $10-088$ & Myers, Kurt & $\begin{array}{l}\text { CAES Vertical Axis } \\
\text { Wind Turbine }\end{array}$ & & $\mathrm{P}$ & & & & & & & & & & \\
\hline $10-090$ & $\begin{array}{l}\text { Mattson, } \\
\text { Earl }\end{array}$ & $\begin{array}{l}\text { Development of } \\
\text { Thermally Generated In } \\
\text { Situ Precipitation } \\
\text { Barriers due to } \\
\text { Subsurface Heat } \\
\text { Injection }\end{array}$ & & & $\mathrm{S}$ & $\mathrm{S}$ & $\mathrm{P}$ & & & & $\mathrm{S}$ & & & \\
\hline $10-092$ & $\begin{array}{l}\text { Stewart, } \\
\text { Frederick }\end{array}$ & $\begin{array}{l}\text { Hybrid Osmosis Water } \\
\text { Purification Systems } \\
\text { Research }\end{array}$ & & $\mathrm{P}$ & $\mathrm{S}$ & $\mathrm{S}$ & & $\mathrm{S}$ & & & & & & \\
\hline $10-093$ & $\begin{array}{l}\text { Mousseau, } \\
\text { Vince }\end{array}$ & $\begin{array}{l}\text { Nuclear Reactor Safety } \\
\text { Case }\end{array}$ & & & & & $\mathrm{S}$ & $\mathrm{P}$ & $\mathrm{S}$ & & & & & \\
\hline $10-094$ & $\begin{array}{l}\text { Whiting, } \\
\text { Eric }\end{array}$ & $\begin{array}{l}\text { Advanced Visualization } \\
\text { using Immersive } \\
\text { Environments }\end{array}$ & $\mathrm{S}$ & $\mathrm{S}$ & $\mathrm{S}$ & $\mathrm{S}$ & $\mathrm{S}$ & $\mathrm{P}$ & & & & $\mathrm{S}$ & & \\
\hline $10-095$ & $\begin{array}{l}\text { Pernice, } \\
\text { Michael }\end{array}$ & $\begin{array}{l}\text { Error Estimation for } \\
\text { stochastic uncertainty } \\
\text { quantification }\end{array}$ & $\mathrm{S}$ & & $\mathrm{S}$ & $\mathrm{S}$ & $\mathrm{S}$ & $\mathrm{P}$ & & & & & & \\
\hline $10-096$ & $\begin{array}{l}\text { Van Siclen, } \\
\text { Clinton }\end{array}$ & $\begin{array}{l}\text { Object Reconstruction } \\
\text { Technique for use in } \\
\text { Radiography }\end{array}$ & $\mathrm{P}$ & & & & & & & & & & & \\
\hline $11-000$ & $\begin{array}{l}\text { Crawford, } \\
\text { Anthony }\end{array}$ & $\begin{array}{l}\text { Haptic Tele-Operated } \\
\text { End Effector for } \\
\text { Hazardous } \\
\text { Environments }\end{array}$ & $\mathrm{S}$ & & $\mathrm{S}$ & & & $\mathrm{P}$ & S & & $\mathrm{S}$ & & S & \\
\hline
\end{tabular}




\section{Appendix B - Relevance to Major National Programs}

\begin{tabular}{|c|c|c|c|c|c|c|c|c|c|c|c|c|c|c|}
\hline $\begin{array}{l}\text { Project } \\
\text { ID }\end{array}$ & PI & Title & 茫 & 펌 & $\sum_{I I}$ & 椞 & U & 罗 & 点 & Z & $\sum_{\substack{0 \\
0}}$ & 띵 & $\begin{array}{l}\text { 吾 } \\
\text { 工ు } \\
\end{array}$ & $\sum_{J}$ \\
\hline 11-001 & $\begin{array}{l}\text { Glazoff, } \\
\text { Michael }\end{array}$ & $\begin{array}{l}\text { Innovative } \\
\text { Mathematical } \\
\text { Morphology Approach } \\
\text { to Optical Color Image } \\
\text { Analysis and Pattern } \\
\text { Recognition }\end{array}$ & & & $\mathrm{S}$ & & $\mathrm{P}$ & $\mathrm{S}$ & & & & & & \\
\hline $11-005$ & $\begin{array}{l}\text { Riddle, } \\
\text { Catherine }\end{array}$ & $\begin{array}{l}\text { Speciation Behavior of } \\
\text { Americium Oxidation } \\
\text { States for the } \\
\text { Separation of } \\
\text { Americium from } \\
\text { Curium in Nuclear } \\
\text { Processing }\end{array}$ & & $\mathrm{S}$ & & & & $\mathrm{P}$ & & & & & & \\
\hline 11-006 & $\begin{array}{l}\text { Mincher, } \\
\text { Bruce }\end{array}$ & $\begin{array}{l}\text { Supercritical Fluid } \\
\text { Extraction of Actinides } \\
\text { from TRISO Reactor } \\
\text { Fuels }\end{array}$ & & & $\mathrm{S}$ & & & $\mathrm{P}$ & & & $\mathrm{S}$ & & & \\
\hline $11-007$ & $\begin{array}{l}\text { Baldwin, } \\
\text { Thoma }\end{array}$ & $\begin{array}{l}\text { Integration of } \\
\text { Renewable Energy } \\
\text { Resources through } \\
\text { Energy Storage and } \\
\text { SuperGrid }\end{array}$ & & $\mathrm{P}$ & & & & & & & & $\mathrm{S}$ & & \\
\hline 11-019 & Lister, Tedd & $\begin{array}{l}\text { Electrochemical } \\
\text { Production of Synthesis } \\
\text { Gas From CO2 }\end{array}$ & & $\mathrm{P}$ & & & & & & & & & & \\
\hline $11-022$ & Welker, Kurt & $\begin{array}{l}\text { Electronic Warfare } \\
\text { Digital } \\
\text { Communications and } \\
\text { Network Traffic } \\
\text { Modeling }\end{array}$ & & & & & & $P$ & & & & & & \\
\hline $11-023$ & $\begin{array}{l}\text { Martin, } \\
\text { Leigh }\end{array}$ & $\begin{array}{l}\text { Development of a } \\
\text { simplified soft donor } \\
\text { technique for trivalent } \\
\text { lanthanide actinide } \\
\text { separations }\end{array}$ & & & $\mathrm{S}$ & & $\mathrm{S}$ & $\mathrm{P}$ & & & $\mathrm{S}$ & & & S \\
\hline $11-031$ & $\begin{array}{l}\text { Ginosar, } \\
\text { Daniel }\end{array}$ & $\begin{array}{l}\text { Mechanisms of Self } \\
\text { Inhibition of Catalytic } \\
\text { Conversion of } \mathrm{CO} 2 \text { to } \\
\text { Methanol }\end{array}$ & & $\mathrm{P}$ & & $\mathrm{S}$ & $\mathrm{S}$ & & & & & & & \\
\hline $11-032$ & $\begin{array}{l}\text { Ogden, } \\
\text { Mark }\end{array}$ & $\begin{array}{l}\text { Ammonia-based } \\
\text { Extraction Methods for } \\
\text { the Separation and } \\
\text { Purification of Metals }\end{array}$ & $\mathrm{S}$ & $\mathrm{S}$ & $\mathrm{S}$ & & $\mathrm{S}$ & $\mathrm{S}$ & & & $\mathrm{S}$ & & & S \\
\hline $11-033$ & $\begin{array}{l}\text { Pepper, } \\
\text { Sarah }\end{array}$ & $\begin{array}{l}\text { Reduction and } \\
\text { Retention of } \\
\text { Technetium using } \\
\text { Fe(II) Hydroxides } \\
\end{array}$ & & & $\mathrm{S}$ & & $\mathrm{P}$ & $\mathrm{S}$ & & & $\mathrm{S}$ & & & \\
\hline
\end{tabular}




\section{Appendix B - Relevance to Major National Programs}

\begin{tabular}{|c|c|c|c|c|c|c|c|c|c|c|c|c|c|c|}
\hline $\begin{array}{l}\text { Project } \\
\text { ID }\end{array}$ & PI & Title & $\begin{array}{l}\mathbb{2} \\
\text { 台 } \\
\end{array}$ & 聓 & $\sum_{I I}$ & 聓 & 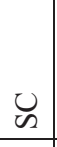 & 崩 & 号 & Z & $\sum_{\substack{\infty \\
0}}^{\substack{0 \\
0}}$ & 됭 & 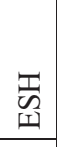 & $\sum_{J}$ \\
\hline $11-034$ & $\begin{array}{l}\text { Daniels, } \\
\text { Michael }\end{array}$ & $\begin{array}{l}\text { Thermal Burn } \\
\text { Characteristics in } \\
\text { Oxygen-less } \\
\text { Environment }\end{array}$ & & & & & $P$ & & & & & & & \\
\hline $11-036$ & $\begin{array}{l}\text { Wilson, } \\
\text { Aaron }\end{array}$ & $\begin{array}{l}\text { Kolbe Electrolysis of } \\
\text { Bio-Oils for the } \\
\text { Production of Diesel } \\
\text { and Aviation Fuels }\end{array}$ & & $\mathrm{P}$ & & & S & & & & & & & \\
\hline $11-037$ & $\begin{array}{l}\text { Schley, } \\
\text { Robert }\end{array}$ & $\begin{array}{l}\text { Development of In } \\
\text { Reactor Experiment } \\
\text { Configuration for Laser } \\
\text { Based Material } \\
\text { Properties } \\
\text { Measurement } \\
\end{array}$ & & & & & & $\mathrm{P}$ & & & & & & \\
\hline $11-038$ & Harris, Brian & $\begin{array}{l}\text { Dissolution, Extraction, } \\
\text { and Separation of Rare } \\
\text { Earth Elements using } \\
\text { Soft Donor Ligands in } \\
\text { Conjunction with Ionic } \\
\text { Liquids and } \\
\text { Supercritical Carbon } \\
\text { Dioxide }\end{array}$ & & $\mathrm{P}$ & & & & S & & & & & & \\
\hline $11-041$ & $\begin{array}{l}\text { Khafizov, } \\
\text { Marat }\end{array}$ & $\begin{array}{l}\text { In-situ Micro-Raman } \\
\text { Spectroscopy and } \\
\text { Modeling of } \\
\text { Breakaway Oxidation } \\
\text { ofZzircaloy Cladding }\end{array}$ & $\mathrm{S}$ & $\mathrm{S}$ & $\mathrm{S}$ & & S & $\mathrm{P}$ & S & & S & & $\mathrm{S}$ & \\
\hline $11-042$ & $\begin{array}{l}\text { McQueen, } \\
\text { Miles }\end{array}$ & $\begin{array}{l}\text { Experimental Cyber } \\
\text { Security Vulnerability } \\
\text { Prediction, Discovery } \\
\text { and Mitigation }\end{array}$ & & & & & S & $\mathrm{S}$ & & & & $P$ & & \\
\hline $11-043$ & $\begin{array}{l}\text { Meyer, } \\
\text { Mitchell }\end{array}$ & $\begin{array}{l}\text { Characterization of } \\
\text { Nanomechanical } \\
\text { Response of Material to } \\
\text { Determine Key } \\
\text { Variables in Stress } \\
\text { Corrosion Cracking }\end{array}$ & & & & & S & $P$ & S & & & & & \\
\hline $11-045$ & Huang, Hai & $\begin{array}{l}\text { Multiscale Coupled } \\
\text { Hydrogeophysics } \\
\text { Modeling - Advancing } \\
\text { Understanding of } \\
\text { Reactive Transport and } \\
\text { Effective Subsurface } \\
\text { Environmental } \\
\text { Monitoring }\end{array}$ & & & $\mathrm{P}$ & S & S & & & & & & & \\
\hline
\end{tabular}




\section{Appendix B - Relevance to Major National Programs}

\begin{tabular}{|c|c|c|c|c|c|c|c|c|c|c|c|c|c|c|}
\hline $\begin{array}{l}\text { Project } \\
\text { ID }\end{array}$ & PI & Title & $\begin{array}{l}\mathbb{Z} \\
Z \\
Z\end{array}$ & 됲 & $\sum_{I I}$ & 떧 & U & 㞱 & 寽 & Z & $\sum_{\substack{\infty \\
0}}$ & 띵 & $\begin{array}{c}\mathbb{v} \\
\text { 工I } \\
\end{array}$ & $\sum$ \\
\hline $11-046$ & $\begin{array}{l}\text { McQueen, } \\
\text { Miles }\end{array}$ & $\begin{array}{l}\text { Known Secure Sensor } \\
\text { Measurements for } \\
\text { Detecting Unauthorized } \\
\text { Process Manipulation } \\
\text { and Falsification of } \\
\text { State }\end{array}$ & & & & & $\mathrm{S}$ & $\mathrm{S}$ & & & & $\mathrm{P}$ & & \\
\hline $11-051$ & $\begin{array}{l}\text { Lessing, } \\
\text { Paul }\end{array}$ & $\begin{array}{l}\text { Alternative Processing } \\
\text { for Improved UO2 } \\
\text { Based Fuels }\end{array}$ & & & & & & $\mathrm{P}$ & & & & & & \\
\hline $11-053$ & $\begin{array}{l}\text { Kinlaw, } \\
\text { Matthew }\end{array}$ & $\begin{array}{l}\text { High Energy } \\
\text { Photofission Signatures } \\
\text { Study }\end{array}$ & $\mathrm{P}$ & & & & & & & & & & & \\
\hline $11-054$ & $\begin{array}{l}\text { Kinlaw, } \\
\text { Matthew }\end{array}$ & $\begin{array}{l}\text { Treaty Verification } \\
\text { with Photofission- } \\
\text { Induced Prompt } \\
\text { Signatures } \\
\end{array}$ & $\mathrm{P}$ & & & & & & & & & & & \\
\hline $11-055$ & $\begin{array}{l}\text { Akers, } \\
\text { Douglas }\end{array}$ & $\begin{array}{l}\text { Spatially Resolved } \\
\text { Positron Analysis of } \\
\text { Hydride Formation in } \\
\text { LWR Fuels with GE - } \\
\text { Gloval Nuclear Nuclear } \\
\text { Fuels }\end{array}$ & & & & & & $\mathrm{P}$ & & & $\mathrm{S}$ & & & \\
\hline $11-059$ & $\begin{array}{l}\text { Chichester, } \\
\text { David }\end{array}$ & $\begin{array}{l}\text { Unconventional } \\
\text { Radiation Detection } \\
\text { Methods for Nuclear } \\
\text { Nonproliferation, } \\
\text { Safeguards and Treaty } \\
\text { Verification }\end{array}$ & $\mathrm{P}$ & & & & $\mathrm{S}$ & $\mathrm{S}$ & & & & & & \\
\hline $11-060$ & $\begin{array}{l}\text { Thomas } \\
\text { Zagula }\end{array}$ & $\begin{array}{l}\text { SMC Advanced Armor } \\
\text { Materials and Systems } \\
\text { R\&D and D\&D }\end{array}$ & $\mathrm{S}$ & & & & $\mathrm{S}$ & $\mathrm{S}$ & & & & & & \\
\hline $11-061$ & $\begin{array}{l}\text { Martineau, } \\
\text { Richard }\end{array}$ & $\begin{array}{l}\text { Advanced Modeling } \\
\text { and Simulation } \\
\text { Concepts for ATR }\end{array}$ & & & & & & $\mathrm{P}$ & S & & & & & \\
\hline $11-062$ & $\begin{array}{l}\text { Hugo, } \\
\text { Jacques }\end{array}$ & $\begin{array}{l}\text { Full-Scope Human } \\
\text { System Simulation } \\
\text { Laboratory for Plant- } \\
\text { Specific and Generic } \\
\text { NPP Simulator Models } \\
\text { Studies } \\
\end{array}$ & & & & & $\mathrm{S}$ & $\mathrm{P}$ & & & & & & \\
\hline $11-064$ & $\begin{array}{l}\text { Grauer, } \\
\text { Diana }\end{array}$ & $\begin{array}{l}\text { Dynamic Thermo- } \\
\text { Mechanical Energy } \\
\text { Conversion Simulation }\end{array}$ & & $\mathrm{P}$ & & & $\mathrm{S}$ & & & & $\mathrm{S}$ & & & \\
\hline $11-065$ & $\begin{array}{l}\text { Gertman, } \\
\text { David }\end{array}$ & $\begin{array}{l}\text { Development and } \\
\text { Demonstration for } \\
\text { National Fuel } \\
\text { Efficiency }\end{array}$ & & S & & $\mathrm{S}$ & & & & & & & $\mathrm{P}$ & \\
\hline
\end{tabular}




\section{Appendix B - Relevance to Major National Programs}

\begin{tabular}{|c|c|c|c|c|c|c|c|c|c|c|c|c|c|c|}
\hline $\begin{array}{l}\text { Project } \\
\text { ID }\end{array}$ & PI & Title & $\begin{array}{l}\mathbb{4} \\
\text { Z } \\
\text { Z }\end{array}$ & 띺 & $\sum_{I I}$ & 덛 & U & 崩 & 总 & Z & $\sum_{\substack{\infty \\
0}}$ & 뒹 & 壳 & $\sum_{S}$ \\
\hline $11-066$ & $\begin{array}{l}\text { Rieger, } \\
\text { Craig }\end{array}$ & $\begin{array}{l}\text { Targeted Energy } \\
\text { Management Toolset } \\
\text { for Comfort and } \\
\text { Savings Based on } \\
\text { Advanced } \\
\text { Computational } \\
\text { Intelligence Techniques }\end{array}$ & & $\mathrm{P}$ & & $\mathrm{S}$ & & & & & & $\mathrm{S}$ & & \\
\hline $11-067$ & West, James & $\begin{array}{l}\text { Pyro Processing } \\
\text { Facility (FCF) Nuclear } \\
\text { Material and } \\
\text { Environmental } \\
\text { Signature Baseliine for } \\
\text { MCNP Modeling } \\
\text { Verification }\end{array}$ & $\mathrm{P}$ & & & & & $\mathrm{S}$ & & & & & & \\
\hline $11-068$ & Kelly, Dana & $\begin{array}{l}\text { Science Based } \\
\text { Simulation Model of } \\
\text { Human Performance } \\
\text { for Human Reliability } \\
\text { Analysis }\end{array}$ & & & & & & $\mathrm{P}$ & $\mathrm{S}$ & & & & & \\
\hline $11-069$ & $\begin{array}{l}\text { Gasper, } \\
\text { Peter }\end{array}$ & $\begin{array}{l}\text { Industrial Control } \\
\text { System Threat Axis } \\
\text { Analysis to } \\
\text { Characterize and } \\
\text { Quantify Malicious } \\
\text { Intent from a Threat } \\
\text { Actor }\end{array}$ & $\mathrm{S}$ & & & & & & & & & $\mathrm{P}$ & & \\
\hline 11-074 & $\begin{array}{l}\text { Pernice, } \\
\text { Michael }\end{array}$ & $\begin{array}{l}\text { Uncertainty Analysis } \\
\text { for Multiscale Models } \\
\text { of Nuclear Fuel } \\
\text { Performance }\end{array}$ & $\mathrm{S}$ & & $\mathrm{S}$ & $\mathrm{S}$ & $\mathrm{S}$ & $\mathrm{P}$ & & & & & & \\
\hline $11-075$ & $\begin{array}{l}\text { Pernice, } \\
\text { Michael }\end{array}$ & $\begin{array}{l}\text { Uncertainty Analysis } \\
\text { for Mesoscale Models } \\
\text { of Radiation Effects in } \\
\text { Materials }\end{array}$ & $\mathrm{S}$ & & & & $\mathrm{S}$ & $\mathrm{P}$ & $\mathrm{S}$ & & & & & \\
\hline $11-076$ & $\begin{array}{l}\text { Pernice, } \\
\text { Michael }\end{array}$ & $\begin{array}{l}\text { Exploratory Nuclear } \\
\text { Reactor Safety Analysis } \\
\text { and Visualization via } \\
\text { Integrated Topological } \\
\text { and Geometric } \\
\text { Technqiues }\end{array}$ & $\mathrm{S}$ & & & $\mathrm{S}$ & $\mathrm{S}$ & $\mathrm{P}$ & & & & & & \\
\hline $11-077$ & $\begin{array}{l}\text { Hruska, } \\
\text { Ryan }\end{array}$ & $\begin{array}{l}\text { Prototype INL } \\
\text { Wildland Fire Early } \\
\text { Warning and } \\
\text { Situational Awareness } \\
\text { System }\end{array}$ & & & $\mathrm{S}$ & & $\mathrm{S}$ & $\mathrm{P}$ & & & & & $\mathrm{S}$ & \\
\hline
\end{tabular}





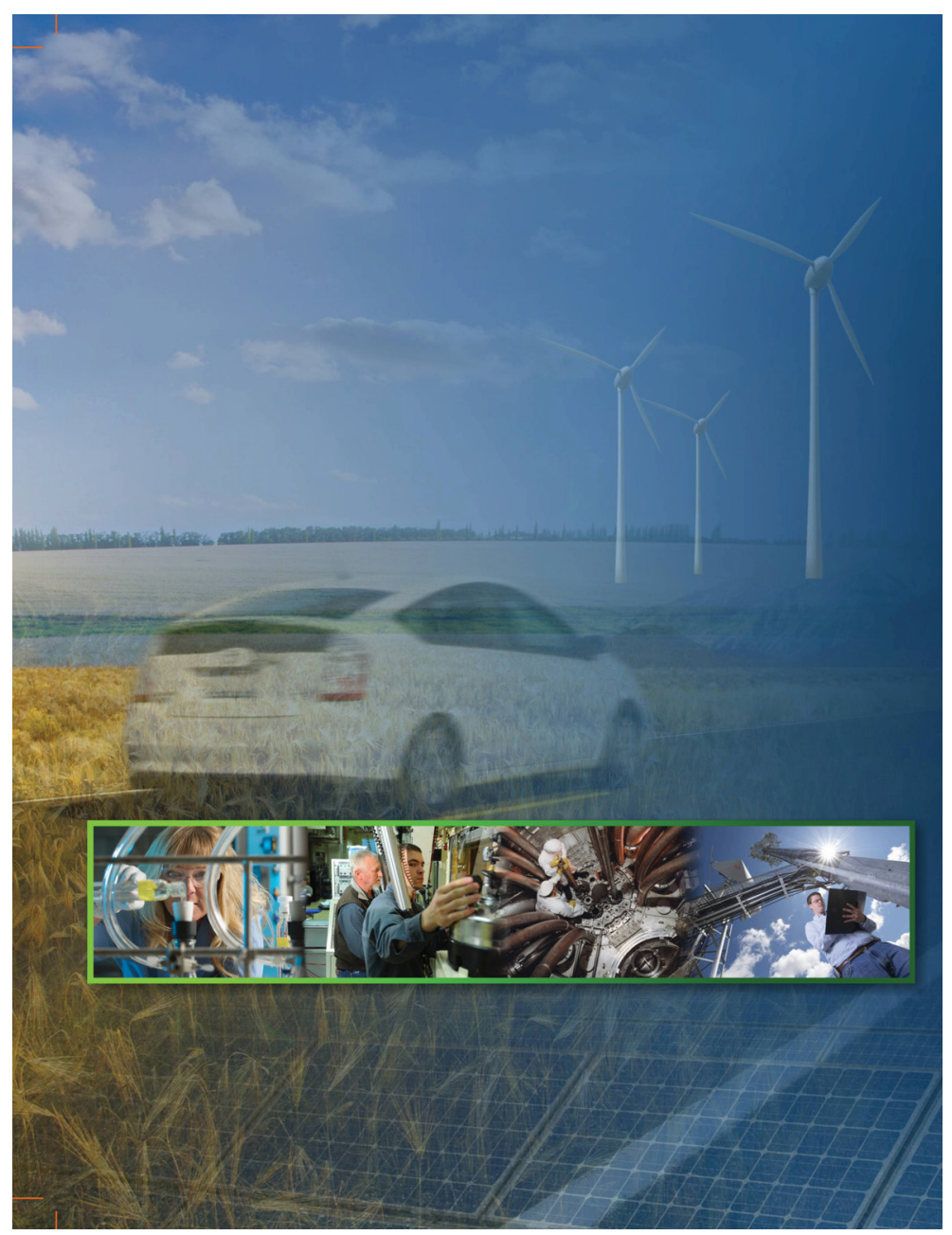

